\section{Pacific Northwest}

National Laboratory

Operated by Battelle for the

U.S. Department of Energy

\title{
Borehole Gravity Meter Surveys at the Waste Treatment Plant, Hanford, Washington
}

J. D. MacQueen

E. Mann

March 2007

Prepared by Microg-LaCoste

for the Pacific Northwest National Laboratory under Contract DE-AC05-76RL01830

with the U.S. Department of Energy 


\section{Borehole Gravity Meter Surveys at the Waste Treatment Plant, Hanford, Washington}
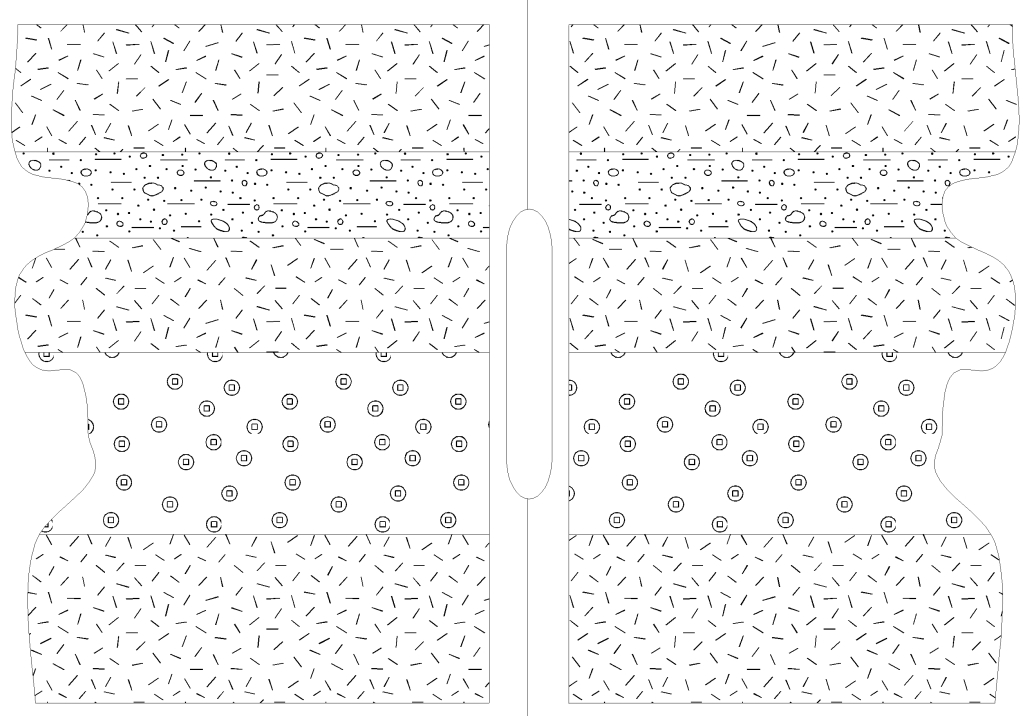

Report MGL-2007-001

Jeffrey D. MacQueen

Ethan Mann

Microg-LaCoste

March 30, 2007

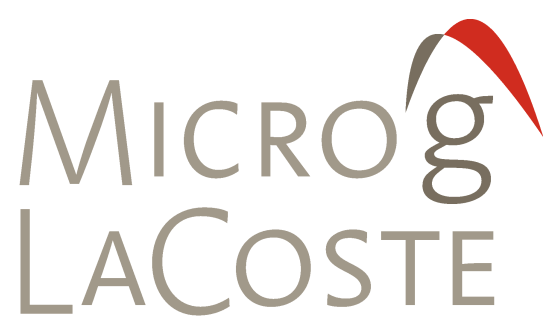

A DIVISION OF LRS 


\title{
DISCLAIMER
}

This report was prepared as an account of work sponsored by an agency of the United States

Government. Neither the United States Government nor any agency thereof, nor Battelle Memorial Institute, nor any of their employees, makes any warranty, express or implied, or assumes any legal liability or responsibility for the accuracy, completeness, or usefulness of any information, apparatus, product, or process disclosed, or represents that its use would not infringe privately owned rights. Reference herein to any specific commercial product, process, or service by trade name, trademark, manufacturer, or otherwise does not necessarily constitute or imply its endorsement, recommendation, or favoring by the United States Government or any agency thereof, or Battelle Memorial Institute. The views and opinions of authors expressed herein do not necessarily state or reflect those of the United States Government or any agency thereof.

\author{
PACIFIC NORTHWEST NATIONAL LABORATORY \\ operated by \\ BATTELLE \\ for the \\ UNITED STATES DEPARTMENT OF ENERGY \\ under Contract DE-AC05-76RL01830
}

Printed in the United States of America
Available to DOE and DOE contractors from the
Office of Scientific and Technical Information,
P.O. Box 62, Oak Ridge, TN 37831-0062;
ph: (865) 576-8401
fax: $(865) 576-5728$
email: mailto:reports@adonis.osti.gov

\author{
Available to the public from the National Technical Information Service, \\ U.S. Department of Commerce, 5285 Port Royal Rd., Springfield, VA 22161 \\ ph: (800) 553-6847 \\ fax: (703) 605-6900 \\ email: orders@ntis.fedworld.gov \\ online ordering: http://www .ntis.gov/ordering.htm
}




\section{Contents}

\begin{tabular}{lll}
\hline 1 & Survey Overview & 1
\end{tabular}

$\begin{array}{lll}2 & \text { Data processing } & \mathbf{1}\end{array}$

2.1 Data editing and reading corrections . . . . . . . . . . . . . . . . . 1

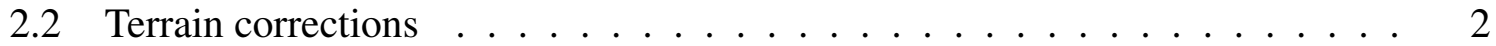

3 The Microg-LaCoste Borehole Gravity Inversion Method 6

4 Density inversion results 11

$4.1 \mathrm{C} 4993 \ldots \ldots \ldots \ldots \ldots \ldots$

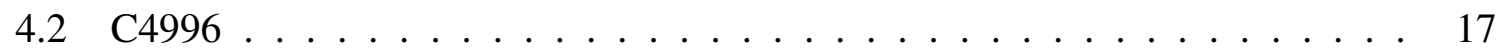

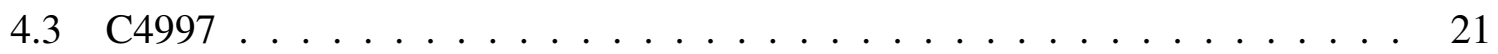

4.4 Well-to-well correlation . . . . . . . . . . . . . . . . . 25

\begin{tabular}{lr}
\hline References & 26
\end{tabular}

\begin{tabular}{|l|l}
\hline Appendices & 27
\end{tabular}

\begin{tabular}{|l|l}
\hline Appendix: A & Processed gravity data
\end{tabular}

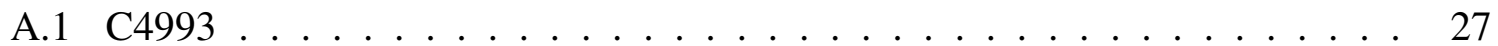

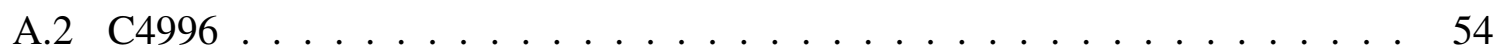

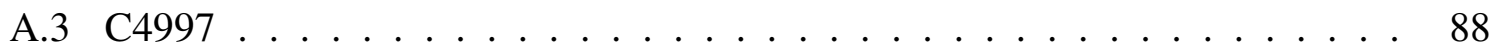

\begin{tabular}{lll}
\hline Appendix: B Inversion density tables & 115
\end{tabular}

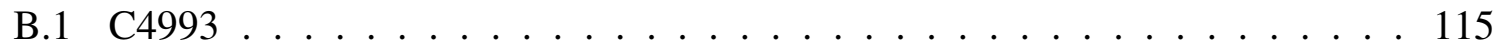

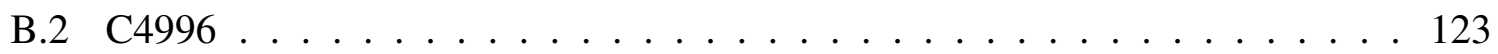

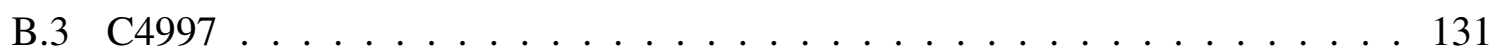

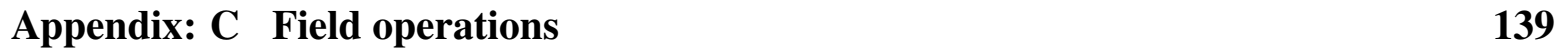

C.1 Equipment . . . . . . . . . . . . . . . . . . . . . . . . . . . . . . . . . . . . . . . . . . . . . . .

C.2 Mobilization . . . . . . . . . . . . . . . . . . . 139

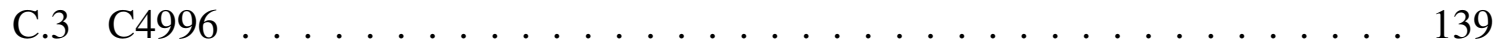

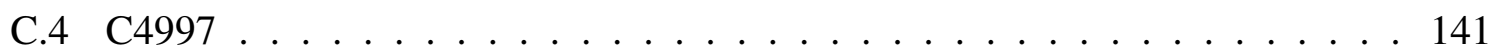

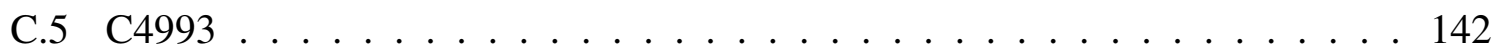

C.6 De-mobilization . . . . . . . . . . . . . . . . . . . . . . 142

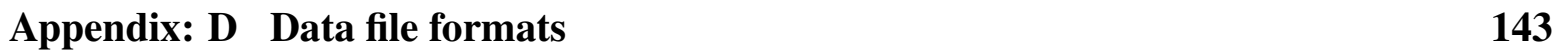

D.1 .dat file format . . . . . . . . . . . . . . . . . . . . . . . . . . . . . . . . . . . . . . . . . . .

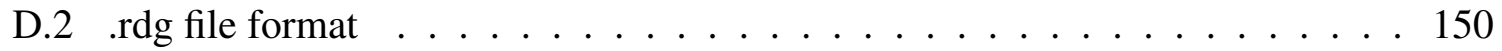

\begin{tabular}{|ll|}
\hline Appendix: E & Calibration and test procedures \\
\hline
\end{tabular}

\begin{tabular}{|ll|}
\hline Appendix: F & Gravity acquisition procedure \\
\hline
\end{tabular}

\begin{tabular}{|ll|}
\hline Appendix: G Non-Conformance reports (scanned) & 157
\end{tabular} 


\section{List of Figures}

$1 \quad$ First editing pass (x-axis is depth, $\mathrm{y}$-axis is BHGM gravity in milliGals) . . . 3

$2 \quad$ Second editing pass ( $\mathrm{x}$-axis is depth, $\mathrm{y}$-axis is BHGM gravity in milliGals) . . 4

$3 \quad$ Well C4993: terrain corrections . . . . . . . . . . . . . 7

4 Well C4996: terrain corrections . . . . . . . . . . . . . . . . . . 8

5 Well C4997: terrain corrections . . . . . . . . . . . . . . . . . . . 9

$6 \quad$ Near zone terrain . . . . . . . . . . . . . . . . . . . . . . 10

7 Well C4993: BHGM inversion results . . . . . . . . . . . . . . 13

$8 \quad$ Well C4993: comparison of BHGM and $\gamma-\gamma$ densities . . . . . . . . . . . 14

$9 \quad$ Well C4993: comparison of BHGM and blocked $\gamma-\gamma$ densities . . . . . . . 15

10 Well C4993: Z-score distribution of inversion fit . . . . . . . . . . . . . . . 16

11 Well C4996: BHGM inversion results . . . . . . . . . . . . . . . . 17

$12 \quad$ Well C4996: comparison of BHGM and $\gamma-\gamma$ densities . . . . . . . . . . . 18

13 Well C4996: comparison of BHGM and blocked $\gamma-\gamma$ densities . . . . . . . 19

14 Well C4996: Z-score distribution of inversion fit . . . . . . . . . . . . . . 20

15 Well C4997: BHGM inversion results . . . . . . . . . . . . . . . . 21

$16 \quad$ Well C4997: comparison of BHGM and $\gamma-\gamma$ densities . . . . . . . . . . . . 22

17 Well C4997: comparison of BHGM and blocked $\gamma-\gamma$ densities . . . . . . . 23

18 Well C4997: Z-score distribution of inversion fit . . . . . . . . . . . . . . . . 24

19 Well-to-well correlations . . . . . . . . . . . . . . . . 25

\section{List of Tables}

$1 \quad$ Acquisition statistics $\ldots \ldots \ldots \ldots \ldots$. . . . . . . . . . . . . . . . . . . . . . . . . . . . .

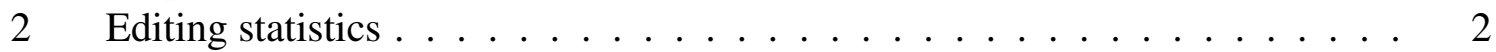

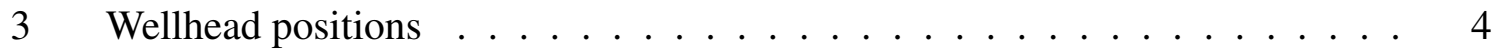

$4 \quad$ Well C4993 Processed gravity . . . . . . . . . . . . . . . . . . . . . . . . . . . . . . . . . . .

5 Well C4996 Processed gravity . . . . . . . . . . . . . . . . . . 55

$6 \quad$ Well C4997 Processed gravity . . . . . . . . . . . . . . . . . . . . . . . 89

$7 \quad$ Well C4993 Inversion densities . . . . . . . . . . . . . . . . . . . . . 115

$8 \quad$ Well C4996 Inversion densities . . . . . . . . . . . . . . . . . . . 123

$9 \quad$ Well C4997 Inversion densities . . . . . . . . . . . . . . . . . . . . 131

10 BHGM \#14 Screw K-factors . . . . . . . . . . . . . . . . . . . . . 155 


\section{Survey Overview}

Microg-LaCoste (MGL) was contracted by Pacfic Northwest National Laboratory (PNNL) to record borehole gravity density data in 3 wells at the Hanford Waste Treatment Plant (WTP) site. The survey was designed to provide highly accurate density information for use in seismic modeling (see McCulloh [1966] and Beyer [1983] for an introduction to borehole gravity surveying). The borehole gravity meter (BHGM) tool has a very large depth of investigation (hundreds of feet) compared to other density tools so it is not influenced by casing or near welbore effects, such as washouts. The survey goal was to provide rock densities with an estimated error of $\pm 0.05 \mathrm{~g} / \mathrm{cm}^{3}$ or less for use in the seismic modeling. The final survey design used 10 foot station intervals and depth control by surface wireline odometer. Applying inversion techniques to the BHGM data, it proved possible to calculate densities with estimated errors of less than $\pm 0.02 \mathrm{~g} / \mathrm{cm}^{3}$ over almost all intervals, which significantly exceeded the survey goal.

This survey probably constitutes the largest BHGM survey ever done. The largest number of readings ever recorded before this in a single BHGM survey was about 450 readings, to the best of our knowledge. This survey almost undoubtably contains the longest section (approx. 4200 feet in the three wells) of BHGM data taken at such a small station spacing (10 feet).

\begin{tabular}{|r|r|r|r|}
\hline Well name & $\begin{array}{r}\text { number of } \\
\text { gravity readings }\end{array}$ & $\begin{array}{r}\text { Hours spent } \\
\text { in well }\end{array}$ & $\begin{array}{r}\text { Average gravity station } \\
\text { accuracy (mGals) }\end{array}$ \\
\hline C4993 & 741 & 115 & 0.008 \\
\hline C4996 & 1006 & 214 & 0.015 \\
\hline C4997 & 747 & 137 & 0.008 \\
\hline
\end{tabular}

Table 1: Acquisition statistics

These stations were acquired from Nov. 11th, 2006 to Dec. 10th, 2006. Details of field operations are included in Appendix $\mathrm{C}$.

\section{Data processing}

A good general introduction to BHGM data processing can be found in Beyer [1983].

\subsection{Data editing and reading corrections}

Processing of the BHGM data, after the in-field acquisition and processing described in Appendices D.1, D.2, and F, consisted of four steps:

1. The field data was loaded into a binary file, with first-order checking applied (rejecting values obviously out of range).

2. The data was then plotted as density versus depth, and inspected visually, with obvious large outliers (the red x's in figure 1) removed. 
3. The density-depth plot was zoomed in to cover a few stations at a time. Smaller outliers, which were not obvious at the scale of the whole well, were visually detected and removed (figure 2).

4. The edited data was processed to remove linear time drifts. For the purpose of drift removal, any time gap in the acquisition greater than two hours started a new linear drift segment. The drift segments were determined by least-squares, to minimize the scatter of the gravity readings at each depth.

The steps listed above used a set of MGL-developed scripts written in the MATLAB (C) The Mathworks, Inc.) programming language. These scripts were tested by run-time inspection within MATLAB, and by tests with synthetic data where appropriate.

Table 2 shows the number of original field records (Raw), the number which passed the first-order loading checks (see processing step 1) (Records read), the number which passed the visual inspections (see processing steps 2 and 3 ) (Accepted records), and the percentage of the original data accepted for further processing.

\begin{tabular}{|r|r|r|r|r|}
\hline Well & Raw records & Records read & Accepted records & Final percentage \\
\hline \hline C4993 & 741 & 740 & 721 & $97.3 \%$ \\
\hline C4996 & 1006 & 1003 & 904 & $89.9 \%$ \\
\hline C4997 & 747 & 729 & 700 & $93.7 \%$ \\
\hline
\end{tabular}

Table 2: Editing statistics

The field data showed small depth deviations (usually on the order of \pm 0.05 feet) around the nominal station depths. A depth correction is often applied to bring all of the stations to their nominal depths. Application of depth corrections made essentially no difference in the station repeat statistics, so these were not done. This indicates that the actual error in depth measurements was of the order of the nominal error.

MGL calculated corrections for circular error (systematic errors in the gravity reading as a function of the screw position, caused by small mechanical imperfections) and residual tide errors (small errors due to imperfect calculation of the true local earth tides). These errors proved to be quite small, generally $<0.001 \mathrm{mGal}$, and made no significant difference to the repeatability of the final gravity data, so they have not been included in this report.

\subsection{Terrain corrections}

An underlying assumption in the process of calculating density from the gravity values measured in the boreholes is that the surrounding earth is composed of homogeneous flat layers. One obvious departure from this assumption is the earth air interface. Terrain corrections are made to correct for the departures of the surrounding land surface from a level plane passing through ground level at the well head.

Terrain correction methods adopted by the geophysical community for surface gravity surveys generally follow a scheme originally presented by Hayford and Bowie [1912]. This methods divide the land surface into a series of rings centered on the gravity station position. 


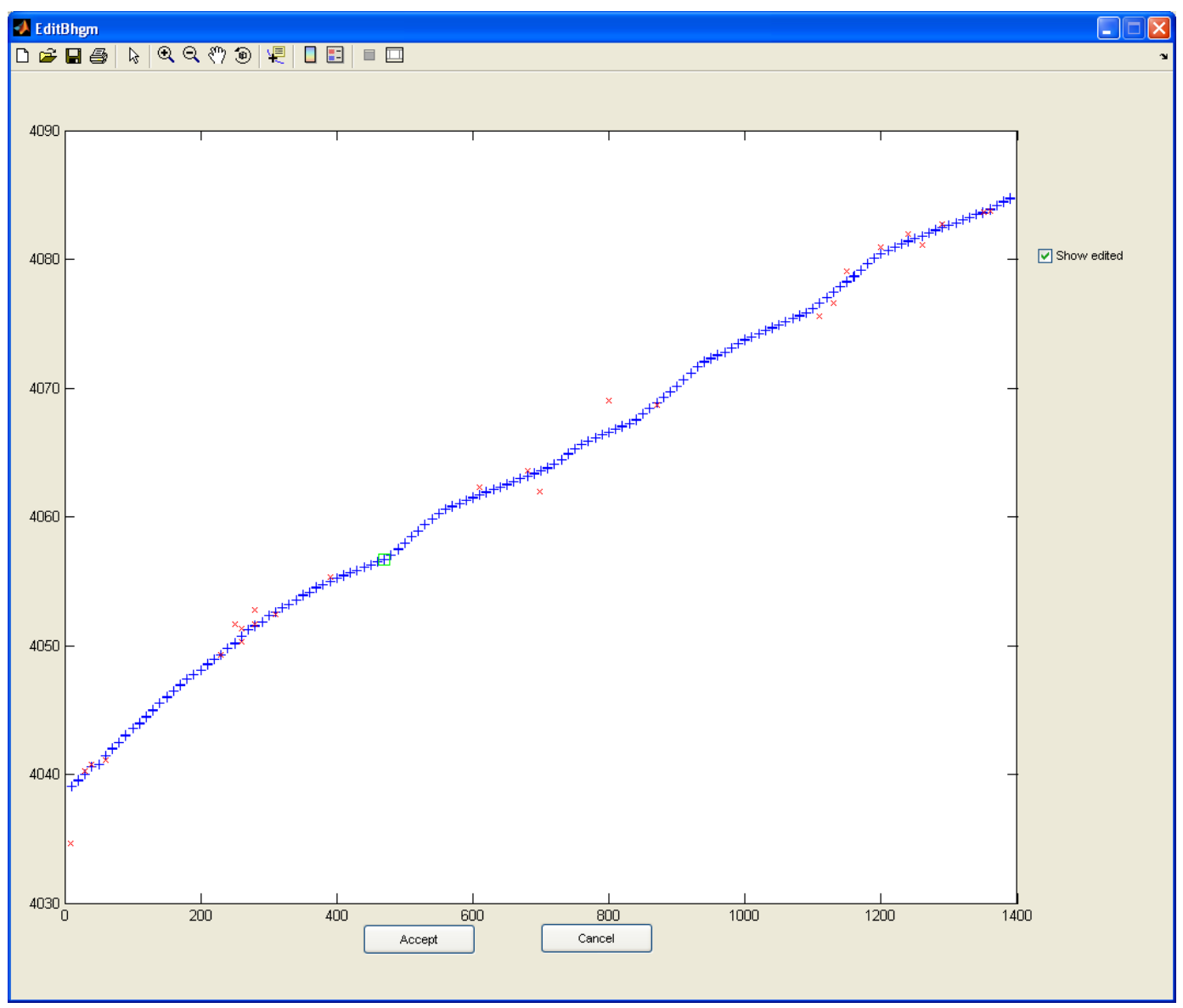

Figure 1: First editing pass (x-axis is depth, $\mathrm{y}$-axis is BHGM gravity in milliGals)

Each of these rings is further divided into equal sized compartments bounded by radials from the gravity station position and assigned an elevation. This arrangement is convenient because the gravitational attraction of the compartments can be calculated exactly using the equation

$g_{i}=\frac{2 \pi G \rho}{N}\left(\sqrt{r_{1}^{2}+(h-\Delta h)^{2}}-\sqrt{r_{1}^{2}+\left(h_{1}-\Delta h\right)^{2}}-\sqrt{r_{2}^{2}+(h-\Delta h)^{2}}+\sqrt{r_{2}^{2}+\left(h_{1}-\Delta h\right)^{2}}\right)$

where $G$ is the gravitational constant, $r$ is the average density of the rocks enclosed by the compartment, called Terrain Density, $r_{1}$ is the inner radius distance of the zone measured from the well, $r_{2}$ is the outer radius distance of the zone measured from the well, $h$ is the difference between the station elevation and the compartment elevation, $h_{1}$ is the difference between the station elevation and ground level, and $\Delta h$ is the correction for the earth's curvature given by

$$
\Delta h=\frac{d^{2}}{2 R}
$$

where $d$ is the mean radial distance of the zone and $R$ is the mean radius of the earth.

The near zone terrain corrections (closer than 1280 meters to the wellhead) were calculated using the method of Krohn [1976] in order to fully use the detailed near-well topographic survey data. 


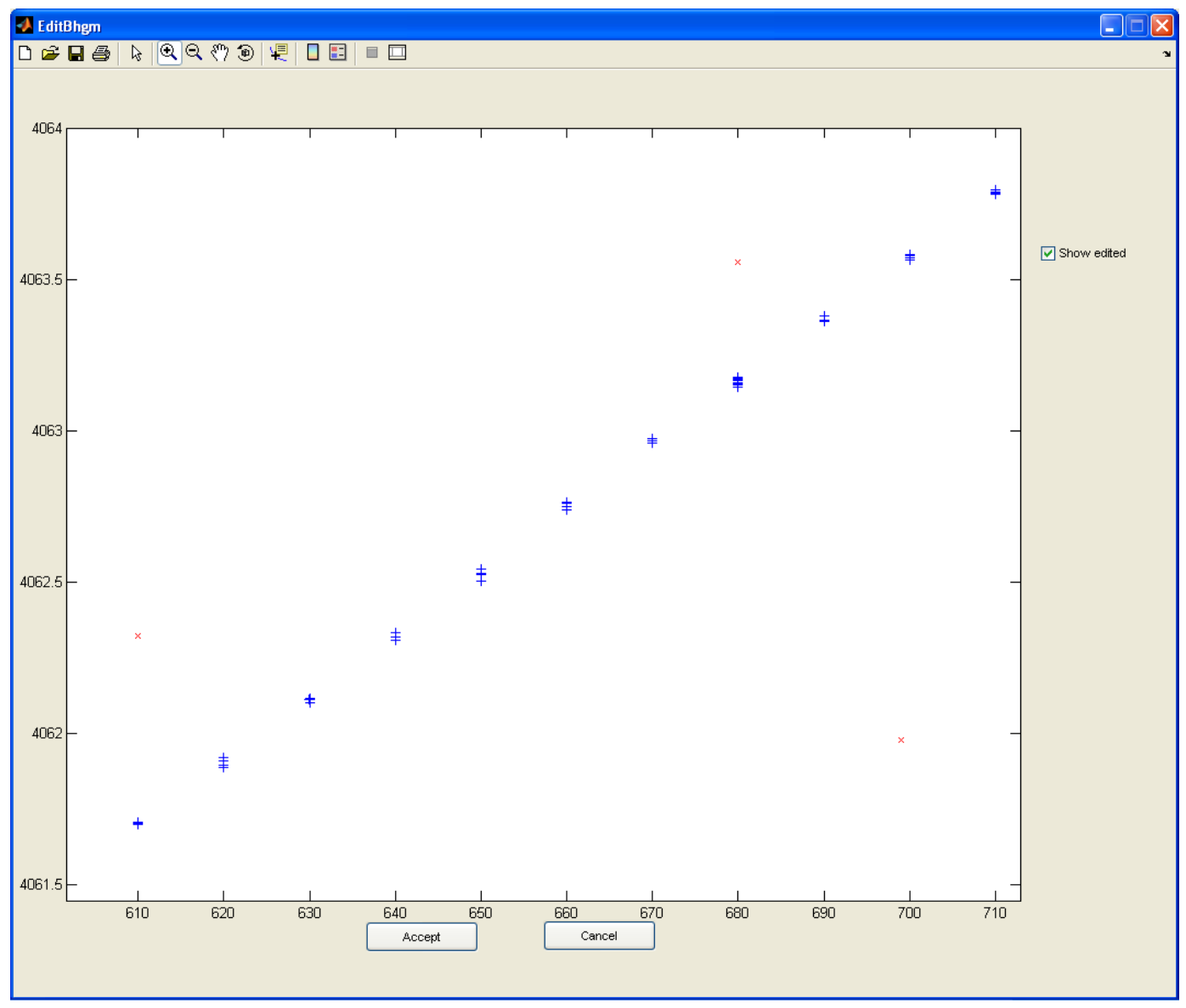

Figure 2: Second editing pass (x-axis is depth, y-axis is BHGM gravity in milliGals)

Three sets of digital elevation data (DEM's) were used. The far zone terrain data was obtained from the w140n90 Gtopo30 data file downloaded from the USGS seamless web site, http://seamless.usgs.gov/. Gtopo30 data is gridded at 30 arc seconds or approximately 1 kilometer, the horizontal datum is WGS84 and the elevations are referenced to sea level. The original data file positions use units of latitude, longitude and meters. We projected this data to UTM Zone 11 North using the NAD83 horizontal datum with all units in meters. The positions of the three wells using the NADV88 vertical datum are given in table 3

\begin{tabular}{|r|r|r|r|}
\hline Well & Easting & Northing & Height \\
\hline \hline C4993 & $307755.440 \mathrm{~m}$ & $5158260.448 \mathrm{~m}$ & $200.499 \mathrm{~m}$ \\
\hline $\mathrm{C} 4996$ & $307826.310 \mathrm{~m}$ & $5158555.282 \mathrm{~m}$ & $204.079 \mathrm{~m}$ \\
\hline $\mathrm{C} 4997$ & $307977.327 \mathrm{~m}$ & $5158249.429 \mathrm{~m}$ & $206.406 \mathrm{~m}$ \\
\hline
\end{tabular}

Table 3: Wellhead positions

Hanford personnel provided the data used for the near and intermediate zones. All Hanford data was projected to UTM zone 11 North, with NAD83 horizontal datum and NAVD88 
vertical datum. All horizontal and vertical units are meters.

The intermediate zone DEM file, Hanford_dem_10m_utm83_navd88.asc, is gridded at $10 \mathrm{~m}$ intervals and is contained in the following rectangle:

$$
\begin{array}{lr}
\text { Western-most easting: } & 261508.7 \mathrm{~m} \\
\text { Eastern-most easting: } & 348847.8 \mathrm{~m} \\
\text { Northen-most northing: } & 5210501.7 \mathrm{~m} \\
\text { Southern-most northing: } & 5210501.7 \mathrm{~m}
\end{array}
$$

The Gtopo30 data was truncated at an outer radius of 166700 meters and an inner rectangle corresponding to the outer dimensions of the Hanford 10m DEM file. The combined Gtopo30 and Hanford 10m DEM data sets was used to generate the compartment elevations for the Hayford-Bowie scheme from an inner radius of 1280 meters to 166700 meters from each well position.

Hanford personnel supplied two files for the near zone terrain corrections; wtpdem_gnd_utm11.xyz and wtpdem_bld_utm11.xyz. Both files contained current ground elevations from GPS survey work around the WTP site. One file, wtpdem_bld_utm11.xyz, included the elevations of the floors of the basements of the WTP buildings and the vaults in the pit surrounding the C4993 well. This data file was used for the near zone terrain corrections from the well site positions to a radius of 1280 meters.

The wtpdem_bld_utm11.xyz file is gridded at 0.6 meter intervals and is contained in the following rectangle:

$$
\begin{array}{lr}
\text { Western-most easting: } & 306149.0 \mathrm{~m} \\
\text { Eastern-most easting: } & 309745.7 \mathrm{~m} \\
\text { Northen-most northing: } & 5159672.7 \mathrm{~m} \\
\text { Southern-most northing: } & 5156825.2 \mathrm{~m}
\end{array}
$$

To fully utilize the near terrain data that included the basement and vault details, the number of rings and compartments was increased dramatically from the original HayfordBowie scheme.

The terrain correction compartment elevations for each well are contained in the files

C4993_BHGM_terrain_elevations.txt

C4996_BHGM_terrain_elevations.txt

C4997_BHGM_terrain_elevations.txt

The most frequently used terrain correction density is $2.67 \mathrm{~g} / \mathrm{cm}^{3}$. This has been used as the terrain density for the intermediate and far zones terrain but the terrain immediately surrounding the wells is definitely of lower density. For example, well C4993 is within a large pit completely within alluvial deposits. The density of these deposits is close to $2.0 \mathrm{~g} / \mathrm{cm}^{3}$, as shown by the BHGM data. The deviations from the well head elevations within a radius of 1280 meters are probably all contained within alluvium above the shallowest basalt layers. Therefore, $2.0 \mathrm{~g} / \mathrm{cm}^{3}$ is a reasonable density to use for the near zone out to 1280 meters from each well. 
The software used for the terrain corrections (and other processing tasks) is derived from the quality controlled software used by EDCON (Exploration Data Consultants, Inc.) for two Borehole Gravity surveys associated with the Yucca Mountain project in Nevada.

The first of these surveys was conducted on September 22 and 23, 2000 in the NC-EWDP19D well in Nye County Nevada approximately 90 miles North West of Las Vegas. The survey was performed under Purchase Order Number A17368GJ0A of TRW Environmental Safety System Inc. of 1261 Town Center Dr., Las Vegas, NV 89134.

The second survey was conducted on February 25 and 26, 2002 in the NC-EWDP-22S well in Nye County Nevada approximately 90 miles North West of Las Vegas. The survey was performed under Technical Services Agreement TA001821 on behalf of Bechtel SAIC Company, LLC of 1180 Town Center Dr., Las Vegas, NV 89144.

The original software was called R7.EXE and ran on the Microsoft DOS operating system. The same code was used in the software program called R8.EXE used with the Hanford WTP Borehole Gravity surveys except the user interface was adapted so the software would run on Microsoft Windows XP operating system.

The terrain corrections for each well have been converted to density corrections, and are plotted in figures 3, 4, and 5. Well C4997 shows the effect, in the shallowest part of the well, of the proximity of the large basement excavations. The near-zone corrections for well C4993 show the effect of the well location in the center of a large and deep excavation. These relationships between close near-surface topography and the density corrections can be understood from the near-zone topography map in figure 6 .

\section{The Microg-LaCoste Borehole Gravity Inversion Method}

The recently-developed Microg-LaCoste BHGM inversion method, based on work originally presented in MacQueen [1989], allows stable calculation of interval densities over much closer station spacings than are feasible using the traditional method. The damped leastsquares techniques [Aster et al., 2005] used in the inversion stabilize the density calculations in three ways:

1. The observed gravity data, which we know contain some amount of error, are not fit exactly, but only to within a tolerance determined by the noise level of the data.

2. The inherent redundancy of borehole gravity data further stabilizes the calculations. Each interval density influences the measured value at all the borehole gravity stations, so that each gravity value helps to constrain all the interval densities in the logged interval. The rocks in a given interval will increase the gravity for all stations above the interval, and decrease the gravity for all stations below the interval. The inversion algorithm uses all the gravity data to help estimate the density in a given interval, not just the data from the two stations immediately above and below the interval.

An example might make this redundancy more obvious. Assume we have eleven stations at 10 foot intervals, spanning 100 feet of the well, with four repeats at each station (44 measurements). The traditional method of calculating densities would use the four gravity measurements immediately above and below each interval, which gives 16 differences to determine the interval density. Statistical analysis ot the correlation induced 


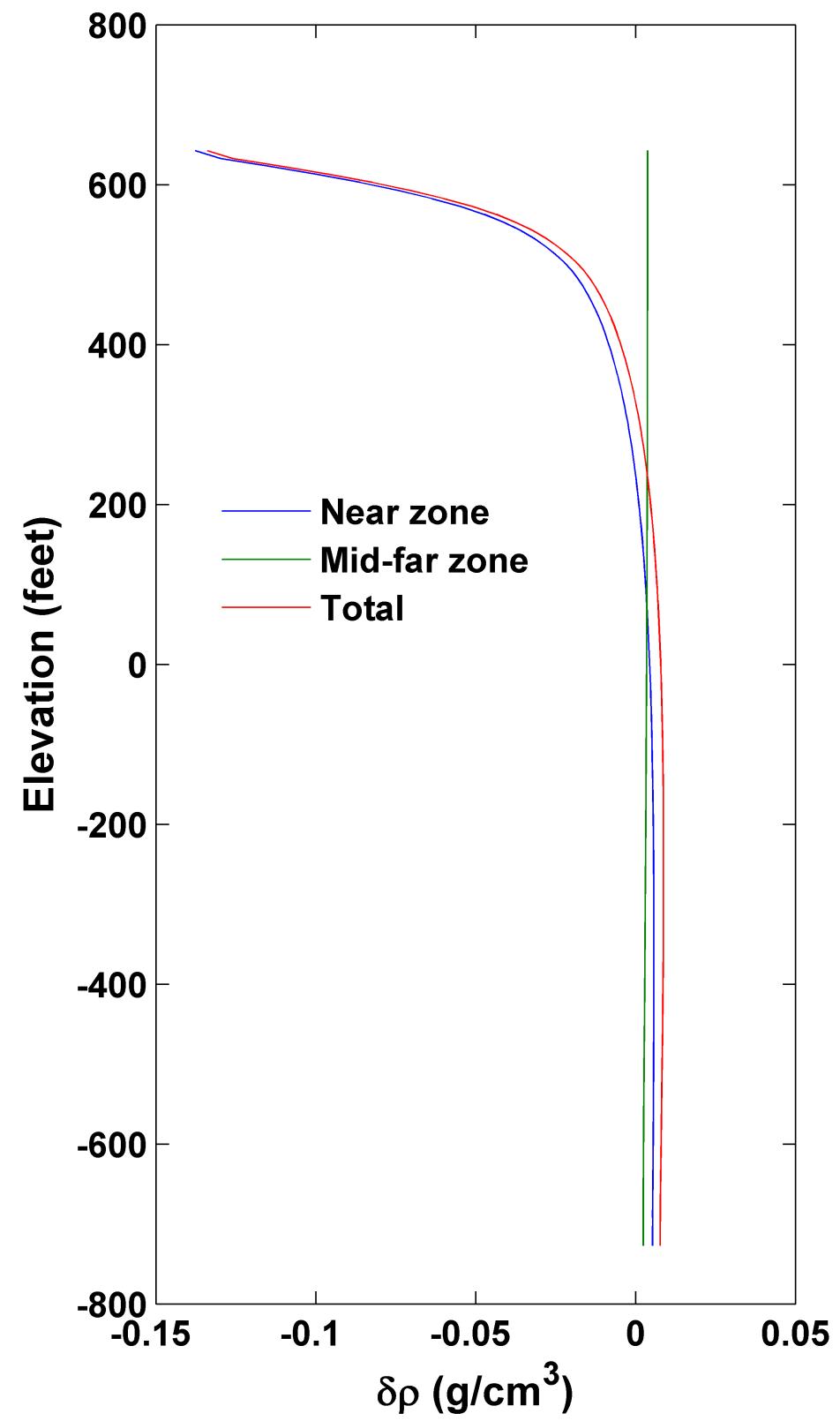

Figure 3: Well C4993: terrain corrections 


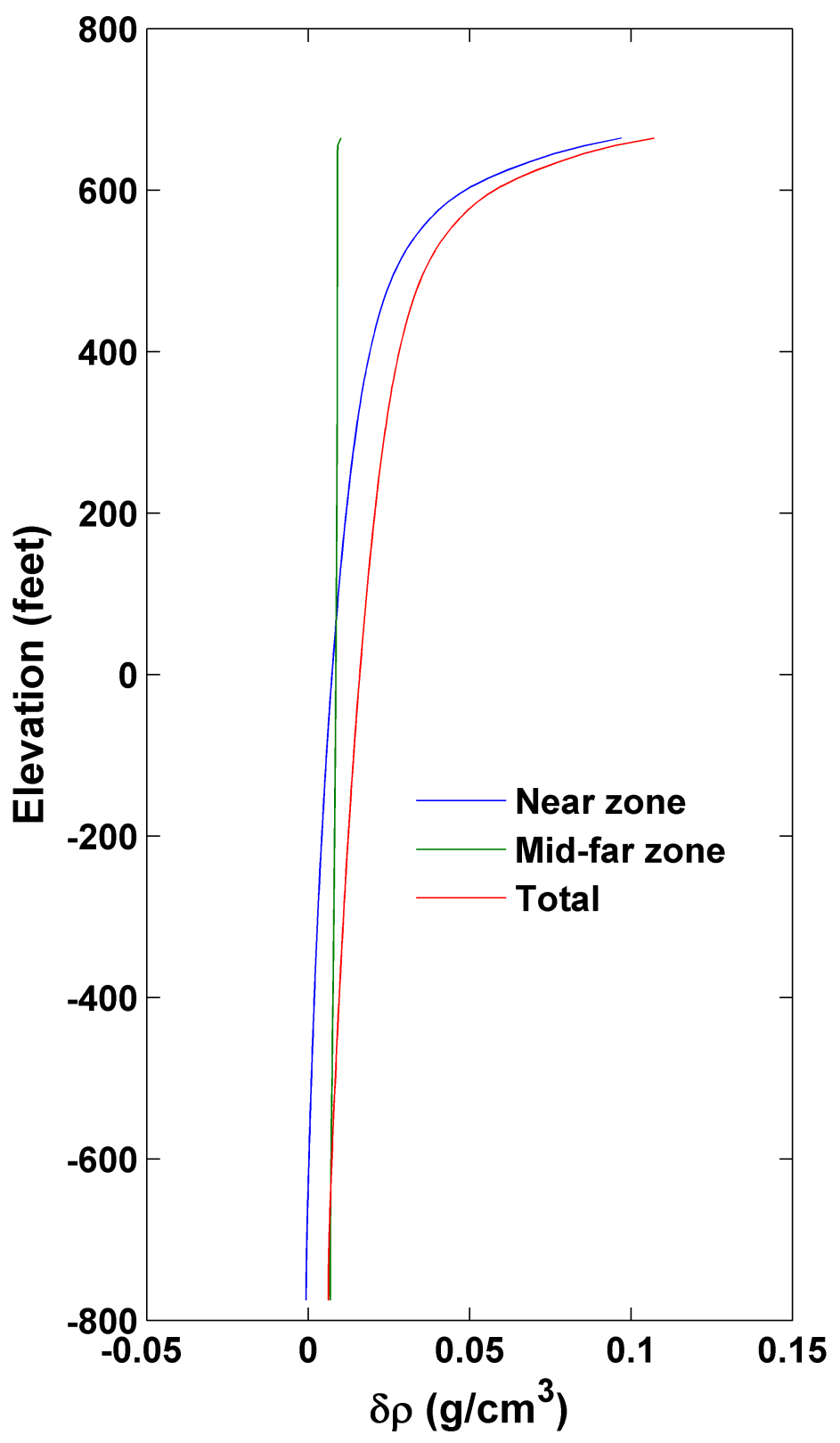

Figure 4: Well C4996: terrain corrections 


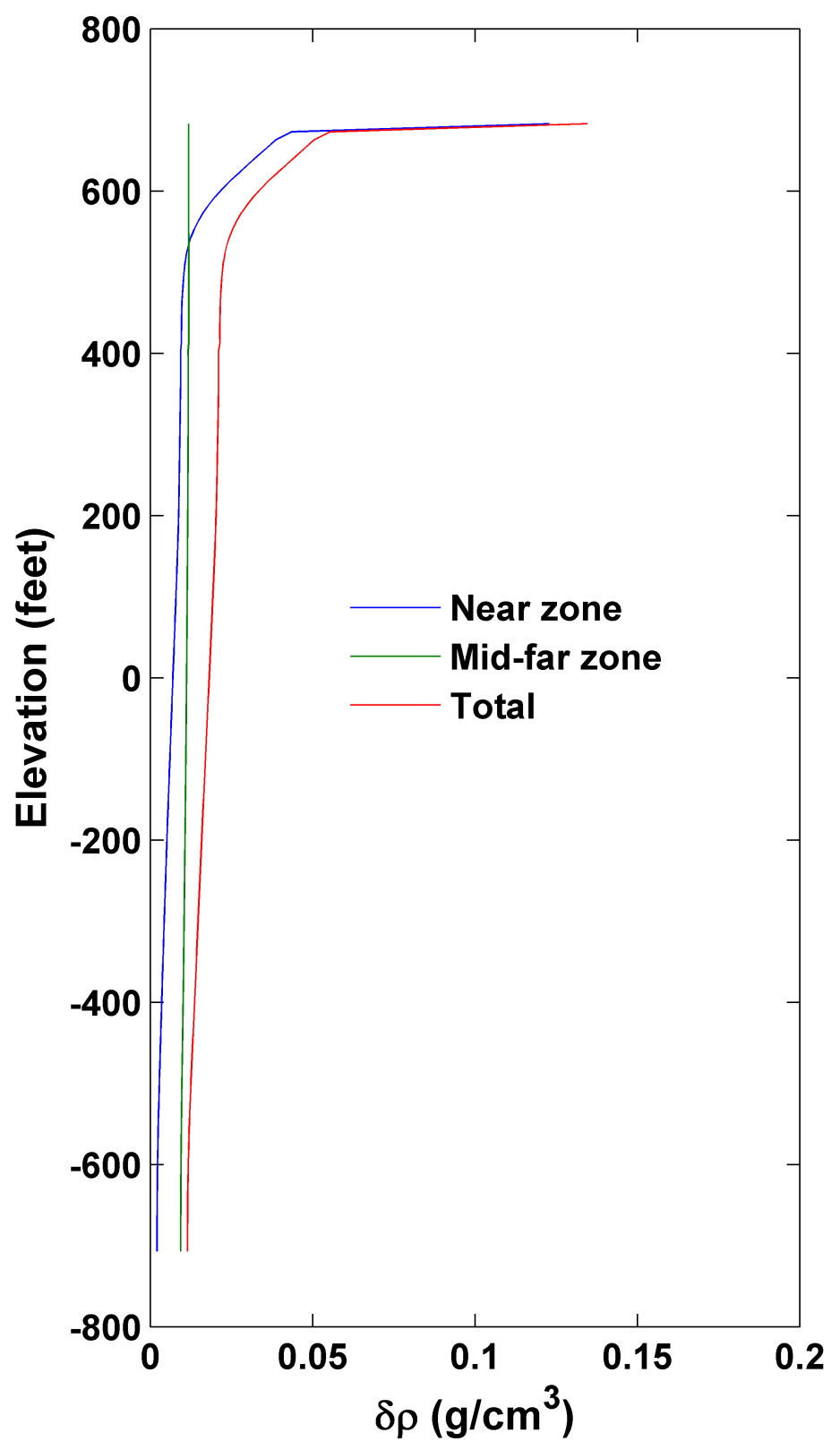

Figure 5: Well C4997: terrain corrections 


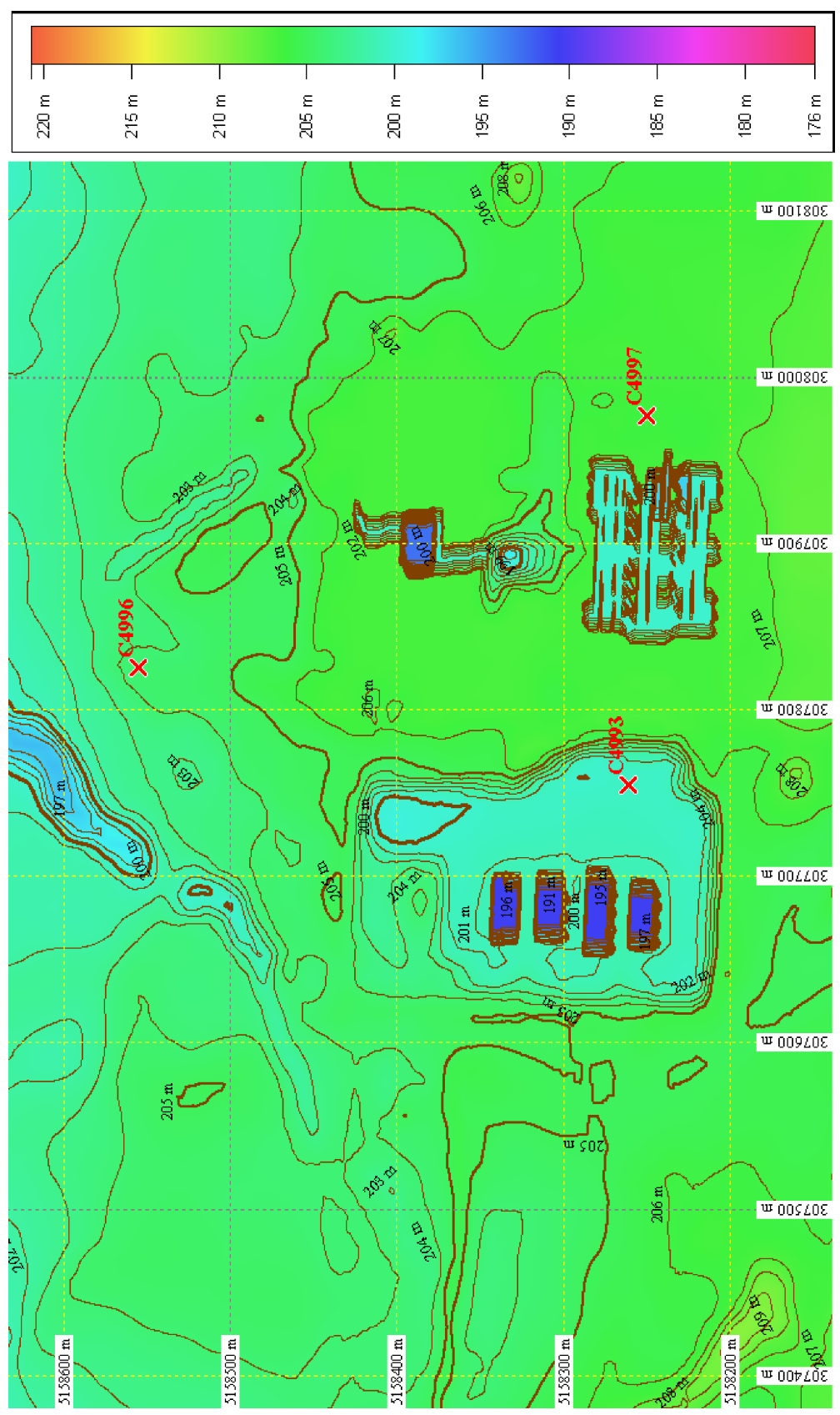

Figure 6: Near zone terrain 
by the differencing shows that only seven combinations of these differences are independent. The inversion uses all 44 gravity measurements to determine each interval density.

3. Given the inherent non-uniqueness of gravity inversions, there are an infinite number of density models which reproduce the observed gravity data to within a specified tolerance. Out of this infinite number of models, the inversion algorithm calculates the one having the smallest deviation (in a least-squares sense) from a constant density. This has been called "the principle of least astonishment".

The density inversion was implemented by MGL as a MATLAB script. The script was suhjected to extensive testing, using synthetic data with realistic random errors added to demonstrate satisfactory recovery of the synthetic model.

\section{Density inversion results}

The inversion results for each well are plotted in the following figures. There are four plots for each well:

1. A plot of the BHGM inversion density, with a $\pm 1 \sigma$ uncertainty plotted using thinner lines. For most of these plots, the uncertainty is so low that the uncertainties are not distinguishable.

2. A plot of the $\gamma-\gamma$ density log overlaid on the BHGM inversion density.

3. A plot of the $\gamma-\gamma$ density log, blocked to the gravity station intervals, overlaid on the BHGM inversion density.

4. A plot of the inversion fit to the data, summarized by the distribution of Z-scores. The $\mathrm{Z}$ score (a standardized measure of misfit) for the $\mathrm{i}$ 'th gravity measurement is defined as

$$
Z(i)=\frac{g(i)-\hat{g}(i)}{\sigma_{g}(i)}
$$

where $g(i)$ is the observed gravity, $\hat{g}(i)$ is the gravity predicted by the inversion densities, and $\sigma_{g}(i)$ is the uncertainty of the gravity measurement. If the data has been fit properly, the $\mathrm{Z}$ scores should have a Gaussian distribution with standard deviation of 1. In the plots, the gray bars are the calculated $\mathrm{Z}$ score distribution, and the red line is the theoretical standard Gaussian distribution. The $\mathrm{Z}$ score distributions for all wells is very close to the target Gaussian.

Note that the logs are plotted in elevation relative to sea level, not depth relative to the wellhead.

In general, the BHGM and $\gamma-\gamma$ densities agree quite well, particularly when the $\gamma-\gamma$ densities are blocked. There are some areas of significant differences:

- The $\gamma-\gamma$ densities are obviously unreliable in the cased shallower sections of the wells (generally surface to 350-400 foot depth) 
- The $\gamma-\gamma$ densities are possibly suspect in the sedimentary packages interbedded with the basalts. Inspection of the caliper logs indicates a tendency towards washout in these zones, which would interfere with the accurate functioning of the $\gamma-\gamma$ tool.

Overall, the geology of the wells appears to be accurately reflected in the BHGM densities. The density logs show

- A shallow, low-density section with compaction-induced increased density with depth

- A series of high-density massive basalt flow units, with lower densities towards the top of each flow. This may be a product of increased weathering, increased fracturing, or increased vesicularization (or any combination of these) towards the tops of the flows.

- Relatively low-density sedimentary packages interbedded with the basalt flows.

The well-to-well correlation is excellent, as shown in figure 19. 


\subsection{C4993}

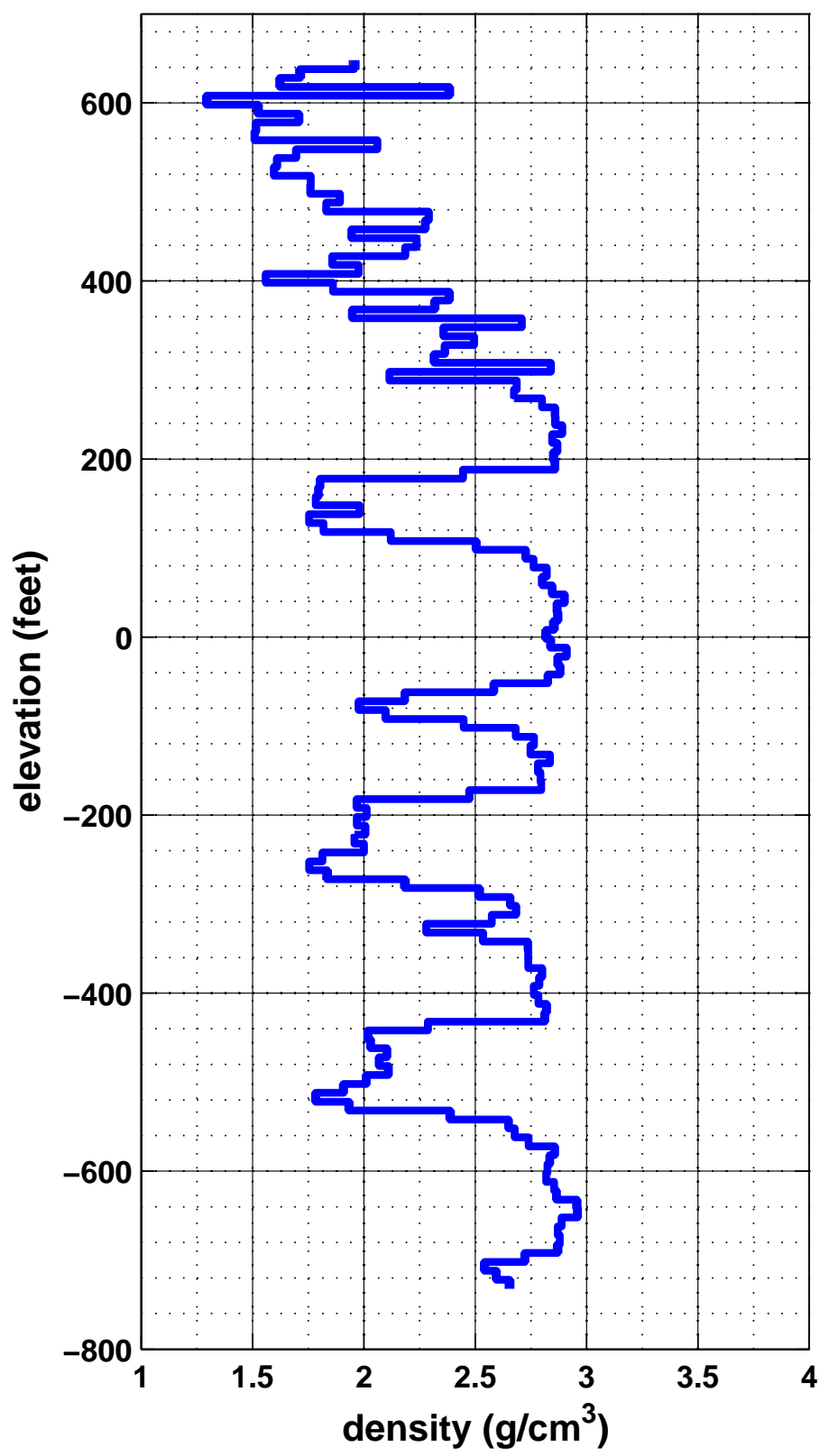

Figure 7: Well C4993: BHGM inversion results 


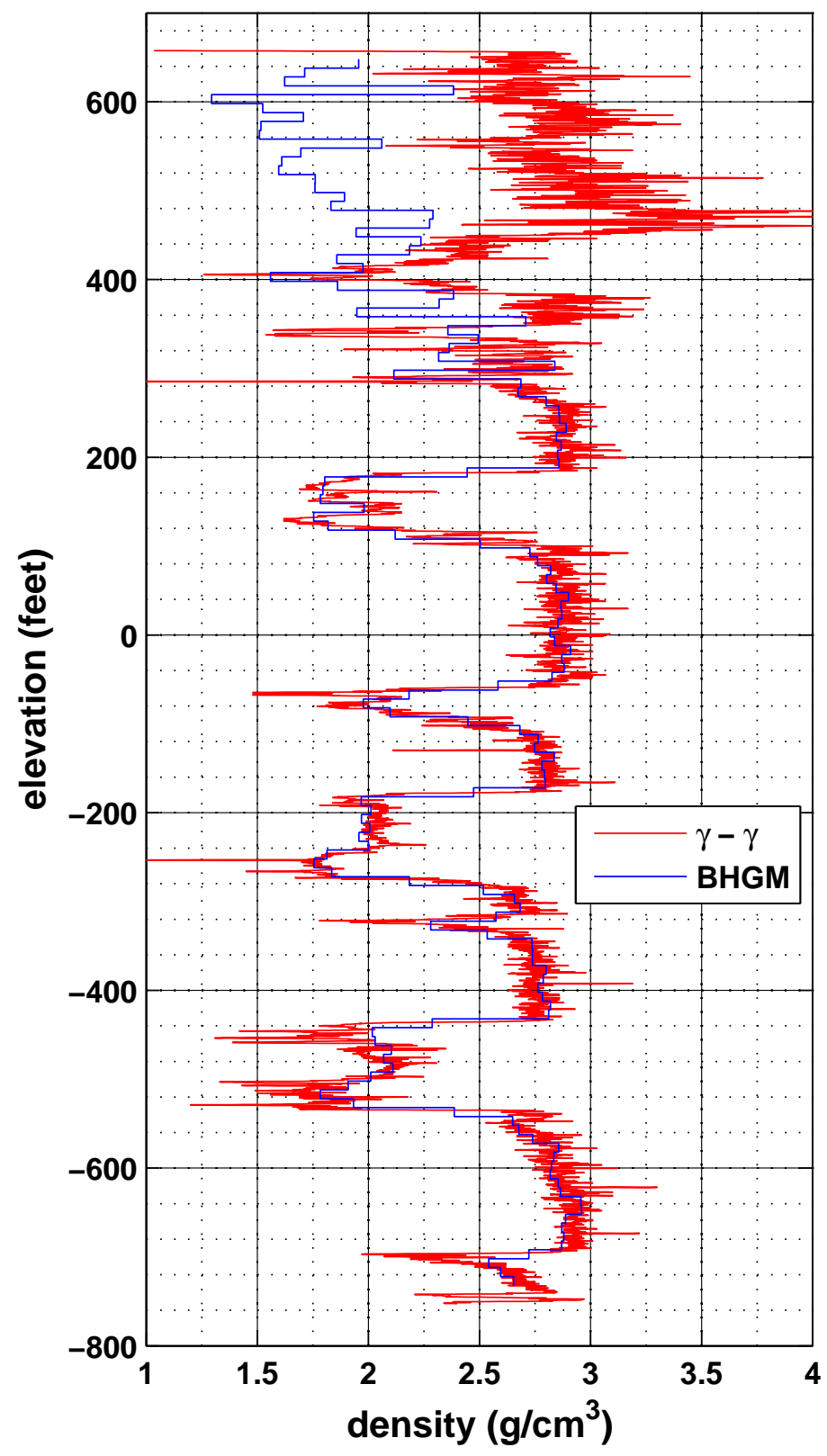

Figure 8: Well C4993: comparison of BHGM and $\gamma-\gamma$ densities 


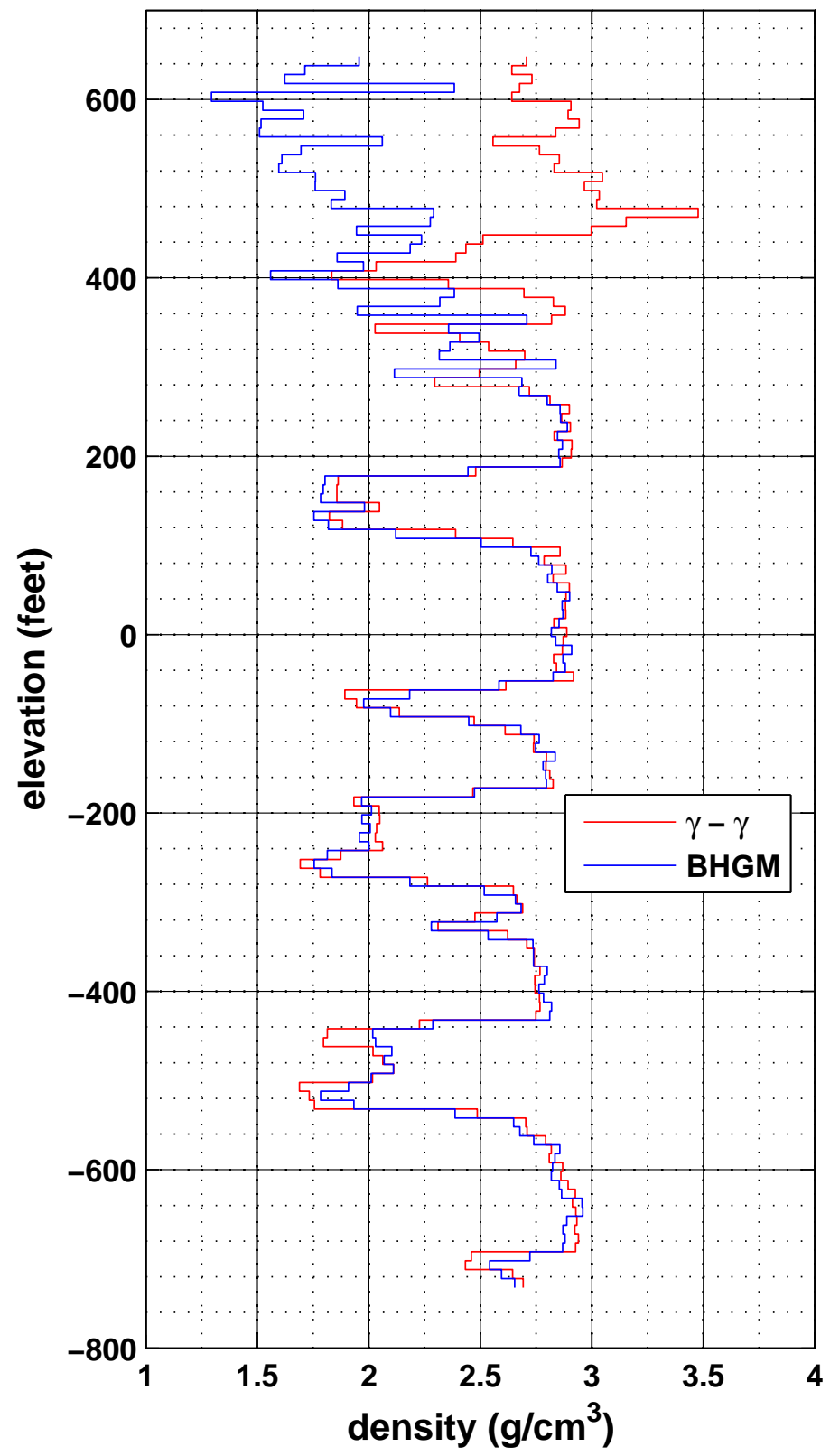

Figure 9: Well C4993: comparison of BHGM and blocked $\gamma-\gamma$ densities 


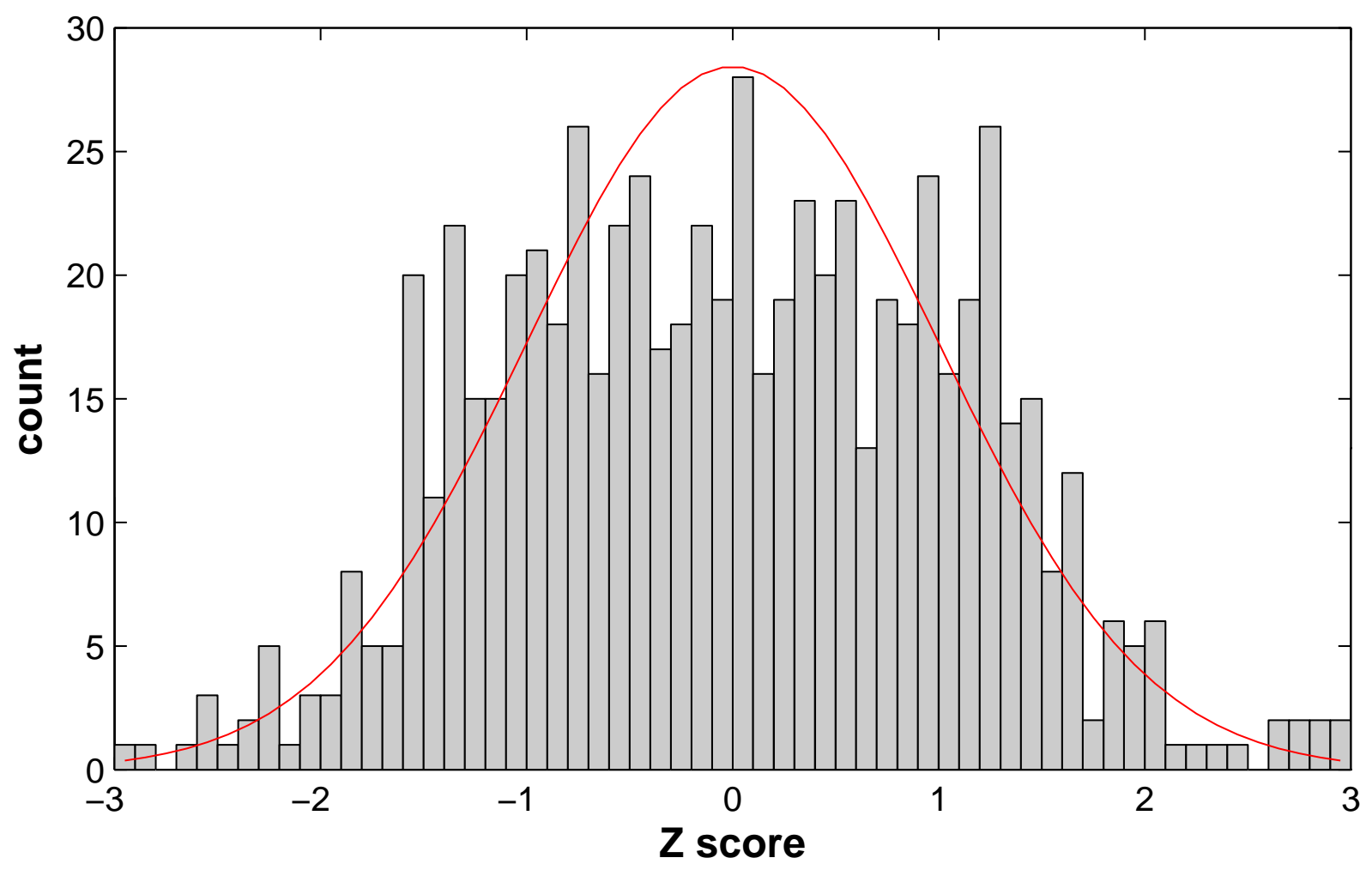

Figure 10: Well C4993: Z-score distribution of inversion fit 


\section{$4.2 \quad \mathbf{C 4 9 9 6}$}

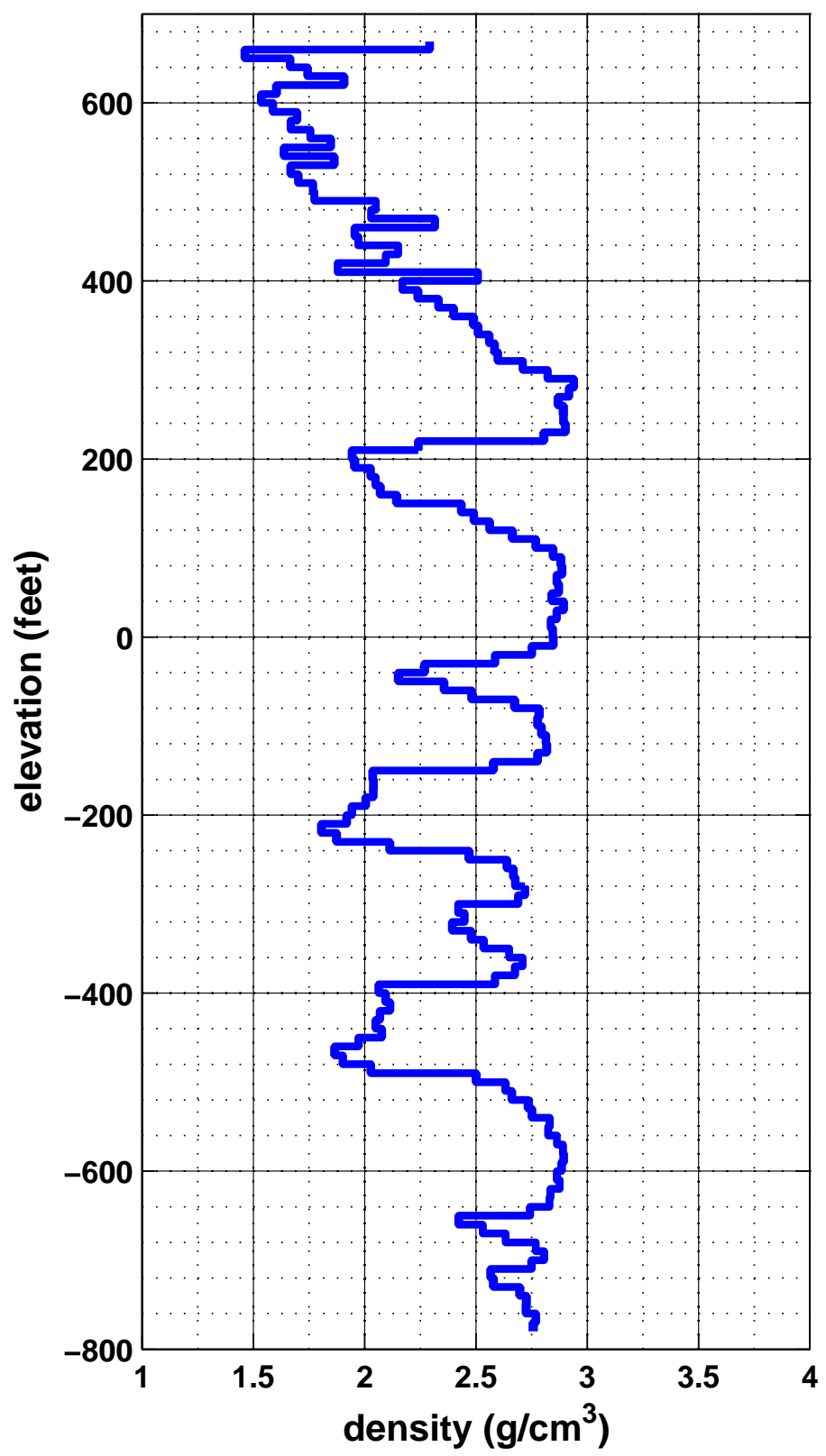

Figure 11: Well C4996: BHGM inversion results 


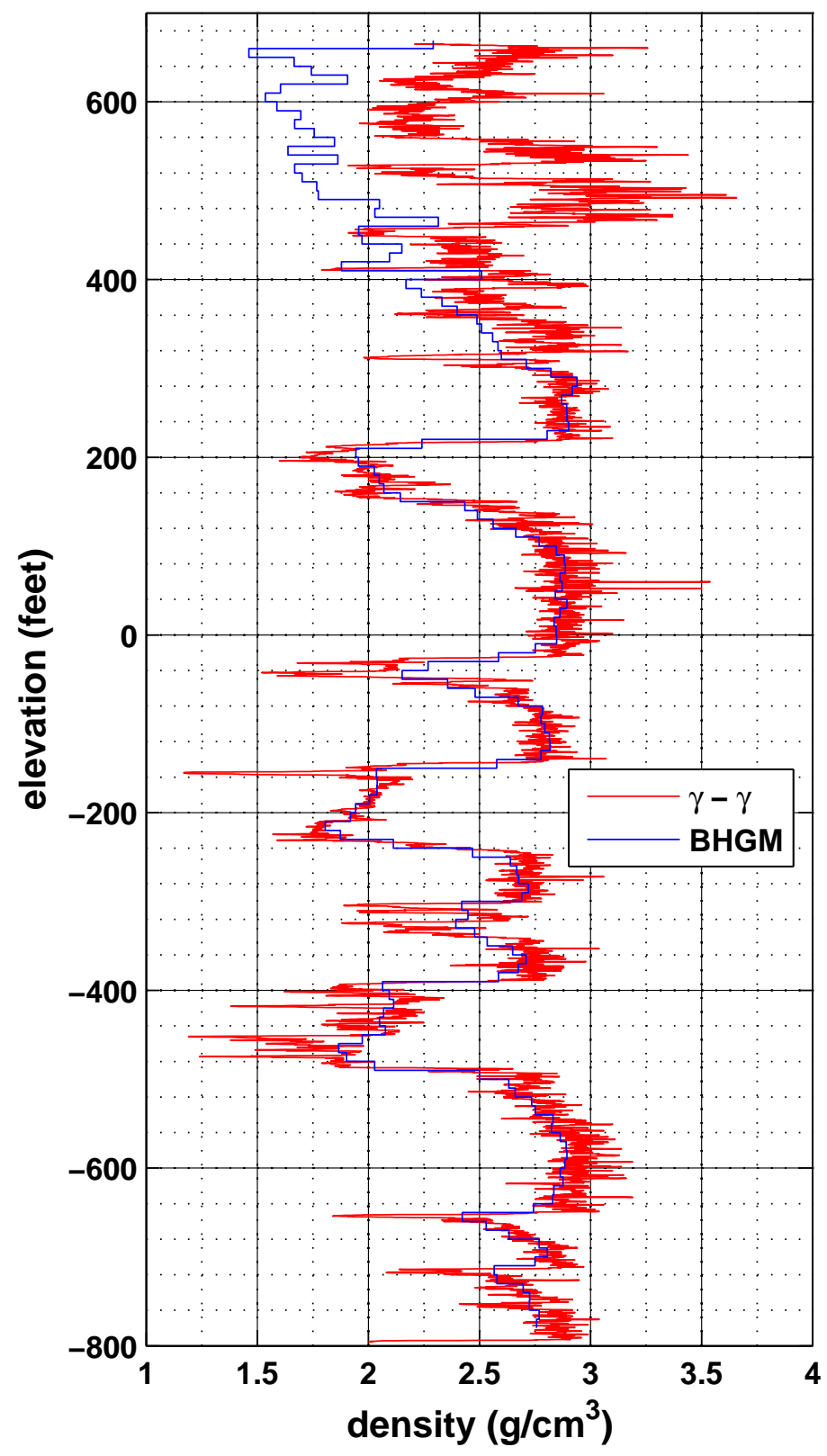

Figure 12: Well C4996: comparison of BHGM and $\gamma-\gamma$ densities 


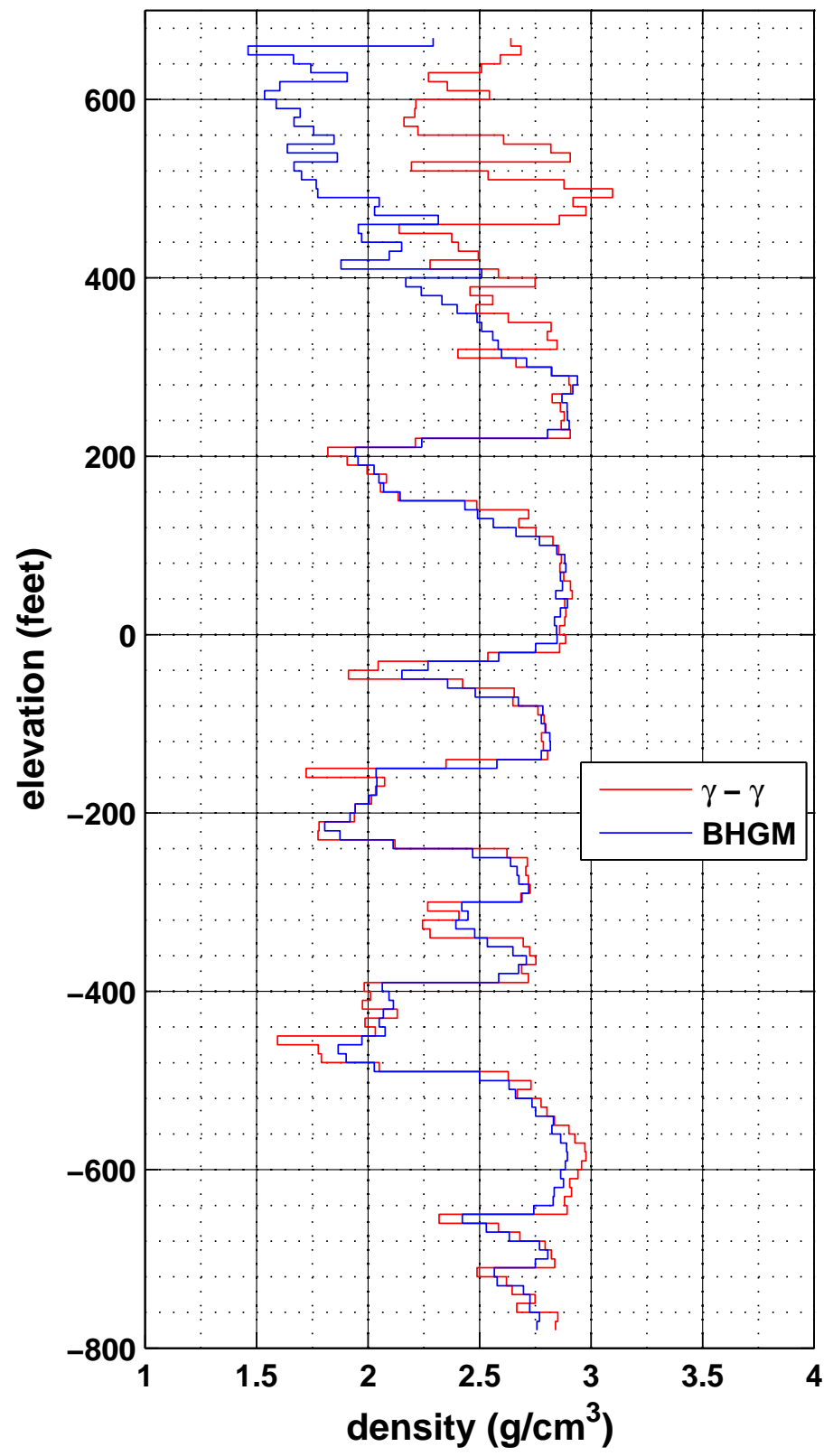

Figure 13: Well C4996: comparison of BHGM and blocked $\gamma-\gamma$ densities 


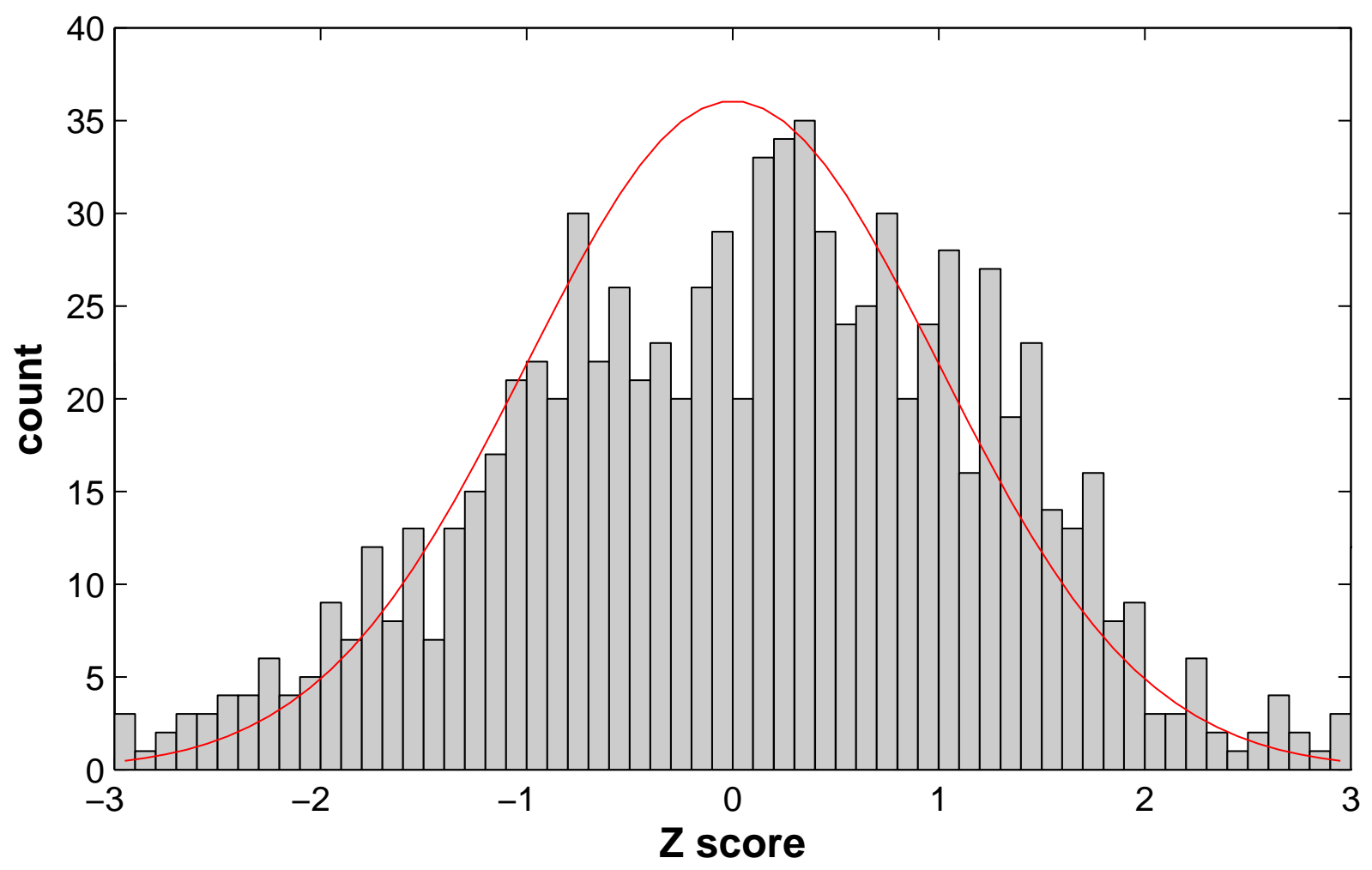

Figure 14: Well C4996: Z-score distribution of inversion fit 


\section{$4.3 \quad \mathbf{C 4 9 9 7}$}

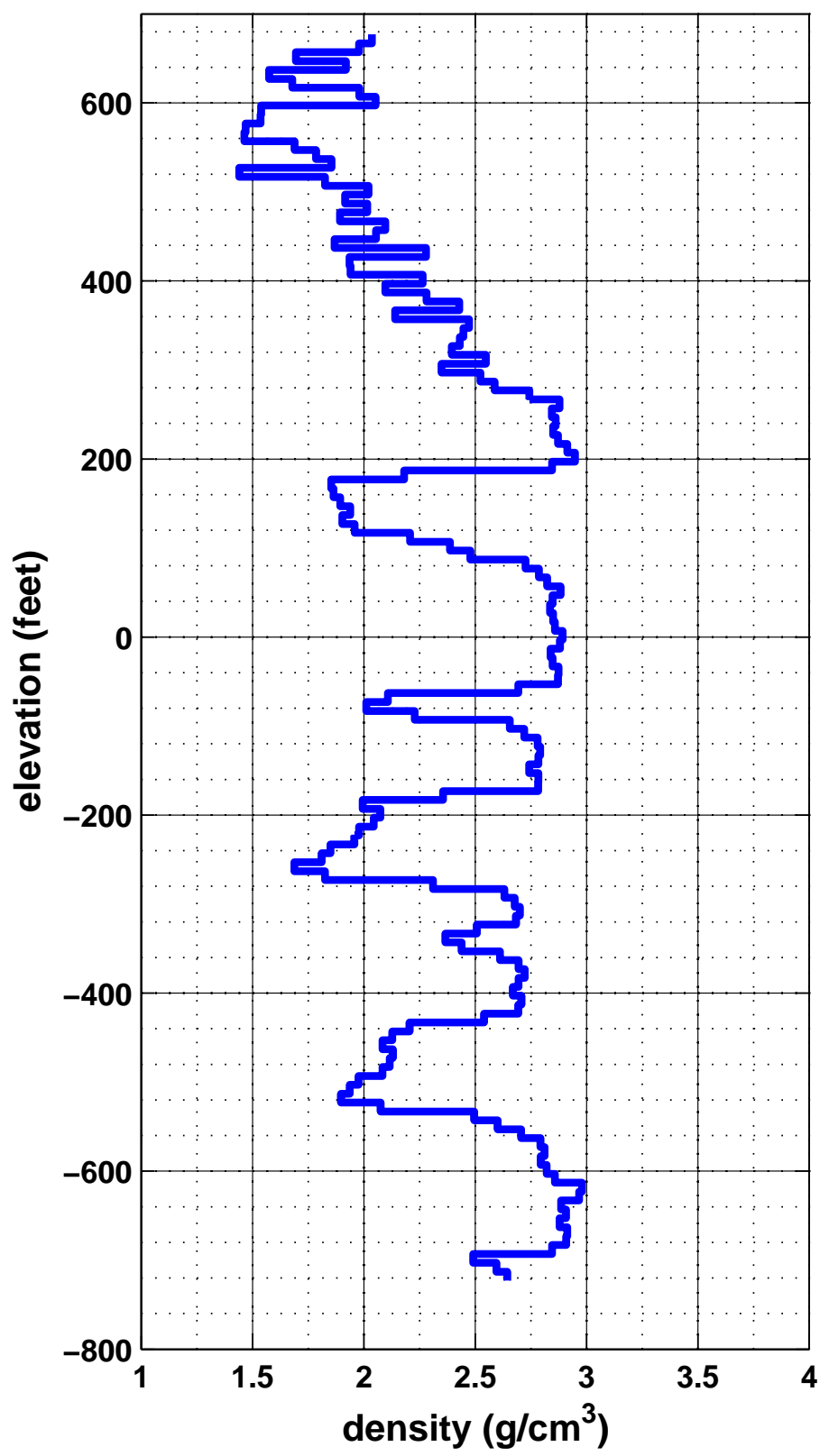

Figure 15: Well C4997: BHGM inversion results 


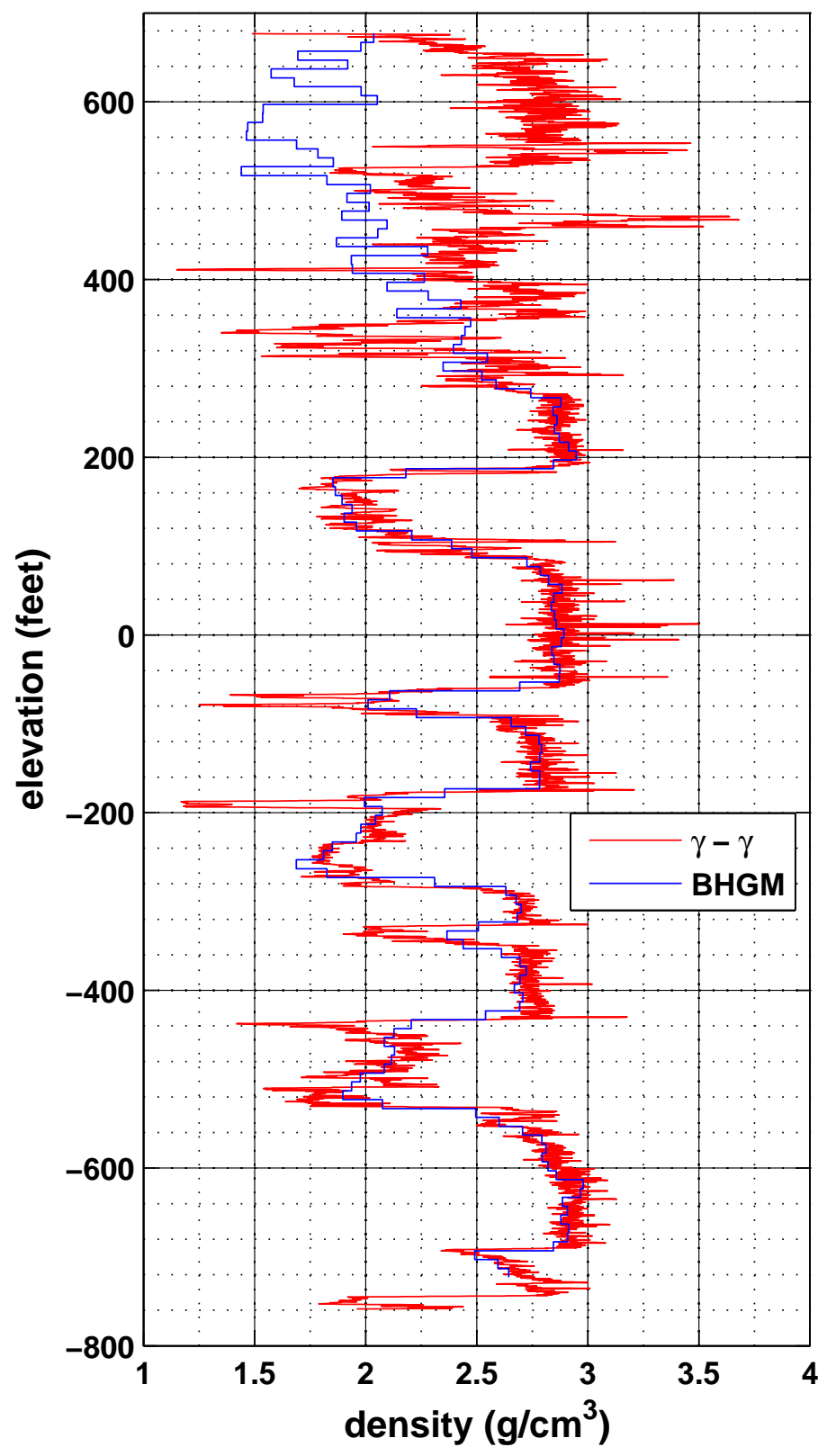

Figure 16: Well C4997: comparison of BHGM and $\gamma-\gamma$ densities 


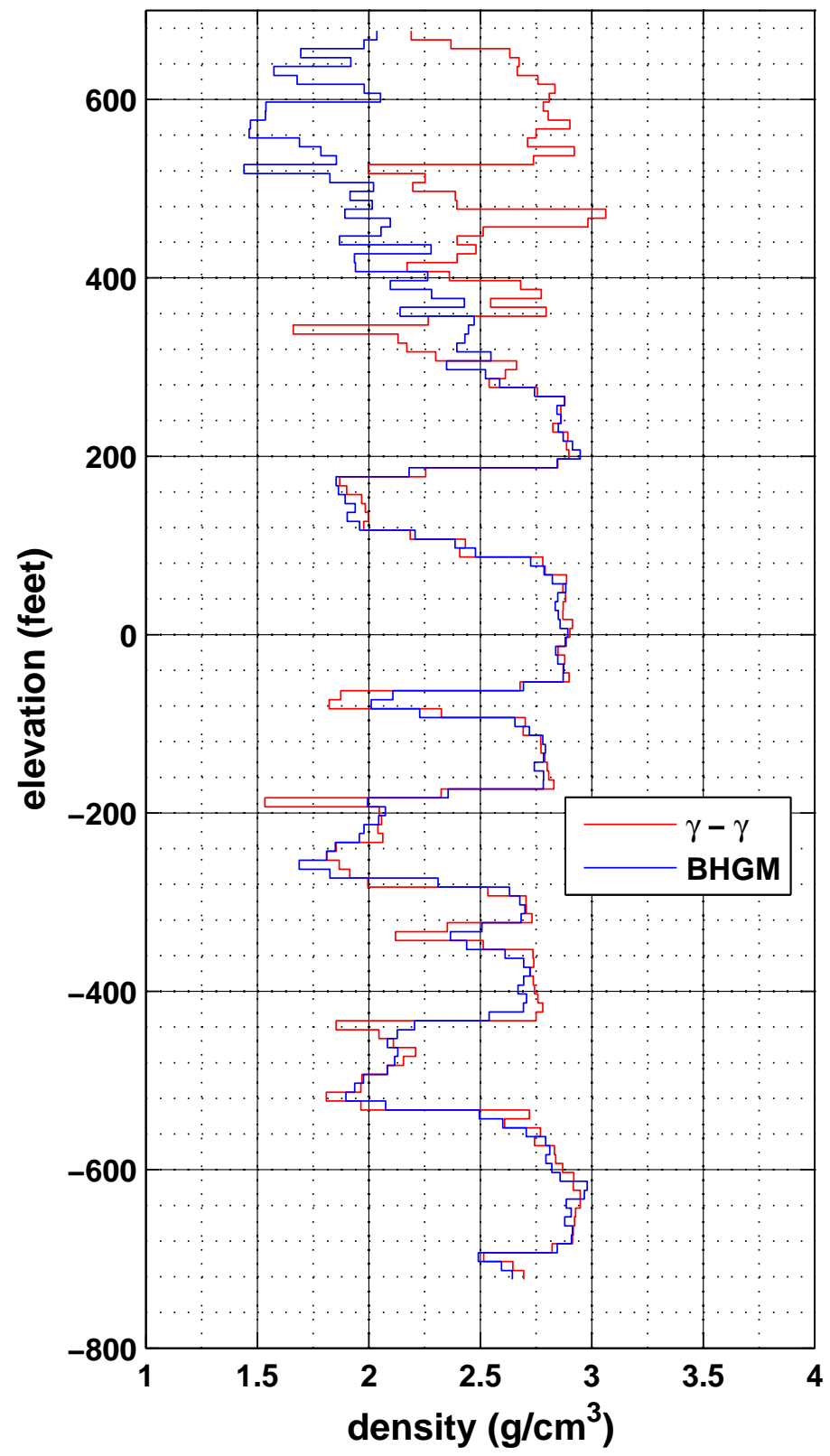

Figure 17: Well C4997: comparison of BHGM and blocked $\gamma-\gamma$ densities 


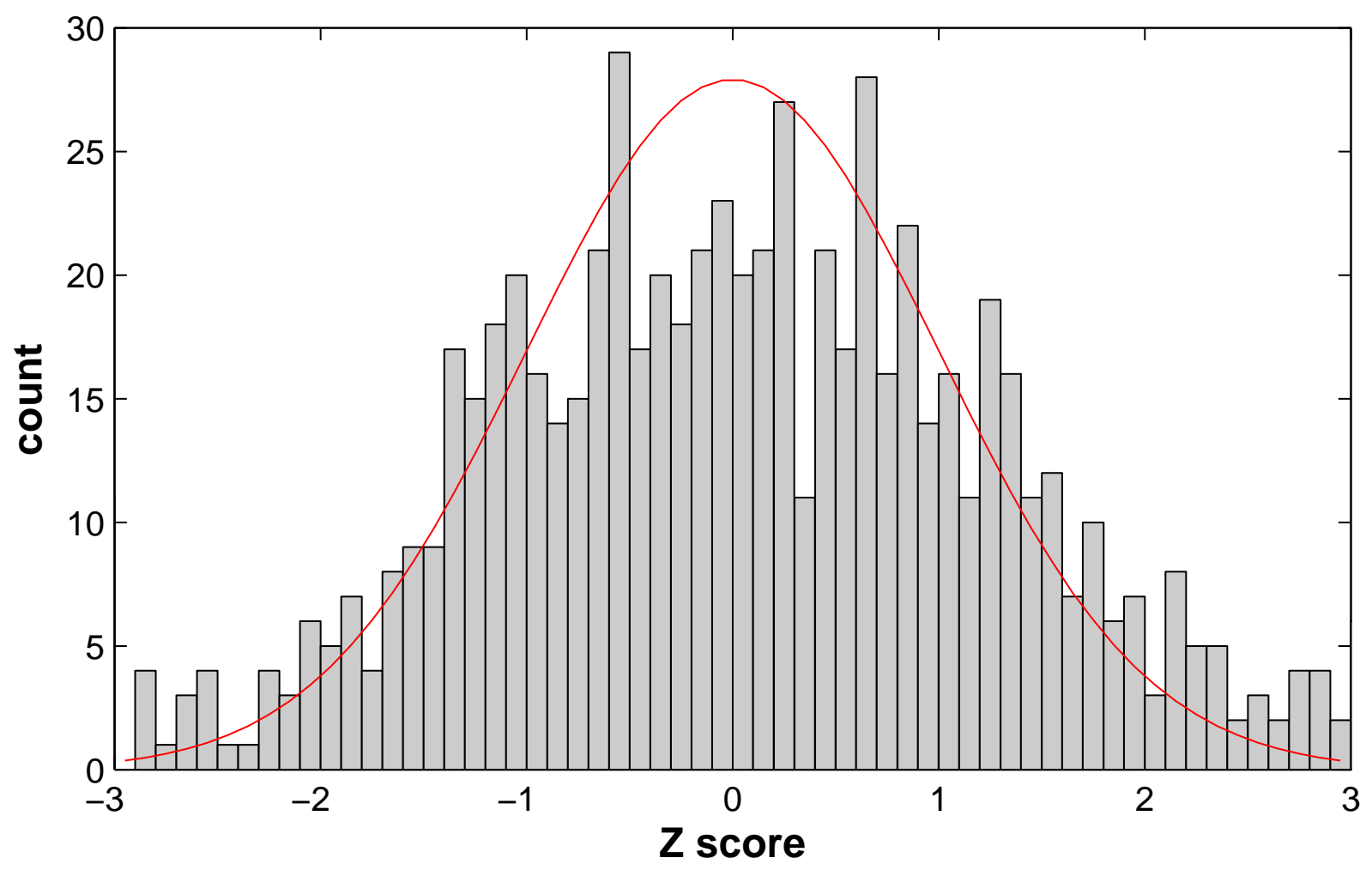

Figure 18: Well C4997: Z-score distribution of inversion fit 


\subsection{Well-to-well correlation}

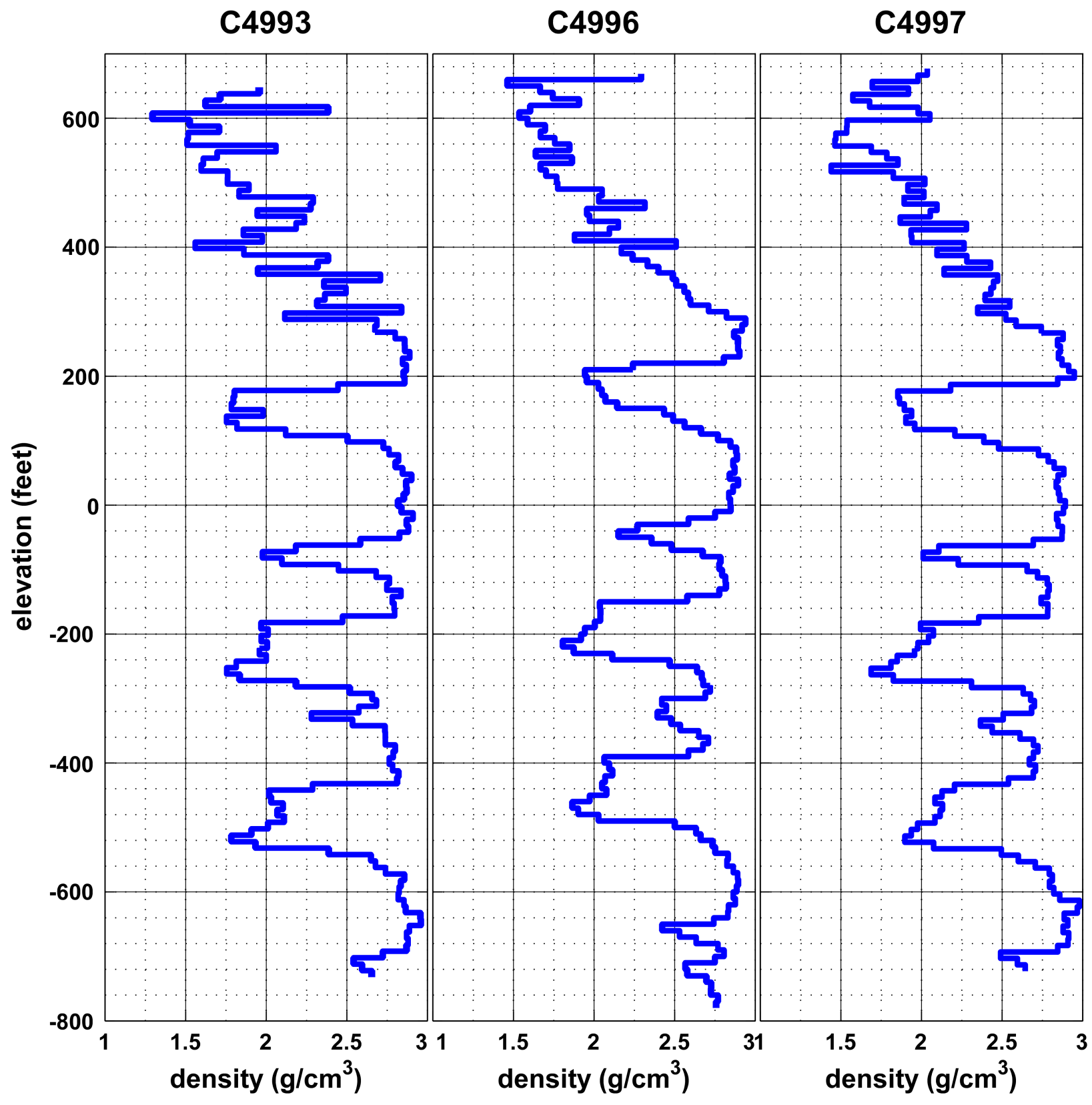

Figure 19: Well-to-well correlations 


\section{References}

Richard C. Aster, Brian Borchers, and Clifford H. Thurber. Parameter Estimation and Inverse Problems. Number 90 in the International Geophysics Series. Elsevier Academic Press, 2005.

Larry A. Beyer. Borehole Gravity Surveys: Theory, Mechanics, and Nature of Measurements. Number 83-76 in the Open-File Report Series. U. S. Geological Survey, 1983.

J.F. Hayford and William Bowie. The effect of topography and isostatic compensation upon the intensity of gravity. Number 10 in the Special Publication Series. U.S. Coast and Geodetic Survey, 1912.

Douglas H. Krohn. Gravity terrain corrections using multiquadric equations. Geophysics, 41 (2), 1976.

Jeffrey D. MacQueen. Inversion of borehole gravimeter data. In Expanded Abstracts of the Technical Program, number 59. Society of Exploration Geophysicists, 1989.

Thane H. McCulloh. The promise of precise borehole gravity in petroleum exploration and exploitation. Number 531 in the Circular Series. U. S. Geological Survey, 1966. 


\section{Appendices}

\section{Appendix: A Processed gravity data}

The processed gravity data for each well is presented in the following tables. The columns are:

Reading number: the reading number from the .rdg file.

File ID: the subfile identifier for the reading (file C4993a.rdg, C4993b.rdg, etc).

Reading depth: the reading depth in feet, relative to the wellhead.

Reading time: the reading time in days and fractional days, relative to 12:00:00, May 22, 1960.

gravity: the logged gravity data in milliGals, extracted from the .dat file (see Appendices D.1 and D.2. This is relative to an arbitrary initial value.

Tidal corr: the tidal correction in milliGals. Note that this correction has already been applied to the gravity values in the .rdg file, and is included here for reference.

Free-air corr: free-air correction, in milliGals. This correction is not applied until the inversion step, to facilitate using standard formulas for estimating densities. These formulas usually include a term for the free-air effect.

Near-zone TC: the near-zone terrain correction, in milliGals.

Mid-far zone TC: the mid- and far-zone terrain correction, in milliGals.

Drift corr: the drift correction, in milliGals.

Corrected gravity: the final corrected gravity, in milliGals. This is defined as Corrected gravity $=$ gravity + Near-zone TC + Mid-far zone TC + Drift corr .

\section{A.1 C4993}




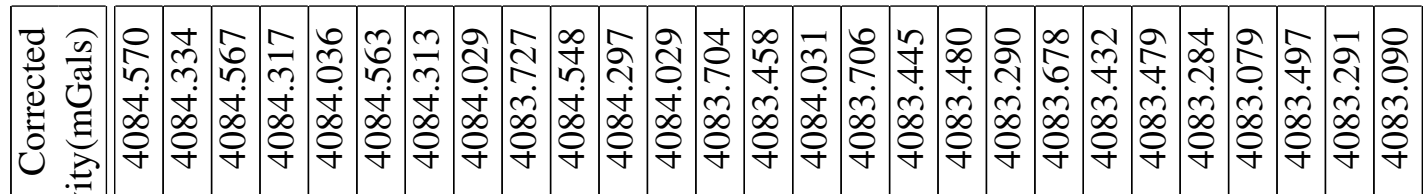
b.

ڤే ठำ

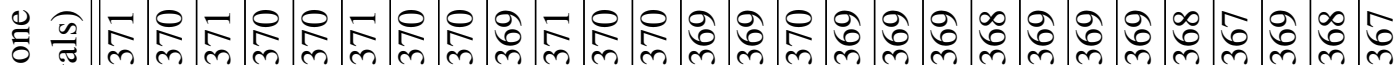

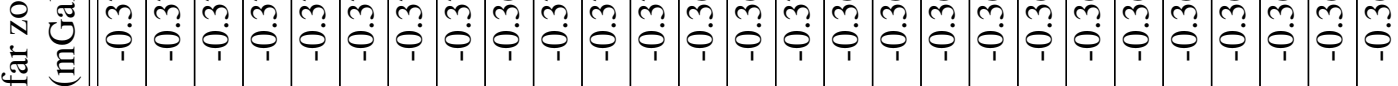
$\sum_{\substack{1 \\ \hdashline}}^{U}$

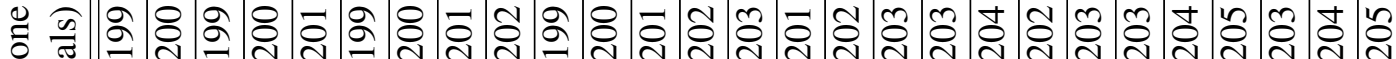

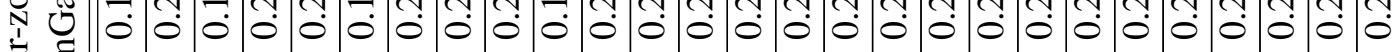
สี $\ddot{z}$

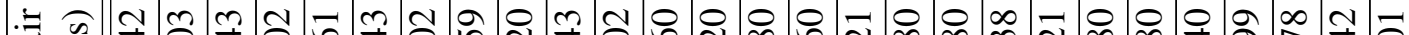
i స

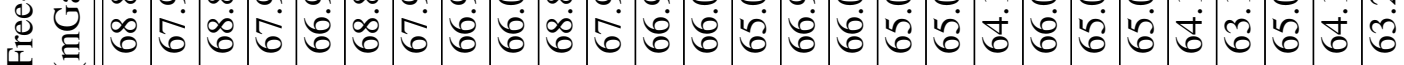
ठำ

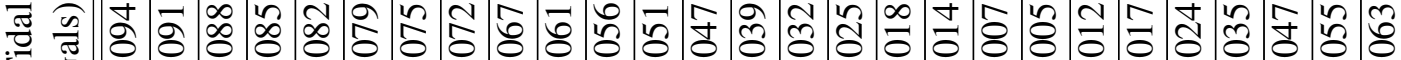

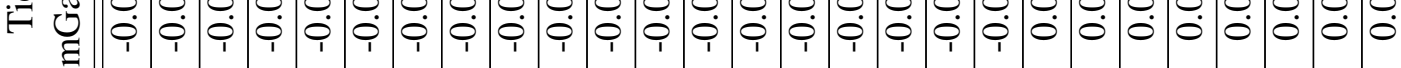

วิก

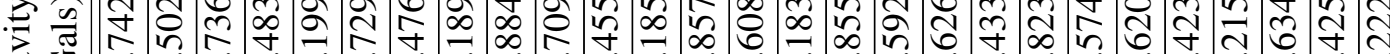
ت

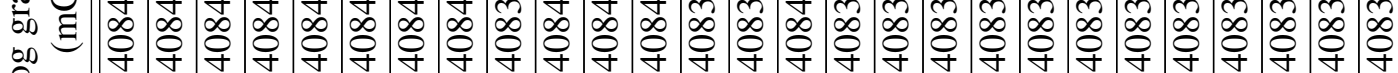
은

b ๙ 吾

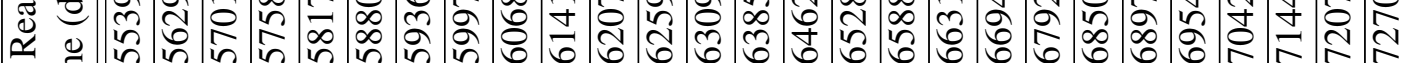

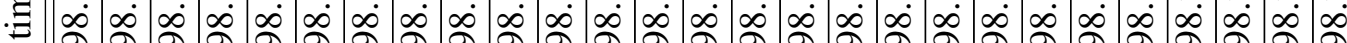
مे

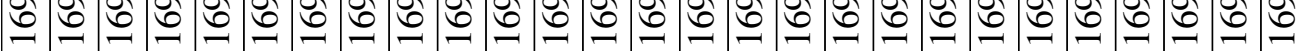

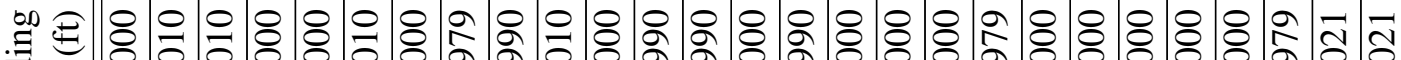
च

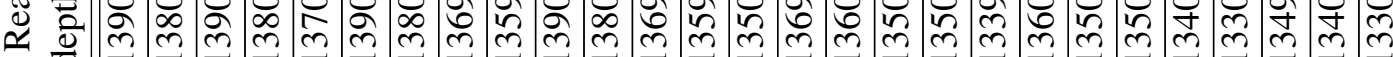

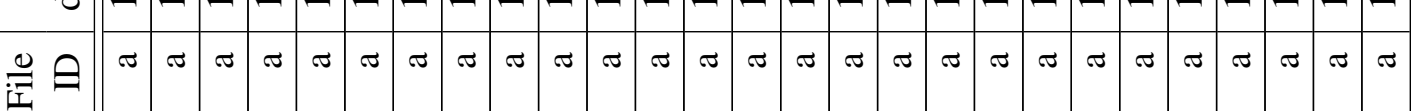

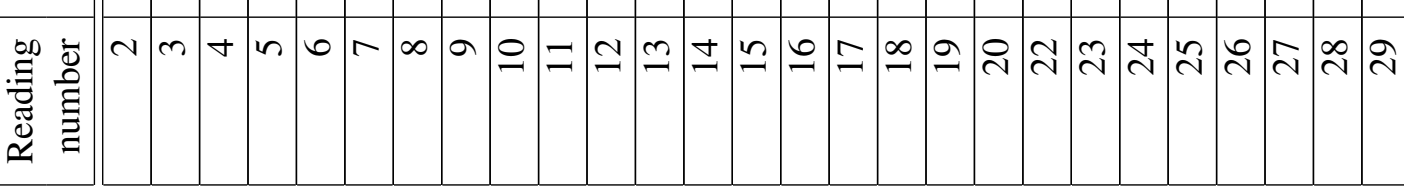




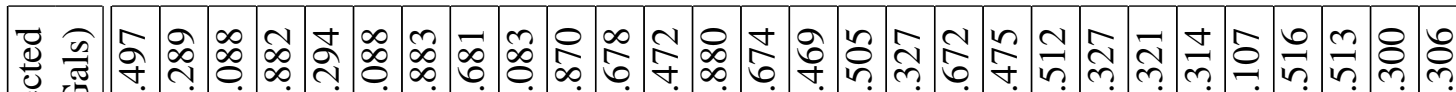

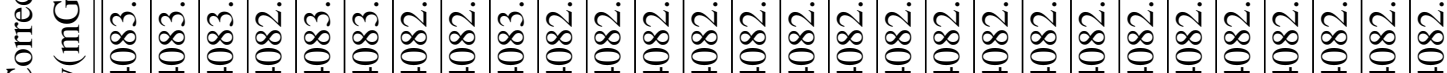
U

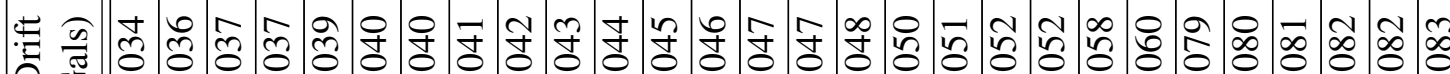
A

‡

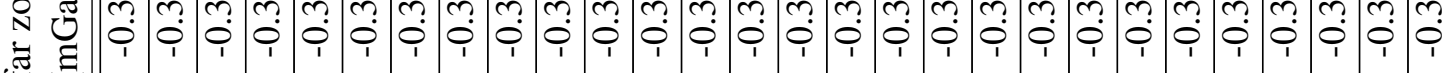

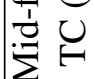

\&

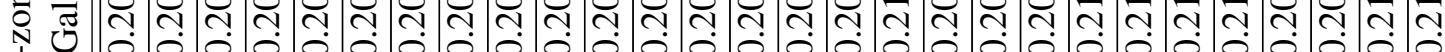
艺 ॠ $\mathrm{Z} \cup$

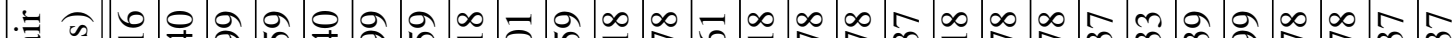

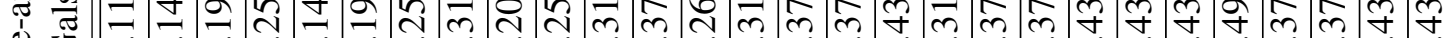
递 0

สే శิ

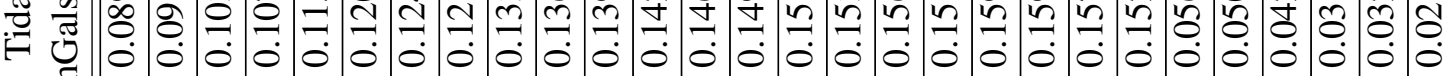
$\Xi$ ठั

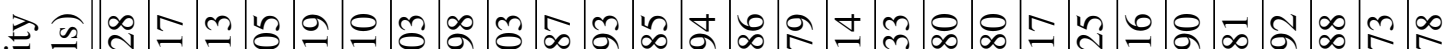
च च m m m

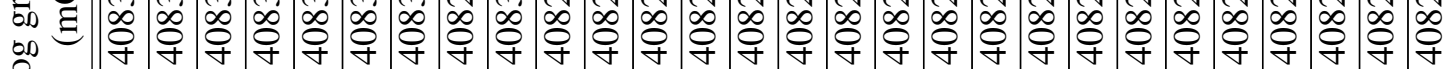
으

正

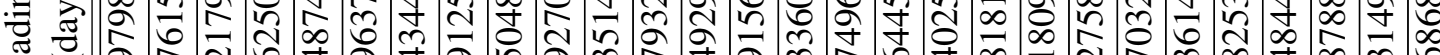

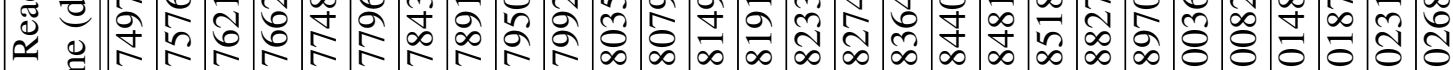

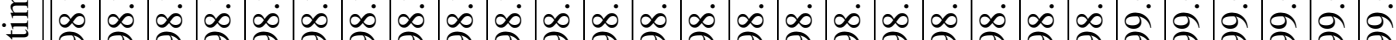

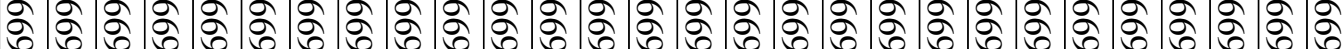

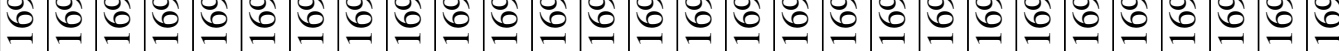

正 ஓ થ 2 d 养

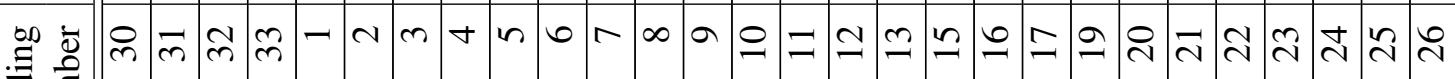
(ี) 
包

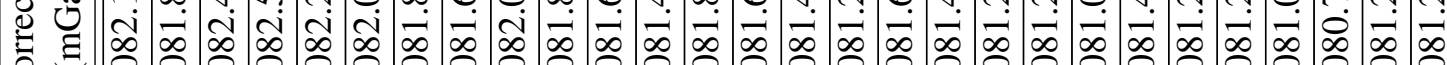

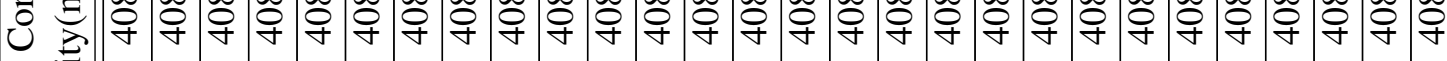
ț

正 :

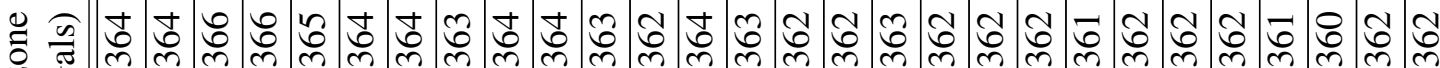

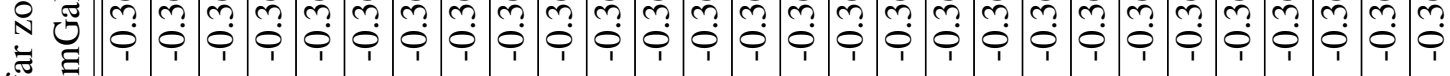

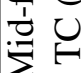

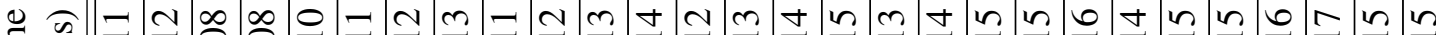

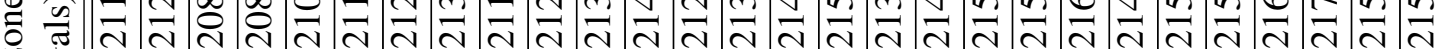

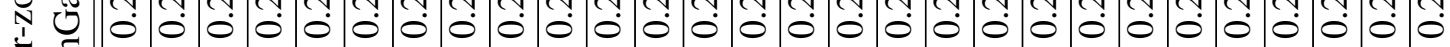
卷 $\ddot{z}$

\begin{tabular}{|c|c|c|c|c|c|c|c|c|c|c|c|c|c|c|c|c|c|}
\hline 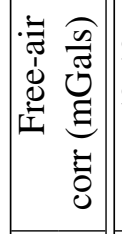 & 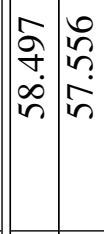 & 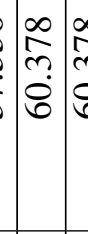 & 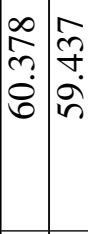 & & & 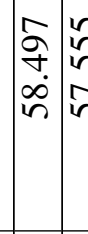 & 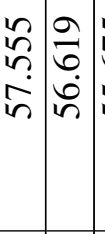 & $\begin{array}{ll}0 & n \\
\text { in } \\
\text { in }\end{array}$ & 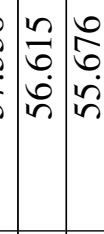 & 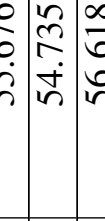 & & & & & & & in \\
\hline $\begin{array}{l}\widetilde{a} \\
\tilde{J} \\
\vdots \\
\Xi \\
\vdots \\
0 \\
0\end{array}$ & 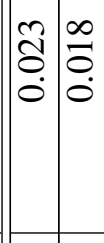 & $\stackrel{0}{O}$ & & & 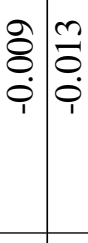 & 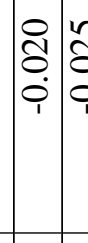 & 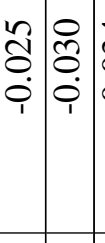 & 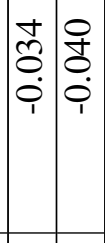 & {$\left[\begin{array}{ll}n & 1 \\
0 & 2 \\
0 & 0 \\
0 & 0 \\
1 & 1\end{array}\right.$} & 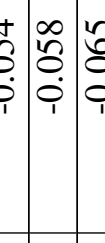 & 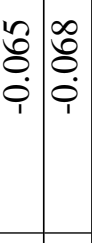 & & 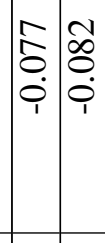 & 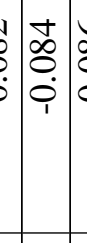 & \begin{tabular}{l|l}
0 & \\
0 & $\infty$ \\
0 & 0 \\
0 & 0 \\
0 & 0 \\
1 & 1
\end{tabular} & 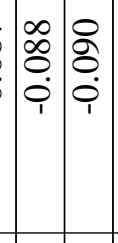 & $\begin{array}{l}\bar{a} \\
\dot{8} \\
\dot{0} \\
\dot{0}\end{array}$ \\
\hline : & 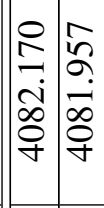 & 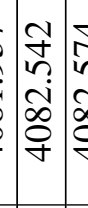 & 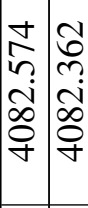 & & 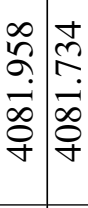 & 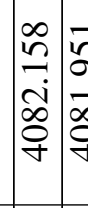 & 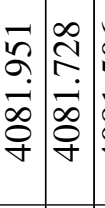 & 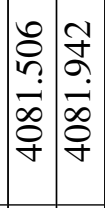 & 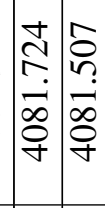 & 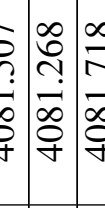 & 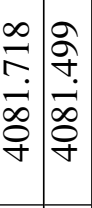 & 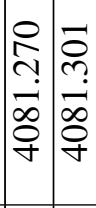 & 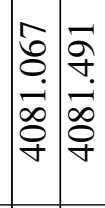 & 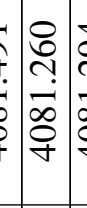 & 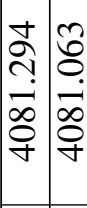 & 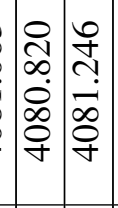 & 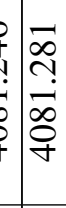 \\
\hline 6 & 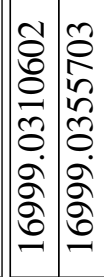 & 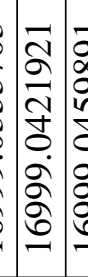 & 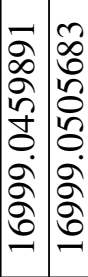 & & 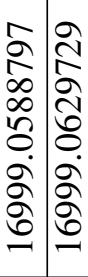 & 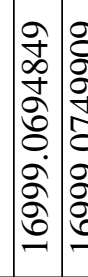 & 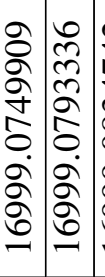 & 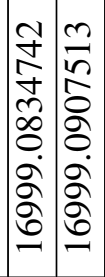 & 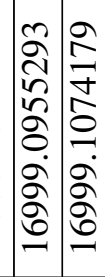 & 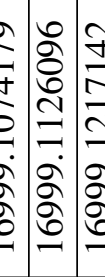 & 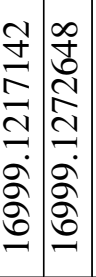 & 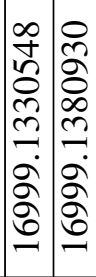 & 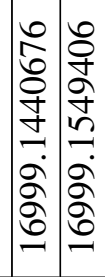 & 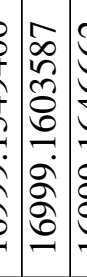 & 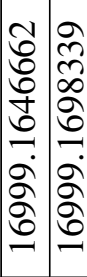 & 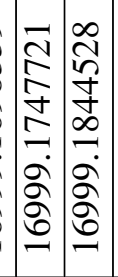 & 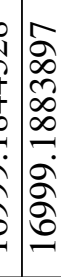 \\
\hline $\begin{array}{l}\vec{E} \\
\vec{E} \\
\underline{\Xi}\end{array}$ & 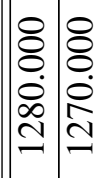 & 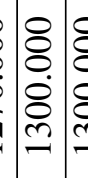 & 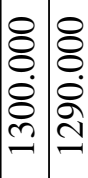 & & 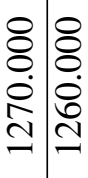 & 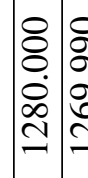 & 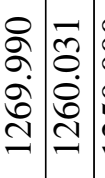 & 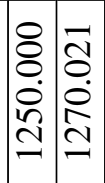 & 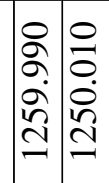 & 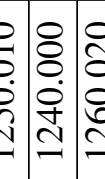 & 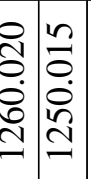 & 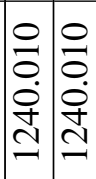 & 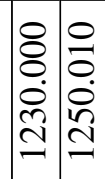 & 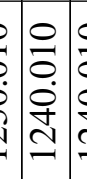 & 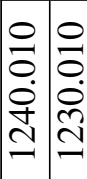 & 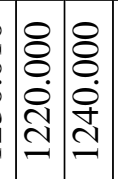 & 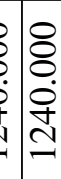 \\
\hline & & ـ م. & مـ مـ & & م مـ & & . م م. & مـ مـ & مـ م. & ـ & مـ مـ & م مـ & & م. & م. م. & م. م. & م. \\
\hline $\begin{array}{l}\vec{D} \\
\vec{\Xi}\end{array}$ & & iे & $\dot{m} \bar{m}$ & & m & $\stackrel{m}{\infty}$ & $\stackrel{\infty}{m}$ & $\infty$ m) & F F & $\bar{f} F$ & $\begin{array}{l} \\
7\end{array}$ & 果 & 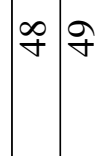 & fin & $\bar{n} \approx$ & $\approx n$ & in \\
\hline
\end{tabular}




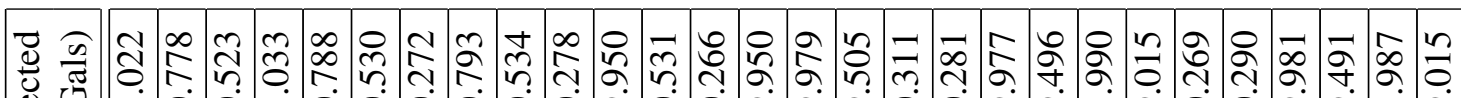
范

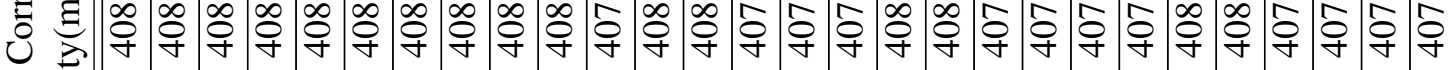

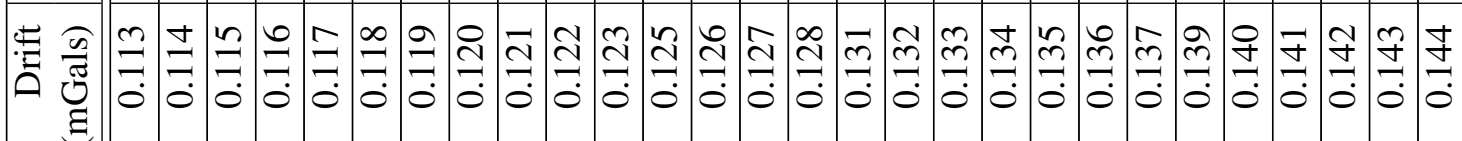
:

ప

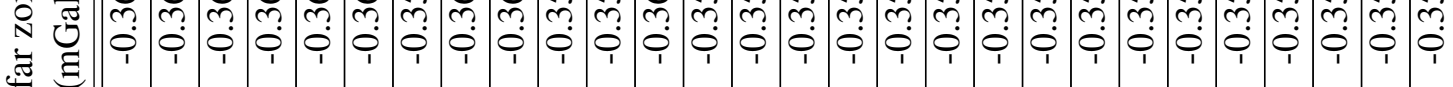
穿

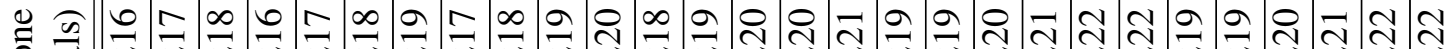

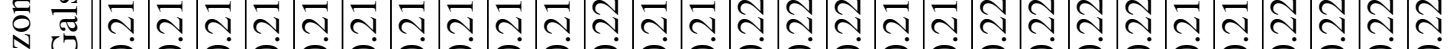
要 覀 $\mathrm{Z}$

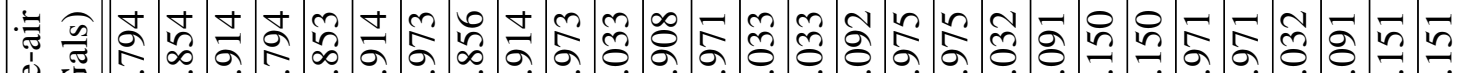
i 0 j 垔 ठํ.

चี

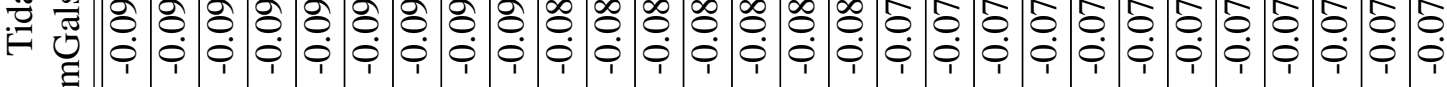

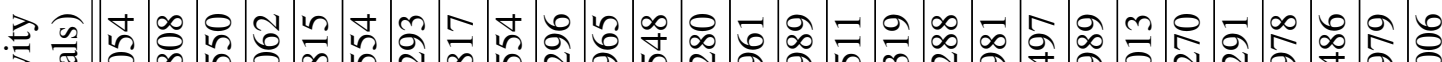
च ש क 足 舟

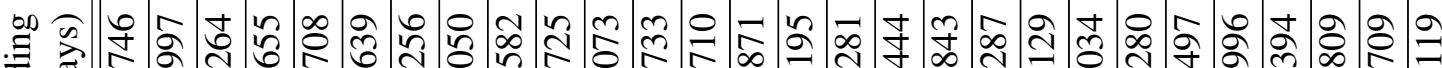

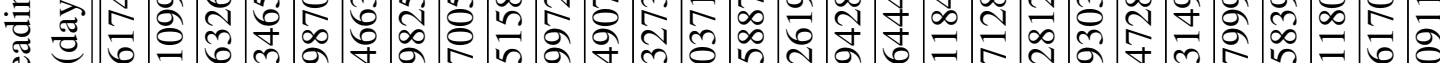
$\approx$ ๘

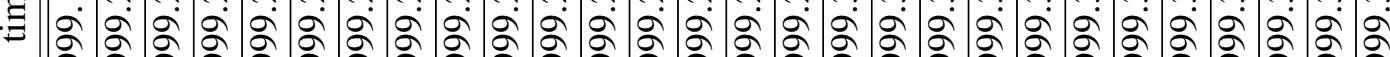

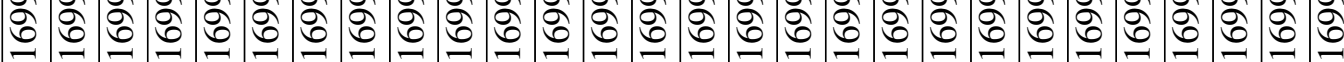
骂 ๘ $\approx$ ฯ

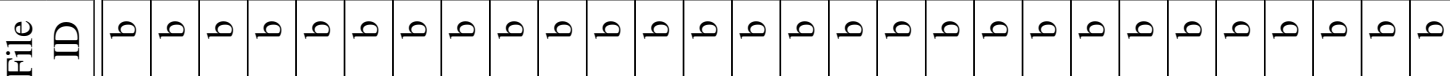

$\sin$

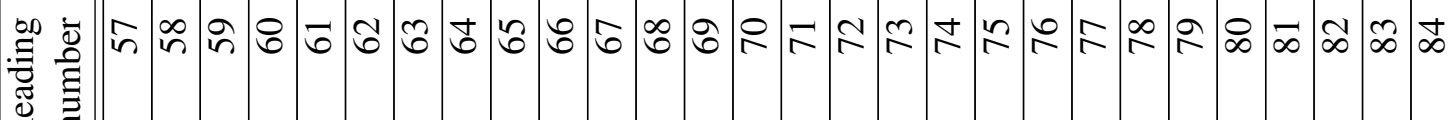




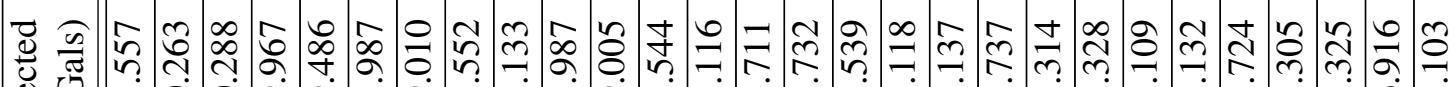
热 U

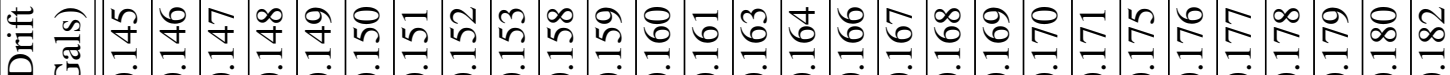

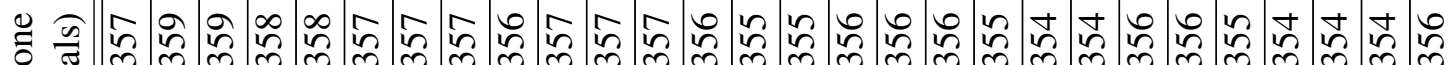

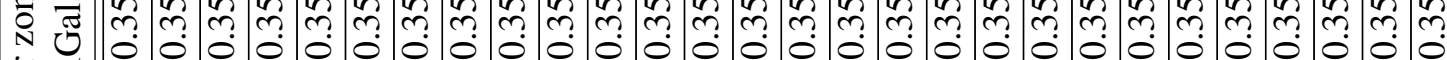
$\exists$ 皇

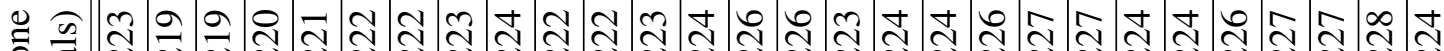
สึ

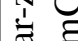
बi $\mathrm{Z}$,

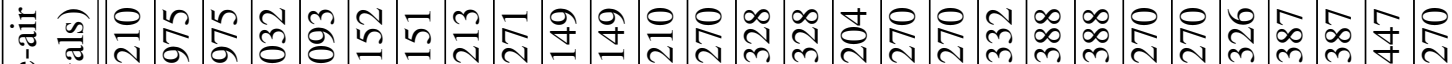

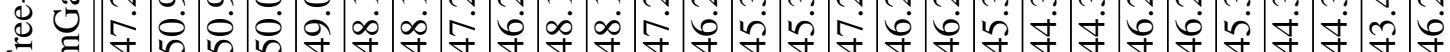
童 ठํ.

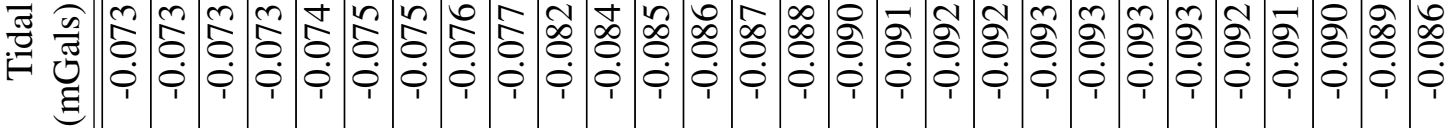

రํ.

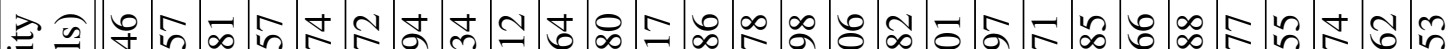
خ

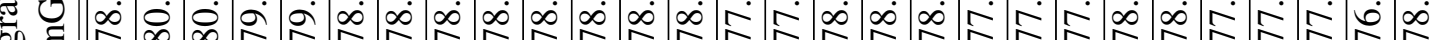

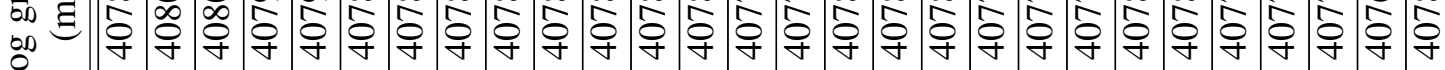
으

航

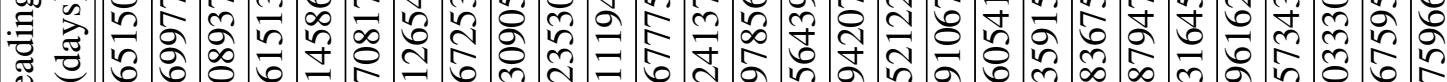
உ

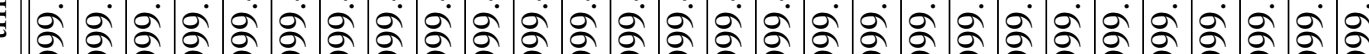
б。

包 च $=0.0$ ळ

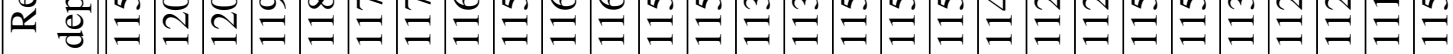
م. 正 


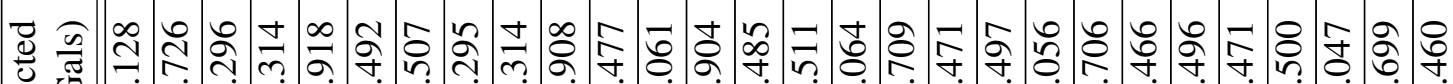
送

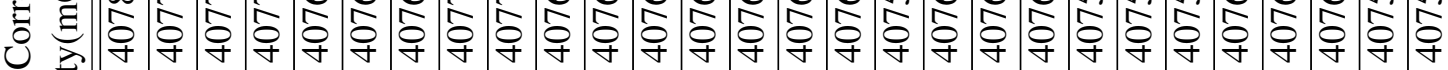

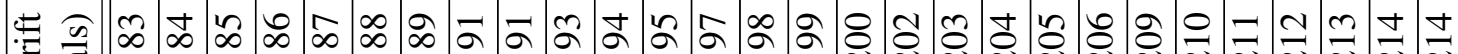

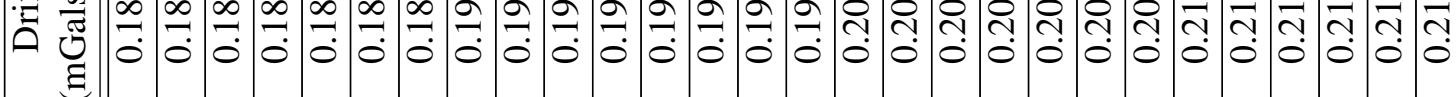

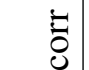

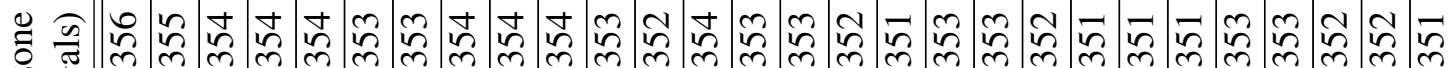

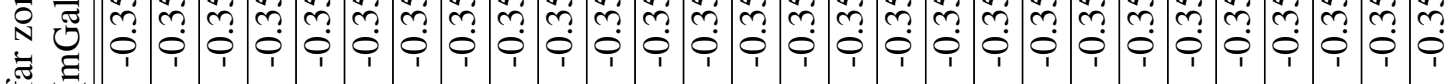
竞

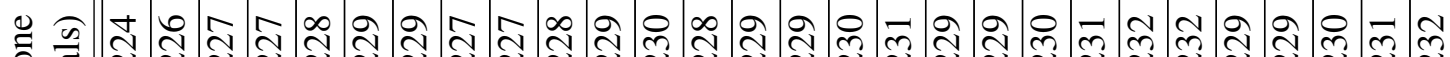
व $\stackrel{1}{=}$ ๘ $\mathrm{Z} \cup$

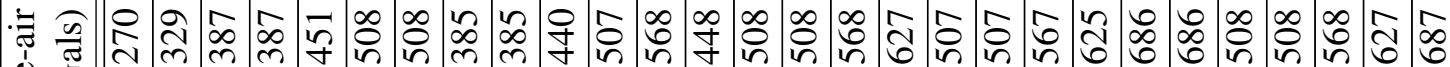
党

¿

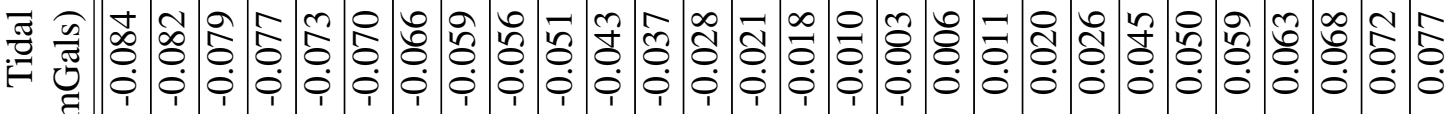
$\Xi$

ठํ

: 菊

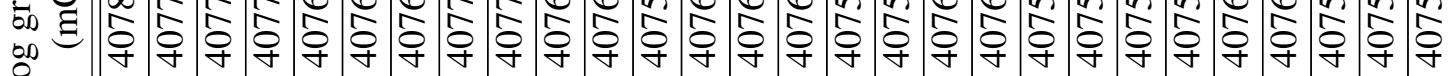
으

\begin{tabular}{|c|c|c|c|c|c|c|c|c|c|c|c|c|c|c|c|c|c|c|c|c|c|c|c|c|c|c|c|c|}
\hline 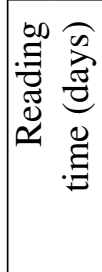 & 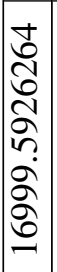 & 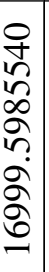 & 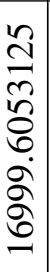 & 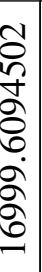 & 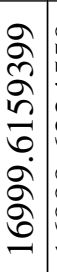 & 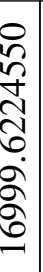 & 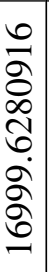 & $\begin{array}{l}0 \\
0 \\
\hat{2} \\
\dot{2} \\
2 \\
\hat{\sigma} \\
\end{array}$ & 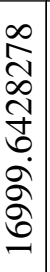 & 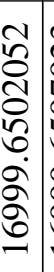 & 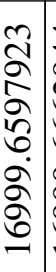 & 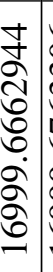 & 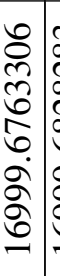 & 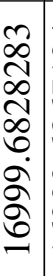 & 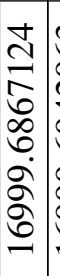 & שర & & $\begin{array}{l}g \\
g \\
g \\
g \\
g\end{array}$ & & 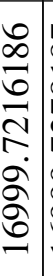 & $\begin{array}{l}\stackrel{2}{\circ} \\
\text { बे }\end{array}$ & 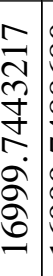 & 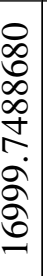 & פ̇. & 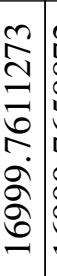 & 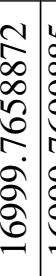 & 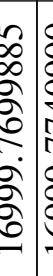 & 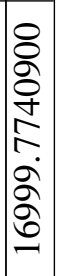 \\
\hline 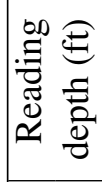 & $\begin{array}{l}8 \\
8 \\
0 \\
i \\
= \\
=\end{array}$ & হু & $\begin{array}{l}a \\
a \\
\grave{a} \\
=\end{array}$ & $\begin{array}{l}\frac{a}{a} \\
\vec{a} \\
\grave{2}\end{array}$ & $\begin{array}{l}\bar{\Xi} \\
0 \\
\dot{\delta} \\
\vdots \\
=\end{array}$ & $\begin{array}{l}8 \\
8 \\
0 \\
\vdots \\
= \\
\end{array}$ & $\begin{array}{l}8 \\
8 \\
0 \\
\vdots \\
=\end{array}$ & $\begin{array}{l}\infty \\
2 \\
\vdots \\
\\
=\end{array}$ & $\begin{array}{l}\infty \\
2 \\
2 \\
\\
=\end{array}$ & $\begin{array}{l}8 \\
ٌ \\
a \\
\varrho \\
=\end{array}$ & $\begin{array}{l}8 \\
\delta \\
2 \\
\delta \\
=\end{array}$ & $\begin{array}{l}8 \\
8 \\
\dot{8} \\
0 \\
=\end{array}$ & $\begin{array}{l}8 \\
2 \\
2 \\
= \\
=\end{array}$ & $\begin{array}{l}8 \\
8 \\
\dot{0} \\
= \\
=\end{array}$ & $\begin{array}{l}8 \\
8 \\
0 \\
0 \\
= \\
=\end{array}$ & 8 & & 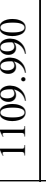 & $\underline{8}$ & $\begin{array}{l}8 \\
\delta \\
2 \\
\delta\end{array}$ & $\begin{array}{l}\hat{\sigma} \\
\varrho \\
\grave{a} \\
0 \\
0\end{array}$ & $\begin{array}{l}8 \\
2 \\
\hat{\sigma} \\
\hat{0}\end{array}$ & 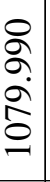 & & $\begin{array}{l}8 \\
8 \\
0 \\
\vdots \\
=\end{array}$ & \begin{tabular}{l|l}
8 & \\
8 & \\
$\dot{8}$ & \\
$=$ &
\end{tabular} & 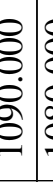 & $\begin{array}{l}\dot{0} \\
\infty \\
0\end{array}$ \\
\hline$\frac{0}{12} \ominus$ & م & م & مـ & مـ & مـ & مـ & مـ & م & م & مـ & مـ & م & مـ & م & م & م & e & مـ & م & م & م & م & مـ & م. & م & . . م. & . . م. & 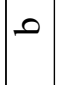 \\
\hline 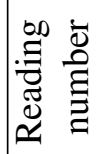 & $\underline{=}$ & I & $\infty$ & 9 & 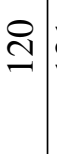 & $\vec{\beth}$ & ป & $\stackrel{\Xi}{\beth}$ & $\stackrel{\Omega}{\simeq}$ & ㄴ) & $\widehat{\simeq}$ & 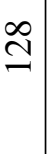 & ปे & $\stackrel{\circ}{-}$ & $\bar{n}$ & 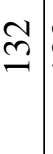 & & $\dot{m}$ & & $\stackrel{0}{2}$ & $\hat{n}$ & 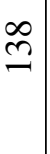 & ले & $\bar{\nabla}$ & $\stackrel{\mathcal{I}}{ \pm}$ & $\stackrel{m}{ \pm}$ & 过 & $\stackrel{\curvearrowleft}{ \pm}$ \\
\hline
\end{tabular}




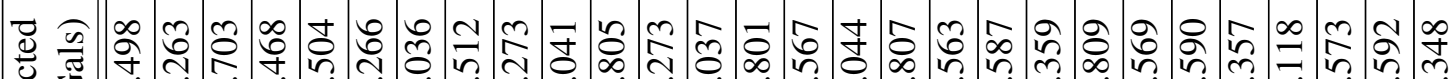
d 整

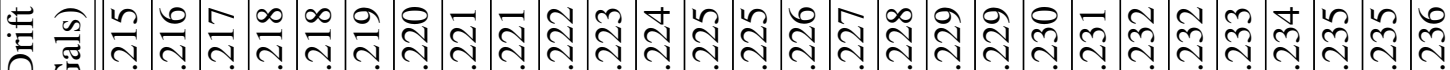

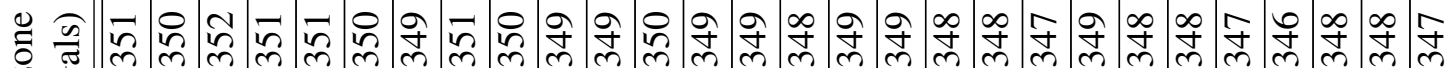

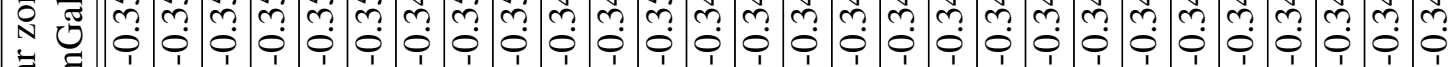
苛

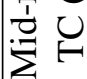

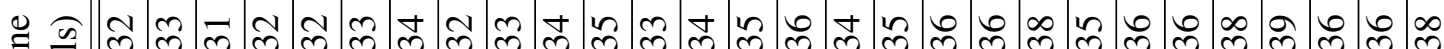

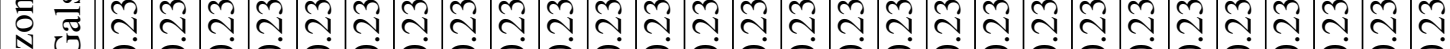

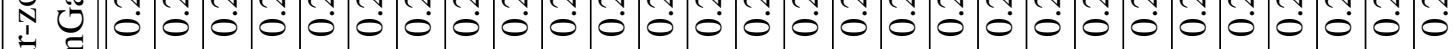
ซี Z

\begin{tabular}{|c|c|c|c|c|c|c|c|c|c|c|c|c|c|c|c|c|c|c|c|c|c|c|c|}
\hline 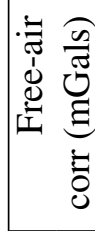 & $\begin{array}{l}\infty \\
0 \\
0 \\
\infty \\
\tilde{n}\end{array}$ & $\begin{array}{c}\tilde{J} \\
\underset{\sim}{\infty} \\
\infty \\
m\end{array}$ & 8 & $\begin{array}{l}\hat{\infty} \\
0 \\
\dot{\sigma} \\
\tilde{m}\end{array}$ & $\begin{array}{l}\text { ld } \\
\text { c }\end{array}$ & 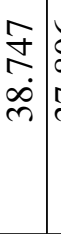 & $\begin{array}{c}\infty \\
\infty \\
m \\
m \\
m\end{array}$ & r & $\underset{\sim}{\infty}$ & $\begin{array}{l}0 \\
\vdots \\
0 \\
0 \\
0 \\
0 \\
0\end{array}$ & 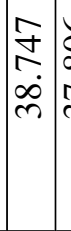 & 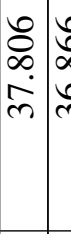 & 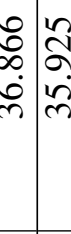 & 㐫 & 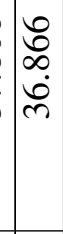 & $\begin{array}{c}\tilde{\sigma} \\
\tilde{n} \\
\tilde{n}\end{array}$ & & $\begin{array}{l}0 \\
\dot{0} \\
\dot{0}\end{array}$ & 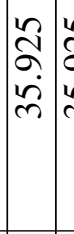 & $\begin{array}{l}\text { ๙े } \\
\text { n் }\end{array}$ & $\begin{array}{l}\dot{v} \\
\dot{b} \\
\dot{d}\end{array}$ & $\begin{array}{l}\hat{\sigma} \\
\tilde{n} \\
\tilde{n}\end{array}$ & \\
\hline 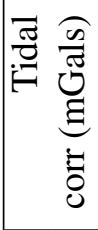 & $\begin{array}{l}0 \\
\infty \\
0 \\
0 \\
0\end{array}$ & $\begin{array}{l}n \\
\infty \\
0 \\
0 \\
0\end{array}$ & $\bar{c}$ & $\begin{array}{l} \pm \\
\dot{o} \\
\dot{0}\end{array}$ & $\begin{array}{l}\infty \\
\stackrel{\infty}{o} \\
0 \\
0\end{array}$ & $\begin{array}{l}\overline{0} \\
\dot{0} \\
0\end{array}$ & $\begin{array}{ll}\stackrel{c}{0} \\
\vdots \\
0\end{array}$ & $\frac{e}{2} \stackrel{0}{=}$ & 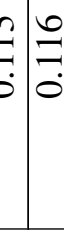 & $\stackrel{9}{\Xi}$ & 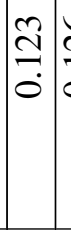 & 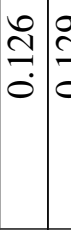 & 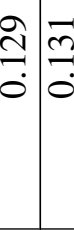 & $\stackrel{\vec{m}}{\stackrel{+}{\circ}}$ & $\frac{n}{0}$ & $\frac{\tilde{m}}{0}$ & $\stackrel{\vec{m}}{\overrightarrow{0}} \frac{O}{0}$ & 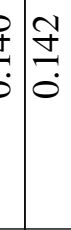 & $\stackrel{m}{\dot{\sigma}}$ & 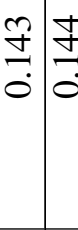 & $\frac{J}{\square}$ & 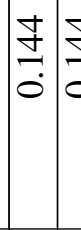 & $\begin{array}{l} \pm \\
\\
\end{array}$ \\
\hline 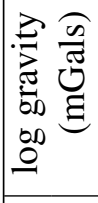 & 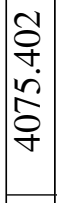 & 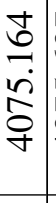 & $\begin{array}{l}\hat{\delta} \\
\delta \\
\dot{n} \\
\hat{\delta} \\
\dot{y}\end{array}$ & $\begin{array}{l}0 \\
0 \\
n \\
n \\
\delta \\
\dot{y}\end{array}$ & & 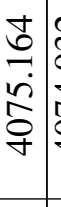 & 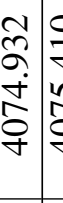 & 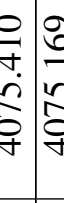 & 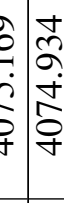 & 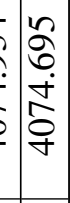 & 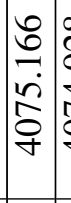 & 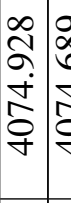 & 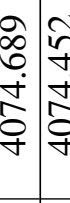 & 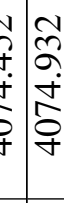 & 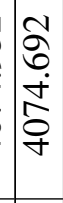 & 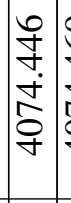 & 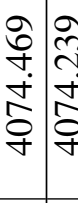 & 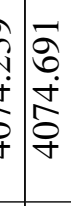 & 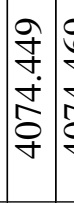 & 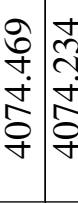 & $\begin{array}{l}\alpha \\
\delta \\
\alpha \\
\tilde{r} \\
\delta \\
\sigma\end{array}$ & 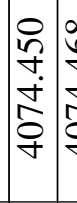 & 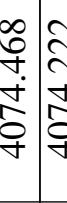 \\
\hline 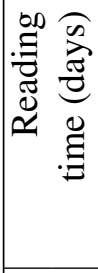 & $\begin{array}{l}\infty \\
i \\
i \\
N \\
i \\
\hat{N} \\
2 \\
2 \\
\delta \\
0 \\
-\end{array}$ & 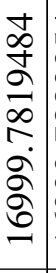 & 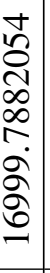 & 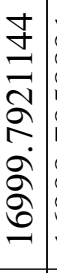 & $\begin{array}{l}\bar{a} \\
\infty \\
\infty \\
2 \\
2 \\
2 \\
2 \\
0 \\
0\end{array}$ & $\begin{array}{l}0 \\
+ \\
\infty \\
2 \\
2 \\
2 \\
2 \\
2 \\
2 \\
0 \\
- \\
-\end{array}$ & 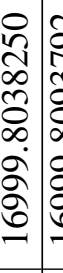 & 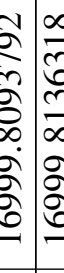 & 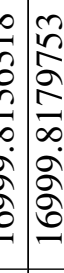 & 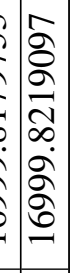 & 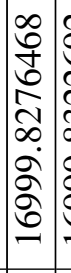 & 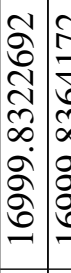 & 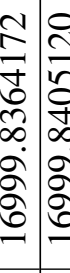 & 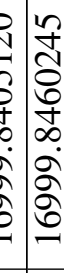 & 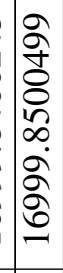 & 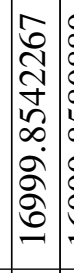 & 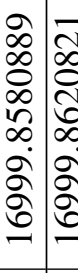 & 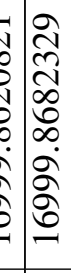 & $\begin{array}{l}\bar{N} \\
\mathbf{N} \\
\mathbf{N} \\
0 \\
2 \\
2 \\
0 \\
0\end{array}$ & 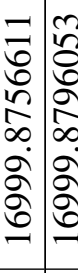 & 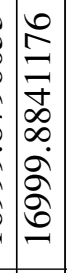 & 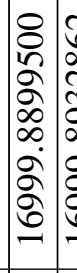 & 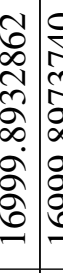 \\
\hline 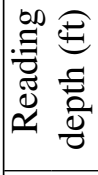 & $\begin{array}{l}8 \\
8 \\
0 \\
0 \\
\infty \\
0 \\
-\end{array}$ & $\begin{array}{l}8 \\
8 \\
0 \\
0 \\
0 \\
\end{array}$ & $\begin{array}{l}8 \\
\varnothing \\
\varnothing \\
\dot{8}\end{array}$ & $\begin{array}{l}8 \\
8 \\
0 \\
\dot{0} \\
0 \\
0\end{array}$ & $\begin{array}{l}8 \\
\varnothing \\
\\
\varnothing\end{array}$ & $\begin{array}{c}8 \\
8 \\
0 \\
0 \\
0 \\
0\end{array}$ & 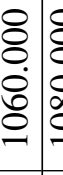 & 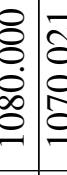 & 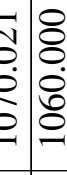 & 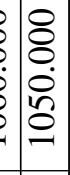 & 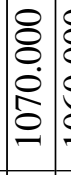 & 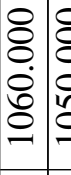 & 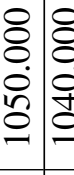 & 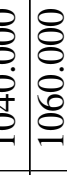 & \begin{tabular}{l}
8 \\
$\vdots$ \\
$\dot{8}$ \\
\hdashline \\
0
\end{tabular} & $\begin{array}{l}0 \\
0 \\
0 \\
\dot{0} \\
0 \\
0\end{array}$ & 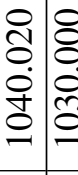 & 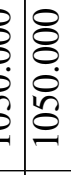 & 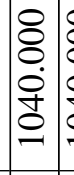 & $\begin{array}{ll}8 & 8 \\
8 & 8 \\
\dot{0} & 0 \\
0 & 0 \\
0 & \end{array}$ & 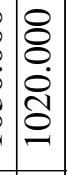 & $\mid$\begin{tabular}{l}
8 \\
$\delta$ \\
$\dot{0}$ \\
$\dot{+}$ \\
\hdashline \\
\hdashline
\end{tabular} & $\begin{array}{l}8 \\
8 \\
0 \\
+ \\
0 \\
\end{array}$ \\
\hline & م & م & م & مـ & م & م & كـ مـ & ك & م م م & م & | & ــ مـ & o 0 & م. & م & . & 0 0 - & م. & . & م 0 م & مـ & . & م \\
\hline 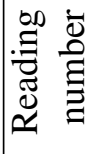 & 号 & 卞 & $\stackrel{\infty}{+\infty}$ & $\stackrel{g}{ \pm}$ & $\stackrel{8}{n}$ & $\sqrt{n}$ & กี & $\stackrel{n}{2}$ & ป $2 \stackrel{2}{2}$ & 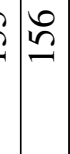 & 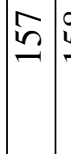 & 必 & $\stackrel{2}{2}$ & 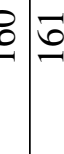 & $\widetilde{\sigma}$ & $\widehat{6}$ & $\underset{0}{t}$ & 6 & $\underline{6}$ & $\stackrel{8}{0}:$ & 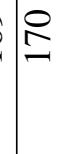 & $\Xi$ & $\stackrel{N}{I}$ \\
\hline
\end{tabular}




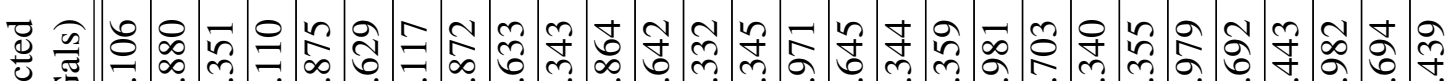
ש

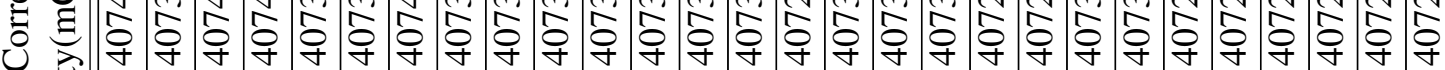

Е चี $\Xi$

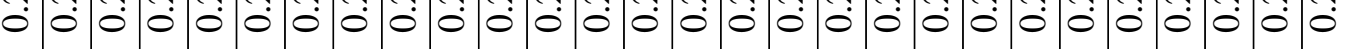

芩 a

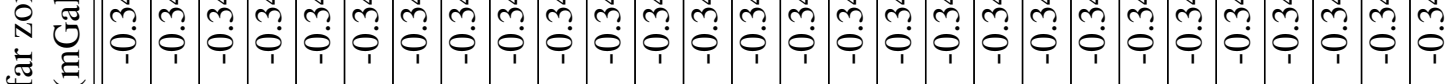

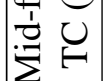

ஜ

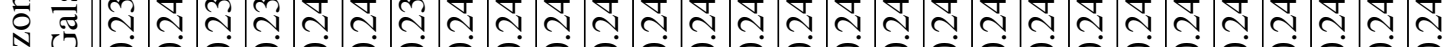
$\stackrel{1}{\ddots}$ ซี Z

\begin{tabular}{|c|c|c|c|c|c|c|c|c|c|c|c|c|c|c|c|c|c|c|c|c|c|c|c|c|c|c|c|c|}
\hline 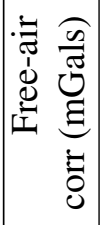 & 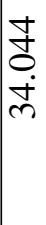 & $\begin{array}{l}\mathscr{0} \\
\dot{0} \\
\dot{m}\end{array}$ & $\begin{array}{l}\infty \\
\infty \\
\sigma \\
\dot{j}\end{array}$ & \begin{tabular}{l}
\multirow{Z}{*}{} \\
0 \\
$\dot{J}$ \\
m.
\end{tabular} & 吉 & $\begin{array}{c}\tilde{\sigma} \\
\\
\tilde{m}\end{array}$ & m & 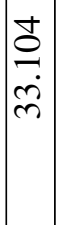 & $\begin{array}{c}\tilde{\sigma} \\
\underset{\sim}{\sim} \\
\tilde{m}\end{array}$ & 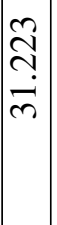 & 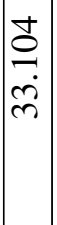 & 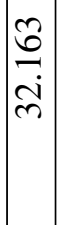 & 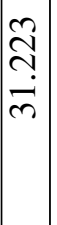 & 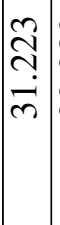 & 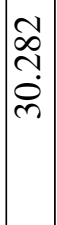 & $\begin{array}{l}\tilde{b} \\
\mathfrak{v} \\
\tilde{n}\end{array}$ & 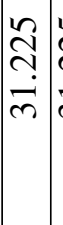 & กิ & $\left|\begin{array}{c}\tilde{N} \\
\infty \\
\\
\tilde{n}\end{array}\right|$ & 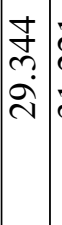 & $\frac{\vec{N}}{\tilde{N}}$ & $\frac{\vec{N}}{\tilde{N}}$ & $\left|\begin{array}{c}N \\
\infty \\
N \\
\vdots \\
\infty\end{array}\right|$ & $\begin{array}{l}\mathcal{N} \\
\tilde{J} \\
\stackrel{\gamma}{\sim}\end{array}$ & $\begin{array}{l}\bar{\delta} \\
\stackrel{+}{*} \\
\infty \\
\sim\end{array}$ & $\left|\begin{array}{c}+ \\
\infty \\
\\
\vdots \\
m\end{array}\right|$ & 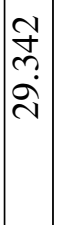 & \begin{tabular}{c}
$\overline{0}$ \\
\multirow{+}{*}{} \\
$\infty$ \\
$\sim$ \\
$\sim$
\end{tabular} \\
\hline 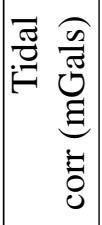 & $\frac{8}{\dot{\Xi}}$ & 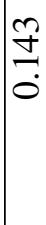 & 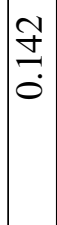 & $\begin{array}{l}\vec{\Xi} \\
\dot{0}\end{array}$ & $\stackrel{0}{\stackrel{0}{ \pm}}$ & $\underset{\infty}{\infty}$ & $\frac{0}{0}$ & $\frac{⿱}{m}$ & $\frac{\tilde{\sigma}}{\tilde{\sigma}}$ & $\frac{0}{\stackrel{m}{0}}$ & $\frac{\widehat{N}}{\dot{0}}$ & 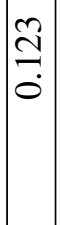 & 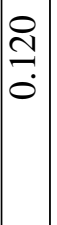 & 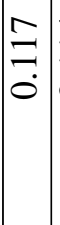 & 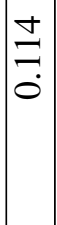 & $\begin{array}{l}8 \\
\stackrel{0}{0}\end{array}$ & $\stackrel{n}{0}$ & \begin{tabular}{c}
$\mathcal{O}$ \\
\hdashline \\
$\dot{0}$
\end{tabular} & \begin{tabular}{|l}
$\infty$ \\
0 \\
0 \\
0 \\
0
\end{tabular} & $\begin{array}{l}\dot{J} \\
\stackrel{2}{0} \\
\dot{0}\end{array}$ & $\begin{array}{l}2 \\
\infty \\
0 \\
0\end{array}$ & $\begin{array}{l}2 \\
\infty \\
0 \\
0 \\
0\end{array} \mid$ & $\begin{array}{c}0 \\
\infty \\
0 \\
0 \\
0\end{array}$ & $\begin{array}{c}0 \\
0 \\
0\end{array}$ & 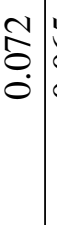 & $\mid \begin{array}{l}n \\
0 \\
0 \\
0 \\
0\end{array}$ & \begin{tabular}{l}
$\overline{8}$ \\
\hdashline \\
$\dot{0}$
\end{tabular} & ڤ̊ \\
\hline 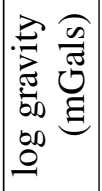 & 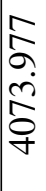 & 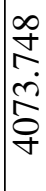 & 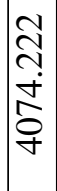 & $\begin{array}{l}a \\
\hat{\sigma} \\
\hat{n} \\
\hat{S} \\
\dot{y}\end{array}$ & 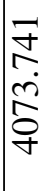 & $\begin{array}{l}\tilde{\sigma} \\
\dot{\gamma} \\
\tilde{\sigma} \\
\tilde{g}\end{array}$ & $\begin{array}{l}2 \\
\infty \\
\sigma \\
\tilde{N} \\
\hat{\sigma} \\
\gamma\end{array}$ & $\begin{array}{l}0 \\
\tilde{r} \\
\dot{r} \\
\tilde{\sigma} \\
\dot{q}\end{array}$ & 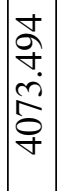 & 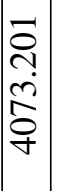 & 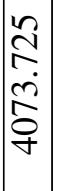 & 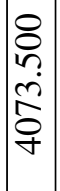 & 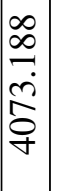 & 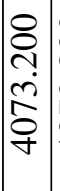 & 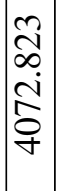 & 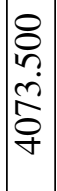 & 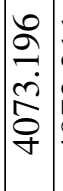 & 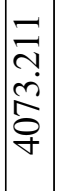 & 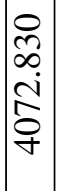 & 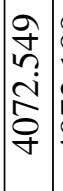 & 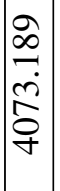 & 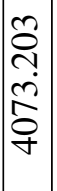 & 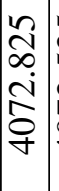 & 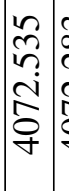 & 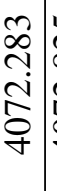 & 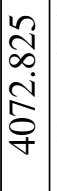 & 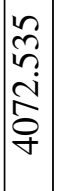 & 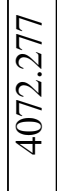 \\
\hline 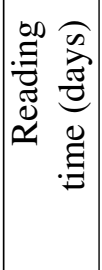 & 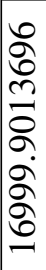 & 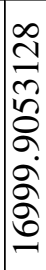 & 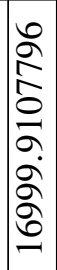 & 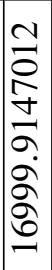 & 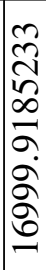 & 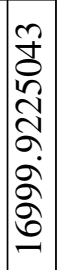 & 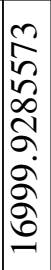 & $\begin{array}{l}n \\
\tilde{n} \\
\tilde{n} \\
\tilde{\sigma} \\
\sigma \\
\sigma \\
\sigma \\
\sigma\end{array}$ & 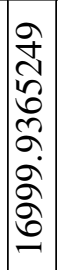 & 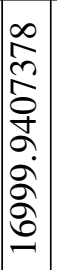 & 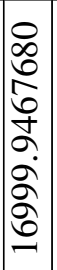 & 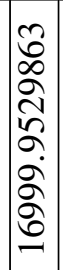 & 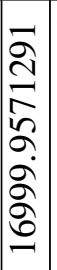 & 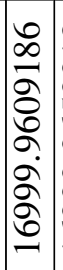 & 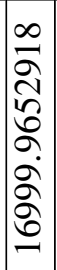 & $\begin{array}{l}\bar{\sigma} \\
\infty \\
\sigma \\
\hat{\sigma} \\
\hat{\sigma} \\
\Omega \\
\sigma \\
\sigma\end{array}$ & 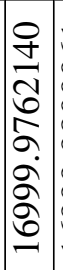 & $\begin{array}{l}\bar{\delta} \\
\delta \\
\delta \\
\delta \\
\infty \\
\delta \\
\delta \\
\delta \\
\delta\end{array}$ & 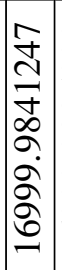 & 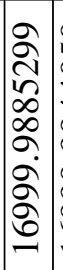 & 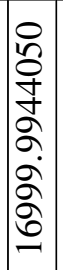 & 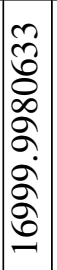 & 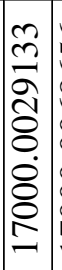 & 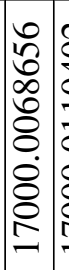 & 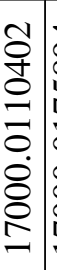 & 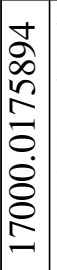 & 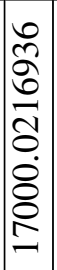 & 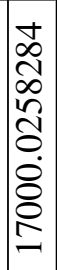 \\
\hline 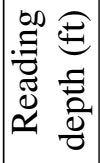 & 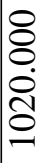 & $\begin{array}{l}0 \\
0 \\
0 \\
0 \\
0\end{array}$ & $\begin{array}{l}\bar{m} \\
0 \\
0 \\
0 \\
0\end{array}$ & 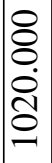 & $\begin{array}{l}8 \\
8 \\
0 \\
0 \\
0\end{array}$ & 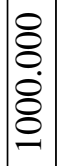 & 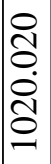 & $\begin{array}{l}8 \\
8 \\
0 \\
0 \\
0 \\
0\end{array}$ & \begin{tabular}{l}
8 \\
8 \\
0 \\
$\dot{8}$ \\
8 \\
\hdashline
\end{tabular} & $\begin{array}{l}8 \\
8 \\
8 \\
8 \\
\delta \\
2\end{array}$ & $\begin{array}{l}8 \\
8 \\
0 \\
0 \\
0 \\
0\end{array}$ & \begin{tabular}{l}
8 \\
$\varnothing$ \\
8 \\
$\stackrel{8}{8}$ \\
$\varnothing$ \\
\hdashline
\end{tabular} & 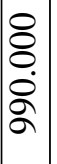 & 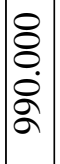 & 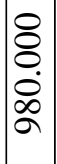 & $\begin{array}{l}8 \\
8 \\
8 \\
8 \\
8 \\
\varrho\end{array}$ & $\begin{array}{l}\bar{ত} \\
\delta \\
\dot{\delta} \\
\delta\end{array}$ & $\begin{array}{l}\overline{\text { ㄱ}} \\
\delta \\
\check{\delta} \\
\Omega\end{array}$ & $\begin{array}{l}\text { ஓ } \\
\delta \\
\dot{\delta} \\
\infty \\
\varnothing\end{array}$ & $\begin{array}{l}\bar{\sigma} \\
0 \\
\dot{0} \\
a\end{array}$ & $\begin{array}{l}a \\
a \\
2 \\
\infty \\
a\end{array}$ & $\begin{array}{l}a \\
a \\
2 \\
\infty \\
a\end{array}$ & 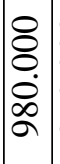 & 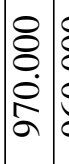 & 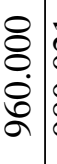 & $\begin{array}{l}\bar{N} \\
\delta \\
\dot{0} \\
\infty \\
\sigma\end{array}$ & 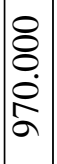 & \begin{tabular}{l}
8 \\
8 \\
0 \\
0 \\
0 \\
\hdashline
\end{tabular} \\
\hline$\stackrel{0}{\frac{1}{2}} \Theta$ & م & م & ص & مـ & م & م & م & م & م & م & مـ & م & م & مـ & م & م & م & م & م & م & م & مـ & م & - & . & م & مـ & م \\
\hline 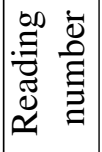 & $r$ & $\stackrel{n}{\sim}$ & $\stackrel{0}{2}$ & $\approx$ & $\stackrel{\infty}{\Sigma}$ & 2 & $\infty$ & $\infty$ & 스 & $\infty$ & $\stackrel{+}{\infty}$ & $\stackrel{\infty}{\infty}$ & $\infty$ & $\infty$ & $\begin{array}{l}\infty \\
\infty\end{array}$ & ळ & § & $\bar{\Omega}$ & ๙ & ă & 孛 & ๙2 & 은 & $\hat{a}$ & $\stackrel{\infty}{\varrho}$ & 2) & 尺্ণ & $\overline{\mathrm{N}}$ \\
\hline
\end{tabular}




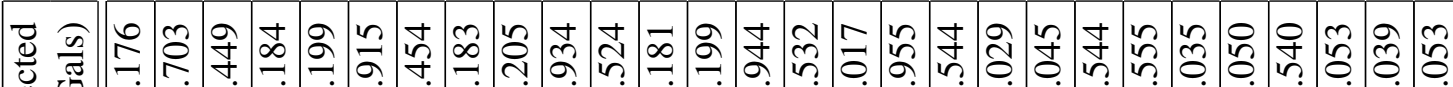

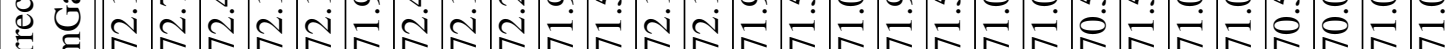

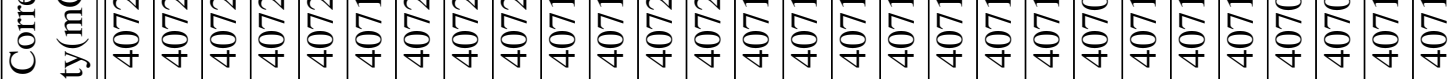

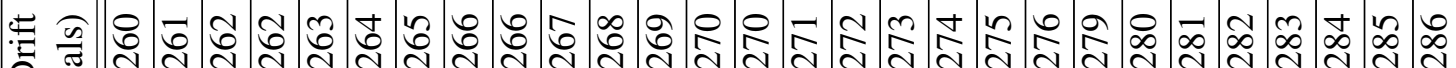
मी ठ․

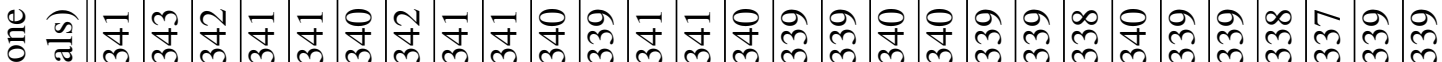

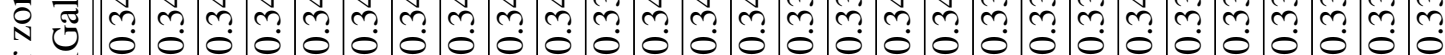
$\exists$ 皇

:

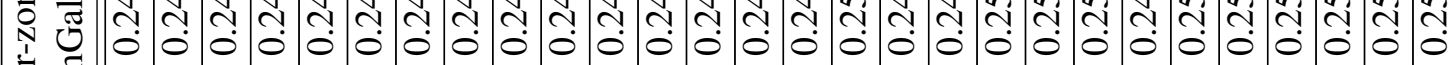
๘ Z U

\begin{tabular}{|c|c|c|c|c|c|c|c|c|c|c|c|c|c|c|c|c|c|c|c|c|c|c|c|c|}
\hline 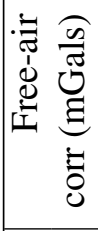 & 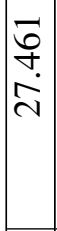 & & 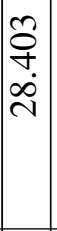 & $\begin{array}{l}m \\
\dot{b} \\
\dot{v} \\
\hat{v}\end{array}$ & ร & & 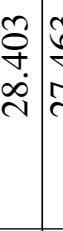 & & 6 & 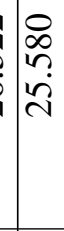 & 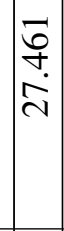 & & & $\begin{array}{l}0 \\
\infty \\
n \\
n \\
\\
\end{array}$ & & 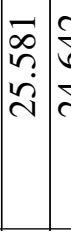 & & & 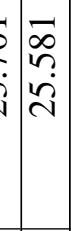 & & & \begin{tabular}{l}
0 \\
\multirow{2}{*}{} \\
\end{tabular} & $\begin{array}{l}\vec{U} \\
\stackrel{+}{d}\end{array}$ & \\
\hline 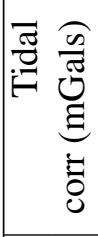 & $\begin{array}{l}\bar{n} \\
0 \\
0 \\
0\end{array}$ & $\begin{array}{l}\text { 只 } \\
0 \\
0\end{array}$ & 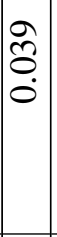 & \begin{tabular}{l}
\multirow{2}{*}{} \\
0 \\
0
\end{tabular} & $\begin{array}{l}0 \\
\tilde{\delta} \\
0 \\
0\end{array}$ & 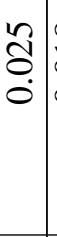 & $\begin{array}{c}\infty \\
\vdots \\
0 \\
0\end{array}$ & $\frac{m}{6}$ & 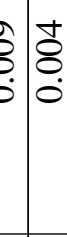 & : & $\mid \begin{array}{l}0 \\
8 \\
0 \\
0 \\
1\end{array}$ & $\mid \begin{array}{l}8 \\
\dot{8} \\
0 \\
0\end{array}$ & 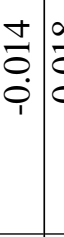 & 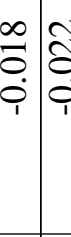 & $\mid \begin{array}{c}\stackrel{\overbrace{}}{0} \\
0 \\
0 \\
1\end{array}$ & 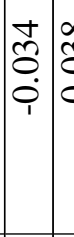 & 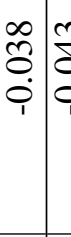 & 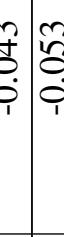 & $\hat{b}$ & 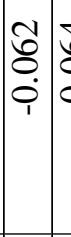 & 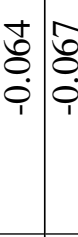 & 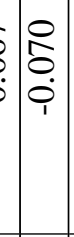 & 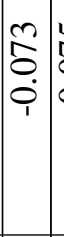 & \\
\hline 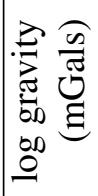 & 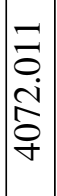 & 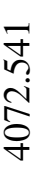 & 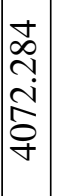 & 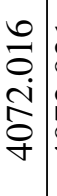 & $\begin{array}{l}\bar{c} \\
\hat{0} \\
\dot{N} \\
\hat{o} \\
+\end{array}$ & 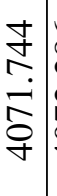 & 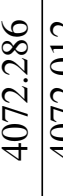 & 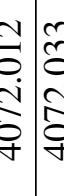 & 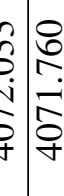 & $\frac{\tilde{f}}{\tilde{T}}$ & $\mid \begin{array}{l}\hat{\delta} \\
0 \\
i \\
\hat{i} \\
\dot{y}\end{array}$ & 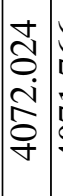 & 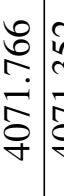 & 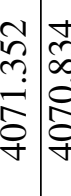 & $\frac{n}{r}$ & 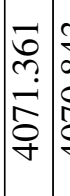 & 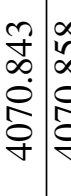 & 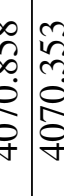 & 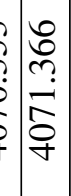 & 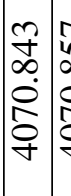 & 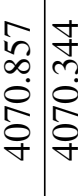 & 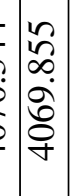 & 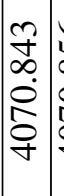 & 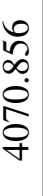 \\
\hline 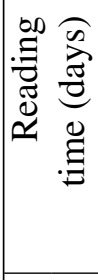 & 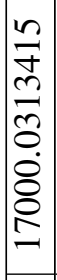 & 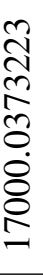 & 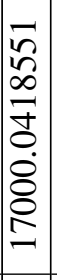 & 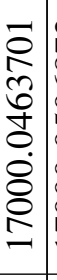 & 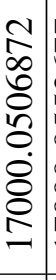 & 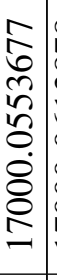 & 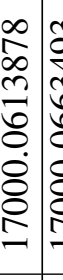 & 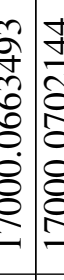 & 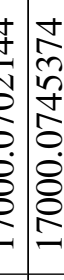 & 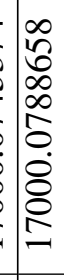 & 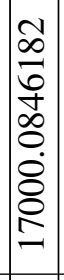 & 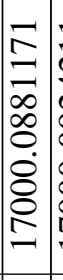 & 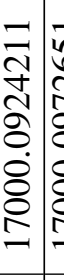 & 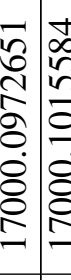 & $\mid \begin{array}{l}\infty \\
\infty \\
n \\
\\
8 \\
- \\
8 \\
8 \\
2 \\
\end{array}$ & 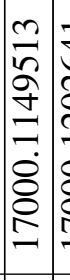 & 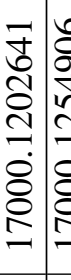 & 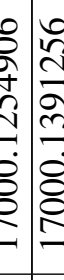 & 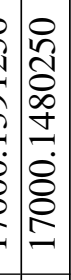 & 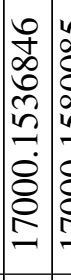 & 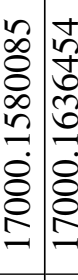 & 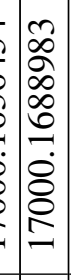 & 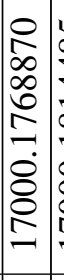 & 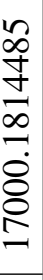 \\
\hline 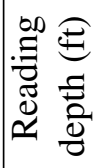 & $\begin{array}{l}8 \\
8 \\
8 \\
\check{n} \\
\swarrow\end{array}$ & $\begin{array}{l}8 \\
8 \\
\vdots \\
\vdots \\
\text { â }\end{array}$ & 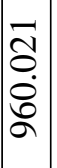 & $\begin{array}{l}\bar{\sigma} \\
0 \\
0 \\
\sigma\end{array}$ & 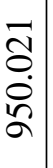 & 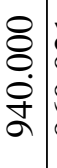 & 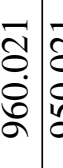 & 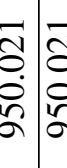 & 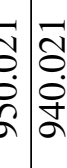 & 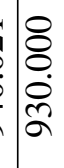 & 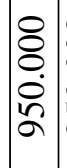 & 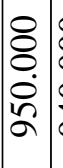 & 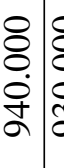 & 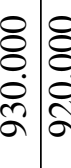 & $\mid \begin{array}{l}0 \\
0 \\
0 \\
\dot{g} \\
\sigma\end{array}$ & \begin{tabular}{l|l}
0 & \\
$\vdots$ & \\
0 & \\
0 & \\
$\sigma$ &
\end{tabular} & 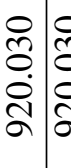 & 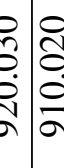 & $\begin{array}{l}0 \\
\dot{0} \\
\dot{0} \\
\vdots \\
\sigma \\
\sigma\end{array}$ & 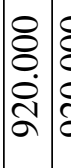 & 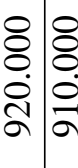 & 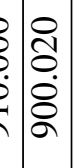 & 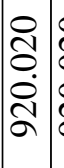 & 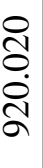 \\
\hline & مـ & مـ & م & مـ & مـ & مـ & كـ مـ & ـ & o & م & مـ & . مـ & ك. & ـ & م & . & ـ مـ & م. & م & ــ مـ & م & مـ| & . مـ & مـ \\
\hline 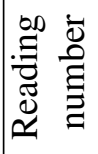 & 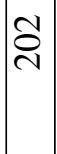 & 气ิ & ষ্ণ & $\stackrel{\overbrace{}}{\overbrace{}}$ & 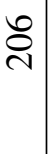 & $\hat{\text { sิ }}$ & 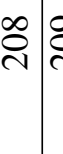 & 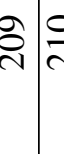 & $\vec{v} \bar{\lambda}$ & $\frac{\sim}{N}$ & $\frac{m}{\sim}$ & $\stackrel{\nabla}{\sim}$ & $\frac{n}{\sim}$ & $\frac{0}{\sim} \frac{}{\pi}$ & $\frac{\infty}{\sim}$ & $\frac{\partial}{\lambda}$ & 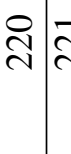 & $\vec{N}$ & 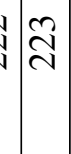 & 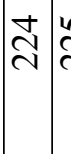 & 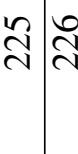 & ন & $\begin{array}{l}\infty \\
\stackrel{\sim}{N}\end{array}$ & సે \\
\hline
\end{tabular}




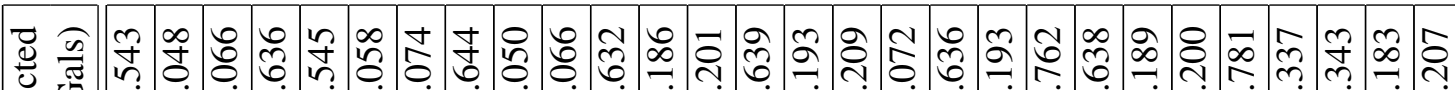
过

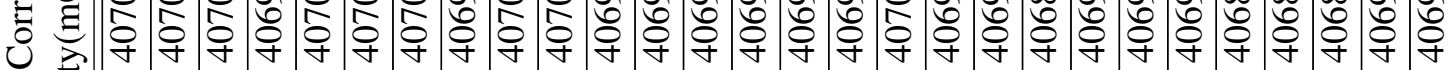

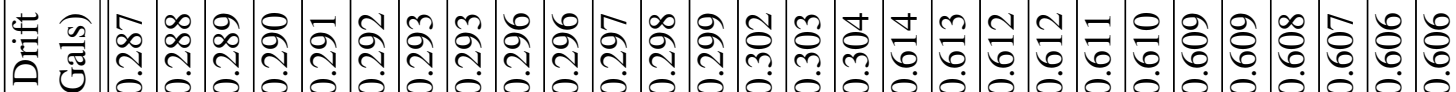

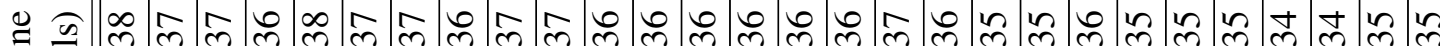
ก

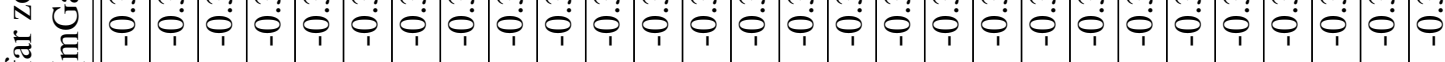
坐 党

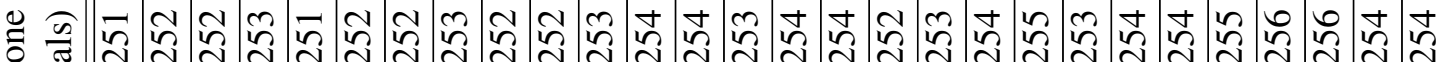

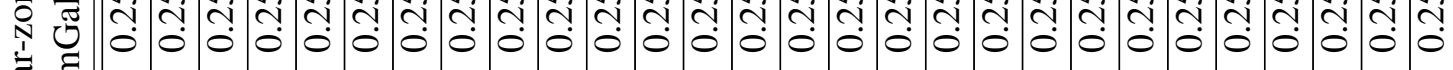
刍 Z

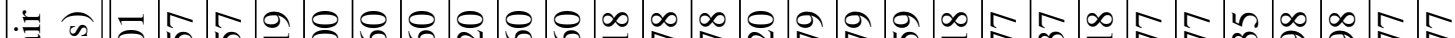

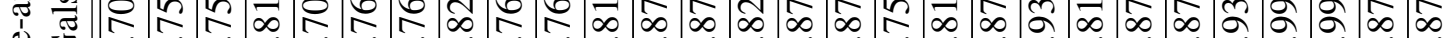

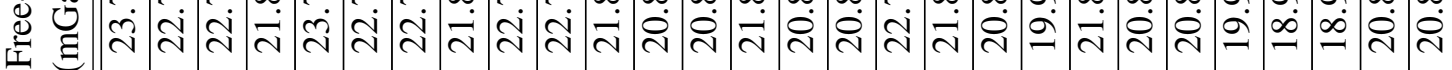
8.

플

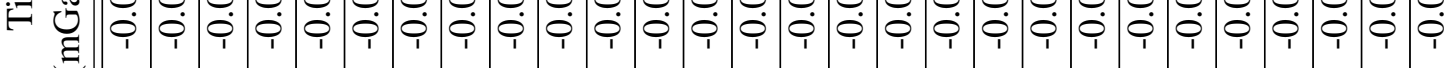
8ั

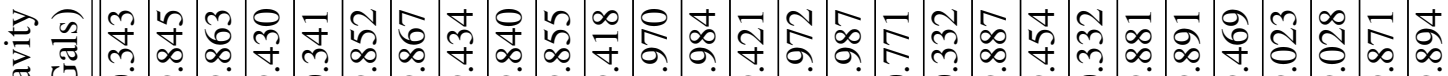
ธ ๘

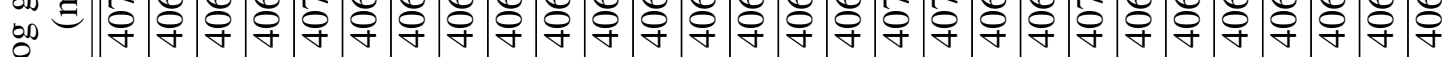

舫

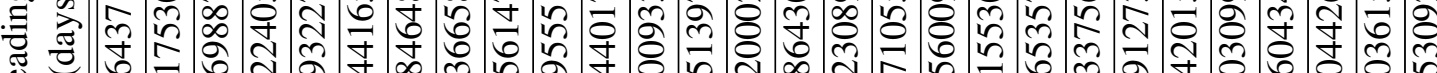

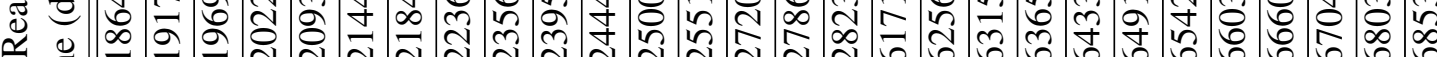

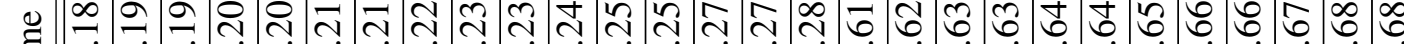
ઇ

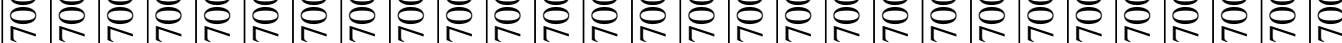

. च «

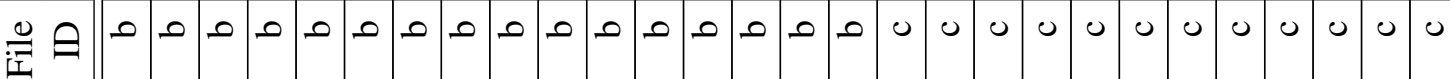

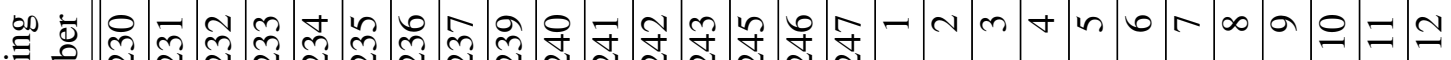
ซ్. 


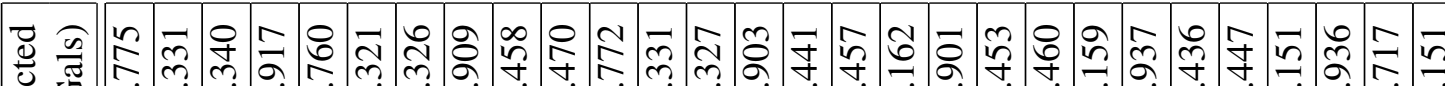
包

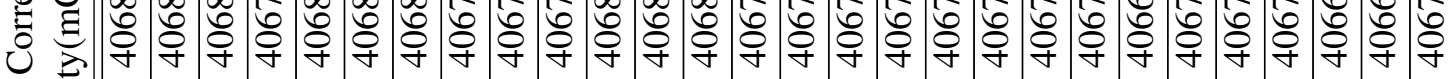

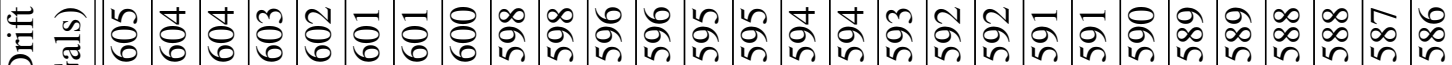
-

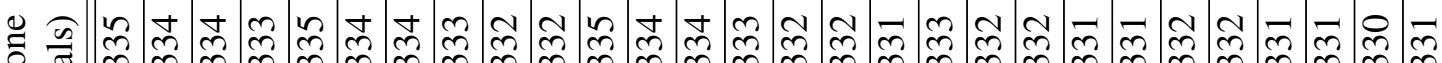

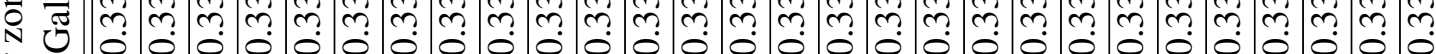
类 皇

๖

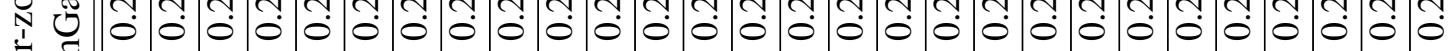
ส Z

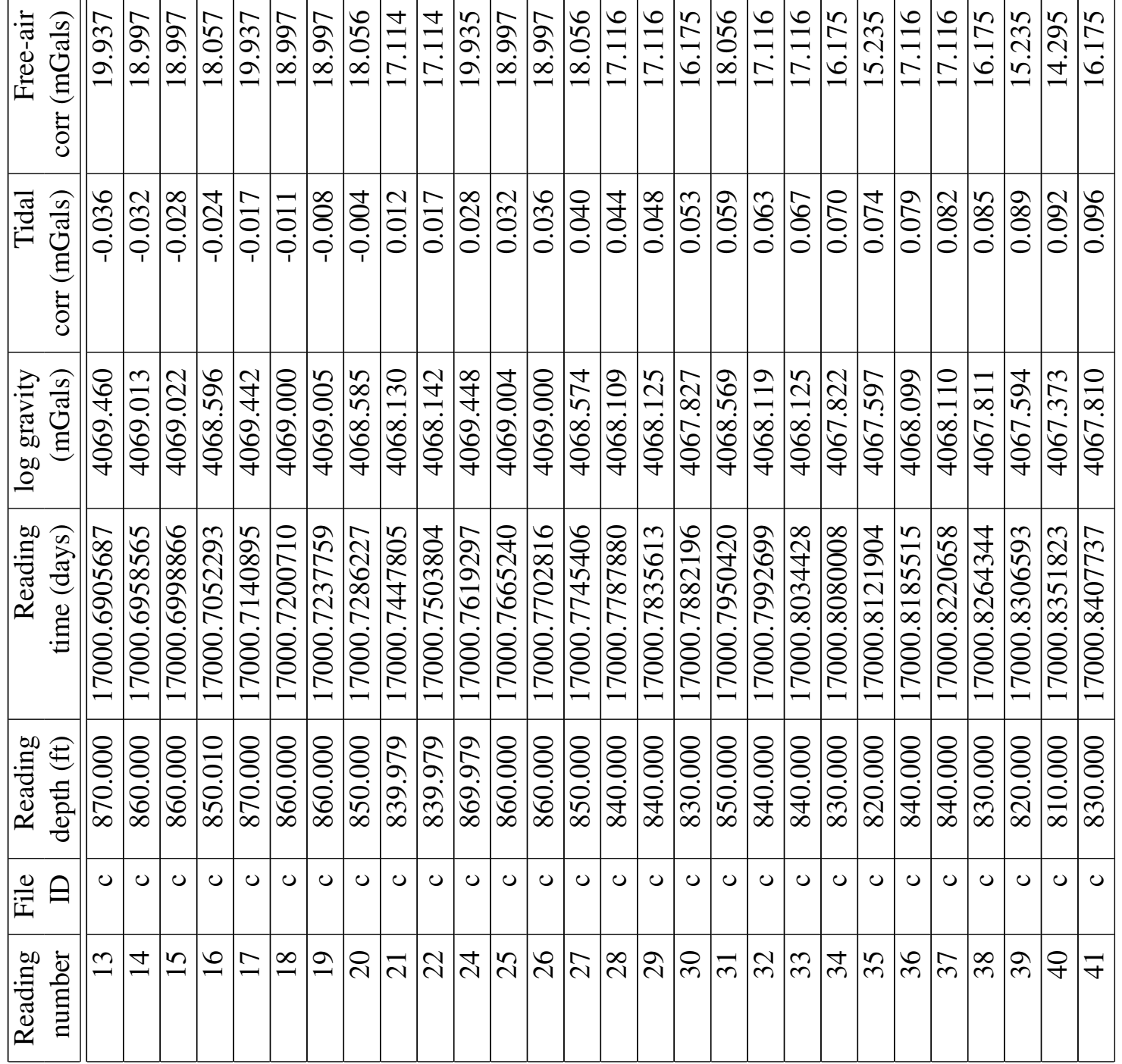




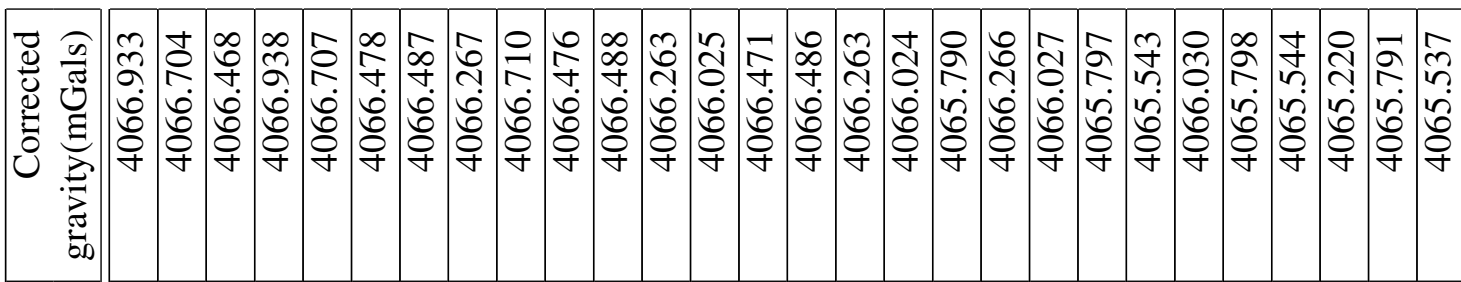

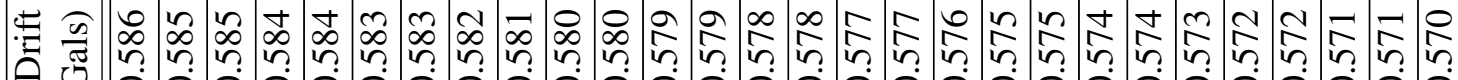
¿

च

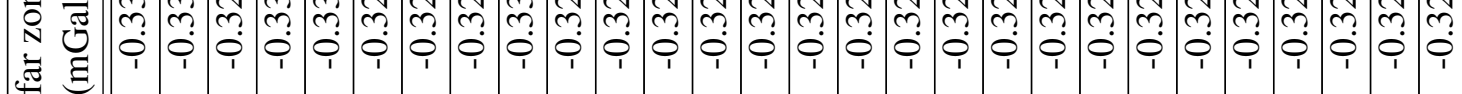

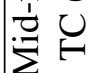

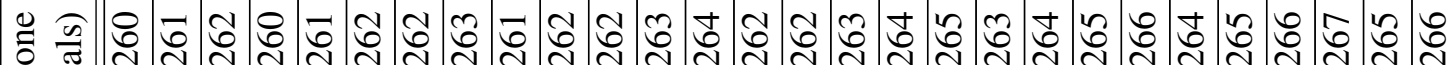

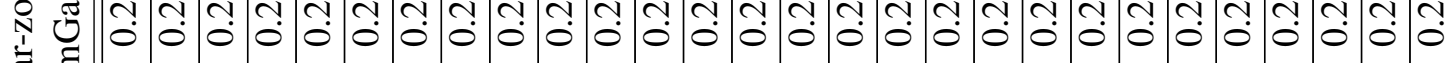
₹ Z

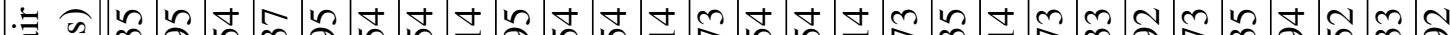

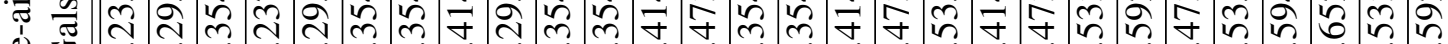

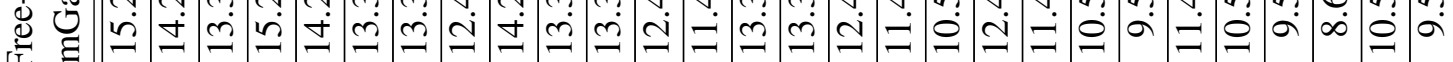
ठั

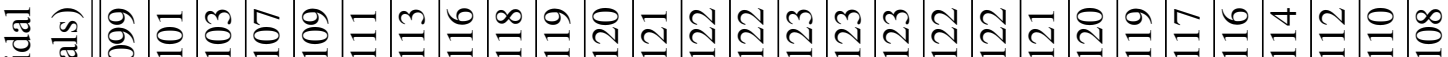

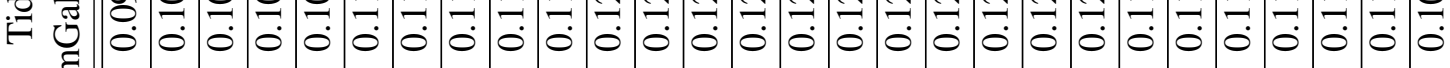
.

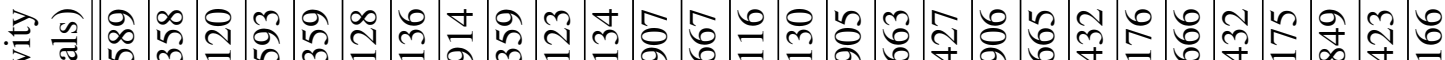
๘ 更 으

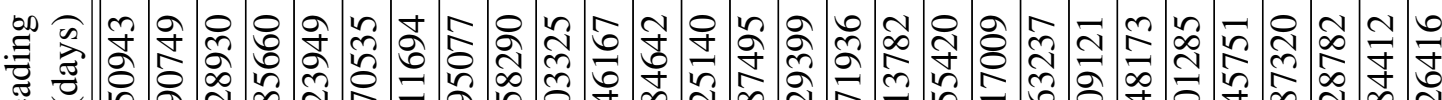

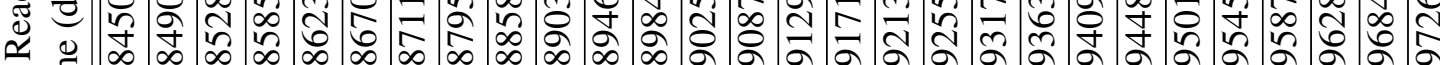

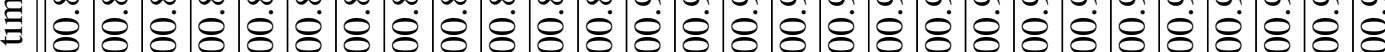

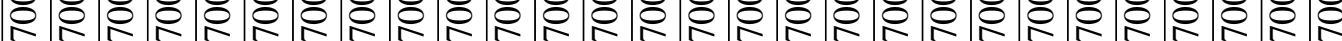

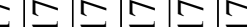

. ब च

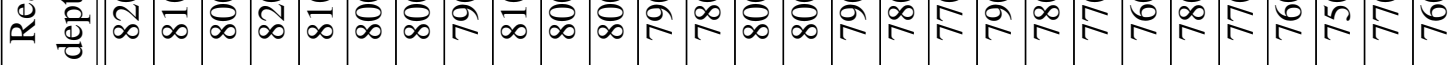
$\stackrel{\oplus}{=} \theta$ $\stackrel{00}{\Xi}$

. 


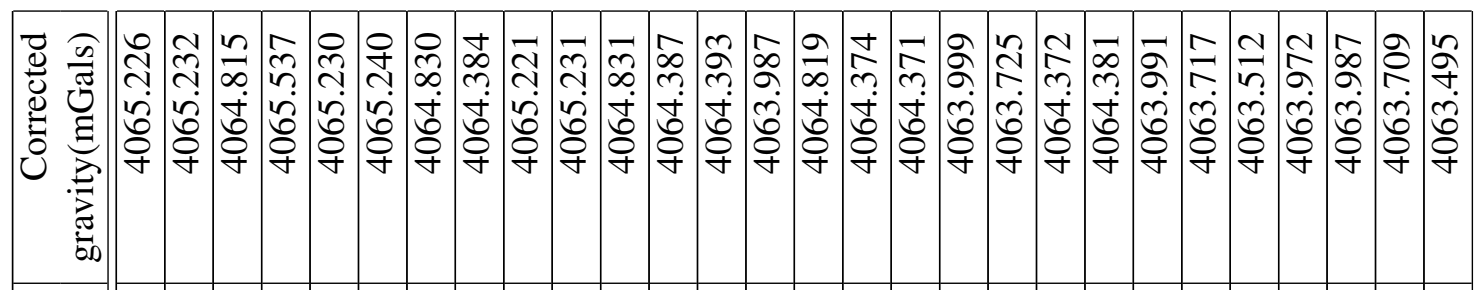

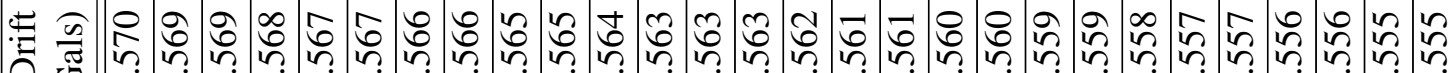
西

z

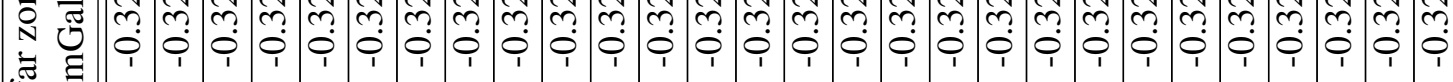
쿄

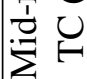

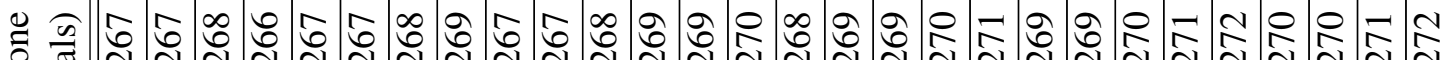
年 ‡

Z U

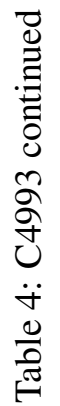

\begin{tabular}{|c|c|c|c|c|c|c|c|c|c|c|c|c|c|c|c|c|c|c|c|c|c|c|c|c|c|}
\hline 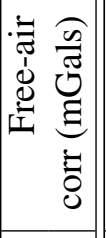 & $\mid \begin{array}{c}0 \\
n \\
6 \\
\infty \\
\infty\end{array}$ & & $\frac{\pi}{\pi}$ & & $\mid \begin{array}{c}n \\
0 \\
\infty \\
\infty\end{array}$ & & & $\underset{\sigma}{\tilde{R}}$ & & & & & $\dot{b}$ & $\stackrel{乛}{\sim}$ & $\frac{\hat{\sigma}}{6}$ & & & 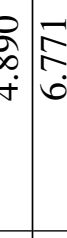 & $\frac{\pi}{\sigma}$ & $\begin{array}{l}0 \\
\infty \\
\dot{n}\end{array}$ & $\begin{array}{l}\infty \\
\infty \\
\dot{\forall}\end{array}$ & 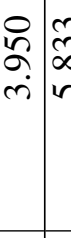 & $\dot{i}$ & & $\hat{\sigma}$ \\
\hline 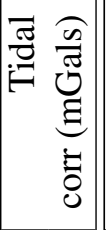 & $\frac{8}{0}$ & $\frac{n}{0}$ & $\frac{8}{0}$ & $\begin{array}{l}0 \\
8 \\
0 \\
0\end{array}$ & $\begin{array}{l}\mathscr{2} \\
\dot{o} \\
0\end{array}$ & $\begin{array}{l}8 \\
8 \\
0 \\
0\end{array}$ & $\begin{array}{l}\hat{\infty} \\
0 \\
0 \\
0\end{array}$ & 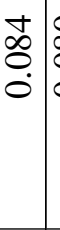 & \begin{tabular}{l|l}
0 & 1 \\
0 & \\
0 & \\
0 &
\end{tabular} & 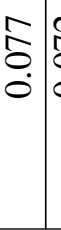 & 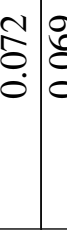 & 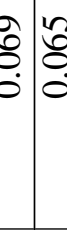 & : & ஜ̊ & $\begin{array}{l}1 \\
\tilde{0} \\
0 \\
0\end{array}$ & $\underset{0}{0}$ & 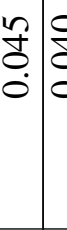 & $\begin{array}{l}0 \\
\stackrel{0}{0} \\
\stackrel{0}{0}\end{array}$ & סृ. & 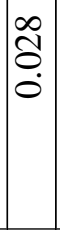 & 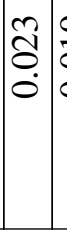 & $\frac{a}{0} \cdot \frac{1}{c}$ & $\stackrel{8}{8}$ & & $\frac{1}{8}$ \\
\hline 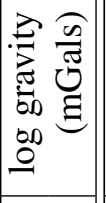 & 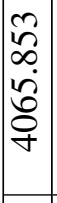 & $\begin{array}{l}0 \\
2 \\
\infty \\
n \\
0 \\
\delta \\
y\end{array}$ & 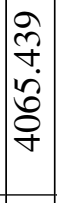 & 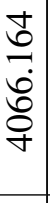 & 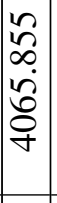 & 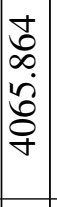 & 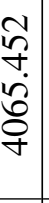 & 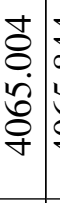 & 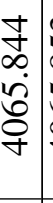 & $\begin{array}{ll}n & \\
n & \\
\infty & \\
\dot{2} & \\
\delta & \\
\vdots & \end{array}$ & 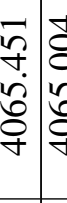 & 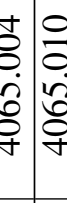 & 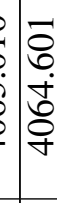 & 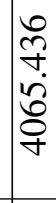 & $\begin{array}{l}2 \\
2 \\
o \\
\dot{d} \\
\dot{+}\end{array}$ & $\begin{array}{l}0 \\
\infty \\
\alpha \\
\dot{d} \\
\delta \\
\dot{y}\end{array}$ & 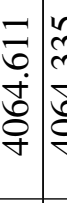 & 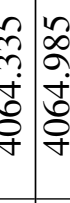 & $\begin{array}{l}0 \\
\sigma \\
\dot{\delta} \\
\delta \\
\dot{+}\end{array}$ & $\begin{array}{l}\bar{\delta} \\
\delta \\
\dot{d} \\
o \\
\dot{q}\end{array}$ & 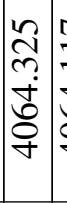 & 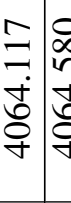 & 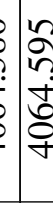 & & $\left\{\begin{array}{l}\infty \\
8 \\
8 \\
\dot{8} \\
\delta\end{array}\right.$ \\
\hline 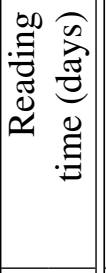 & 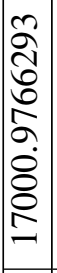 & $\begin{array}{l}2 \\
8 \\
0 \\
\delta \\
\infty \\
0 \\
8 \\
8\end{array}$ & $\begin{array}{l}n \\
\hat{0} \\
\infty \\
2 \\
2 \\
0 \\
0 \\
8 \\
0 \\
1 \\
\end{array}$ & 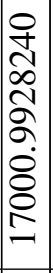 & 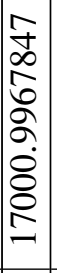 & $\begin{array}{l}0 \\
2 \\
0 \\
8 \\
8 \\
0 \\
\dot{8} \\
8 \\
2 \\
-1\end{array}$ & $\begin{array}{l}2 \\
0 \\
0 \\
0 \\
\vdots \\
\dot{8} \\
\vdots \\
\end{array}$ & 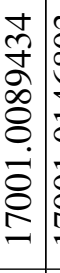 & $\begin{array}{l}0 \\
0 \\
\infty \\
0 \\
+ \\
0 \\
\vdots \\
\delta \\
\delta\end{array}$ & 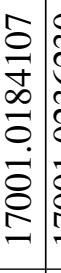 & 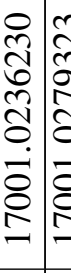 & 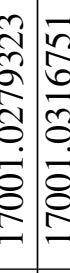 & 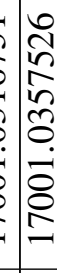 & 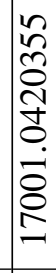 & 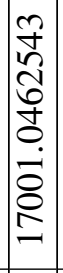 & 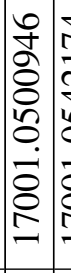 & 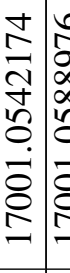 & 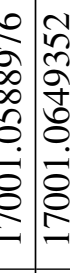 & 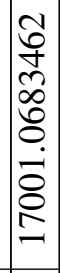 & 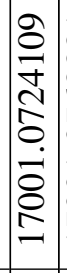 & 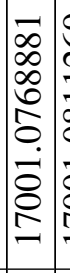 & 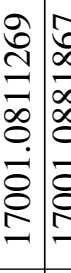 & 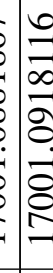 & 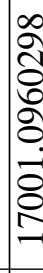 & 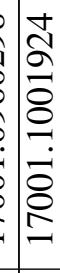 \\
\hline 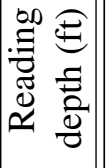 & \begin{tabular}{|l}
$\vec{\delta}$ \\
0 \\
$\dot{n}$ \\
\\
\end{tabular} & $\begin{array}{l}\bar{\delta} \\
\dot{0} \\
\stackrel{2}{n}\end{array}$ & 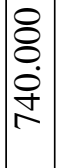 & 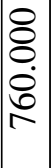 & 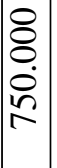 & 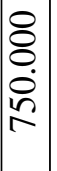 & 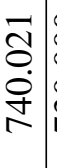 & 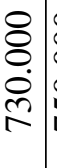 & $\begin{array}{l}8 \\
8 \\
\vdots \\
\dot{n} \\
\end{array}$ & $\begin{array}{l}8 \\
8 \\
\vdots \\
\check{0} \\
\end{array}$ & 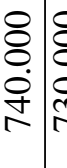 & 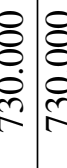 & ?. & $\begin{array}{l}8 \\
8 \\
0 \\
\dot{8} \\
\end{array}$ & \begin{tabular}{l}
8 \\
8 \\
0 \\
0 \\
\hdashline \\
\end{tabular} & 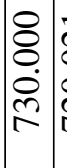 & 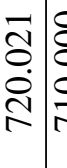 & 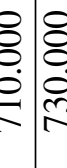 & $\begin{array}{l}8 \\
\vdots \\
0 \\
\dot{2} \\
\end{array}$ & 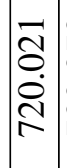 & $\begin{array}{l}\frac{9}{5} \\
\hat{2} \\
\text { 2}\end{array}$ & 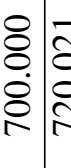 & 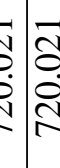 & & : \\
\hline$\stackrel{\oplus}{=}$ & 0 & 0 & 0 & 0 & 0 & 0 & 0 & 0 & 0 & 0 & 0 & o & 0 & 0 & 0 & 0 & 0 & ن 0 & 0 & 0 & 0 & 0 & 0 & 0 & 0 \\
\hline 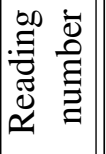 & $?$ & $\nabla$ & 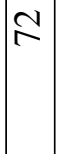 & $m$ & 咅 & $n$ & $\div$ & $尺$ & $\infty$ & 2 & $\triangleright \bar{\alpha}$ & $\bar{\infty} \propto$ & $\infty$ & $\infty$ & 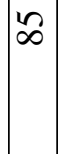 & $\infty$ & $\infty)$ & $\infty \gg$ & প & $\bar{a}$ & 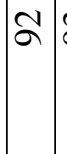 & $\sigma \delta$ & $t$ & ะ & $\hat{a}$ \\
\hline
\end{tabular}




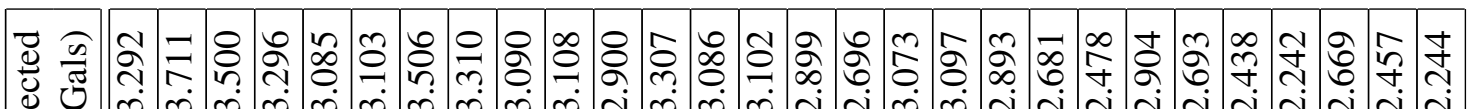

氙

U

.

के

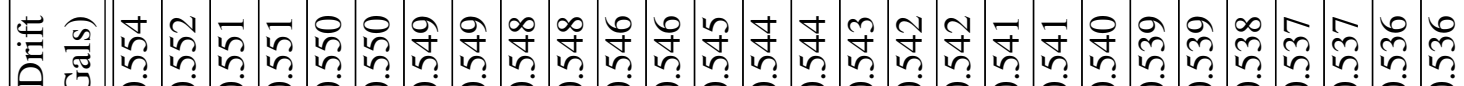
$\Xi$

ठํ.

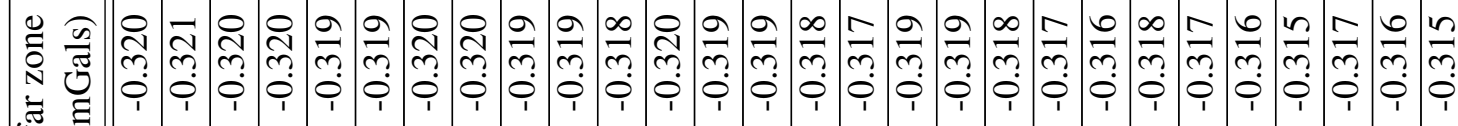
䨔

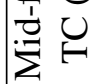

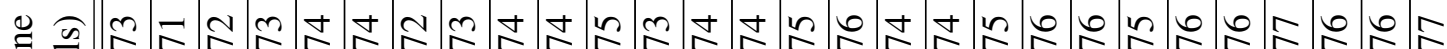

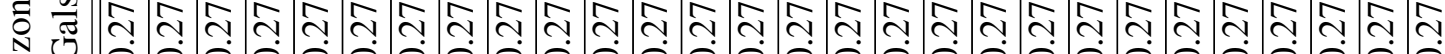
尝 そ̇

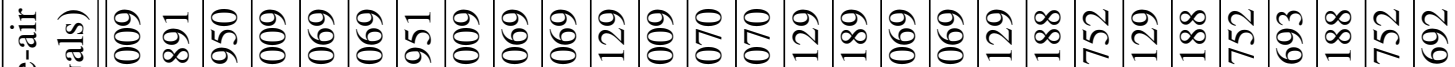
岂 ठํ.

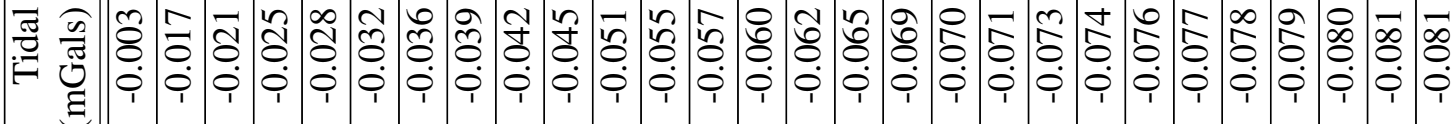

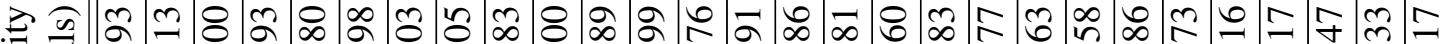
च क ह

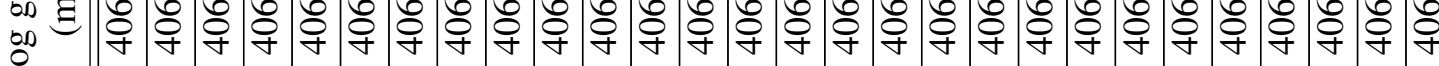

舟 司

5.

의 艺

\begin{tabular}{|c|c|c|c|c|c|c|c|c|c|c|c|c|c|c|c|c|c|c|c|c|c|c|c|c|c|c|c|c|}
\hline$\frac{0}{1}$ & 0 & 0 & 0 & 0 & 0 & 0 & 0 & 0 & 0 & 0 & 0 & 0 & 0 & 0 & 0 & 0 & 0 & 0 & 0 & 0 & o 0 & 0 & 0 & & u & & u & 0 \\
\hline 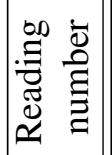 & $\approx$ & হे & ○ & 으 & 으 & $\hat{0}$ & 吾 & $\underline{\underline{a}}$ & $\stackrel{8}{\circ}$ & $\underline{\underline{\theta}}$ & $\stackrel{\infty}{0}$. & 8 & $\stackrel{ }{=}$ & $\exists$ & $\cong$ & $\stackrel{m}{=}$ & $\cong$ & $\stackrel{0}{=}$ & $\Xi$ & & & & & & & & & 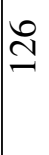 \\
\hline
\end{tabular}


勿 ๖

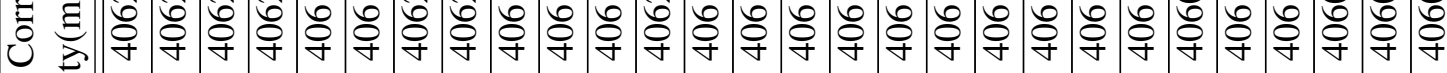
on

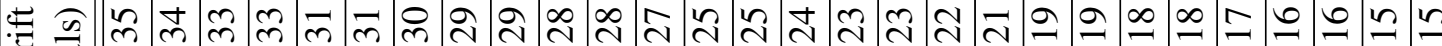
ลี

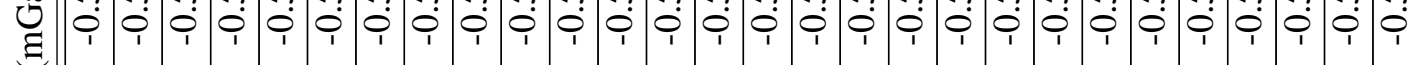

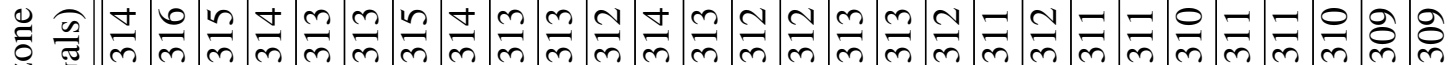

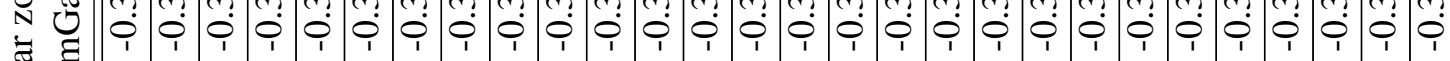
‡్

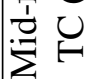

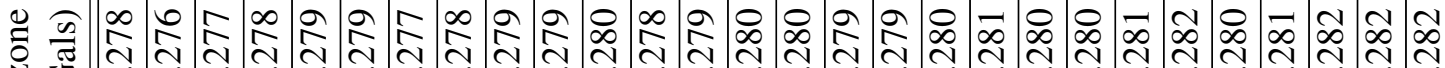

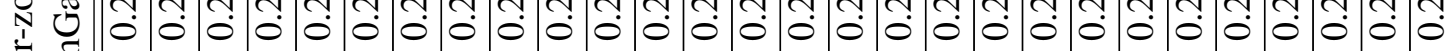
สี 乙 U

\begin{tabular}{|c|c|c|c|c|c|c|c|c|c|c|c|c|c|c|c|c|c|c|c|c|c|c|c|c|c|c|c|c|}
\hline 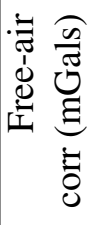 & $\mid \begin{array}{c}2 \\
\hat{b} \\
\text { id } \\
1\end{array}$ & 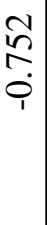 & 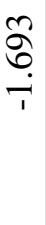 & 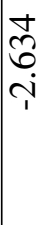 & 敨 & $\begin{array}{l} \pm \\
n \\
n \\
r\end{array}$ & $\begin{array}{l}2 \\
2 \\
0 \\
-1\end{array}$ & $\begin{array}{l}m \\
\tilde{\sigma} \\
0 \\
i \\
1\end{array}$ & $\begin{array}{l} \pm \\
n \\
n \\
r\end{array}$ & $\begin{array}{l} \pm \\
n \\
n \\
m \\
\eta\end{array}$ & $\begin{array}{l}\frac{1}{n} \\
+ \\
+\end{array}$ & 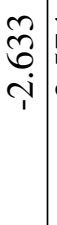 & $\begin{array}{l} \pm \\
\tilde{n} \\
n \\
\eta \\
1\end{array}$ & $\begin{array}{l}n \\
n \\
+ \\
\dot{r}\end{array}$ & $\begin{array}{l}n \\
\tilde{n} \\
\sim \\
i\end{array}$ & $\begin{array}{l}m \\
n \\
n \\
m\end{array}$ & $\begin{array}{l}m \\
\tilde{n} \\
m\end{array}$ & $\begin{array}{c}n \\
\tilde{n} \\
\dot{r}\end{array}$ & $\begin{array}{l}2 \\
\tilde{n} \\
\tilde{n} \\
1\end{array}$ & 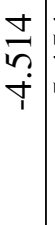 & 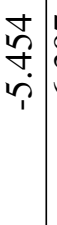 & $\begin{array}{c}\hat{2} \\
\text { స్ } \\
\vdots \\
1\end{array}$ & $\begin{array}{l}0 \\
m \\
m \\
r \\
1\end{array}$ & \begin{tabular}{l}
\multirow{2}{*}{} \\
$\stackrel{+}{*}$ \\
$\ddot{1}$
\end{tabular} & 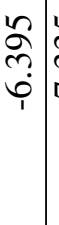 & $\begin{array}{l}n \\
\text { ñ. } \\
r\end{array}$ & 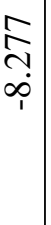 & v \\
\hline 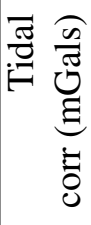 & $\begin{array}{c}1 \\
\infty \\
0 \\
0 \\
0\end{array}$ & $\begin{array}{l}N \\
\infty \\
0 \\
0 \\
1\end{array}$ & $\begin{array}{l}\text { No } \\
0 \\
0 \\
\end{array}$ & $\begin{array}{l}N \\
\infty \\
0 \\
0 \\
1\end{array}$ & $\begin{array}{l}N \\
\infty \\
0 \\
0 \\
1\end{array}$ & $\begin{array}{c}\vec{\infty} \\
0 \\
0 \\
0\end{array}$ & \begin{tabular}{|c}
$\infty$ \\
$\infty$ \\
0 \\
0 \\
1
\end{tabular} & $\begin{array}{l}0 \\
\infty \\
0 \\
0 \\
0 \\
1\end{array}$ & $\begin{array}{c}0 \\
\infty \\
0 \\
\dot{0} \\
1\end{array}$ & $\begin{array}{l}0 \\
\hat{0} \\
\vdots \\
0 \\
1\end{array}$ & $\begin{array}{l}0 \\
0 \\
0 \\
0\end{array}$ & 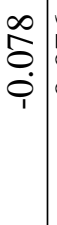 & $\mid \begin{array}{c}0 \\
0 \\
0 \\
0 \\
1\end{array}$ & $\begin{array}{l}n \\
\hat{0} \\
\dot{0}\end{array}$ & 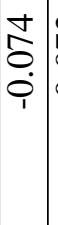 & 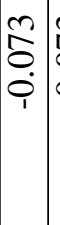 & $\begin{array}{l}m \\
\hat{0} \\
\dot{1}\end{array}$ & 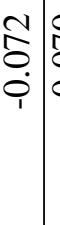 & 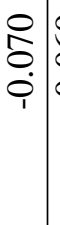 & $\begin{array}{l}9 \\
0 \\
0 \\
0 \\
1\end{array}$ & 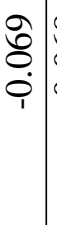 & 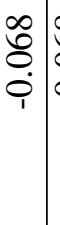 & 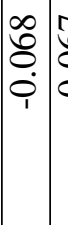 & $\begin{array}{l}\hat{\sigma} \\
0 \\
0 \\
\end{array}$ & $\begin{array}{l}\hat{\sigma} \\
0 \\
0 \\
\end{array}$ & $\begin{array}{l}0 \\
0 \\
0 \\
0 \\
1\end{array}$ & $\begin{array}{l}0 \\
0 \\
0 \\
0 \\
1\end{array}$ & \\
\hline 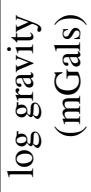 & 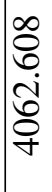 & 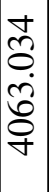 & 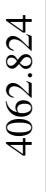 & 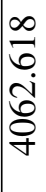 & 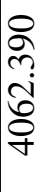 & 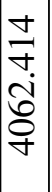 & $\begin{array}{l}n \\
\tilde{\infty} \\
\infty \\
i \\
\delta \\
\delta \\
\dot{q}\end{array}$ & 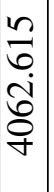 & 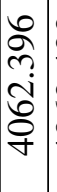 & 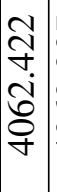 & 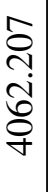 & 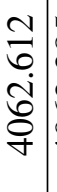 & 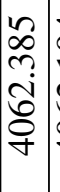 & 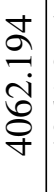 & $\begin{array}{l}n \\
\hat{a} \\
\frac{n}{6} \\
\vdots \\
y\end{array}$ & 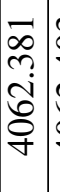 & 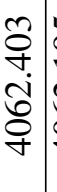 & 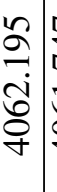 & 守 & 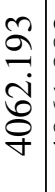 & 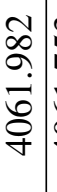 & 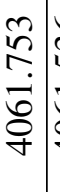 & 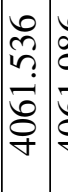 & 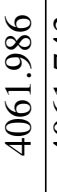 & 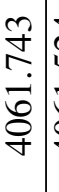 & 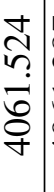 & 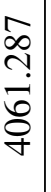 & \\
\hline 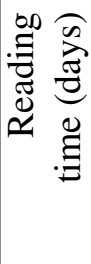 & 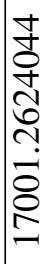 & 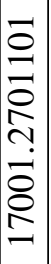 & 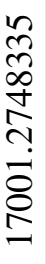 & 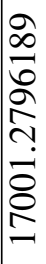 & 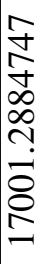 & 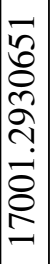 & 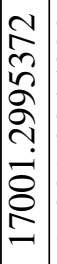 & 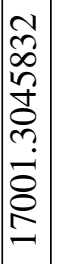 & 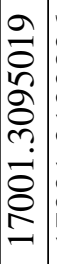 & 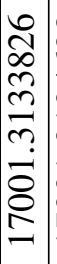 & 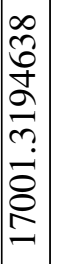 & 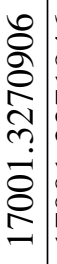 & 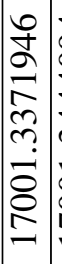 & 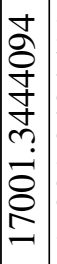 & 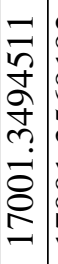 & 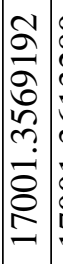 & 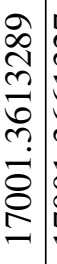 & 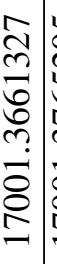 & 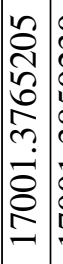 & 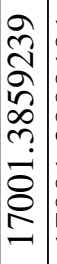 & 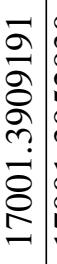 & 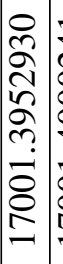 & 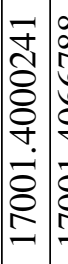 & 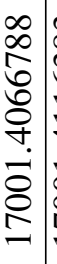 & 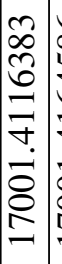 & 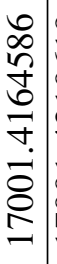 & 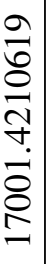 & $\Phi$ \\
\hline 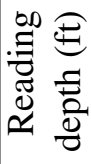 & 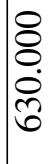 & 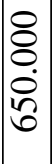 & 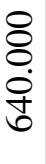 & 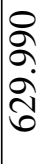 & 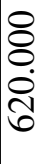 & $\begin{array}{l}8 \\
8 \\
0 \\
\dot{0} \\
\text { స్ }\end{array}$ & $\begin{array}{l}0 \\
\infty \\
\sigma \\
\hat{\sigma} \\
\tilde{b}\end{array}$ & 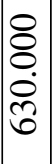 & 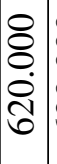 & $\begin{array}{l}8 \\
\delta \\
\delta \\
\dot{0} \\
\text { త్ }\end{array}$ & 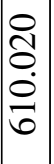 & 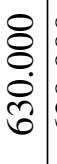 & 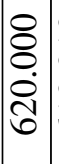 & $\mid \begin{array}{l}0 \\
0 \\
0 \\
0 \\
\sigma \\
0\end{array}$ & \begin{tabular}{l}
0 \\
0 \\
0 \\
$\dot{8}$ \\
\hdashline \\
\hdashline
\end{tabular} & 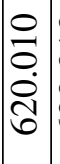 & 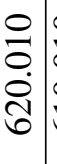 & 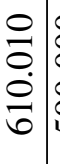 & 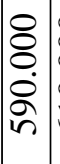 & $\begin{array}{l}8 \\
8 \\
0 \\
0 \\
6 \\
0\end{array}$ & 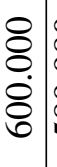 & $\begin{array}{l}\curvearrowright \\
\infty \\
a \\
\infty \\
\infty \\
n\end{array}$ & $\begin{array}{l}\Omega \\
\Omega \\
\sigma \\
2 \\
n \\
n\end{array}$ & $\begin{array}{l}8 \\
8 \\
8 \\
8 \\
\varnothing \\
\end{array}$ & 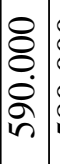 & $\begin{array}{l}8 \\
\varnothing \\
\varnothing \\
\infty \\
\infty \\
n\end{array}$ & 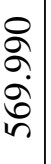 & \\
\hline$\stackrel{0}{\dot{\mid c}} \Theta$ & u & ט & 0 & 0 & 0 & 0 & 0 & 0 & u & 0 & 0 & 0 & ن & u & u & 0 & ن & 0 & 0 & ن & 0 & u & 0 & 0 & u & 0 & 0 & \\
\hline 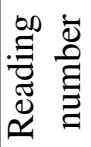 & ㄱ & $\stackrel{\infty}{\underset{c}{d}}$ & 그 & 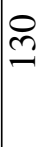 & $m$ & ๙2 & $\stackrel{m}{m}$ & $\stackrel{+}{m}$ & $\tilde{m}$ & @ & $\underline{m}$ & 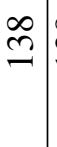 & $\stackrel{2}{\beth}$ & 士 & $\stackrel{\mathcal{I}}{ \pm}$ & $\stackrel{\Re}{\Xi}$ & さ & $\stackrel{\curvearrowleft}{ \pm}$ & Ð & $\underset{+}{\infty}$ & 守 & $\stackrel{\oslash}{n}$ & $\bar{n}$ & N & n & $\stackrel{+}{n}$ & in & \\
\hline
\end{tabular}




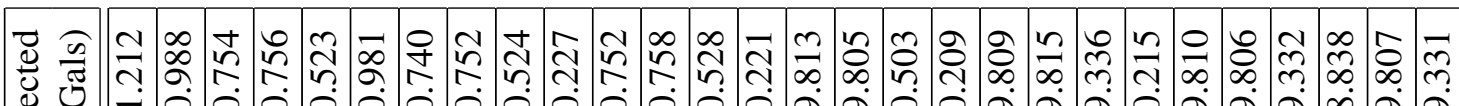
包 U गٕ

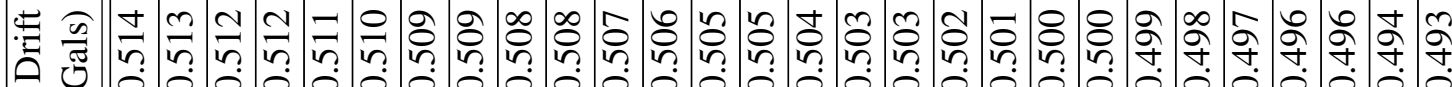
¿ำ

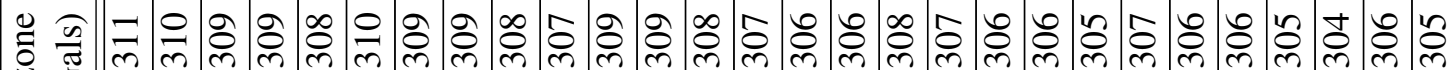

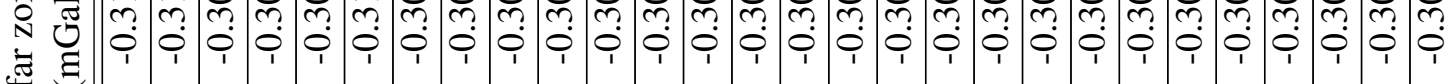

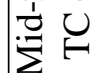

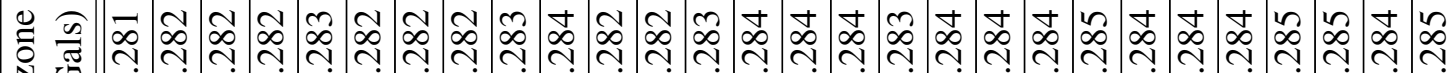

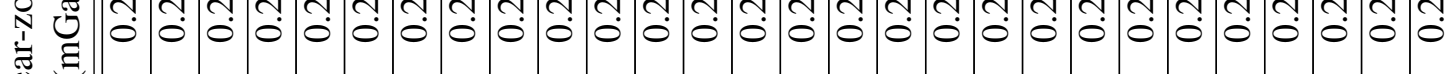
ङ $\mathrm{Z}$,

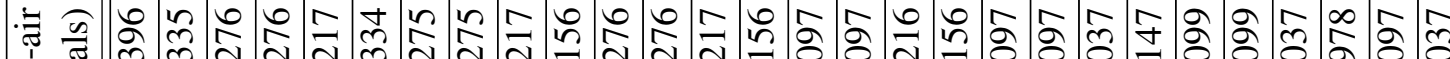
ذ่⿱丶万⿱⿰㇒一乂 龸 ఏँ

สี ชิ

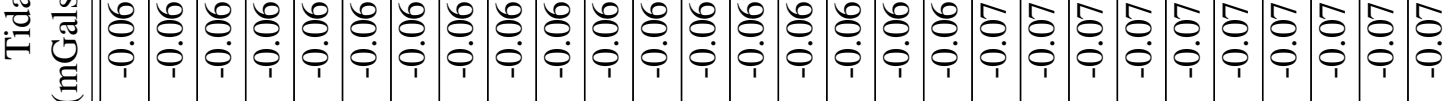
. 8

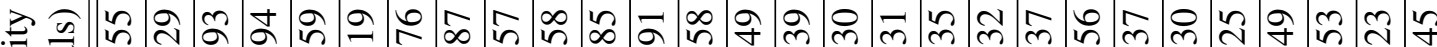
च कo

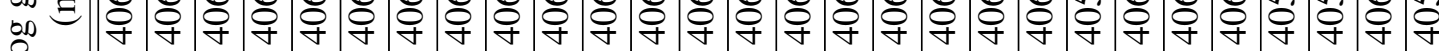

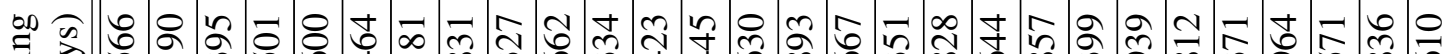

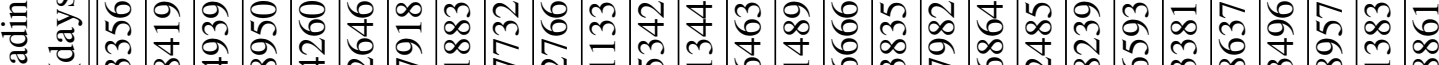

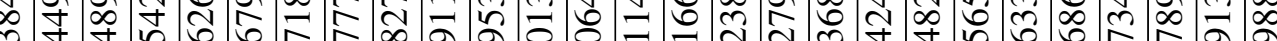

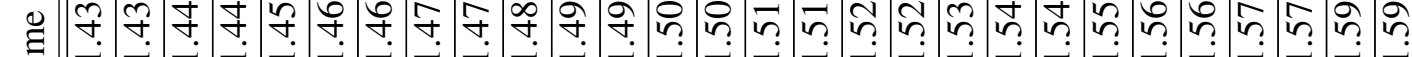

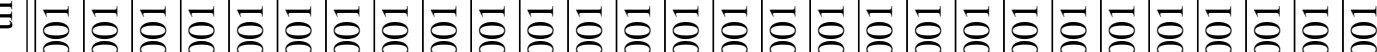
5.5555 .555555555

.

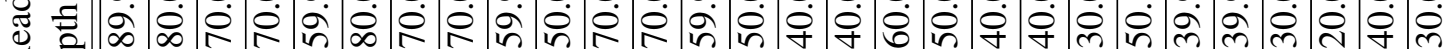

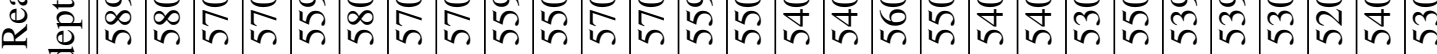
$\stackrel{0}{*}$

.  $\stackrel{\simeq}{\Xi}$ 


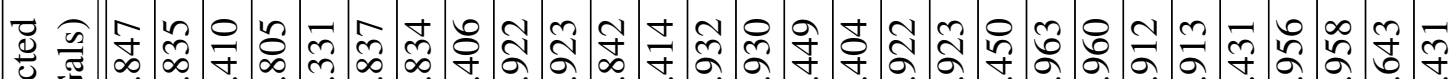

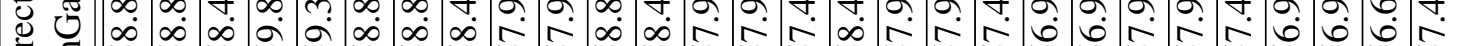

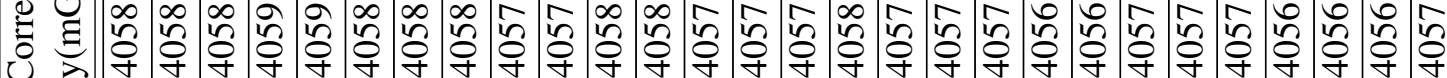

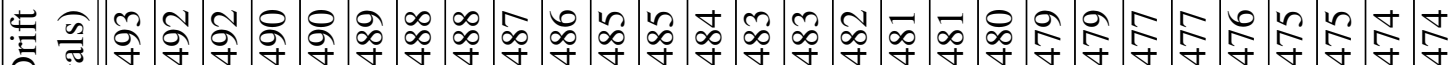
A

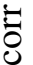

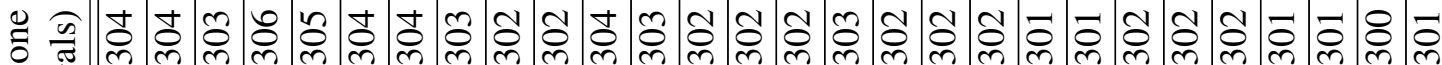

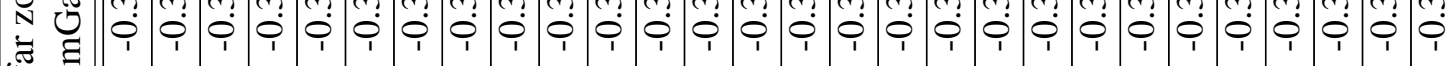
旁

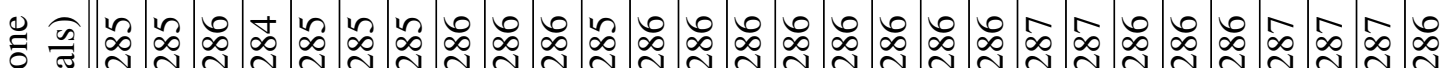

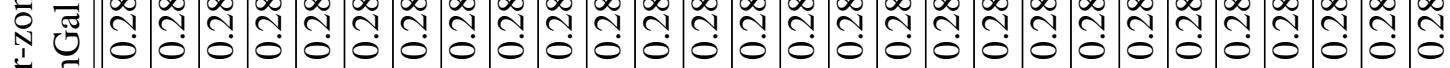
ซี $\ddot{Z} \cup$

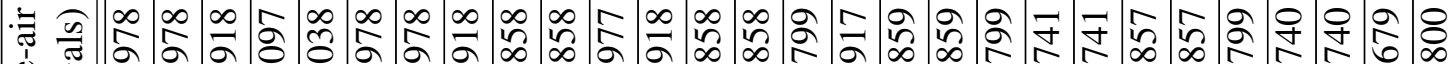

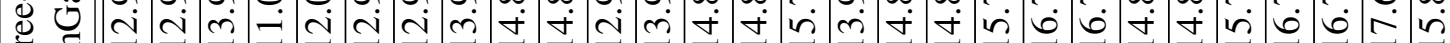

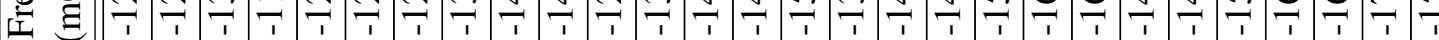

\section{ठํ.}

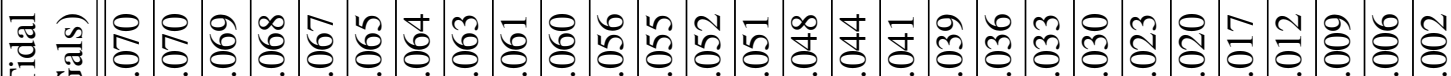

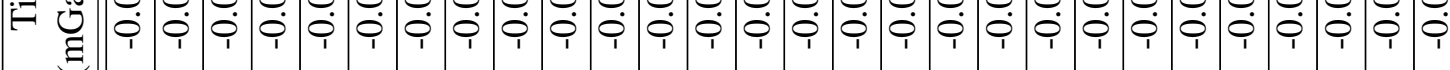
ठํ.

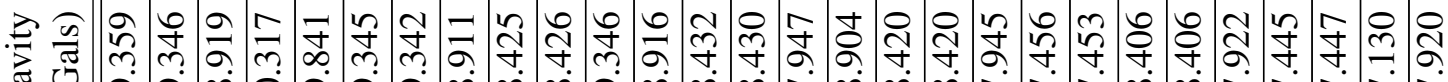
ప্ D舟 으

. 录 胥 $\approx$ « ヨ

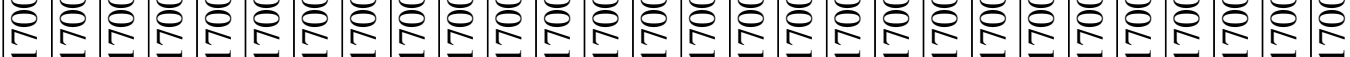

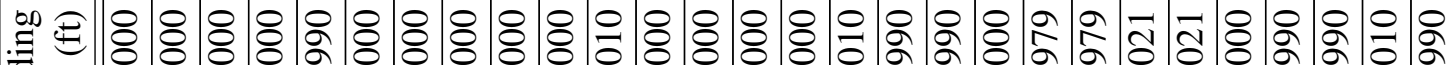

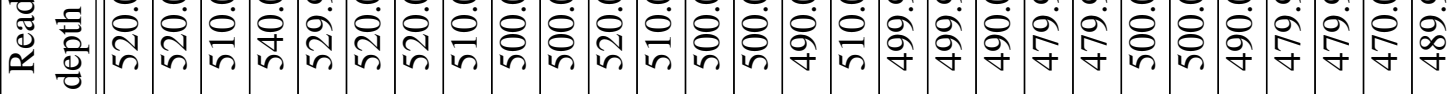
$\stackrel{0}{2}$

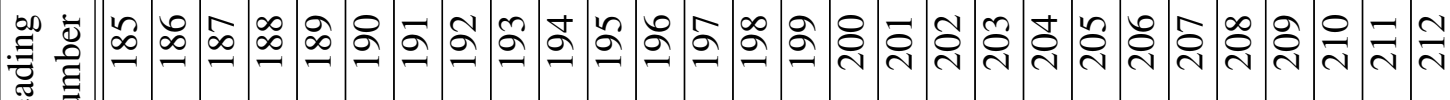
苞 


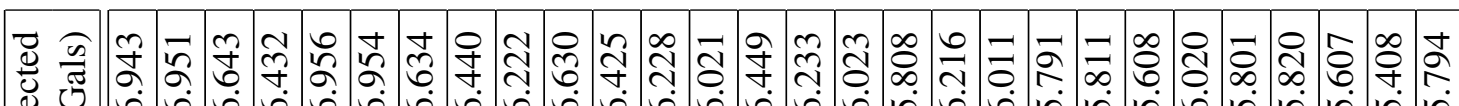
Ð o

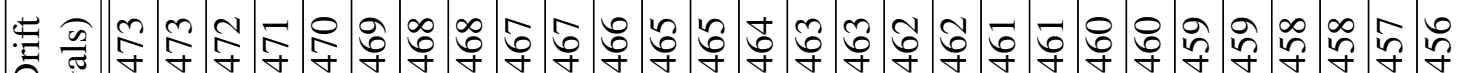
○ i

อิ

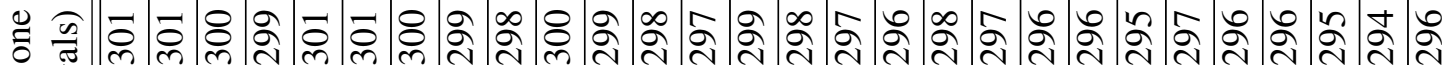

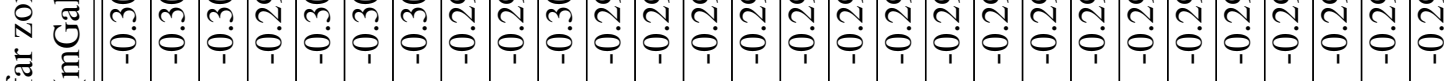

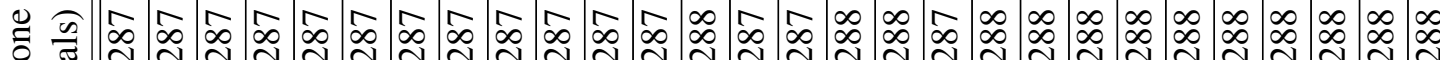

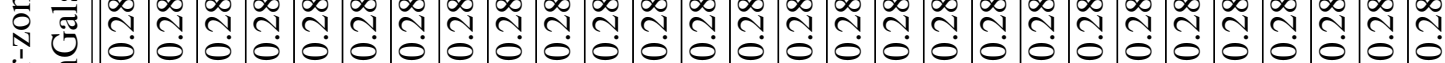
है $\ddot{z}$ U

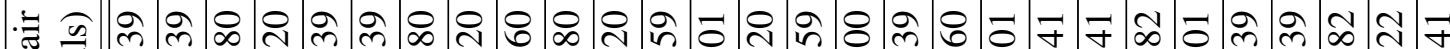

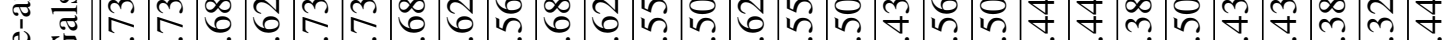

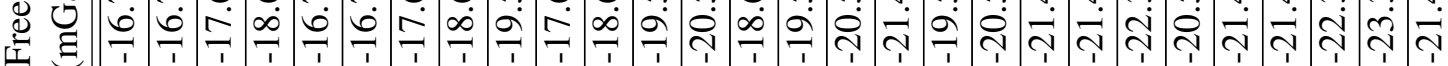
כั

즘

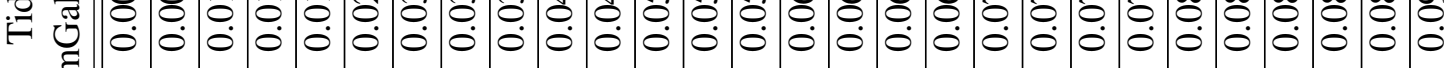

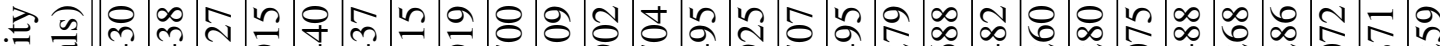

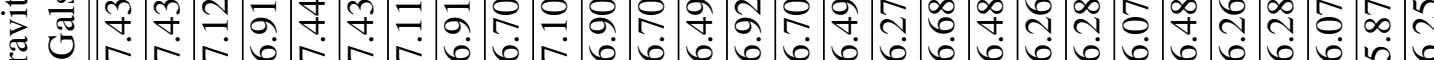

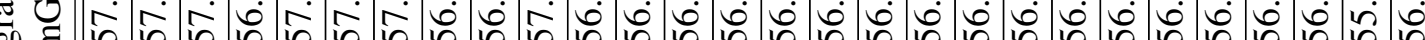

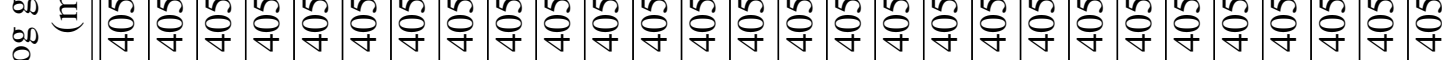

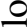

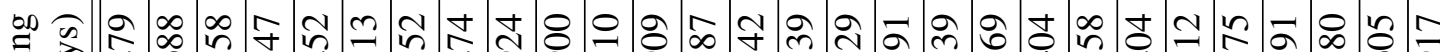

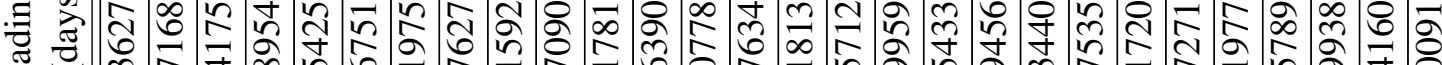

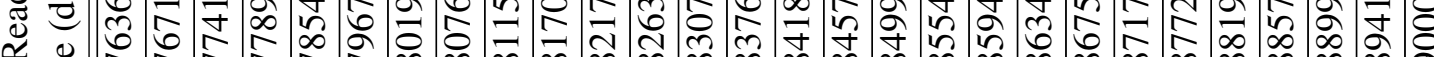
E

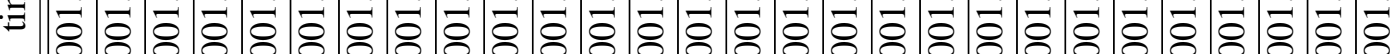

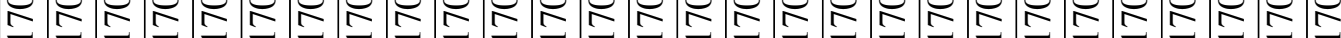

:

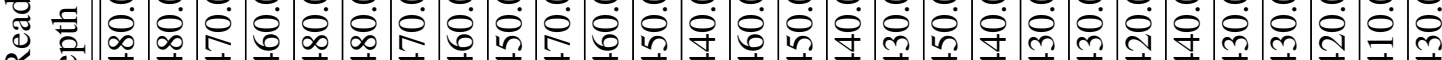

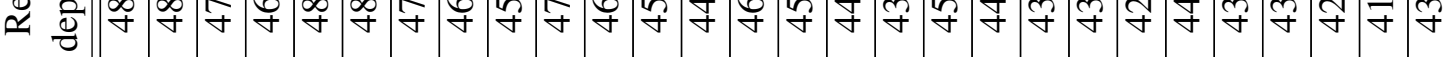
$\stackrel{\circ}{\circ}$ 然

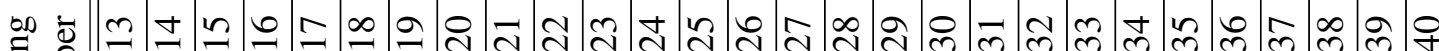
: 


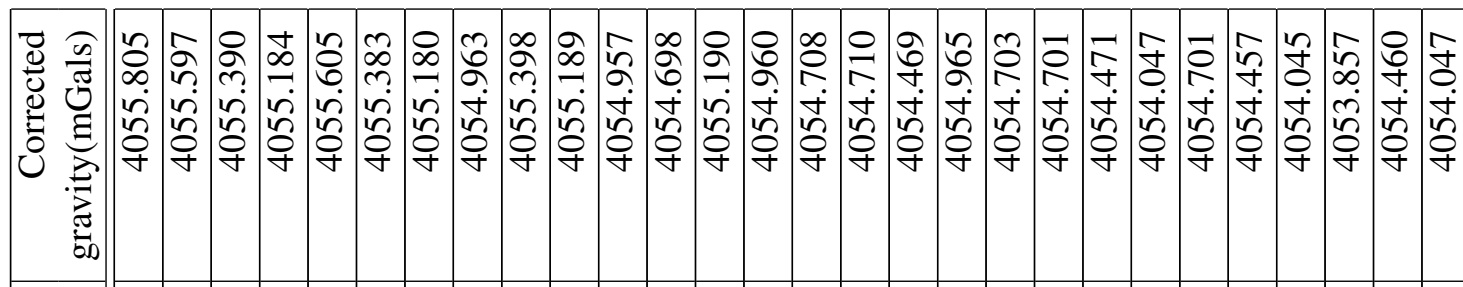

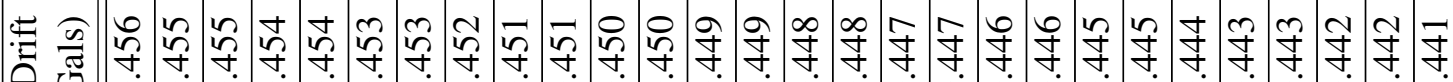

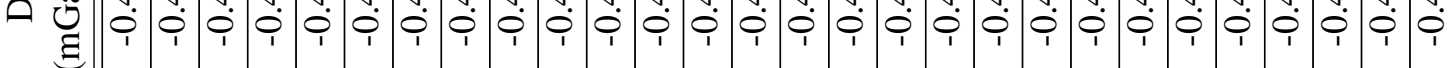

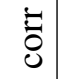

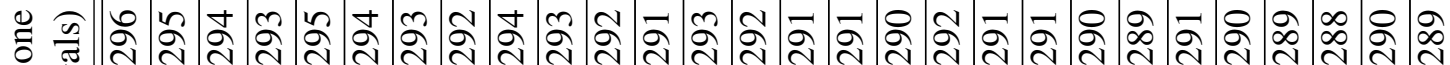

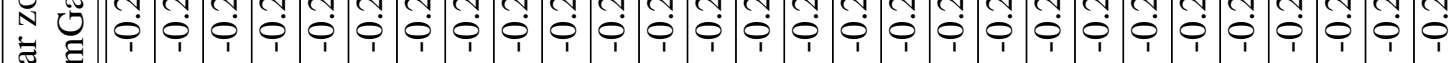
쿄 $\sum_{\ominus}$

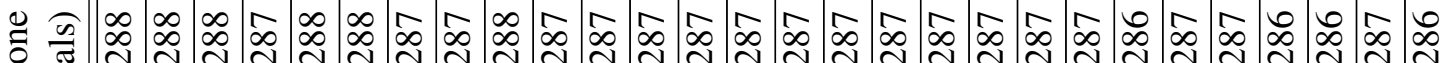

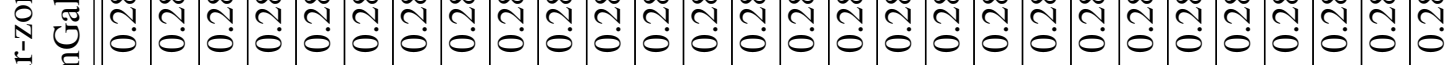
ซี

Z U

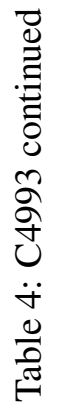

\begin{tabular}{|c|c|c|c|c|c|c|c|c|c|c|c|c|c|c|c|c|c|c|c|c|c|c|c|c|}
\hline 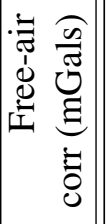 & 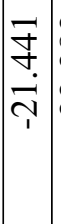 & 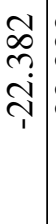 & है & & & & & & & & & & 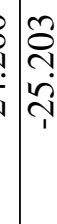 & $\begin{array}{l}\stackrel{m}{ \pm} \\
\overrightarrow{ \pm} \\
\stackrel{1}{1}\end{array}$ & 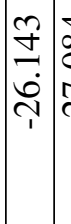 & 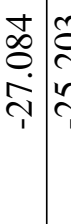 & & 8 & & & & 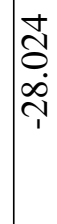 & $\left|\begin{array}{l}0 \\
0 \\
\infty \\
i \\
1\end{array}\right|$ & \\
\hline 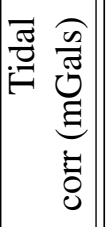 & $\bar{a}$ & 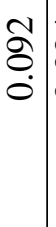 & $\begin{array}{l}\dot{a} \\
\stackrel{a}{0} \\
\dot{0}\end{array}$ & \begin{tabular}{l|l}
$\dot{2}$ \\
$\dot{g}$ \\
$\dot{0}$
\end{tabular} & $\begin{array}{l}0 \\
2 \\
\vdots \\
0\end{array}$ & ô. & $\begin{array}{l}\hat{g} \\
\dot{0}\end{array}$ & $\begin{array}{l}\hat{8} \\
\dot{0} \\
\dot{0}\end{array}$ & $\hat{8}$ & 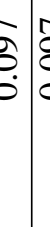 & 8 & 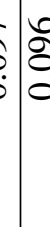 & : & 囟 & 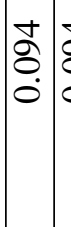 & $\begin{array}{l}\dot{g} \\
\stackrel{\partial}{0}\end{array}$ & 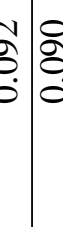 & $\begin{array}{l}0 \\
\dot{0} \\
0\end{array}$ & $\left|\begin{array}{l}\infty \\
\infty \\
0 \\
0 \\
0\end{array}\right|$ & \begin{tabular}{l|l}
0 & \\
$\infty$ & 0 \\
0 & 0 \\
0 & 5
\end{tabular} & & : & 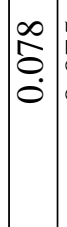 & 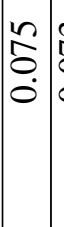 \\
\hline 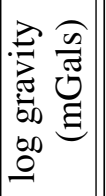 & 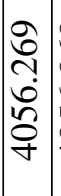 & 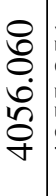 & 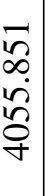 & 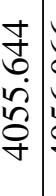 & & 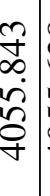 & $\begin{array}{l}\infty \\
n \\
0 \\
\dot{n} \\
n \\
0 \\
y\end{array}$ & 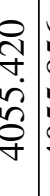 & 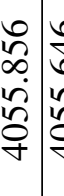 & 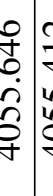 & 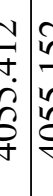 & 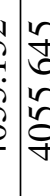 & 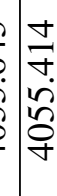 & 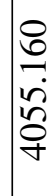 & 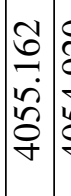 & 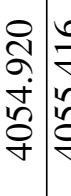 & 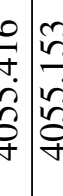 & 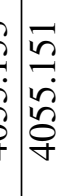 & 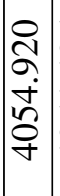 & 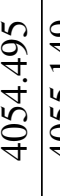 & 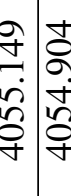 & 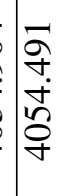 & 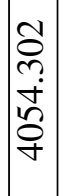 & 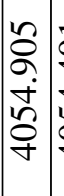 \\
\hline 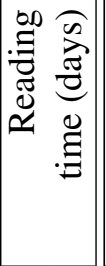 & 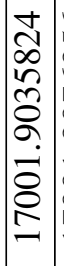 & $\begin{array}{l}0 \\
2 \\
2 \\
8 \\
\vdots \\
\dot{8} \\
2\end{array}$ & 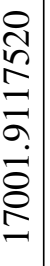 & 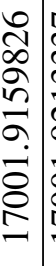 & 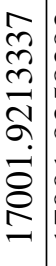 & $\begin{array}{l}n \\
2 \\
\infty \\
\infty \\
\vdots \\
\vdots \\
\vdots \\
\vdots\end{array}$ & 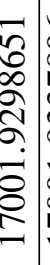 & 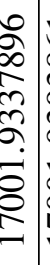 & 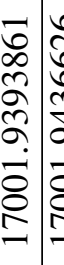 & 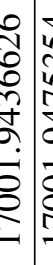 & 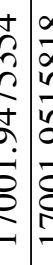 & 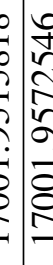 & 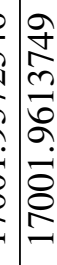 & 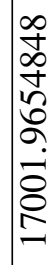 & 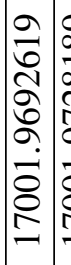 & 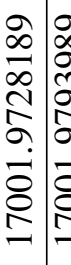 & 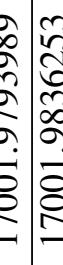 & 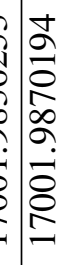 & 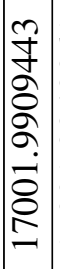 & 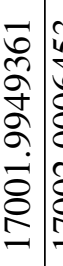 & 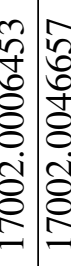 & 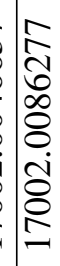 & 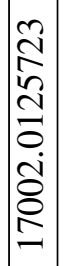 & 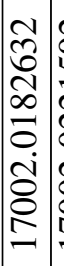 \\
\hline 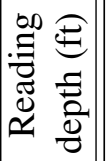 & $\begin{array}{l}8 \\
\delta \\
8 \\
\dot{0} \\
\mathscr{\sigma}\end{array}$ & $\begin{array}{l}\stackrel{8}{8} \\
\stackrel{0}{\dot{\leftrightarrow}} \\
\stackrel{+}{+}\end{array}$ & \begin{tabular}{l}
8 \\
8 \\
0 \\
0 \\
\hdashline \\
$\forall$
\end{tabular} & \begin{tabular}{l|l}
8 \\
8 \\
$\dot{8}$ \\
8 \\
+
\end{tabular} & 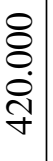 & $\begin{array}{l}8 \\
8 \\
\dot{0} \\
\dot{7} \\
\nabla\end{array}$ & $\begin{array}{l}8 \\
8 \\
\\
8 \\
8 \\
+\end{array}$ & 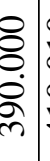 & 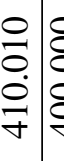 & 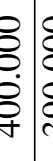 & 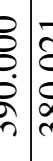 & 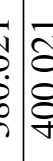 & i. & $\begin{array}{l}8 \\
8 \\
0 \\
\dot{8} \\
\infty \\
m\end{array}$ & 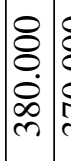 & 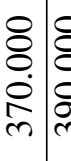 & 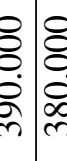 & 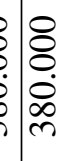 & & 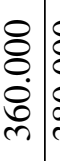 & 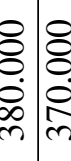 & $\begin{array}{l}8 \\
8 \\
0 \\
0 \\
0\end{array}$ & 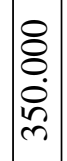 & $\begin{array}{l}8 \\
8 \\
\dot{0} \\
\text { m }\end{array}$ \\
\hline$\frac{0}{i} \theta$ & v & 0 & 0 & 0 & 0 & u & 0 & 0 & 0 & u & ن & e & 0 & 0 & 0 & 0 & ن & 0 & 0 & 0 & 0 & 0 & 0 & 0 \\
\hline 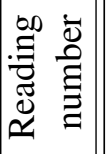 & $\underset{\sim}{\beth}$ & $\stackrel{\stackrel{\sim}{\sim}}{\sim}$ & $\stackrel{m}{\sim}$ & 守 & $\stackrel{n}{\stackrel{\sim}{d}}$ & $\begin{array}{l}\text { o } \\
\text { d }\end{array}$ & $\vec{\sim}$ & $\stackrel{\infty}{\sim}$ & $\underset{\sim}{\stackrel{a}{v}}$ & 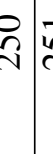 & $\vec{a} \mid$ & 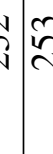 & $\stackrel{\sim}{\sim}$ & in & 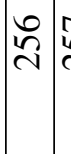 & $\hat{n}$ & 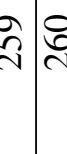 & $\bar{d}$ & స్ & ֶి & 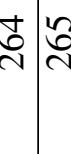 & i & $\widehat{i}$ & 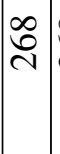 \\
\hline
\end{tabular}


竎 U J

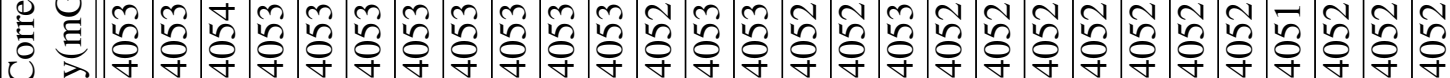

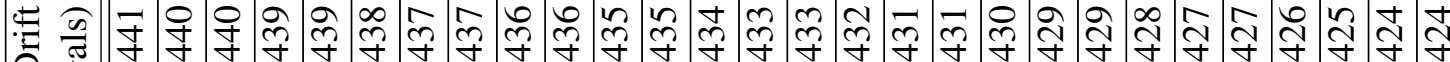

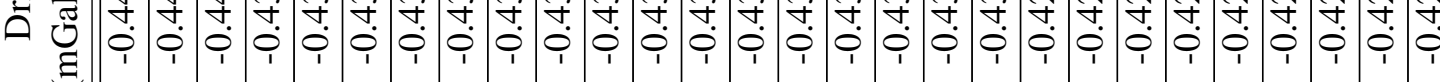

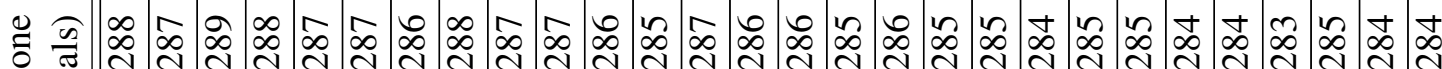

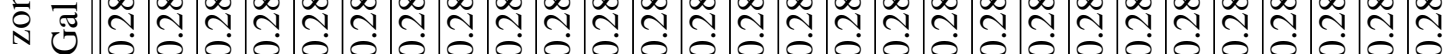
छ 旁

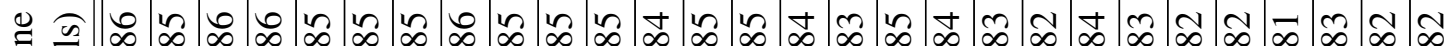

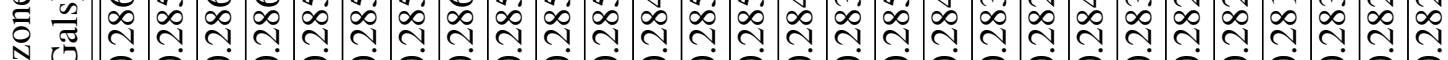
$\stackrel{1}{=}$ ङ $\mathrm{Z} \cup$

\begin{tabular}{|c|c|c|c|c|c|c|c|c|c|c|c|c|c|c|c|c|c|c|c|c|c|c|c|c|}
\hline 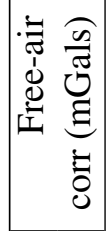 & \begin{tabular}{l}
0 \\
0 \\
$\infty$ \\
\multirow{1}{1}{} \\
1
\end{tabular} & 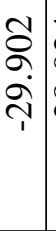 & 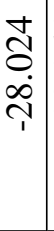 & 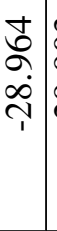 & 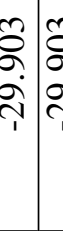 & 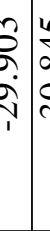 & 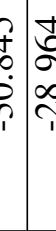 & & : & 年 & 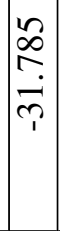 & 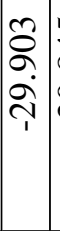 & & & & 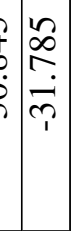 & & & & & 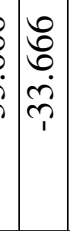 & $\begin{array}{c}\hat{8} \\
\mathbf{8} \\
\dot{+} \\
\tilde{1} \\
1\end{array}$ & & \\
\hline 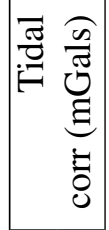 & $\begin{array}{l}0 \\
\\
0 \\
0 \\
0\end{array}$ & $\begin{array}{l}\infty \\
0 \\
0 \\
0 \\
0\end{array}$ & $\begin{array}{l}1 \\
0 \\
0 \\
0 \\
0\end{array}$ & $\begin{array}{l}- \\
0 \\
0 \\
0\end{array}$ & 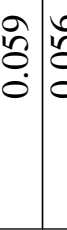 & 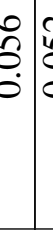 & 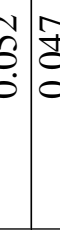 & $\stackrel{g}{g}$ & : & 官 & 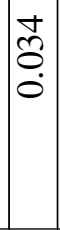 & ठे. & 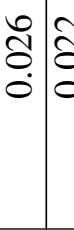 & $\stackrel{\infty}{a}$ & $\begin{array}{l}0 \\
\\
\end{array}$ & 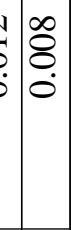 & $\begin{array}{l}1 \\
8 \\
0 \\
0\end{array}$ & 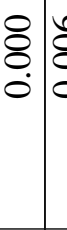 & & 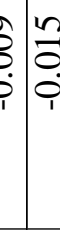 & $\begin{array}{l}\infty \\
0 \\
0 \\
0 \\
1\end{array}$ & $\begin{array}{l}\tilde{N} \\
0 \\
0 \\
1 \\
1\end{array}$ & 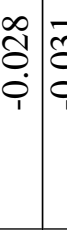 & \\
\hline 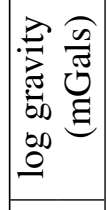 & 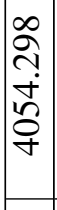 & 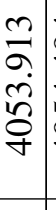 & 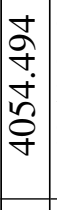 & 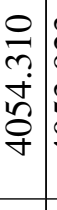 & 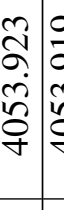 & & 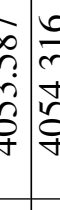 & 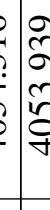 & $\left\{\begin{array}{l}\tilde{n} \\
\tilde{\sigma} \\
\tilde{n} \\
\tilde{o} \\
\gamma\end{array}\right.$ & $\begin{array}{l}n \\
\tilde{n} \\
\tilde{n} \\
n \\
\dot{y}\end{array}$ & 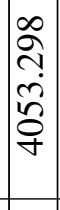 & 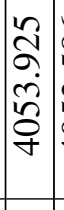 & 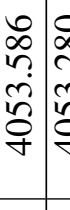 & 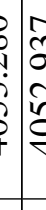 & 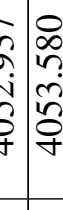 & 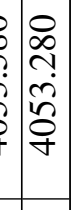 & 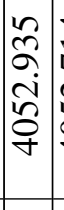 & 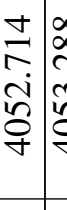 & 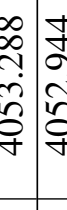 & 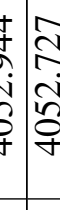 & 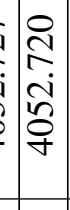 & 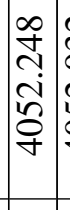 & 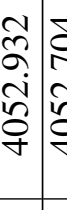 & \\
\hline 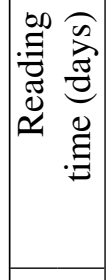 & 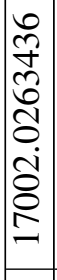 & 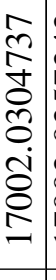 & 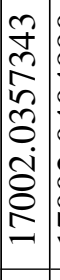 & 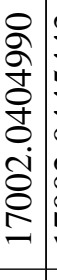 & 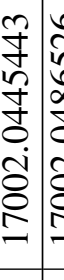 & 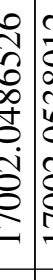 & 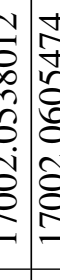 & 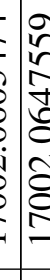 & 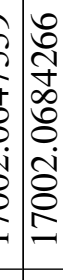 & 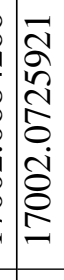 & 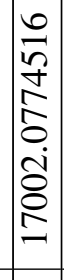 & 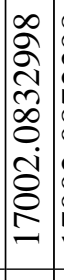 & 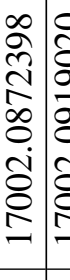 & 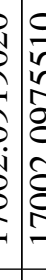 & 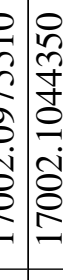 & 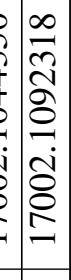 & 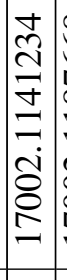 & 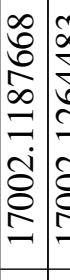 & 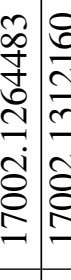 & 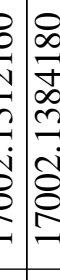 & 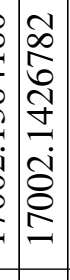 & 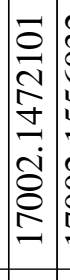 & 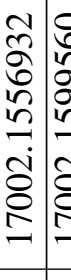 & 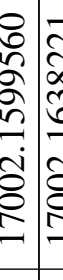 \\
\hline 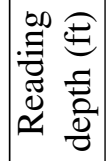 & $\begin{array}{l}8 \\
8 \\
8 \\
\dot{0} \\
\check{n} \\
m\end{array}$ & $\begin{array}{l}\vec{m} \\
0 \\
\dot{+} \\
m\end{array}$ & $\begin{array}{c}8 \\
8 \\
0 \\
\dot{8} \\
i \\
\infty\end{array}$ & $\begin{array}{l}8 \\
8 \\
\vdots \\
\dot{n} \\
\end{array}$ & 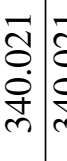 & 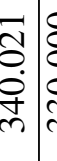 & 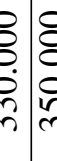 & 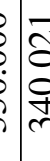 & 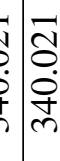 & 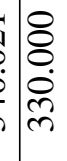 & 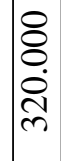 & $\begin{array}{c}\overrightarrow{\widehat{d}} \\
\dot{0} \\
\dot{0} \\
\mathrm{~m}\end{array}$ & 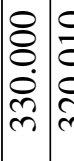 & 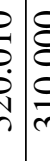 & 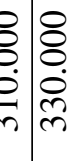 & 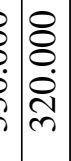 & $\begin{array}{c}\stackrel{0}{0} \\
0 \\
\stackrel{0}{0} \\
\end{array}$ & 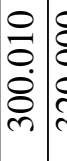 & 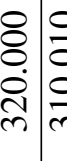 & 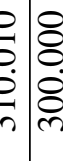 & 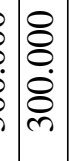 & $\begin{array}{l}8 \\
8 \\
\dot{8} \\
\AA\end{array}$ & $\begin{array}{l}0 \\
0 \\
0 \\
\stackrel{0}{m}\end{array}$ & ְُ \\
\hline$\stackrel{\theta}{\theta}$ & 0 & 0 & 0 & 0 & 0 & o & o & 0 & ט & 0 & 0 & 0 & $\begin{array}{lll}0 & 0\end{array}$ & & 0 & 0 & 0 & 0 & 0 & \begin{tabular}{l|l}
0 & 0
\end{tabular} & 0 & 0 & 0 & 0 \\
\hline 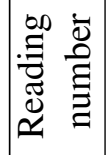 & $\stackrel{尺}{\stackrel{2}{*}}$ & $\bar{\nabla}$ & $\stackrel{\mathbb{N}}{\mathbf{N}}$ & $\frac{n}{\lambda}$ & $\stackrel{\Delta}{\Delta}$ & $\hat{\imath}$ & $\bar{v}$ & i & $\hat{v} \underset{\sim}{\infty}$ & $\stackrel{\infty}{\stackrel{\infty}{\sim}}$ & $\underset{\sim}{\infty}$ & $\begin{array}{c}\infty \\
\infty \\
\sim\end{array}$ & 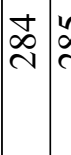 & 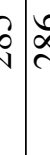 & $\begin{array}{l}\stackrel{\infty}{\infty} \\
\stackrel{\infty}{N}\end{array}$ & 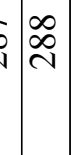 & 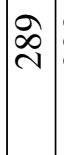 & ৯े & $\overline{\grave{\lambda}}$ & va & 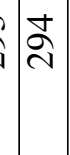 & 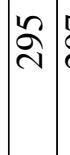 & $\hat{\check{\nu}}$ & \\
\hline
\end{tabular}




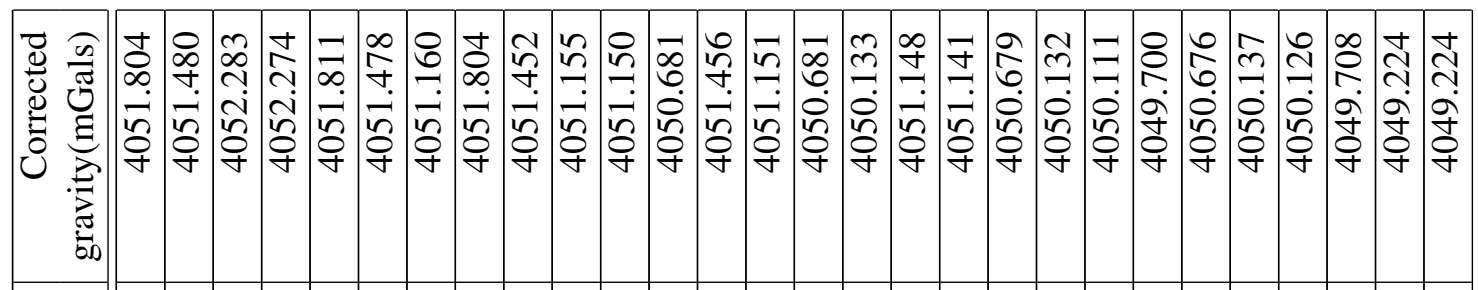

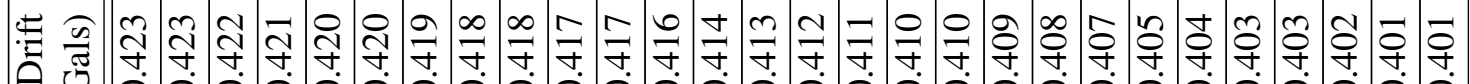

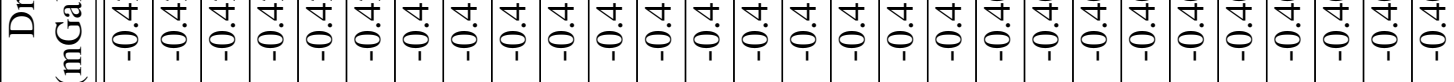

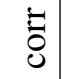

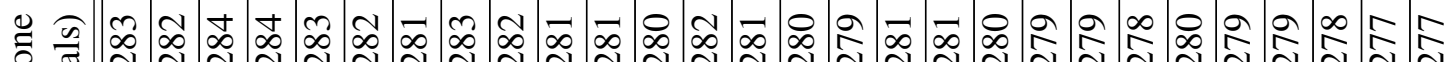

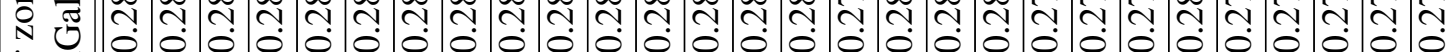
音 $\sum_{i}^{\prime}$

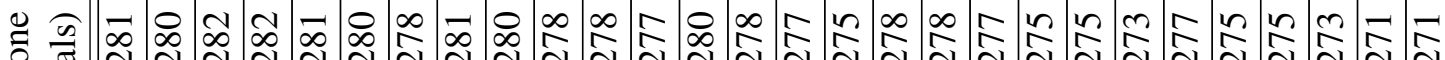

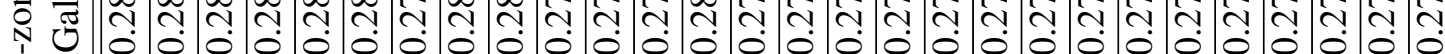
클

,

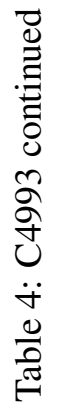

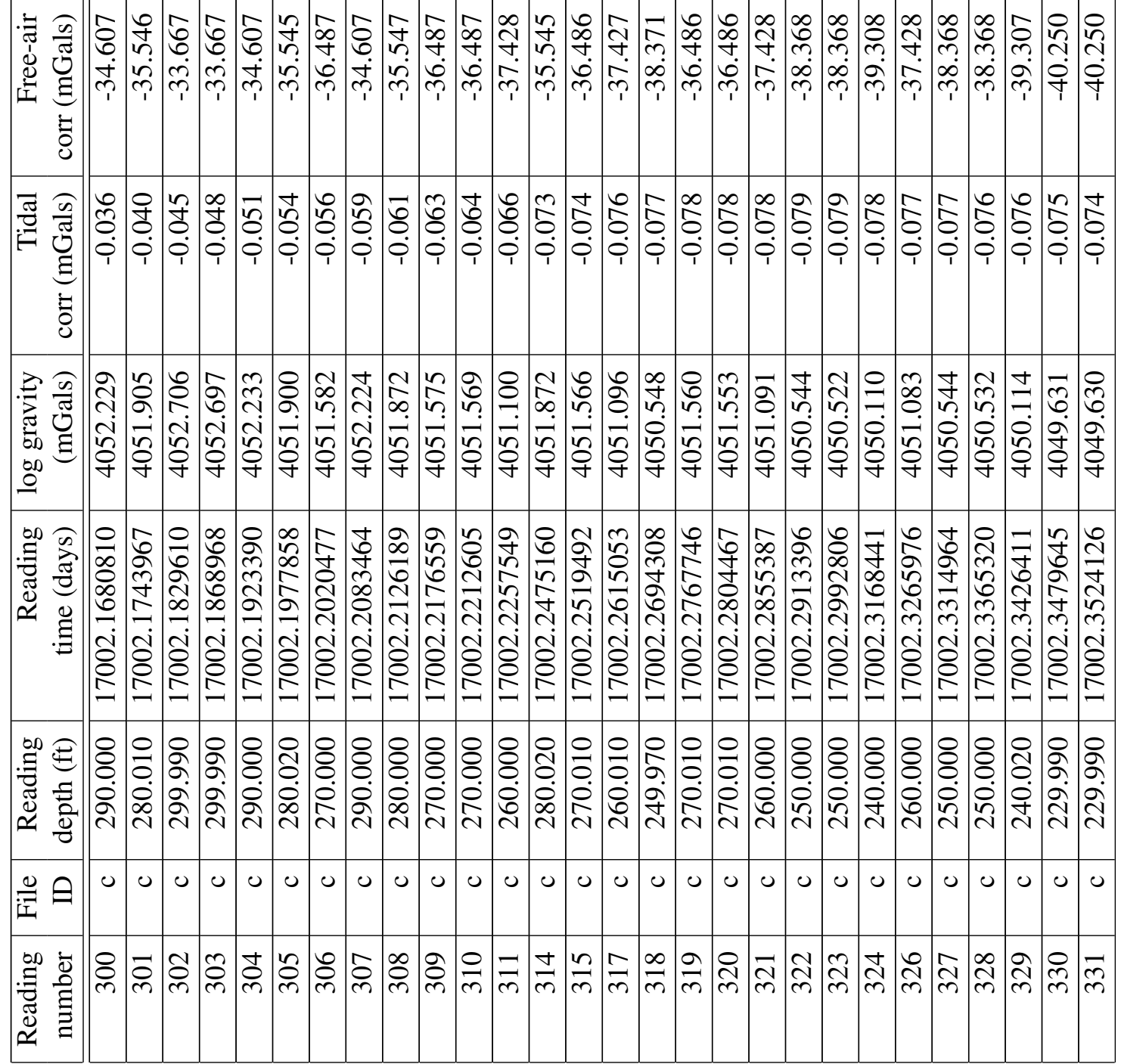




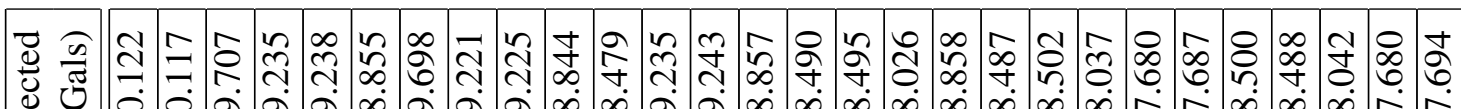

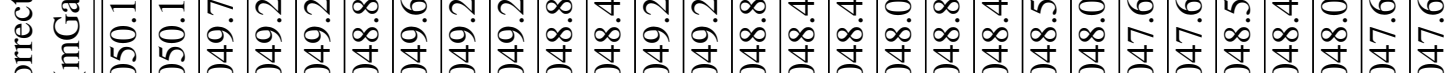
U

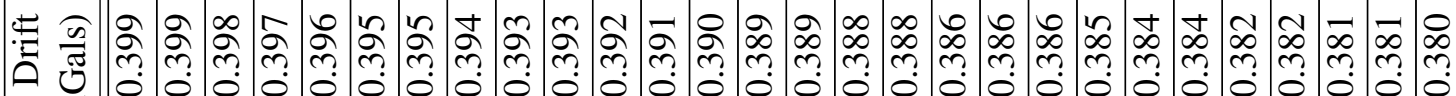

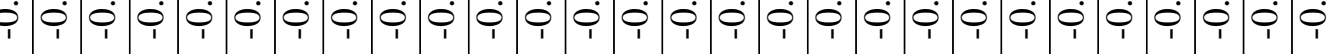

ठำ

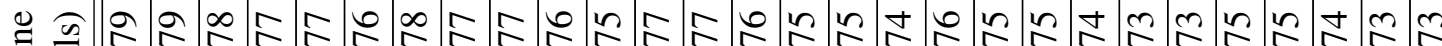

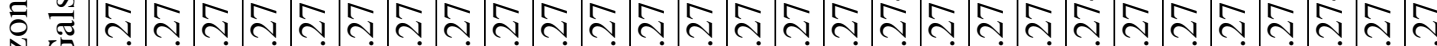

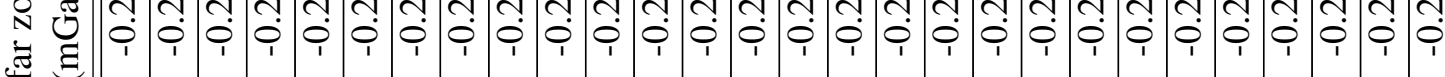

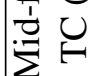

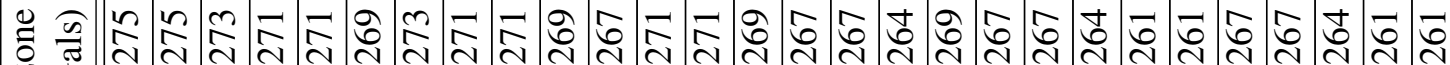

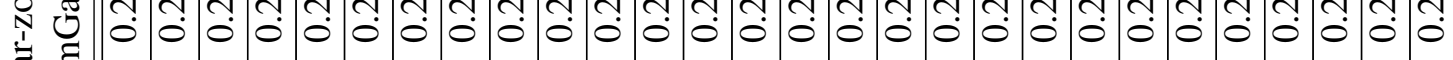
范 Z

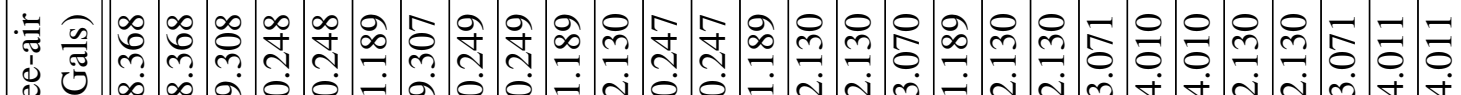
䓛

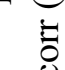

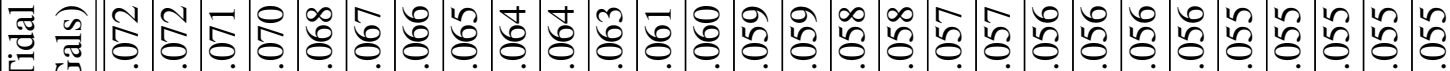

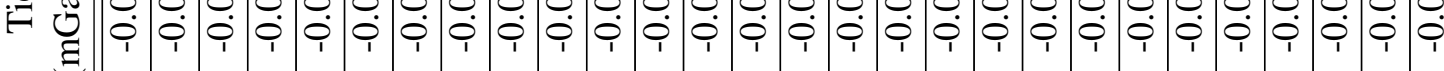

司

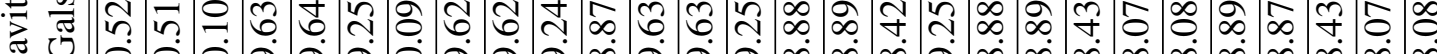
毛

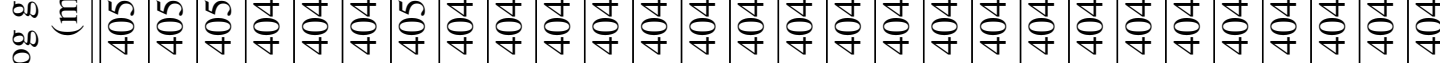
으

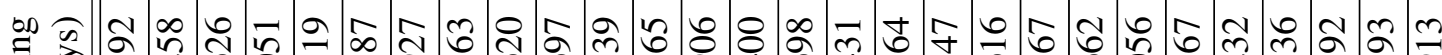

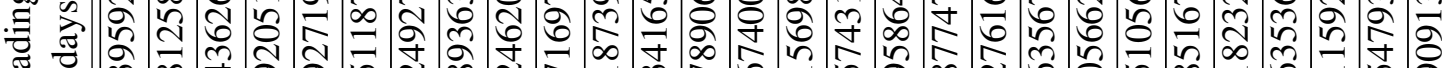
$\approx$ ֻ

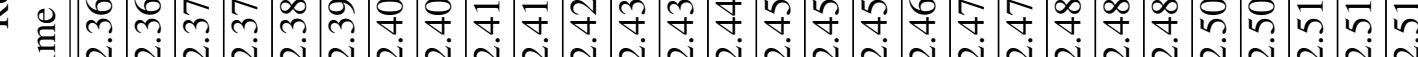
$\exists$ ¿

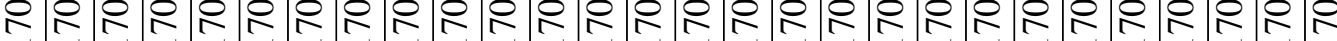

"ᄂ : ळ

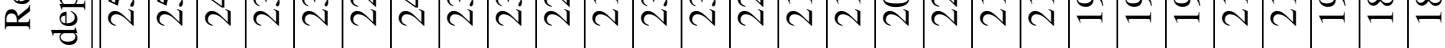
$\stackrel{0}{=} \theta$

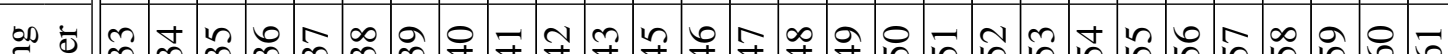

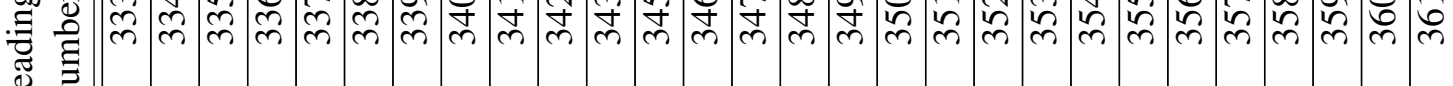
$\simeq$ 


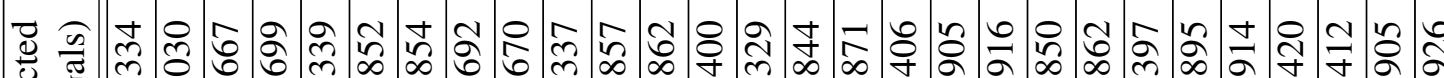

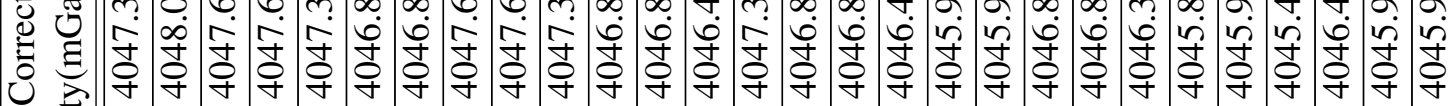

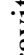
की

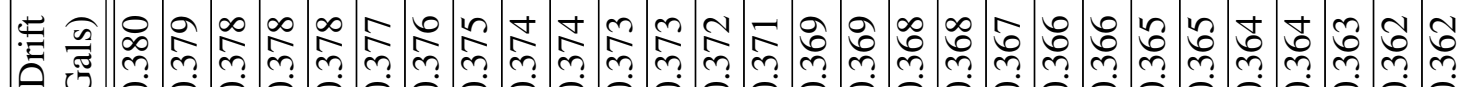
A :

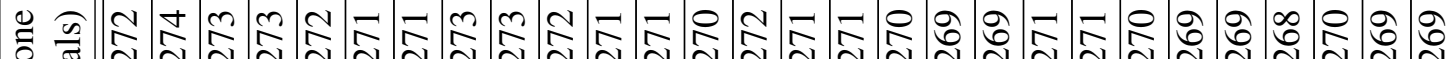

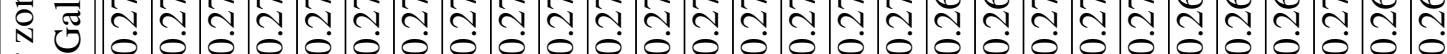
范 齐

๘

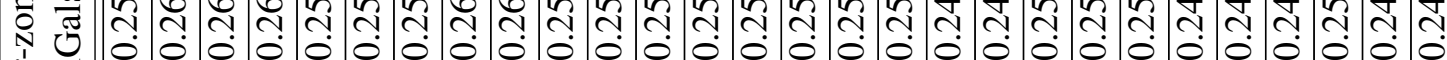
竎节 乙u

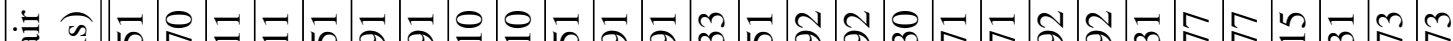

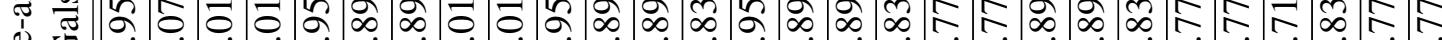

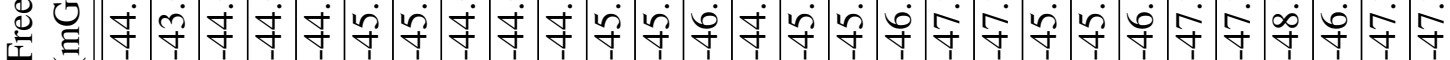
ठัँ

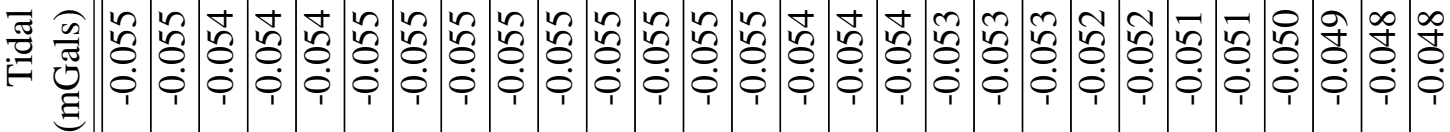
ठ․

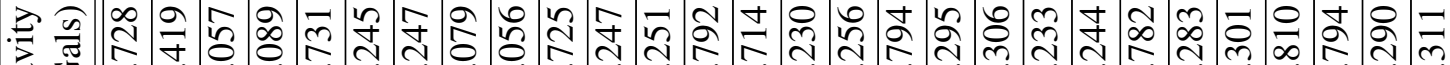
政

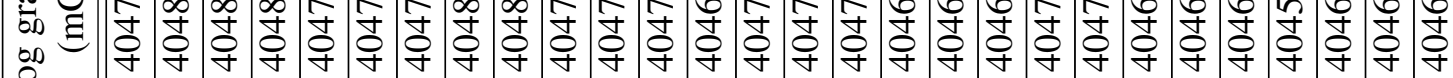
西

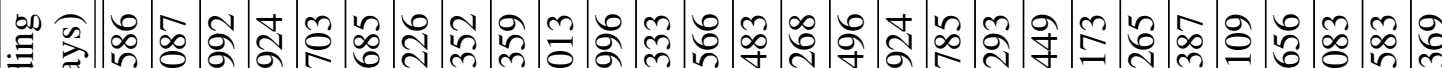
च चٓच

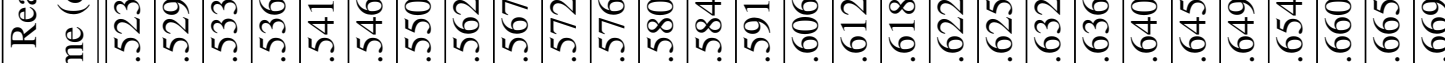

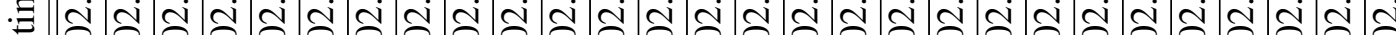

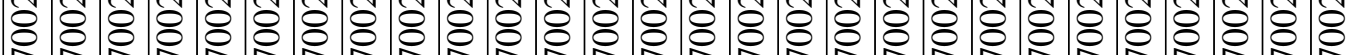

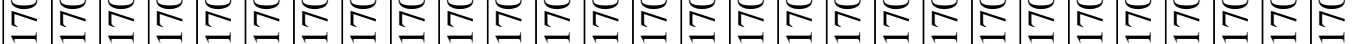

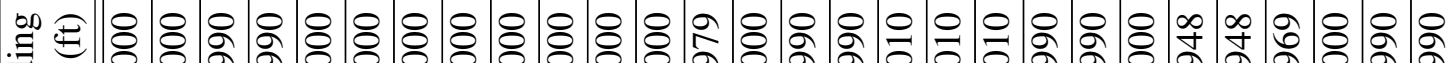

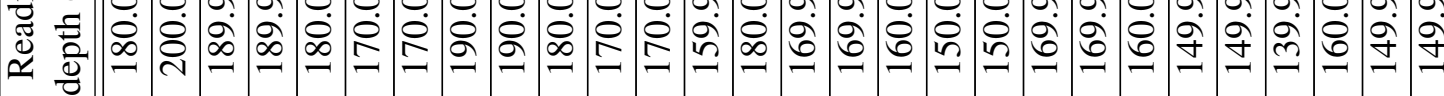
$\frac{0}{2}$

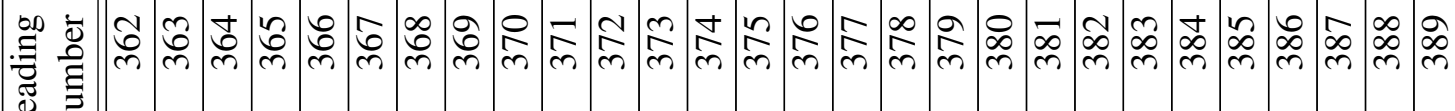

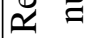




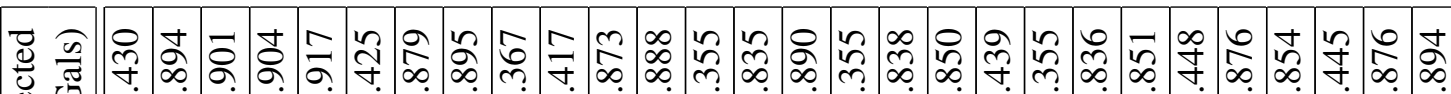

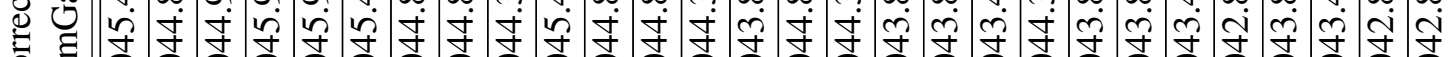

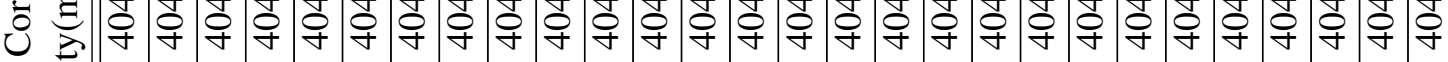

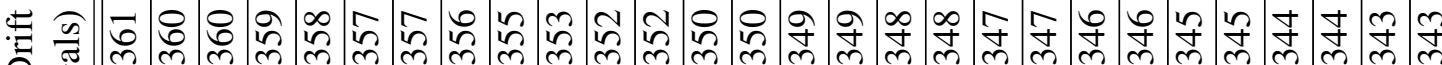

荎

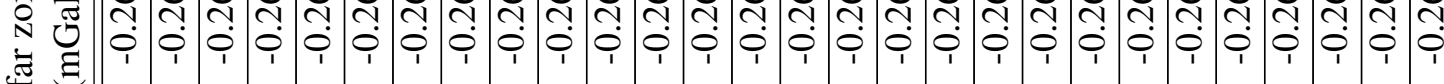

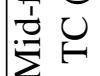

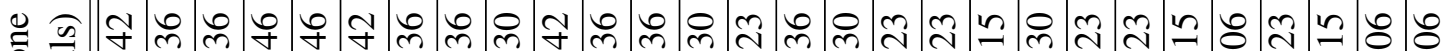

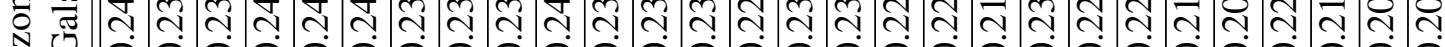
1 ฮै $\mathrm{Z}$

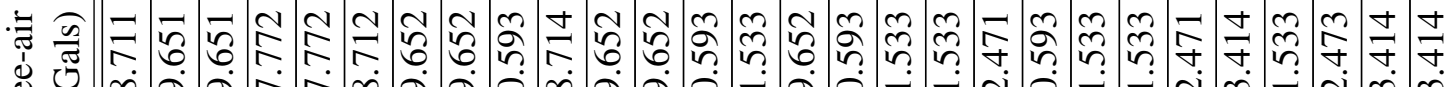

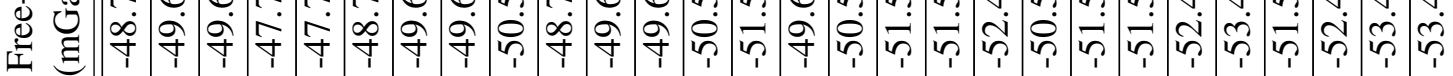
อั

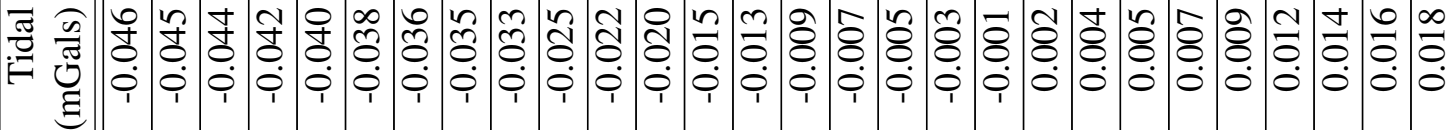
ठํ

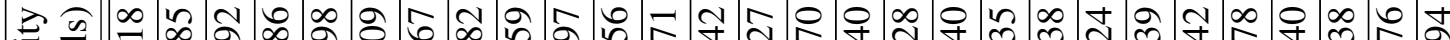
ट्च - nं ń

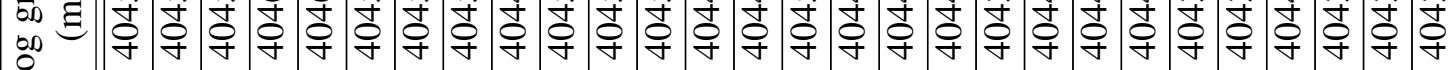

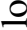

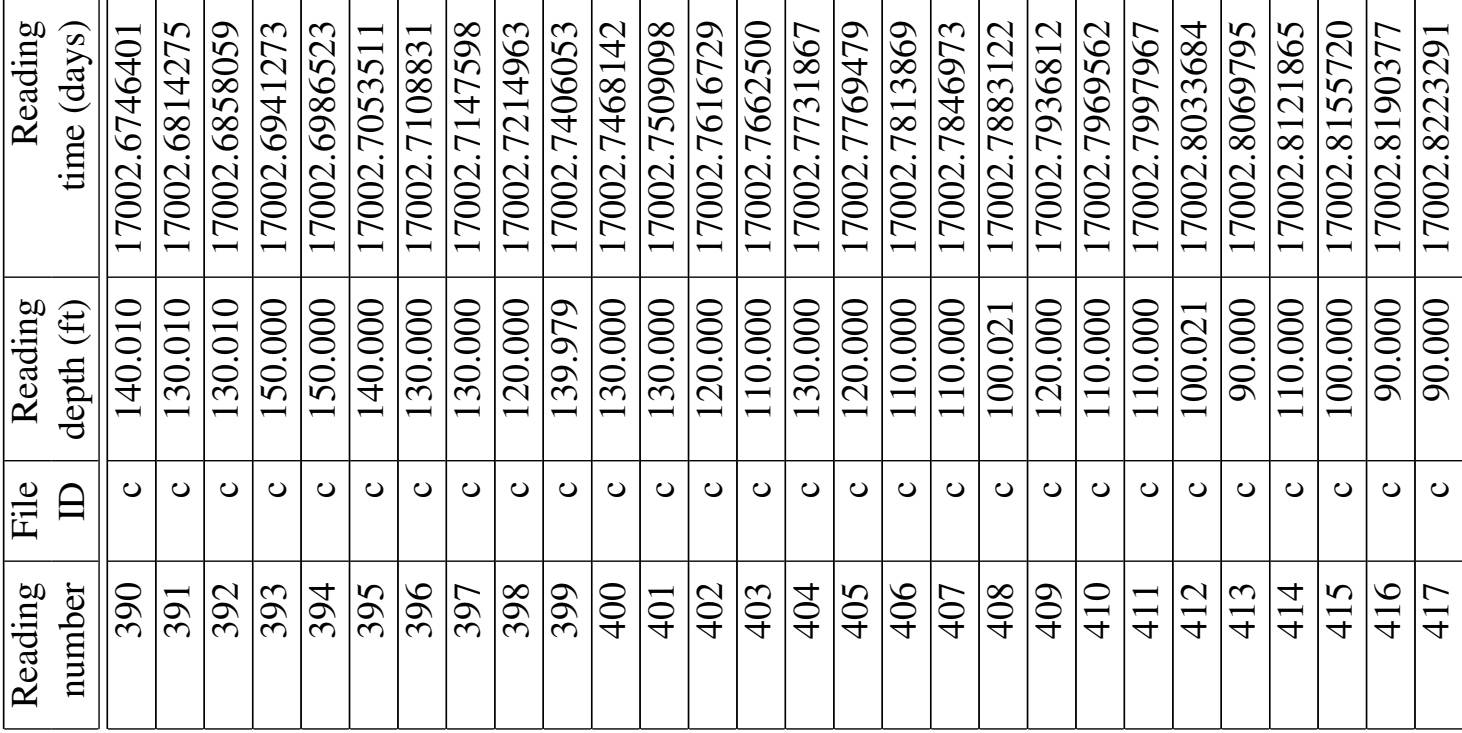


ఫ U :

:

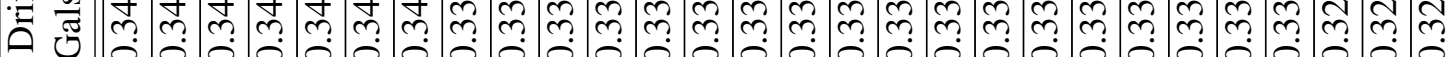
Z ¿ั0

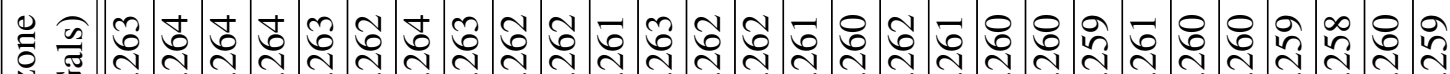

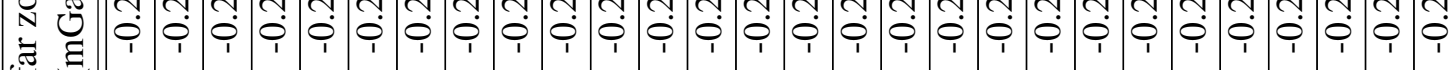
齐

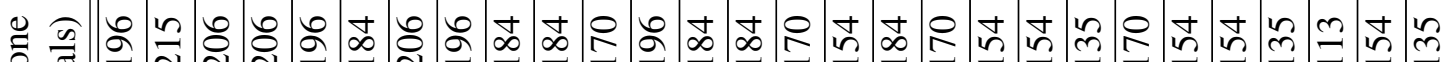
苾 ซี Z

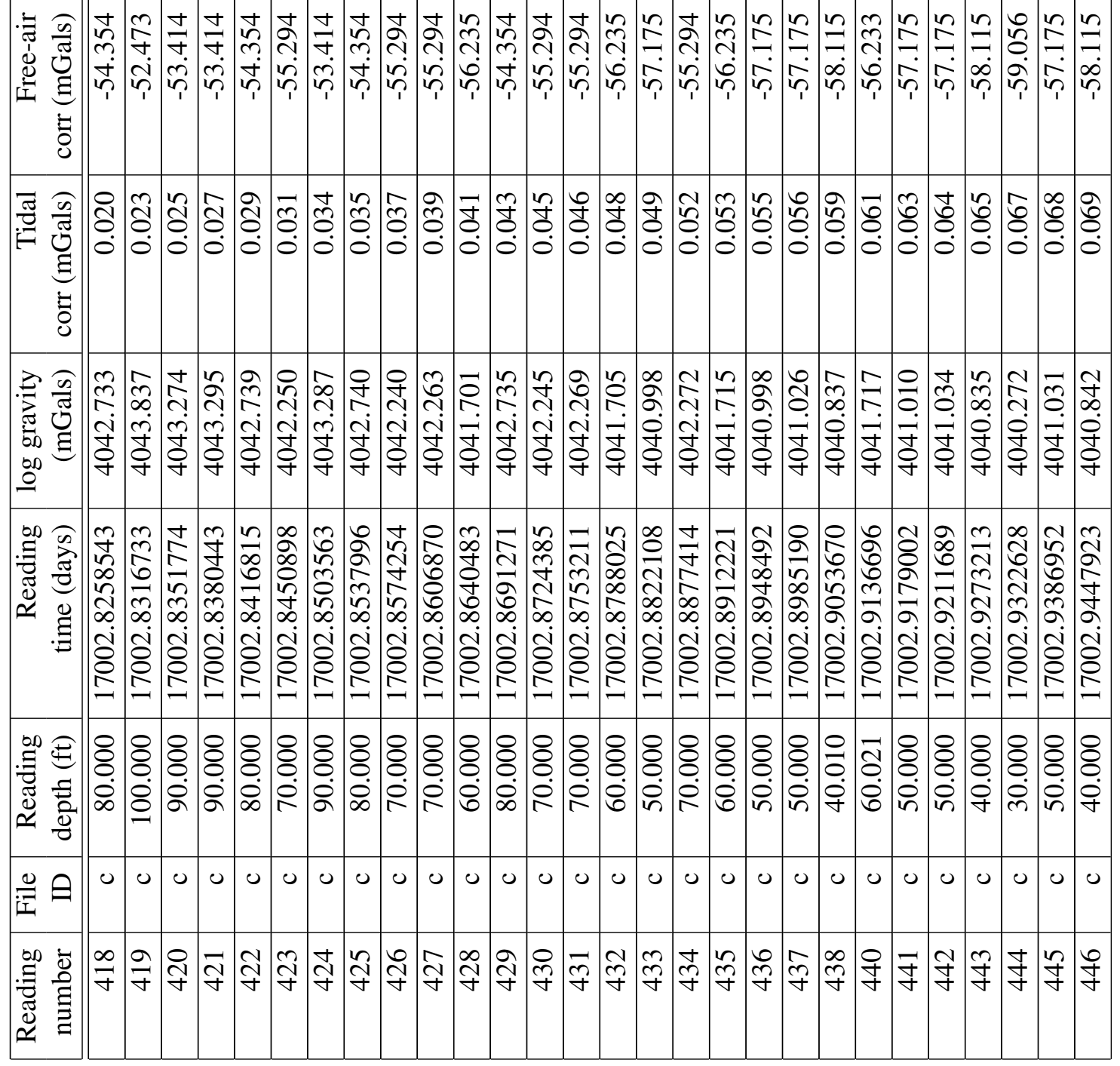




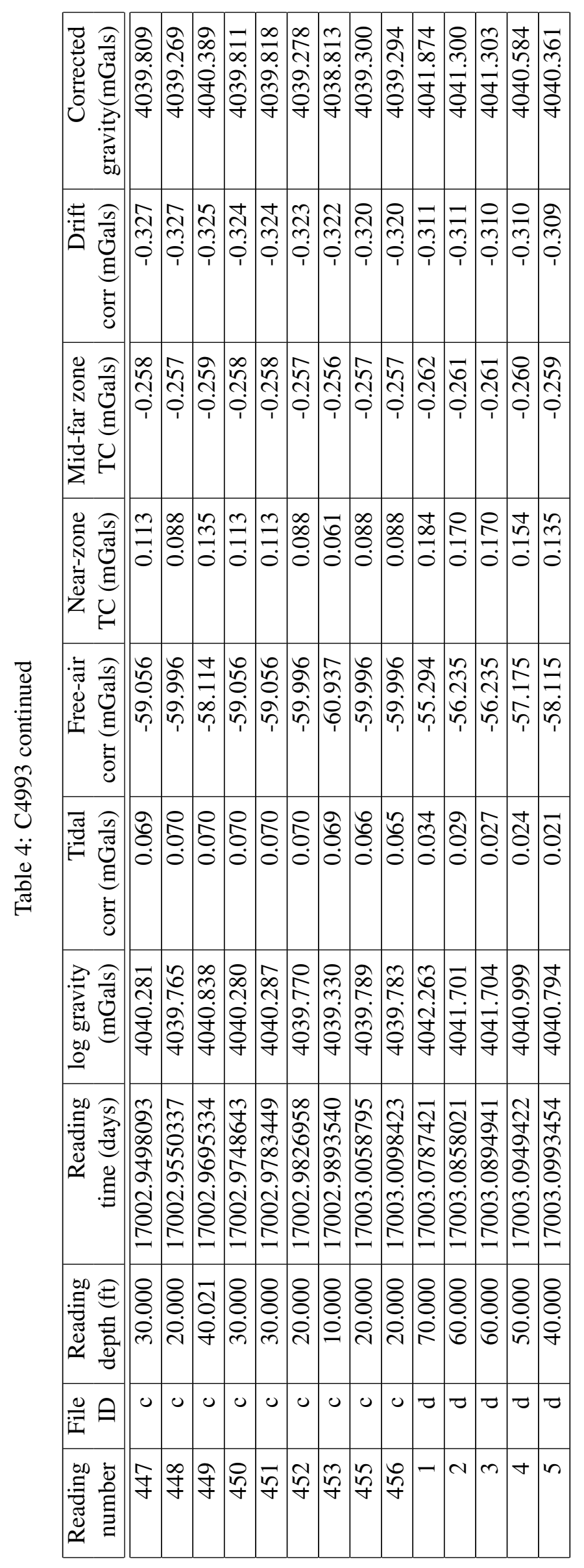


A.2 $\mathbf{C 4 9 9 6}$ 


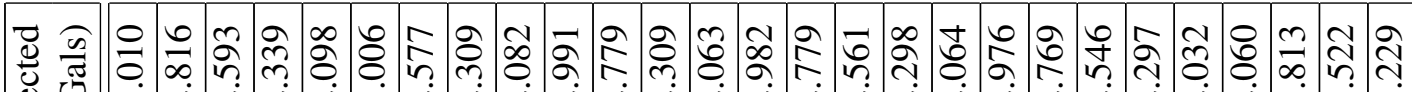

d

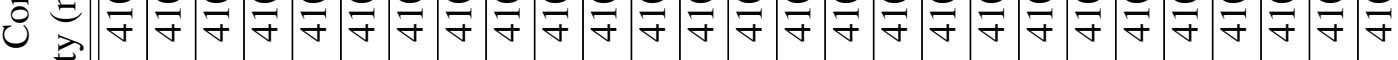
晃

है

๘ 官 总

8

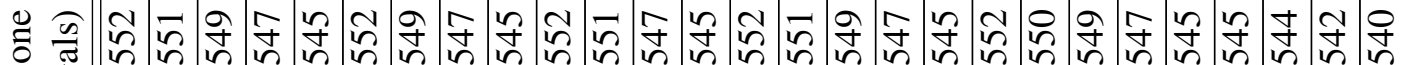

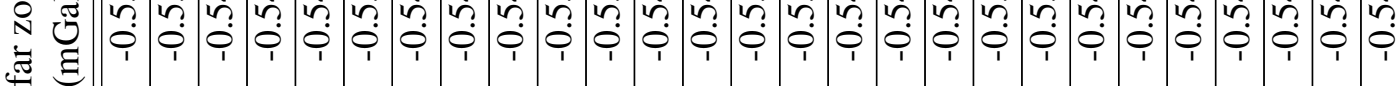

$\underset{\sim}{\mathcal{2}}$

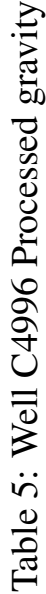

泀 向

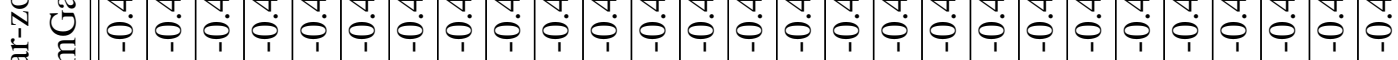

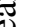

Z

ఈ

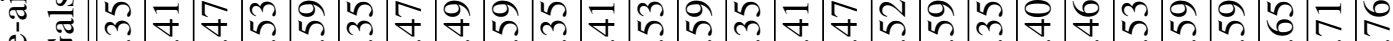
年 ○ั

苑 茪

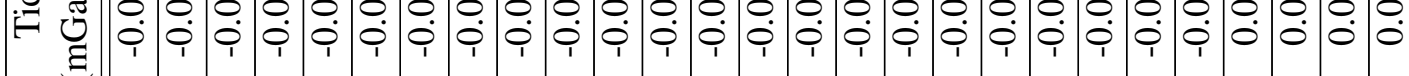
:

当 命 च j

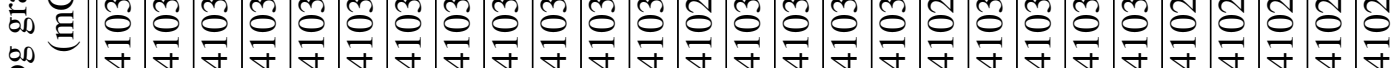
잉

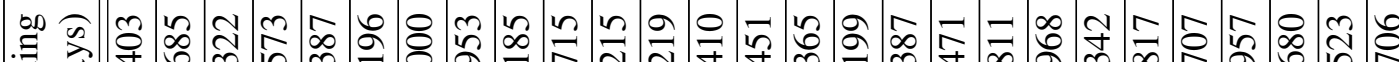
శ

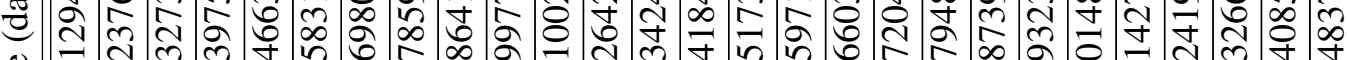

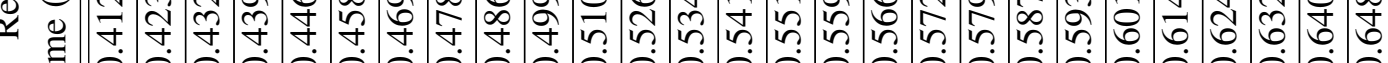

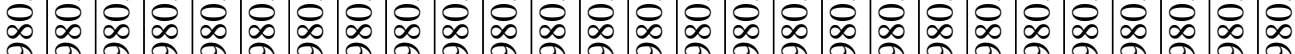

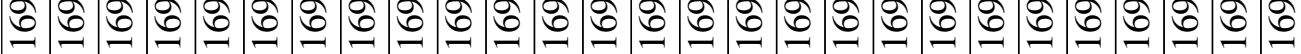

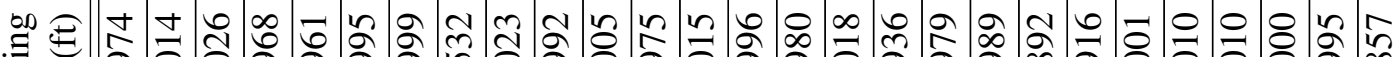

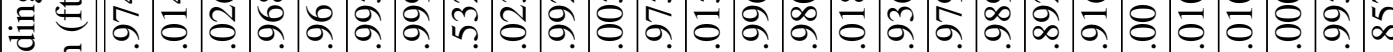
ت

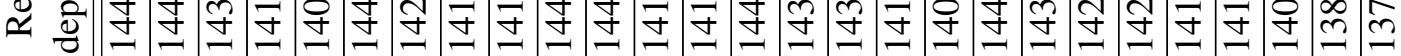
م 远

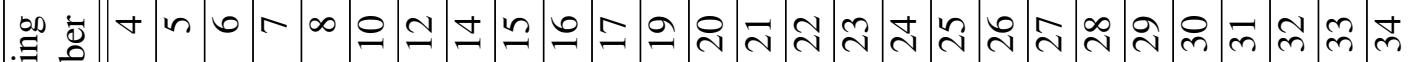
刍 


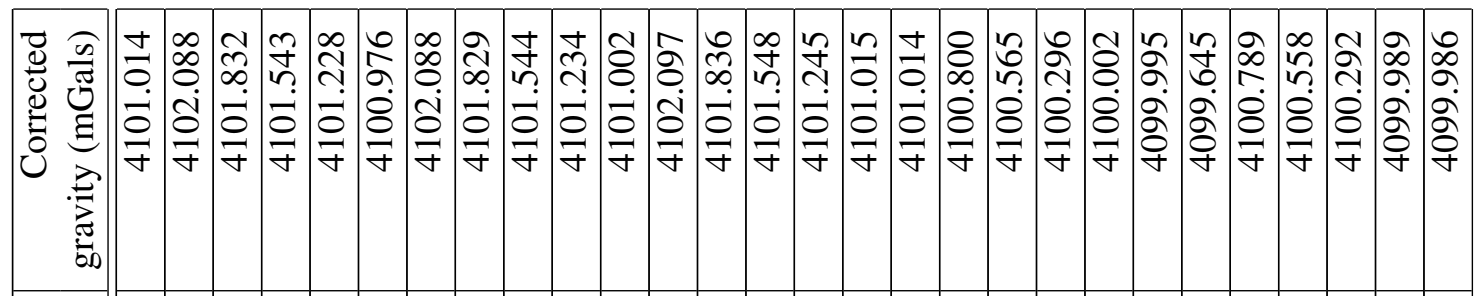

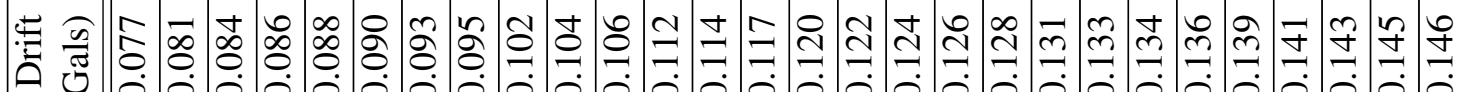

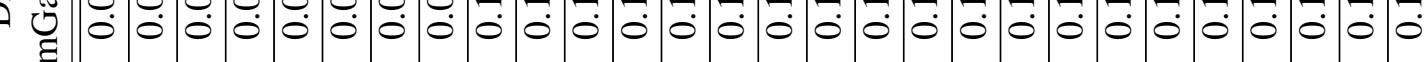

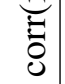

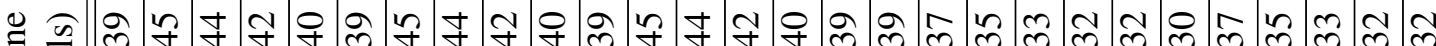

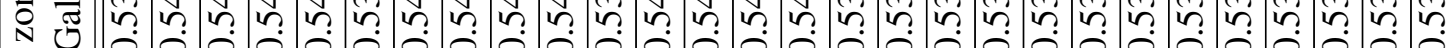
节

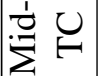

苛 向 N 范告

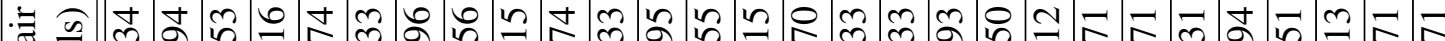

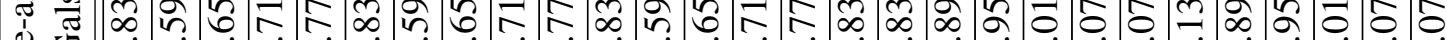
党 ठํำ

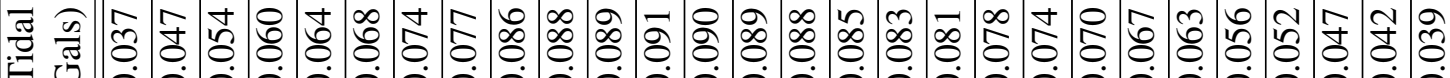
F ¿ัర

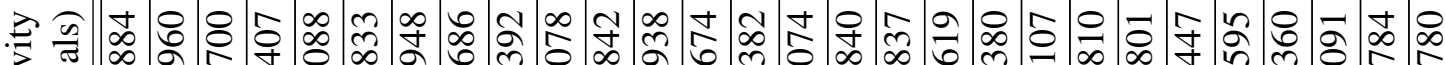
च

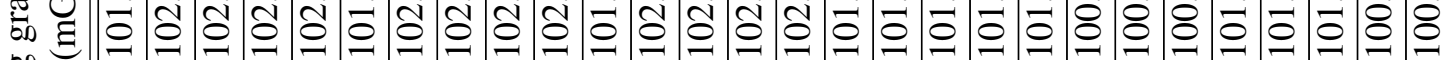
呬

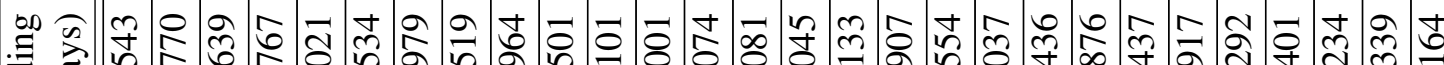
胥

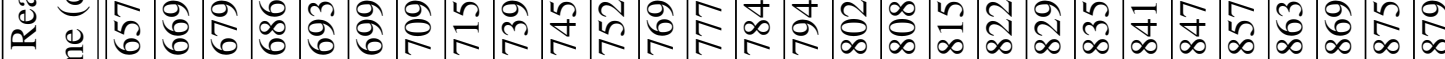

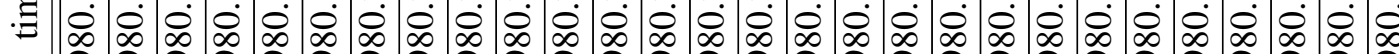
bे

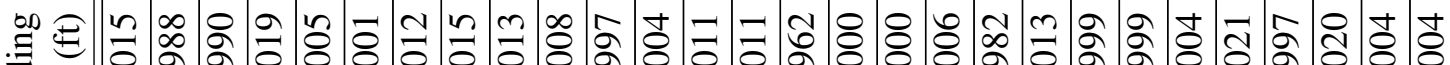
च

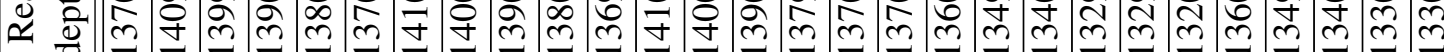

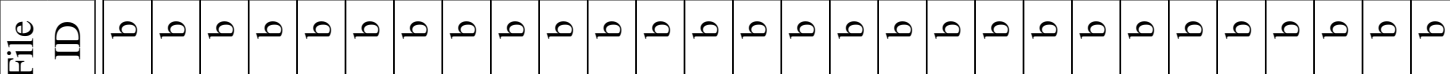

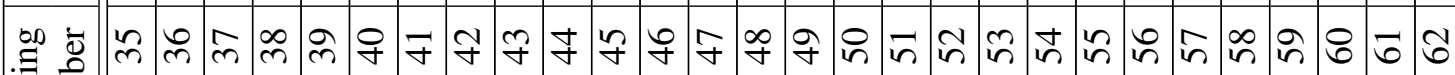
急 


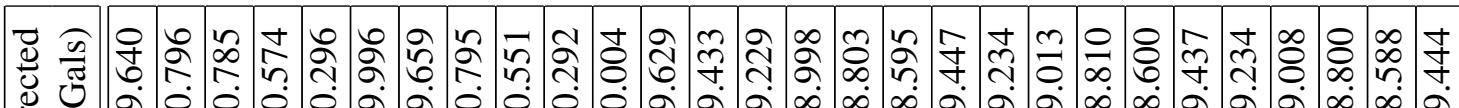

b

U

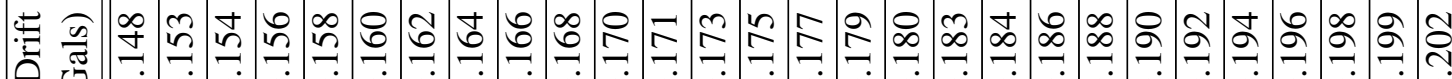

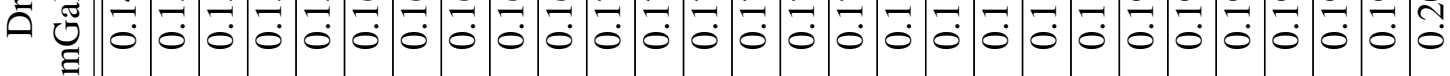

ஜ

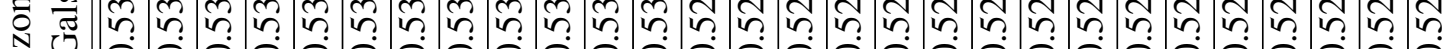
z

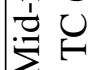

苽

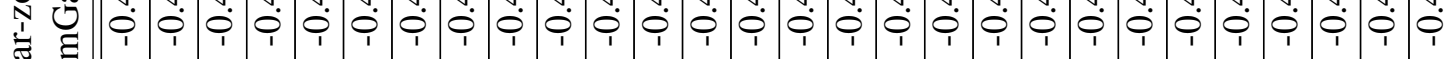
ฮ $\mathrm{Z}$

药

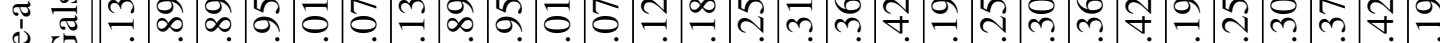

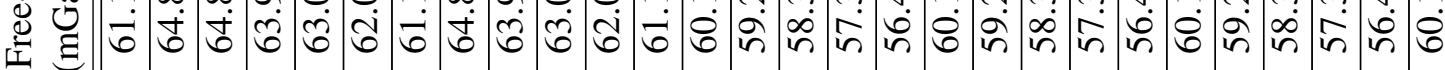
:

沓

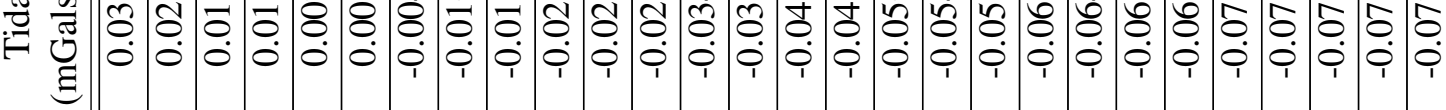

8

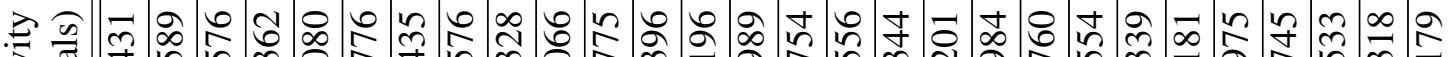
द्य 茂 인

我 万人 - 馬 ๘

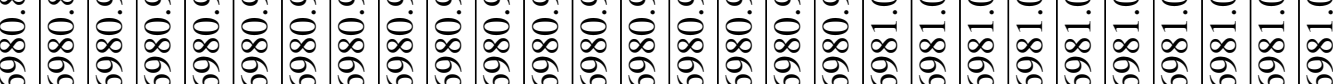

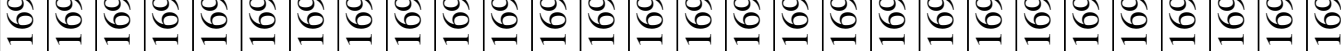
告 ๘ ๘ م

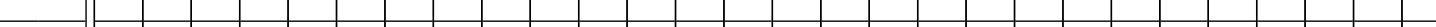

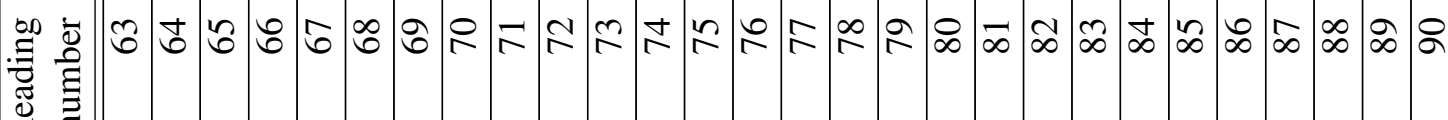




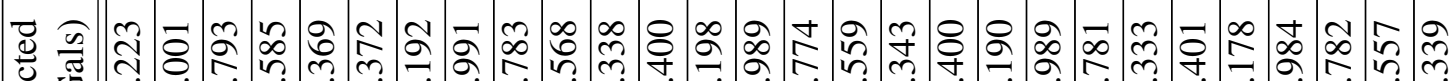

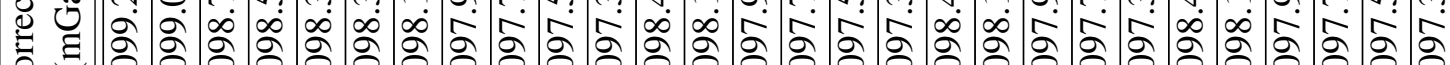
U $\begin{aligned} & \text { u } \\ & \text { o }\end{aligned}$

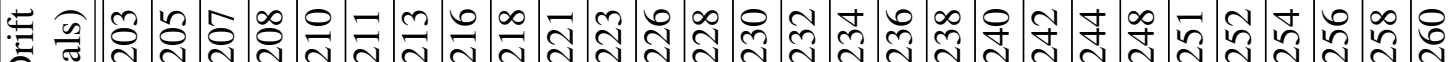

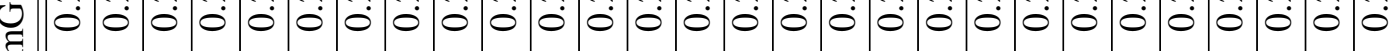
:

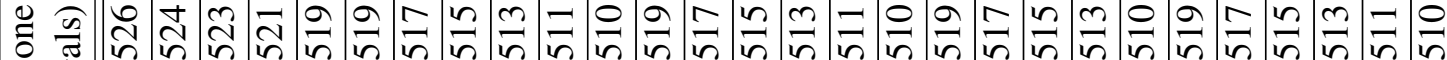

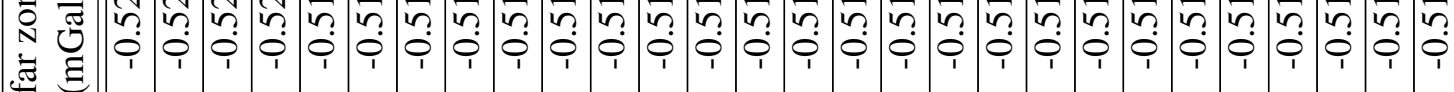

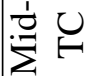

节 向

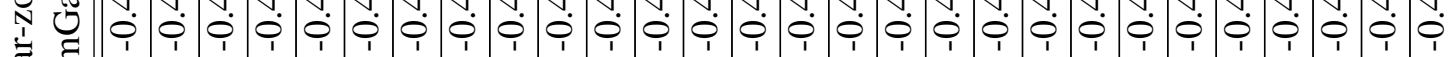
范告 $\mathrm{Z}$,

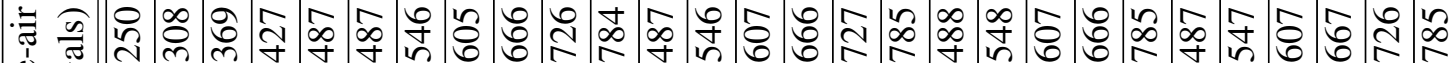

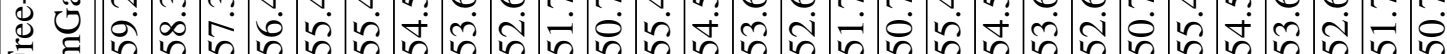
空 ठํำ

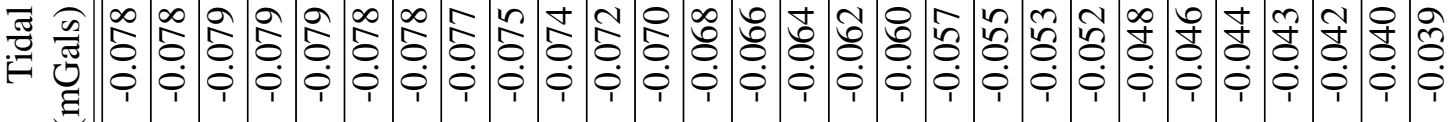
2

ઊa 元 फ

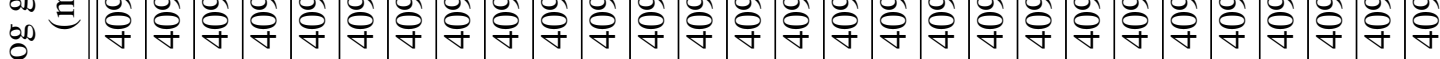
으

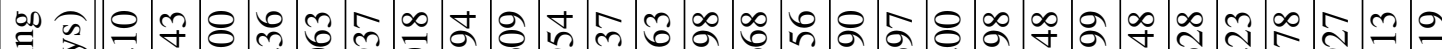

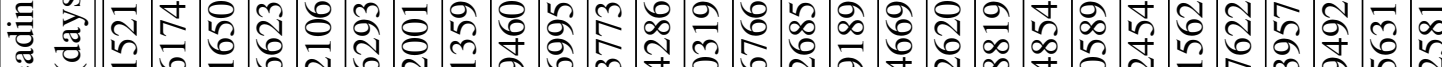
¿

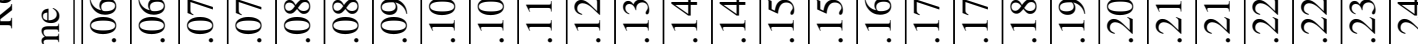

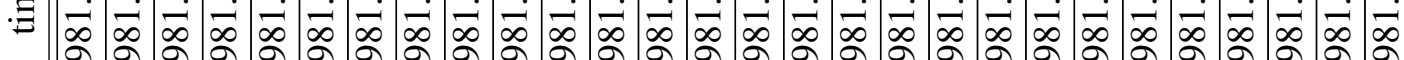
ல

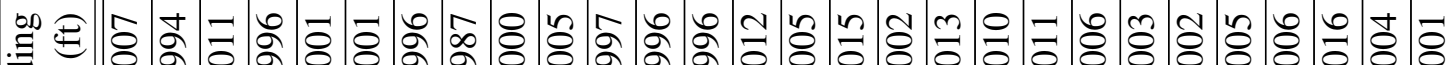
च $\approx$ :

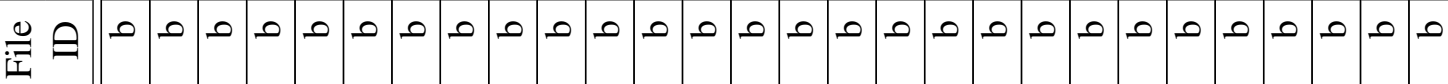

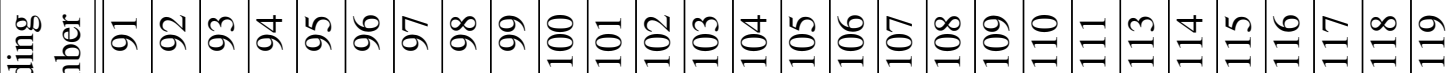

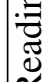


绨 至 己

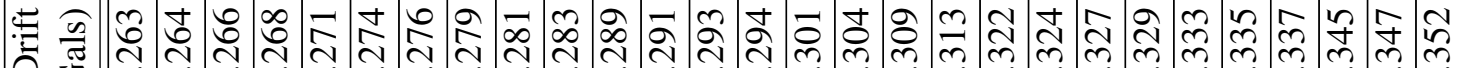

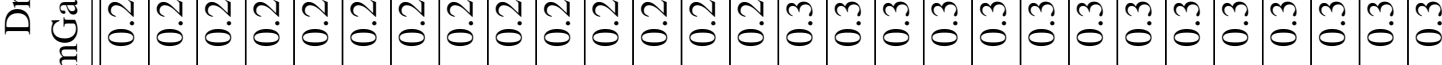

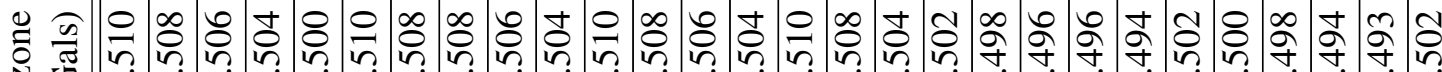
ñ $\sum$

亲全 苟 ฮ $Z$ U

: ن

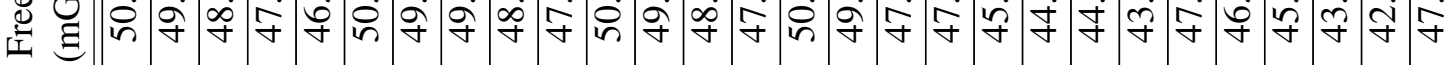
ठํำ

丞 F

¿ั

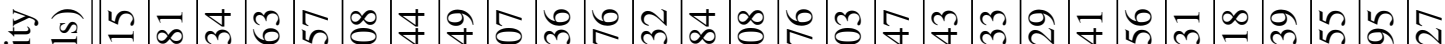

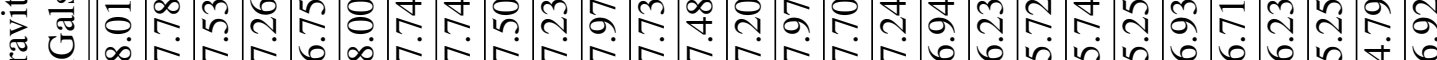

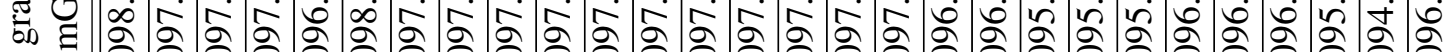

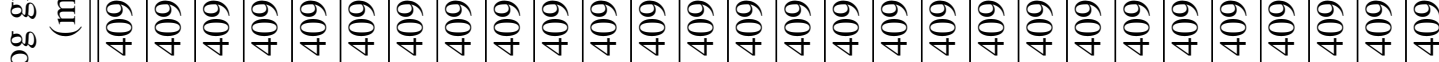

舟

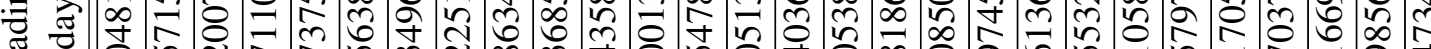

-

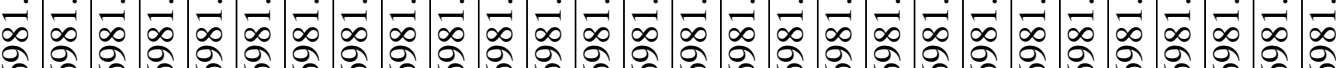

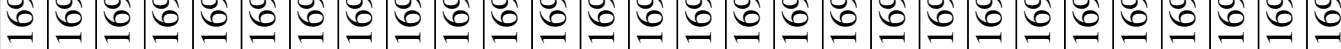

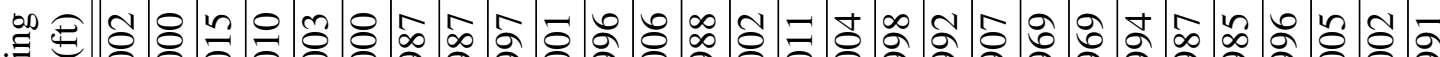
$\because$ - 0 б \&

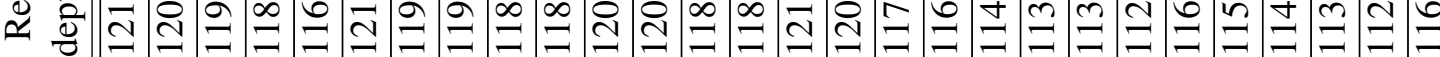
$\cong \mathrm{O}$

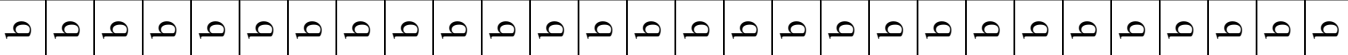
$\Xi$

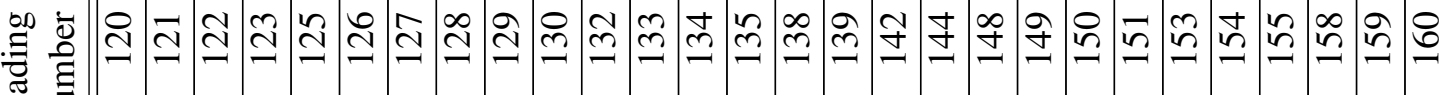
$\simeq$ 


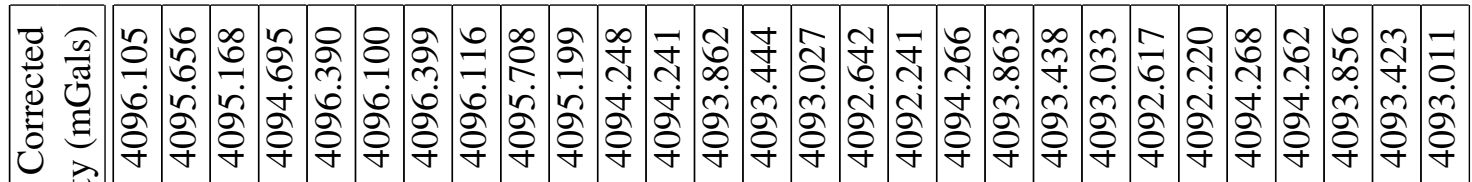

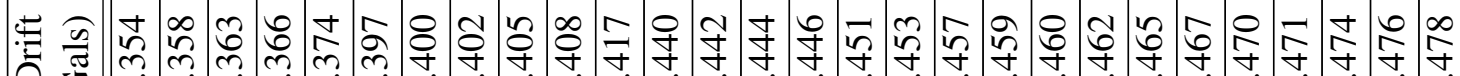

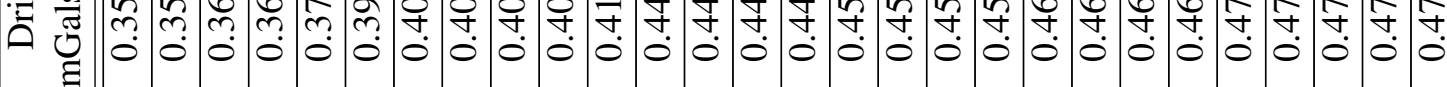

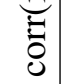

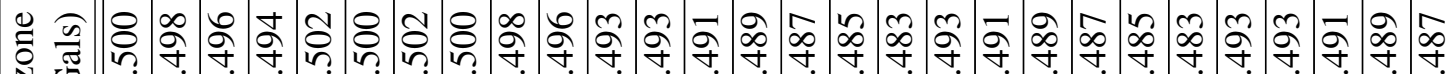

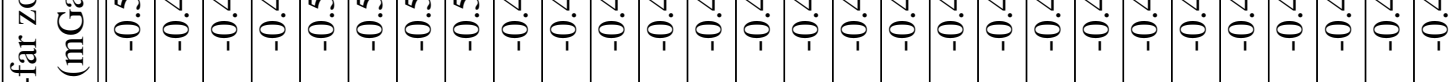

$\sum_{\ominus}$

司 毛 N ฮ Z

\begin{tabular}{|c|c|c|c|c|c|c|c|c|c|c|c|c|c|c|c|c|c|c|c|c|c|c|c|c|}
\hline 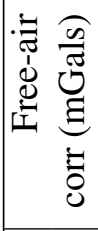 & $\mid \begin{array}{l}1 \\
0 \\
0 \\
\dot{0} \\
+\end{array}$ & & $\begin{array}{l}\vec{D} \\
\stackrel{1}{ } \\
\dot{f}\end{array}$ & & $\begin{array}{l}\text { है } \\
\text { f }\end{array}$ & & $\frac{\sigma}{\sigma}$ & & & & & & $\begin{array}{l}\text { ? } \\
\text { : }\end{array}$ & $\begin{array}{l}\stackrel{2}{a} \\
\stackrel{+}{\sigma}\end{array}$ & $\begin{array}{l}\infty \\
n \\
n \\
\infty \\
n\end{array}$ & & 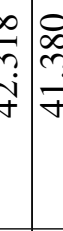 & $\begin{array}{l}\hat{\tilde{\sigma}} \\
\dot{\sigma} \\
\dot{q}\end{array}$ & $\begin{array}{l}\hat{a} \\
\hat{q} \\
\hat{m}\end{array}$ & $\begin{array}{c}\infty \\
m\end{array}$ & 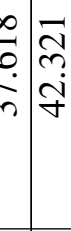 & $\mid \begin{array}{c}\vec{N} \\
\tilde{j} \\
\stackrel{\sim}{f}\end{array}$ & 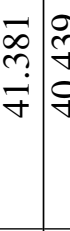 & i \\
\hline 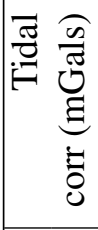 & $\begin{array}{l}n \\
0 \\
0 \\
0 \\
1 \\
1\end{array}$ & 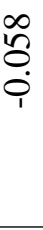 & $\begin{array}{l}0 \\
0 \\
0 \\
0 \\
1\end{array}$ & $\begin{array}{l}\infty \\
⿱ \\
0 \\
0 \\
1\end{array}$ & $\begin{array}{l}- \\
0 \\
0 \\
\dot{1}\end{array}$ & $\begin{array}{l}\tilde{n} \\
0 \\
0\end{array}$ & $\overline{8}$ & 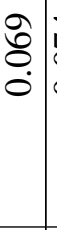 & $\vec{J}$ & \begin{tabular}{c|c}
$N$ & \\
0 & \\
0 & \\
0 &
\end{tabular} & 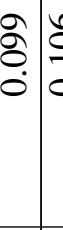 & $\frac{0}{0}$ & $\frac{\sigma}{0}$ & 文 & $\begin{array}{l}\vec{\sigma} \\
\dot{8} \\
0\end{array}$ & & 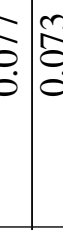 & 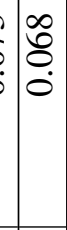 & 잉 & & 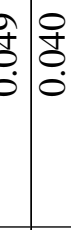 & ڤె. & & \begin{tabular}{l|l} 
\\
$\dot{0}$
\end{tabular} \\
\hline 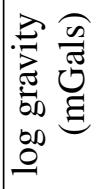 & 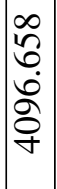 & 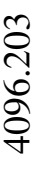 & $\begin{array}{l}\infty \\
\stackrel{2}{2} \\
\stackrel{2}{2} \\
2 \\
\dot{y}\end{array}$ & 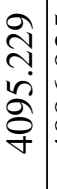 & 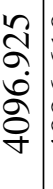 & $\begin{array}{l}0 \\
0 \\
0 \\
\dot{0} \\
\stackrel{0}{o} \\
\dot{q}\end{array}$ & 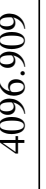 & 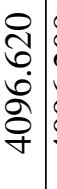 & & 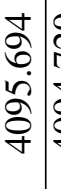 & 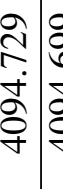 & 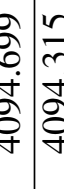 & 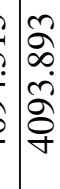 & 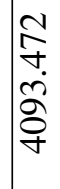 & $\begin{array}{c}0 \\
\infty \\
0 \\
0 \\
2 \\
\delta \\
\dot{y}\end{array}$ & 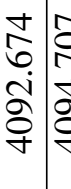 & 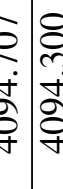 & $\left\{\begin{array}{l}\vec{\alpha} \\
\infty \\
\dot{\alpha} \\
\partial \\
\dot{\gamma}\end{array}\right.$ & $\begin{array}{l}\bar{\sigma} \\
+ \\
\dot{\gamma} \\
\hat{\sigma} \\
\dot{q}\end{array}$ & 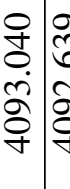 & 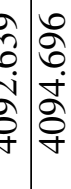 & $\begin{array}{l}\infty \\
\infty \\
0 \\
\dot{+} \\
\dot{g} \\
\dot{f}\end{array}$ & 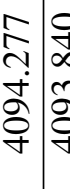 & 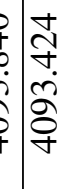 \\
\hline 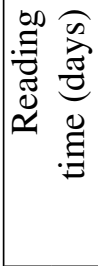 & 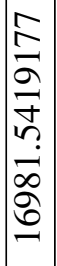 & $\begin{array}{l}n \\
0 \\
\infty \\
2 \\
n \\
n \\
\infty \\
\infty \\
0 \\
0 \\
-1\end{array}$ & 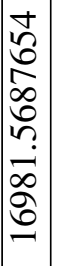 & 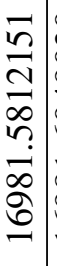 & 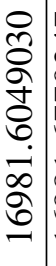 & 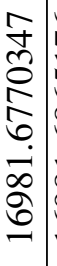 & 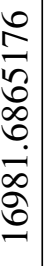 & 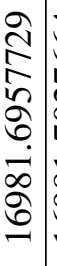 & 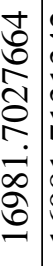 & 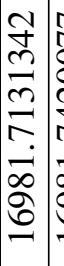 & 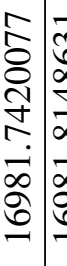 & 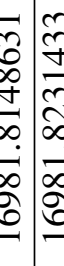 & 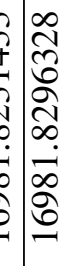 & 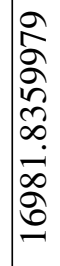 & 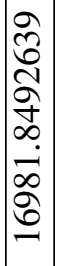 & 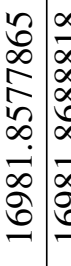 & 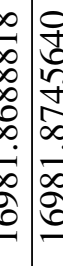 & $\left\{\begin{array}{l}\sqrt{\sqrt{n}} \\
\infty \\
\infty \\
\infty \\
\infty \\
\infty \\
\infty \\
0 \\
0 \\
0\end{array}\right.$ & 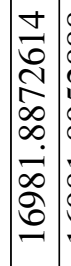 & 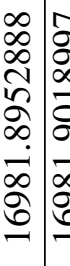 & 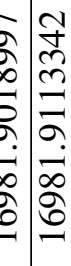 & $\mid \begin{array}{l} \pm \\
0 \\
0 \\
\sigma \\
\sigma \\
\infty \\
\delta \\
-0\end{array}$ & 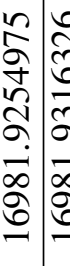 & 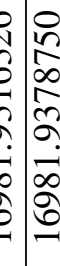 \\
\hline 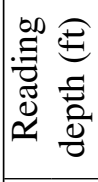 & $\begin{array}{l}2 \\
\Omega \\
\Omega \\
= \\
=\end{array}$ & $\begin{array}{l}8 \\
8 \\
0 \\
\\
= \\
=\end{array}$ & $\begin{array}{l}\stackrel{8}{2} \\
2 \\
2 \\
= \\
=\end{array}$ & $\begin{array}{l} \pm \\
\infty \\
a \\
\grave{\Xi} \\
=\end{array}$ & $\begin{array}{l}\dot{\partial} \\
\hat{\sigma} \\
\hat{\sigma} \\
= \\
=\end{array}$ & $\begin{array}{l}m \\
8 \\
0 \\
\dot{0} \\
= \\
=\end{array}$ & $\begin{array}{l}\dot{8} \\
8 \\
\dot{9} \\
= \\
=\end{array}$ & 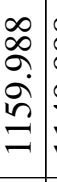 & $\begin{array}{l}\infty \\
\sigma \\
\sigma \\
\dot{\sigma} \\
\Xi\end{array}$ & $\begin{array}{l}\hat{\sigma} \\
\hat{\sigma} \\
\hat{\sigma} \\
=\end{array}$ & 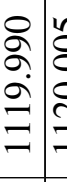 & 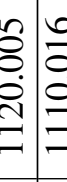 & 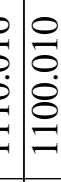 & $\begin{array}{l}\circ \\
2 \\
2 \\
2 \\
0 \\
0\end{array}$ & $\begin{array}{l}8 \\
8 \\
0 \\
\dot{0} \\
\infty \\
0 \\
-\end{array}$ & 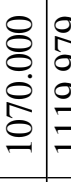 & 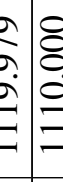 & $\begin{array}{l}8 \\
8 \\
\dot{8} \\
\dot{8} \\
\stackrel{8}{8}\end{array}$ & $\begin{array}{l}\Omega \\
\hat{\sigma} \\
2 \\
\infty \\
0 \\
0\end{array}$ & 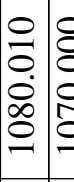 & 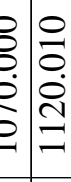 & $\begin{array}{c}0 \\
0 \\
0 \\
\dot{\Xi} \\
= \\
=\end{array}$ & 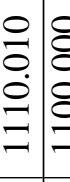 & 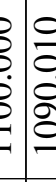 \\
\hline & م & مـ & مـ & مـ & مـ & مـ & م & م & مـ & مـ & ـ مـ & ـ م & م. & م & م & ـ مـ & م. & م & مـ & S & م. م & مـ & o 0 & o \\
\hline 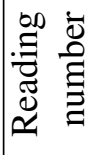 & $\overline{0}$ & ర్ర & $\underline{6}$ & 6 & 6 & bे & $\stackrel{2}{2}$ & $\Sigma$ & $\mathbb{N}$ & I & $\Sigma$ & $\infty ?$ & $1 \approx$ & $\infty$ & $\mathscr{\infty}$ & $\underset{\infty}{\infty} \alpha$ & $\begin{array}{ll}\infty & \infty\end{array}$ & 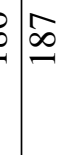 & $\infty$ & 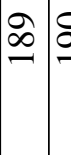 & $2 \bar{\sigma}$ & $\sigma$ & $\stackrel{\sigma}{\sigma}$ & $\stackrel{0}{O}$ \\
\hline
\end{tabular}




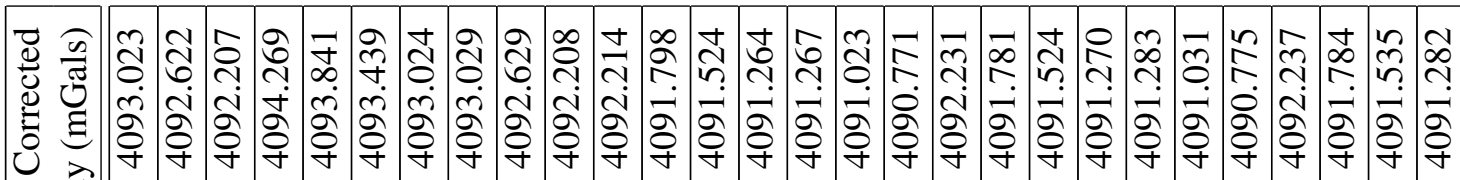
.

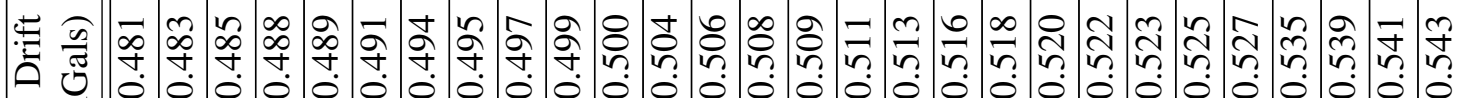
A ठำ

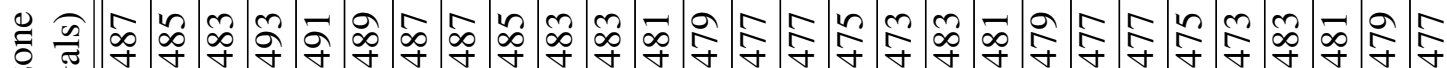

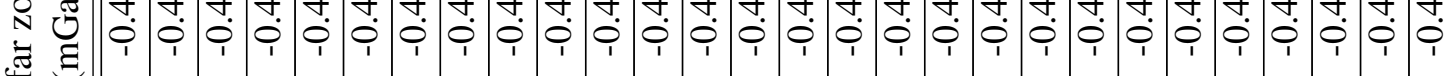

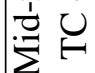

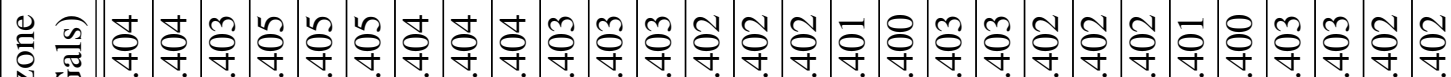
N ङ $\mathrm{Z} \cup$

ఈ ส

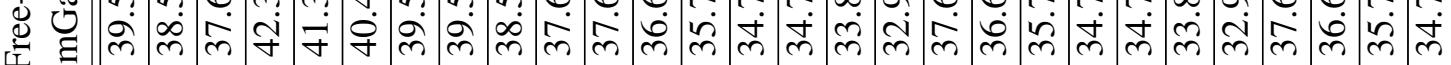
ठำ

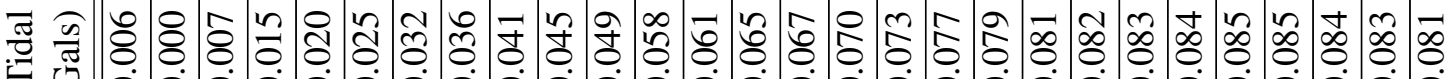

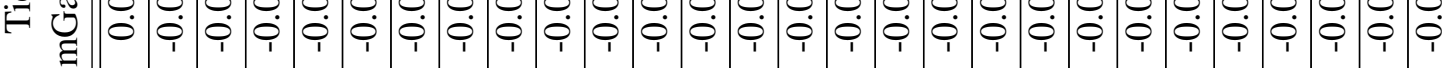
¿

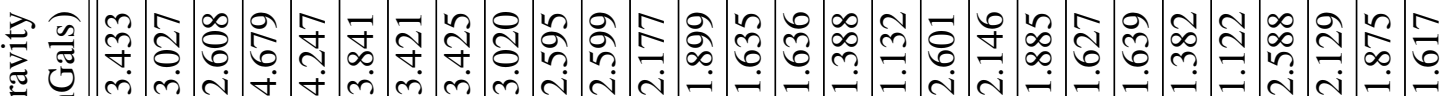

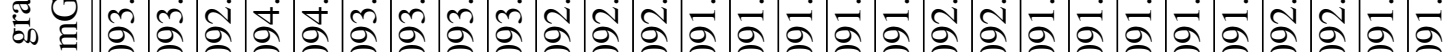

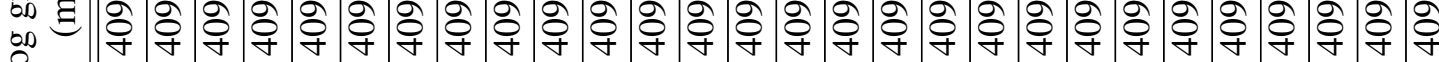

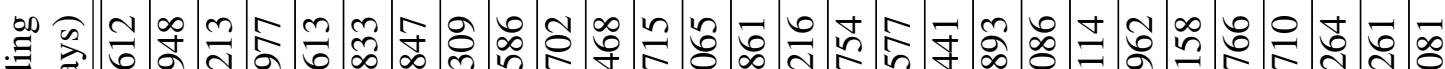

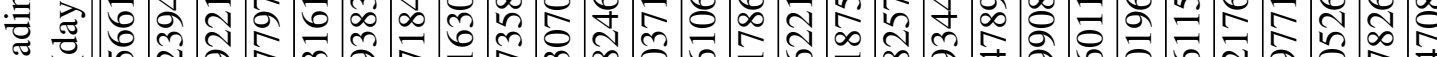
¿ .

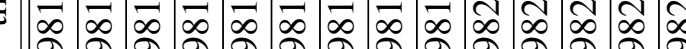
6

\begin{tabular}{|c|c|c|c|c|c|c|c|c|c|c|c|c|c|c|c|c|c|c|c|c|c|c|c|c|c|}
\hline 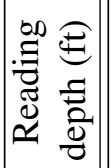 & \begin{tabular}{|l|} 
\\
0 \\
0 \\
$\dot{0}$ \\
8 \\
0 \\
\end{tabular} & \begin{tabular}{|l|} 
\\
8 \\
8 \\
0 \\
$\infty$ \\
0 \\
0 \\
\end{tabular} & $\begin{array}{l}8 \\
8 \\
0 \\
0 \\
0\end{array}$ & 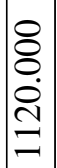 & $\begin{array}{l}0 \\
0 \\
0 \\
\dot{0} \\
= \\
=\end{array}$ & \begin{tabular}{|l|} 
\\
8 \\
0 \\
8 \\
0 \\
$=$
\end{tabular} & $\begin{array}{l}\vec{Z} \\
\dot{0} \\
\dot{\dot{g}} \\
\dot{0}\end{array}$ & 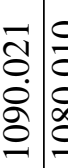 & 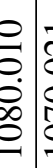 & 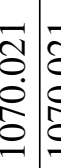 & & 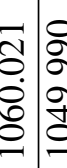 & 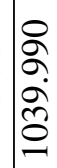 & 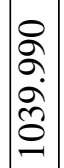 & 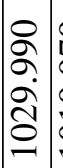 & 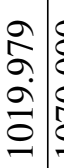 & & 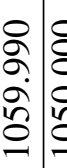 & 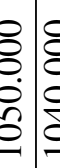 & 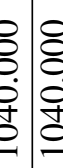 & 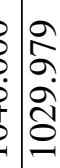 & $\begin{array}{l}\frac{8}{\sigma} \\
\frac{\sigma}{\sigma}\end{array}$ & $\begin{array}{l}0 \\
0 \\
0 \\
0 \\
0 \\
0\end{array}$ & & 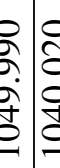 \\
\hline$\Theta$ & مـ & مـ & مـ & مـ & مـ & مـ & مـ & كـ م & ــ م. & ك مـ & S & ـ 0 م & م & م & م & ــ مـ & م & o 0 & S & o & مـ & م & مـ & ـ & م \\
\hline $\begin{array}{l}0 \\
\text { है } \\
\end{array}$ & $\hat{a}$ & $\stackrel{\infty}{\varrho}$ & 玉 & ঠ্ণ & $\overline{\tilde{N}}$ & 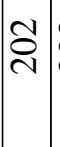 & ڤి & 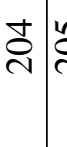 & $\stackrel{n}{\mathrm{c}}$ & 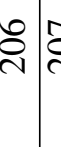 & 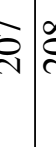 & 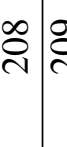 & $\frac{O}{2}$ & $\bar{\nabla}$ & $\stackrel{\sim}{\sim}$ & $\frac{m}{\sim}$ & $\frac{n}{\sqrt{n}}$ & \begin{tabular}{l|l}
0 & $\frac{1}{2}$ \\
\end{tabular} & $\vec{\nabla} \mid \frac{\alpha}{\sigma}$ & $\frac{\infty}{\sim} \frac{O}{\sim}$ & ì & $\overline{\mathrm{N}}$ & $\overrightarrow{\mathrm{A}}$ & $\stackrel{\sim}{\sim}$ & \begin{tabular}{c|c}
$\hat{v}$ & \multirow{2}{*}{}
\end{tabular} \\
\hline
\end{tabular}




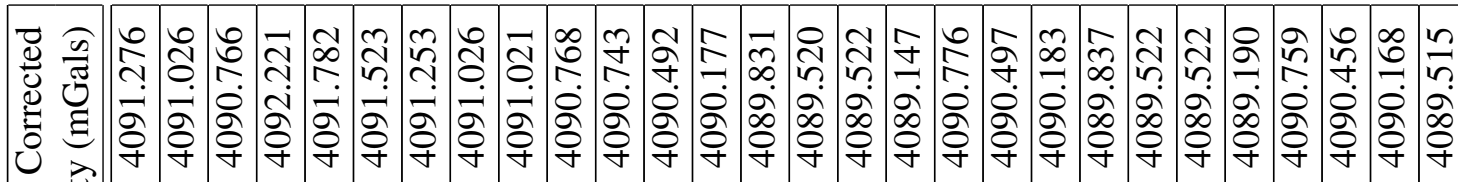

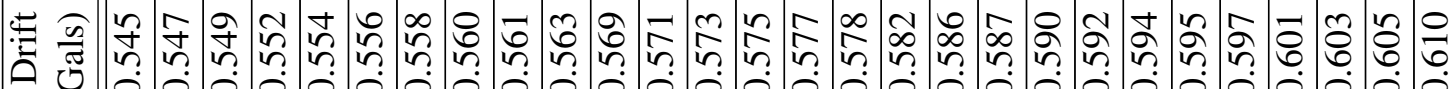

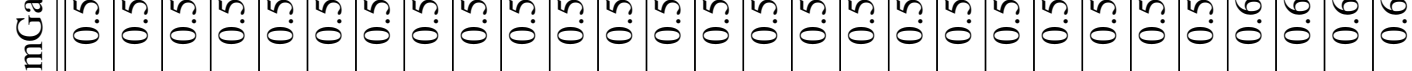
¿ำ

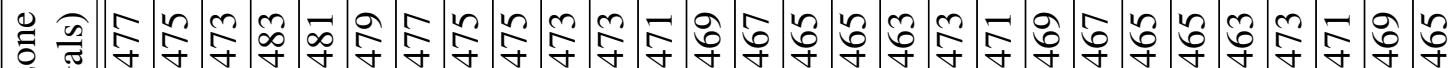

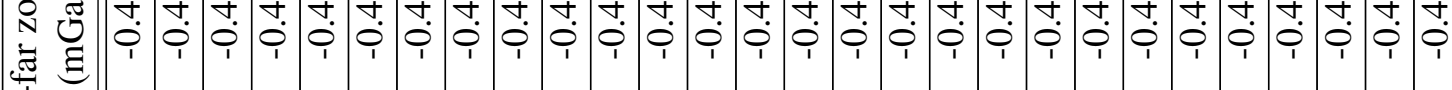

$\sum_{\ominus}$

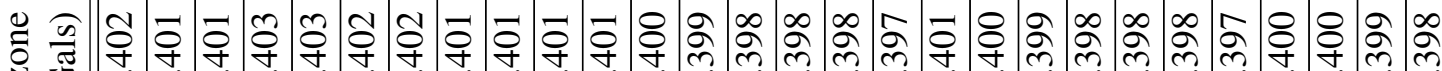

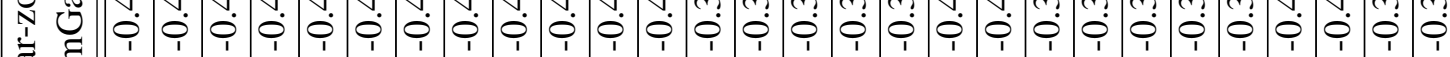
ซ 乙

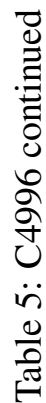

\begin{tabular}{|c|c|c|c|c|c|c|c|c|c|c|c|c|c|c|c|c|c|c|c|c|c|c|c|c|}
\hline 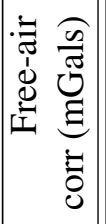 & \begin{tabular}{l}
$\infty$ \\
2 \\
\multirow{1}{j}{} \\
$m$
\end{tabular} & $\begin{array}{l}\infty \\
\infty \\
\infty \\
\dot{m} \\
m\end{array}$ & & $\begin{array}{l}\bar{b} \\
\bar{m}\end{array}$ & & & $\begin{array}{rl}r & a \\
\dot{m} & \alpha \\
d & d\end{array}$ & $\begin{array}{l}\vdots \\
\vdots \\
\infty \\
\infty \\
\infty\end{array}$ & $\begin{array}{l}\frac{b}{a} \\
i \\
\tilde{n}\end{array}$ & & & 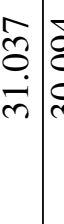 & & $\dot{0}$ & \begin{tabular}{l} 
\pm \\
\multirow{2}{*}{} \\
$\infty$ \\
$i$
\end{tabular} & $\begin{array}{ll}\bar{a} & 0 \\
\tilde{a} & 0 \\
\tilde{n} & 0\end{array}$ & & & $\frac{n}{2}$ & ڤ̊ & ते & $\tilde{N}$ & & \\
\hline 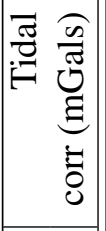 & $\begin{array}{c}0 \\
\infty \\
0 \\
0 \\
1\end{array}$ & $\begin{array}{l}\infty \\
0 \\
0 \\
0 \\
1\end{array}$ & $\begin{array}{l}\hat{a} \\
\hat{a} \\
0 \\
\end{array}$ & 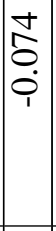 & 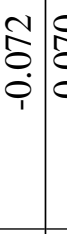 & & 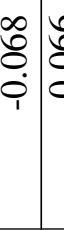 & 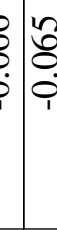 & : & $\begin{array}{l}\infty \\
0 \\
0 \\
0 \\
1 \\
1\end{array}$ & $\begin{array}{l}0 \\
2 \\
0 \\
0 \\
1\end{array}$ & 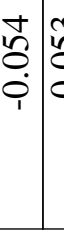 & 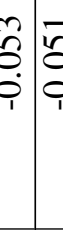 & $\begin{array}{l}5 \\
0 \\
0 \\
0\end{array}$ & 守 & $\begin{array}{l}0 \\
0 \\
0 \\
0 \\
1 \\
1\end{array}$ & 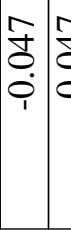 & 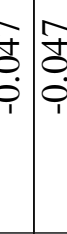 & 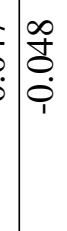 & $\mid \begin{array}{l}0 \\
0 \\
0 \\
0 \\
1\end{array}$ & $\begin{array}{l}0 \\
\dot{J} \\
\dot{0} \\
\dot{1}\end{array}$ & $\begin{array}{l}0 \\
\stackrel{R}{0} \\
\dot{0} \\
1\end{array}$ & $\circ 0$ & \\
\hline 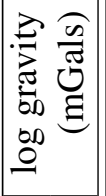 & 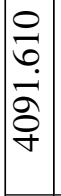 & 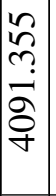 & 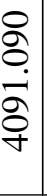 & $\begin{array}{l}n \\
n \\
n \\
i \\
\delta \\
\partial \\
f\end{array}$ & 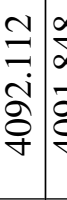 & 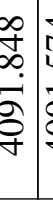 & 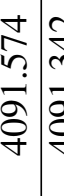 & 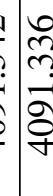 & 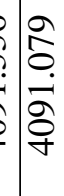 & 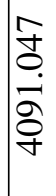 & $\begin{array}{l}\overline{2} \\
\hat{2} \\
\dot{8} \\
\dot{8} \\
y\end{array}$ & 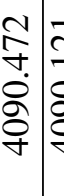 & 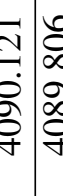 & 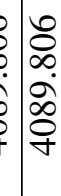 & 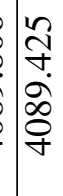 & 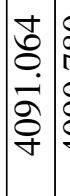 & 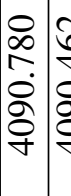 & 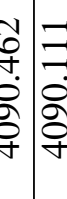 & $\left\{\begin{array}{l}\bar{a} \\
\vec{a} \\
\infty \\
0 \\
+\end{array}\right.$ & $\begin{array}{l}2 \\
\infty \\
\dot{0} \\
\infty \\
\infty \\
\dot{y}\end{array}$ & 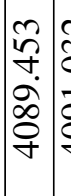 & 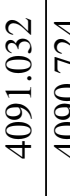 & 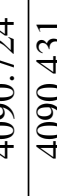 & \\
\hline 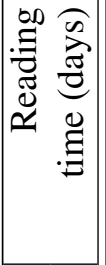 & 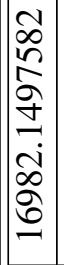 & 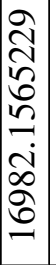 & 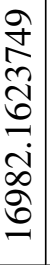 & 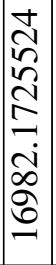 & 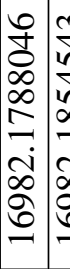 & 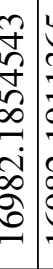 & 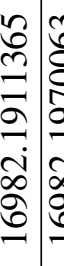 & \begin{tabular}{l}
$\infty$ \\
\multirow{3}{8}{} \\
8 \\
8 \\
8 \\
$\alpha$ \\
0 \\
0
\end{tabular} & 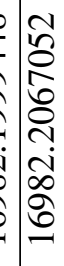 & 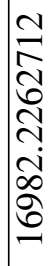 & 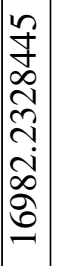 & 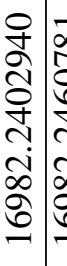 & 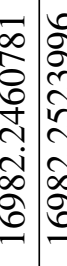 & 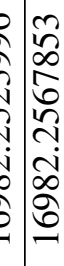 & $\left\{\begin{array}{l}2 \\
\hat{0} \\
2 \\
0 \\
1 \\
1 \\
\infty \\
0 \\
0 \\
1\end{array}\right.$ & 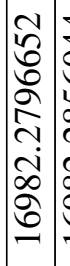 & 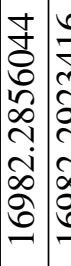 & 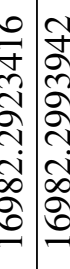 & 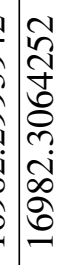 & $\begin{array}{l}n \\
0 \\
\infty \\
0 \\
\sigma \\
i \\
\infty \\
0 \\
0 \\
-1\end{array}$ & 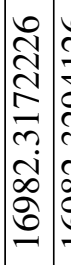 & 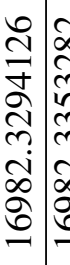 & 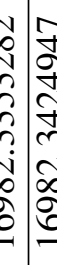 & 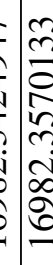 \\
\hline 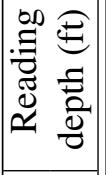 & 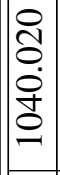 & 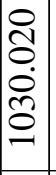 & 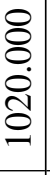 & $\begin{array}{l}2 \\
\infty \\
\alpha \\
0 \\
\delta \\
0\end{array}$ & 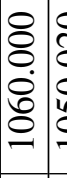 & 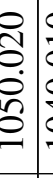 & 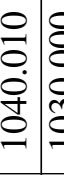 & 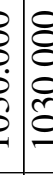 & $\begin{array}{l}8 \\
8 \\
0 \\
0 \\
0\end{array}$ & 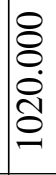 & $\begin{array}{l}8 \\
\infty \\
\hat{\sigma} \\
\hat{8} \\
0 \\
\end{array}$ & 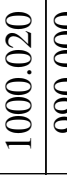 & 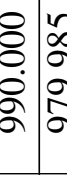 & 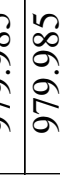 & $\left\{\begin{array}{l}0 \\
0 \\
\dot{\rho} \\
a\end{array}\right.$ & 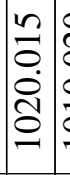 & 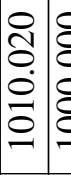 & 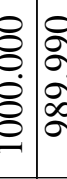 & 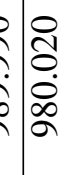 & 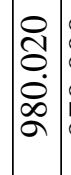 & 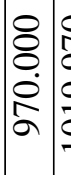 & 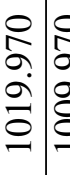 & 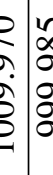 & \\
\hline$\frac{0}{i} \theta$ & م & م & م & مـ & ـ & . 0. & ك 0 م & o & م & مـ & م & ــ مـ & ـ مـ & مـ م & مـ & ـ مـ & S & ـ م & مـ D & م. & ـ ـ م & ـ. & 0 & \\
\hline 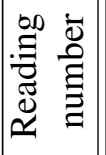 & $\overrightarrow{\widehat{N}}$ & \begin{tabular}{l}
$\infty$ \\
\multirow{N}{N}{}
\end{tabular} & તે & 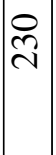 & $\overline{\widehat{\lambda}}$ & $\begin{array}{c}\mathrm{N} \\
\mathrm{N}\end{array}$ & 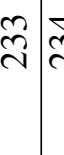 & $\hat{\imath}$ & $\begin{array}{l}\infty \\
\curvearrowright \\
\end{array}$ & $\hat{\widehat{\lambda}}$ & $\begin{array}{l}\infty \\
\tilde{\lambda}\end{array}$ & $\stackrel{\vec{\nu}}{\stackrel{\overbrace{}}{c}}$ & 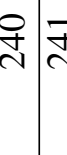 & $\stackrel{\sim}{\stackrel{\sim}{\sim}}$ & $\underset{\sim}{\stackrel{g}{\sim}}$ & $\underset{J}{\stackrel{J}{Z}}$ & $\stackrel{n}{\stackrel{\sim}{N}}$ & \begin{tabular}{l|r}
\multirow{2}{*}{} \\
\multirow{2}{*}{}
\end{tabular} & 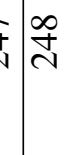 & $\underset{\sim}{\stackrel{a}{\sim}}$ & 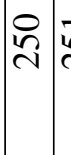 & $\bar{n}$ & 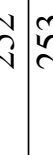 & \\
\hline
\end{tabular}




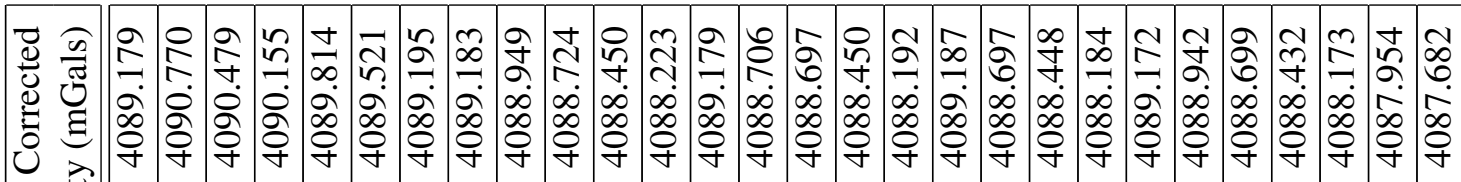

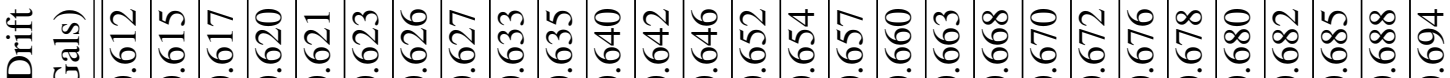
A

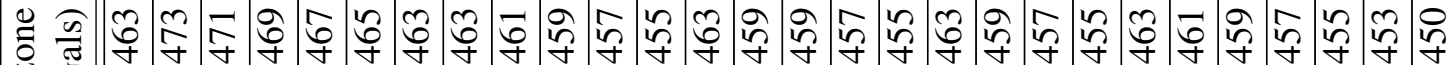
N 串

‡

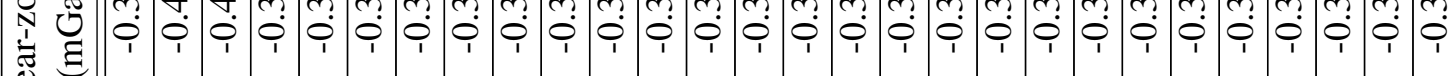
Z U : a \& is ठํ.

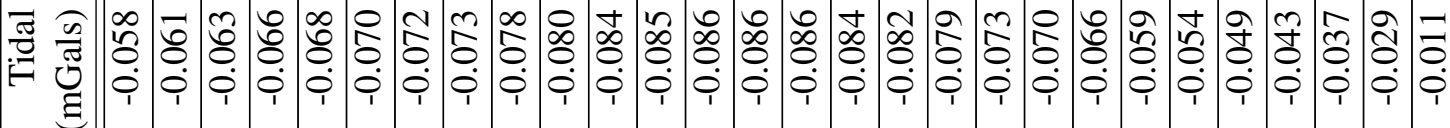
ठํ.

츨 胥

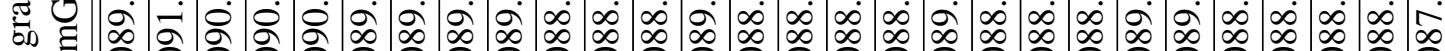

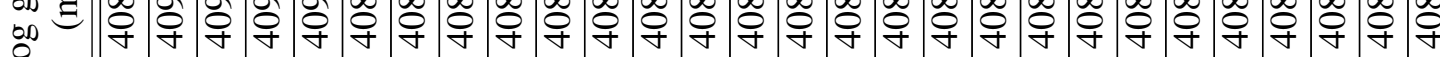

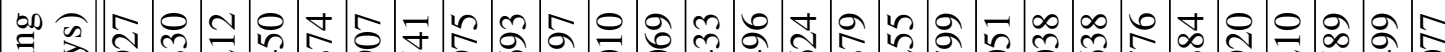

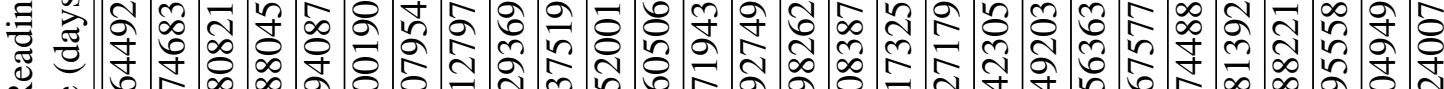

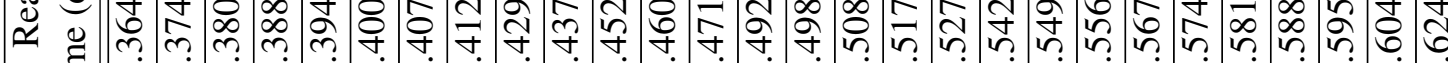

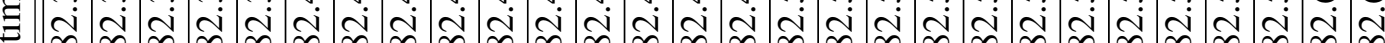

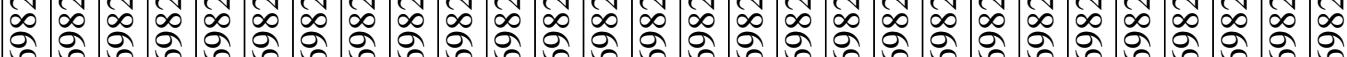

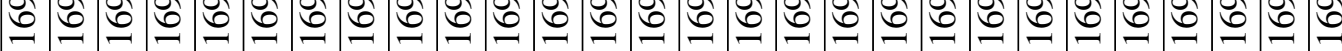

車 च « ప্ট

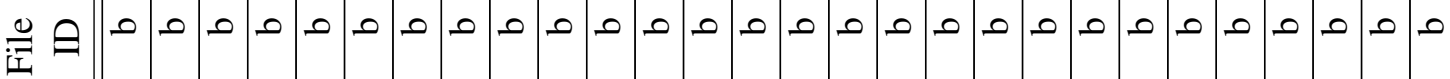

m

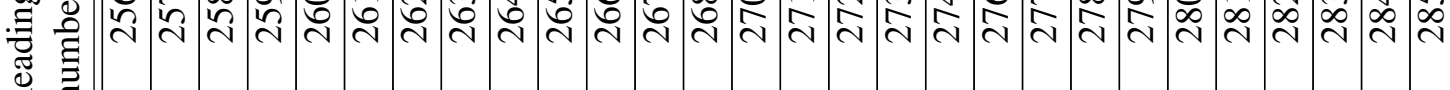
$\simeq$ 


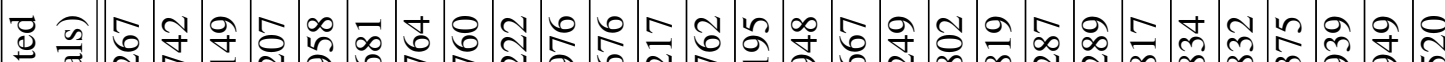

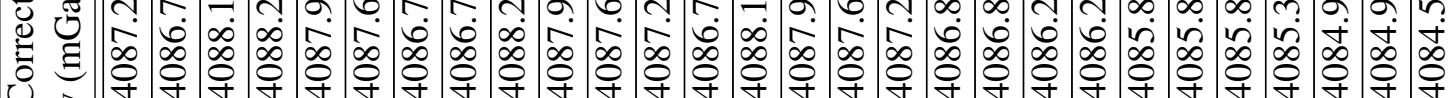

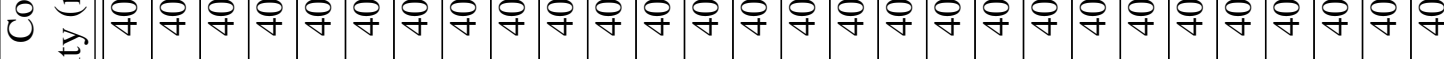
焉

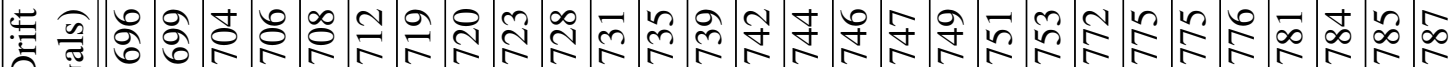
西

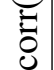

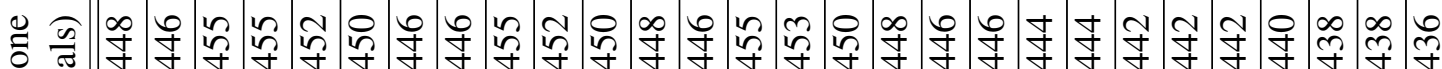

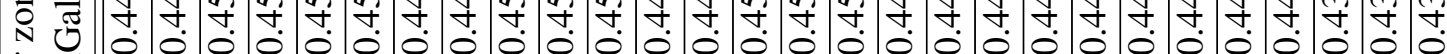
: $\sum_{2}^{\prime}$

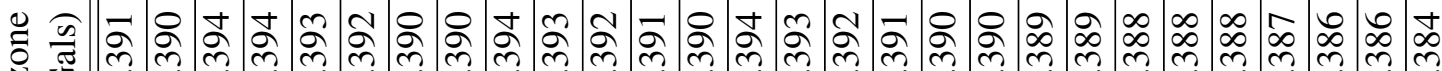

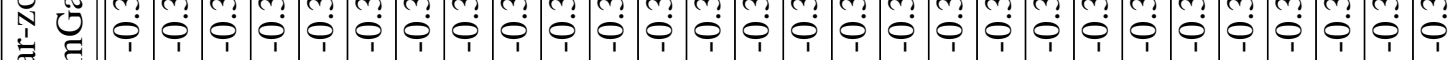
है 乙

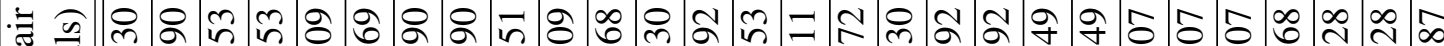

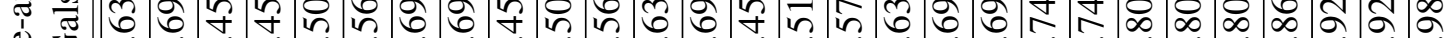
包 I ठํ.

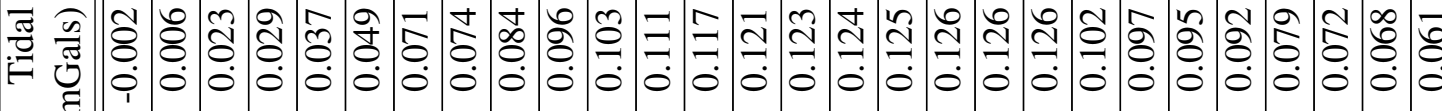
ठํ.

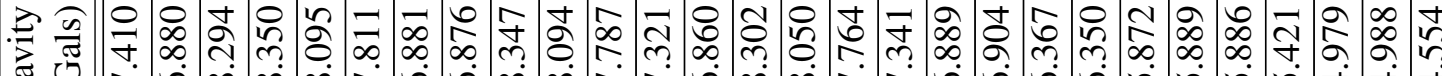

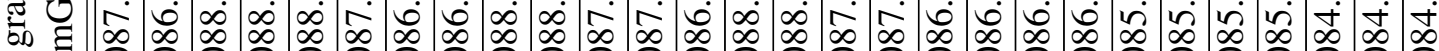

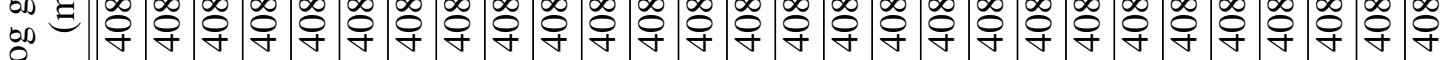
으

正 手

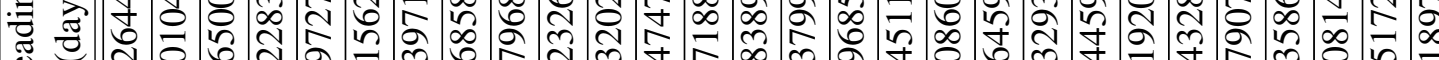
$\approx$ ¿

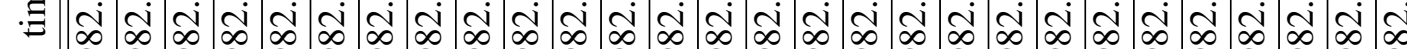

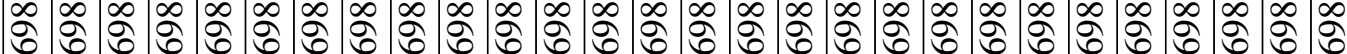

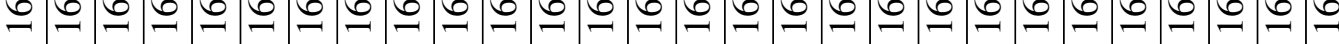

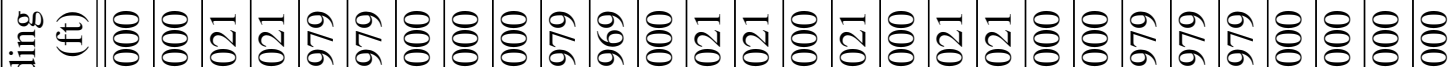
ॠ

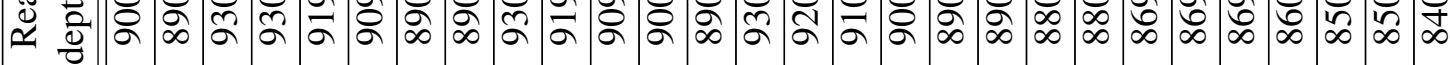

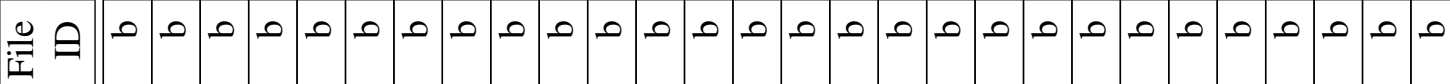

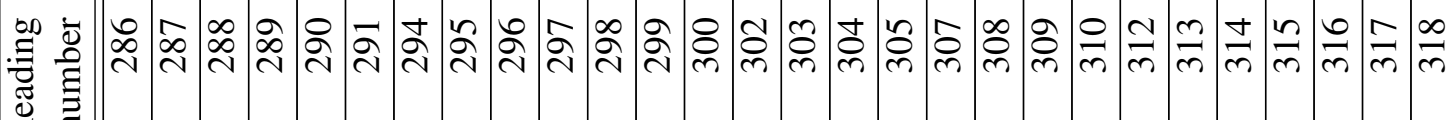
$\approx$ 


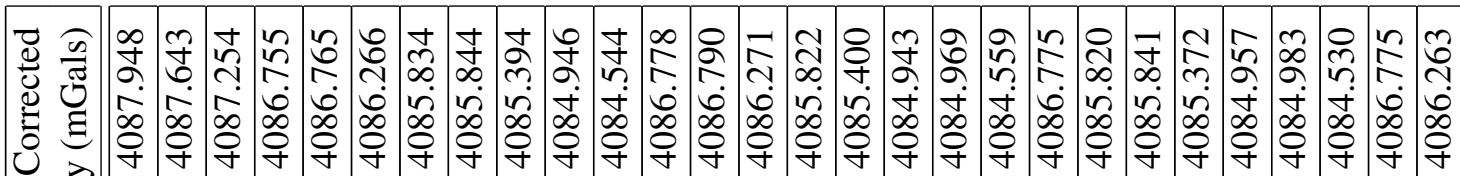
.

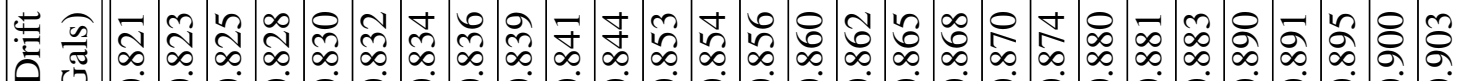
D ठำ

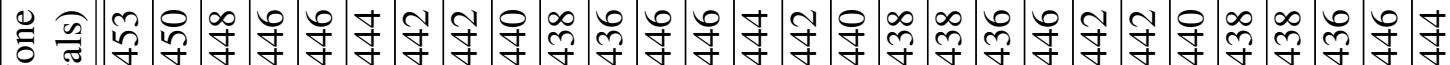
节

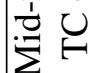

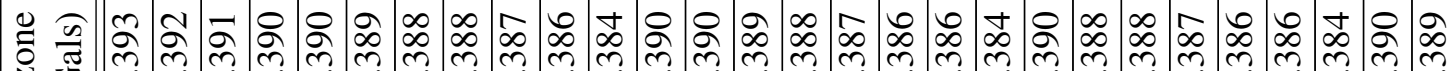

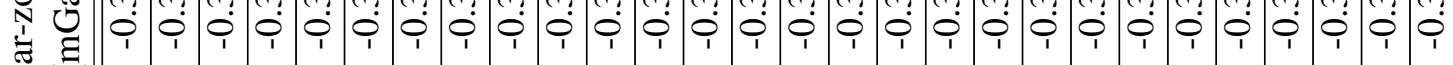
承 乙

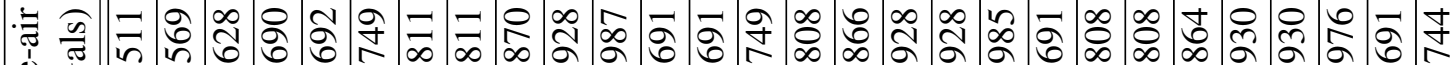

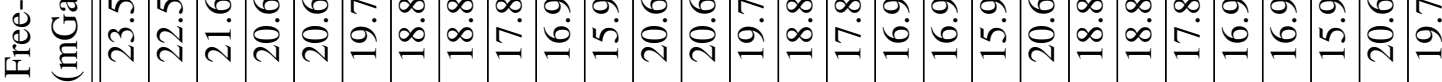
ఏँ

సే

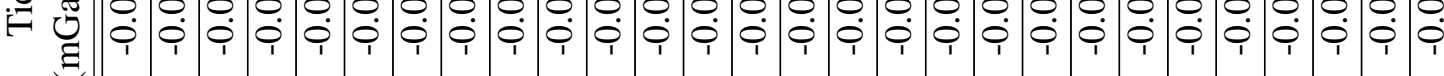
¿

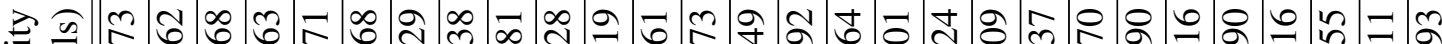

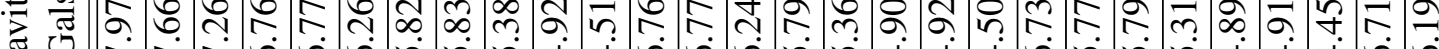

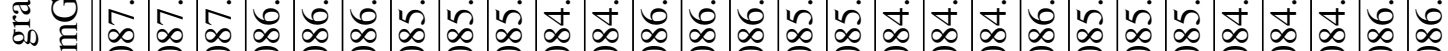

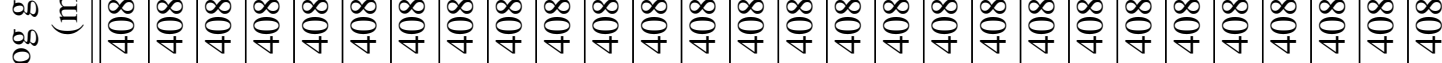

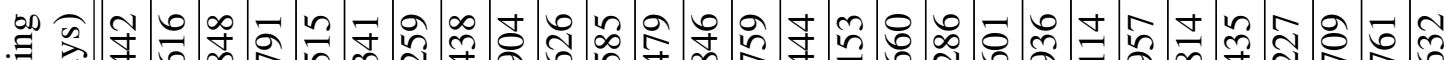

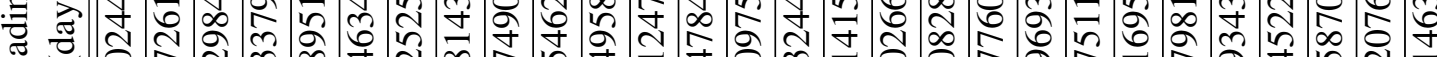

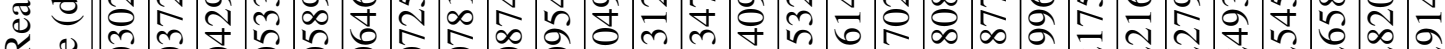
\& 0 .

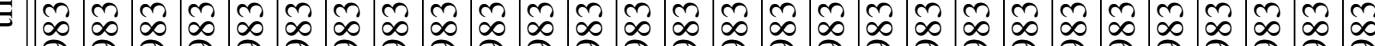

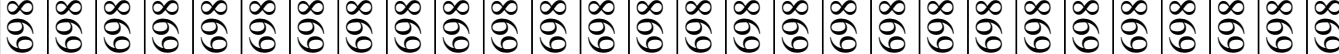

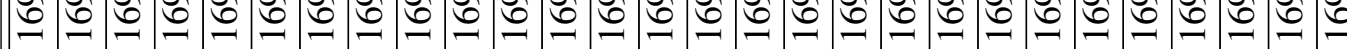

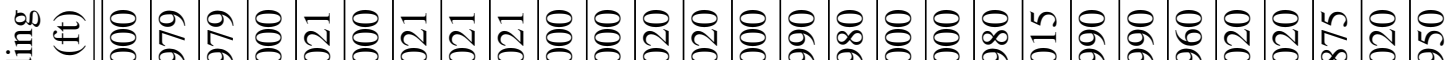

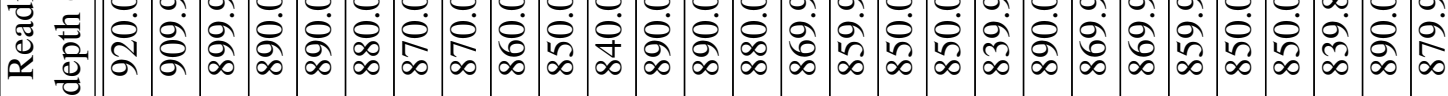
堂

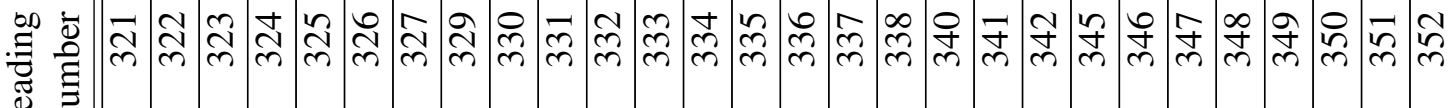
$\simeq$ 


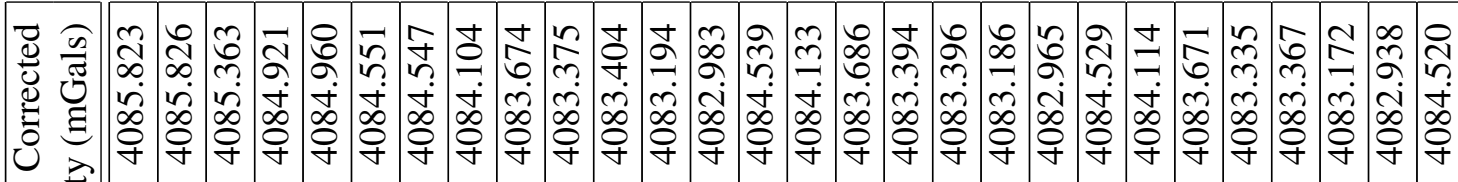
离

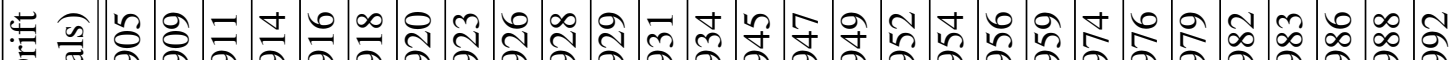
ద

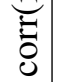

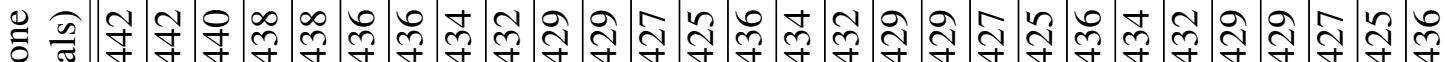
品

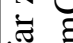
皇

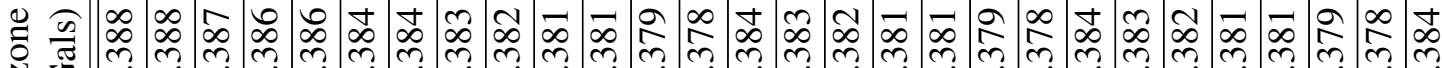
光 胥 乙

$\because$ ส ঊ

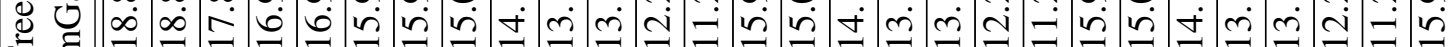
空 ठํ.

สี

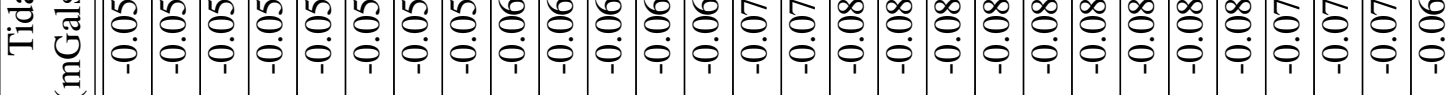
$\stackrel{\Xi}{0}$

. च

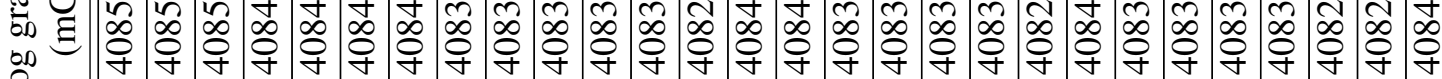
으

紫 茪 胥

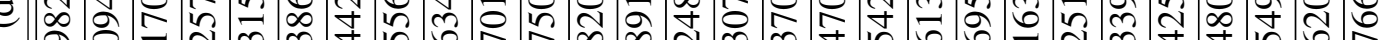

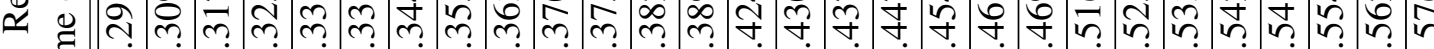

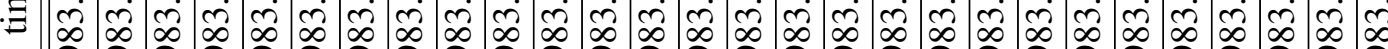

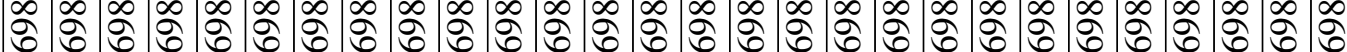

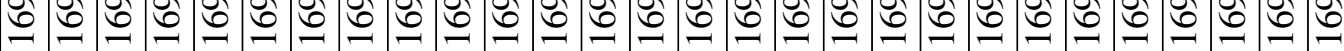

•

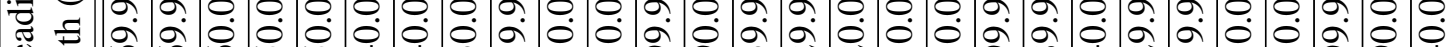

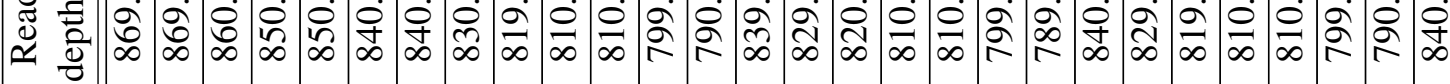

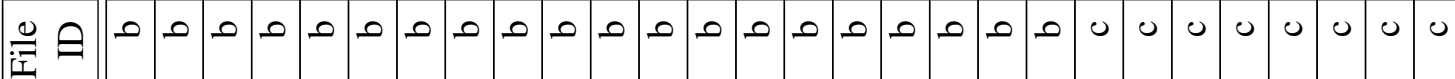

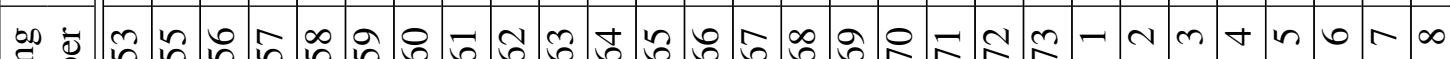

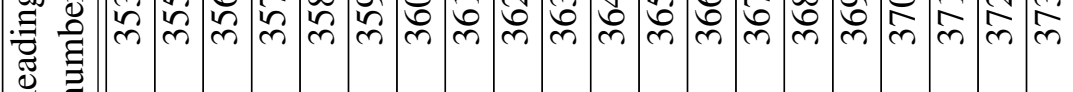




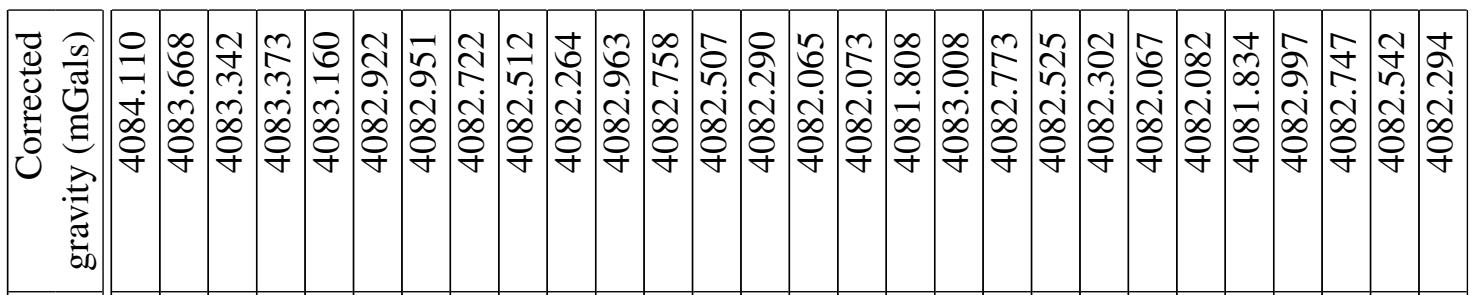

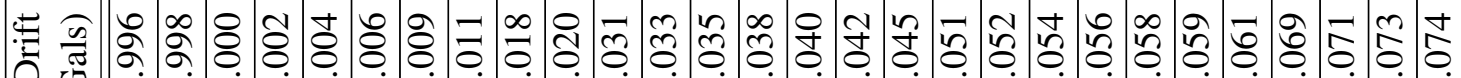
ద

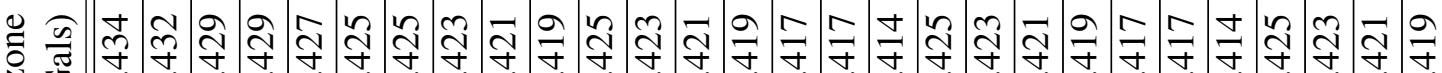

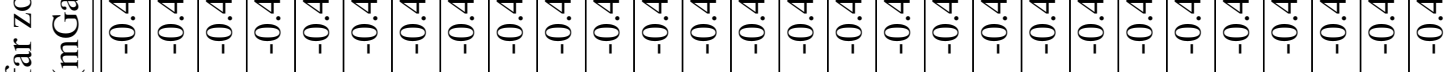
过

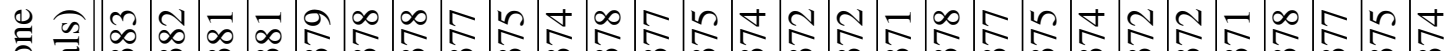
व न

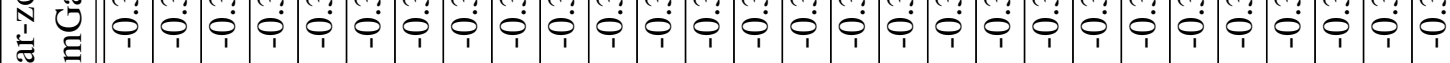
ङ $\mathrm{Z}$,

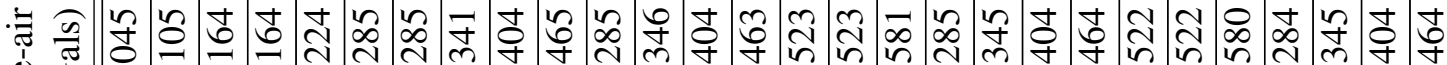
总 .

8

สే

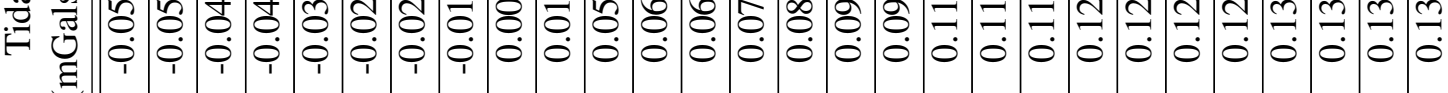
ठำ

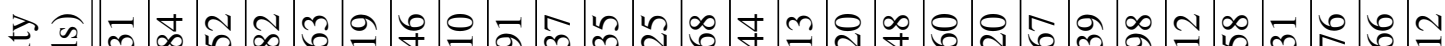

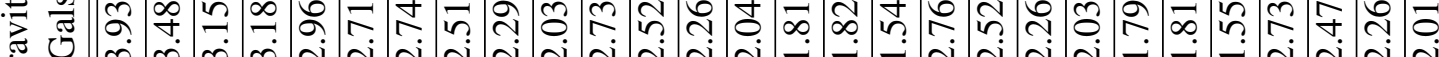
Б0

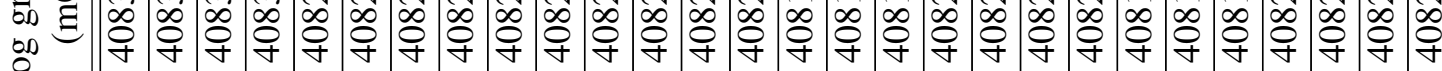

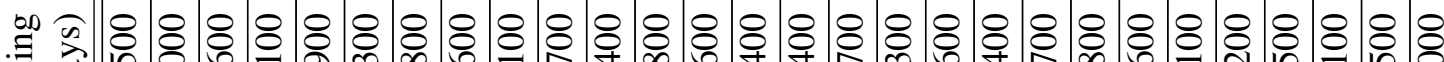

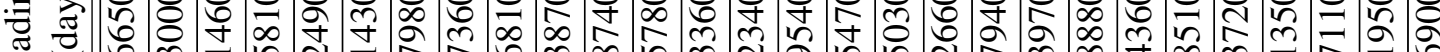

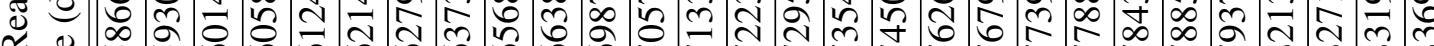

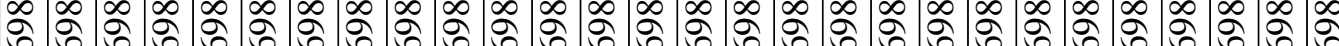

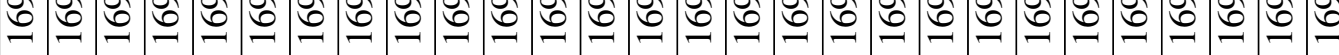

. थ $\stackrel{\oplus}{=}$ $\begin{array}{lllllllllllllllllllllllllllllll}0 & 0 & 0 & 0 & 0 & 0 & 0 & 0 & 0 & 0 & 0 & 0 & 0 & 0 & 0 & 0 & 0 & 0 & 0 & 0 & 0 & 0 & 0 & 0 & 0 & 0 & 0 & 0\end{array}$

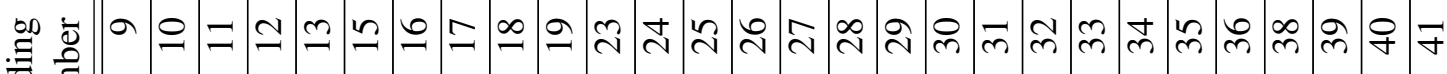
覂 


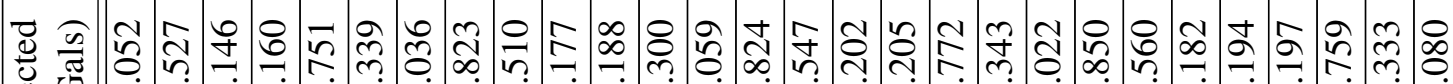

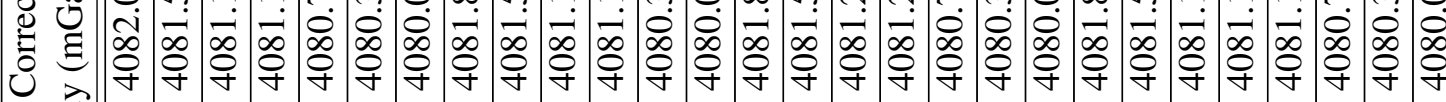

50

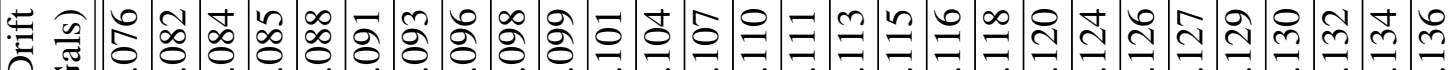
A

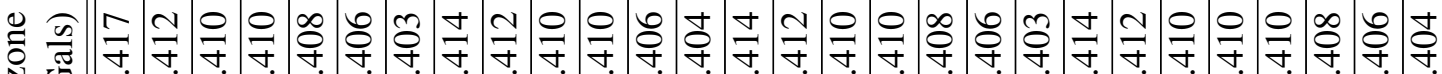

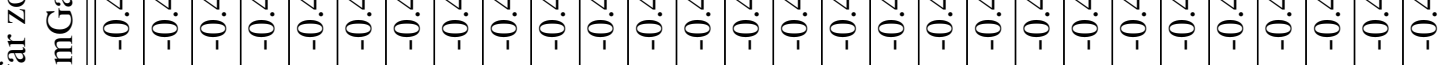
๘

$\sum$

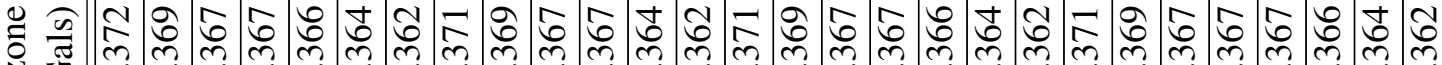
茪 శี 乙

\begin{tabular}{|c|c|c|c|c|c|c|c|c|c|c|c|c|c|c|c|c|c|c|c|c|c|c|c|c|c|}
\hline 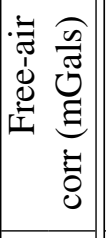 & $\begin{array}{l}\widetilde{N} \\
\text { ָุ } \\
\sim\end{array}$ & 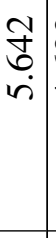 & 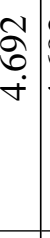 & $\begin{array}{l}\tilde{\sigma} \\
\dot{f} \\
\stackrel{+}{*}\end{array}$ & & \begin{tabular}{|l}
$\vec{\lambda}$ \\
$\infty$ \\
$i$ \\
$ن$
\end{tabular} & & $\begin{array}{l}\infty \\
n \\
n \\
n\end{array}$ & & & $\begin{array}{l}\bar{D} \\
\text { id } \\
\text { int }\end{array}$ & $\begin{array}{l}\infty \\
\infty \\
\infty \\
\infty\end{array}$ & 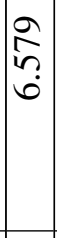 & & & & & & $\left|\begin{array}{l}n \\
\infty \\
n \\
0 \\
0\end{array}\right|$ & 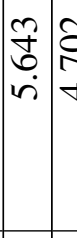 & 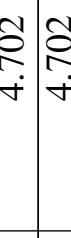 & 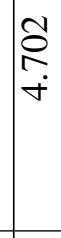 & $\dot{r}$ & $\begin{array}{c}\vec{\lambda} \\
\infty \\
i \\
\vec{i}\end{array}$ & \\
\hline 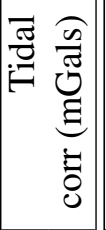 & \begin{tabular}{l}
0 \\
$\dddot{m}$ \\
\hdashline \\
0
\end{tabular} & $\frac{0}{\stackrel{0}{0}}$ & $\stackrel{\infty}{\stackrel{\infty}{J}}$ & $\begin{array}{l}0 \\
\stackrel{\sim}{J} \\
0\end{array}$ & $\frac{N}{\mathfrak{O}}$ & $\begin{array}{l}0 \\
= \\
0 \\
0\end{array}$ & $\begin{array}{l}\frac{N}{=} \\
0\end{array}$ & 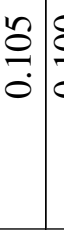 & $\begin{array}{c}0 \\
0 \\
0\end{array}$ & : & 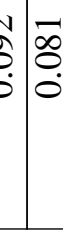 & 咅 & \begin{tabular}{|l|} 
\\
0 \\
0 \\
0
\end{tabular} & $\mid \begin{array}{l}\infty \\
2 \\
0 \\
0 \\
0\end{array}$ & $\begin{array}{lll}N & \alpha \\
\vdots & c & c \\
0 & c & c\end{array}$ & & 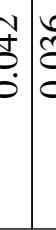 & $\begin{array}{l}0 \\
\substack{0 \\
0} \\
0\end{array}$ & $\begin{array}{l}n \\
0 \\
0 \\
0\end{array}$ & $\stackrel{g}{\delta}$ & 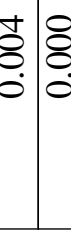 & $\begin{array}{l}2 \\
8 \\
0 \\
0\end{array}$ & $\begin{array}{l}Z \\
\overline{0} \\
0 \\
0 \\
1\end{array}$ & \begin{tabular}{l|}
0 \\
0 \\
0 \\
$\dot{1}$ \\
1
\end{tabular} & 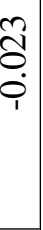 \\
\hline 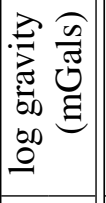 & $\begin{array}{l}n \\
6 \\
\gamma \\
\dot{\infty} \\
0 \\
\dot{f}\end{array}$ & 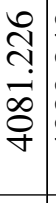 & $\begin{array}{l}0 \\
+ \\
\infty \\
\dot{0} \\
\infty \\
\delta \\
\dot{+}\end{array}$ & 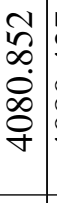 & \begin{tabular}{l}
$\hat{m}$ \\
\multirow{t}{*}{} \\
$\dot{\delta}$ \\
0 \\
$\dot{+}$
\end{tabular} & \begin{tabular}{|c|}
$\infty$ \\
0 \\
0 \\
$\dot{0}$ \\
0 \\
0 \\
$y$
\end{tabular} & & 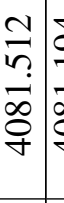 & 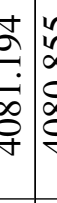 & 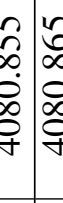 & 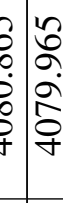 & 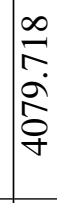 & 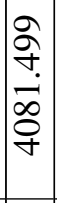 & 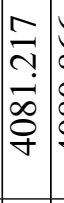 & 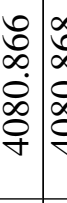 & & 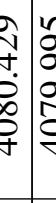 & 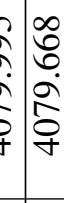 & $\begin{array}{l}= \\
\bar{n} \\
\vdots \\
\infty \\
o \\
q\end{array}$ & 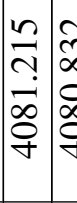 & 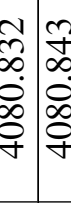 & $\begin{array}{l}n \\
f \\
\infty \\
0 \\
0 \\
0 \\
o\end{array}$ & 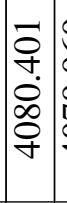 & 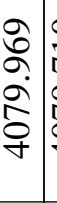 & 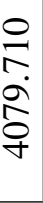 \\
\hline 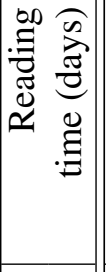 & $\begin{array}{l}8 \\
8 \\
2 \\
21 \\
\infty \\
\infty \\
\infty \\
\infty \\
0 \\
0 \\
- \\
\end{array}$ & 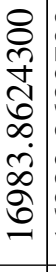 & 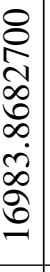 & $\begin{array}{l}8 \\
0 \\
1 \\
i \\
\infty \\
\infty \\
\infty \\
\infty \\
0 \\
0\end{array}$ & $\begin{array}{l}8 \\
8 \\
2 \\
\delta \\
\infty \\
\infty \\
\dot{\infty} \\
\infty \\
0 \\
0\end{array}$ & $\begin{array}{l}8 \\
0 \\
0 \\
2 \\
\infty \\
\infty \\
\infty \\
0 \\
0 \\
- \\
\end{array}$ & $\begin{array}{l}8 \\
0 \\
\hat{0} \\
2 \\
\infty \\
\infty \\
0 \\
0 \\
0 \\
-\end{array}$ & 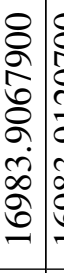 & 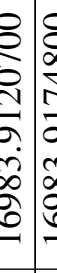 & 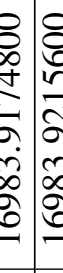 & 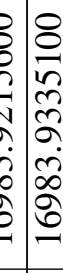 & 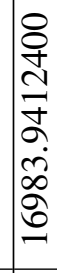 & 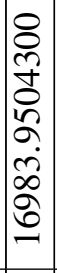 & 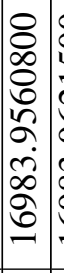 & 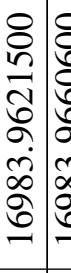 & 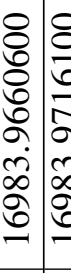 & 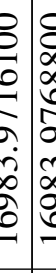 & $\begin{array}{ll}3 & 8 \\
0 & + \\
0 \\
0 \\
0 \\
0 \\
0 \\
0 \\
0 \\
0 \\
0 \\
0 \\
0 \\
0 \\
0 \\
0\end{array}$ & $\begin{array}{l}8 \\
8 \\
8 \\
0 \\
2 \\
2 \\
1 \\
0 \\
0 \\
0 \\
-\end{array}$ & 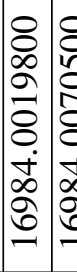 & 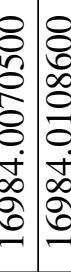 & $\begin{array}{l}8 \\
0 \\
n \\
n \\
0 \\
\dot{+} \\
\infty \\
0 \\
0 \\
-1\end{array}$ & $\begin{array}{l}8 \\
0 \\
0 \\
0 \\
0 \\
0 \\
0 \\
\dot{+} \\
\infty \\
0 \\
0 \\
0 \\
-1 \\
\end{array}$ & 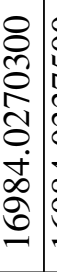 & 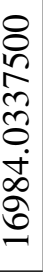 \\
\hline 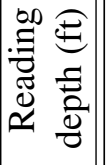 & 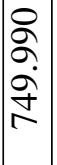 & 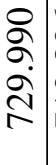 & $\begin{array}{l}0 \\
2 \\
\infty \\
0 \\
0\end{array}$ & $\begin{array}{l}0 \\
2 \\
\infty \\
\dot{2} \\
\end{array}$ & $\begin{array}{l}8 \\
8 \\
\vdots \\
\vdots \\
\end{array}$ & \begin{tabular}{l}
8 \\
8 \\
8 \\
8 \\
\hdashline
\end{tabular} & $\begin{array}{l}a \\
\hat{\sigma} \\
\vdots \\
\infty \\
0\end{array}$ & 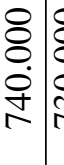 & 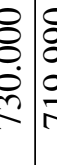 & 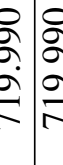 & م. & $\begin{array}{l}8 \\
\delta \\
\alpha \\
\infty \\
0\end{array}$ & $\begin{array}{l}\infty \\
\curvearrowleft \\
2 \\
\curvearrowright \\
\approx \\
\approx\end{array}$ & $\begin{array}{l}a \\
\hat{a} \\
a \\
\text { N }\end{array}$ & 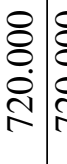 & 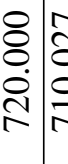 & 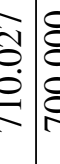 & 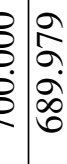 & 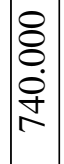 & 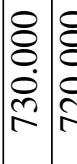 & 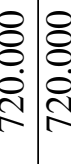 & $\begin{array}{l}8 \\
8 \\
0 \\
0 \\
i\end{array}$ & \begin{tabular}{l}
8 \\
8 \\
0 \\
0 \\
\hdashline \\
\end{tabular} & $\begin{array}{l}8 \\
8 \\
8 \\
8\end{array}$ & के \\
\hline$\stackrel{\theta}{=}$ & 0 & 0 & 0 & 0 & 0 & 0 & 0 & 0 & v & o c & ن & 0 & 0 & 0 & 0 & 0 & 0 & u 0 & 0 & 0 & 0 & 0 & 0 & 0 & 0 \\
\hline 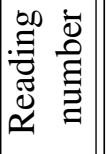 & $\stackrel{F}{f}$ & $\mathscr{f}$ & o & $\mathcal{F}$ & $\stackrel{\infty}{+}$ & $g$ & $\therefore$ & $\bar{n} \delta$ & 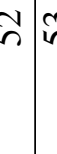 & $\tilde{n}$ is & j & in & $\infty$ & in & 8 & $\sigma 3$ & 0 & $\hat{b}$ & $\approx$ & 87 & $6 \approx$ & 8 & 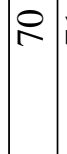 & $\nabla$ & 2 \\
\hline
\end{tabular}




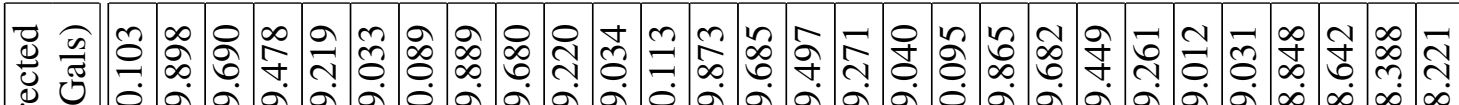
过

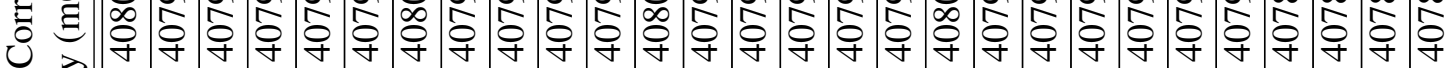
(

言

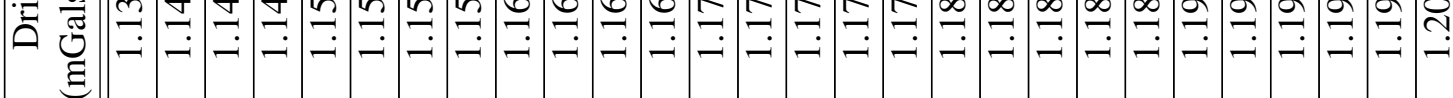

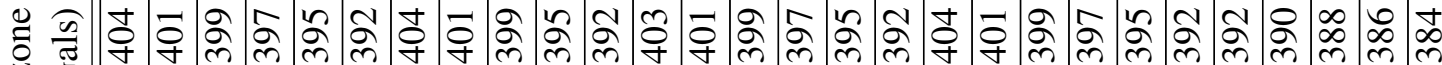
ñ

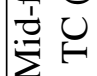

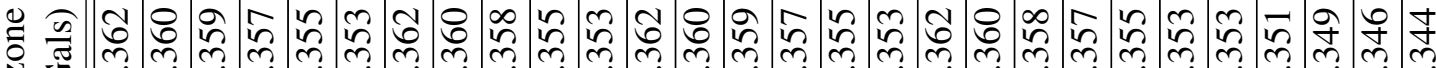
先 $\ddot{z}$

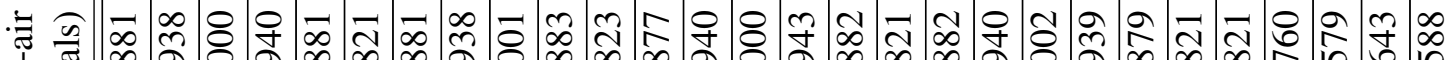

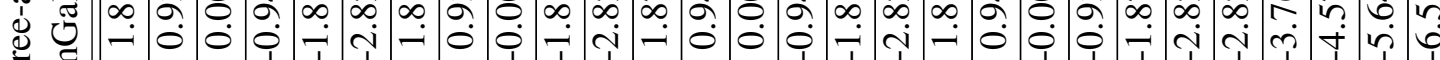
is :

可 $⿹$

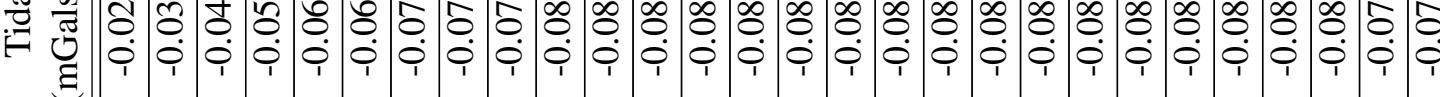
¿

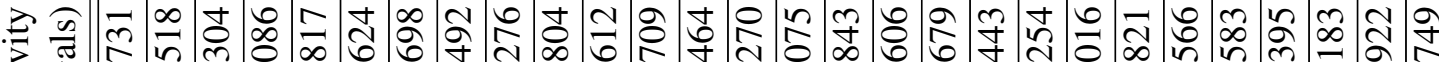

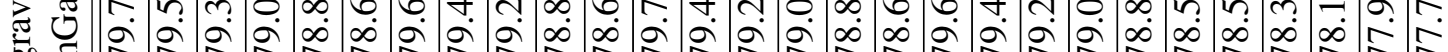

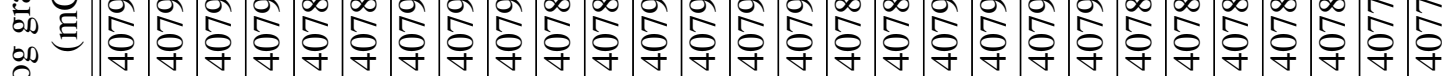
$\stackrel{90}{a}$

에

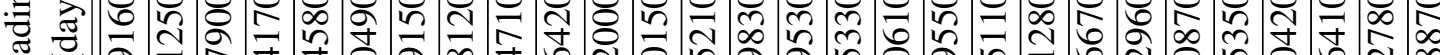

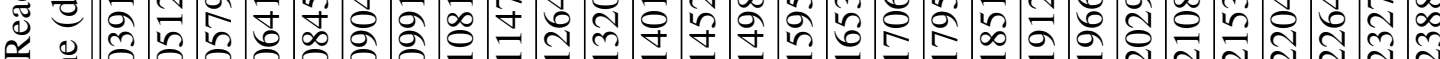

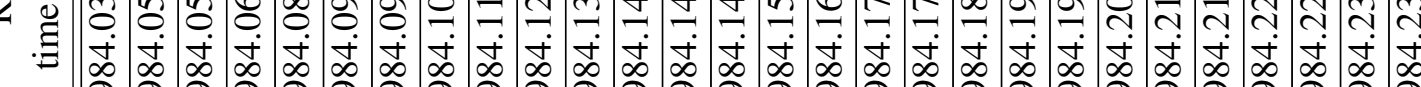

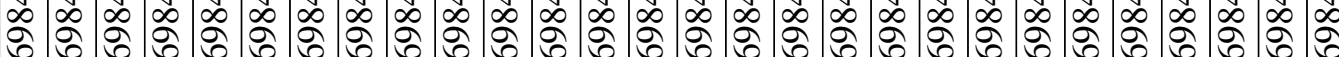

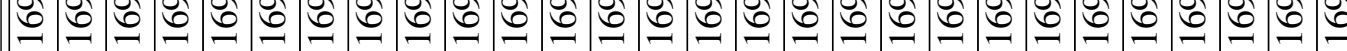

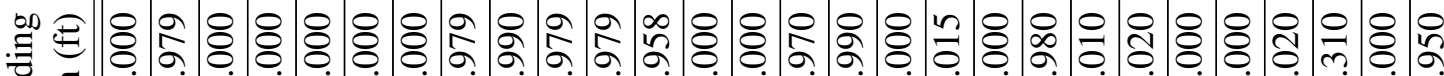

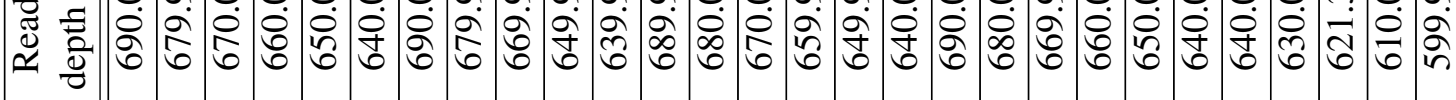
$\frac{\circ}{2}$

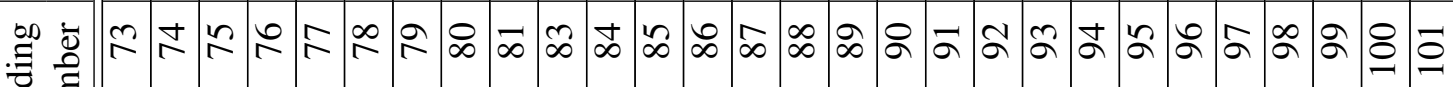
.

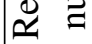




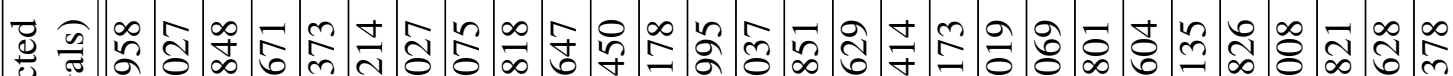

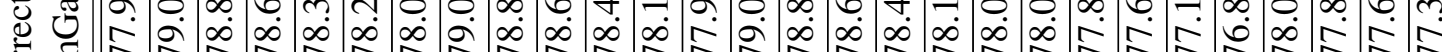

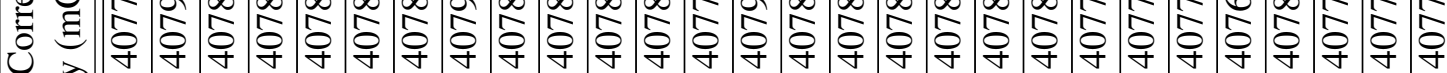

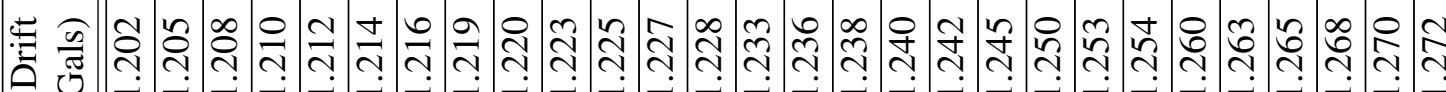

‡

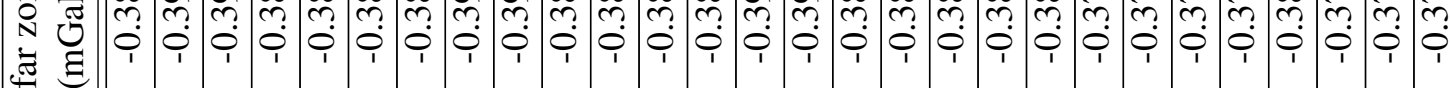

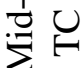

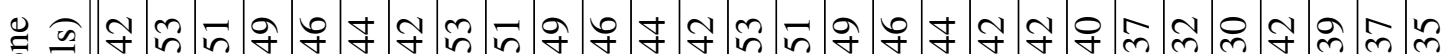
ప न N ซึ 乙

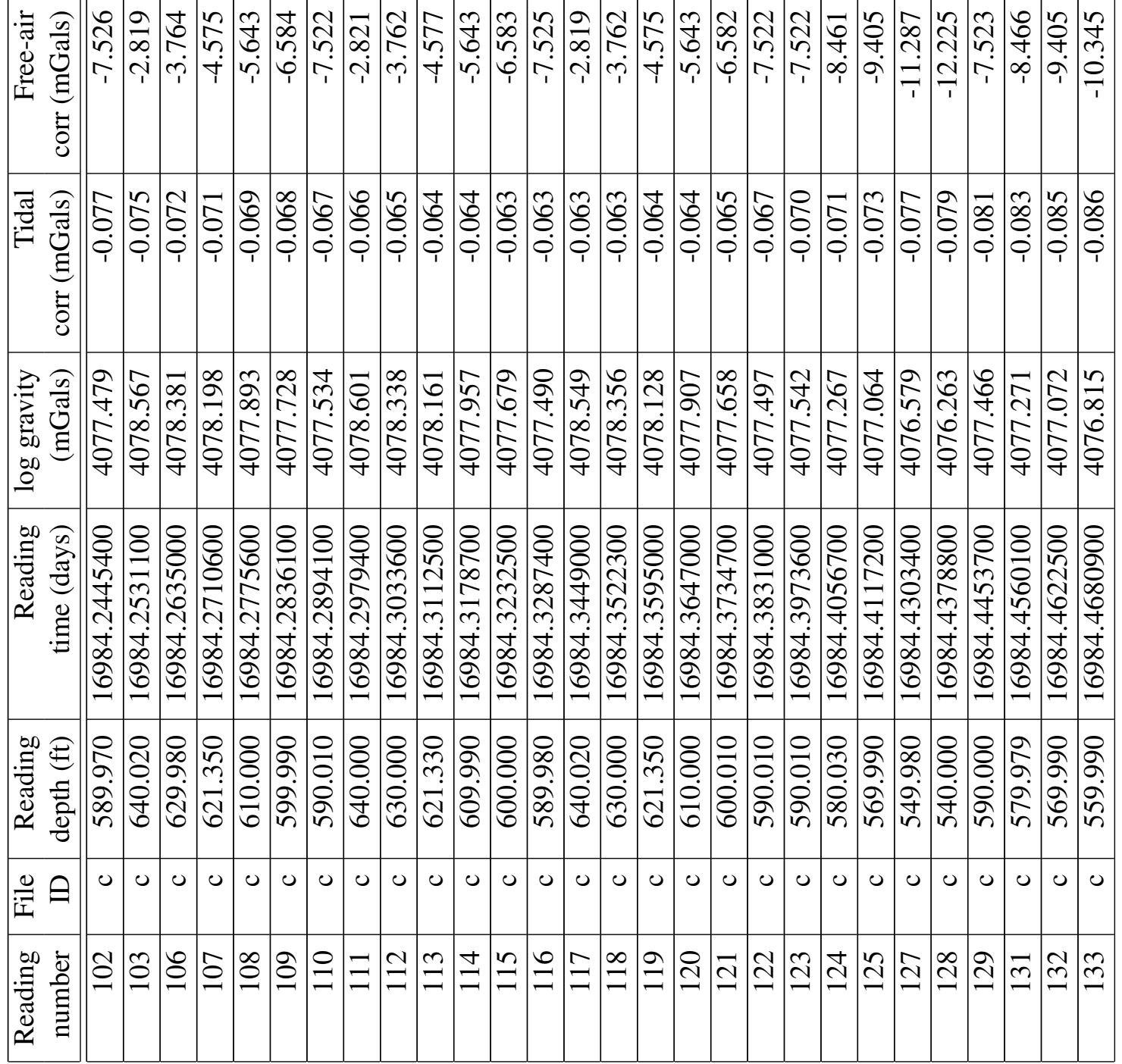




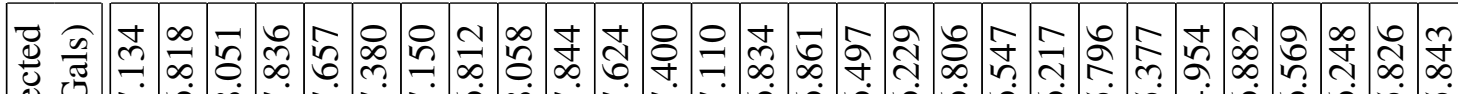
过

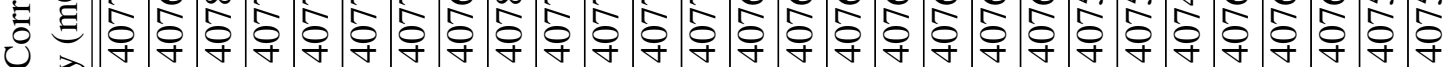
.

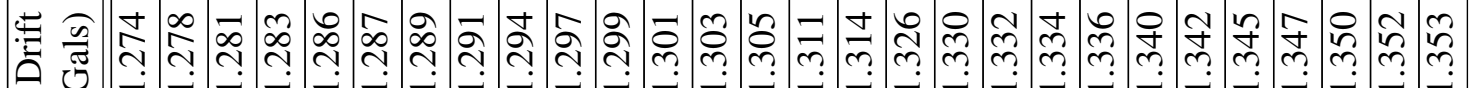
$-$

ร

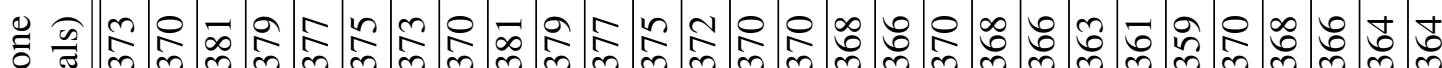

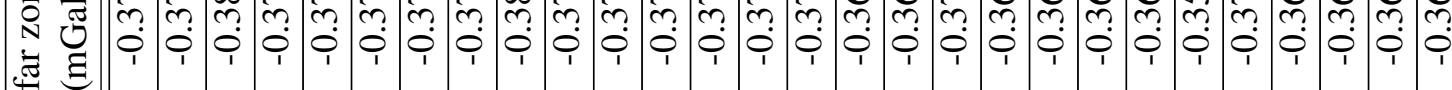

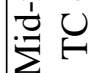

들

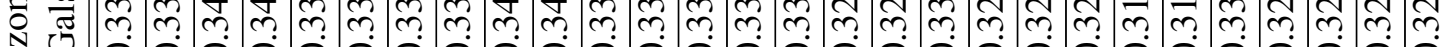

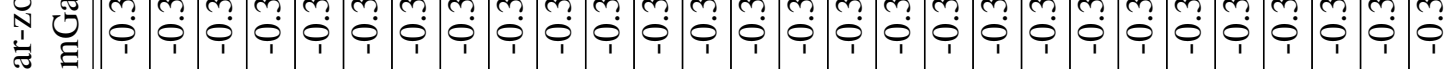
Ð $\mathrm{U}$

:

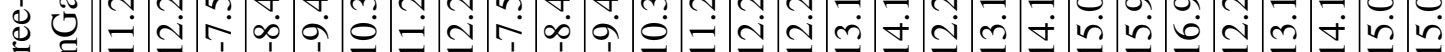
在

ठ

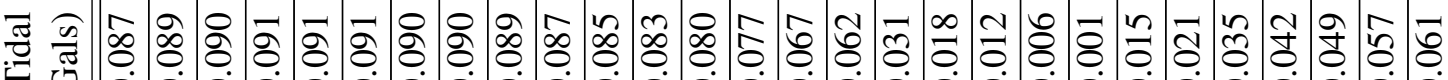
F

¿ั

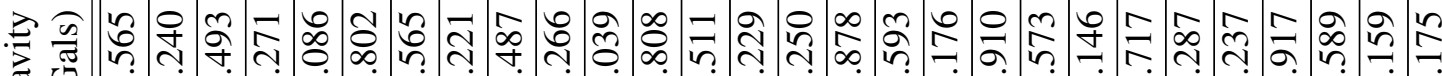

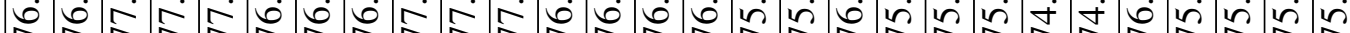

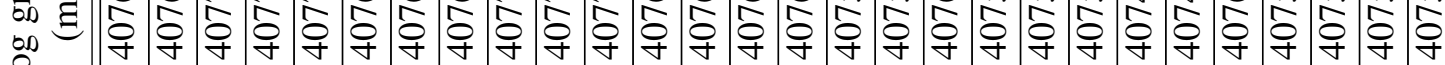

品

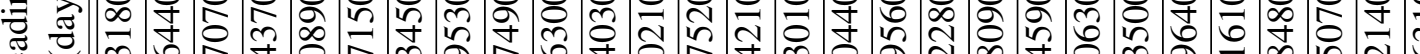
4

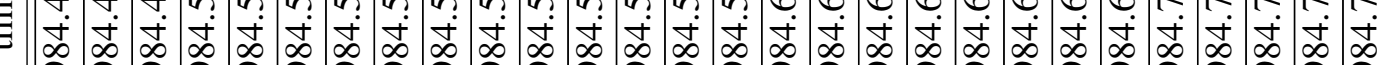

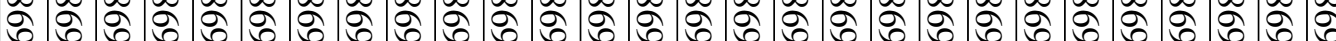

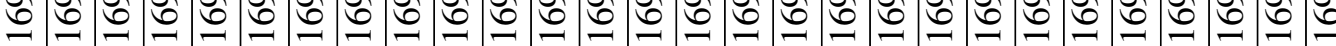

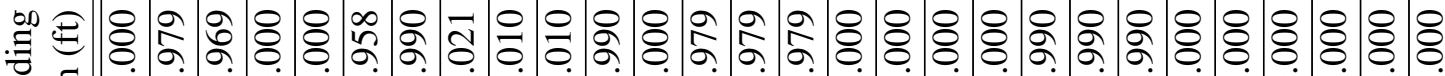
产

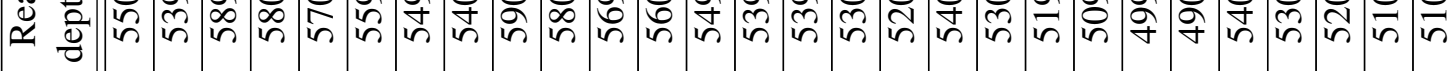
$\stackrel{\circ}{\circ}$

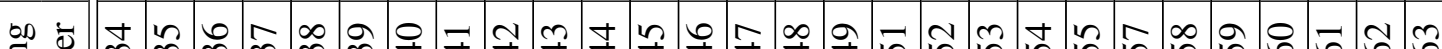

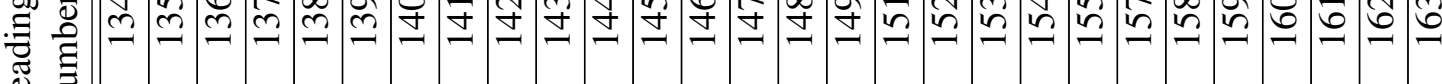

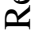




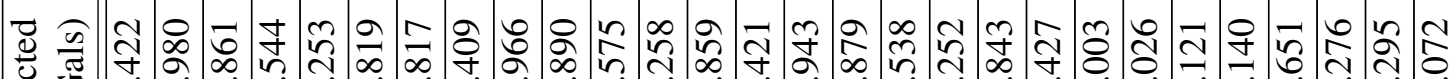

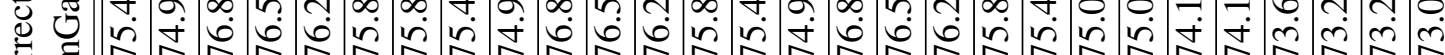

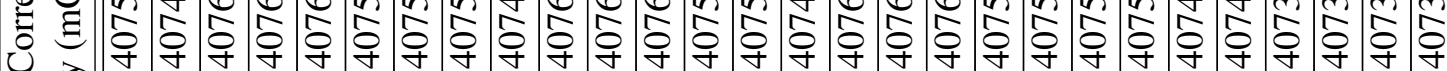
$\circlearrowleft \geqslant$ 党 言 竞

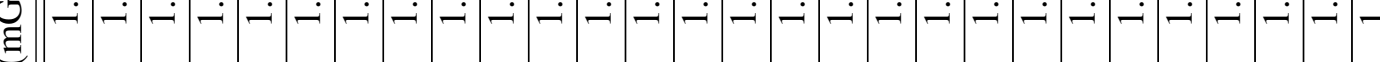
ठ

苛 苟 章

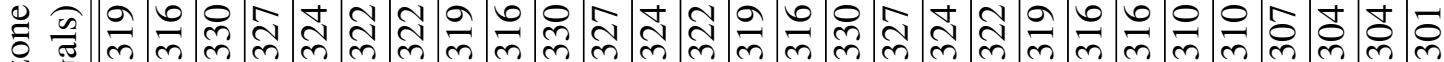
N

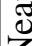
$\mathrm{Z} \cup$

\begin{tabular}{|c|c|c|c|c|c|c|c|c|c|c|c|c|c|c|c|c|c|c|c|c|}
\hline 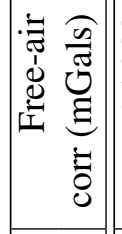 & $\mid \begin{array}{l}2 \\
2 \\
a \\
n \\
1 \\
1\end{array}$ & & & & & & & & & & & & & & & & $T$ & & i̊. & \\
\hline$\frac{\widehat{a}}{\sqrt[\pi]{0}}$ & 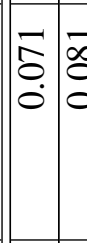 & & & $\begin{array}{c}\bar{c} \\
\overline{0} \\
\dot{0}\end{array}$ & $=\frac{}{0}$ & $\stackrel{\infty}{\infty}$ & & & & & $\begin{array}{l}\infty \\
0 \\
0 \\
0\end{array}$ & & & q & 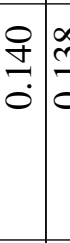 & & & 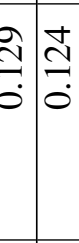 & $\vec{\Xi}$ & \\
\hline \begin{tabular}{ll|}
50 & 0 \\
00 & \\
0 & \\
\end{tabular} & 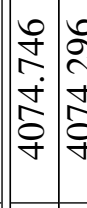 & $\begin{array}{l}0 \\
\hat{a} \\
0 \\
0\end{array}$ & & 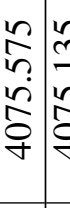 & 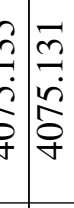 & 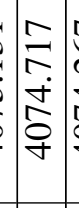 & 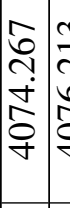 & & 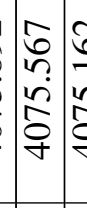 & & 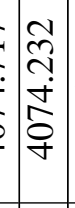 & & 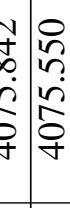 & \begin{tabular}{l}
$\dot{D}$ \\
\hdashline \\
\\
$\hat{\sigma}$ \\
$\dot{q}$ \\
\end{tabular} & 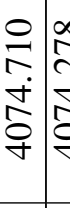 & & 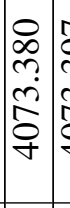 & 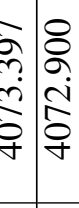 & 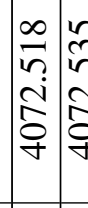 & \\
\hline 2 & 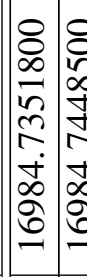 & 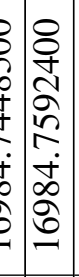 & 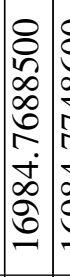 & 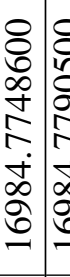 & 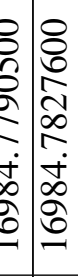 & 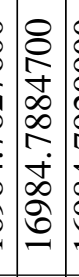 & 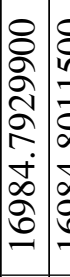 & 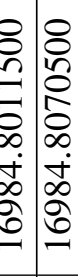 & 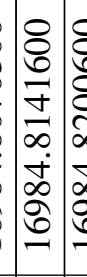 & 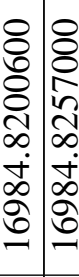 & 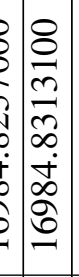 & 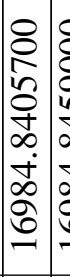 & 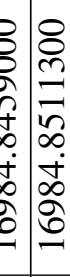 & 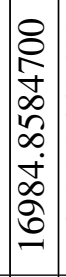 & 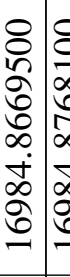 & & 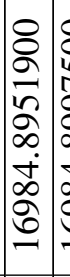 & 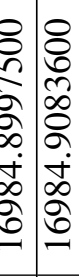 & 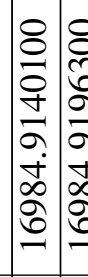 & \\
\hline 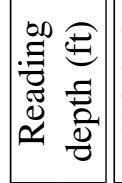 & 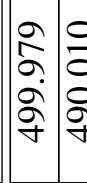 & 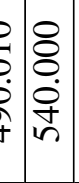 & 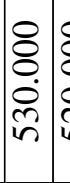 & 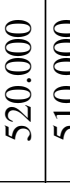 & \begin{tabular}{lll}
8 & 8 \\
\hdashline & 8 \\
\hdashline & 0 \\
\hdashline
\end{tabular} & $\begin{array}{l}8 \\
\vdots \\
\dot{0} \\
\vdots \\
\vdots\end{array}$ & 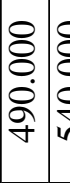 & \begin{tabular}{lll}
8 & 8 \\
8 \\
\hdashline \\
\hdashline \\
\hdashline
\end{tabular} & 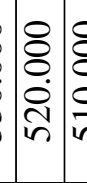 & \begin{tabular}{l|l}
8 & 8 \\
$\vdots$ & 8 \\
$\vdots$ \\
$\vdots$ \\
$\vdots$
\end{tabular} & 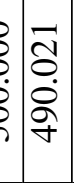 & & 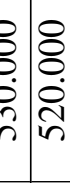 & $\varnothing$ & 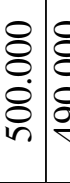 & & $\begin{array}{c}0 \\
0 \\
0 \\
\dot{P} \\
\dot{q} \\
0\end{array}$ & 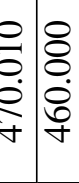 & 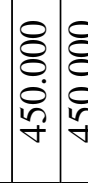 & \\
\hline & 00 & 0 & 0 & 00 & $\begin{array}{lll}0 & 0\end{array}$ & 0 & 0 & 0 & 0 & \begin{tabular}{l|l}
0 & 0
\end{tabular} & 0 & 0 & 0 & 0 & 0 & & 0 & 0 & 0 & \\
\hline $\begin{array}{l}\overrightarrow{0} \\
\text { हो }\end{array}$ & (2) & 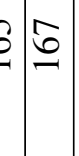 & $\infty$ & 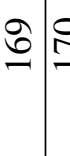 & $2 I$ & $\stackrel{I}{I}$ & $\stackrel{\Xi}{I}$ & 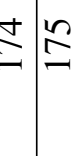 & I & $\Sigma \stackrel{\infty}{\Xi}$ & 2. & $\infty$ & $\underset{\infty}{0} \underset{\infty}{0}$ & $\infty$ & \begin{tabular}{l|l}
$\infty$ \\
$\infty$ & $\mathscr{\alpha}$
\end{tabular} & $\stackrel{\infty}{0}$ & $\stackrel{\infty}{\infty} \underset{\infty}{\infty}$ & 2 & $\bar{\Xi} \delta$ & 2 \\
\hline
\end{tabular}




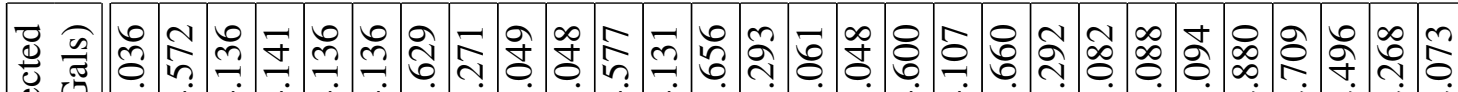

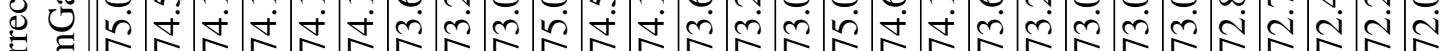

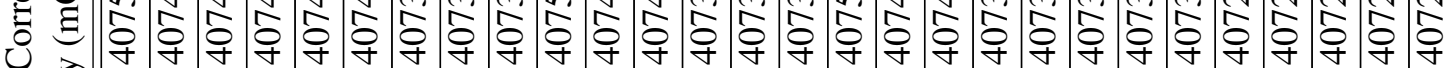

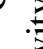

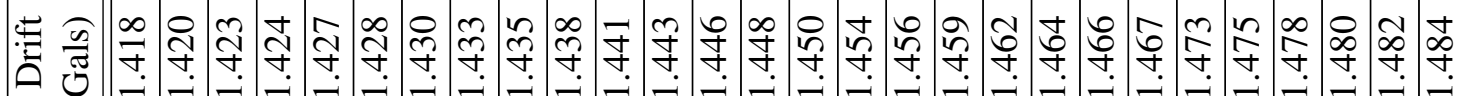
ठํㅠㅇ

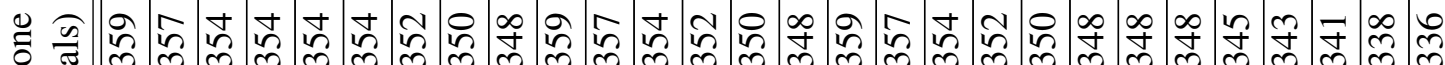

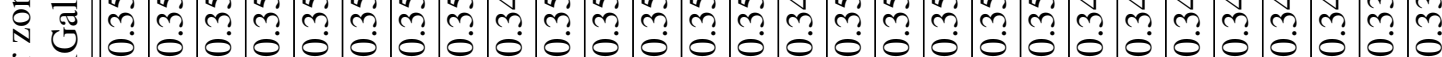
范

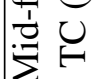

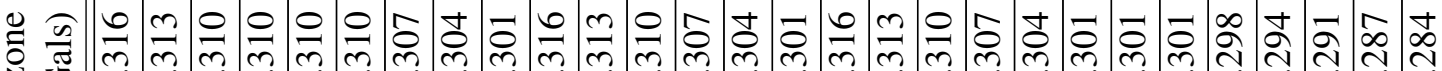
告 ॠ $\mathrm{Z}$

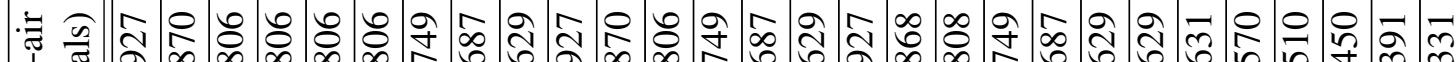
递 ڤั

चี

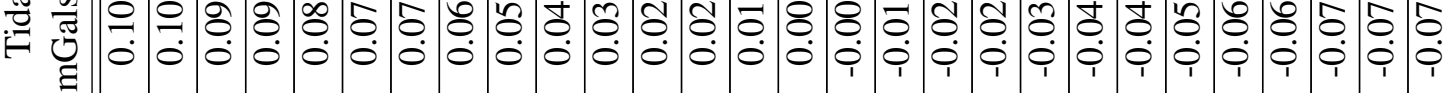
¿ั่

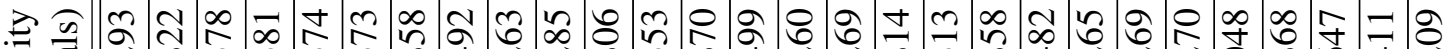
च छٓ क on

no

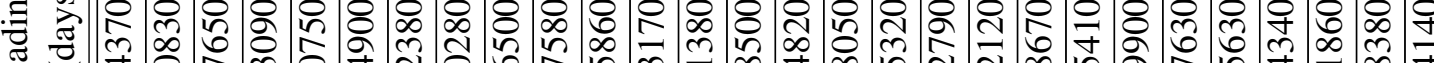
$\approx$ U

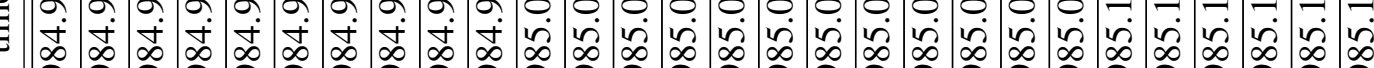
○े

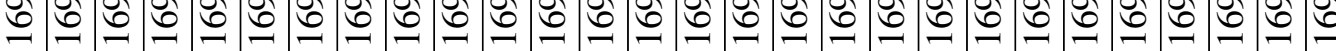

告

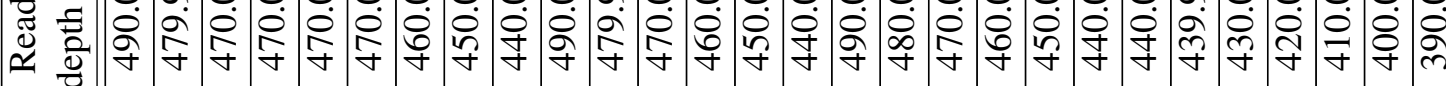
$\frac{0}{1} \mathrm{a}$

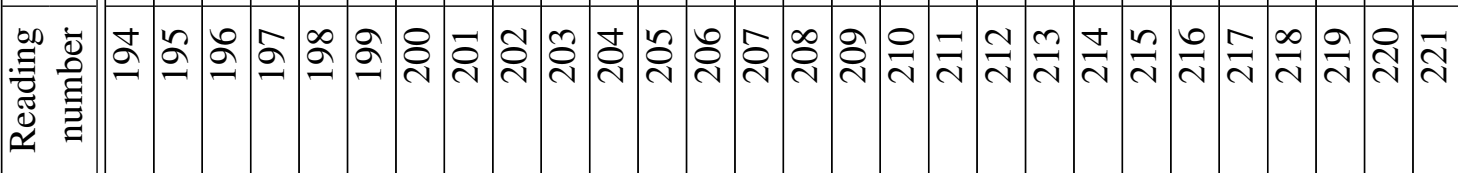




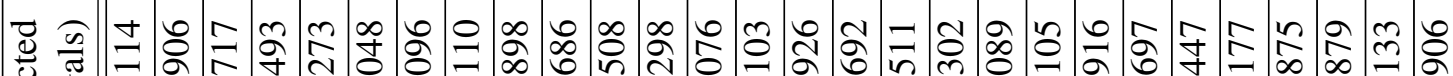

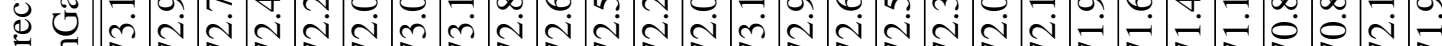
0
0
0 5

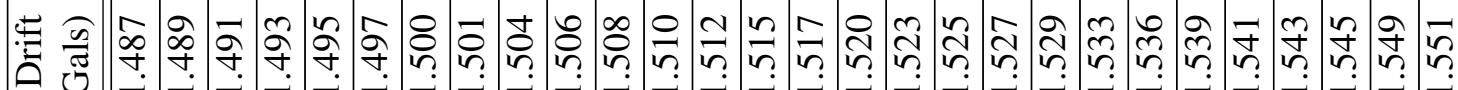

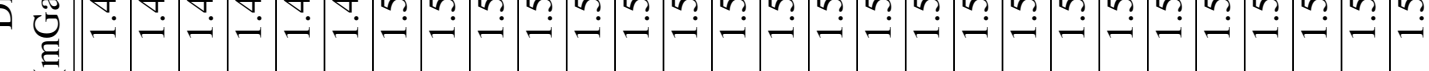

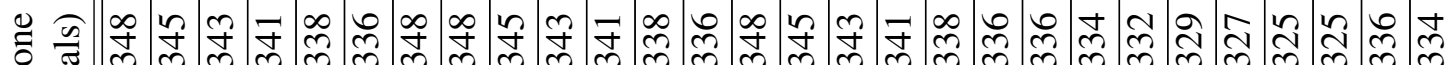

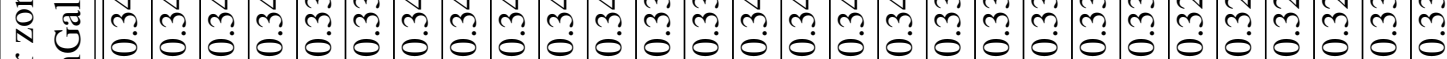
卷 竞

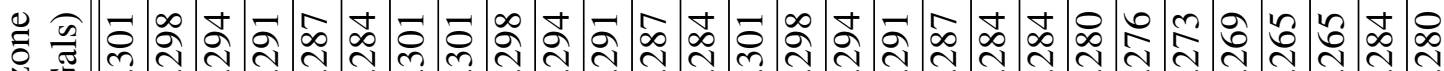

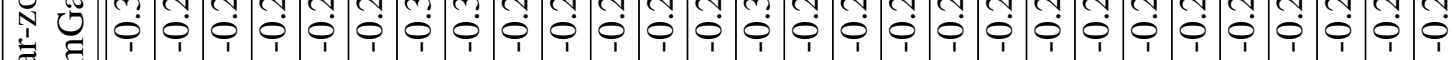
ซี Z 茫 •

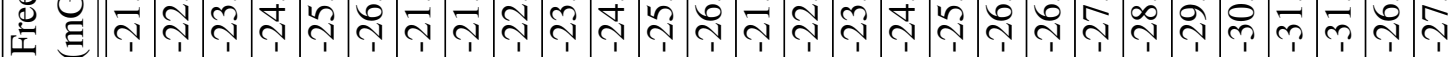
ठั)

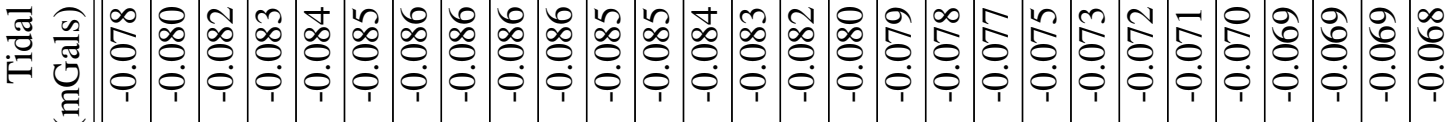
局

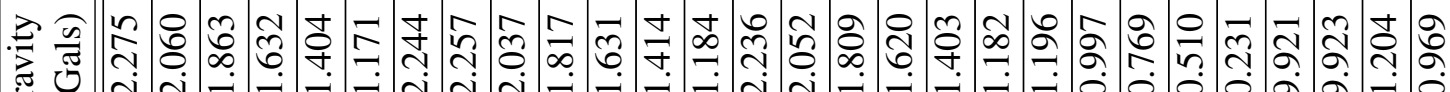
m ह 00
0

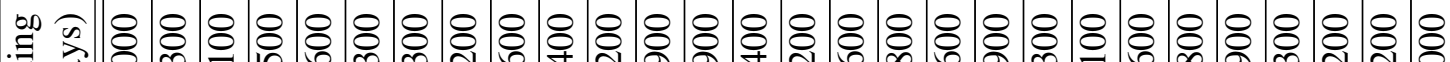

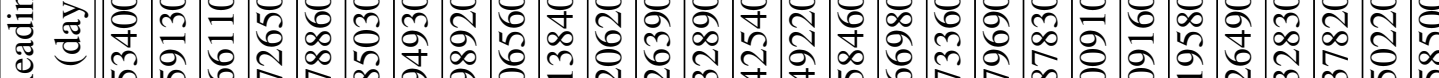

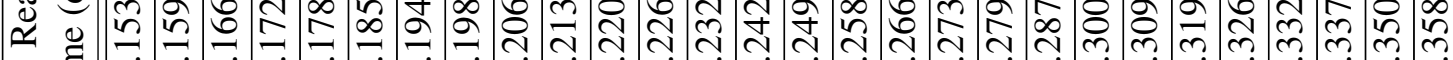

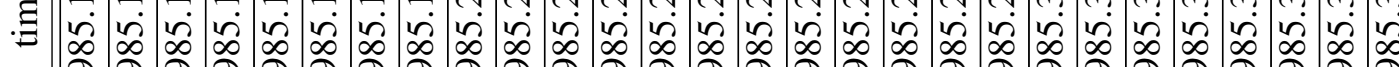

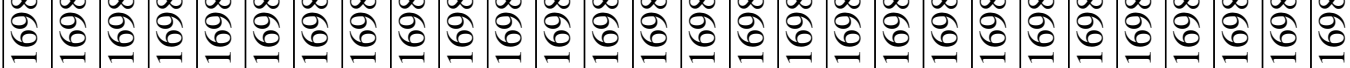

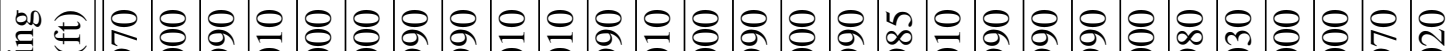
:

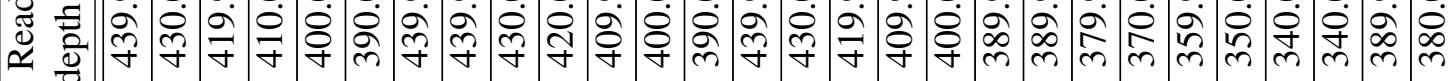
$\stackrel{0}{=}$

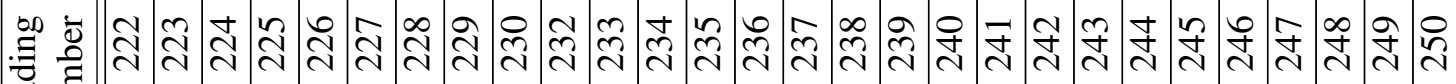

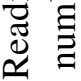




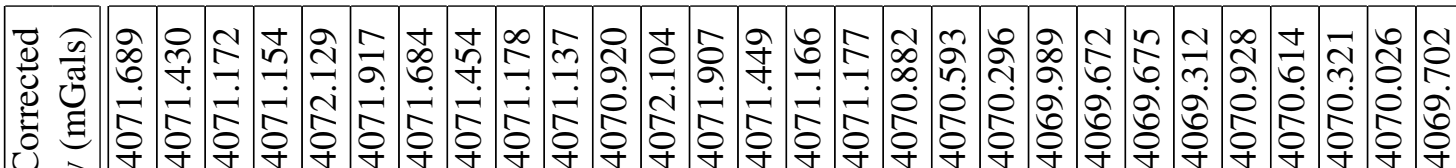
药

Е

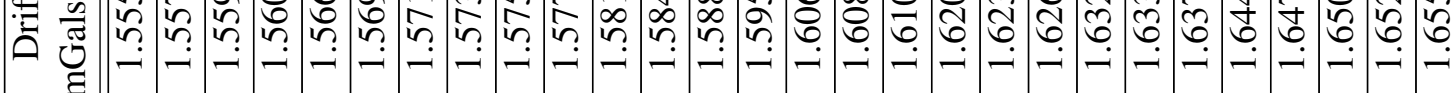

ठำ

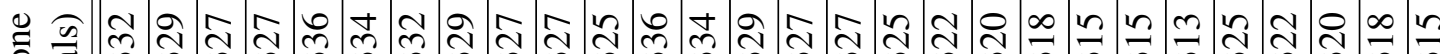

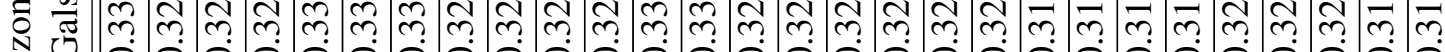
节

承

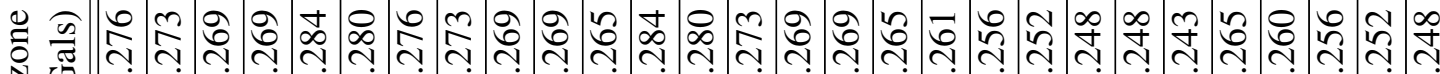

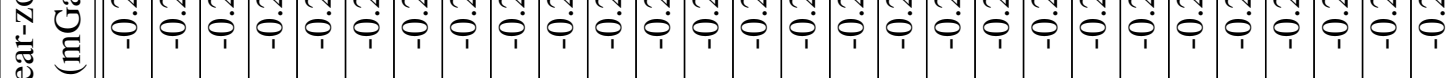
乙

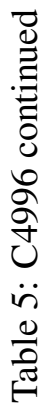

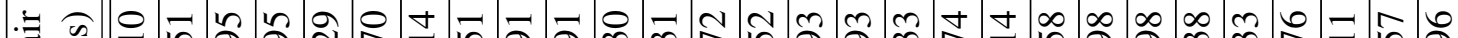

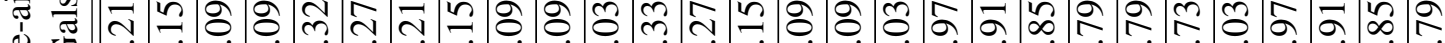
d

8

范

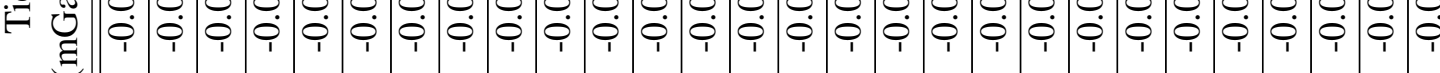

8

: T 勿

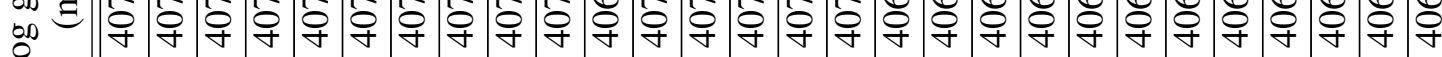

告 च च च

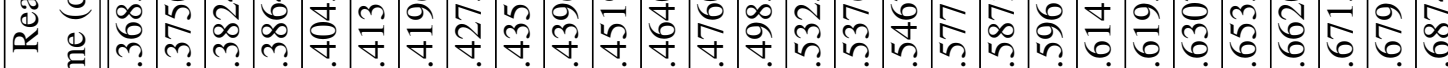

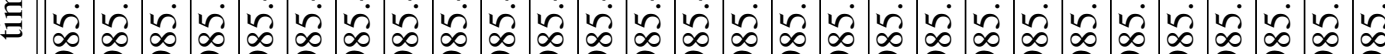

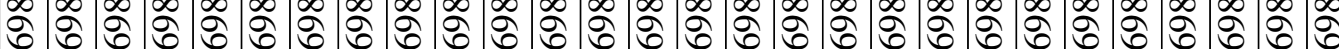

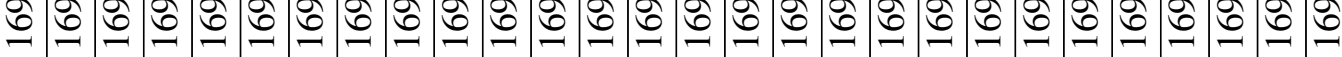

.

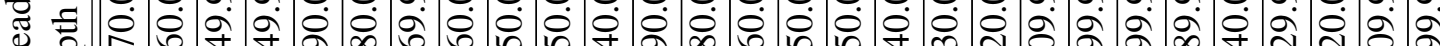
थ द्वे $\stackrel{0}{=} \theta$ : $\simeq$ 


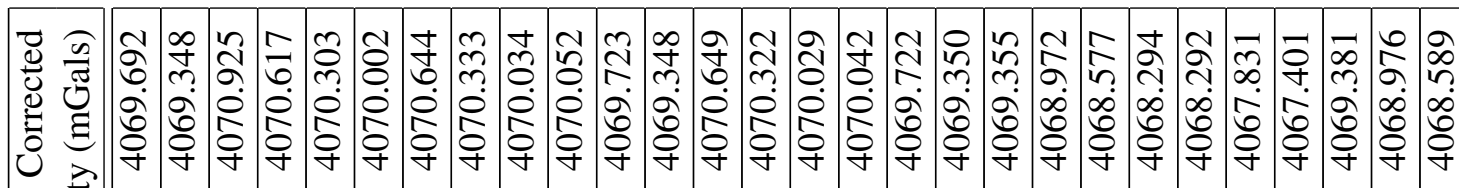

\# a J

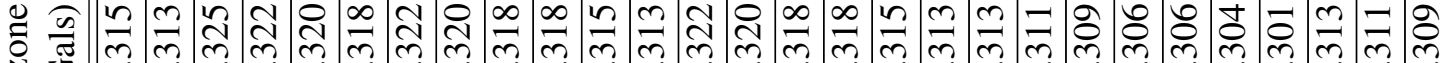

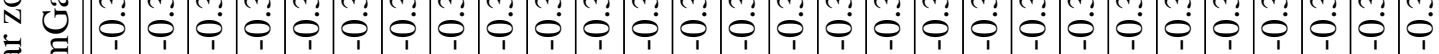
串

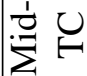

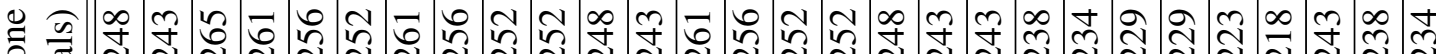

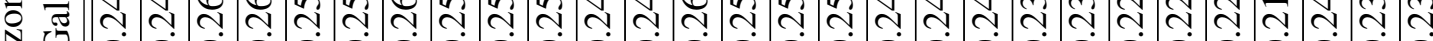

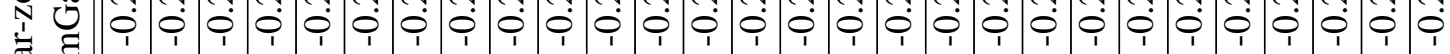
胥 乙

\begin{tabular}{|c|c|c|c|c|c|c|c|c|c|c|c|c|c|c|c|c|c|c|c|c|c|c|c|c|}
\hline 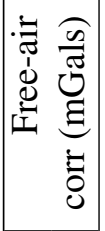 & $\begin{array}{l}2 \\
2 \\
\dot{m} \\
\dot{m}\end{array}$ & $\begin{array}{l}m \\
m \\
\vec{n} \\
m \\
r\end{array}$ & & & & & & & $\underset{\infty}{\infty}$ & & $\mid \begin{array}{c}m \\
\stackrel{n}{2} \\
\tilde{r} \\
1\end{array}$ & $\mid$ & \begin{tabular}{l|l}
$\bar{\sigma}$ & 0 \\
$\tilde{j}$ & $\vdots$ \\
$\tilde{1}$ & $\vdots$
\end{tabular} & & & $f^{\circ}$ & $\begin{array}{l}m \\
\stackrel{n}{\infty} \\
\tilde{r}\end{array}$ & $\left|\begin{array}{l}\hat{6} \\
\dot{0} \\
i \\
1\end{array}\right|$ & & $\begin{array}{l}n \\
\infty \\
\infty \\
m\end{array}$ & 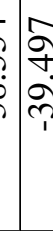 & 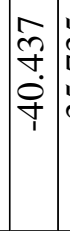 & 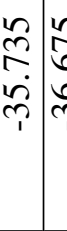 & $\begin{array}{l}0 \\
0 \\
0 \\
? \\
1\end{array}$ \\
\hline 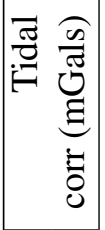 & $\begin{array}{l}1 \\
0 \\
0 \\
0\end{array}$ & $\begin{array}{c}m \\
0 \\
0 \\
0\end{array}$ & $\begin{array}{l}0 \\
\text { ô. } \\
0 \\
0\end{array}$ & 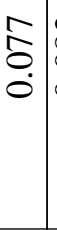 & 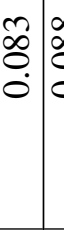 & $\begin{array}{lll}\infty & \\
\infty & \\
0 & \\
0 & & \\
0\end{array}$ & $\stackrel{c}{e}$ & b. & $\frac{1}{0}$ & $\frac{\bar{d}}{0}$ & 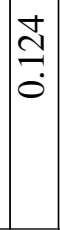 & $\begin{array}{c}2 \\
2 \\
0 \\
0\end{array}$ & $\stackrel{\vec{m}}{\dot{0}}$ & \begin{tabular}{lll}
$m$ & \multicolumn{1}{c}{} \\
$\stackrel{2}{0}$ & $\frac{c}{c}$
\end{tabular} & & $\frac{\infty}{2}$ & $\begin{array}{l}\infty \\
m \\
0\end{array}$ & $\frac{\hat{m}}{2}$ & & $\stackrel{n}{\frac{n}{0}} \frac{\vec{c}}{c}$ & $\frac{\pi}{0}$ & $\frac{0}{2}$ & 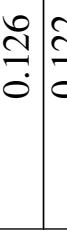 & \\
\hline 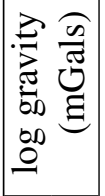 & $\begin{array}{l}\hat{n} \\
\hat{n} \\
\infty \\
0 \\
0 \\
y\end{array}$ & 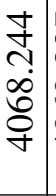 & $\begin{array}{l}\hat{n} \\
\infty \\
\dot{0} \\
b \\
o \\
y\end{array}$ & 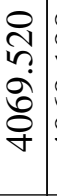 & 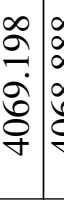 & 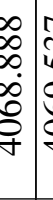 & 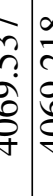 & 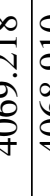 & 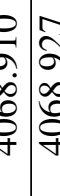 & $\left\{\begin{array}{l}\infty \\
\infty \\
\infty \\
\infty \\
\varnothing \\
\varnothing\end{array}\right.$ & 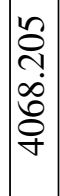 & 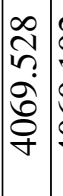 & 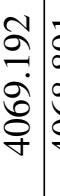 & 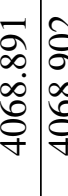 & 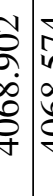 & 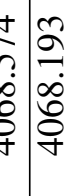 & $\begin{array}{l}\hat{a} \\
\vdots \\
\dot{0} \\
\delta \\
o\end{array}$ & $\begin{array}{l}\infty \\
\delta \\
\infty \\
\hat{\sigma} \\
0 \\
\gamma\end{array}$ & 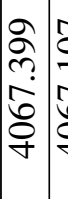 & 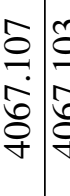 & 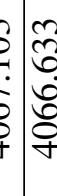 & 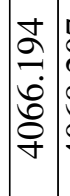 & 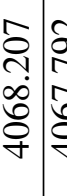 & 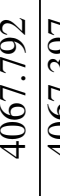 \\
\hline 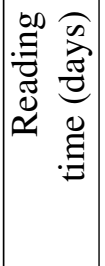 & $\begin{array}{l}8 \\
8 \\
1 \\
0 \\
0 \\
0 \\
1 \\
1 \\
0 \\
0 \\
0 \\
-\end{array}$ & 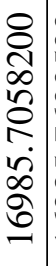 & $\begin{array}{l}8 \\
8 \\
2 \\
8 \\
0 \\
1 \\
\infty \\
\infty \\
2\end{array}$ & 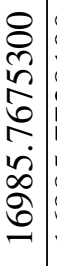 & 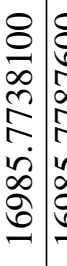 & 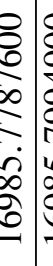 & 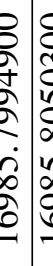 & 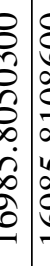 & 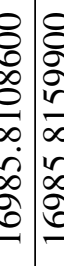 & 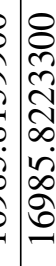 & $\begin{array}{l}8 \\
0 \\
2 \\
\infty \\
0 \\
\infty \\
0 \\
\infty \\
\infty \\
0 \\
0\end{array}$ & $\begin{array}{l}8 \\
0 \\
n \\
2 \\
0 \\
0 \\
\dot{0} \\
\infty \\
0 \\
0 \\
-1\end{array}$ & 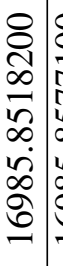 & 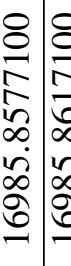 & 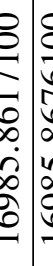 & 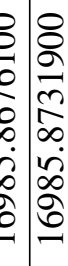 & $\begin{array}{l}8 \\
8 \\
8 \\
\infty \\
0 \\
\infty \\
\infty \\
\infty \\
0 \\
0 \\
0\end{array}$ & $\begin{array}{l}8 \\
0 \\
1 \\
2 \\
\infty \\
\infty \\
1 \\
2 \\
2 \\
0 \\
0\end{array}$ & 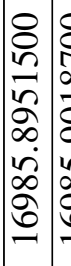 & 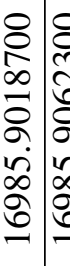 & $\begin{array}{l}b \\
b \\
b \\
b \\
0 \\
0 \\
0 \\
0 \\
0 \\
0 \\
0 \\
0 \\
0\end{array}$ & 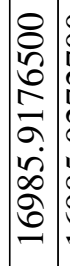 & 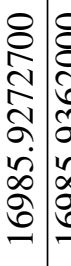 & 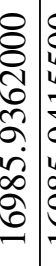 \\
\hline 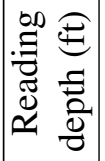 & $\begin{array}{l}\stackrel{2}{\Omega} \\
\text { } \\
\text { নे }\end{array}$ & $\begin{array}{l}0 \\
0 \\
\dot{0} \\
\stackrel{2}{v}\end{array}$ & $\begin{array}{l}8 \\
8 \\
0 \\
\dot{0}\end{array}$ & $\begin{array}{l}8 \\
8 \\
0 \\
\dot{0} \\
m\end{array}$ & 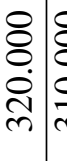 & 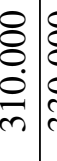 & 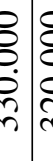 & 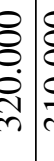 & 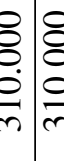 & : & 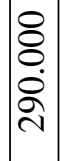 & $\begin{array}{l}8 \\
\delta \\
\vdots \\
0 \\
\infty \\
\tilde{n}\end{array}$ & $\begin{array}{l}8 \\
8 \\
\dot{0} \\
\dot{1} \\
\text { }\end{array}$ & 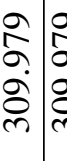 & 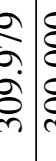 & 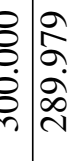 & $\begin{array}{l}2 \\
\hat{\alpha} \\
\vdots \\
\alpha \\
\alpha \\
\end{array}$ & 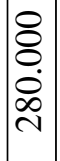 & 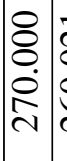 & 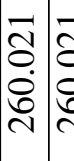 & 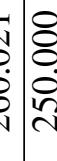 & $\begin{array}{l}8 \\
8 \\
0 \\
\dot{+} \\
\text { ․ }\end{array}$ & 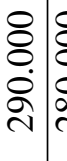 & 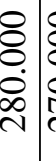 \\
\hline$\stackrel{\theta}{2}$ & 0 & 0 & 0 & 0 & 0 & 0 & 0 & 0 & o & 0 & 0 & 0 & 0 & 0 & v & 0 & 0 & 0 & 0 & 0 & ט & 0 & 0 & 0 \\
\hline 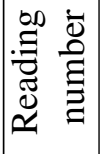 & $\hat{\infty}$ & $\begin{array}{l}\infty \\
\infty \\
\sim\end{array}$ & त & $\overline{\widehat{\lambda}}$ & ๙ે & ה & ถิ & 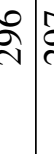 & $\hat{v}$ & i & ষ্লি & రి & లి & 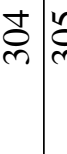 & 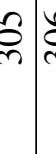 & 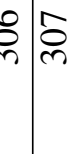 & $\stackrel{\infty}{\infty}$ & ஓి & $\frac{0}{m}$ & $\bar{m} \frac{\Omega}{\sigma}$ & $\frac{m}{n}$ & $\underset{m}{\Xi}$ & $\frac{n}{m}$ & $\frac{0}{m}$ \\
\hline
\end{tabular}




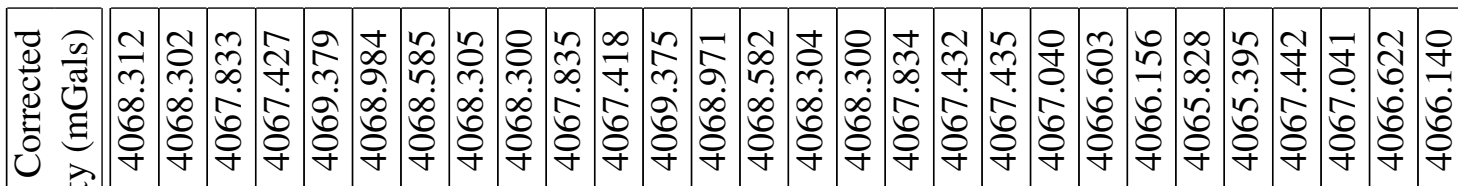
离

Е

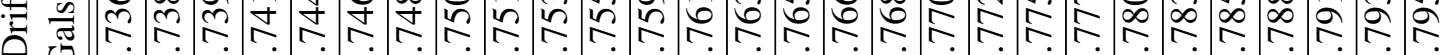

ठั

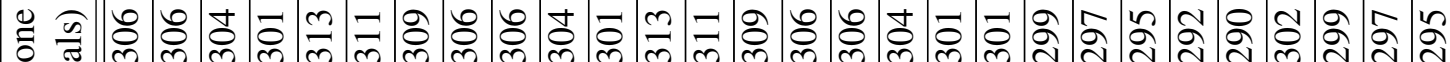

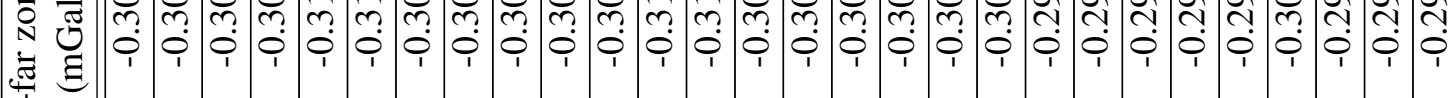

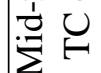

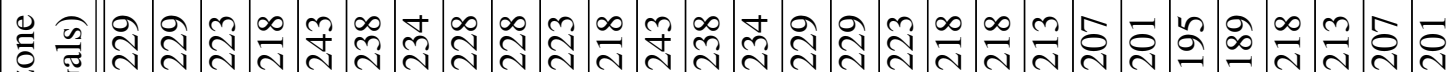

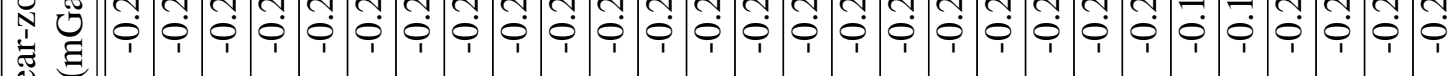
乙

$\exists$ ‡

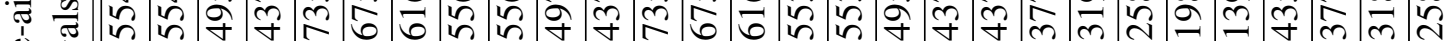

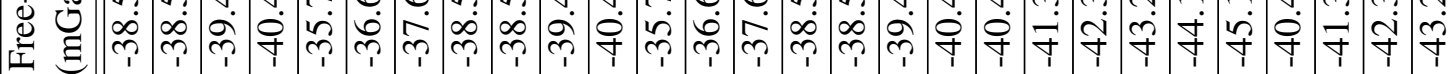
ఫั

สี శิ

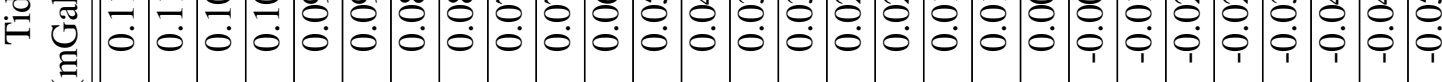
8

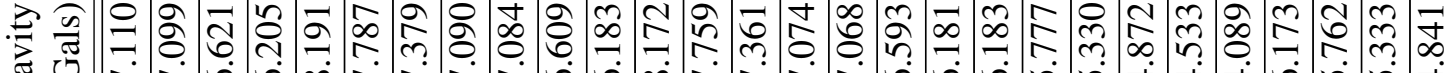
خ

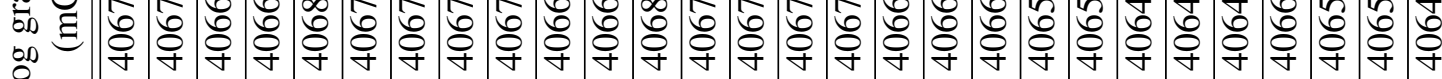

先 \&

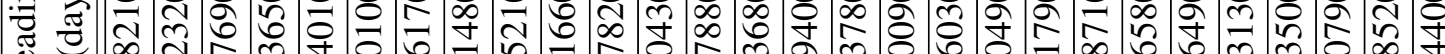

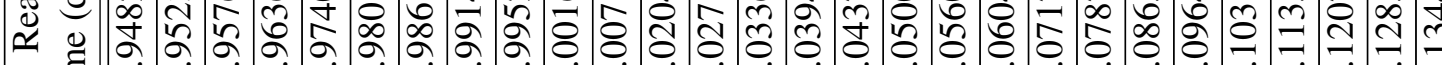

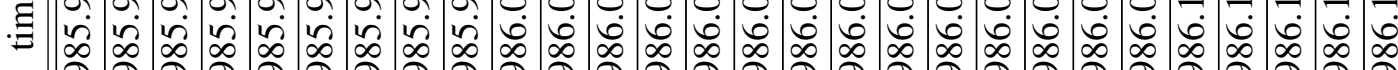

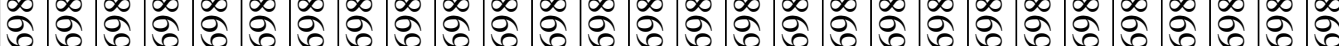

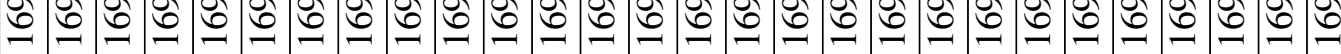

.

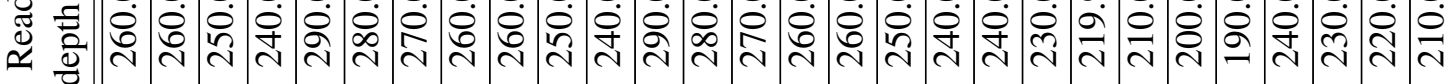

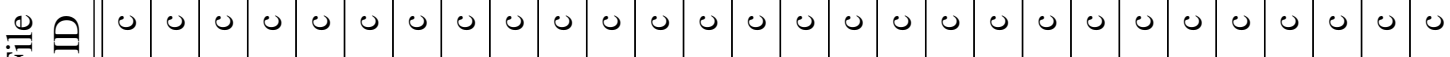
厌

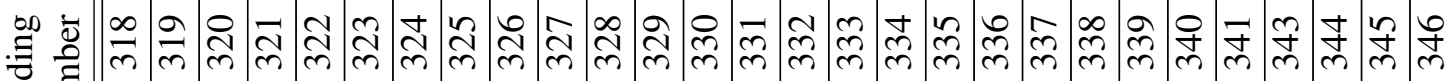
(3) 


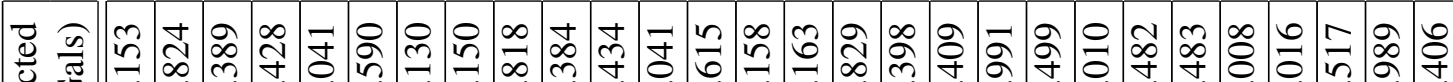
ن U

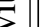
bू

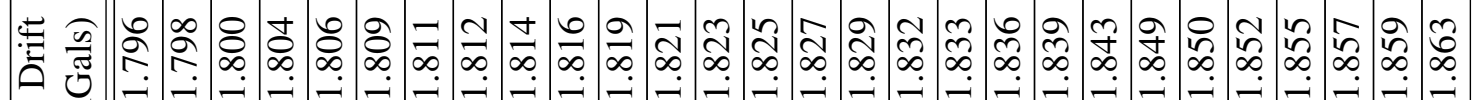

‡

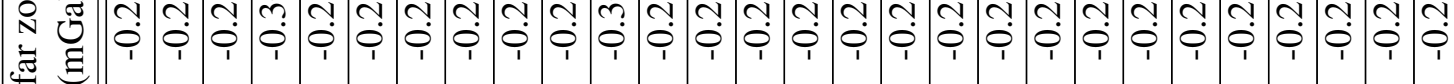

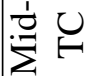

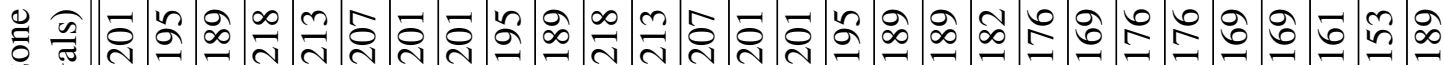
N ซึ 乙

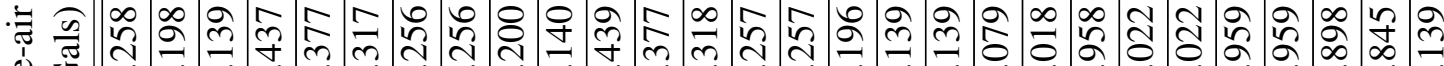
d j

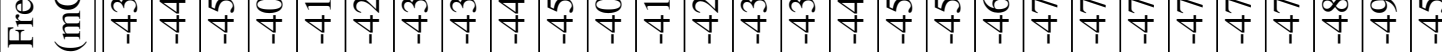

\section{ठํ.}

สే 車 ठํ.

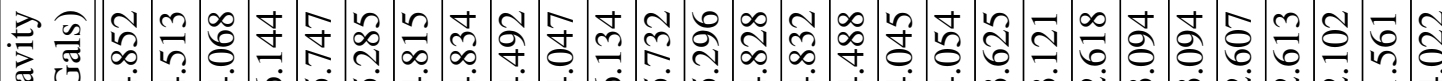
ت

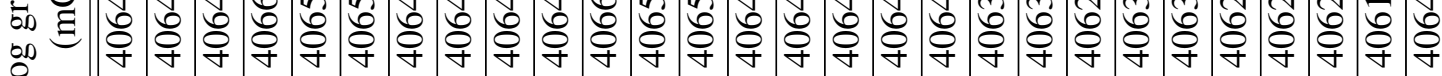
으 :

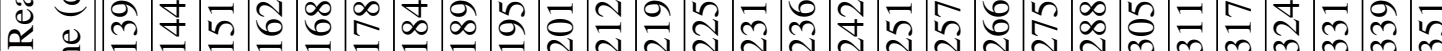
.J bे

의 范 $\stackrel{0}{=}$

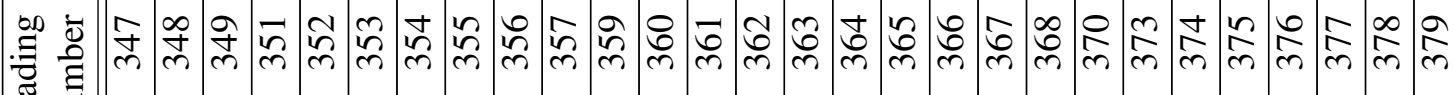
冚 


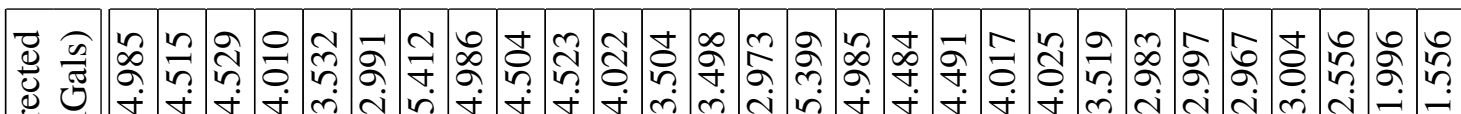

苛

0
0 离

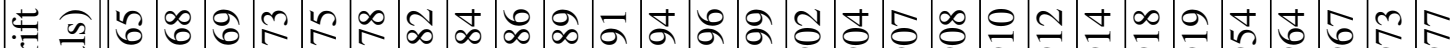
పే

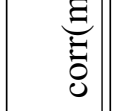

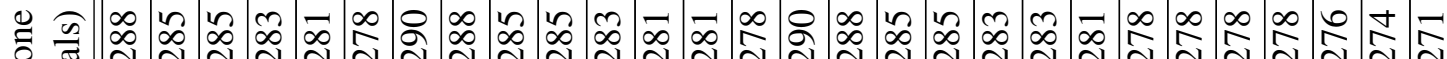

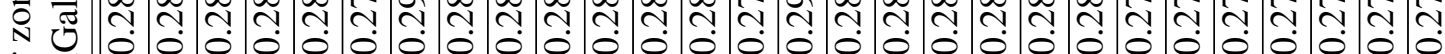

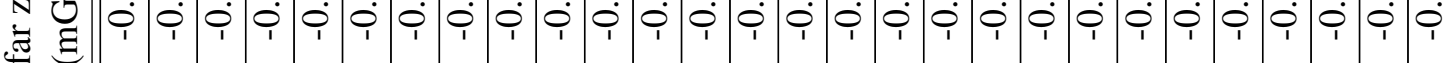
党

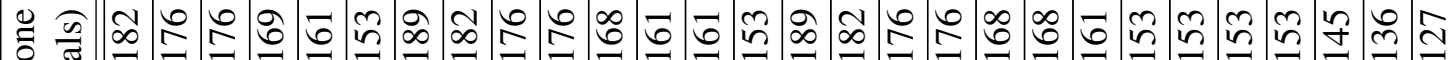

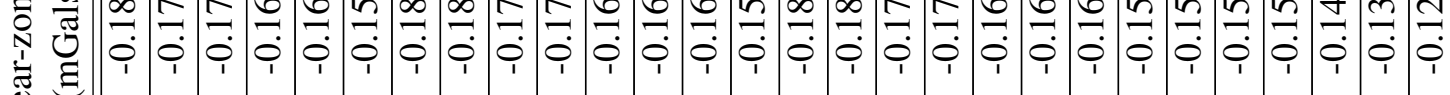
乙

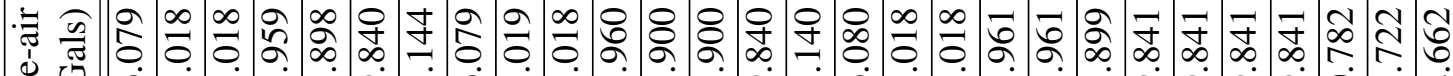
总 ఏँ

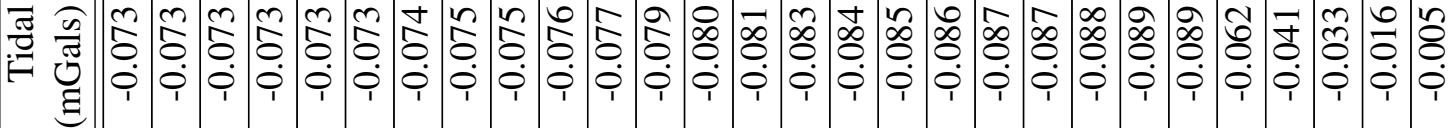
ठำ

증 च

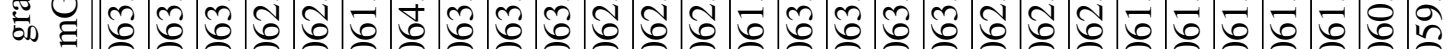

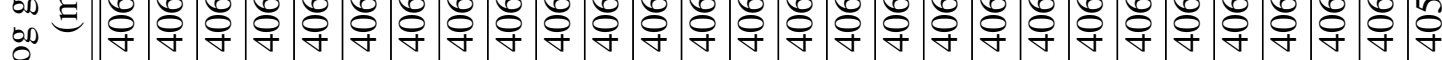
으

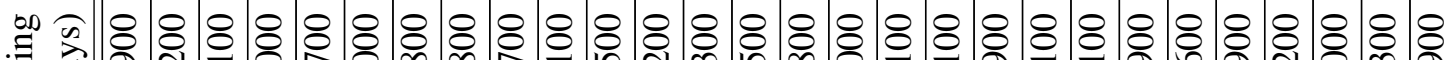
苂 坖 थ

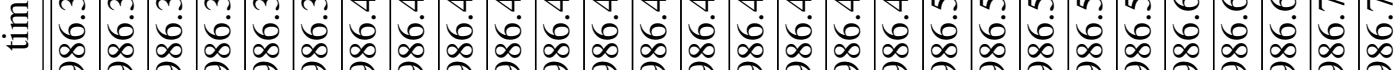
bे 0 b

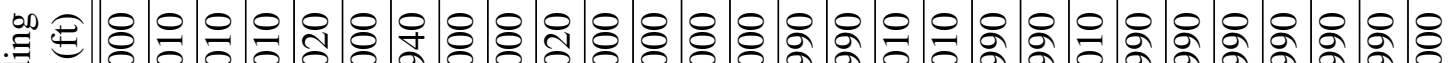
ळ

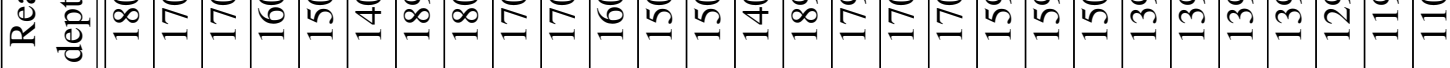
$\frac{0}{*} \theta$

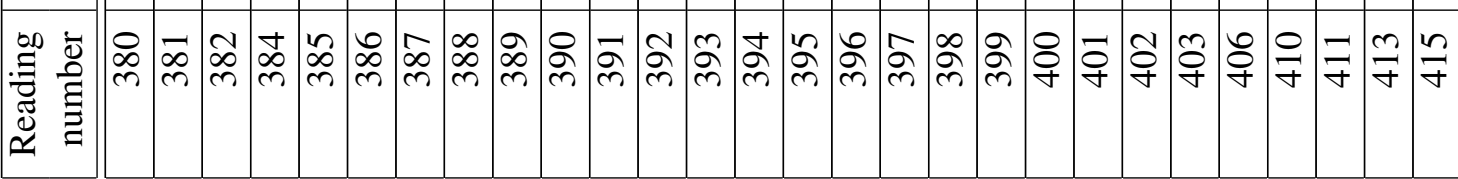




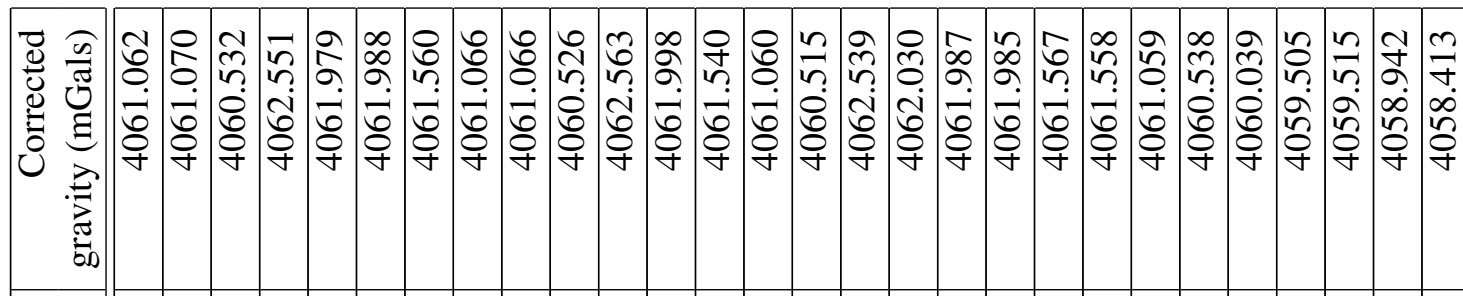

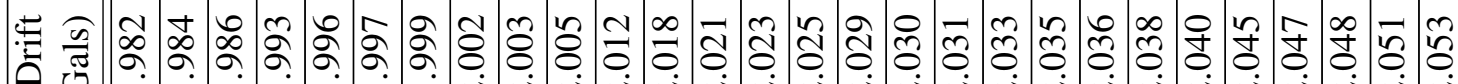

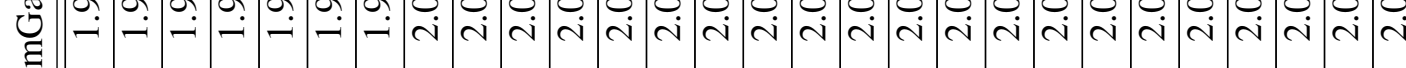

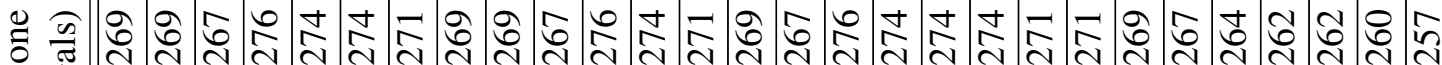

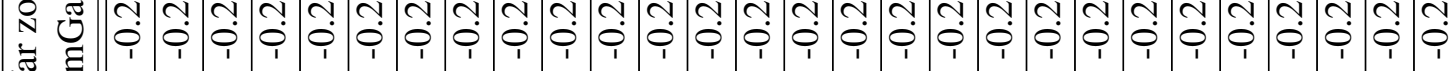
㭉

$\sum_{\ominus}$

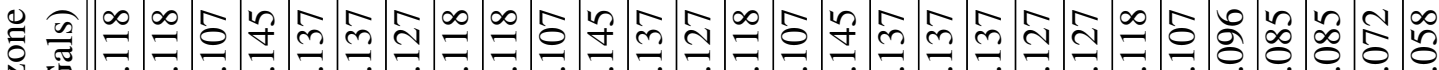
崩 हี 乙

\begin{tabular}{|c|c|c|c|c|c|c|c|c|c|c|c|c|c|c|c|c|c|c|c|c|c|c|c|}
\hline 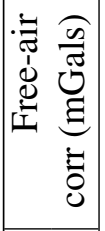 & $\mid \begin{array}{l}c \\
\delta \\
0 \\
\infty \\
n \\
1\end{array}$ & 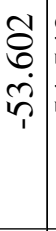 & r & & & $\begin{array}{c}\vec{N} \\
\stackrel{\vec{n}}{i}\end{array}$ & $\begin{array}{l}\text { हु } \\
\text { in }\end{array}$ & & & & & $\begin{array}{c}\tau \\
6 \\
0 \\
i \\
\tilde{n} \\
1\end{array}$ & & & $\frac{\sqrt{n}}{\dot{n}}$ & $\stackrel{\vec{N}}{\stackrel{\vec{n}}{i}}$ & 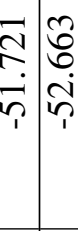 & $\begin{array}{c}m \\
b \\
b \\
i \\
n \\
1\end{array}$ & 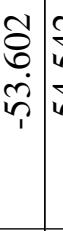 & 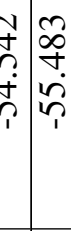 & 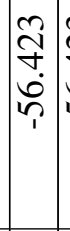 & 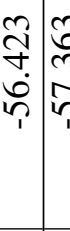 & $\infty$ \\
\hline 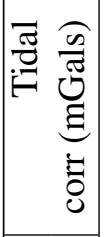 & $\begin{array}{l}1 \\
0 \\
0 \\
0\end{array}$ & $\begin{array}{c}\overline{0} \\
0 \\
0\end{array}$ & 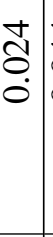 & $\begin{array}{l} \pm \\
\dot{0} \\
\dot{0}\end{array}$ & 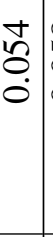 & $\begin{array}{l}\infty \\
2 \\
0 \\
0\end{array}$ & ర্ & $\begin{array}{c}5 \\
0 \\
0\end{array}$ & & 5 & $\begin{array}{l}\stackrel{0}{=} \\
\stackrel{0}{\circ}\end{array}$ & $\stackrel{ \pm}{\Xi}$ & $\bar{z}$ & & 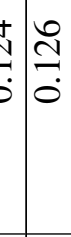 & 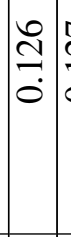 & 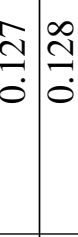 & $\begin{array}{c}\infty \\
\stackrel{\infty}{1} \\
\\
\end{array}$ & 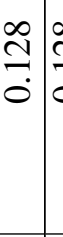 & & $\stackrel{\Xi}{\beth}$ & & $\frac{\infty}{0}$ \\
\hline 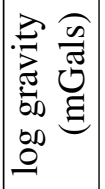 & 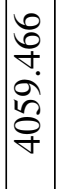 & 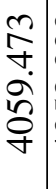 & 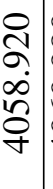 & $\begin{array}{l}0 \\
\infty \\
0 \\
\dot{0} \\
8 \\
0 \\
+\end{array}$ & & $\begin{array}{l}- \\
\dot{0} \\
\dot{+} \\
\dot{0} \\
0 \\
\dot{q}\end{array}$ & & 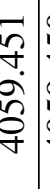 & 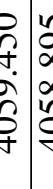 & 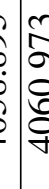 & 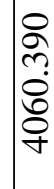 & $\begin{array}{l}\infty \\
\sigma \\
\sigma \\
\tilde{\sigma} \\
\dot{\gamma}\end{array}$ & 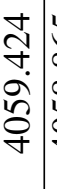 & 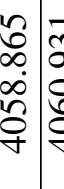 & 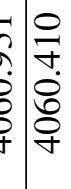 & 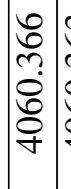 & 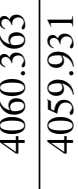 & 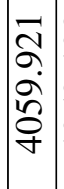 & 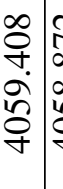 & 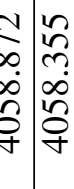 & 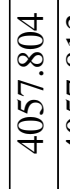 & 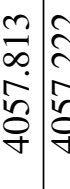 & 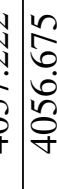 \\
\hline 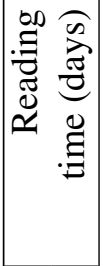 & 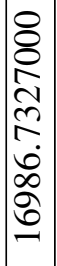 & 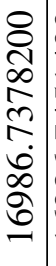 & 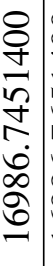 & $\begin{array}{l}8 \\
8 \\
\pm \\
2 \\
2 \\
6 \\
\infty \\
8 \\
8 \\
-1\end{array}$ & 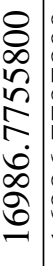 & 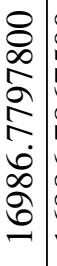 & 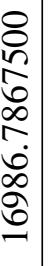 & $\begin{array}{l}8 \\
6 \\
0 \\
2 \\
2 \\
\dot{0} \\
0 \\
6 \\
-\end{array}$ & 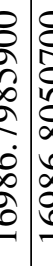 & 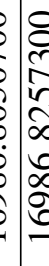 & $\left\{\begin{array}{l}8 \\
8 \\
0 \\
0 \\
0 \\
\infty \\
0 \\
0 \\
\infty \\
0 \\
0 \\
0\end{array}\right.$ & 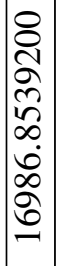 & 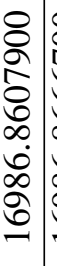 & 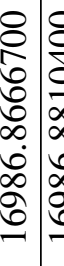 & 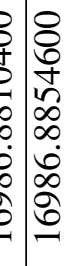 & $\begin{array}{l}8 \\
8 \\
0 \\
2 \\
\infty \\
\infty \\
0 \\
\dot{0} \\
2 \\
0 \\
0 \\
-1\end{array}$ & 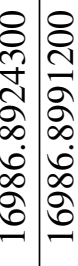 & 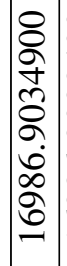 & 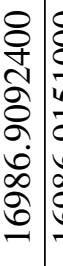 & 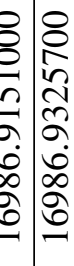 & 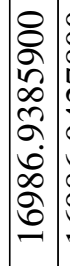 & 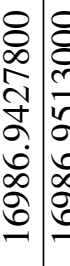 & 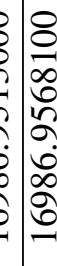 \\
\hline 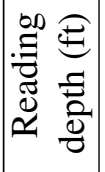 & $\begin{array}{l}8 \\
8 \\
\dot{8} \\
0\end{array}$ & \begin{tabular}{l}
8 \\
$\varnothing$ \\
$\dot{8}$ \\
\hdashline
\end{tabular} & $\begin{array}{l}8 \\
8 \\
\dot{0} \\
\text { ஜ }\end{array}$ & $\begin{array}{l}\stackrel{2}{\alpha} \\
\text { ป் }\end{array}$ & 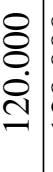 & 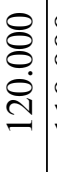 & $\begin{array}{l}8 \\
\delta \\
\vdots \\
0 \\
=\end{array}$ & 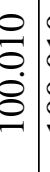 & $\stackrel{f}{\dot{p}}$ & $\xi$ & 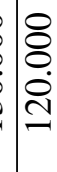 & $\begin{array}{l}8 \\
8 \\
0 \\
0 \\
=\end{array}$ & $\begin{array}{l}8 \\
8 \\
\dot{8} \\
\varnothing\end{array}$ & 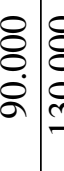 & 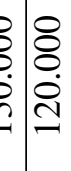 & $\begin{array}{l}8 \\
\delta \\
\dot{\Xi} \\
\end{array}$ & 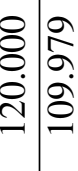 & $\begin{array}{l}a \\
\hat{a} \\
8 \\
8\end{array}$ & \begin{tabular}{l|l}
8 & $\delta$ \\
$\delta$ & 8 \\
$\dot{\delta}$ &
\end{tabular} & 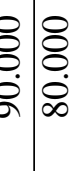 & \begin{tabular}{l}
8 \\
$\delta$ \\
$\dot{0}$ \\
\hdashline
\end{tabular} & 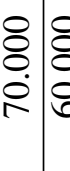 & \\
\hline & 0 & 0 & 0 & 0 & 0 & 0 & 0 & 0 & u & 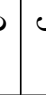 & 0 & 0 & 0 & o & ט & 0 & 0 & 0 & 0 & ט & 0 & 0 & ن ن ט \\
\hline 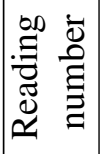 & $\frac{0}{\gamma}$ & $\frac{\bar{\sigma}}{\nabla}$ & $\frac{\infty}{\gamma}$ & 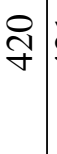 & $\checkmark$ & $\underset{\mathcal{F}}{\stackrel{f}{*}}$ & $\stackrel{\mathcal{Y}}{\mathcal{H}}$ & 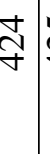 & $\begin{array}{l}0 \\
y\end{array}$ & $\stackrel{\infty}{\jmath}$ & 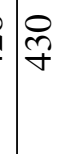 & $\overrightarrow{\tilde{\sigma}}$ & $\stackrel{m}{\sigma}$ & $\stackrel{\vec{f}}{\vec{f}}$ & 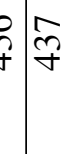 & $\begin{array}{l}\infty \\
\stackrel{\sim}{\sim}\end{array}$ & 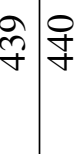 & $尹$ & $\stackrel{\sim}{f}$ & $\stackrel{f}{f} \underset{f}{f}$ & $\stackrel{n}{f}$ & $\stackrel{8}{+} \underset{7}{7}$ & \\
\hline
\end{tabular}




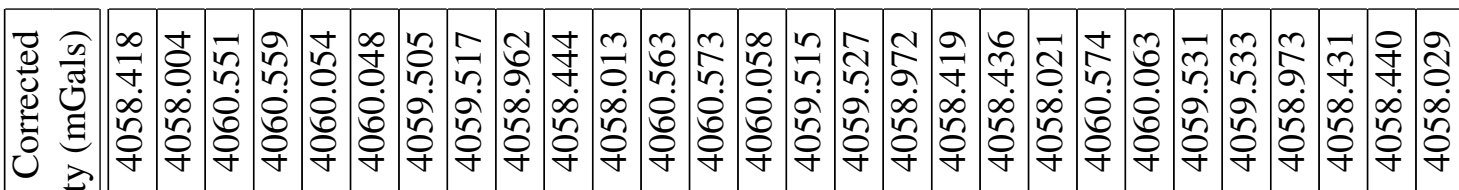
글

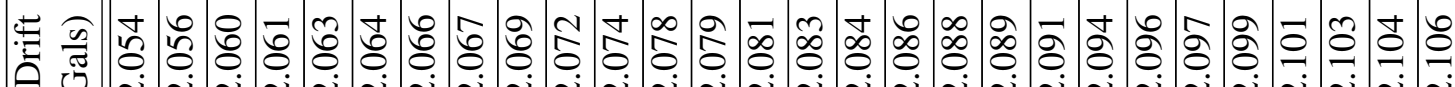
• ठำ

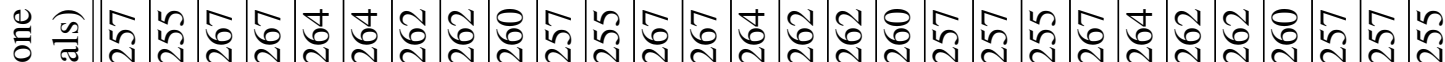

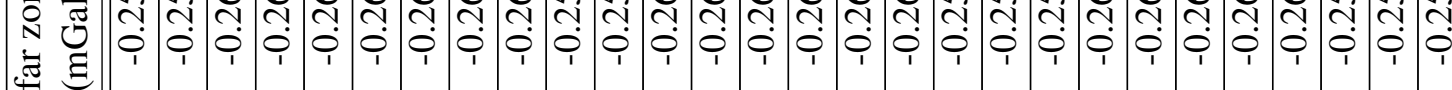

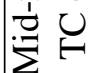

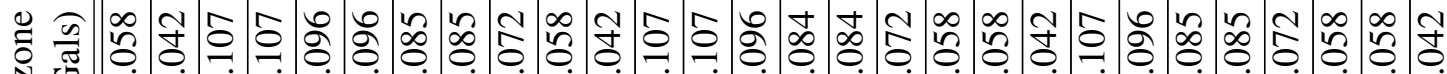

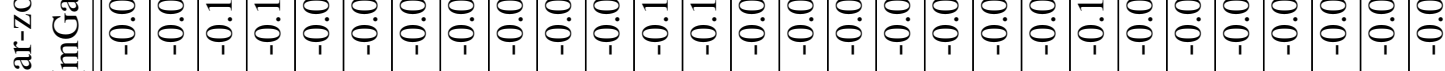
ซึ 乙

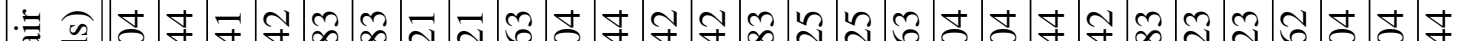
I 远 ठำ

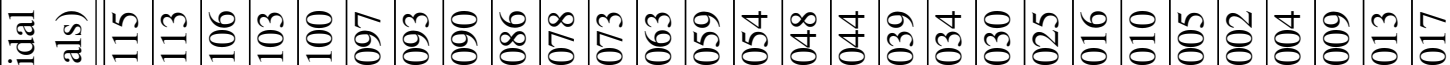

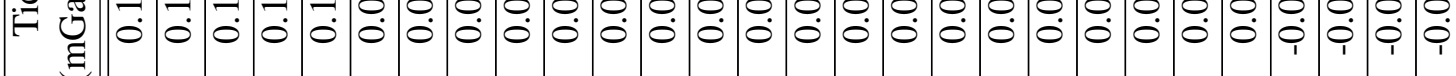
ठำ

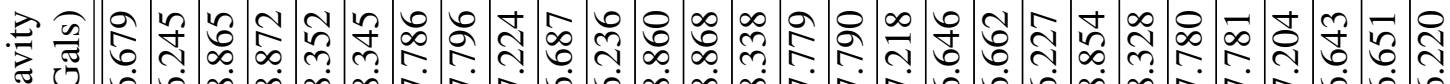
تٓ 0,0
o

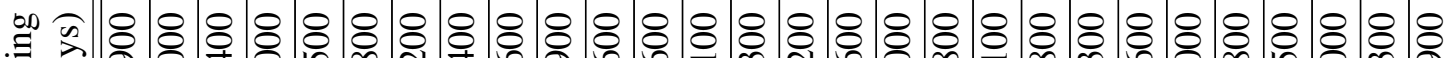

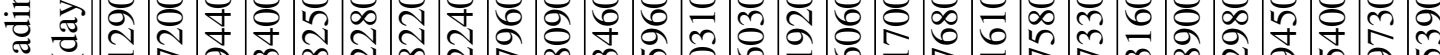

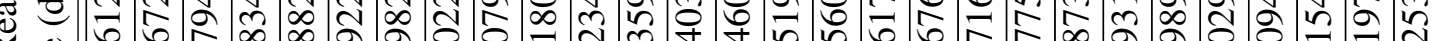

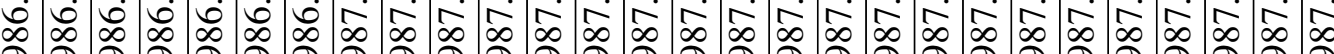
б

告 Є थ $\stackrel{0}{=}$

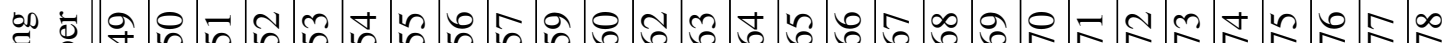

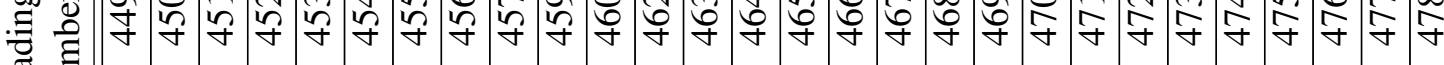
(4) 


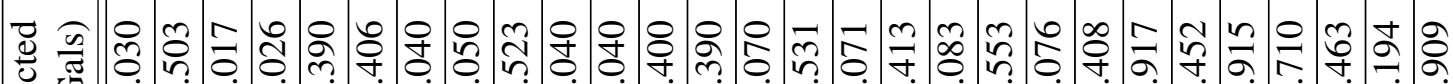

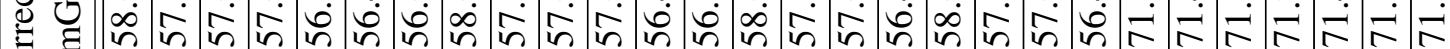

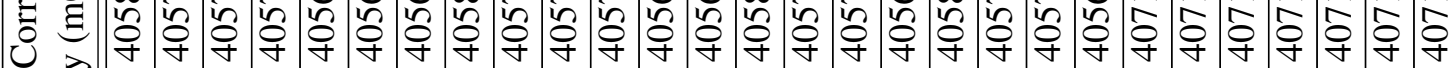

:

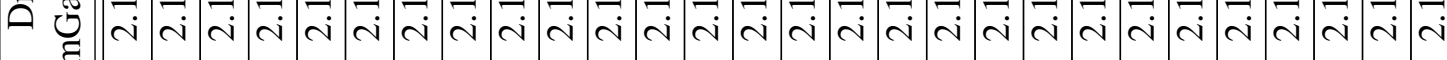

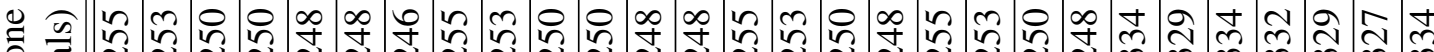
赵 $=$ 荧 $\sum$

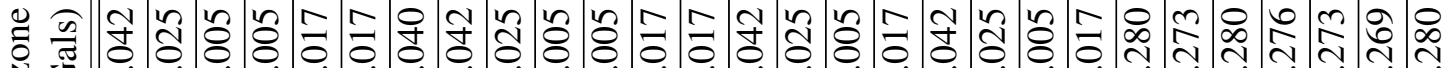

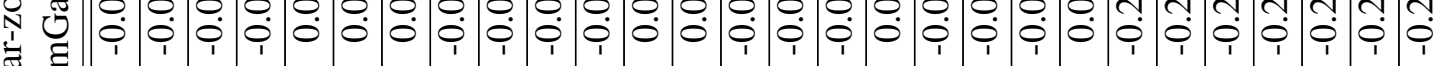
胥 乙

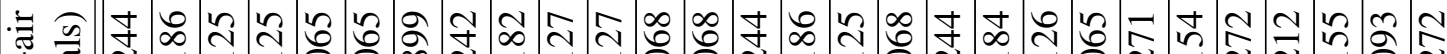
i

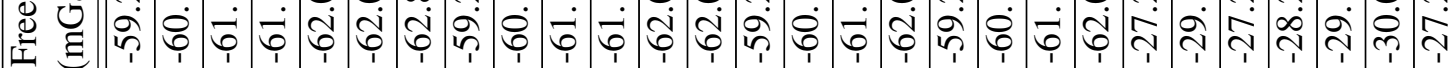

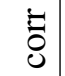

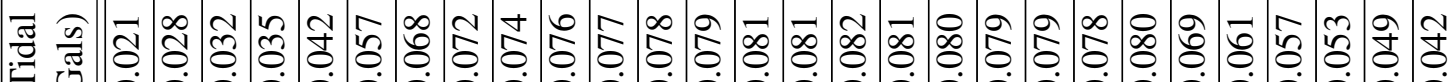

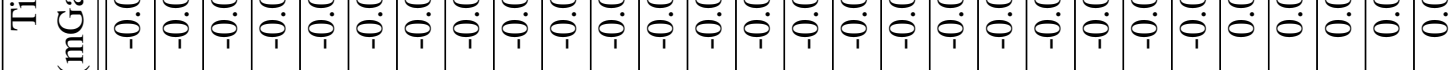

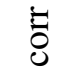

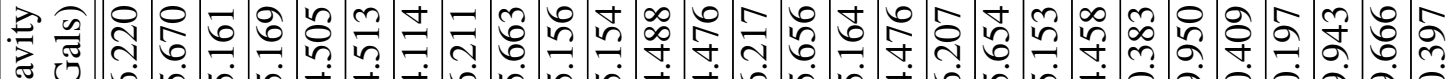
đ

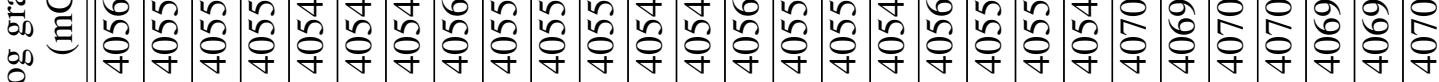
으

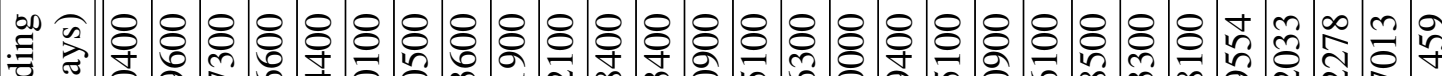
"⿱一兀)

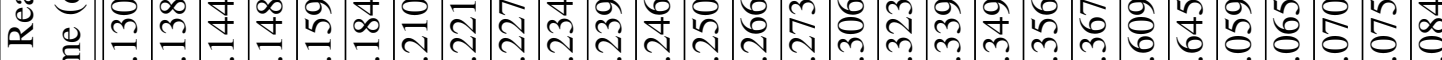

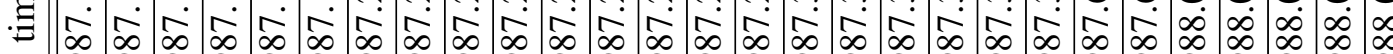
完

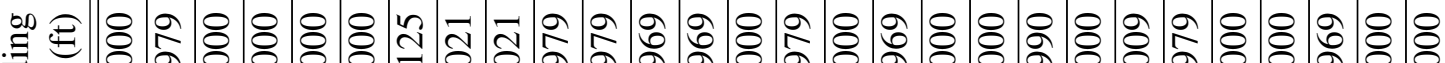

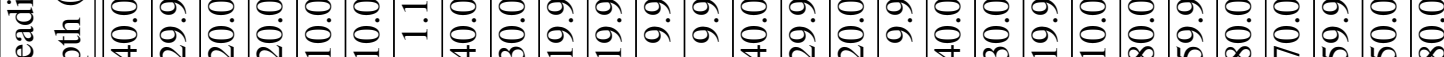
$\simeq$ 常

$\frac{0}{2}$

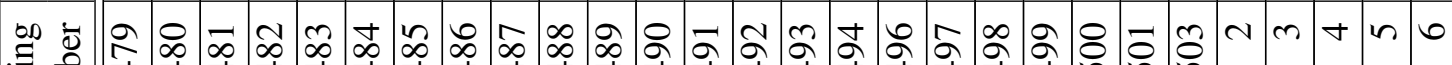
忥 声|f 


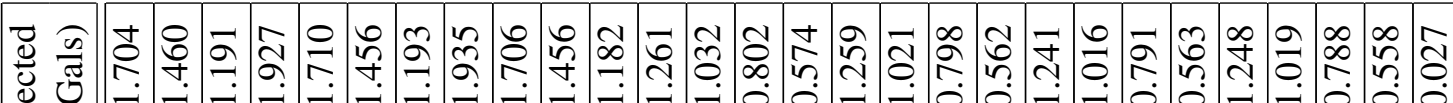

过

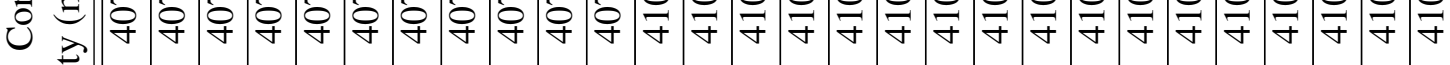

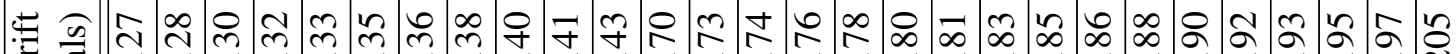

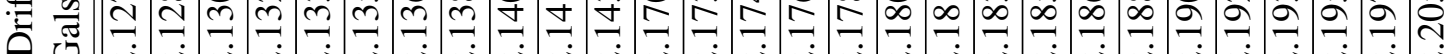
西

ठํ

ఊ

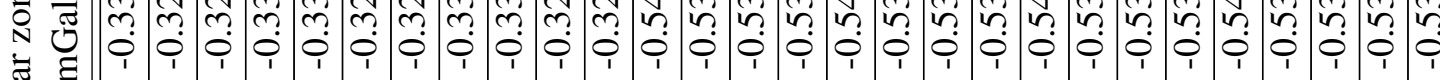

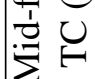

宕全 告 ๘ 乙 U

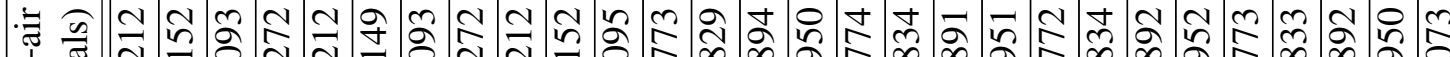
ن

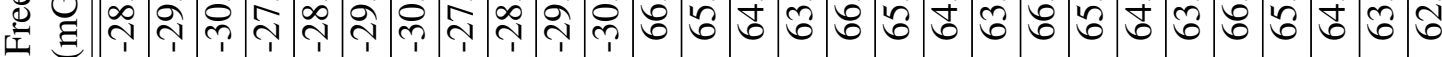
ठ․

శुำ

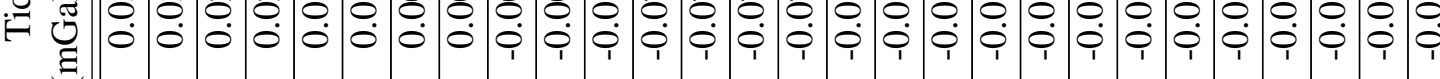
¿ั

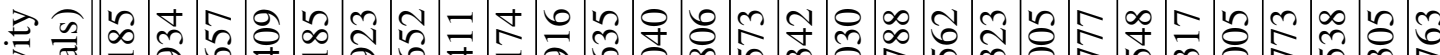
خ

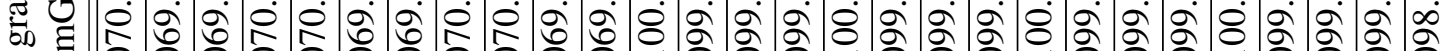
on

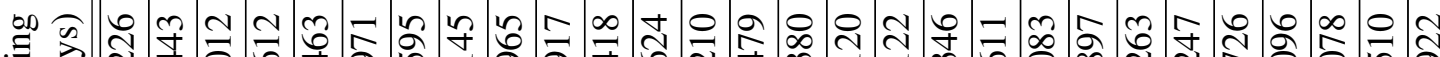

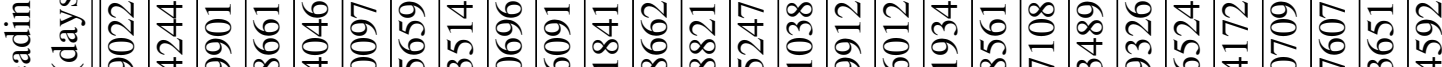
$\simeq$ bे a $a$ a

\begin{tabular}{|c|c|c|c|c|c|c|c|c|c|c|c|c|c|c|c|c|c|c|c|c|c|c|c|c|c|c|c|c|}
\hline 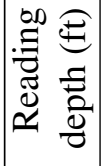 & $\begin{array}{l}8 \\
8 \\
0 \\
0 \\
\text { m }\end{array}$ & 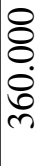 & $\begin{array}{l}8 \\
8 \\
0 \\
0 \\
\infty \\
\text { n. }\end{array}$ & 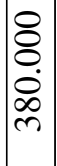 & 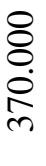 & 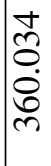 & 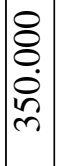 & 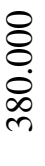 & $\begin{array}{l}8 \\
8 \\
0 \\
\grave{g} \\
\text { m }\end{array}$ & 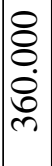 & $\begin{array}{l}a \\
\hat{a} \\
a \\
\text { m }\end{array}$ & $\begin{array}{l}\stackrel{8}{8} \\
0 \\
\dot{8} \\
\infty \\
m\end{array}$ & $\begin{array}{l}0 \\
0 \\
2 \\
0 \\
0 \\
2\end{array}$ & 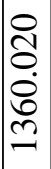 & $\begin{array}{l}0 \\
\infty \\
0 \\
\dot{\sigma} \\
m \\
m\end{array}$ & $\begin{array}{l}0 \\
0 \\
0 \\
0 \\
\infty \\
m\end{array}$ & $\begin{array}{l}0 \\
0 \\
0 \\
\stackrel{0}{0} \\
\dddot{m}\end{array}$ & 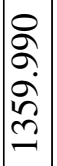 & 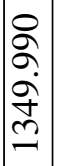 & 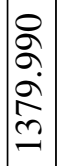 & 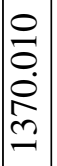 & 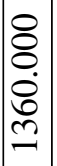 & $\begin{array}{l}8 \\
8 \\
8 \\
\dot{0} \\
\tilde{n} \\
\end{array}$ & 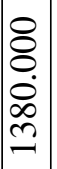 & 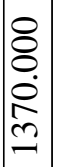 & 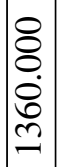 & $\begin{array}{l}a \\
\hat{\sigma} \\
a \\
\text { m }\end{array}$ & 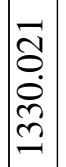 \\
\hline$\frac{0}{2} \theta$ & $\nabla$ & $\nabla$ & ठ & 乙 & $\nabla$ & $\nabla$ & $\nabla$ & $\nabla$ & $\nabla$ & $\nabla$ & $\tau$ & $\nabla$ & $\nabla$ & $\nabla$ & $\nabla$ & $\nabla$ & $\nabla$ & $\nabla$ & $\nabla$ & $\nabla$ & ठ & $\nabla$ & ర & ס & $\nabla$ & $\nabla$ & $\nabla$ & $\nabla$ \\
\hline 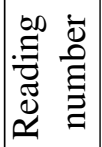 & $r$ & $\infty$ & $a$ & 으 & 二 & $\simeq$ & $m$ & $\Xi$ & $n$ & 0 & 三 & $\infty$ & a & 이 & $\bar{N}$ & N & $\widetilde{\sim}$ & さ & กิ & $\stackrel{\sim}{\sim}$ & $\widehat{\sim}$ & $\stackrel{\infty}{\sim}$ & ภิ & 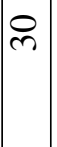 & $\bar{m}$ & $\tilde{m}$ & $m$ & ম \\
\hline
\end{tabular}




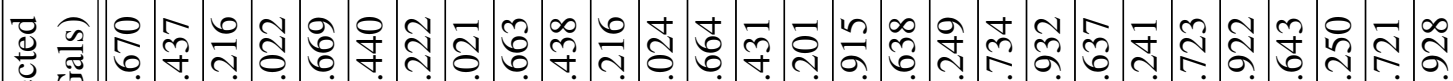
ठ

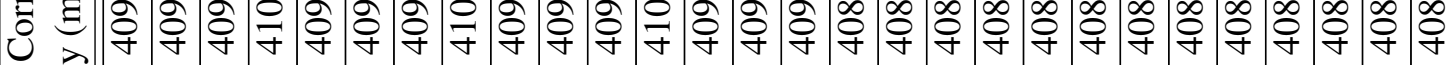



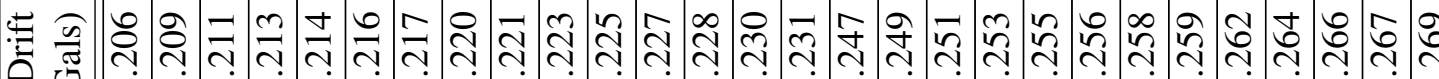
j

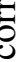

‡

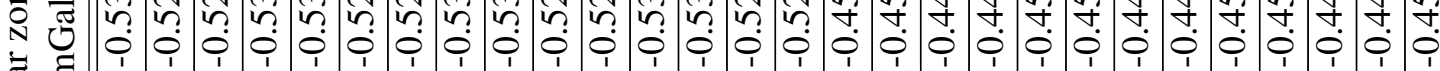
تี है

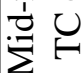

䒘 毛

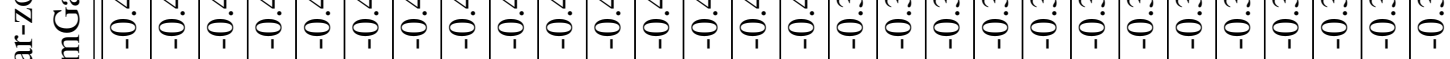
ซึ

Z

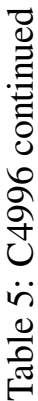

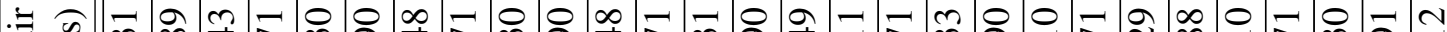

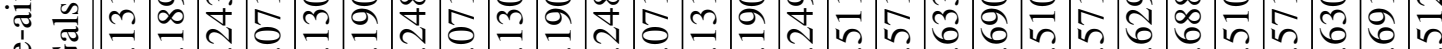
递 ठํ.

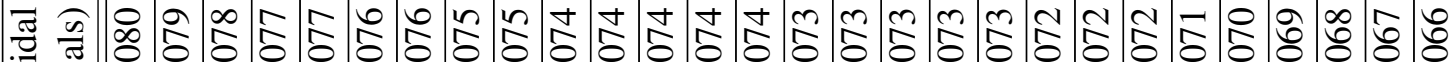

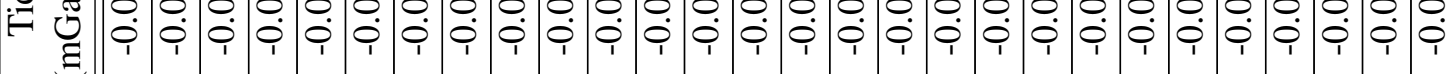
8.

글 䒕

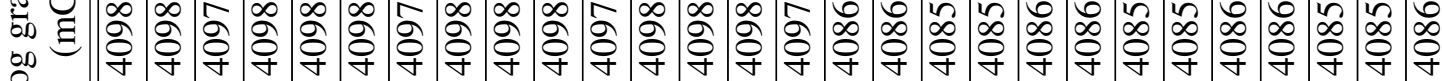
으

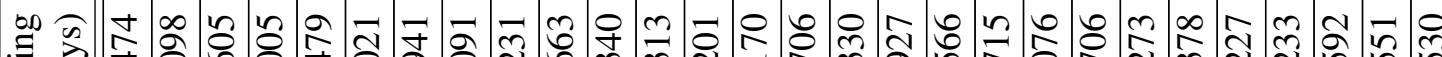

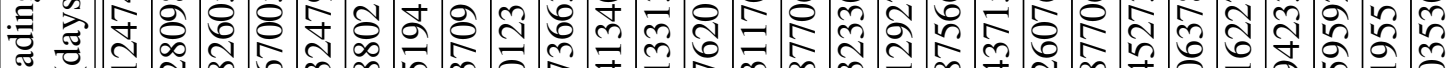

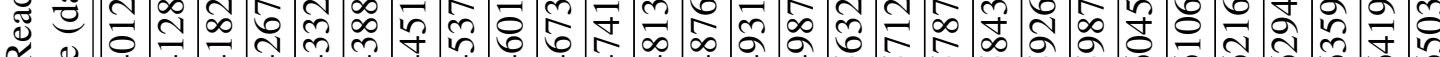

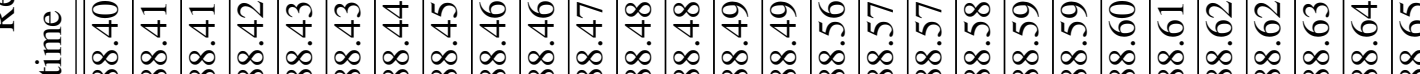

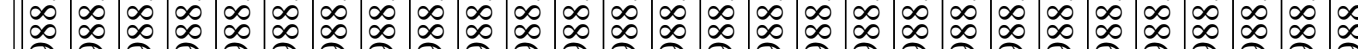

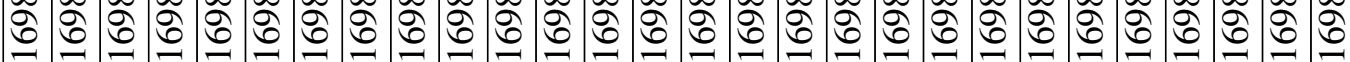

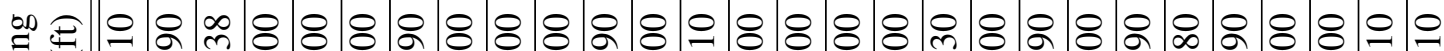

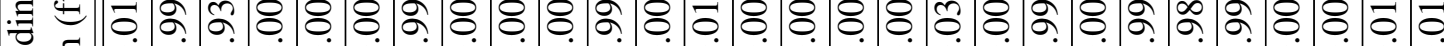
※

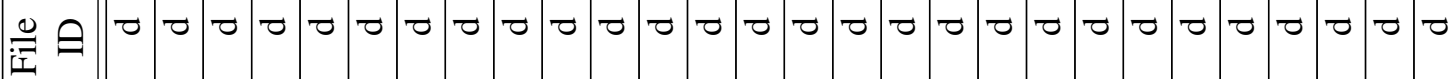
留 $\ddot{\Xi}$ 


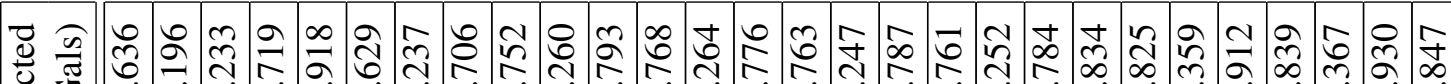

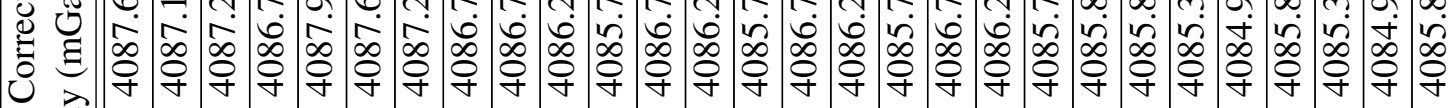
.

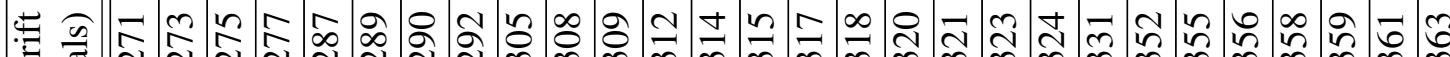
छ ठํ

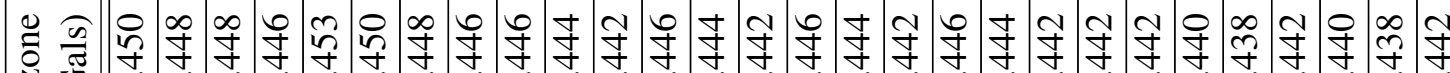
n 党

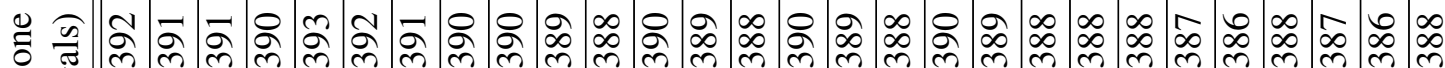

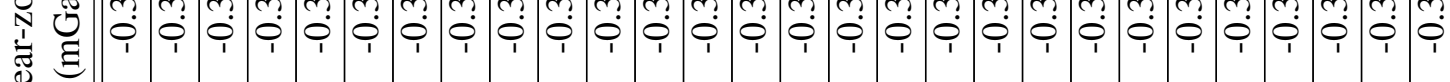
乙 U

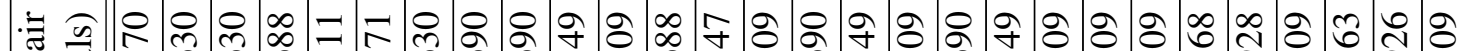

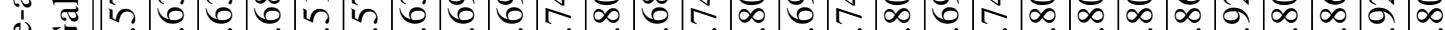

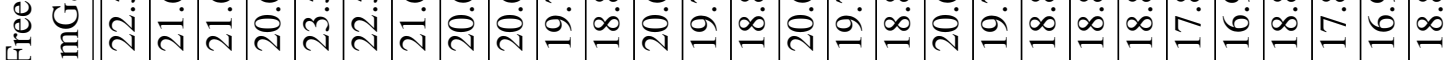
:

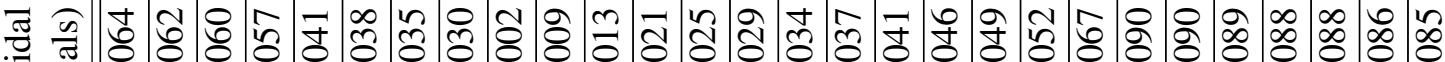

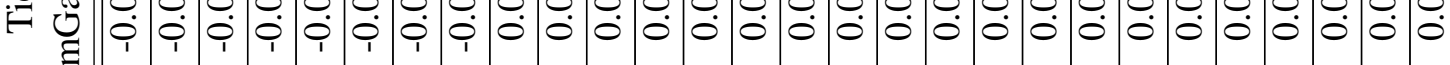

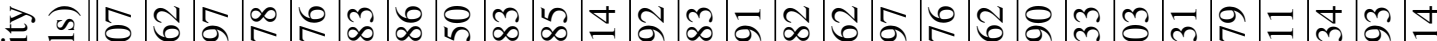

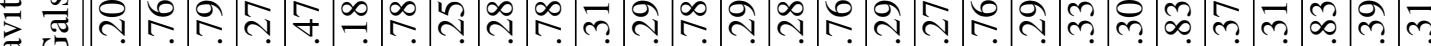
荡

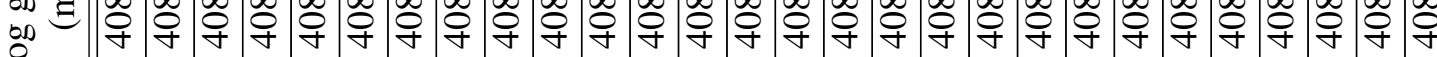

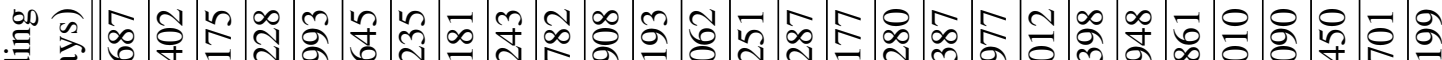

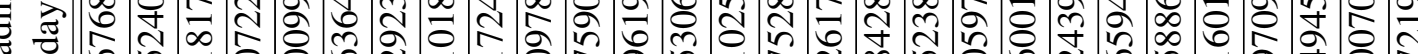
作 光

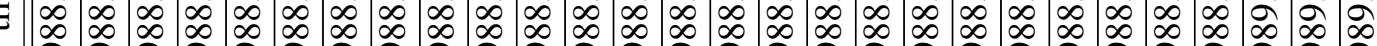
b)

先 I

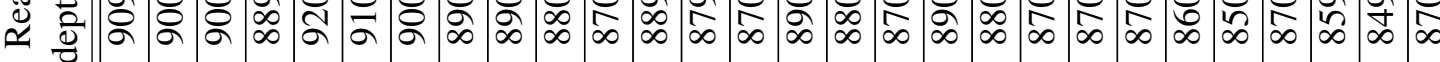

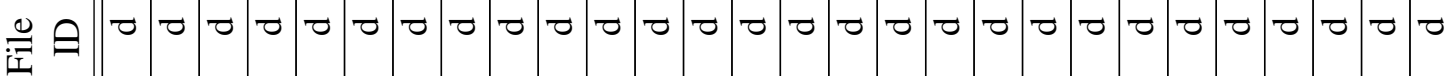

䑻苛 


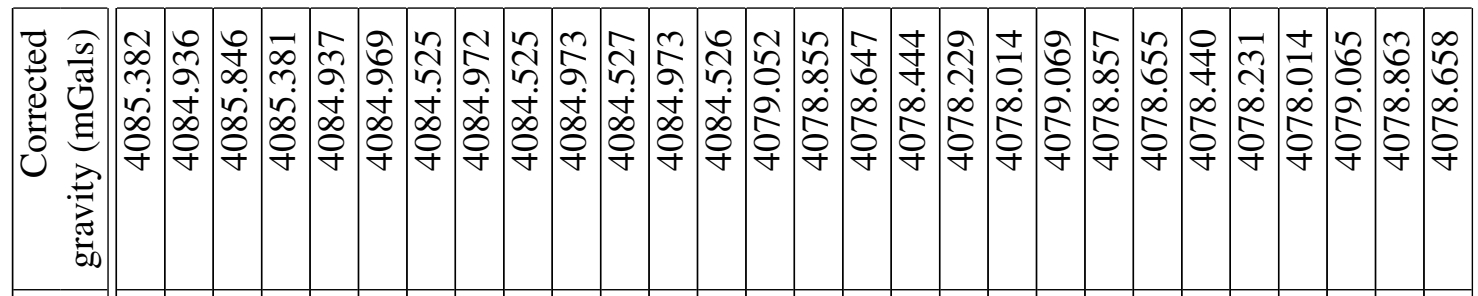

:

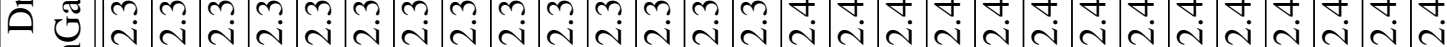

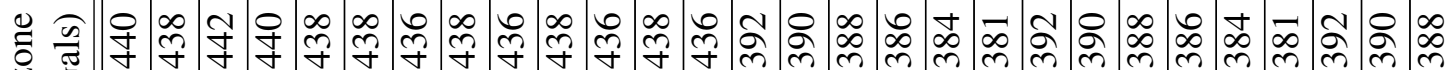

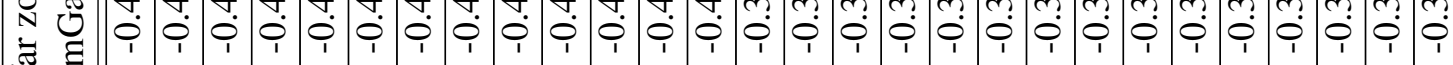
त्ञ

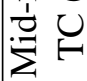

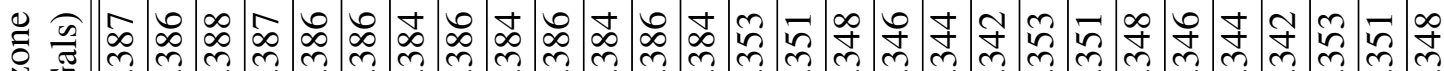
苜 ฮี Z

\begin{tabular}{|c|c|c|c|c|c|c|c|c|c|c|c|c|c|c|c|c|c|c|c|c|c|c|c|c|}
\hline 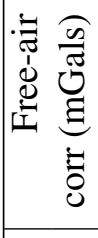 & 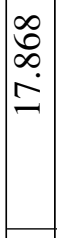 & 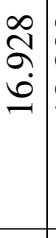 & $\bar{\alpha}$ & & o & & $\begin{array}{l}\hat{\infty} \\
\vdots \\
\tilde{2}\end{array}$ & & & & 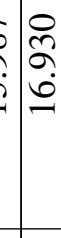 & $\begin{array}{l}\infty \\
\vdots \\
\vdots \\
\vdots\end{array}$ & $\mid \begin{array}{cc}\bar{\gamma} \\
\infty \\
i \\
i \\
i\end{array}$ & & & 6 & $\left|\begin{array}{l}n \\
n \\
n \\
r \\
1\end{array}\right|$ & & $\stackrel{F}{r}$ & $\begin{array}{c}m \\
\dot{J} \\
\dot{p} \\
\dot{1}\end{array}$ & $\begin{array}{c}m \\
\infty \\
n \\
0 \\
1 \\
1\end{array}$ & $\begin{array}{l}\widetilde{N} \\
\infty \\
\infty \\
\sim \\
\sim\end{array}$ & & $\dot{\gamma}$ \\
\hline 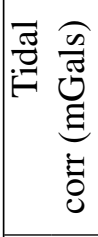 & $\begin{array}{l}2 \\
\infty \\
0 \\
0 \\
0\end{array}$ & $\begin{array}{l}\vec{\infty} \\
0 \\
0\end{array}$ & 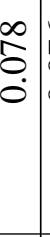 & $\begin{array}{l}0 \\
\vdots \\
0 \\
0\end{array}$ & $\begin{array}{l}\tilde{2} \\
\tilde{o} \\
0\end{array}$ & $\begin{array}{l}\hat{8} \\
0 \\
0\end{array}$ & $\begin{array}{l}2 \\
\delta \\
0 \\
0\end{array}$ & b. & \begin{tabular}{l|l}
0 \\
6 \\
0 \\
0
\end{tabular} & 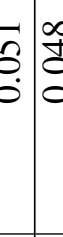 & $\stackrel{0}{0}$ & 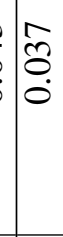 & $\mid$\begin{tabular}{l}
1 \\
8 \\
\hdashline \\
0 \\
0
\end{tabular} & & 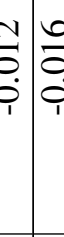 & 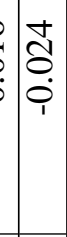 & $\left|\begin{array}{l}\infty \\
\mathbb{1} \\
0 \\
0 \\
1\end{array}\right|$ & $\begin{array}{l}n \\
0 \\
0 \\
0 \\
1\end{array}$ & 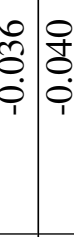 & $\mid \begin{array}{l}8 \\
0 \\
0 \\
0 \\
1\end{array}$ & 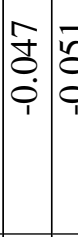 & 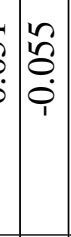 & $\mid \begin{array}{c}1 \\
0 \\
0 \\
0 \\
1\end{array}$ & $\begin{array}{l}n \\
0 \\
0 \\
0 \\
0\end{array}$ \\
\hline 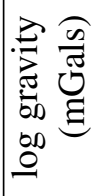 & $\begin{array}{l}n \\
\dot{0} \\
\infty \\
\infty \\
\infty \\
\infty \\
\dot{q}\end{array}$ & 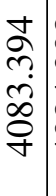 & 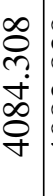 & $\begin{array}{l}2 \\
\hat{\approx} \\
\infty \\
\dot{\hat{\infty}} \\
\infty \\
\dot{\sigma}\end{array}$ & & $\begin{array}{l}\infty \\
\vec{\nabla} \\
\dot{\hat{\theta}} \\
\infty \\
\dot{\sigma}\end{array}$ & & 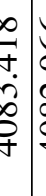 & & 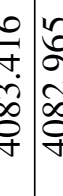 & 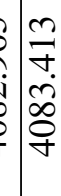 & $\begin{array}{l}8 \\
8 \\
a \\
\infty \\
\infty \\
o \\
+\end{array}$ & 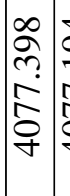 & & 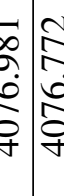 & 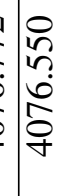 & 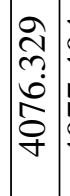 & 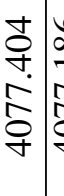 & 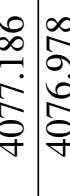 & 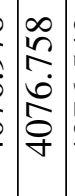 & 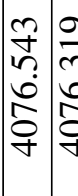 & 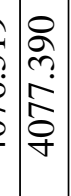 & 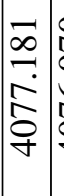 & 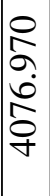 \\
\hline 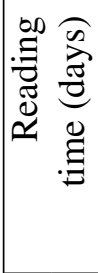 & 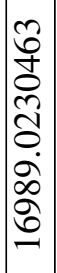 & $\begin{array}{l}n \\
0 \\
0 \\
\infty \\
0 \\
0 \\
\dot{0} \\
0 \\
0 \\
0\end{array}$ & 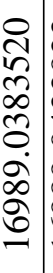 & 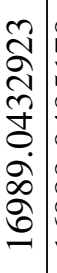 & 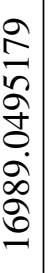 & 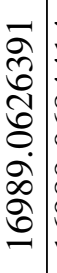 & 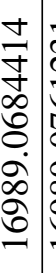 & 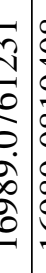 & 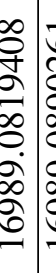 & 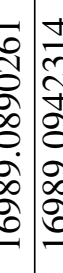 & 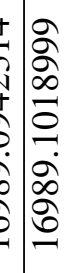 & 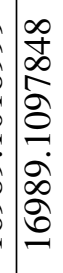 & 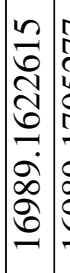 & 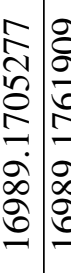 & 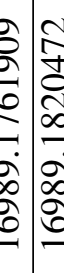 & 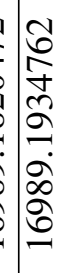 & 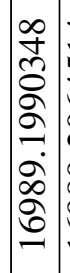 & 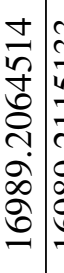 & 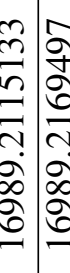 & 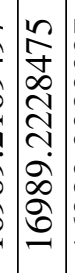 & 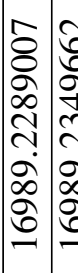 & 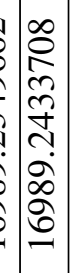 & 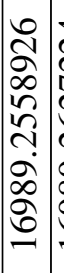 & 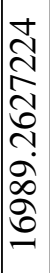 \\
\hline 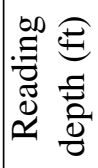 & \begin{tabular}{|l|}
8 \\
8 \\
8 \\
$\dot{0}$ \\
$\infty$
\end{tabular} & $\begin{array}{l}8 \\
\varnothing \\
\check{\delta} \\
\check{n} \\
\infty\end{array}$ & $\begin{array}{l}8 \\
\delta \\
\dot{\delta} \\
\dot{\infty}\end{array}$ & $\begin{array}{l}8 \\
\varnothing \\
0 \\
\dot{8} \\
\infty\end{array}$ & $\begin{array}{l}8 \\
\varnothing \\
\varnothing \\
\dot{\circ} \\
\infty \\
\infty\end{array}$ & $\begin{array}{l}8 \\
\delta \\
\dot{0} \\
\check{n} \\
\infty\end{array}$ & \begin{tabular}{l|l}
8 & \\
$\delta$ & \\
$\dot{\varphi}$ & \\
+ &
\end{tabular} & 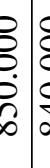 & 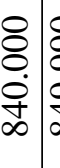 & 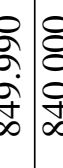 & 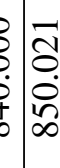 & $\begin{array}{l}8 \\
8 \\
0 \\
\dot{f} \\
\infty\end{array}$ & \begin{tabular}{l}
8 \\
8 \\
$\dot{8}$ \\
\multirow{+}{0}{}
\end{tabular} & 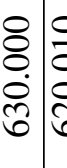 & 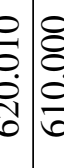 & 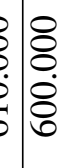 & $\begin{array}{l}\infty \\
\infty \\
2 \\
\infty \\
\infty \\
n\end{array}$ & 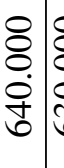 & 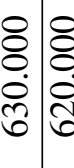 & 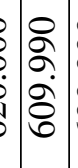 & 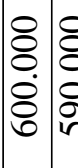 & 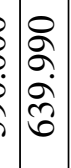 & $\begin{array}{c}0 \\
0 \\
0 \\
\dot{0} \\
0 \\
0\end{array}$ & 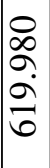 \\
\hline$\cong 0$ & $\nabla$ & $\tau$ & $\tau$ & $\tau$ & $\nabla$ & $\nabla$ & ठ & ठ & $\sigma$ & $\tau$ & 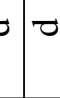 & $\nabla$ & D & $\sigma \tau$ & $\sigma$ & $\sigma$ & 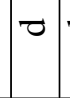 & $\sigma$ & 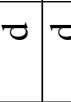 & $\sigma$ & $\sigma=$ & $\nabla$ & $\sigma$ & $\sigma$ \\
\hline 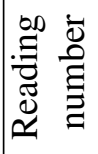 & a & 2 & n & ๙๐ & $a$ & 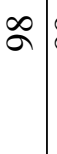 & ন & $\underline{B}$ & 으 & $\hat{b} \underline{c}$ & $\stackrel{t}{O}$ & $\stackrel{n}{0}$ & 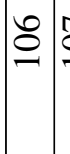 & $\hat{0}$ & $\stackrel{8}{0}$ & 을 & $\equiv$ & $\cong \cong$ & $\stackrel{n}{=}$ & $\cong$ & $\stackrel{0}{=}$ & $\stackrel{\infty}{=}$ & $\Xi$ & 고 \\
\hline
\end{tabular}




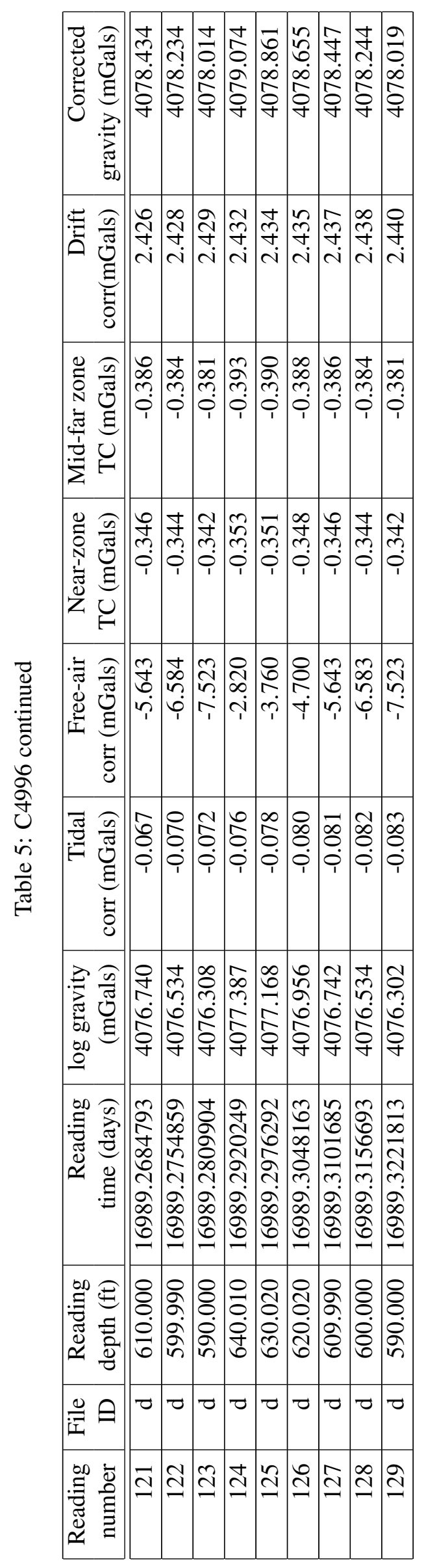


A.3 C4997 


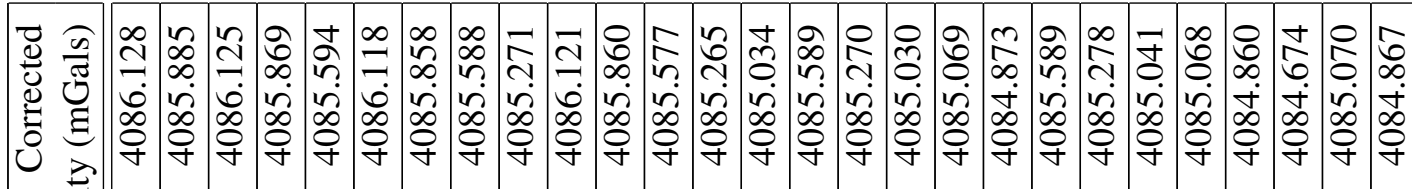
$\sum_{\bar{\pi}}$

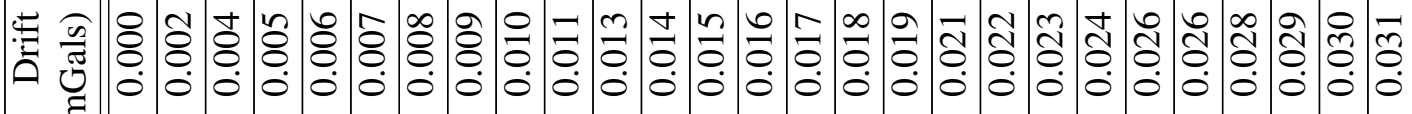
ठั

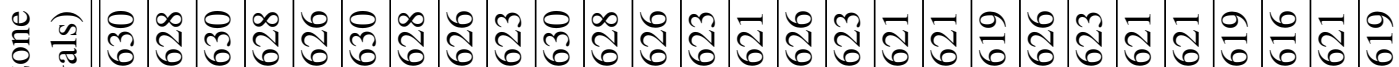

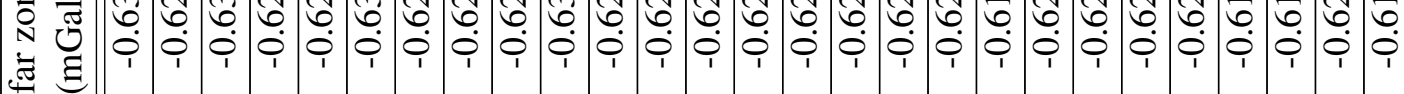

$\underset{\sim}{2}$

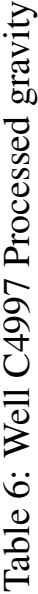

O A N A

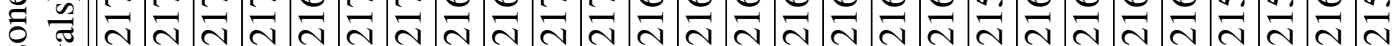

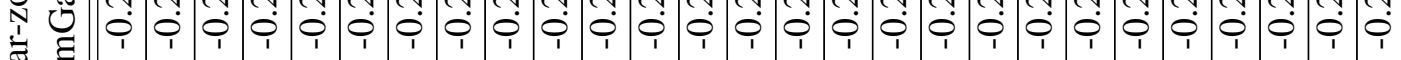
承

Z

$\because$ ‘ ส 离

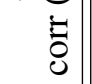

శี

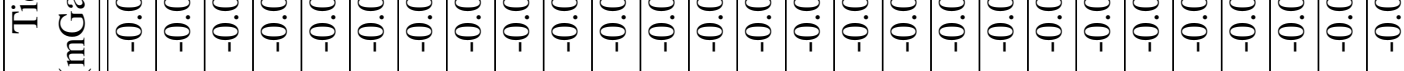
¿ับ

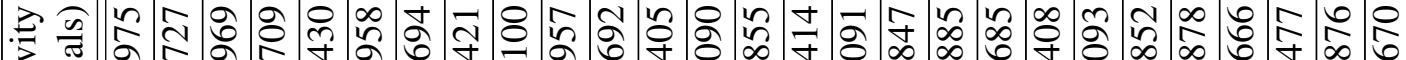

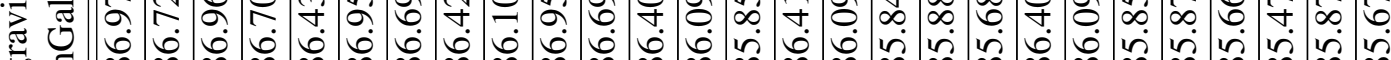

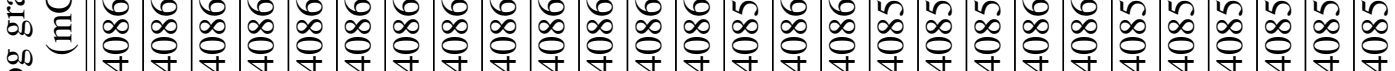
잉

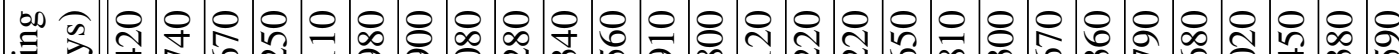

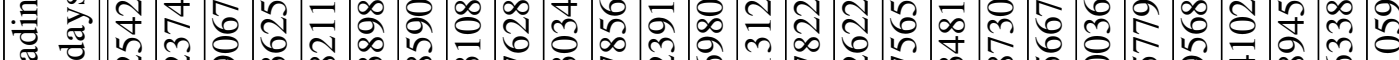

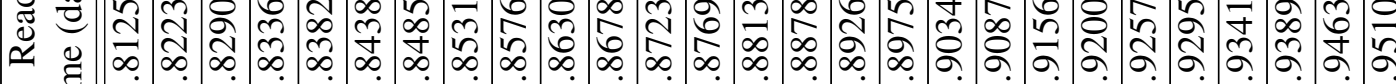

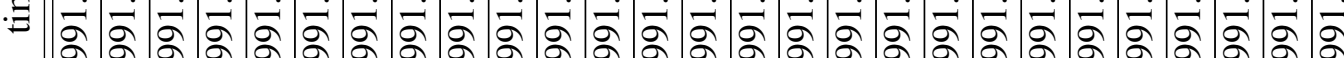

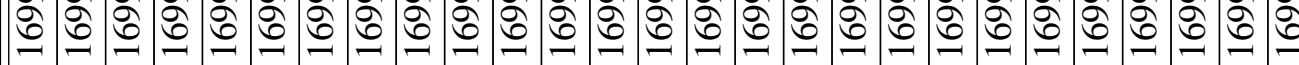

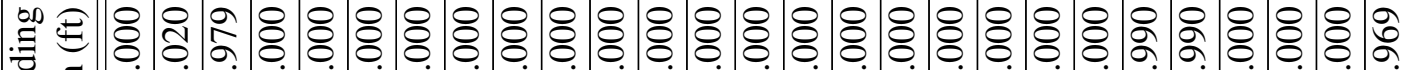
ஓ

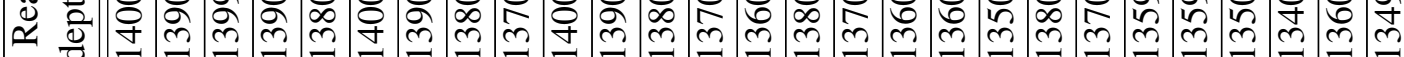

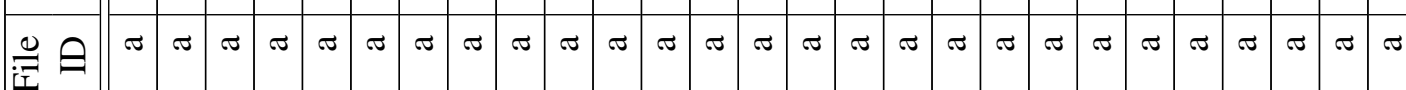

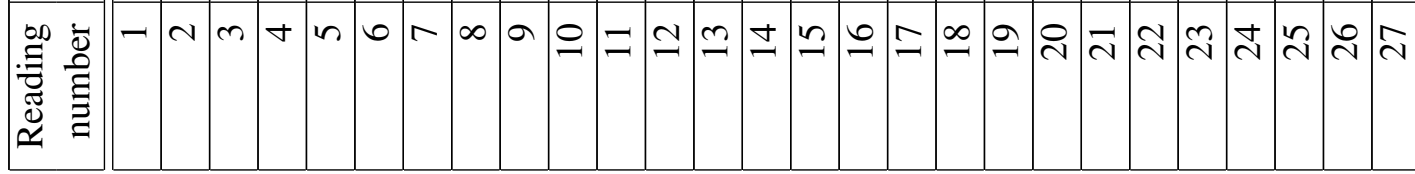




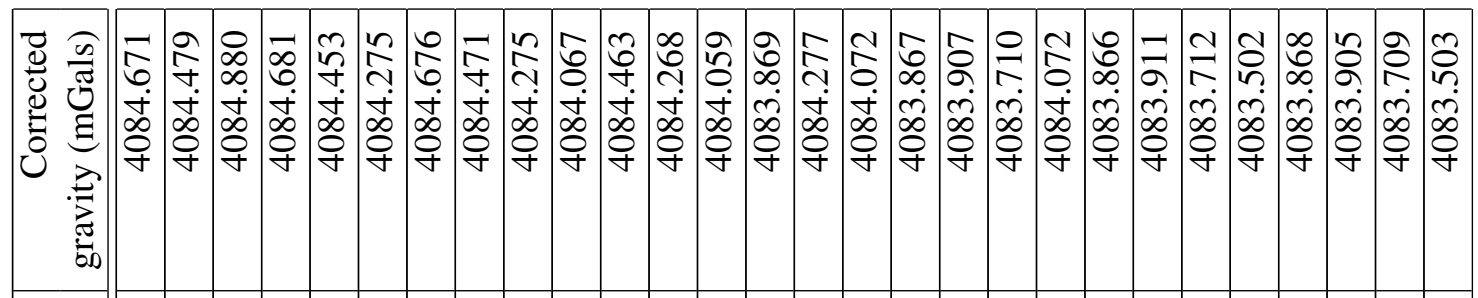

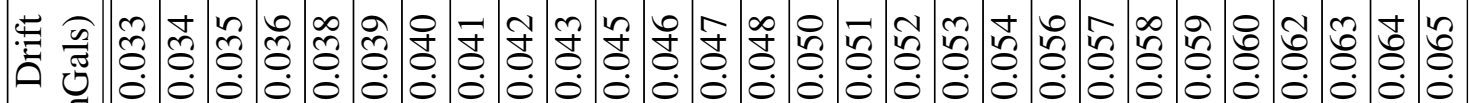
ठํํㄹ

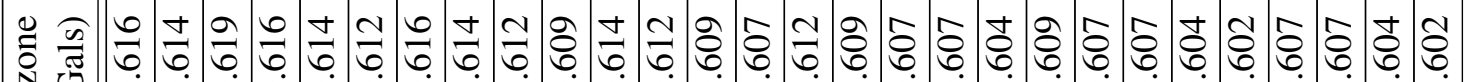

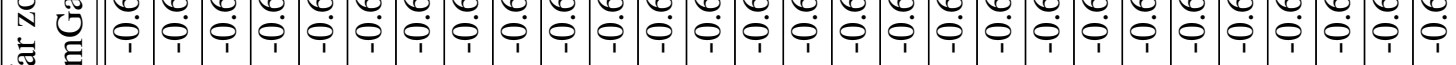
究

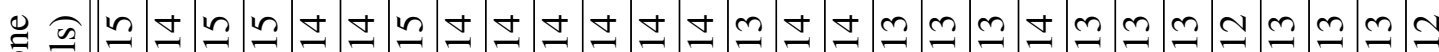

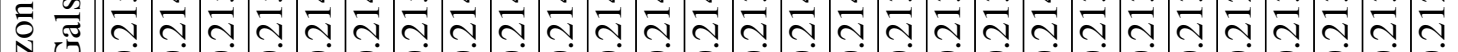

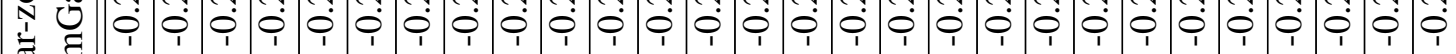
ฮ $\mathrm{Z}$,

\begin{tabular}{|c|c|c|c|c|c|c|c|c|c|c|c|c|c|c|c|c|c|c|c|c|c|c|c|c|}
\hline 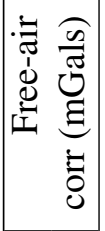 & กิ & & & & & \begin{tabular}{l}
$\tilde{m}$ \\
\multirow{+}{*}{} \\
$\dot{n}$
\end{tabular} & 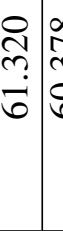 & & 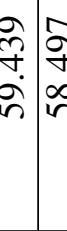 & $\begin{array}{l}\infty \\
\infty \\
\delta\end{array}$ & $\begin{array}{l}m \\
\stackrel{m}{8} \\
\dot{n}\end{array}$ & & & 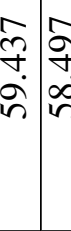 & $\begin{array}{l}n \\
n \\
n \\
n\end{array}$ & 㔯 & & $\tilde{n}$ & $\begin{array}{l}n \\
n \\
n \\
n\end{array}$ & & & $\mid \begin{array}{l}n \\
n \\
n \\
n\end{array}$ & $\mid \begin{array}{l}0 \\
0 \\
0 \\
0 \\
i \\
n\end{array}$ & $\begin{array}{l}0 \\
i n \\
n\end{array}$ \\
\hline 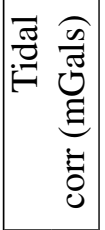 & $\begin{array}{l}0 \\
0 \\
0 \\
0 \\
1 \\
1\end{array}$ & 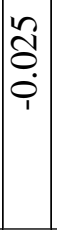 & $\overbrace{}^{c}$ & $\begin{array}{l}\tilde{N} \\
0 \\
0 \\
1\end{array}$ & $\begin{array}{l}\overline{\widehat{D}} \\
0 \\
\dot{0}\end{array}$ & 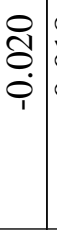 & 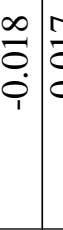 & & 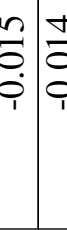 & $\stackrel{1}{\stackrel{1}{0}}$ & $\begin{array}{l}\overline{0} \\
0 \\
0 \\
1\end{array}$ & $\begin{array}{l}0 \\
8 \\
0 \\
0 \\
1\end{array}$ & 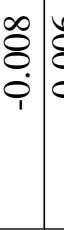 & 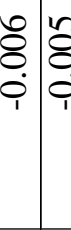 & $\begin{array}{l}1 \\
0 \\
0 \\
0\end{array}$ & $\begin{array}{l}0 \\
8 \\
0 \\
0 \\
1\end{array}$ & & $\begin{array}{l} \\
\end{array}$ & $\mid \begin{array}{l}n \\
\delta \\
0 \\
0\end{array}$ & $\underset{8}{0}$ & & $\mid \begin{array}{l}8 \\
8 \\
0 \\
0\end{array}$ & $\mid \begin{array}{l}8 \\
\vdots \\
0 \\
0\end{array}$ & $\stackrel{1}{8}$ \\
\hline 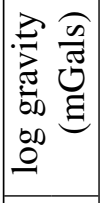 & 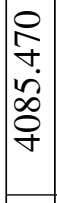 & 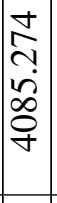 & 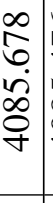 & 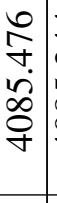 & & $\begin{array}{l}\mathcal{C} \\
\delta \\
0 \\
\dot{\infty} \\
\infty \\
0 \\
+\end{array}$ & 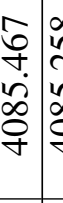 & & 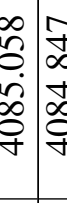 & $:$ & $\begin{array}{l}\infty \\
0 \\
0 \\
\dot{0} \\
0 \\
0 \\
+\end{array}$ & $\begin{array}{l}n \\
\tilde{\infty} \\
\infty \\
\dot{+} \\
\infty \\
\dot{q}\end{array}$ & 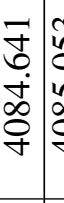 & 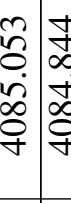 & $\begin{array}{l}n \\
\hat{6} \\
\dot{+} \\
0 \\
o\end{array}$ & 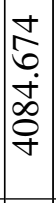 & & 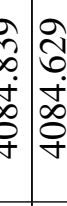 & $\begin{array}{l}n \\
\tilde{b} \\
\dot{+} \\
\infty \\
o \\
\dot{q}\end{array}$ & 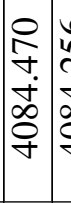 & 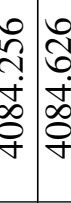 & 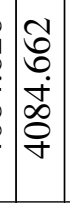 & 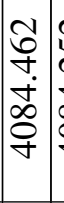 & 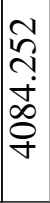 \\
\hline 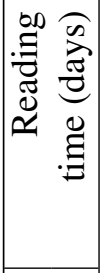 & 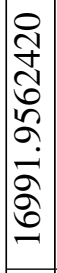 & 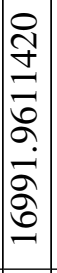 & 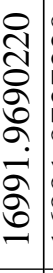 & 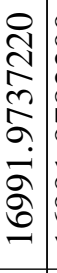 & 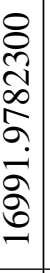 & 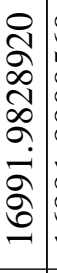 & 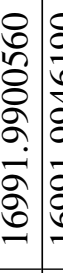 & 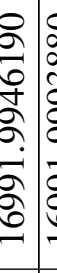 & 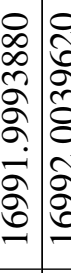 & 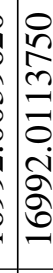 & $\begin{array}{l}8 \\
0 \\
0 \\
0 \\
0 \\
0 \\
i \\
\alpha \\
\delta \\
0 \\
-\end{array}$ & 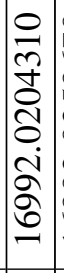 & 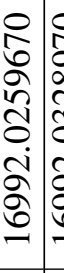 & 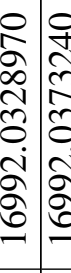 & 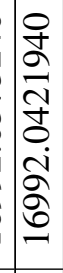 & 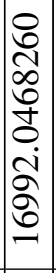 & 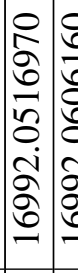 & 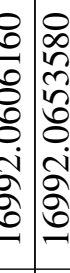 & 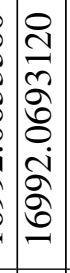 & 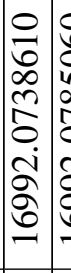 & 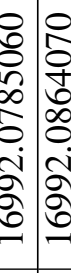 & 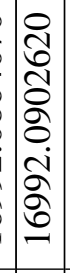 & 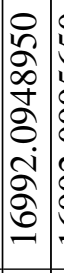 & 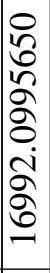 \\
\hline 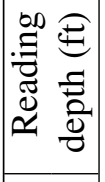 & $\begin{array}{l}\overrightarrow{2} \\
0 \\
\dot{0} \\
\dot{0} \\
- \\
\end{array}$ & $\begin{array}{l}8 \\
8 \\
0 \\
0 \\
\infty \\
\\
-\end{array}$ & 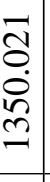 & $\begin{array}{l}8 \\
8 \\
\dot{0} \\
+ \\
0 \\
\end{array}$ & $\begin{array}{l}8 \\
8 \\
\dot{0} \\
m \\
m \\
\end{array}$ & 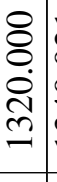 & 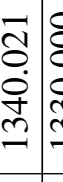 & 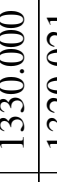 & 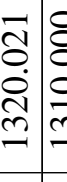 & 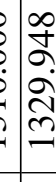 & $\begin{array}{l}8 \\
0 \\
0 \\
0 \\
2 \\
-1\end{array}$ & $\begin{array}{l}8 \\
8 \\
0 \\
0 \\
\\
\end{array}$ & $\begin{array}{l}8 \\
8 \\
\dot{8} \\
8 \\
\end{array}$ & 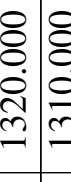 & 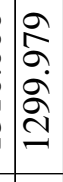 & $\begin{array}{l}2 \\
\hat{a} \\
2 \\
2 \\
-1\end{array}$ & 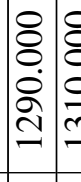 & 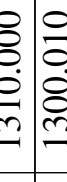 & 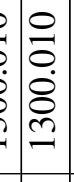 & 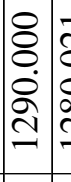 & 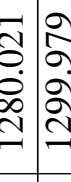 & $\hat{i}$ & 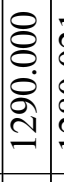 & 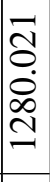 \\
\hline & $\sigma$ & $\pi$ & $\sigma$ & $\pi$ & $\sigma$ & $\pi$ & $\sigma$ & $\sigma$ & $\sigma \mid$ & $\theta$ & $\pi$ & $\approx$ & $\sigma$ & \begin{tabular}{l|l}
$\sigma$ & $\tau$
\end{tabular} & $\pi$ & $\pi$ & $\sigma$ & $\sigma \mid \nabla$ & $\sigma \approx$ & $\sigma$ & \begin{tabular}{l|l}
$\sigma$ & $\sigma$
\end{tabular} & 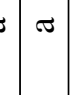 & $\approx$ & $\approx$ \\
\hline 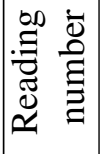 & $\stackrel{\infty}{\sim}$ & શે & లి & $\bar{m}$ & $\approx$ & $m$ & ষ্ & $m$ & 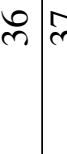 & $i \stackrel{\infty}{\infty}$ & mे & f & F & $\mathcal{F} \underset{\neg}{\mathcal{F}}$ & 寸 & $\mathscr{f}$ & $\stackrel{\circ}{+}$ & ২ & $f i n$ & $\bar{n}$ & $\tilde{n} n$ & in & $\approx n$ & 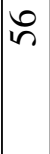 \\
\hline
\end{tabular}




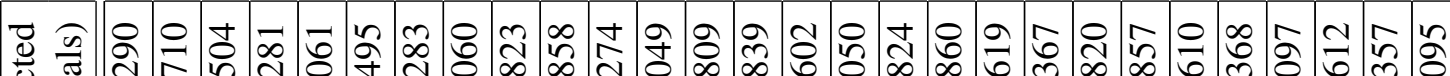
U.

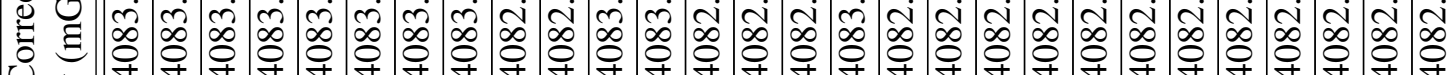

氙 A

节 节

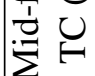

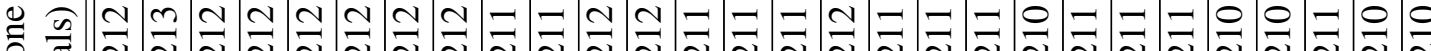

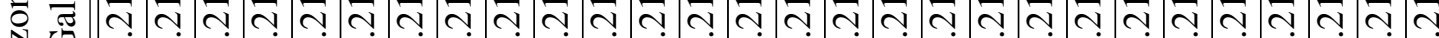

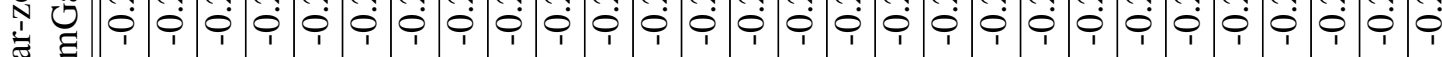
ซึ

$\mathrm{Z} \cup$

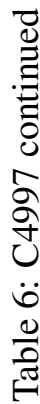

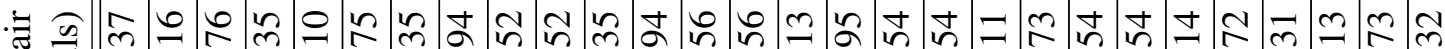

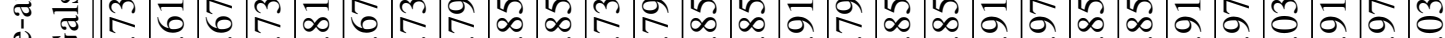

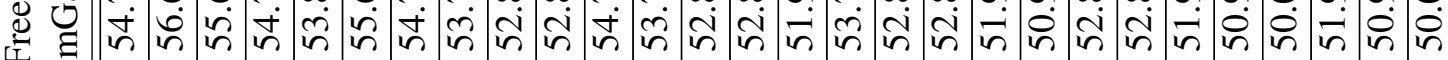

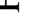

8

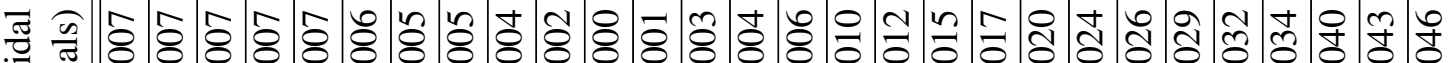

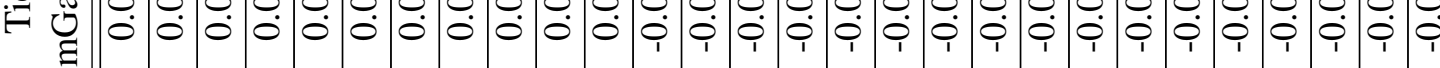

ठ

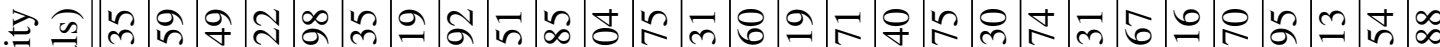

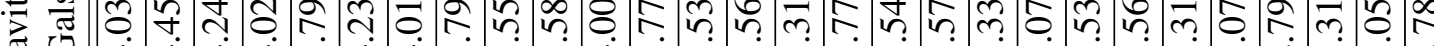

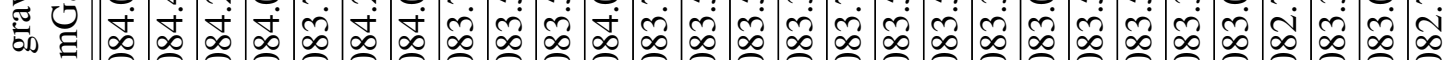

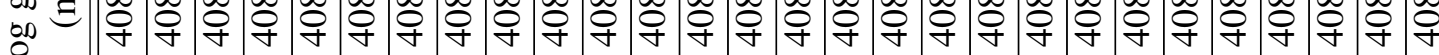

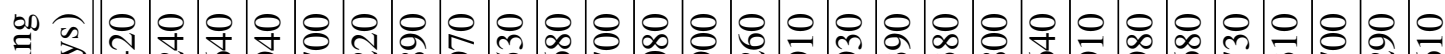

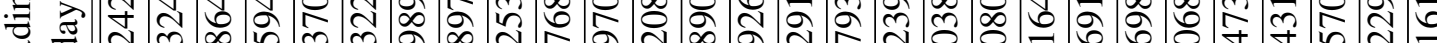

ש

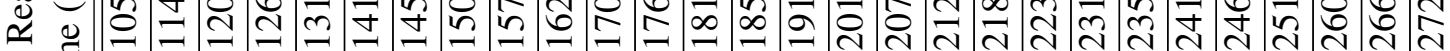

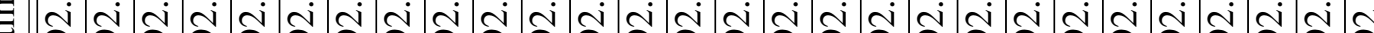

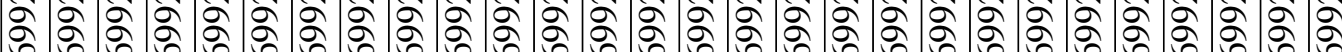

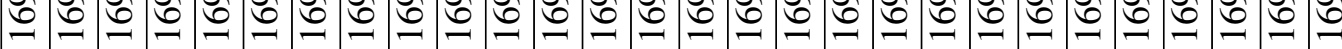

. च च

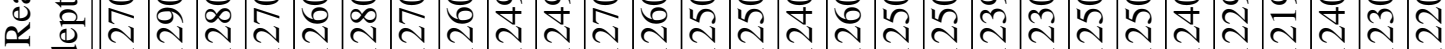

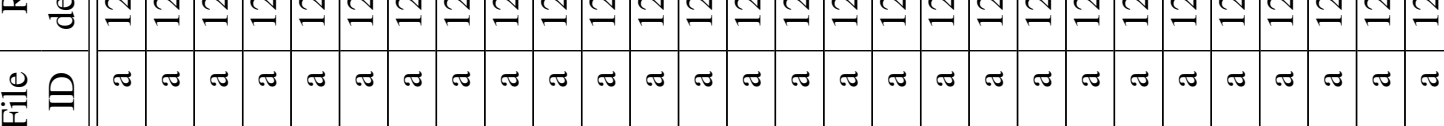

䑻 I 


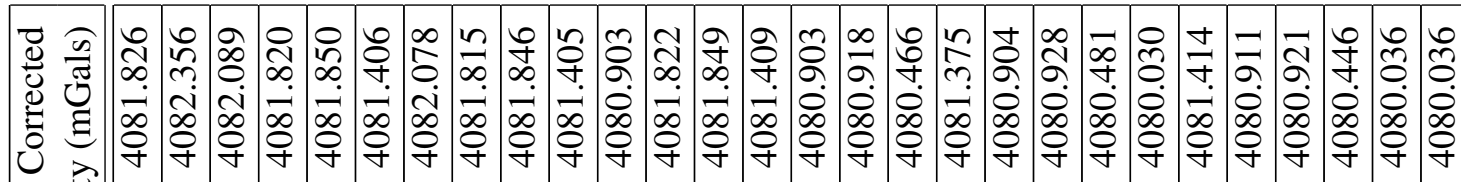

言

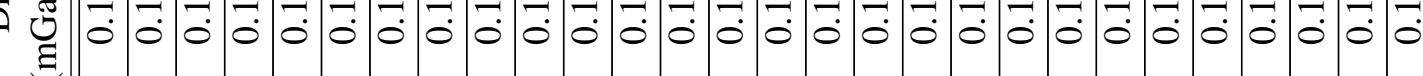

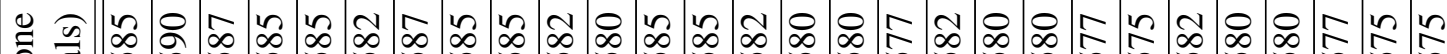

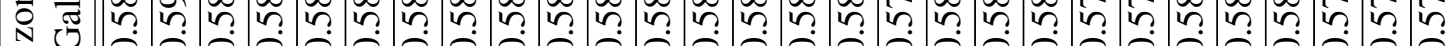
Z E $\sum_{2}^{\prime}$

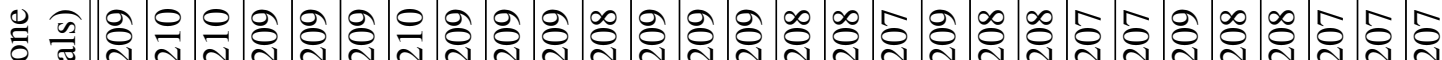

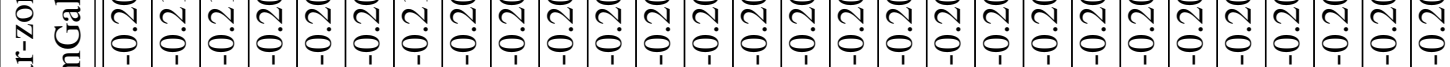
㓂

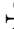

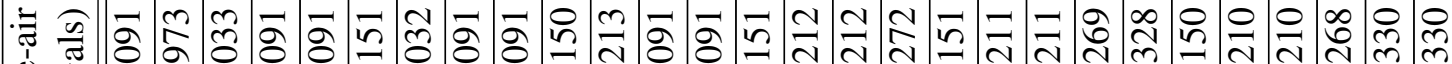
我 空 8

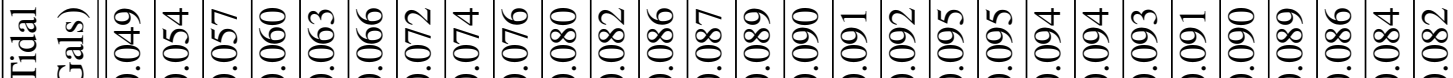

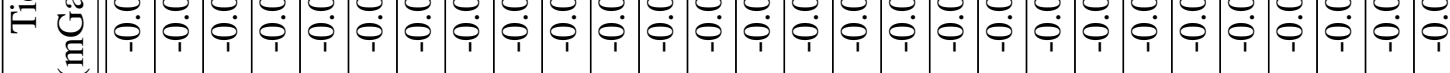
ठํ.

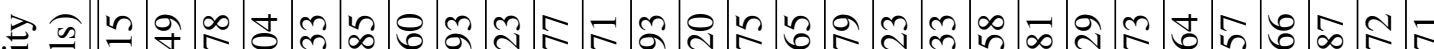

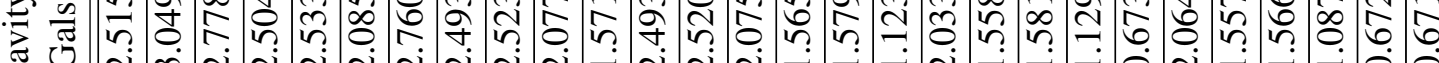

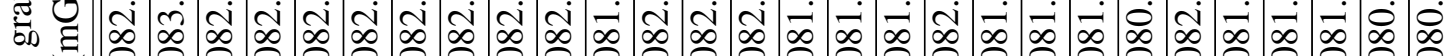

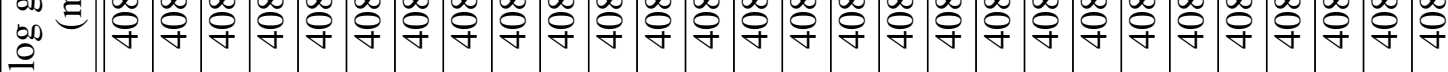

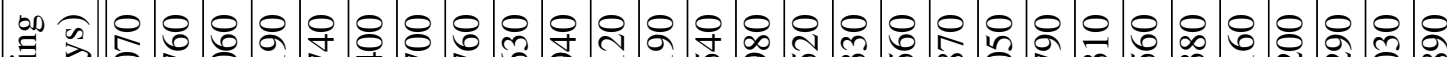

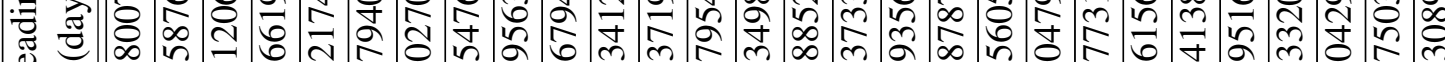
$\approx$ ¿ Đ

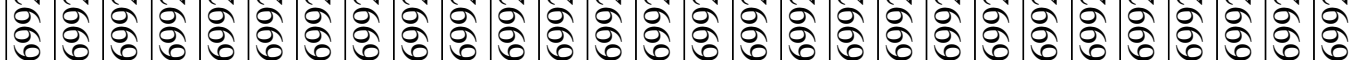

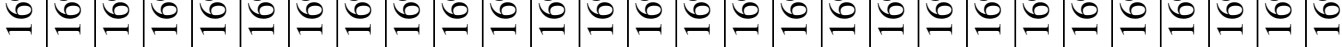

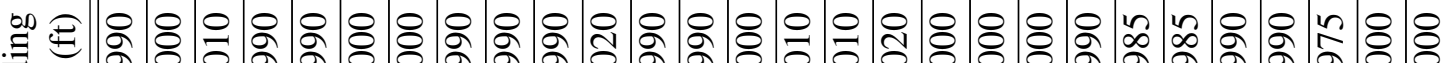
范

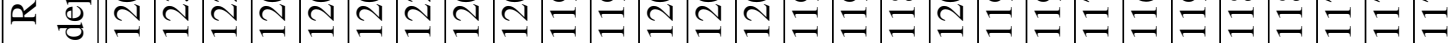
重司

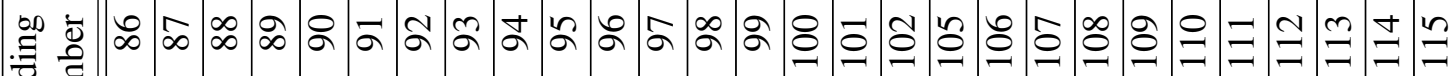
ซ్ 


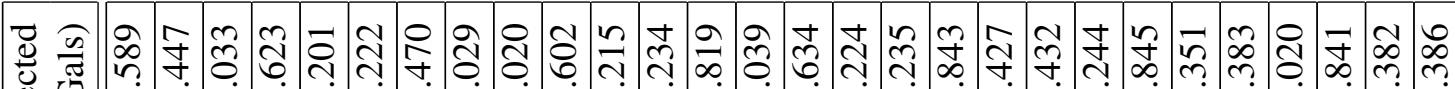
过

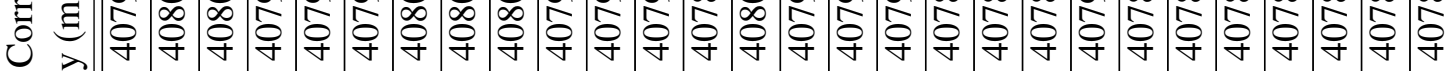
:

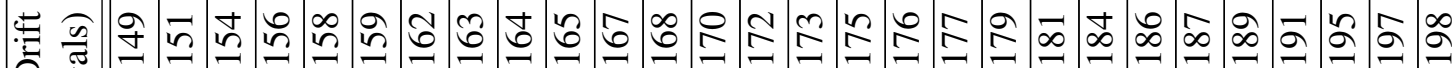

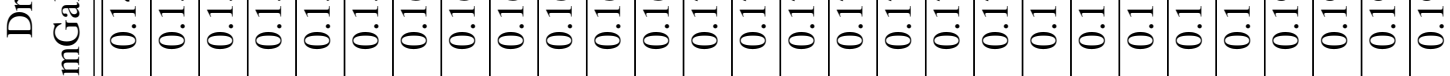
ठำ

mans? 远 : 西

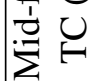

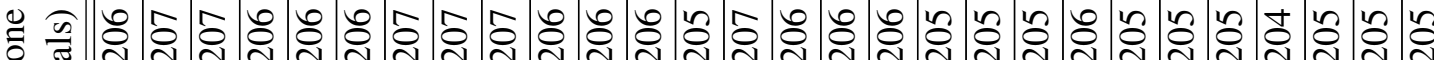

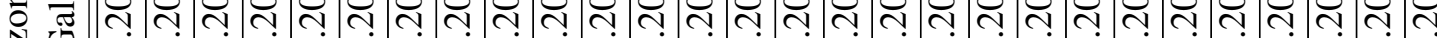
告 Ð 乙 U

등

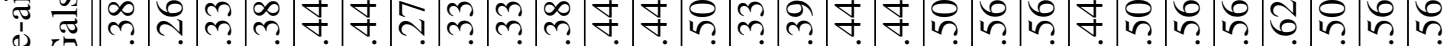

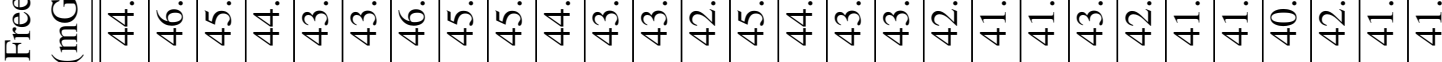
:

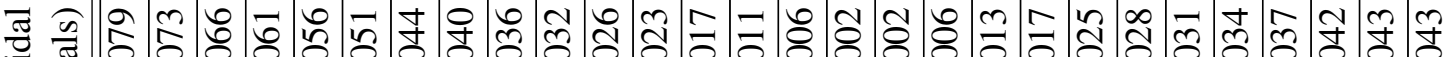
蛋 อั

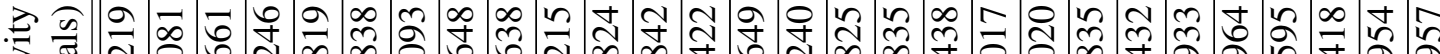

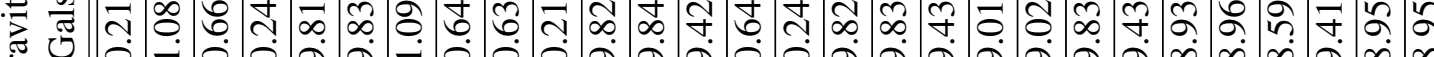

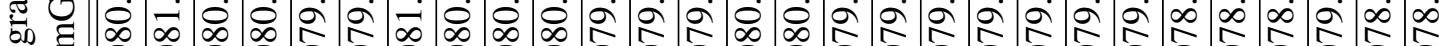

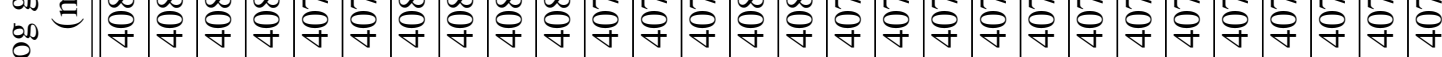

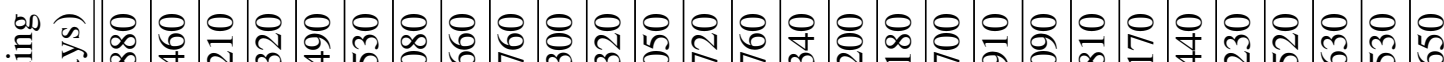

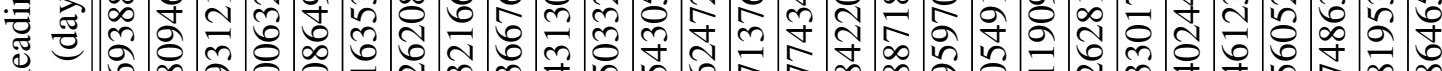

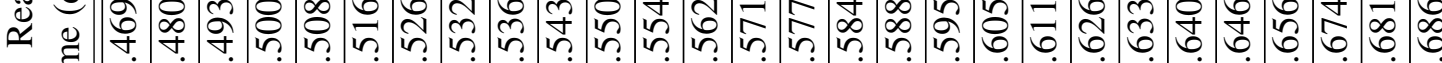

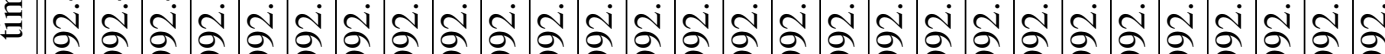

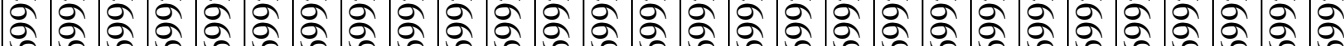

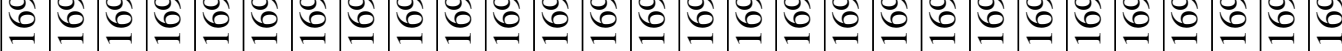

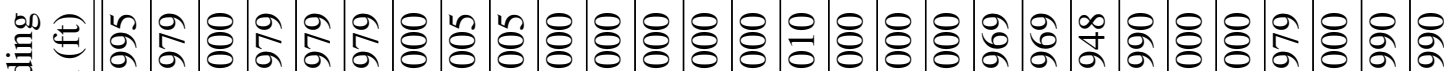
釆

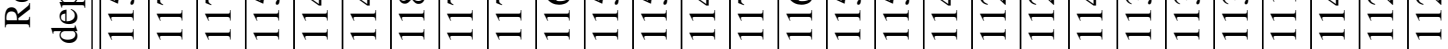

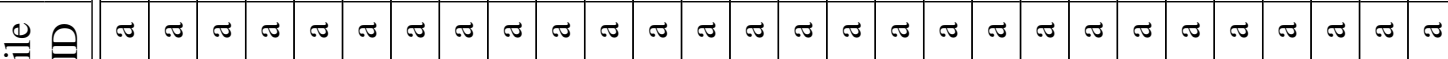

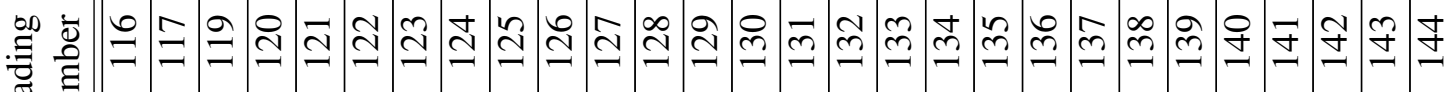
ชี 


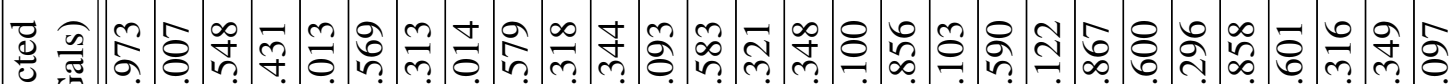

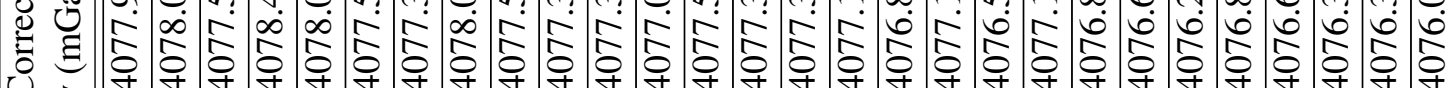
U 夠

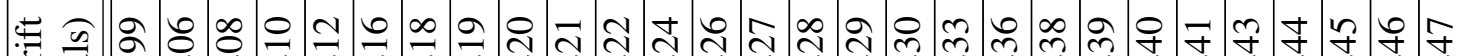
जี

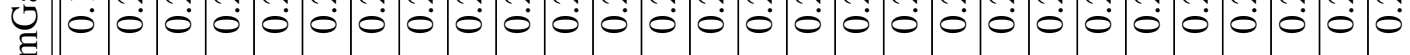
:

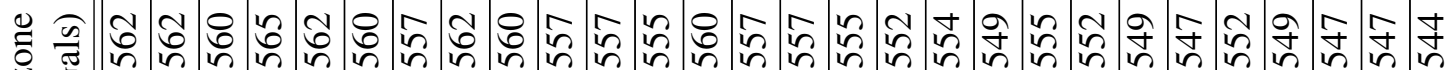

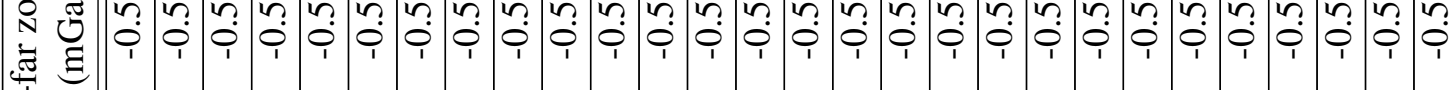

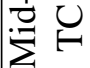

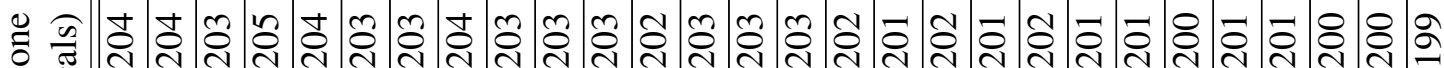

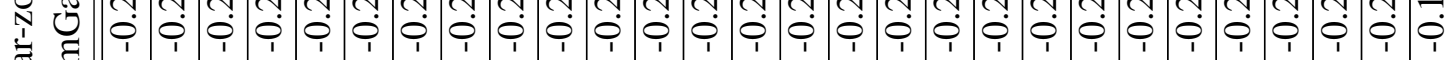
ซี Z

\begin{tabular}{|c|c|c|c|c|c|c|c|c|c|c|c|c|c|c|c|c|c|c|c|c|c|c|c|c|}
\hline 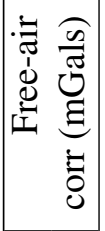 & $\begin{array}{l}\text { î } \\
\dot{0} \\
\dot{q}\end{array}$ & & ले & $\begin{array}{l}0 \\
0 \\
n \\
\dot{\sigma} \\
\end{array}$ & fo & 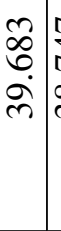 & & & 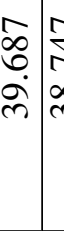 & & $\stackrel{\infty}{\infty}$ & $\begin{array}{l}\infty \\
0 \\
\dot{m} \\
\infty\end{array}$ & 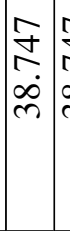 & 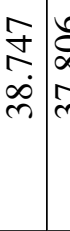 & 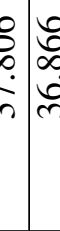 & $\stackrel{\substack{\infty \\
\hdashline}}{\infty}$ & 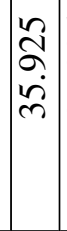 & 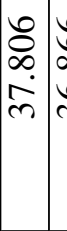 & $\begin{array}{l}0 \\
\infty \\
0 \\
0 \\
ల\end{array}$ & $\begin{array}{l}0 \\
\dot{m} \\
\dot{m}\end{array}$ & $\begin{array}{l}0 \\
0 \\
\infty \\
\dot{0} \\
0\end{array}$ & & & \\
\hline 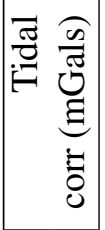 & 导 & $\begin{array}{l}\stackrel{\sim}{J} \\
\stackrel{0}{0} \\
\end{array}$ & $\begin{array}{l}\vec{J} \\
\dot{0} \\
\dot{0}\end{array}$ & 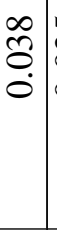 & $\begin{array}{l}n \\
\tilde{n} \\
0\end{array}$ & 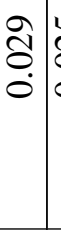 & 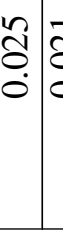 & & 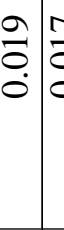 & $\frac{1}{c}$ & $\stackrel{0}{\circ}$ & : & 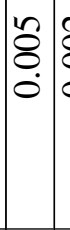 & 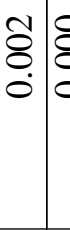 & 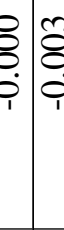 & 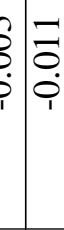 & $\mid \begin{array}{l}\infty \\
0 \\
0 \\
0 \\
1\end{array}$ & 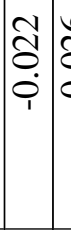 & 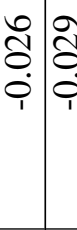 & $\begin{array}{l}\vec{r} \\
\vdots \\
\dot{\varphi} \\
1\end{array}$ & 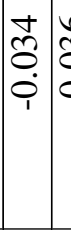 & $\begin{array}{l}0 \\
0 \\
0 \\
0 \\
0\end{array}$ & 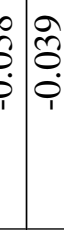 & $\begin{array}{l}\nexists \\
0 \\
0 \\
\end{array}$ \\
\hline 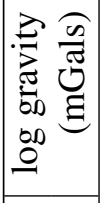 & 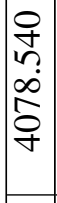 & $\begin{array}{l}\infty \\
0 \\
n \\
\infty \\
5 \\
0 \\
+\end{array}$ & 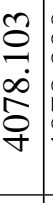 & $\begin{array}{l}8 \\
2 \\
\infty \\
\infty \\
0 \\
+\end{array}$ & & 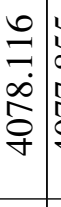 & 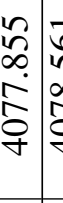 & & 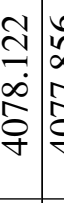 & 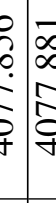 & 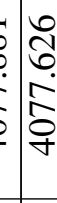 & 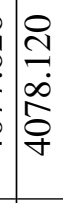 & 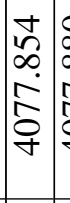 & 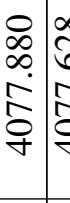 & 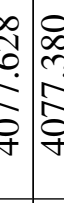 & 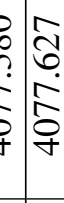 & 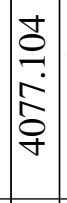 & 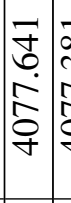 & 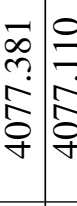 & $\begin{array}{l}\bar{\delta} \\
\infty \\
\dot{0} \\
\dot{0} \\
\dot{q}\end{array}$ & 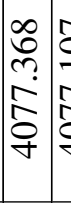 & 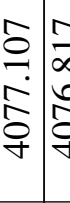 & 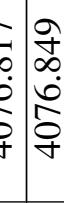 & 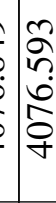 \\
\hline 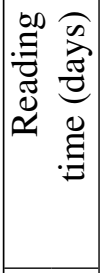 & 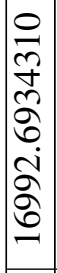 & 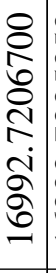 & 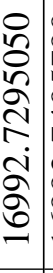 & 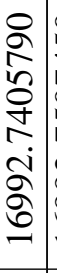 & 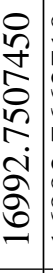 & 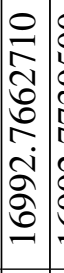 & 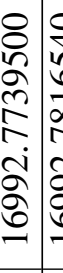 & 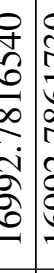 & 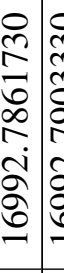 & 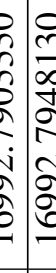 & 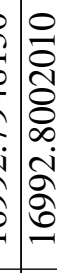 & 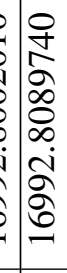 & 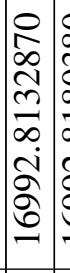 & 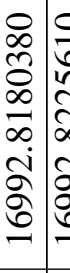 & 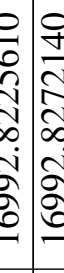 & 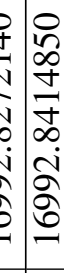 & 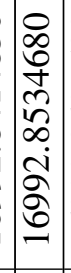 & 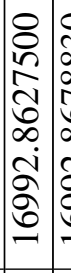 & 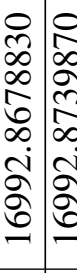 & 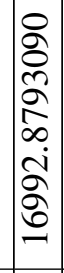 & 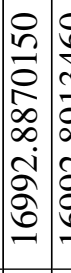 & 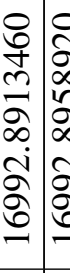 & 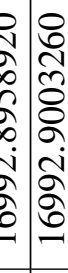 & 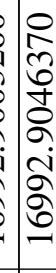 \\
\hline 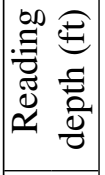 & $\begin{array}{l}a \\
\hat{a} \\
a \\
\Xi \\
=\end{array}$ & 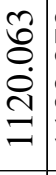 & $\begin{array}{l}\frac{a}{a} \\
\dot{a}\end{array}$ & $\begin{array}{l}a \\
\hat{\sigma} \\
\hat{\jmath} \\
=\end{array}$ & 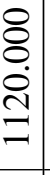 & $\begin{array}{l}\infty \\
2 \\
\alpha \\
o \\
0 \\
=\end{array}$ & 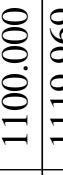 & ڤે & 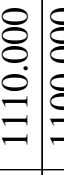 & 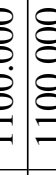 & : & $\left\{\begin{array}{l}8 \\
8 \\
0 \\
0 \\
0 \\
0 \\
0\end{array}\right.$ & $\begin{array}{l}8 \\
8 \\
\dot{8} \\
\varrho \\
\Xi\end{array}$ & 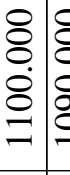 & 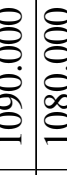 & 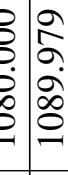 & $\begin{array}{l}8 \\
8 \\
0 \\
0 \\
0\end{array}$ & 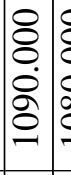 & \begin{tabular}{l|l}
8 & 8 \\
8 & 8 \\
$\dot{0}$ & 0 \\
$\circ$ & 0 \\
$\circ$ &
\end{tabular} & $\begin{array}{l}8 \\
0 \\
0 \\
0 \\
0 \\
0\end{array}$ & 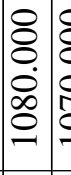 & 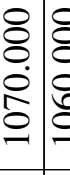 & 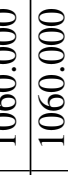 & 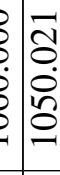 \\
\hline & $\sigma$ & $\pi$ & $\sigma$ & $\pi$ & $\approx$ & $\approx$ & $\sigma$ & $\sigma$ & $\approx$ & $\sigma \pi$ & 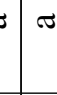 & $\tau$ & $\tau$ & $\pi$ & $\approx$ & $\pi$ & $\sigma$ & $\pi$ & $\sigma$ & $\pi$ & $\pi$ & $\sigma \mid \sigma$ & $\sigma$ & \begin{tabular}{l|l}
$\theta$ & $\sigma$
\end{tabular} \\
\hline 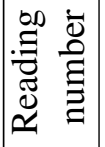 & $\stackrel{n}{ \pm}$ & $\stackrel{0}{ \pm}$ & 巳 & $\stackrel{\infty}{ \pm}$ & 导 & in & $\sqrt{2}$ & है & n & $\begin{array}{l}\text { f } \\
\text { c }\end{array}$ & $\stackrel{2}{2}$ & $n$ & $\stackrel{\infty}{\varrho}$ & is & 8 & $\underline{0}$ & $\mathfrak{6}$ & 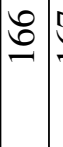 & 5 & 8 & $\stackrel{5}{=}$ & $\Xi \cong$ & $\Sigma \cong$ & $\frac{5}{2}$ \\
\hline
\end{tabular}




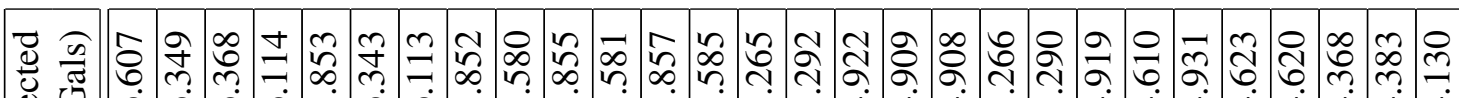

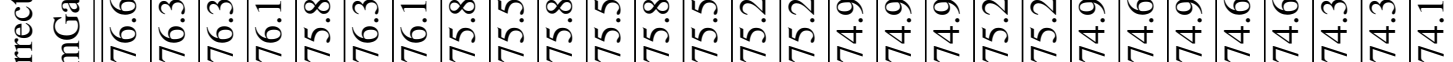

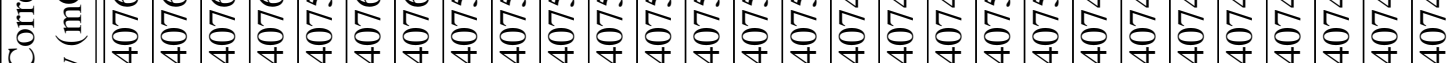

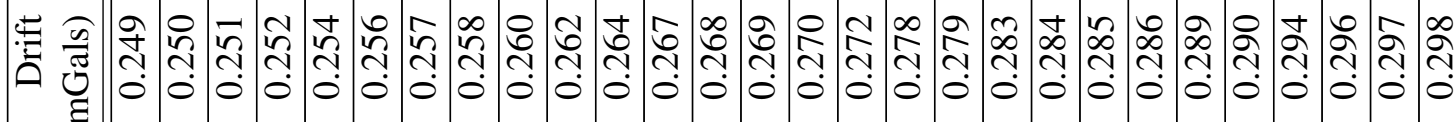
ठํํㄹ

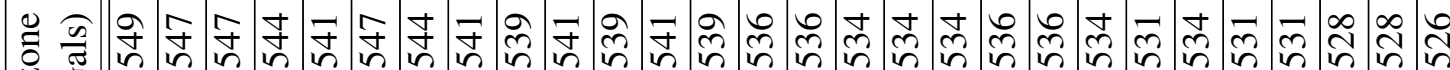

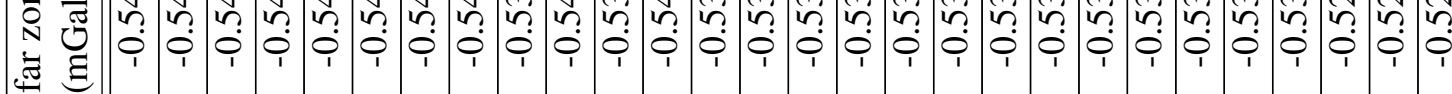

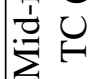

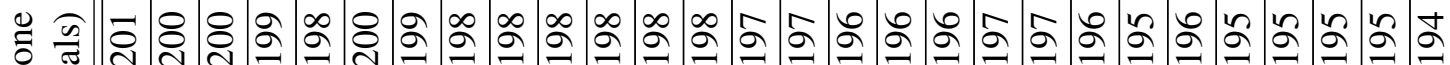
親 乙 U

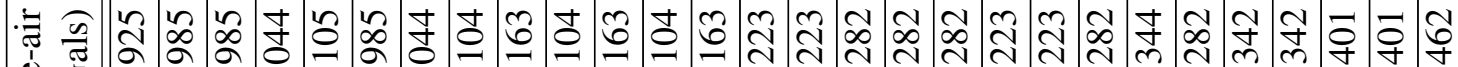
j 空 ठ․

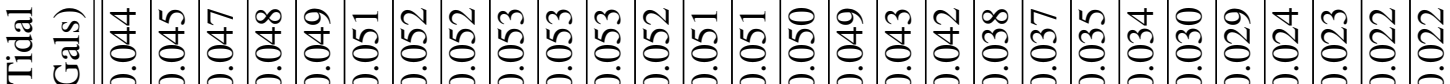

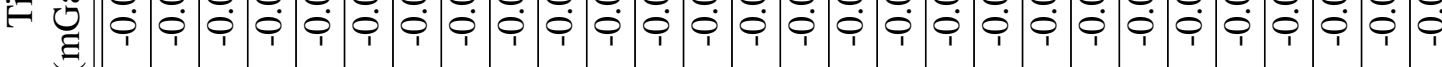
8

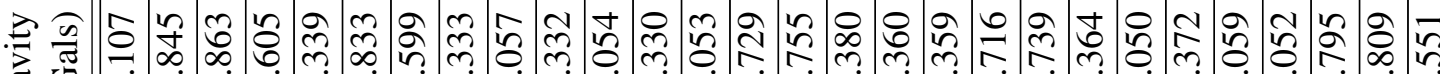

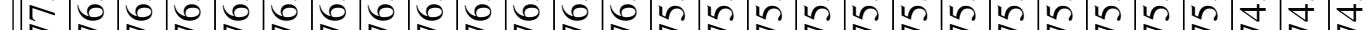

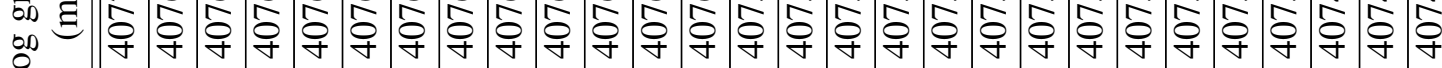

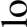

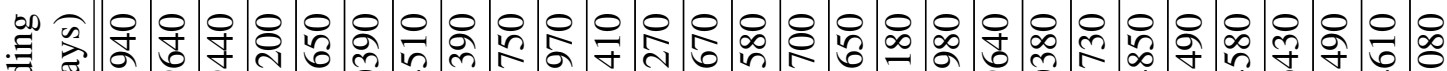

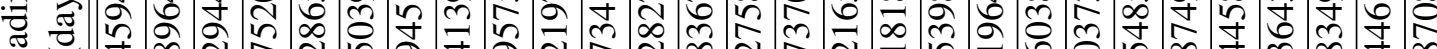
\&

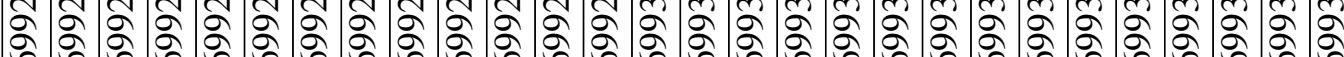

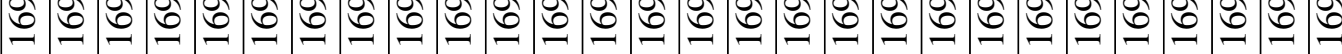

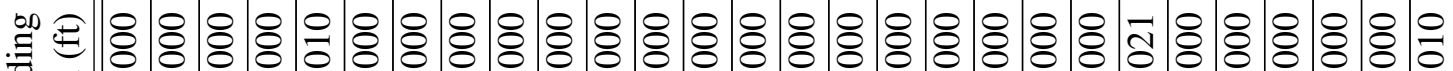

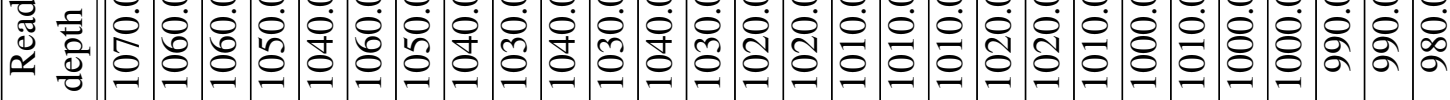
$\frac{\circ}{2}=$

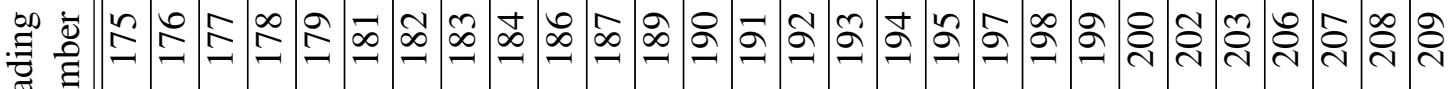
తి 
ठ d 勿

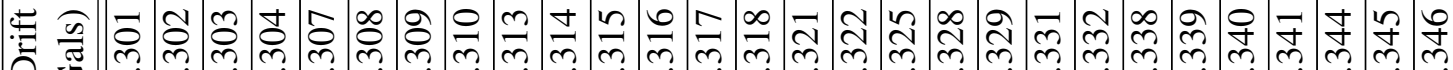

ద

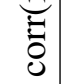

๕ 유 ‡ี $\sum$

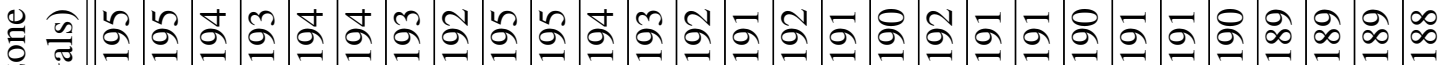
N 疋 Z

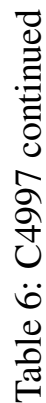

음

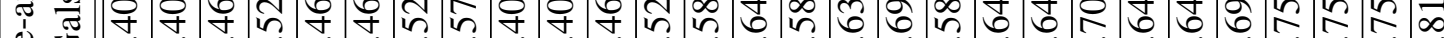

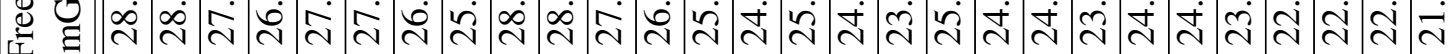

ठํ.

สี

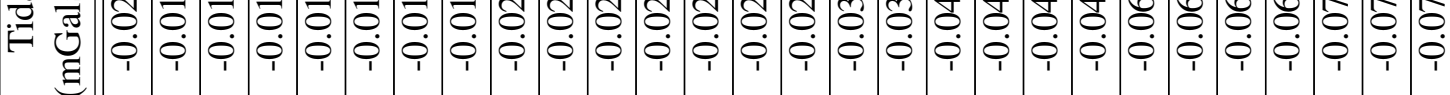
ठำ

증 च $\infty$ n đU

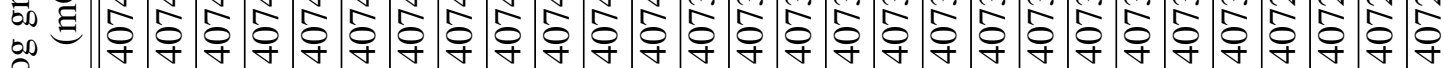
으

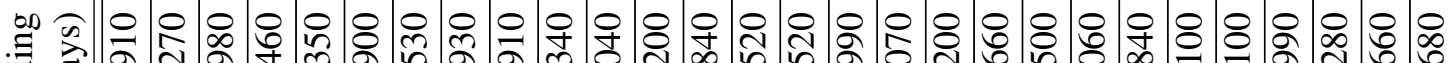

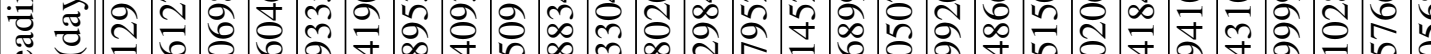

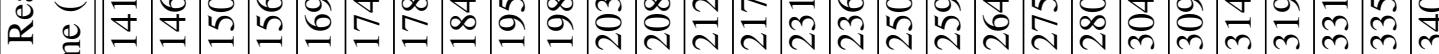

ઇ

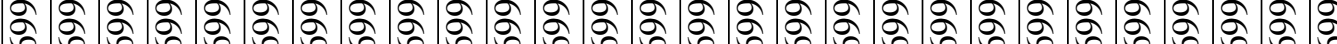
으 000 은 00

. ¿ $\stackrel{0}{\stackrel{\mathrm{i}}{\mathrm{I}}}$

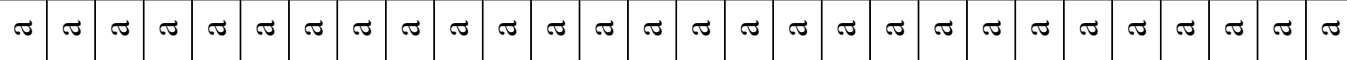

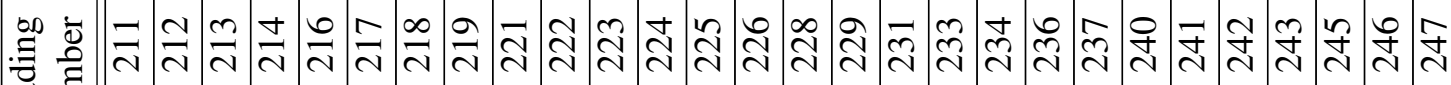
ขึ 


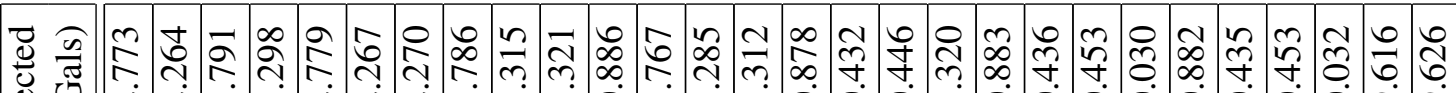
包

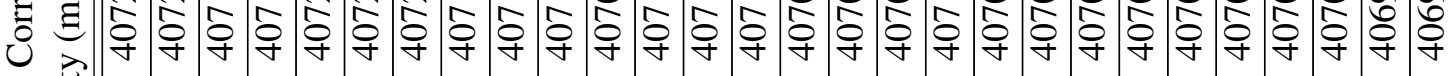
突

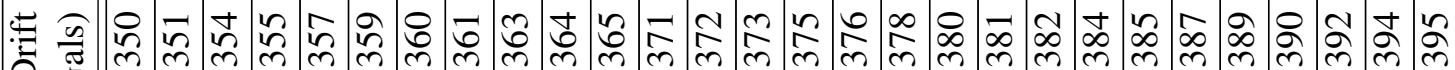
D

ठำ

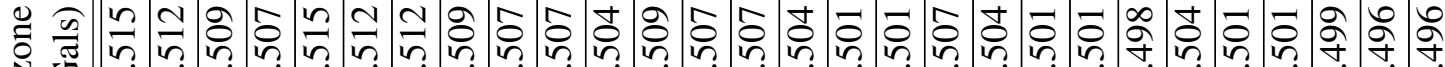

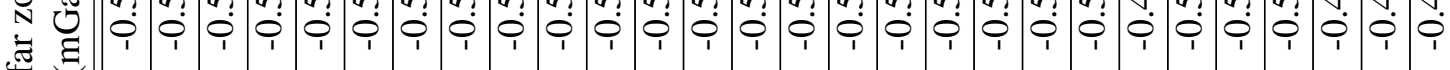
承

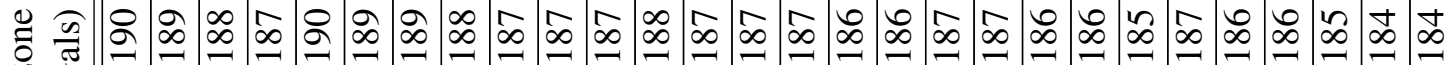

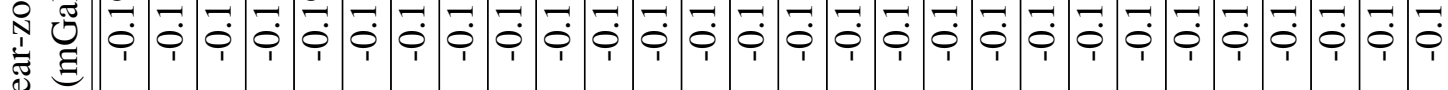
乙

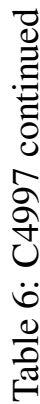

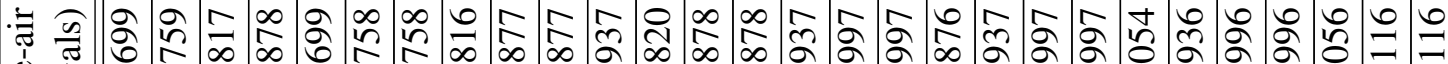
d j

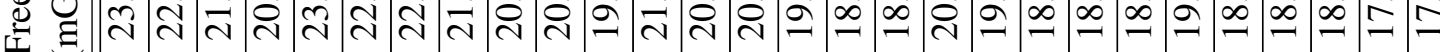

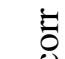

雲

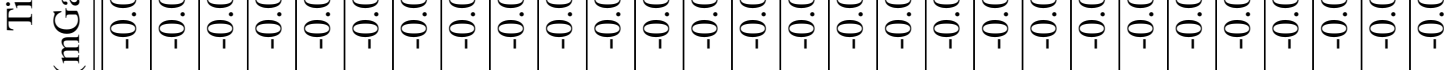

¿

. च

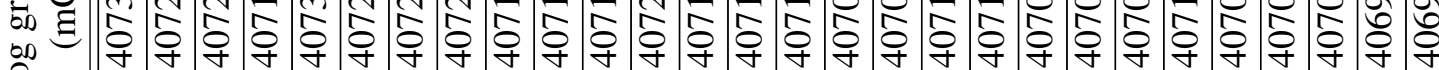
으

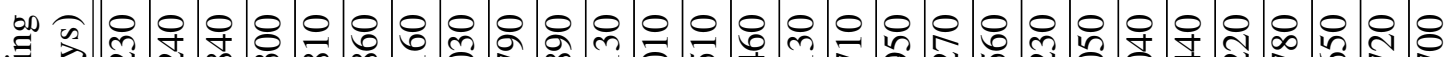
: *

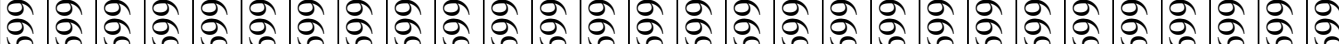

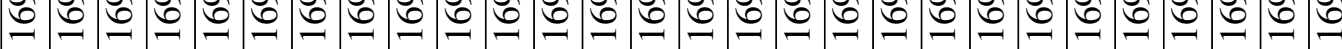

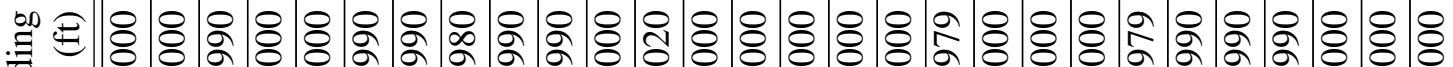
«

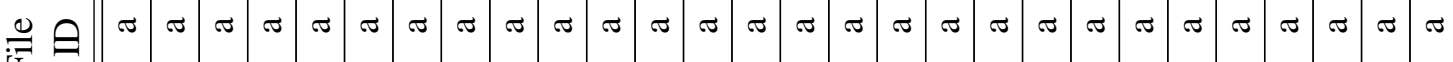
江

ob : 


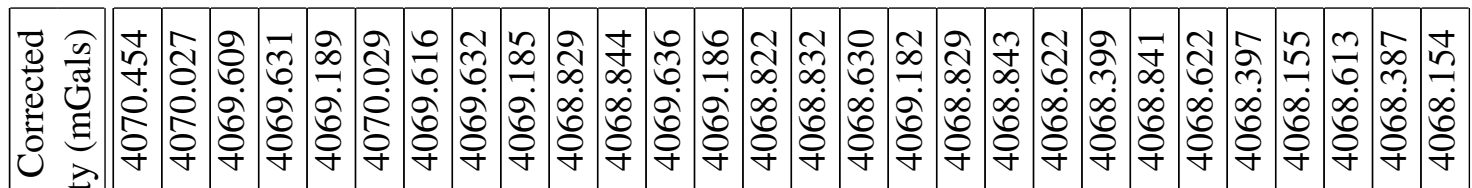
空

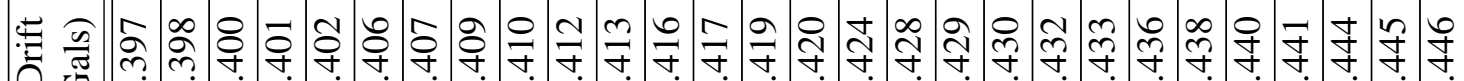
¿ ¿ֶ:

若

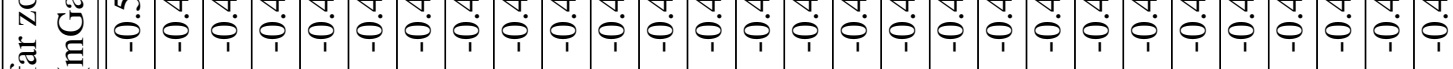
焉

च

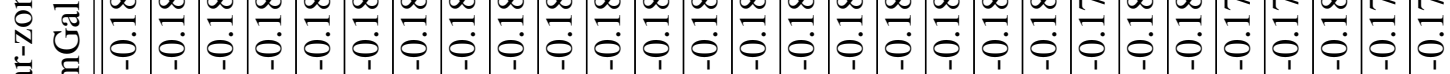
章 乙

$\exists$ ఈ

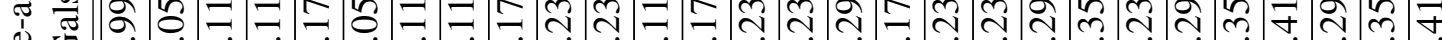
¿ 空 ठํ.

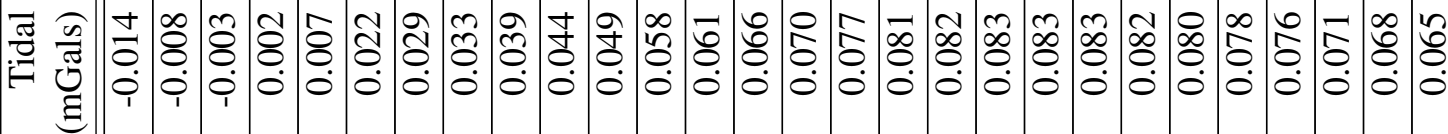
ชั 氙节 क छ श

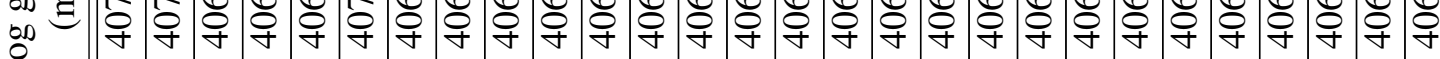
으

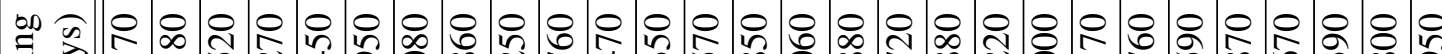

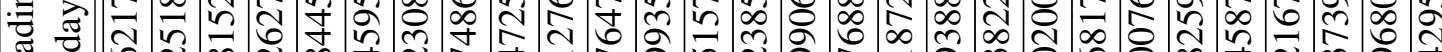
œ

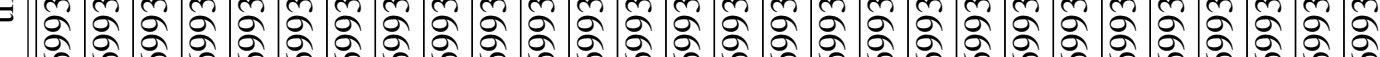

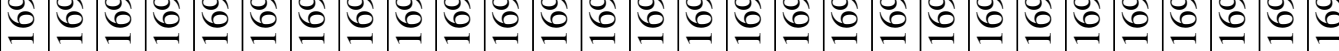

แ ‘

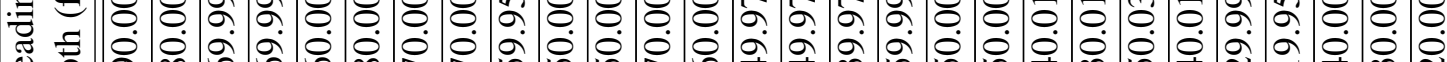
范 $\stackrel{\oplus}{\text { 空 }}$

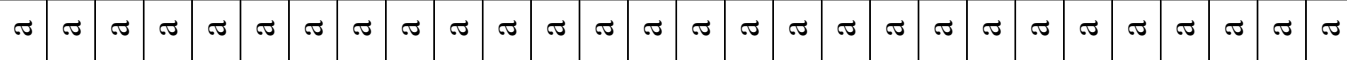

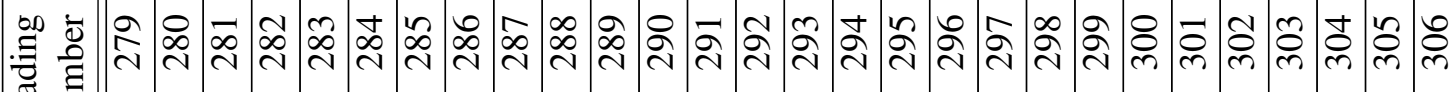
ฮั 


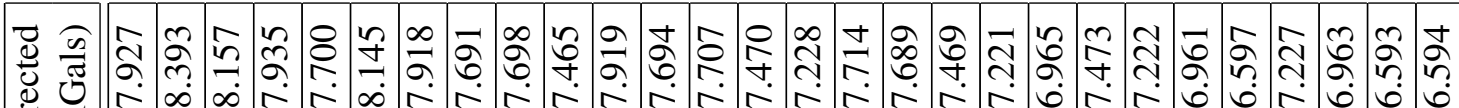
荌

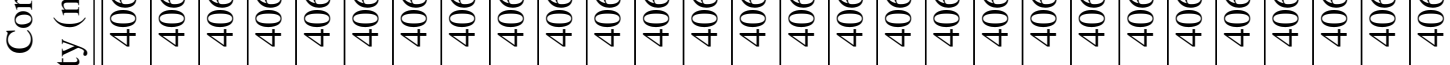
党

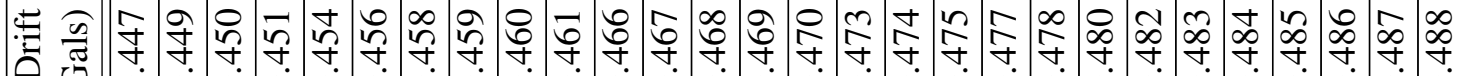

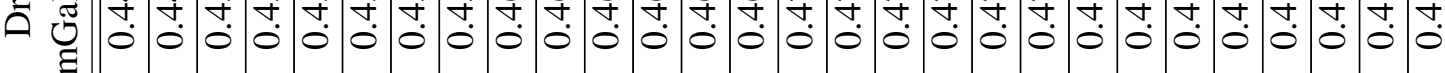
ठำ

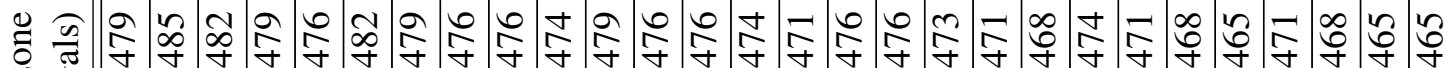

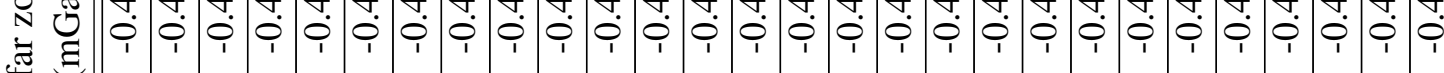

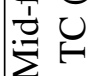

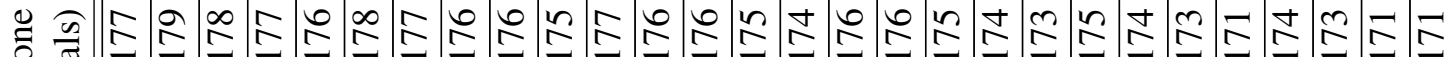

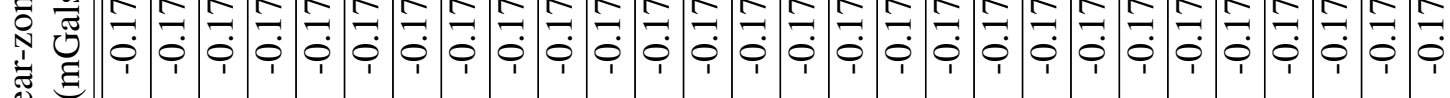
Z U

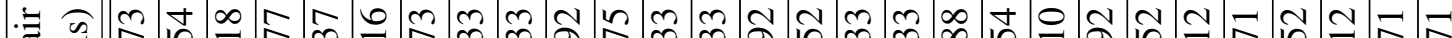

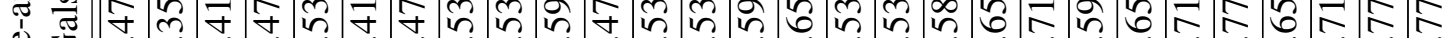

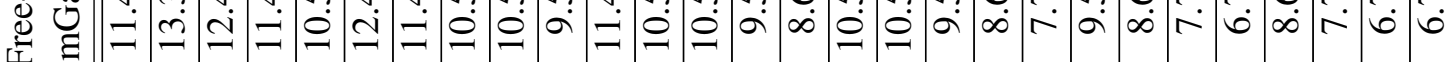

ठั

สี శิ

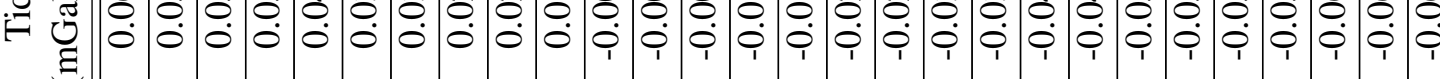
0 . का $\omega_{0} \infty \begin{aligned} & 0 \\ & 0\end{aligned}$

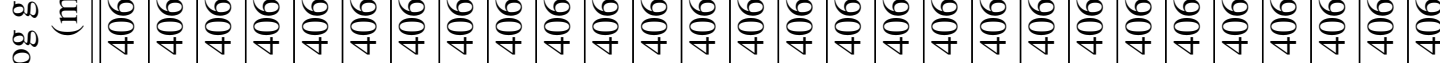

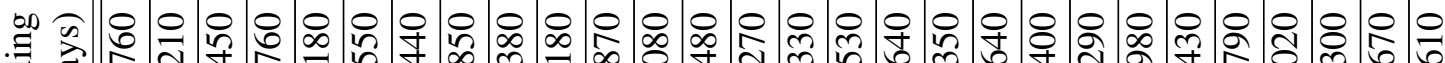
च छ

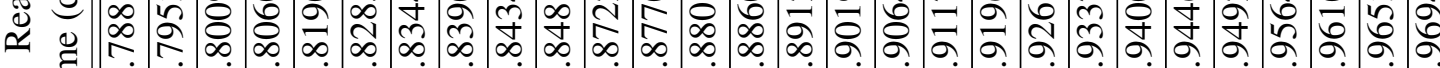
হू்

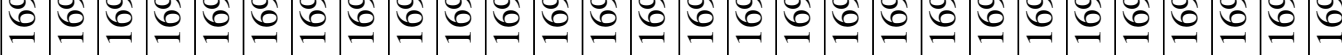

. 凹

共专

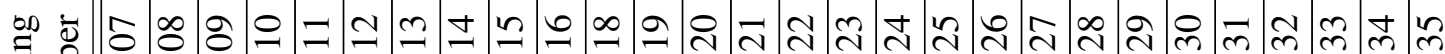
$\exists$ $\approx$

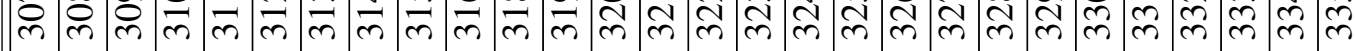




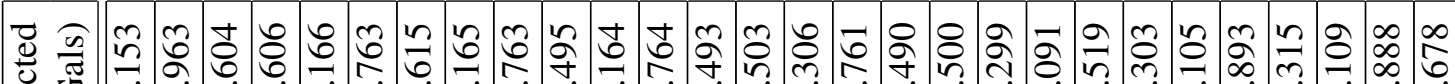

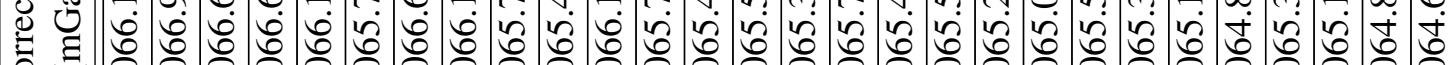
ن कo

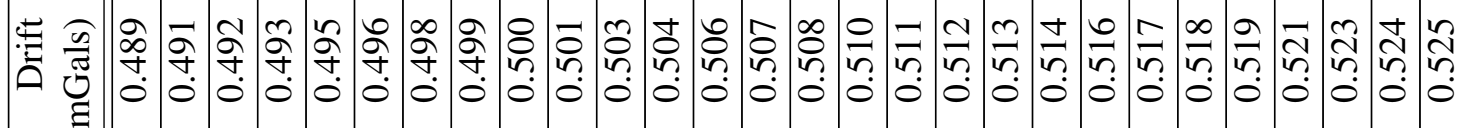
:

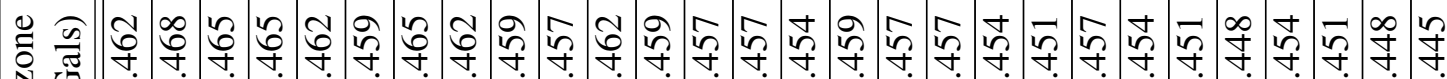

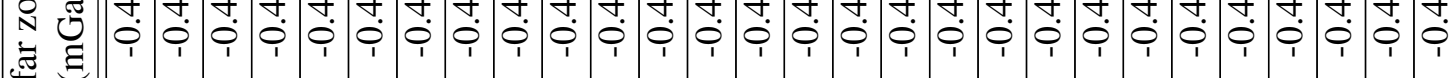
皇

ஜ N ฮँ $\mathrm{Z}$,

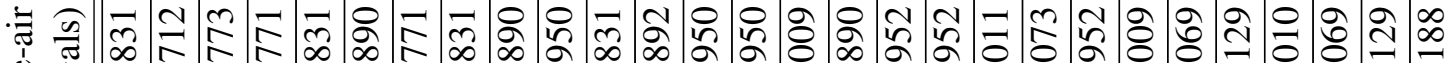
du 至

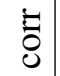

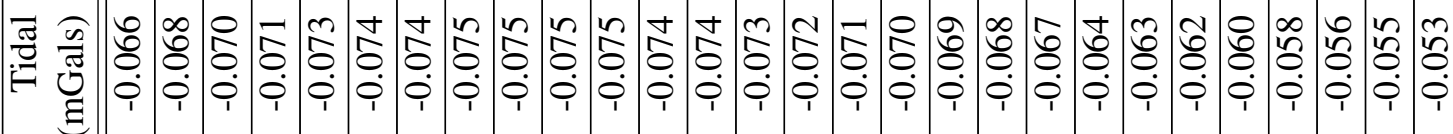
:

. च

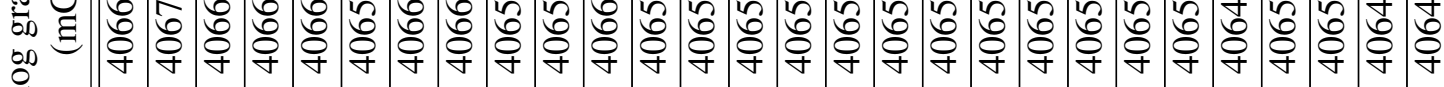
으

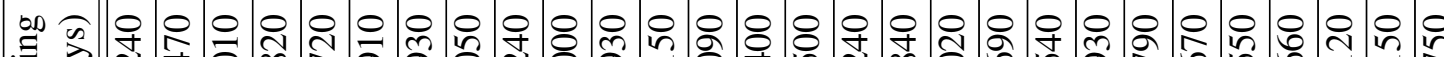

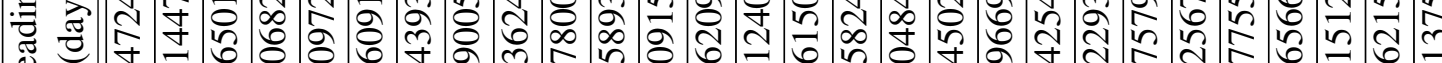
થ ヨ

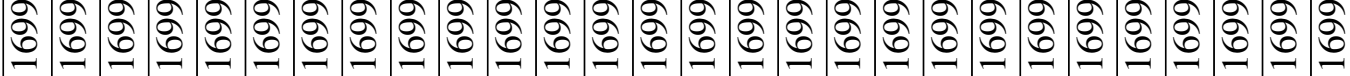

.

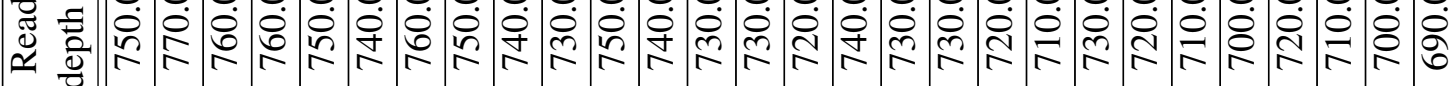

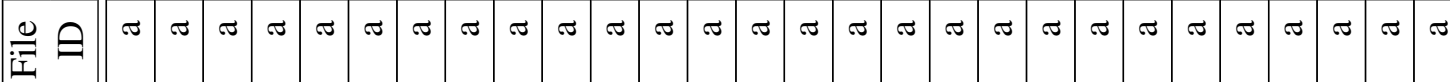

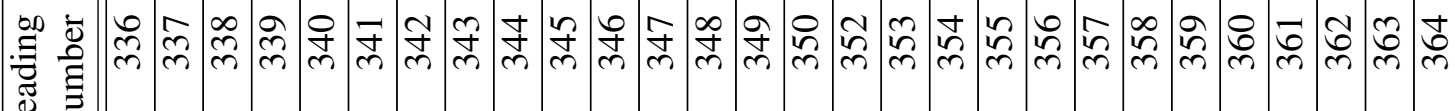
$\simeq \Xi$ 


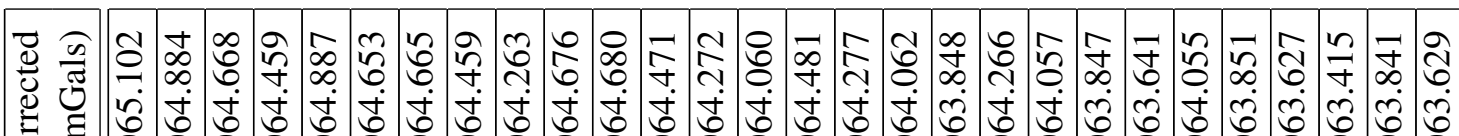
至

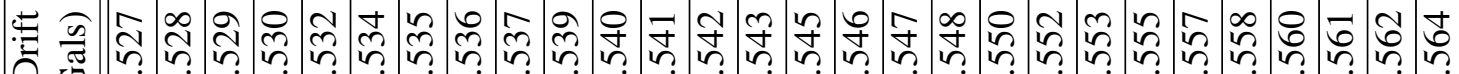
A

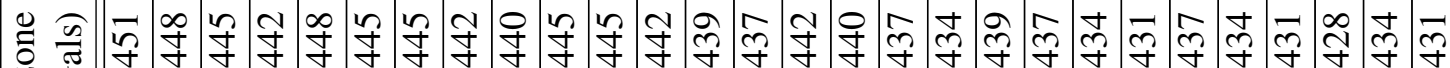

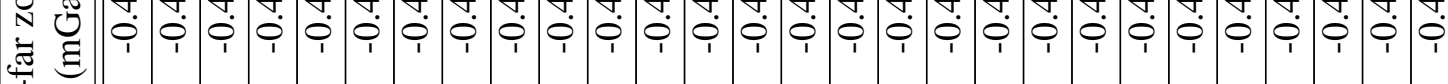

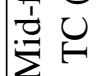

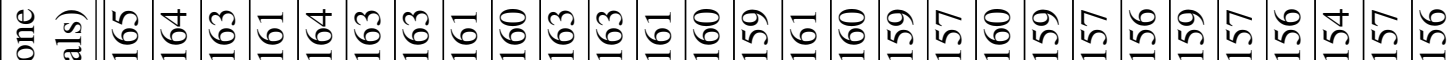

音 ซึ

乙

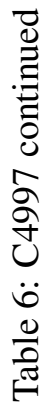

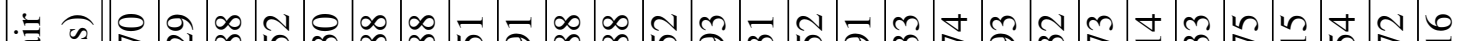

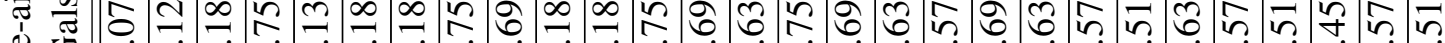

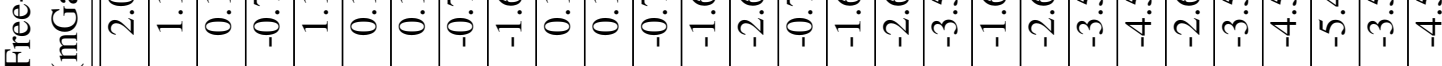

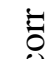

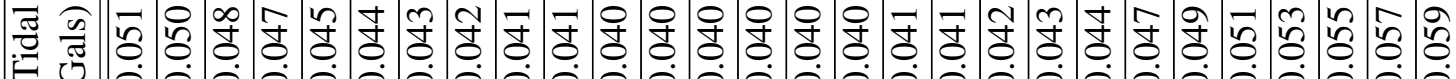

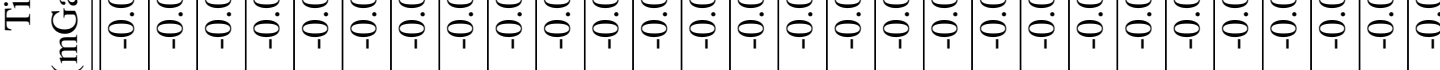

8

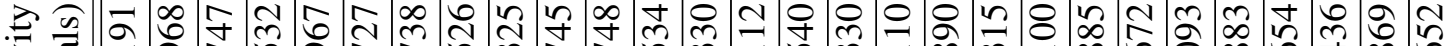

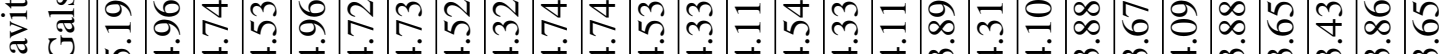
कू

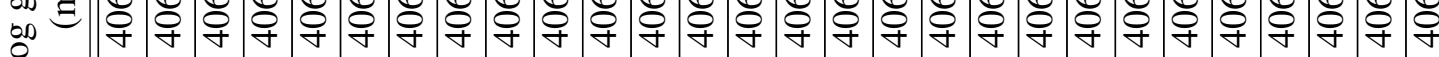

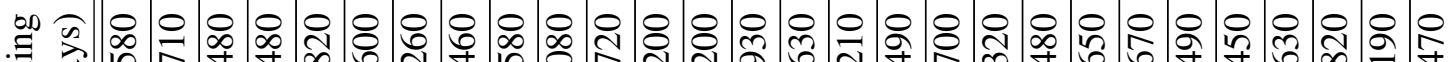

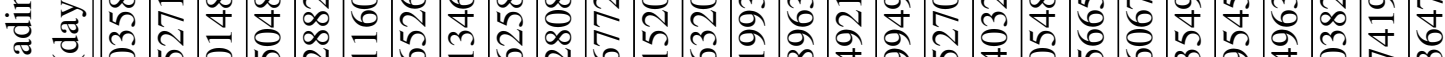

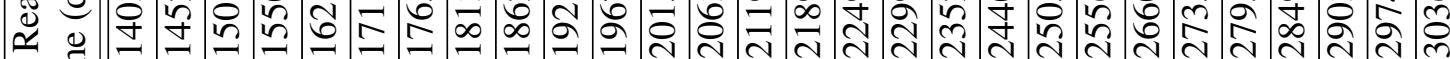

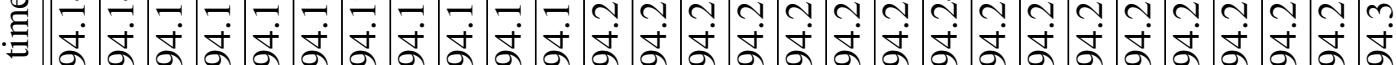
วิ

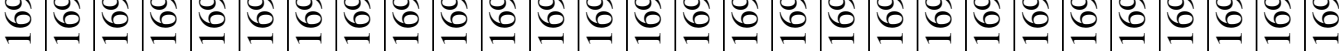

. ت थ $\stackrel{0}{*} \Theta$

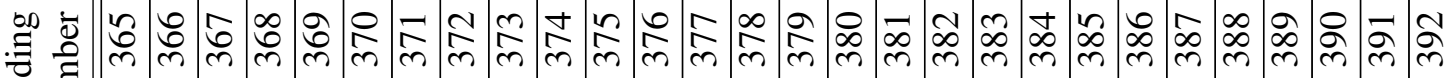
.7. $\simeq$

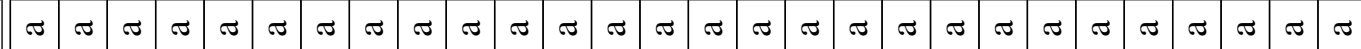




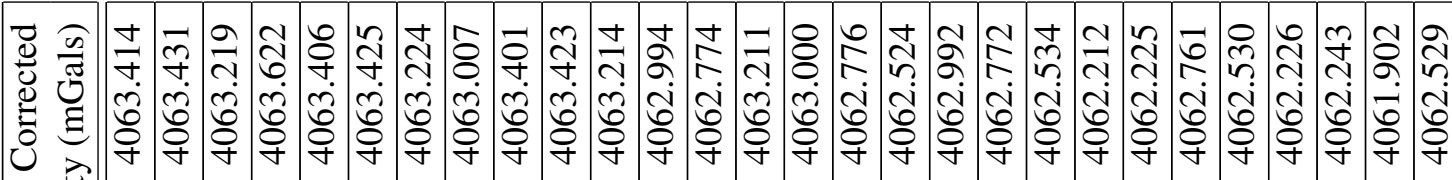
焉

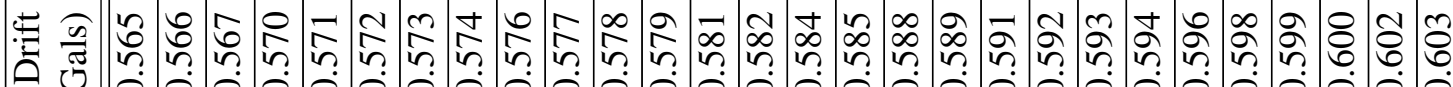
过

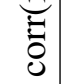

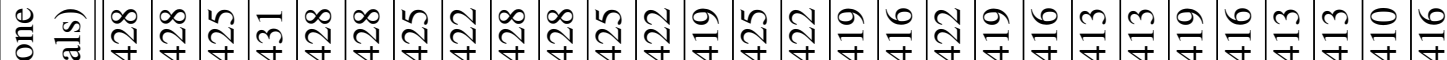

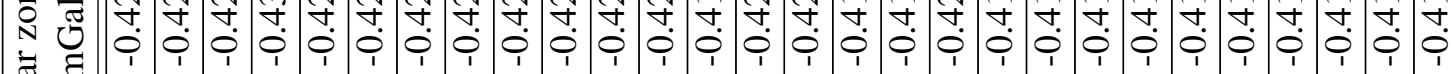
苛 ह

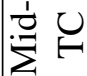

ஜ

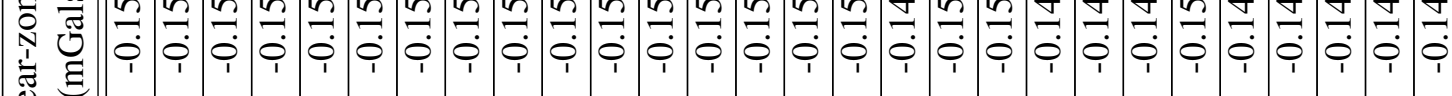
Z

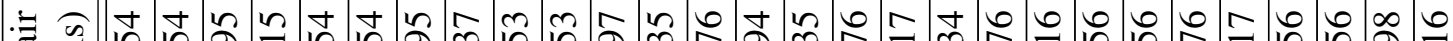

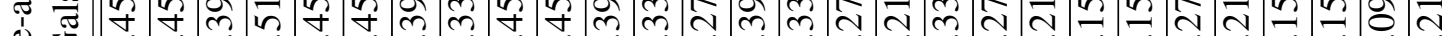
党 ठํ.

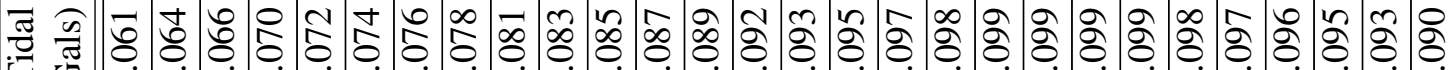

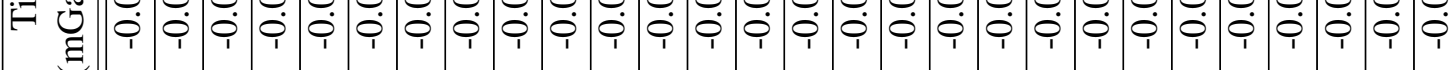
¿ั)

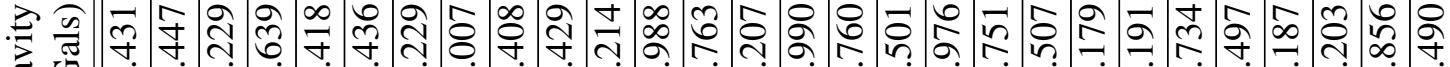
స

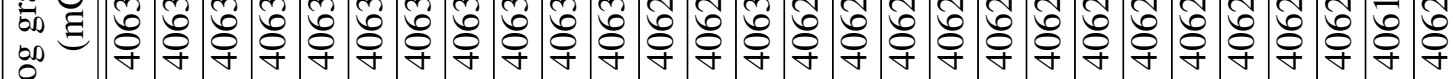
으

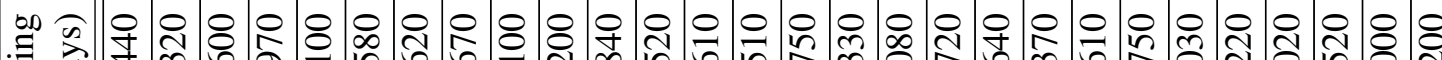

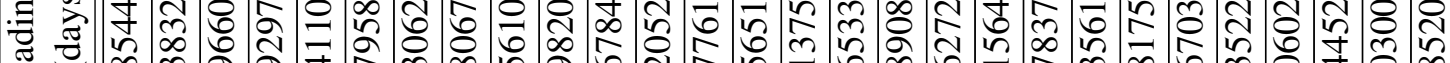

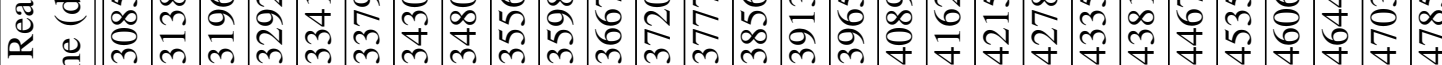
四

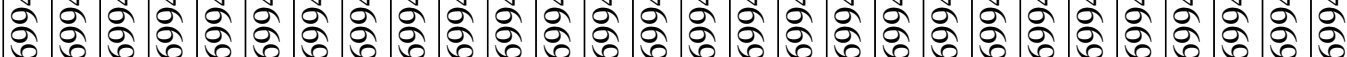

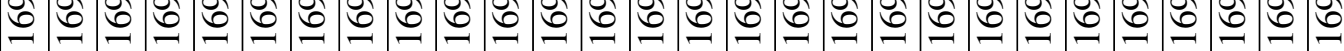

. च

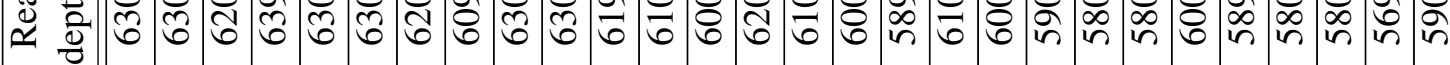
$\stackrel{0}{*}$

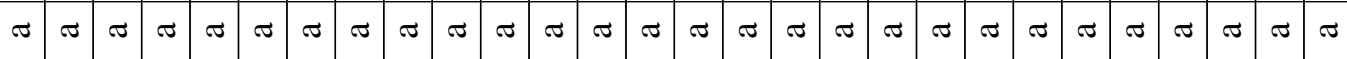
留 㐫 $\simeq$ 


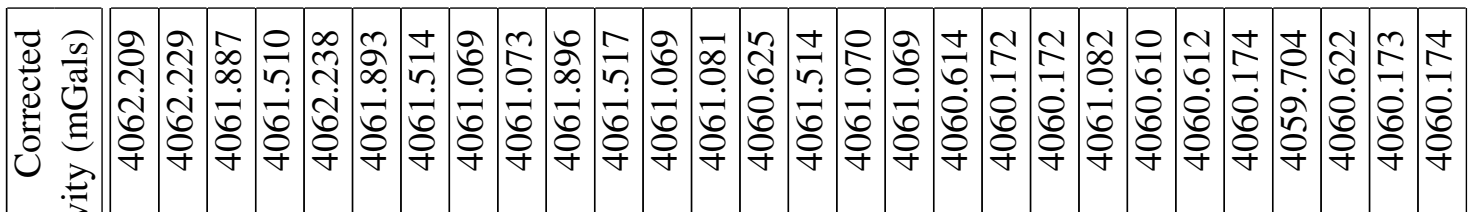

$=$ ప

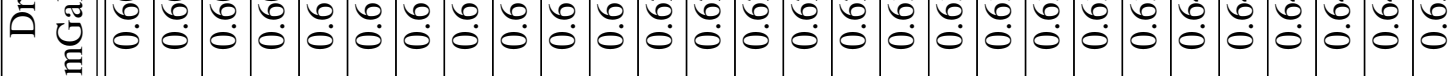

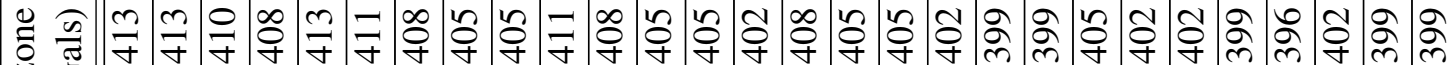
茪

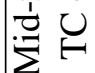

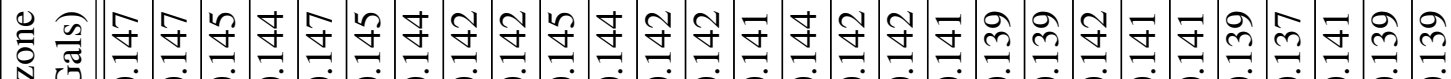
若 Z

$\exists$ ఈ

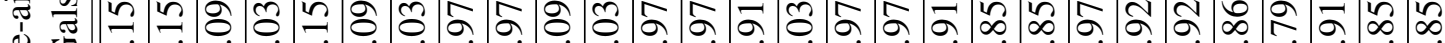

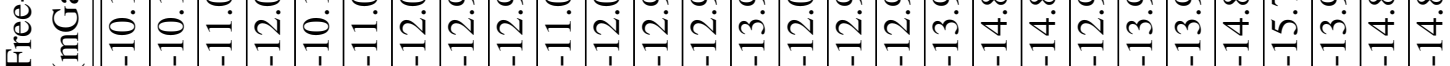

프 (ิ) च च $\Xi$ ठํ.

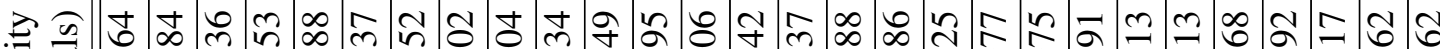

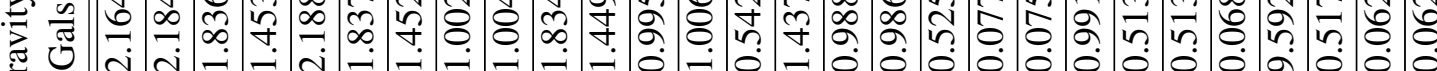
Б

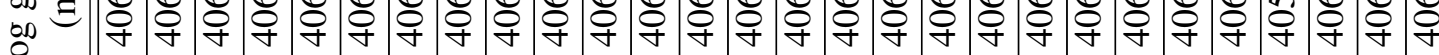

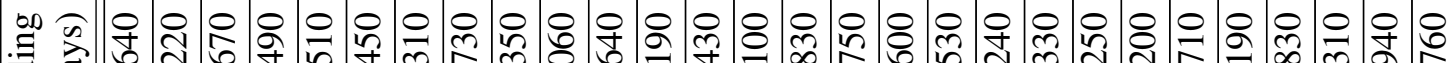

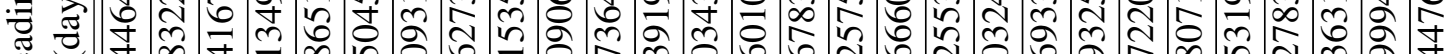

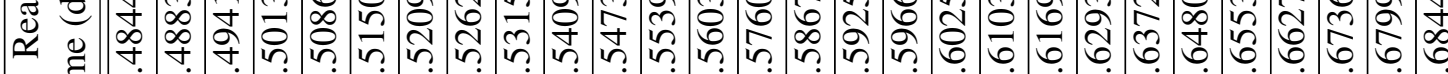

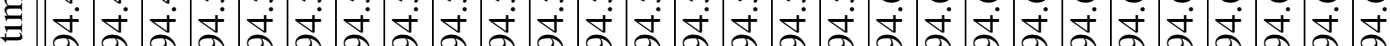

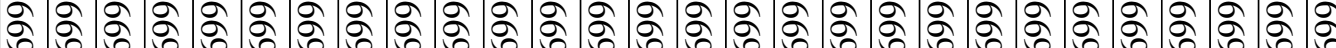

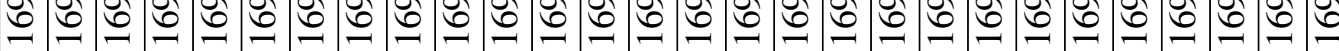

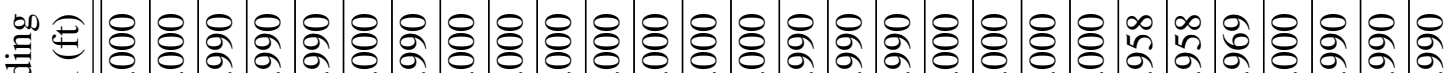

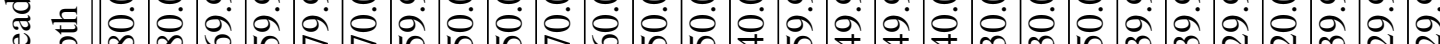
\&

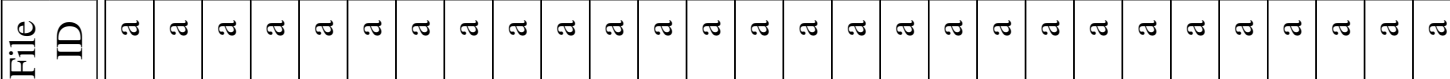

: (4) 


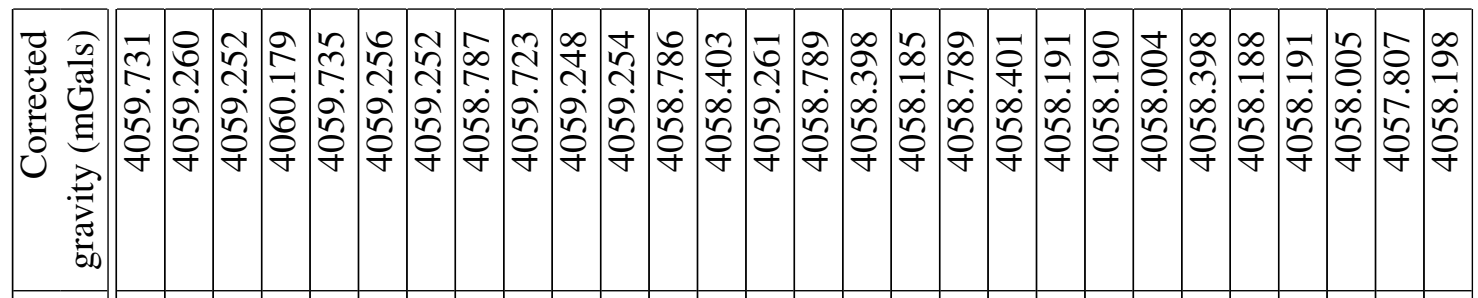

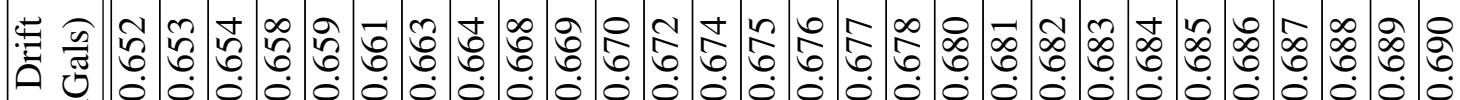

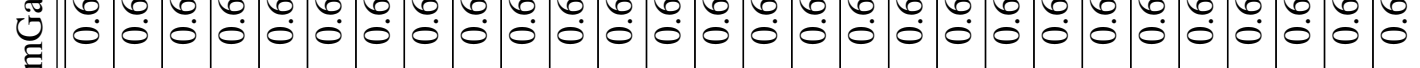
¿ֶ:

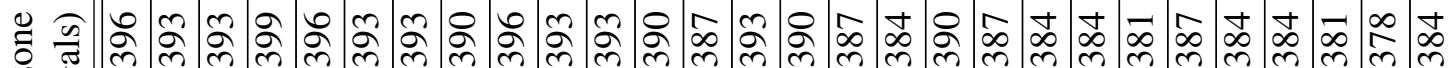

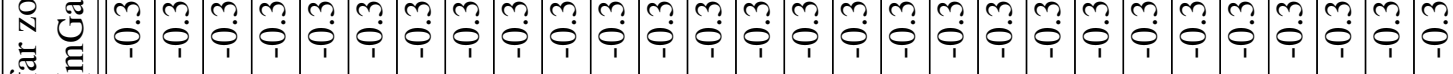

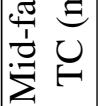

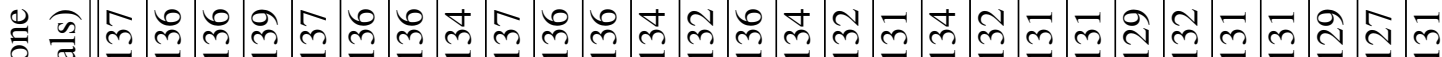
竞 ซี 乙 U

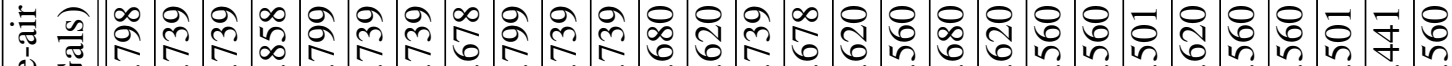

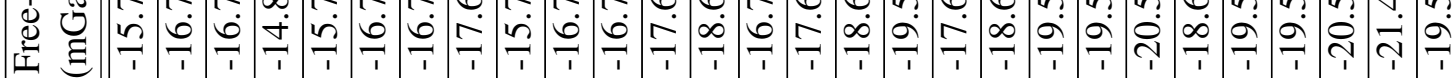

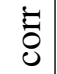

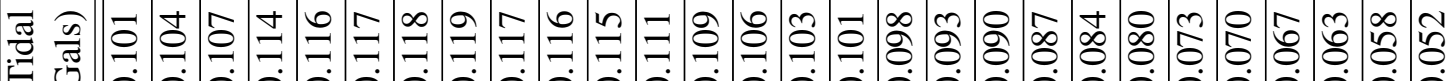

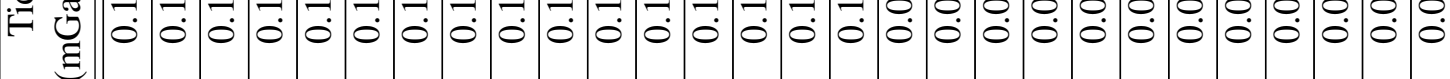
¿ั)

. 苟

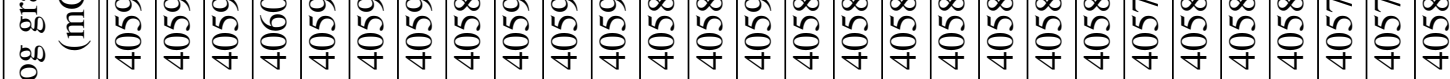
으

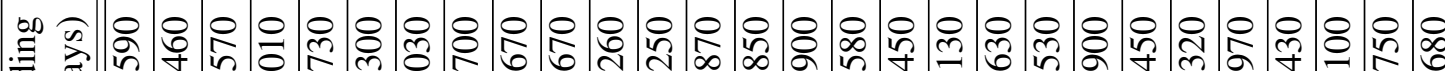

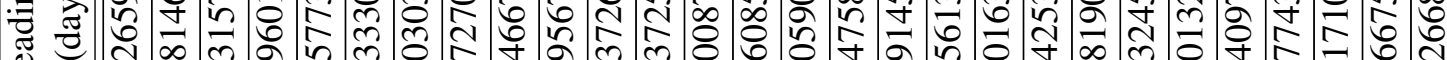

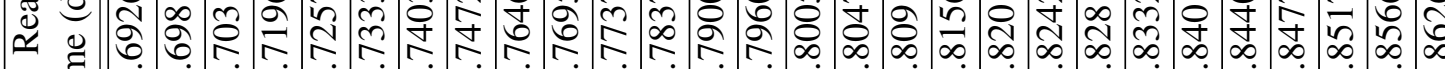

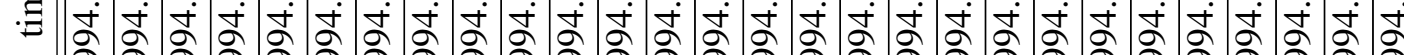

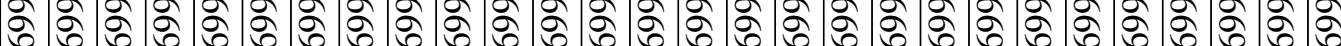

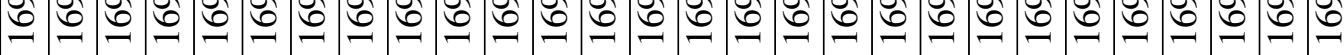

.

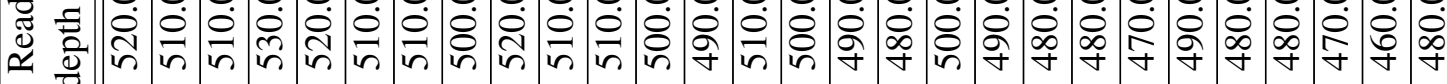

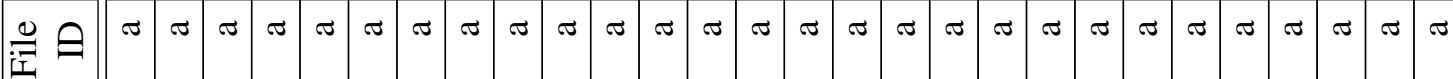
馬

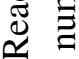




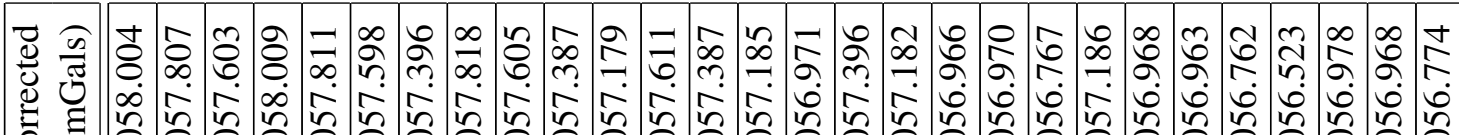

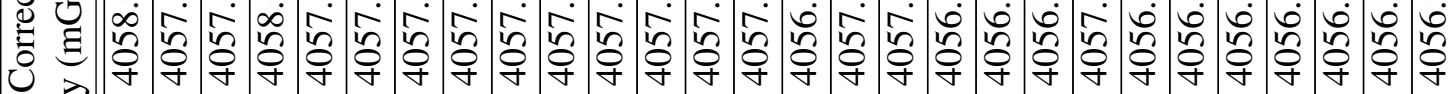
.

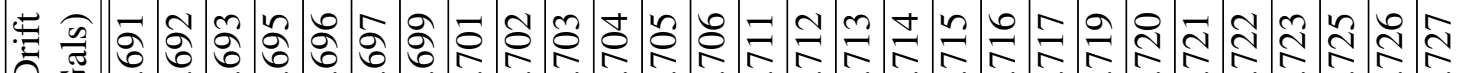
D :

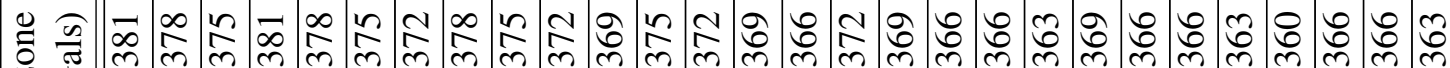

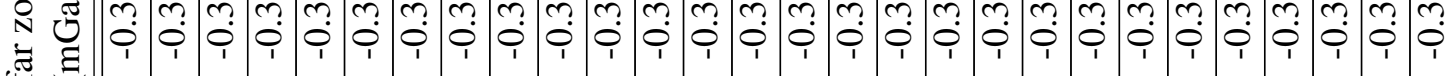
竞

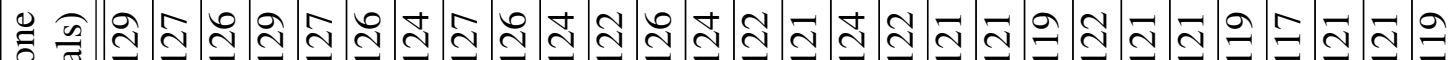

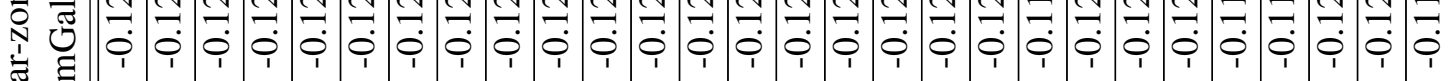
寻 乙

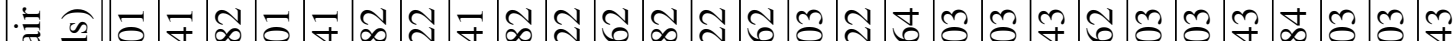
i Ð

สี శิ

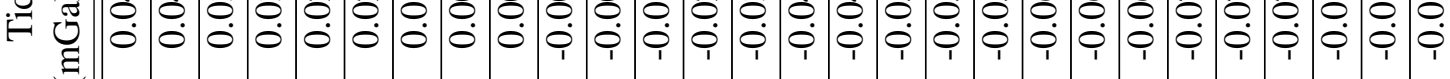

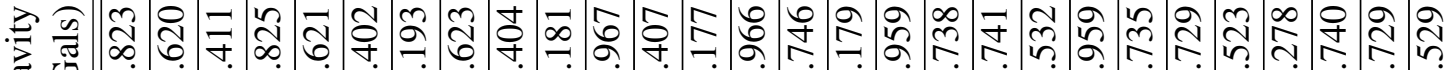

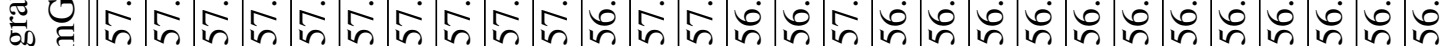

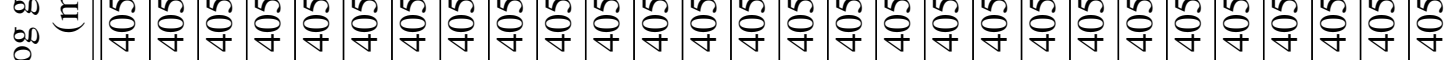

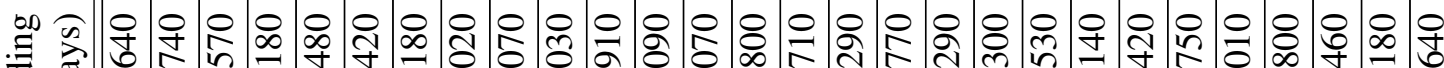

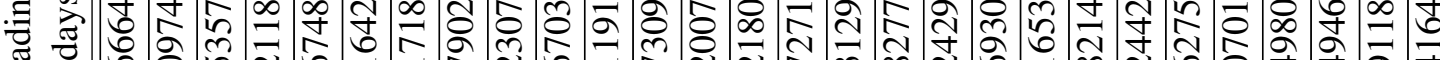

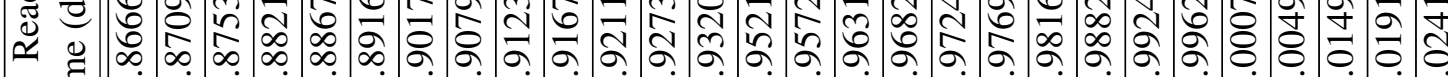

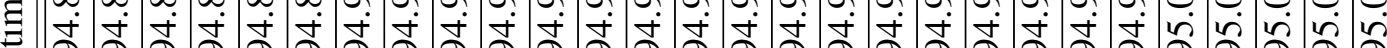

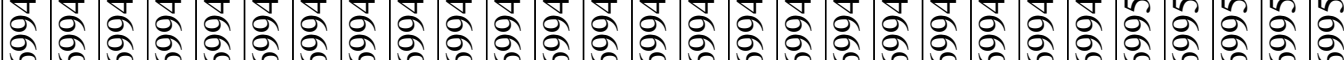

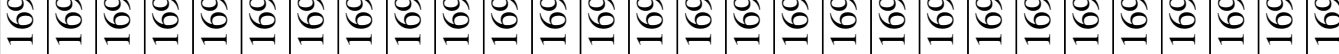

. تै ๙ $\stackrel{0}{*} \Theta$

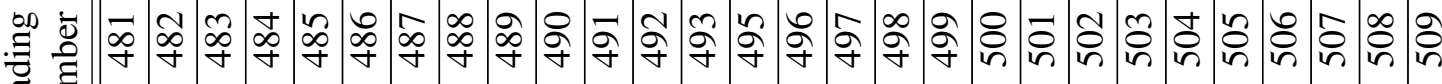
ש 
す্்

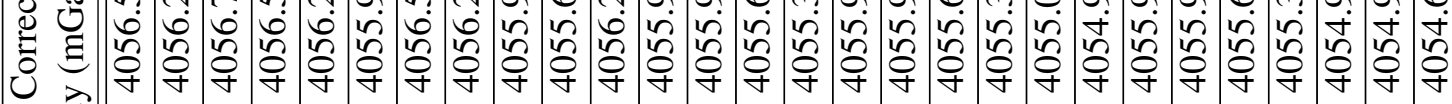

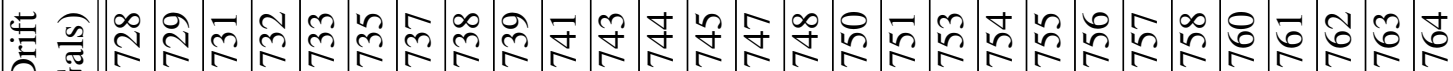

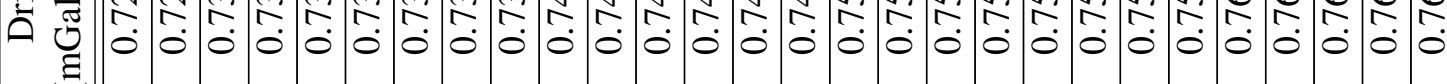
(ְ)

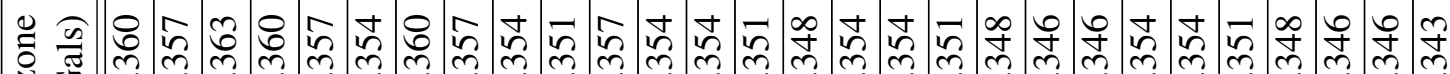

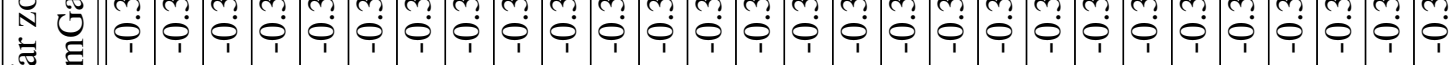
๘ี $\sum^{2}$

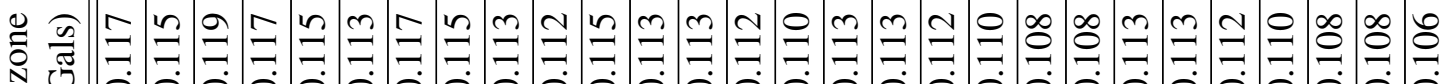
N 造 $\mathrm{Z}$

当 ๘ ส D 8.

उٓ F ठํ.

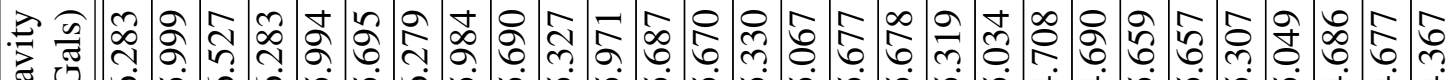

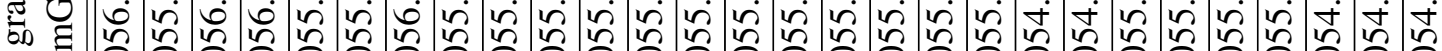

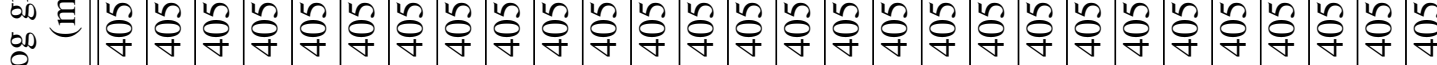

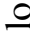

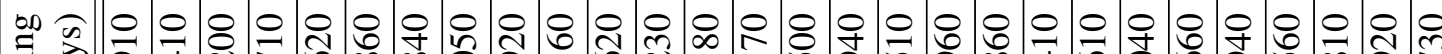

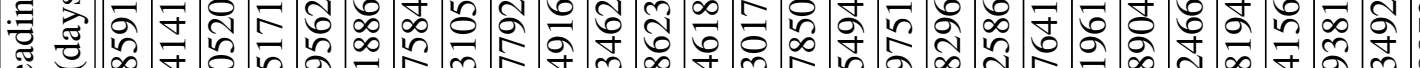

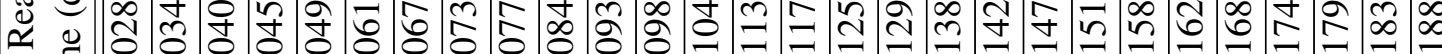

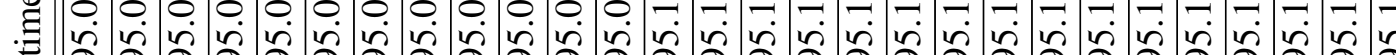

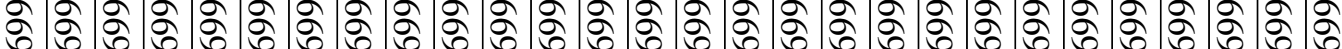

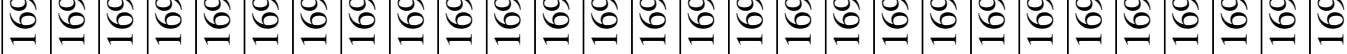

. 芯 空

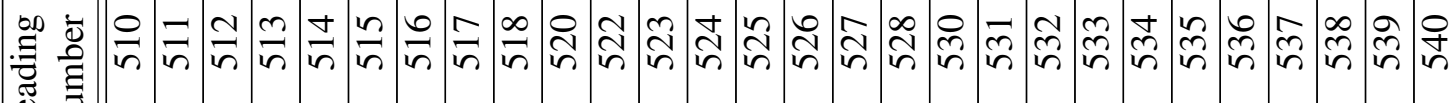
$\simeq$ 


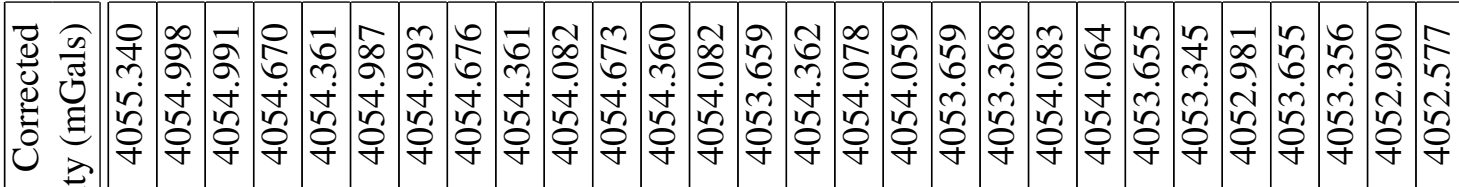
.

•

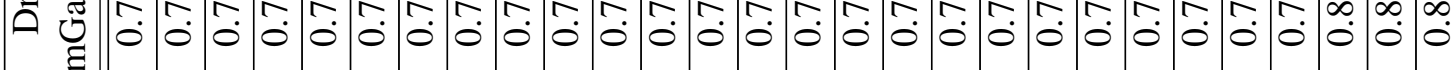

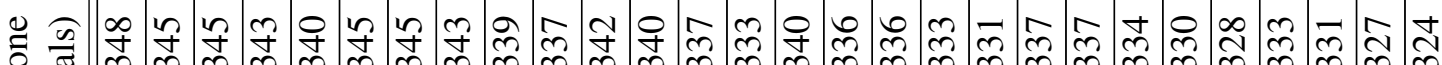
总 ‡ี 竞

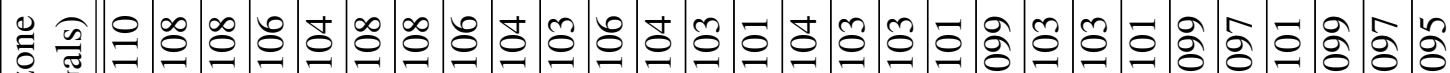

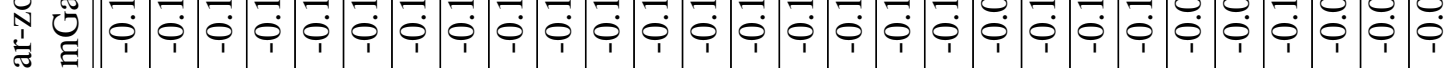
承 乙

$\exists$ ส i 过 ठํำ

సี

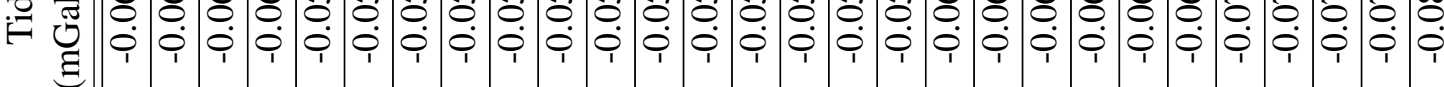

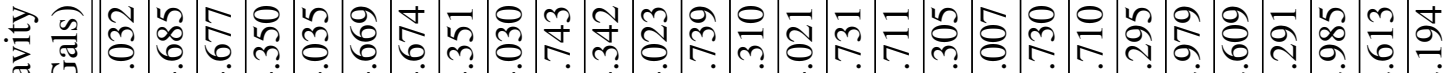

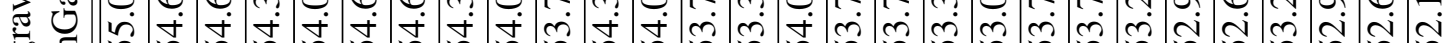

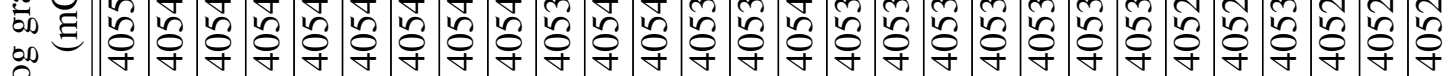
으

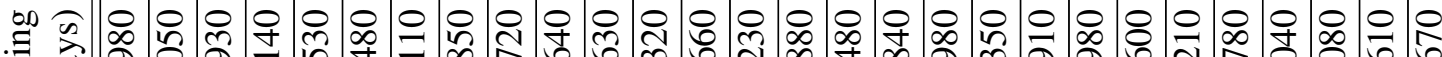

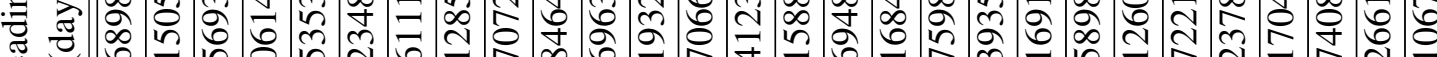

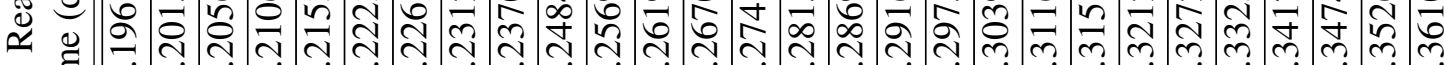

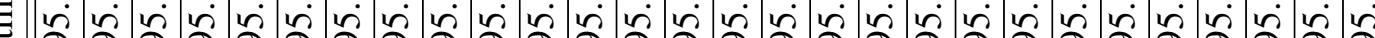

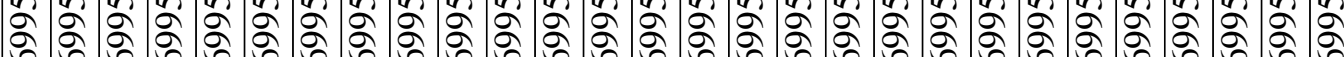

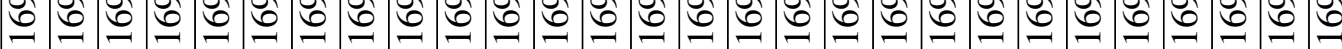

車 થn

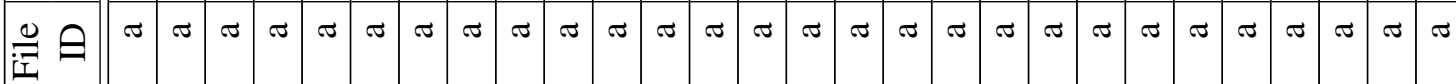

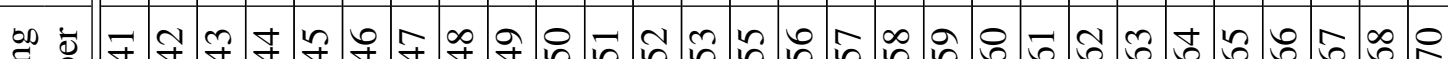

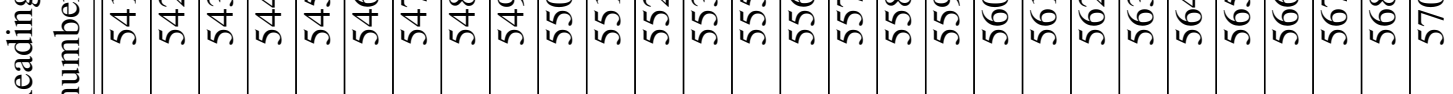
$\simeq$ 
す্்

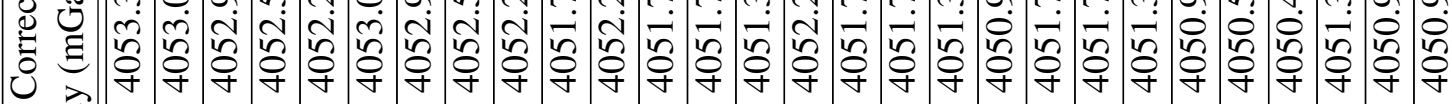

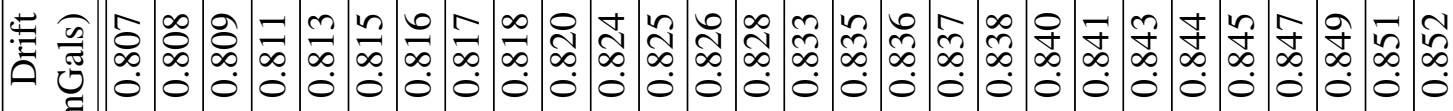
节

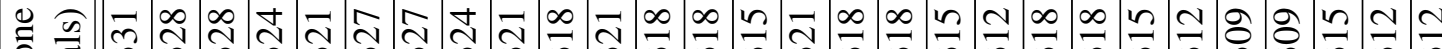

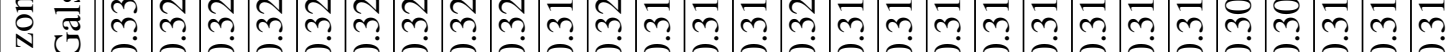
节

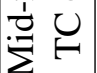

官 党 造 $\mathrm{Z}$

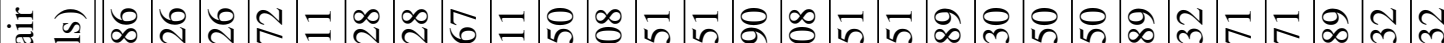

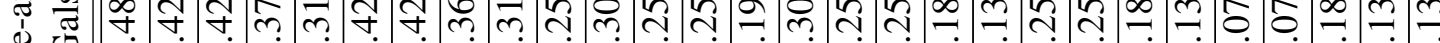
䓛 ठำ

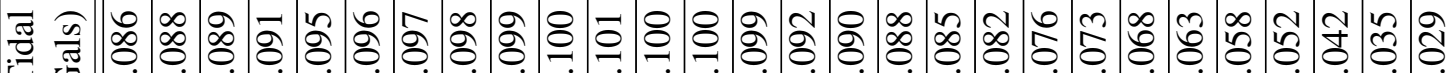

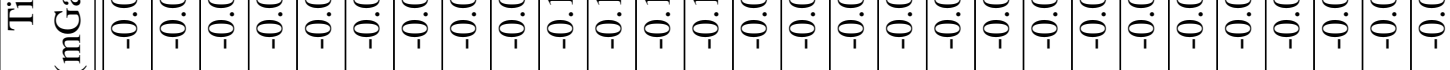
ठํำ

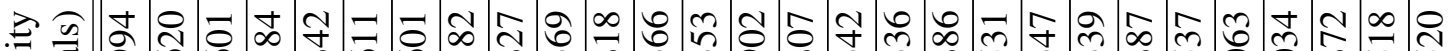

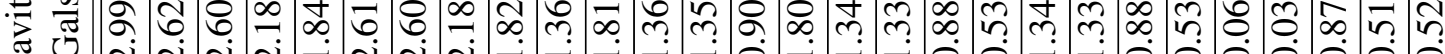
పు

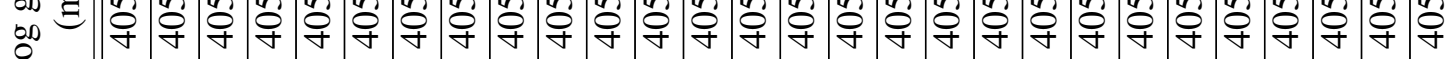
으

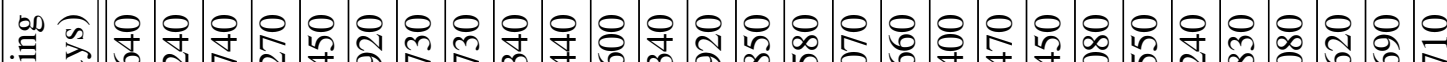

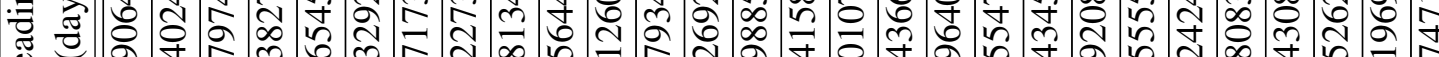

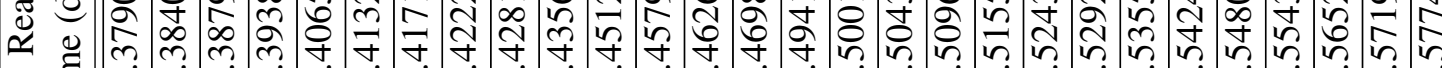

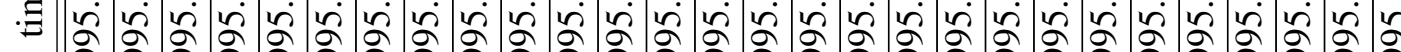

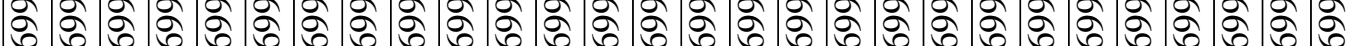

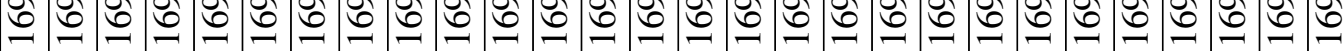

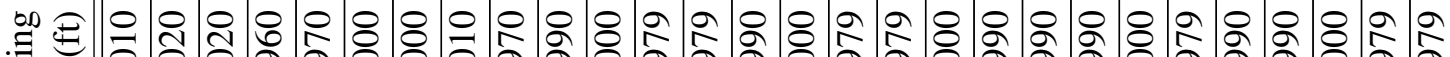

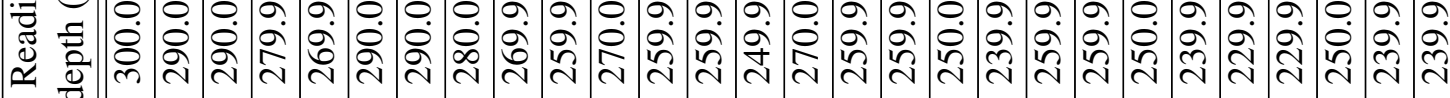

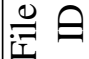

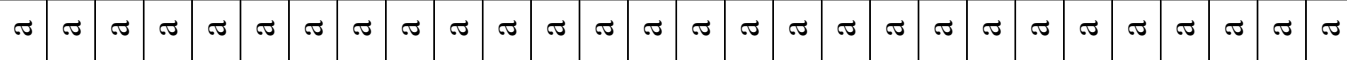

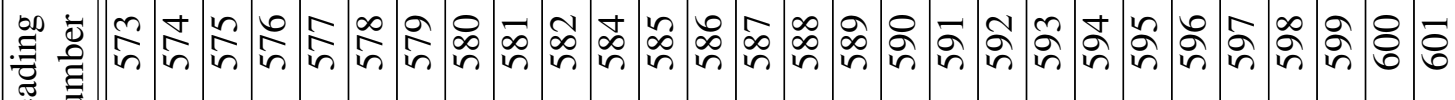
$\simeq \Xi$ 


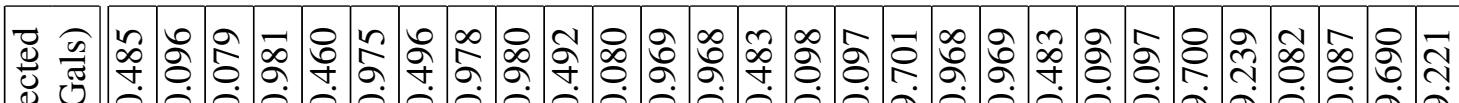
范 U $\begin{aligned} & 0 \\ & 0\end{aligned}$ 离

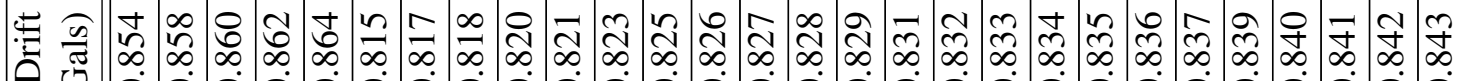

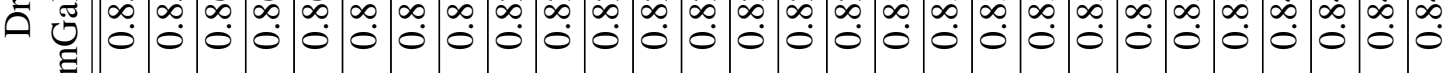
ठํㅇำ

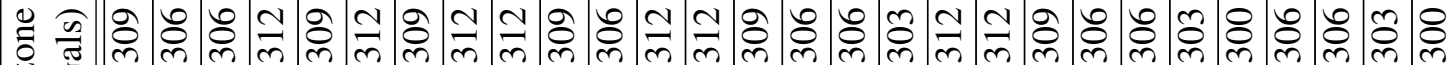

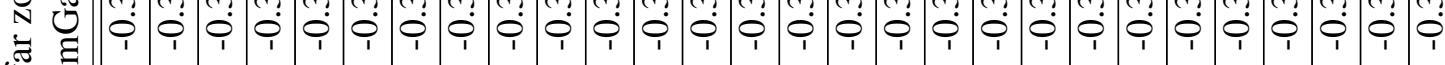
䨔

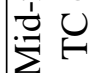

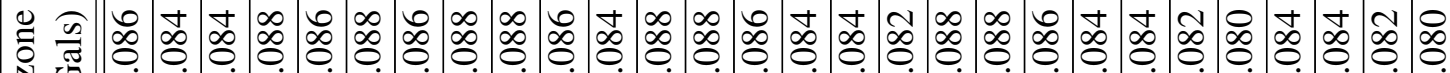
苟 ङ $\mathrm{Z}$,

:

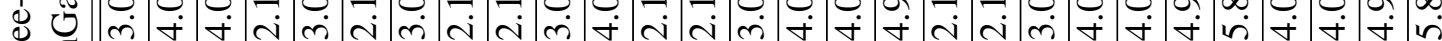

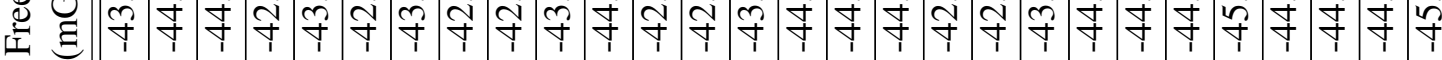
ఏँ

䒕

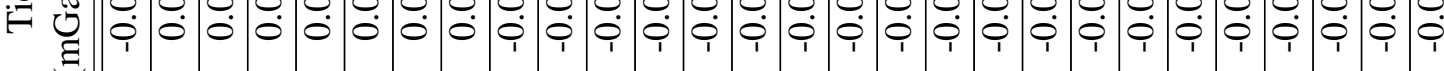
8

Љิ च

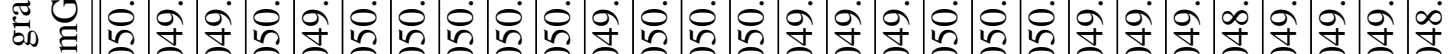
on

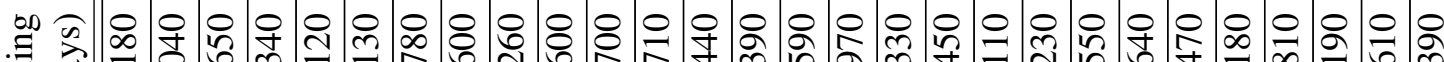
:

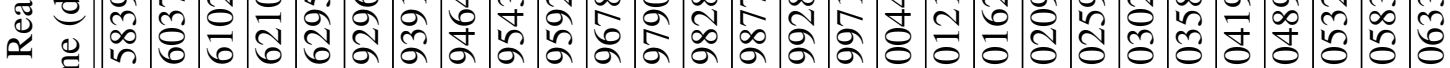

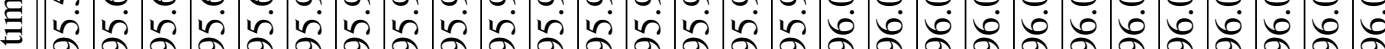

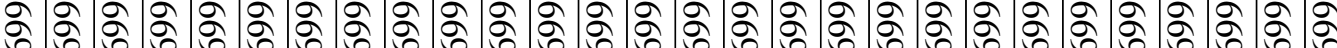

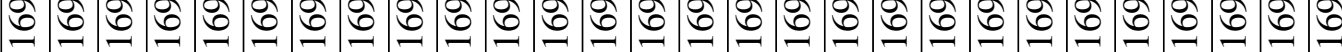

. च च

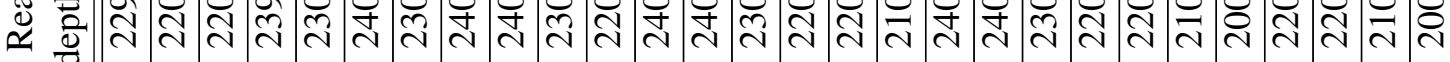

空

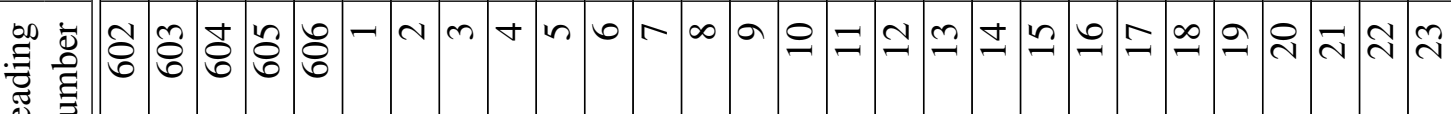
(4) 


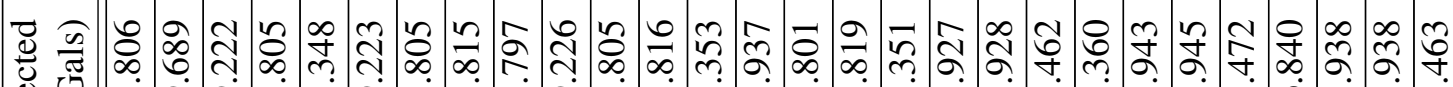

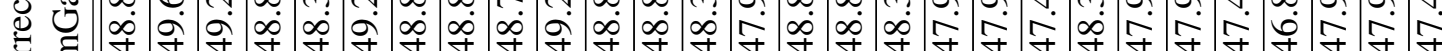

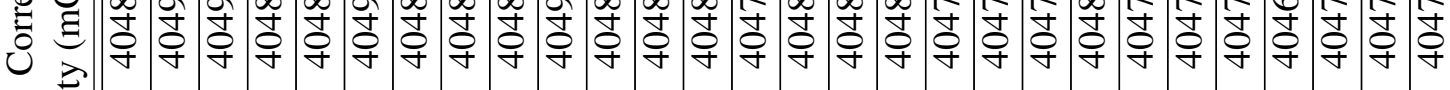

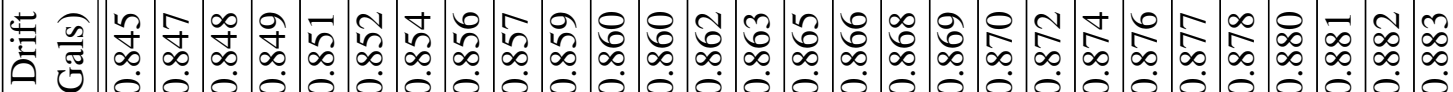

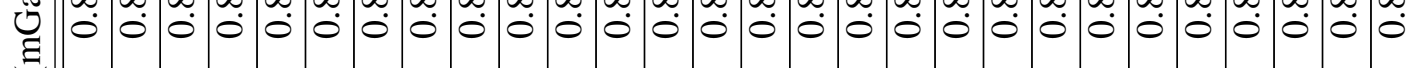
¿ั:

茂

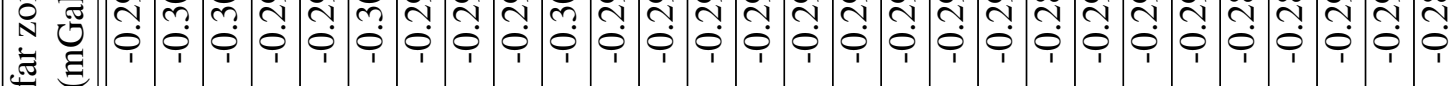

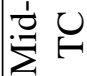

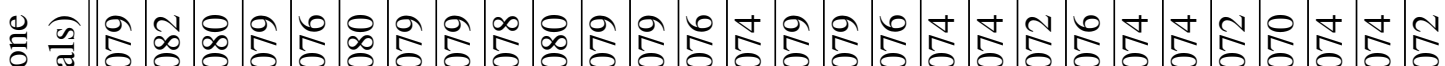

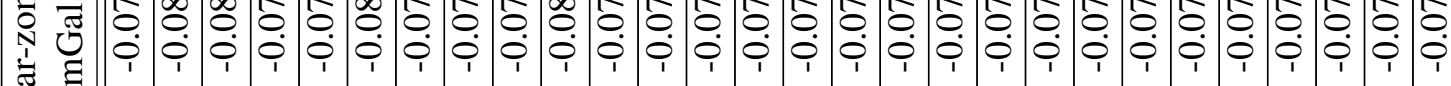
串 Z

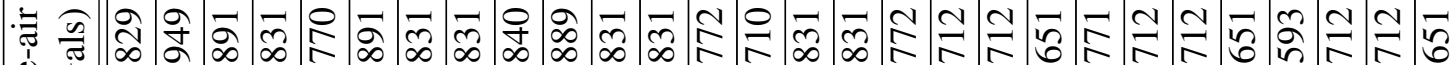
䓛

ठั.

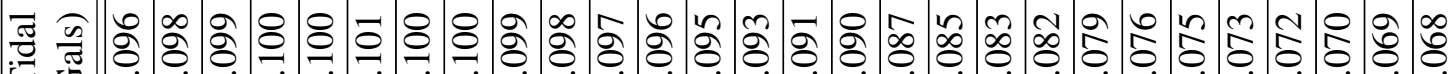

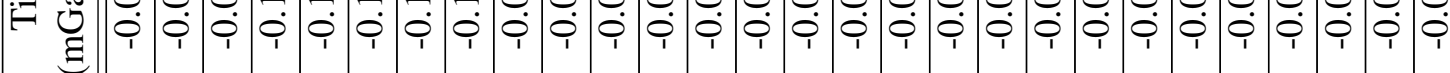
¿ั)

.

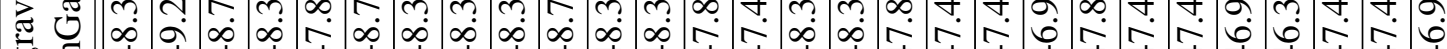

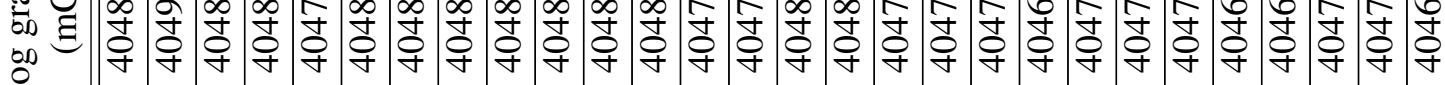
으

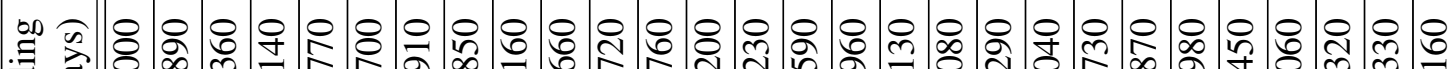
疍 胥 $\simeq$ ¿ - $\mid \begin{aligned} & 0 \\ & 0\end{aligned}$

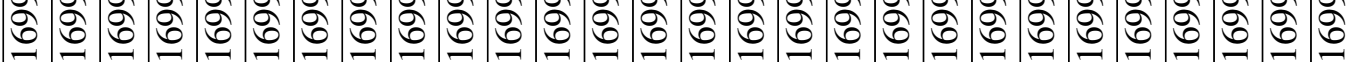

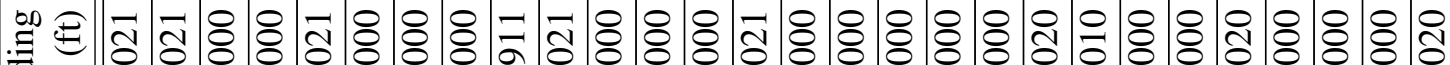
芯 空

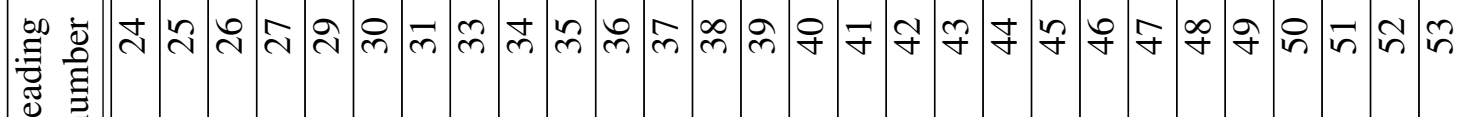
르 


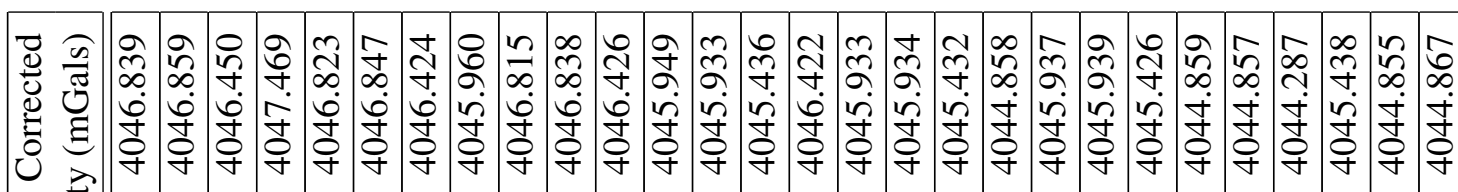
औై

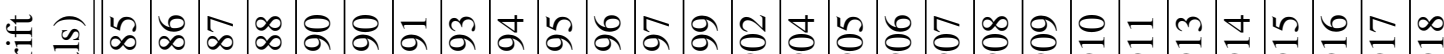

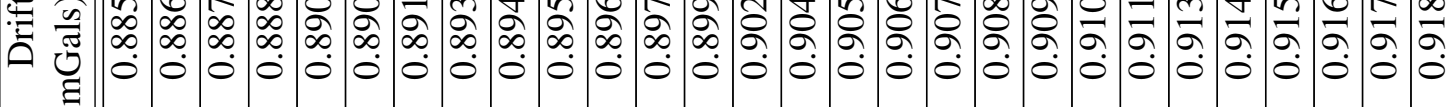
ठั

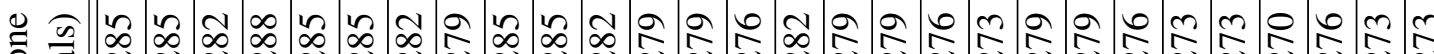
ก

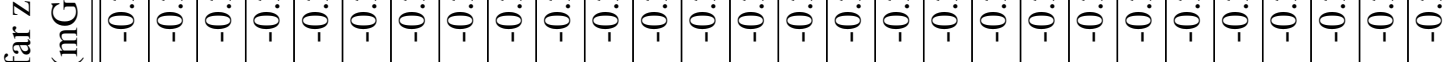
$\underset{\sim}{2}$

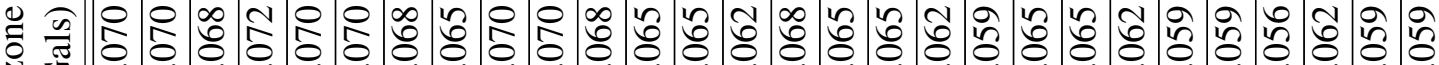

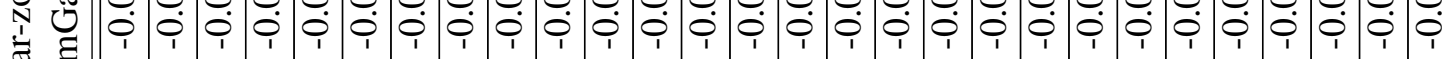
范 Z

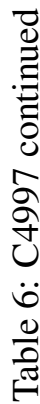

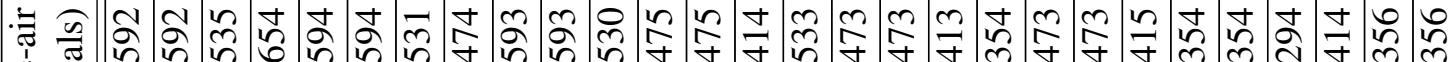
¿ 近 ఏo

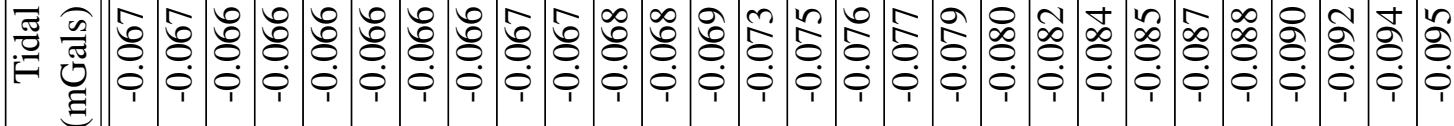

¿̄

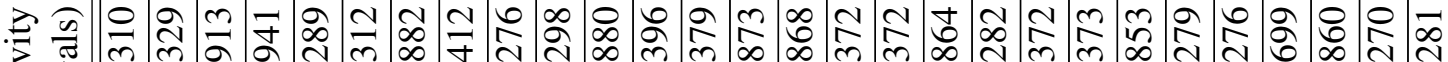
\#

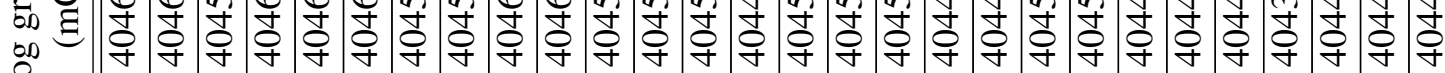

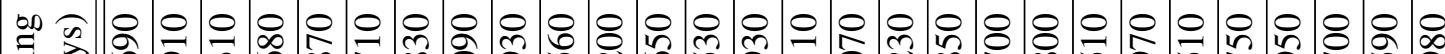

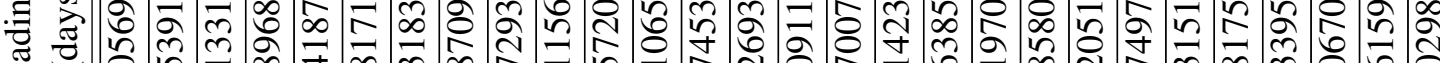

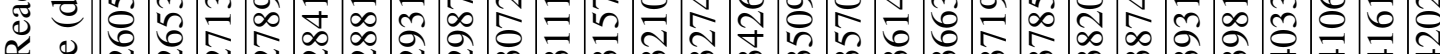

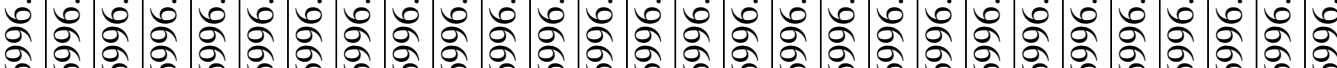

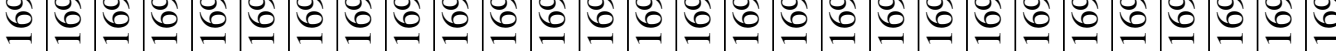

. 芯

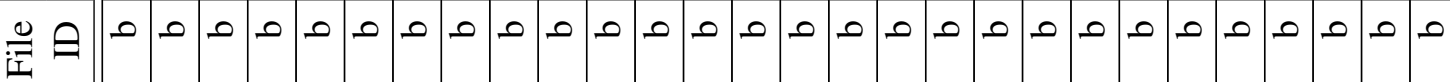

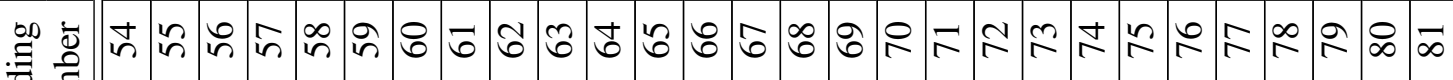
乎 $\simeq \Xi$ 


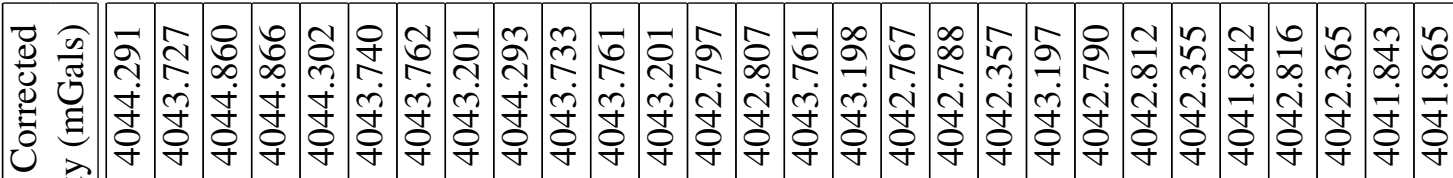

Е

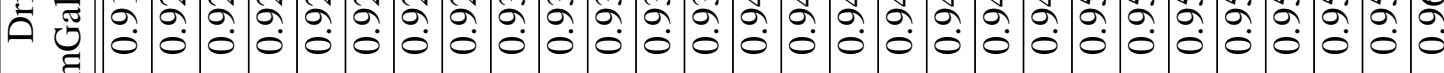
:

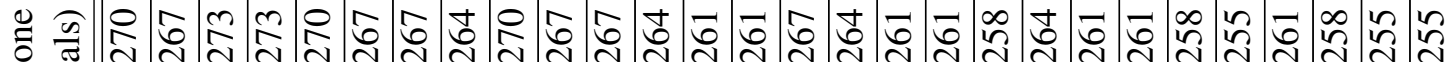

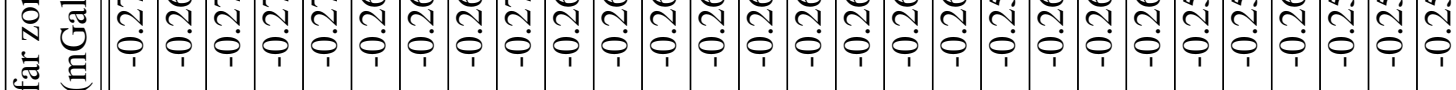

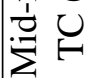

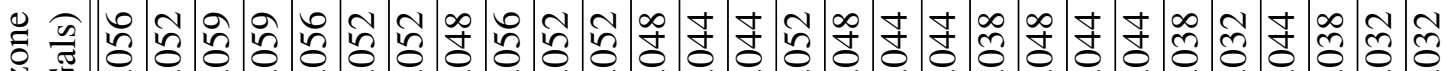

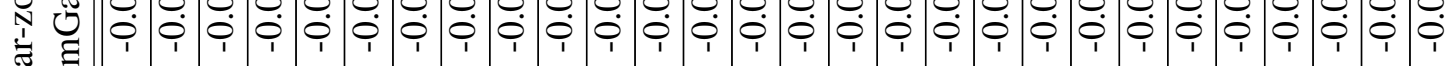
ซึ 乙

$\because$ It ذं సે

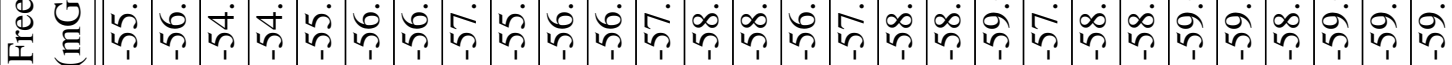
8

䒕 $\frac{a}{\pi} \delta$

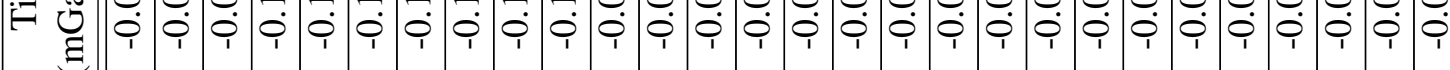
$\stackrel{\Xi}{\circlearrowright}$

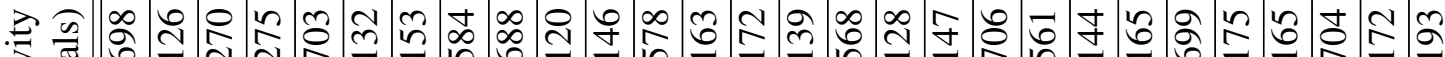
ส

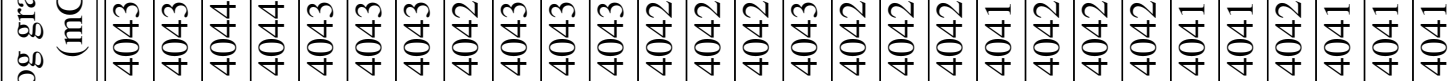
으

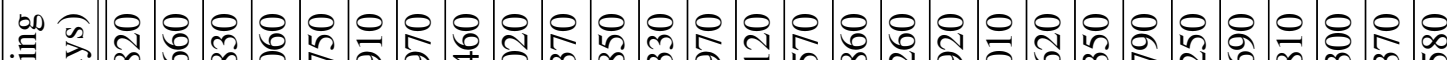

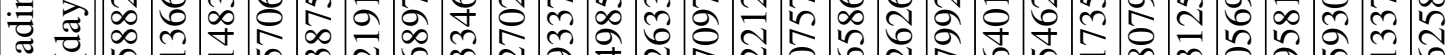

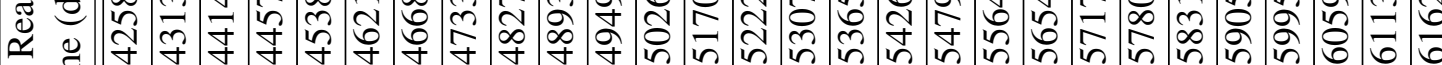
Đ

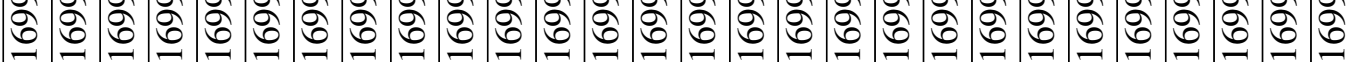

. 竧 $\stackrel{0}{\frac{1}{2}} \Theta$ م. : $\sim=$ 


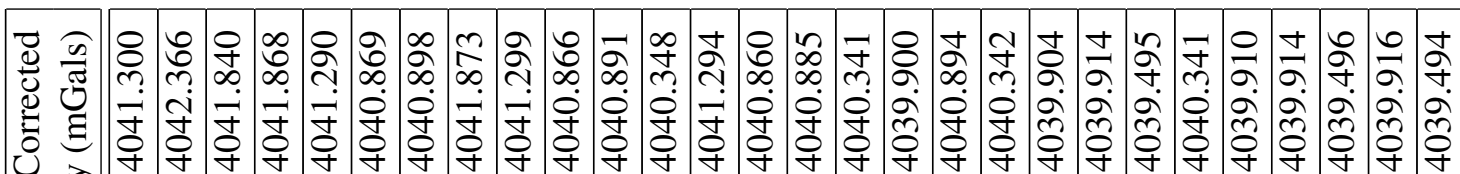
?

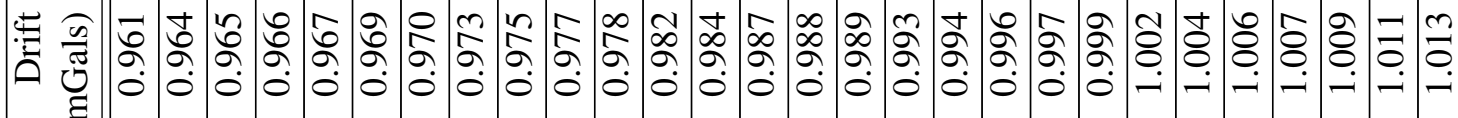

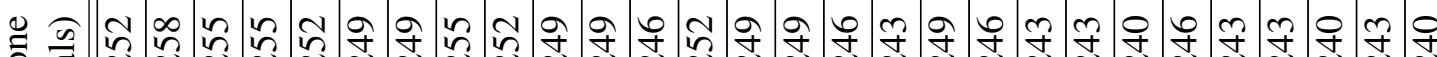

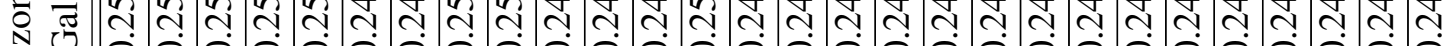

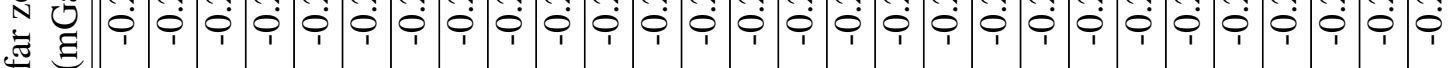
$\stackrel{i}{i}$

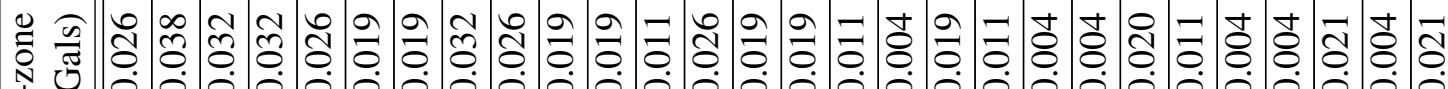

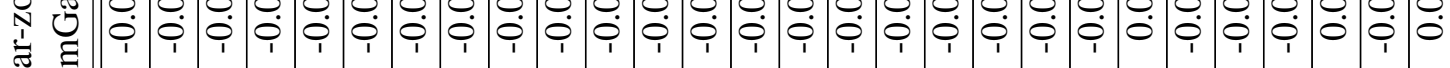
ङ $\mathrm{Z}$,

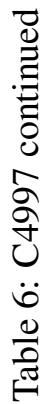

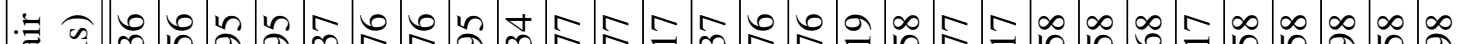

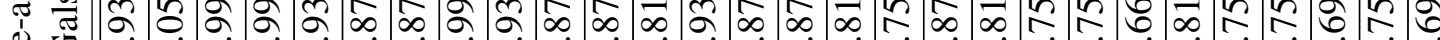

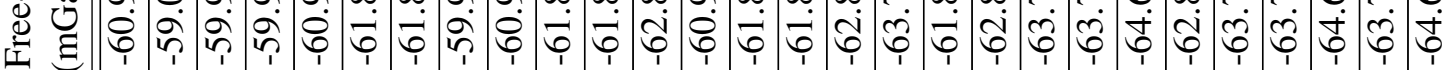
ठำ

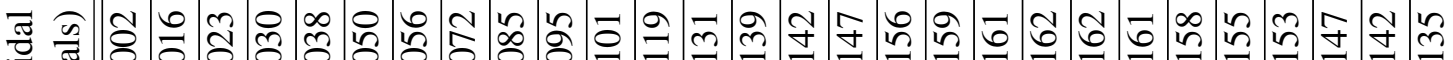

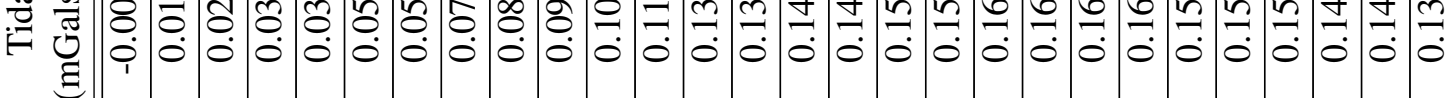
¿ั

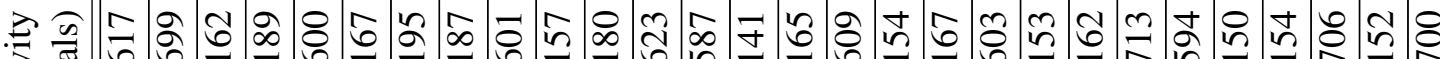
¿ का

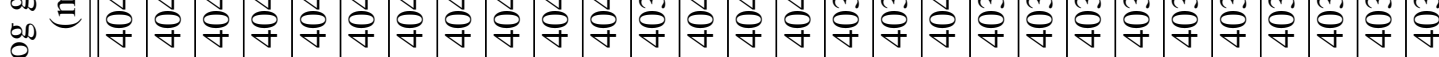

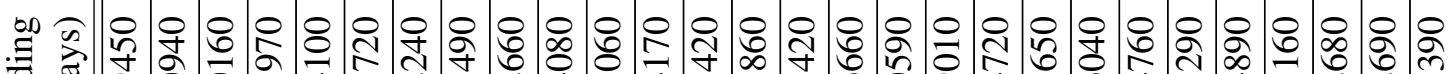

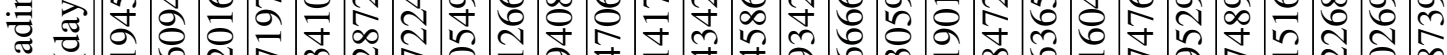

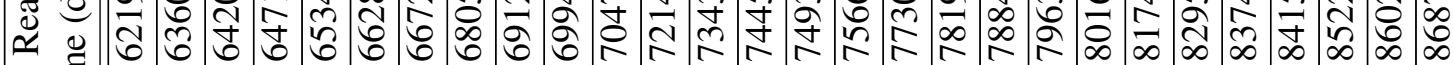
. bे

约 읭 ब $\simeq$ क्षे

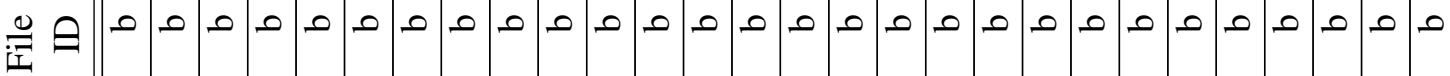

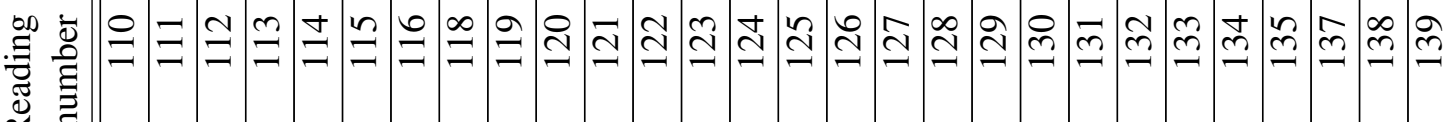
$\simeq$ 


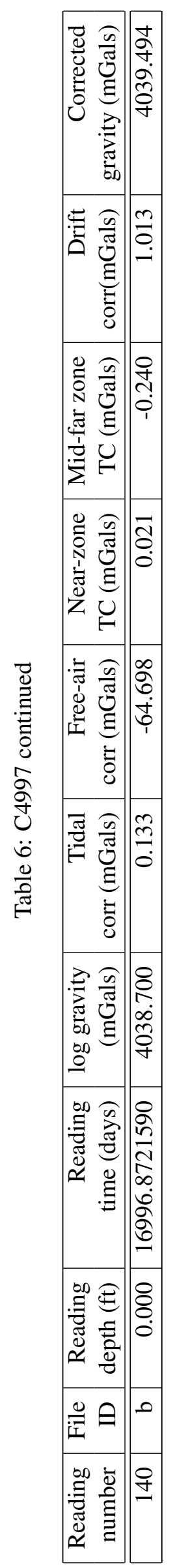




\section{Appendix: B Inversion density tables}

The inversion results for each well are presented in the following tables. The columns are:

depth: the station depth in feet, relative to the wellhead.

$\bar{g}$ : the observed mean gravity at the station, in milliGals.

$\hat{g}:$ the calculated gravity at the station from the inversion model, in milliGals.

$\sigma_{g}:$ the standard deviation of the observed gravity at the station, in milliGals.

Z-score: the standardized error of the inversion fit (dimensionless).

density: interval density calculated by the inversion, in $\mathrm{g} / \mathrm{cm}^{3}$.

$\sigma$ density: the formal uncertainty of the interval density calculated by the inversion, in $\mathrm{g} / \mathrm{cm}^{3}$.

\section{B.1 C4993}

Table 7: Well C4993 Inversion densities

\begin{tabular}{|c|c|c|c|c|c|c|}
\hline $\begin{array}{l}\text { depth } \\
\text { (feet) }\end{array}$ & $\begin{array}{r}\bar{g} \\
\text { (mGals) }\end{array}$ & $\begin{array}{r}\hat{g} \\
(\mathrm{mGals})\end{array}$ & $\begin{array}{r}\sigma_{g} \\
\text { (mGals) }\end{array}$ & Z-score & $\begin{array}{r}\text { density } \\
\left(\mathrm{g} / \mathrm{cm}^{3}\right)\end{array}$ & $\begin{array}{c}\sigma \text { density } \\
\left(\mathrm{g} / \mathrm{cm}^{3}\right)\end{array}$ \\
\hline \multirow[t]{2}{*}{10.0} & 4038.813 & 4038.870 & 0.008 & -6.9 & & \\
\hline & & & & & 1.956 & 0.021 \\
\hline \multirow[t]{2}{*}{20.0} & 4039.285 & 4039.310 & 0.014 & -1.8 & & \\
\hline & & & & & 1.714 & 0.019 \\
\hline \multirow[t]{2}{*}{30.0} & 4039.809 & 4039.812 & 0.009 & -0.4 & & \\
\hline & & & & & 1.623 & 0.018 \\
\hline \multirow[t]{2}{*}{40.0} & 4040.380 & 4040.338 & 0.012 & 3.6 & & \\
\hline & & & & & 2.383 & 0.018 \\
\hline \multirow[t]{2}{*}{50.0} & 4040.579 & 4040.669 & 0.017 & -5.4 & & \\
\hline & & & & & 1.295 & 0.018 \\
\hline \multirow[t]{2}{*}{60.0} & 4041.290 & 4041.278 & 0.012 & 0.9 & & \\
\hline & & & & & 1.525 & 0.018 \\
\hline \multirow[t]{2}{*}{70.0} & 4041.846 & 4041.829 & 0.018 & 1.0 & & \\
\hline & & & & & 1.708 & 0.017 \\
\hline \multirow[t]{2}{*}{80.0} & 4042.330 & 4042.332 & 0.004 & -0.4 & & \\
\hline & & & & & 1.517 & 0.014 \\
\hline \multirow[t]{2}{*}{90.0} & 4042.885 & 4042.885 & 0.010 & -0.0 & & \\
\hline & & & & & 1.509 & 0.014 \\
\hline \multirow[t]{2}{*}{100.0} & 4043.444 & 4043.440 & 0.004 & 1.1 & & \\
\hline & & & & & 2.061 & 0.013 \\
\hline \multirow[t]{2}{*}{110.0} & 4043.844 & 4043.853 & 0.009 & -1.1 & & \\
\hline & & & & & 1.696 & 0.014 \\
\hline
\end{tabular}


Table 7: C4993 continued

\begin{tabular}{|c|c|c|c|c|c|c|}
\hline $\begin{array}{l}\text { depth } \\
\text { (feet) }\end{array}$ & $\begin{array}{r}\bar{g} \\
\text { (mGals) }\end{array}$ & $\begin{array}{r}\hat{g} \\
\text { (mGals) }\end{array}$ & $\begin{array}{r}\sigma_{g} \\
\text { (mGals) }\end{array}$ & Z-score & $\begin{array}{r}\text { density } \\
\left(\mathrm{g} / \mathrm{cm}^{3}\right)\end{array}$ & $\begin{array}{l}\sigma \text { density } \\
\left(\mathrm{g} / \mathrm{cm}^{3}\right)\end{array}$ \\
\hline \multirow[t]{2}{*}{120.0} & 4044.358 & 4044.360 & 0.006 & -0.3 & & \\
\hline & & & & & 1.610 & 0.015 \\
\hline \multirow[t]{2}{*}{130.0} & 4044.888 & 4044.889 & 0.010 & -0.0 & & \\
\hline & & & & & 1.597 & 0.015 \\
\hline \multirow[t]{2}{*}{140.0} & 4045.423 & 4045.420 & 0.006 & 0.5 & & \\
\hline & & & & & 1.761 & 0.014 \\
\hline \multirow[t]{2}{*}{150.0} & 4045.910 & 4045.910 & 0.010 & -0.0 & & \\
\hline & & & & & 1.760 & 0.015 \\
\hline \multirow[t]{2}{*}{160.0} & 4046.404 & 4046.401 & 0.006 & 0.4 & & \\
\hline & & & & & 1.893 & 0.014 \\
\hline \multirow[t]{2}{*}{170.0} & 4046.856 & 4046.857 & 0.008 & -0.1 & & \\
\hline & & & & & 1.832 & 0.013 \\
\hline \multirow[t]{2}{*}{180.0} & 4047.335 & 4047.330 & 0.005 & 1.1 & & \\
\hline & & & & & 2.291 & 0.014 \\
\hline \multirow[t]{2}{*}{190.0} & 4047.684 & 4047.684 & 0.012 & -0.1 & & \\
\hline & & & & & 2.276 & 0.016 \\
\hline \multirow[t]{2}{*}{200.0} & 4048.034 & 4048.043 & 0.007 & -1.2 & & \\
\hline & & & & & 1.945 & 0.015 \\
\hline \multirow{2}{*}{210.0} & 4048.492 & 4048.486 & 0.008 & 0.7 & & \\
\hline & & & & & 2.237 & 0.014 \\
\hline \multirow[t]{2}{*}{220.0} & 4048.853 & 4048.855 & 0.007 & -0.2 & & \\
\hline & & & & & 2.186 & 0.014 \\
\hline \multirow[t]{2}{*}{230.0} & 4049.231 & 4049.236 & 0.008 & -0.7 & & \\
\hline & & & & & 1.858 & 0.013 \\
\hline \multirow[t]{2}{*}{240.0} & 4049.703 & 4049.702 & 0.005 & 0.3 & & \\
\hline & & & & & 1.976 & 0.014 \\
\hline \multirow[t]{2}{*}{250.0} & 4050.125 & 4050.136 & 0.009 & -1.2 & & \\
\hline & & & & & 1.559 & 0.012 \\
\hline \multirow[t]{2}{*}{260.0} & 4050.679 & 4050.679 & 0.002 & 0.3 & & \\
\hline & & & & & 1.862 & 0.010 \\
\hline \multirow[t]{2}{*}{270.0} & 4051.151 & 4051.143 & 0.007 & 1.2 & & \\
\hline & & & & & 2.384 & 0.017 \\
\hline \multirow[t]{2}{*}{280.0} & 4051.467 & 4051.474 & 0.015 & -0.5 & & \\
\hline & & & & & 2.319 & 0.018 \\
\hline \multirow[t]{2}{*}{290.0} & 4051.810 & 4051.821 & 0.008 & -1.5 & & \\
\hline & & & & & 1.948 & 0.016 \\
\hline \multirow[t]{2}{*}{300.0} & 4052.283 & 4052.264 & 0.009 & 2.1 & & \\
\hline & & & & & 2.709 & 0.014 \\
\hline 310.0 & 4052.507 & 4052.512 & 0.005 & -1.0 & & \\
\hline
\end{tabular}


Table 7: C4993 continued

\begin{tabular}{|c|c|c|c|c|c|c|}
\hline $\begin{array}{l}\text { depth } \\
\text { (feet) }\end{array}$ & $\begin{array}{r}\bar{g} \\
\text { (mGals) }\end{array}$ & $\begin{array}{r}\hat{g} \\
(\mathrm{mGals})\end{array}$ & $\begin{array}{r}\sigma_{g} \\
\text { (mGals) }\end{array}$ & Z-score & $\begin{array}{r}\text { density } \\
\left(\mathrm{g} / \mathrm{cm}^{3}\right)\end{array}$ & $\begin{array}{l}\sigma \text { density } \\
\left(\mathrm{g} / \mathrm{cm}^{3}\right)\end{array}$ \\
\hline & & & & & 2.358 & 0.015 \\
\hline \multirow{2}{*}{320.0} & 4052.853 & 4052.849 & 0.008 & 0.5 & & \\
\hline & & & & & 2.495 & 0.014 \\
\hline \multirow[t]{2}{*}{330.0} & 4053.150 & 4053.152 & 0.004 & -0.3 & & \\
\hline & & & & & 2.364 & 0.015 \\
\hline \multirow{2}{*}{340.0} & 4053.486 & 4053.488 & 0.011 & -0.2 & & \\
\hline & & & & & 2.316 & 0.017 \\
\hline \multirow[t]{2}{*}{350.0} & 4053.864 & 4053.836 & 0.010 & 2.8 & & \\
\hline & & & & & 2.839 & 0.015 \\
\hline \multirow[t]{2}{*}{360.0} & 4054.048 & 4054.050 & 0.003 & -1.0 & & \\
\hline & & & & & 2.115 & 0.012 \\
\hline \multirow[t]{2}{*}{370.0} & 4054.464 & 4054.450 & 0.007 & 2.0 & & \\
\hline & & & & & 2.687 & 0.013 \\
\hline \multirow[t]{2}{*}{380.0} & 4054.703 & 4054.704 & 0.005 & -0.0 & & \\
\hline & & & & & 2.674 & 0.009 \\
\hline \multirow{2}{*}{390.0} & 4054.961 & 4054.960 & 0.003 & 0.2 & & \\
\hline & & & & & 2.800 & 0.011 \\
\hline \multirow[t]{2}{*}{400.0} & 4055.186 & 4055.185 & 0.005 & 0.1 & & \\
\hline & & & & & 2.858 & 0.016 \\
\hline \multirow[t]{2}{*}{410.0} & 4055.395 & 4055.395 & 0.010 & -0.0 & & \\
\hline & & & & & 2.860 & 0.016 \\
\hline \multirow[t]{2}{*}{420.0} & 4055.604 & 4055.604 & 0.005 & 0.1 & & \\
\hline & & & & & 2.890 & 0.014 \\
\hline \multirow[t]{2}{*}{430.0} & 4055.804 & 4055.806 & 0.010 & -0.1 & & \\
\hline & & & & & 2.846 & 0.014 \\
\hline \multirow[t]{2}{*}{440.0} & 4056.019 & 4056.018 & 0.005 & 0.0 & & \\
\hline & & & & & 2.869 & 0.014 \\
\hline \multirow[t]{2}{*}{450.0} & 4056.225 & 4056.225 & 0.007 & -0.1 & & \\
\hline & & & & & 2.852 & 0.017 \\
\hline \multirow[t]{2}{*}{460.0} & 4056.437 & 4056.436 & 0.011 & 0.0 & & \\
\hline & & & & & 2.858 & 0.017 \\
\hline \multirow[t]{2}{*}{470.0} & 4056.637 & 4056.646 & 0.006 & -1.4 & & \\
\hline & & & & & 2.445 & 0.013 \\
\hline \multirow[t]{2}{*}{480.0} & 4056.955 & 4056.961 & 0.006 & -1.0 & & \\
\hline & & & & & 1.803 & 0.016 \\
\hline \multirow[t]{2}{*}{490.0} & 4057.440 & 4057.441 & 0.011 & -0.1 & & \\
\hline & & & & & 1.796 & 0.016 \\
\hline \multirow[t]{2}{*}{500.0} & 4057.922 & 4057.922 & 0.007 & -0.0 & & \\
\hline & & & & & 1.784 & 0.011 \\
\hline
\end{tabular}


Table 7: C4993 continued

\begin{tabular}{|c|c|c|c|c|c|c|}
\hline $\begin{array}{l}\text { depth } \\
\text { (feet) }\end{array}$ & $\begin{array}{r}\bar{g} \\
\text { (mGals) }\end{array}$ & $\begin{array}{r}\hat{g} \\
\text { (mGals) }\end{array}$ & $\begin{array}{r}\sigma_{g} \\
\text { (mGals) }\end{array}$ & Z-score & $\begin{array}{r}\text { density } \\
\left(\mathrm{g} / \mathrm{cm}^{3}\right)\end{array}$ & $\begin{array}{l}\sigma \text { density } \\
\left(\mathrm{g} / \mathrm{cm}^{3}\right)\end{array}$ \\
\hline \multirow[t]{2}{*}{510.0} & 4058.408 & 4058.407 & 0.004 & 0.4 & & \\
\hline & & & & & 1.981 & 0.010 \\
\hline \multirow[t]{2}{*}{520.0} & 4058.839 & 4058.841 & 0.005 & -0.4 & & \\
\hline & & & & & 1.754 & 0.008 \\
\hline \multirow[t]{2}{*}{530.0} & 4059.332 & 4059.332 & 0.002 & 0.1 & & \\
\hline & & & & & 1.819 & 0.007 \\
\hline \multirow[t]{2}{*}{540.0} & 4059.809 & 4059.808 & 0.004 & 0.3 & & \\
\hline & & & & & 2.121 & 0.013 \\
\hline \multirow[t]{2}{*}{550.0} & 4060.218 & 4060.207 & 0.008 & 1.5 & & \\
\hline & & & & & 2.505 & 0.017 \\
\hline \multirow[t]{2}{*}{560.0} & 4060.519 & 4060.506 & 0.011 & 1.2 & & \\
\hline & & & & & 2.727 & 0.016 \\
\hline \multirow[t]{2}{*}{570.0} & 4060.750 & 4060.750 & 0.007 & 0.1 & & \\
\hline & & & & & 2.762 & 0.012 \\
\hline \multirow[t]{2}{*}{580.0} & 4060.985 & 4060.984 & 0.005 & 0.1 & & \\
\hline & & & & & 2.821 & 0.014 \\
\hline \multirow[t]{2}{*}{590.0} & 4061.203 & 4061.203 & 0.007 & -0.1 & & \\
\hline & & & & & 2.802 & 0.017 \\
\hline \multirow{2}{*}{600.0} & 4061.430 & 4061.427 & 0.009 & 0.3 & & \\
\hline & & & & & 2.846 & 0.016 \\
\hline \multirow[t]{2}{*}{610.0} & 4061.641 & 4061.641 & 0.004 & 0.1 & & \\
\hline & & & & & 2.901 & 0.016 \\
\hline \multirow[t]{2}{*}{620.0} & 4061.837 & 4061.839 & 0.014 & -0.2 & & \\
\hline & & & & & 2.868 & 0.016 \\
\hline \multirow[t]{2}{*}{630.0} & 4062.046 & 4062.046 & 0.006 & 0.0 & & \\
\hline & & & & & 2.871 & 0.017 \\
\hline \multirow[t]{2}{*}{640.0} & 4062.251 & 4062.253 & 0.012 & -0.1 & & \\
\hline & & & & & 2.853 & 0.018 \\
\hline \multirow[t]{2}{*}{650.0} & 4062.459 & 4062.464 & 0.016 & -0.3 & & \\
\hline & & & & & 2.819 & 0.018 \\
\hline \multirow[t]{2}{*}{660.0} & 4062.685 & 4062.683 & 0.012 & 0.1 & & \\
\hline & & & & & 2.839 & 0.017 \\
\hline \multirow[t]{2}{*}{670.0} & 4062.899 & 4062.898 & 0.004 & 0.2 & & \\
\hline & & & & & 2.911 & 0.014 \\
\hline \multirow[t]{2}{*}{680.0} & 4063.093 & 4063.094 & 0.012 & -0.1 & & \\
\hline & & & & & 2.871 & 0.016 \\
\hline \multirow[t]{2}{*}{690.0} & 4063.301 & 4063.301 & 0.008 & 0.0 & & \\
\hline & & & & & 2.882 & 0.016 \\
\hline 700.0 & 4063.503 & 4063.505 & 0.007 & -0.2 & & \\
\hline
\end{tabular}


Table 7: C4993 continued

\begin{tabular}{|c|c|c|c|c|c|c|}
\hline $\begin{array}{l}\text { depth } \\
\text { (feet) }\end{array}$ & $\begin{array}{r}\bar{g} \\
\text { (mGals) }\end{array}$ & $\begin{array}{r}\hat{g} \\
(\mathrm{mGals})\end{array}$ & $\begin{array}{r}\sigma_{g} \\
\text { (mGals) }\end{array}$ & Z-score & $\begin{array}{r}\text { density } \\
\left(\mathrm{g} / \mathrm{cm}^{3}\right)\end{array}$ & $\begin{array}{l}\sigma \text { density } \\
\left(\mathrm{g} / \mathrm{cm}^{3}\right)\end{array}$ \\
\hline & & & & & 2.826 & 0.016 \\
\hline \multirow[t]{2}{*}{710.0} & 4063.716 & 4063.722 & 0.007 & -0.9 & & \\
\hline & & & & & 2.584 & 0.016 \\
\hline \multirow[t]{2}{*}{720.0} & 4063.987 & 4064.003 & 0.010 & -1.6 & & \\
\hline & & & & & 2.184 & 0.016 \\
\hline \multirow{2}{*}{730.0} & 4064.380 & 4064.385 & 0.008 & -0.5 & & \\
\hline & & & & & 1.978 & 0.015 \\
\hline \multirow[t]{2}{*}{740.0} & 4064.824 & 4064.820 & 0.008 & 0.5 & & \\
\hline & & & & & 2.097 & 0.015 \\
\hline \multirow[t]{2}{*}{750.0} & 4065.229 & 4065.224 & 0.007 & 0.7 & & \\
\hline & & & & & 2.449 & 0.011 \\
\hline \multirow[t]{2}{*}{760.0} & 4065.540 & 4065.538 & 0.004 & 0.5 & & \\
\hline & & & & & 2.682 & 0.010 \\
\hline \multirow[t]{2}{*}{770.0} & 4065.794 & 4065.793 & 0.004 & 0.2 & & \\
\hline & & & & & 2.765 & 0.009 \\
\hline \multirow{2}{*}{780.0} & 4066.027 & 4066.027 & 0.003 & -0.0 & & \\
\hline & & & & & 2.748 & 0.007 \\
\hline \multirow[t]{2}{*}{790.0} & 4066.265 & 4066.265 & 0.002 & 0.1 & & \\
\hline & & & & & 2.836 & 0.011 \\
\hline \multirow[t]{2}{*}{800.0} & 4066.479 & 4066.480 & 0.008 & -0.1 & & \\
\hline & & & & & 2.782 & 0.013 \\
\hline \multirow[t]{2}{*}{810.0} & 4066.709 & 4066.709 & 0.006 & 0.0 & & \\
\hline & & & & & 2.793 & 0.011 \\
\hline \multirow[t]{2}{*}{820.0} & 4066.936 & 4066.936 & 0.002 & 0.0 & & \\
\hline & & & & & 2.797 & 0.011 \\
\hline \multirow[t]{2}{*}{830.0} & 4067.156 & 4067.161 & 0.006 & -0.9 & & \\
\hline & & & & & 2.474 & 0.015 \\
\hline \multirow[t]{2}{*}{840.0} & 4067.453 & 4067.469 & 0.011 & -1.4 & & \\
\hline & & & & & 1.968 & 0.016 \\
\hline \multirow[t]{2}{*}{850.0} & 4067.908 & 4067.907 & 0.007 & 0.1 & & \\
\hline & & & & & 2.012 & 0.014 \\
\hline \multirow[t]{2}{*}{860.0} & 4068.332 & 4068.333 & 0.008 & -0.1 & & \\
\hline & & & & & 1.970 & 0.015 \\
\hline \multirow[t]{2}{*}{870.0} & 4068.770 & 4068.769 & 0.009 & 0.1 & & \\
\hline & & & & & 2.006 & 0.015 \\
\hline \multirow[t]{2}{*}{880.0} & 4069.196 & 4069.197 & 0.009 & -0.1 & & \\
\hline & & & & & 1.958 & 0.012 \\
\hline \multirow[t]{2}{*}{890.0} & 4069.637 & 4069.637 & 0.004 & 0.1 & & \\
\hline & & & & & 2.001 & 0.013 \\
\hline
\end{tabular}


Table 7: C4993 continued

\begin{tabular}{|c|c|c|c|c|c|c|}
\hline $\begin{array}{l}\text { depth } \\
\text { (feet) }\end{array}$ & $\begin{array}{r}\bar{g} \\
\text { (mGals) }\end{array}$ & $\begin{array}{r}\hat{g} \\
\text { (mGals) }\end{array}$ & $\begin{array}{r}\sigma_{g} \\
\text { (mGals) }\end{array}$ & Z-score & $\begin{array}{r}\text { density } \\
\left(\mathrm{g} / \mathrm{cm}^{3}\right)\end{array}$ & $\begin{array}{l}\sigma \text { density } \\
\left(\mathrm{g} / \mathrm{cm}^{3}\right)\end{array}$ \\
\hline \multirow[t]{2}{*}{900.0} & 4070.061 & 4070.066 & 0.010 & -0.5 & & \\
\hline & & & & & 1.814 & 0.012 \\
\hline \multirow{2}{*}{910.0} & 4070.543 & 4070.543 & 0.002 & -0.1 & & \\
\hline & & & & & 1.755 & 0.015 \\
\hline \multirow[t]{2}{*}{920.0} & 4071.038 & 4071.035 & 0.013 & 0.3 & & \\
\hline & & & & & 1.835 & 0.018 \\
\hline \multirow[t]{2}{*}{930.0} & 4071.539 & 4071.506 & 0.014 & 2.4 & & \\
\hline & & & & & 2.185 & 0.018 \\
\hline \multirow[t]{2}{*}{940.0} & 4071.937 & 4071.888 & 0.017 & 2.9 & & \\
\hline & & & & & 2.518 & 0.018 \\
\hline \multirow[t]{2}{*}{950.0} & 4072.190 & 4072.185 & 0.011 & 0.4 & & \\
\hline & & & & & 2.657 & 0.016 \\
\hline \multirow[t]{2}{*}{960.0} & 4072.446 & 4072.446 & 0.007 & 0.1 & & \\
\hline & & & & & 2.684 & 0.014 \\
\hline \multirow[t]{2}{*}{970.0} & 4072.698 & 4072.700 & 0.006 & -0.3 & & \\
\hline & & & & & 2.575 & 0.013 \\
\hline \multirow[t]{2}{*}{980.0} & 4072.978 & 4072.982 & 0.005 & -0.8 & & \\
\hline & & & & & 2.281 & 0.014 \\
\hline \multirow{2}{*}{990.0} & 4073.345 & 4073.339 & 0.009 & 0.7 & & \\
\hline & & & & & 2.535 & 0.015 \\
\hline \multirow[t]{2}{*}{1000.0} & 4073.637 & 4073.632 & 0.007 & 0.7 & & \\
\hline & & & & & 2.737 & 0.015 \\
\hline \multirow[t]{2}{*}{1010.0} & 4073.873 & 4073.873 & 0.007 & -0.0 & & \\
\hline & & & & & 2.739 & 0.014 \\
\hline \multirow[t]{2}{*}{1020.0} & 4074.113 & 4074.113 & 0.006 & -0.0 & & \\
\hline & & & & & 2.739 & 0.013 \\
\hline \multirow[t]{2}{*}{1030.0} & 4074.354 & 4074.353 & 0.005 & 0.2 & & \\
\hline & & & & & 2.800 & 0.015 \\
\hline \multirow[t]{2}{*}{1040.0} & 4074.577 & 4074.578 & 0.012 & -0.1 & & \\
\hline & & & & & 2.788 & 0.014 \\
\hline \multirow[t]{2}{*}{1050.0} & 4074.805 & 4074.805 & 0.003 & -0.0 & & \\
\hline & & & & & 2.763 & 0.009 \\
\hline \multirow[t]{2}{*}{1060.0} & 4075.040 & 4075.040 & 0.003 & 0.0 & & \\
\hline & & & & & 2.785 & 0.011 \\
\hline \multirow[t]{2}{*}{1070.0} & 4075.269 & 4075.268 & 0.005 & 0.1 & & \\
\hline & & & & & 2.820 & 0.017 \\
\hline \multirow[t]{2}{*}{1080.0} & 4075.486 & 4075.488 & 0.021 & -0.1 & & \\
\hline & & & & & 2.812 & 0.016 \\
\hline 1090.0 & 4075.704 & 4075.709 & 0.004 & -1.1 & & \\
\hline
\end{tabular}


Table 7: C4993 continued

\begin{tabular}{|c|c|c|c|c|c|c|}
\hline $\begin{array}{l}\text { depth } \\
\text { (feet) }\end{array}$ & $\begin{array}{r}\bar{g} \\
(\mathrm{mGals})\end{array}$ & $\begin{array}{r}\hat{g} \\
(\mathrm{mGals})\end{array}$ & $\begin{array}{r}\sigma_{g} \\
\text { (mGals) }\end{array}$ & Z-score & $\begin{array}{r}\text { density } \\
\left(\mathrm{g} / \mathrm{cm}^{3}\right)\end{array}$ & $\begin{array}{l}\sigma \text { density } \\
\left(\mathrm{g} / \mathrm{cm}^{3}\right)\end{array}$ \\
\hline & & & & & 2.287 & 0.014 \\
\hline \multirow[t]{2}{*}{1100.0} & 4076.057 & 4076.065 & 0.008 & -1.1 & & \\
\hline & & & & & 2.018 & 0.017 \\
\hline \multirow[t]{2}{*}{1110.0} & 4076.490 & 4076.490 & 0.015 & 0.0 & & \\
\hline & & & & & 2.031 & 0.016 \\
\hline \multirow{2}{*}{1120.0} & 4076.911 & 4076.910 & 0.006 & 0.2 & & \\
\hline & & & & & 2.104 & 0.016 \\
\hline \multirow[t]{2}{*}{1130.0} & 4077.311 & 4077.313 & 0.012 & -0.1 & & \\
\hline & & & & & 2.069 & 0.017 \\
\hline \multirow[t]{2}{*}{1140.0} & 4077.726 & 4077.725 & 0.010 & 0.2 & & \\
\hline & & & & & 2.110 & 0.017 \\
\hline \multirow[t]{2}{*}{1150.0} & 4078.122 & 4078.126 & 0.012 & -0.3 & & \\
\hline & & & & & 2.011 & 0.017 \\
\hline \multirow[t]{2}{*}{1160.0} & 4078.548 & 4078.552 & 0.008 & -0.4 & & \\
\hline & & & & & 1.909 & 0.017 \\
\hline \multirow[t]{2}{*}{1170.0} & 4078.999 & 4079.005 & 0.013 & -0.4 & & \\
\hline & & & & & 1.784 & 0.017 \\
\hline \multirow[t]{2}{*}{1180.0} & 4079.495 & 4079.490 & 0.008 & 0.6 & & \\
\hline & & & & & 1.934 & 0.018 \\
\hline \multirow[t]{2}{*}{1190.0} & 4079.967 & 4079.936 & 0.014 & 2.2 & & \\
\hline & & & & & 2.388 & 0.018 \\
\hline \multirow[t]{2}{*}{1200.0} & 4080.280 & 4080.266 & 0.015 & 0.9 & & \\
\hline & & & & & 2.650 & 0.016 \\
\hline \multirow[t]{2}{*}{1210.0} & 4080.529 & 4080.529 & 0.005 & 0.1 & & \\
\hline & & & & & 2.678 & 0.013 \\
\hline \multirow[t]{2}{*}{1220.0} & 4080.786 & 4080.785 & 0.006 & 0.2 & & \\
\hline & & & & & 2.740 & 0.013 \\
\hline \multirow[t]{2}{*}{1230.0} & 4081.026 & 4081.025 & 0.005 & 0.3 & & \\
\hline & & & & & 2.857 & 0.016 \\
\hline \multirow[t]{2}{*}{1240.0} & 4081.233 & 4081.235 & 0.019 & -0.1 & & \\
\hline & & & & & 2.835 & 0.016 \\
\hline \multirow[t]{2}{*}{1250.0} & 4081.451 & 4081.451 & 0.003 & -0.0 & & \\
\hline & & & & & 2.825 & 0.007 \\
\hline \multirow[t]{2}{*}{1260.0} & 4081.670 & 4081.670 & 0.002 & -0.0 & & \\
\hline & & & & & 2.819 & 0.009 \\
\hline \multirow[t]{2}{*}{1270.0} & 4081.890 & 4081.889 & 0.004 & 0.1 & & \\
\hline & & & & & 2.855 & 0.012 \\
\hline \multirow[t]{2}{*}{1280.0} & 4082.100 & 4082.100 & 0.005 & 0.0 & & \\
\hline & & & & & 2.865 & 0.016 \\
\hline
\end{tabular}


Table 7: C4993 continued

\begin{tabular}{|c|c|c|c|c|c|c|}
\hline $\begin{array}{l}\text { depth } \\
\text { (feet) }\end{array}$ & $\begin{array}{r}\bar{g} \\
(\mathrm{mGals})\end{array}$ & $\begin{array}{r}\hat{g} \\
(\mathrm{mGals})\end{array}$ & $\begin{array}{r}\sigma_{g} \\
(\mathrm{mGals})\end{array}$ & Z-score & $\begin{array}{r}\text { density } \\
\left(\mathrm{g} / \mathrm{cm}^{3}\right)\end{array}$ & $\begin{array}{l}\sigma \text { density } \\
\left(\mathrm{g} / \mathrm{cm}^{3}\right)\end{array}$ \\
\hline \multirow[t]{2}{*}{1290.0} & 4082.313 & 4082.308 & 0.013 & 0.3 & & \\
\hline & & & & & 2.957 & 0.018 \\
\hline \multirow[t]{2}{*}{1300.0} & 4082.493 & 4082.493 & 0.020 & 0.0 & & \\
\hline & & & & & 2.960 & 0.017 \\
\hline \multirow[t]{2}{*}{1310.0} & 4082.676 & 4082.677 & 0.004 & -0.1 & & \\
\hline & & & & & 2.889 & 0.012 \\
\hline \multirow[t]{2}{*}{1320.0} & 4082.879 & 4082.879 & 0.006 & -0.1 & & \\
\hline & & & & & 2.871 & 0.012 \\
\hline \multirow[t]{2}{*}{1330.0} & 4083.086 & 4083.086 & 0.004 & 0.0 & & \\
\hline & & & & & 2.880 & 0.009 \\
\hline \multirow[t]{2}{*}{1340.0} & 4083.290 & 4083.290 & 0.004 & -0.0 & & \\
\hline & & & & & 2.870 & 0.018 \\
\hline \multirow[t]{2}{*}{1350.1} & 4083.470 & 4083.497 & 0.025 & -1.1 & & \\
\hline & & & & & 2.723 & 0.017 \\
\hline \multirow[t]{2}{*}{1360.0} & 4083.704 & 4083.740 & 0.020 & -1.9 & & \\
\hline & & & & & 2.542 & 0.018 \\
\hline \multirow[t]{2}{*}{1370.0} & 4084.031 & 4084.031 & 0.003 & 0.1 & & \\
\hline & & & & & 2.595 & 0.017 \\
\hline \multirow[t]{2}{*}{1380.0} & 4084.315 & 4084.308 & 0.015 & 0.4 & & \\
\hline & & & & & 2.655 & 0.019 \\
\hline 1390.0 & 4084.562 & 4084.570 & 0.010 & -0.8 & & \\
\hline
\end{tabular}




\section{B.2 C4996}

Table 8: Well C4996 Inversion densities

\begin{tabular}{|c|c|c|c|c|c|c|}
\hline $\begin{array}{l}\text { depth } \\
\text { (feet) }\end{array}$ & $\begin{array}{r}\bar{g} \\
\text { (mGals) }\end{array}$ & $\begin{array}{r}\hat{g} \\
(\mathrm{mGals})\end{array}$ & $\begin{array}{r}\sigma_{g} \\
\text { (mGals) }\end{array}$ & Z-score & $\begin{array}{r}\text { density } \\
\left(\mathrm{g} / \mathrm{cm}^{3}\right)\end{array}$ & $\begin{array}{l}\sigma \sigma \text { density } \\
\left(\mathrm{g} / \mathrm{cm}^{3}\right)\end{array}$ \\
\hline \multirow[t]{2}{*}{1.1} & 4056.030 & 4056.117 & 0.015 & -5.8 & & \\
\hline & & & & & 2.293 & 0.016 \\
\hline \multirow[t]{2}{*}{10.0} & 4056.397 & 4056.431 & 0.010 & -3.5 & & \\
\hline & & & & & 1.463 & 0.017 \\
\hline \multirow{2}{*}{20.0} & 4057.046 & 4056.998 & 0.024 & 2.1 & & \\
\hline & & & & & 1.666 & 0.015 \\
\hline \multirow[t]{2}{*}{30.0} & 4057.534 & 4057.512 & 0.021 & 1.0 & & \\
\hline & & & & & 1.744 & 0.015 \\
\hline \multirow[t]{2}{*}{40.0} & 4058.048 & 4058.007 & 0.028 & 1.5 & & \\
\hline & & & & & 1.907 & 0.017 \\
\hline \multirow[t]{2}{*}{50.0} & 4058.443 & 4058.460 & 0.012 & -1.4 & & \\
\hline & & & & & 1.604 & 0.016 \\
\hline \multirow[t]{2}{*}{60.0} & 4058.980 & 4058.990 & 0.015 & -0.7 & & \\
\hline & & & & & 1.536 & 0.016 \\
\hline \multirow[t]{2}{*}{70.0} & 4059.540 & 4059.538 & 0.011 & 0.2 & & \\
\hline & & & & & 1.588 & 0.015 \\
\hline \multirow[t]{2}{*}{80.0} & 4060.077 & 4060.072 & 0.009 & 0.5 & & \\
\hline & & & & & 1.696 & 0.016 \\
\hline \multirow[t]{2}{*}{90.0} & 4060.575 & 4060.579 & 0.021 & -0.2 & & \\
\hline & & & & & 1.668 & 0.015 \\
\hline \multirow[t]{2}{*}{100.0} & 4061.093 & 4061.093 & 0.004 & 0.2 & & \\
\hline & & & & & 1.756 & 0.014 \\
\hline \multirow[t]{2}{*}{110.0} & 4061.588 & 4061.583 & 0.010 & 0.5 & & \\
\hline & & & & & 1.848 & 0.016 \\
\hline \multirow[t]{2}{*}{120.0} & 4062.029 & 4062.052 & 0.017 & -1.3 & & \\
\hline & & & & & 1.638 & 0.016 \\
\hline \multirow[t]{2}{*}{130.0} & 4062.589 & 4062.573 & 0.010 & 1.5 & & \\
\hline & & & & & 1.863 & 0.016 \\
\hline \multirow[t]{2}{*}{140.0} & 4063.025 & 4063.037 & 0.013 & -0.9 & & \\
\hline & & & & & 1.669 & 0.016 \\
\hline \multirow[t]{2}{*}{150.0} & 4063.554 & 4063.552 & 0.013 & 0.2 & & \\
\hline & & & & & 1.702 & 0.015 \\
\hline \multirow[t]{2}{*}{160.0} & 4064.058 & 4064.057 & 0.007 & 0.2 & & \\
\hline & & & & & 1.768 & 0.015 \\
\hline \multirow[t]{2}{*}{170.0} & 4064.545 & 4064.545 & 0.018 & 0.0 & & \\
\hline & & & & & 1.775 & 0.014 \\
\hline 180.0 & 4065.033 & 4065.031 & 0.003 & 0.5 & & \\
\hline
\end{tabular}


Table 8: C4996 continued

\begin{tabular}{|c|c|c|c|c|c|c|}
\hline $\begin{array}{l}\text { depth } \\
\text { (feet) }\end{array}$ & $\begin{array}{r}\bar{g} \\
(\mathrm{mGals})\end{array}$ & $\begin{array}{r}\hat{g} \\
\text { (mGals) }\end{array}$ & $\begin{array}{r}\sigma_{g} \\
\text { (mGals) }\end{array}$ & Z-score & $\begin{array}{r}\text { density } \\
\left(\mathrm{g} / \mathrm{cm}^{3}\right)\end{array}$ & $\begin{array}{c}\sigma \text { density } \\
\left(\mathrm{g} / \mathrm{cm}^{3}\right)\end{array}$ \\
\hline & & & & & 2.050 & 0.012 \\
\hline \multirow[t]{2}{*}{190.0} & 4065.446 & 4065.447 & 0.010 & -0.1 & & \\
\hline & & & & & 2.029 & 0.013 \\
\hline \multirow[t]{2}{*}{200.0} & 4065.874 & 4065.869 & 0.005 & 0.9 & & \\
\hline & & & & & 2.316 & 0.014 \\
\hline \multirow[t]{2}{*}{210.0} & 4066.200 & 4066.218 & 0.011 & -1.5 & & \\
\hline & & & & & 1.956 & 0.015 \\
\hline \multirow[t]{2}{*}{220.0} & 4066.660 & 4066.658 & 0.014 & 0.1 & & \\
\hline & & & & & 1.972 & 0.015 \\
\hline \multirow[t]{2}{*}{230.0} & 4067.094 & 4067.094 & 0.001 & 0.1 & & \\
\hline & & & & & 2.151 & 0.012 \\
\hline \multirow{2}{*}{240.0} & 4067.482 & 4067.485 & 0.012 & -0.2 & & \\
\hline & & & & & 2.096 & 0.013 \\
\hline \multirow[t]{2}{*}{250.0} & 4067.889 & 4067.890 & 0.002 & -0.3 & & \\
\hline & & & & & 1.879 & 0.009 \\
\hline \multirow[t]{2}{*}{260.0} & 4068.358 & 4068.350 & 0.006 & 1.3 & & \\
\hline & & & & & 2.510 & 0.012 \\
\hline \multirow{2}{*}{270.0} & 4068.642 & 4068.648 & 0.005 & -1.2 & & \\
\hline & & & & & 2.170 & 0.013 \\
\hline \multirow[t]{2}{*}{280.0} & 4069.036 & 4069.034 & 0.006 & 0.3 & & \\
\hline & & & & & 2.240 & 0.016 \\
\hline \multirow[t]{2}{*}{290.0} & 4069.417 & 4069.402 & 0.023 & 0.7 & & \\
\hline & & & & & 2.332 & 0.016 \\
\hline \multirow[t]{2}{*}{300.0} & 4069.760 & 4069.746 & 0.022 & 0.6 & & \\
\hline & & & & & 2.400 & 0.016 \\
\hline \multirow[t]{2}{*}{310.0} & 4070.088 & 4070.072 & 0.022 & 0.7 & & \\
\hline & & & & & 2.489 & 0.017 \\
\hline \multirow[t]{2}{*}{320.0} & 4070.379 & 4070.377 & 0.015 & 0.2 & & \\
\hline & & & & & 2.510 & 0.016 \\
\hline \multirow[t]{2}{*}{330.0} & 4070.689 & 4070.675 & 0.023 & 0.6 & & \\
\hline & & & & & 2.560 & 0.015 \\
\hline \multirow[t]{2}{*}{340.0} & 4070.968 & 4070.962 & 0.025 & 0.2 & & \\
\hline & & & & & 2.584 & 0.016 \\
\hline \multirow[t]{2}{*}{350.0} & 4071.242 & 4071.241 & 0.017 & 0.0 & & \\
\hline & & & & & 2.598 & 0.016 \\
\hline \multirow[t]{2}{*}{360.0} & 4071.520 & 4071.517 & 0.010 & 0.3 & & \\
\hline & & & & & 2.711 & 0.015 \\
\hline \multirow[t]{2}{*}{370.0} & 4071.769 & 4071.765 & 0.011 & 0.4 & & \\
\hline & & & & & 2.822 & 0.015 \\
\hline
\end{tabular}


Table 8: C4996 continued

\begin{tabular}{|c|c|c|c|c|c|c|}
\hline $\begin{array}{l}\text { depth } \\
\text { (feet) }\end{array}$ & $\begin{array}{r}\bar{g} \\
(\mathrm{mGals})\end{array}$ & $\begin{array}{r}\hat{g} \\
(\mathrm{mGals})\end{array}$ & $\begin{array}{r}\sigma_{g} \\
\text { (mGals) }\end{array}$ & Z-score & $\begin{array}{r}\text { density } \\
\left(\mathrm{g} / \mathrm{cm}^{3}\right)\end{array}$ & $\begin{array}{c}\sigma \text { density } \\
\left(\mathrm{g} / \mathrm{cm}^{3}\right)\end{array}$ \\
\hline \multirow[t]{2}{*}{380.0} & 4071.987 & 4071.984 & 0.009 & 0.3 & & \\
\hline & & & & & 2.940 & 0.016 \\
\hline \multirow{2}{*}{390.0} & 4072.166 & 4072.173 & 0.029 & -0.2 & & \\
\hline & & & & & 2.918 & 0.015 \\
\hline \multirow[t]{2}{*}{400.0} & 4072.357 & 4072.367 & 0.017 & -0.6 & & \\
\hline & & & & & 2.869 & 0.017 \\
\hline \multirow[t]{2}{*}{410.0} & 4072.575 & 4072.574 & 0.009 & 0.1 & & \\
\hline & & & & & 2.893 & 0.017 \\
\hline \multirow[t]{2}{*}{420.0} & 4072.775 & 4072.775 & 0.014 & -0.0 & & \\
\hline & & & & & 2.894 & 0.016 \\
\hline \multirow[t]{2}{*}{430.0} & 4072.977 & 4072.976 & 0.019 & 0.1 & & \\
\hline & & & & & 2.902 & 0.016 \\
\hline \multirow[t]{2}{*}{440.0} & 4073.162 & 4073.174 & 0.021 & -0.6 & & \\
\hline & & & & & 2.805 & 0.017 \\
\hline \multirow[t]{2}{*}{450.0} & 4073.362 & 4073.398 & 0.011 & -3.3 & & \\
\hline & & & & & 2.241 & 0.016 \\
\hline \multirow[t]{2}{*}{460.0} & 4073.726 & 4073.765 & 0.014 & -2.7 & & \\
\hline & & & & & 1.944 & 0.016 \\
\hline \multirow[t]{2}{*}{470.0} & 4074.209 & 4074.209 & 0.011 & 0.0 & & \\
\hline & & & & & 1.956 & 0.017 \\
\hline \multirow[t]{2}{*}{480.0} & 4074.662 & 4074.648 & 0.015 & 0.9 & & \\
\hline & & & & & 2.027 & 0.015 \\
\hline \multirow[t]{2}{*}{490.0} & 4075.080 & 4075.071 & 0.041 & 0.2 & & \\
\hline & & & & & 2.049 & 0.015 \\
\hline \multirow[t]{2}{*}{500.0} & 4075.491 & 4075.487 & 0.020 & 0.2 & & \\
\hline & & & & & 2.071 & 0.017 \\
\hline \multirow[t]{2}{*}{510.0} & 4075.910 & 4075.898 & 0.021 & 0.6 & & \\
\hline & & & & & 2.144 & 0.017 \\
\hline \multirow[t]{2}{*}{520.0} & 4076.324 & 4076.291 & 0.016 & 2.1 & & \\
\hline & & & & & 2.435 & 0.016 \\
\hline \multirow[t]{2}{*}{530.0} & 4076.627 & 4076.609 & 0.028 & 0.7 & & \\
\hline & & & & & 2.491 & 0.015 \\
\hline \multirow[t]{2}{*}{540.0} & 4076.930 & 4076.912 & 0.031 & 0.6 & & \\
\hline & & & & & 2.562 & 0.016 \\
\hline \multirow[t]{2}{*}{550.0} & 4077.215 & 4077.197 & 0.017 & 1.1 & & \\
\hline & & & & & 2.663 & 0.017 \\
\hline \multirow[t]{2}{*}{560.0} & 4077.470 & 4077.457 & 0.012 & 1.1 & & \\
\hline & & & & & 2.769 & 0.016 \\
\hline 570.0 & 4077.713 & 4077.689 & 0.022 & 1.1 & & \\
\hline
\end{tabular}


Table 8: C4996 continued

\begin{tabular}{|c|c|c|c|c|c|c|}
\hline $\begin{array}{l}\text { depth } \\
\text { (feet) }\end{array}$ & $\begin{array}{r}\bar{g} \\
\text { (mGals) }\end{array}$ & $\begin{array}{r}\hat{g} \\
(\mathrm{mGals})\end{array}$ & $\begin{array}{r}\sigma_{g} \\
\text { (mGals) } \\
\end{array}$ & Z-score & $\begin{array}{r}\text { density } \\
\left(\mathrm{g} / \mathrm{cm}^{3}\right)\end{array}$ & $\begin{array}{l}\sigma \text { density } \\
\left(\mathrm{g} / \mathrm{cm}^{3}\right)\end{array}$ \\
\hline & & & & & 2.847 & 0.016 \\
\hline \multirow[t]{2}{*}{580.0} & 4077.910 & 4077.902 & 0.019 & 0.4 & & \\
\hline & & & & & 2.882 & 0.017 \\
\hline \multirow[t]{2}{*}{590.0} & 4078.106 & 4078.106 & 0.029 & 0.0 & & \\
\hline & & & & & 2.887 & 0.017 \\
\hline \multirow[t]{2}{*}{600.0} & 4078.302 & 4078.308 & 0.027 & -0.2 & & \\
\hline & & & & & 2.863 & 0.016 \\
\hline \multirow{2}{*}{610.0} & 4078.511 & 4078.517 & 0.029 & -0.2 & & \\
\hline & & & & & 2.872 & 0.016 \\
\hline \multirow[t]{2}{*}{620.7} & 4078.738 & 4078.737 & 0.012 & 0.1 & & \\
\hline & & & & & 2.842 & 0.017 \\
\hline \multirow{2}{*}{630.0} & 4078.938 & 4078.937 & 0.014 & 0.1 & & \\
\hline & & & & & 2.894 & 0.017 \\
\hline \multirow[t]{2}{*}{640.0} & 4079.134 & 4079.137 & 0.021 & -0.1 & & \\
\hline & & & & & 2.863 & 0.015 \\
\hline \multirow[t]{2}{*}{650.0} & 4079.332 & 4079.346 & 0.027 & -0.5 & & \\
\hline & & & & & 2.836 & 0.013 \\
\hline \multirow[t]{2}{*}{660.0} & 4079.564 & 4079.561 & 0.024 & 0.1 & & \\
\hline & & & & & 2.845 & 0.015 \\
\hline \multirow[t]{2}{*}{670.0} & 4079.774 & 4079.774 & 0.004 & 0.0 & & \\
\hline & & & & & 2.848 & 0.017 \\
\hline \multirow[t]{2}{*}{680.0} & 4079.972 & 4079.986 & 0.015 & -1.0 & & \\
\hline & & & & & 2.751 & 0.016 \\
\hline \multirow[t]{2}{*}{690.0} & 4080.165 & 4080.223 & 0.032 & -1.8 & & \\
\hline & & & & & 2.586 & 0.015 \\
\hline \multirow[t]{2}{*}{700.0} & 4080.420 & 4080.503 & 0.020 & -4.2 & & \\
\hline & & & & & 2.269 & 0.017 \\
\hline \multirow[t]{2}{*}{710.0} & 4080.852 & 4080.864 & 0.010 & -1.1 & & \\
\hline & & & & & 2.152 & 0.017 \\
\hline \multirow[t]{2}{*}{720.0} & 4081.276 & 4081.253 & 0.020 & 1.2 & & \\
\hline & & & & & 2.357 & 0.016 \\
\hline \multirow[t]{2}{*}{730.0} & 4081.629 & 4081.591 & 0.022 & 1.7 & & \\
\hline & & & & & 2.481 & 0.016 \\
\hline \multirow[t]{2}{*}{740.0} & 4081.921 & 4081.897 & 0.015 & 1.5 & & \\
\hline & & & & & 2.674 & 0.017 \\
\hline \multirow[t]{2}{*}{750.0} & 4082.161 & 4082.154 & 0.011 & 0.6 & & \\
\hline & & & & & 2.784 & 0.017 \\
\hline \multirow[t]{2}{*}{760.0} & 4082.381 & 4082.383 & 0.016 & -0.1 & & \\
\hline & & & & & 2.777 & 0.017 \\
\hline
\end{tabular}


Table 8: C4996 continued

\begin{tabular}{|c|c|c|c|c|c|c|}
\hline $\begin{array}{l}\text { depth } \\
\text { (feet) }\end{array}$ & $\begin{array}{r}\bar{g} \\
(\mathrm{mGals})\end{array}$ & $\begin{array}{r}\hat{g} \\
(\mathrm{mGals})\end{array}$ & $\begin{array}{r}\sigma_{g} \\
\text { (mGals) }\end{array}$ & Z-score & $\begin{array}{r}\text { density } \\
\left(\mathrm{g} / \mathrm{cm}^{3}\right)\end{array}$ & $\begin{array}{c}\sigma \text { density } \\
\left(\mathrm{g} / \mathrm{cm}^{3}\right)\end{array}$ \\
\hline \multirow[t]{2}{*}{770.0} & 4082.616 & 4082.613 & 0.016 & 0.2 & & \\
\hline & & & & & 2.795 & 0.016 \\
\hline \multirow{2}{*}{780.0} & 4082.845 & 4082.839 & 0.022 & 0.2 & & \\
\hline & & & & & 2.815 & 0.016 \\
\hline \multirow[t]{2}{*}{790.0} & 4083.061 & 4083.060 & 0.029 & 0.0 & & \\
\hline & & & & & 2.818 & 0.017 \\
\hline \multirow[t]{2}{*}{800.0} & 4083.273 & 4083.280 & 0.015 & -0.4 & & \\
\hline & & & & & 2.777 & 0.016 \\
\hline \multirow[t]{2}{*}{810.0} & 4083.469 & 4083.511 & 0.025 & -1.7 & & \\
\hline & & & & & 2.579 & 0.017 \\
\hline \multirow[t]{2}{*}{820.0} & 4083.770 & 4083.792 & 0.008 & -2.8 & & \\
\hline & & & & & 2.036 & 0.016 \\
\hline \multirow[t]{2}{*}{830.0} & 4084.212 & 4084.211 & 0.013 & 0.0 & & \\
\hline & & & & & 2.040 & 0.016 \\
\hline \multirow[t]{2}{*}{840.0} & 4084.630 & 4084.631 & 0.013 & -0.0 & & \\
\hline & & & & & 2.039 & 0.016 \\
\hline \multirow{2}{*}{850.0} & 4085.048 & 4085.050 & 0.021 & -0.1 & & \\
\hline & & & & & 2.006 & 0.016 \\
\hline \multirow[t]{2}{*}{860.0} & 4085.474 & 4085.478 & 0.014 & -0.3 & & \\
\hline & & & & & 1.943 & 0.016 \\
\hline \multirow[t]{2}{*}{870.0} & 4085.920 & 4085.921 & 0.022 & -0.1 & & \\
\hline & & & & & 1.919 & 0.016 \\
\hline \multirow[t]{2}{*}{880.0} & 4086.364 & 4086.371 & 0.014 & -0.5 & & \\
\hline & & & & & 1.806 & 0.017 \\
\hline \multirow[t]{2}{*}{890.0} & 4086.857 & 4086.850 & 0.028 & 0.2 & & \\
\hline & & & & & 1.874 & 0.017 \\
\hline \multirow[t]{2}{*}{900.0} & 4087.337 & 4087.311 & 0.020 & 1.3 & & \\
\hline & & & & & 2.113 & 0.017 \\
\hline \multirow[t]{2}{*}{910.0} & 4087.751 & 4087.711 & 0.021 & 1.9 & & \\
\hline & & & & & 2.469 & 0.017 \\
\hline \multirow[t]{2}{*}{920.0} & 4088.039 & 4088.021 & 0.020 & 0.9 & & \\
\hline & & & & & 2.640 & 0.016 \\
\hline \multirow[t]{2}{*}{930.0} & 4088.292 & 4088.286 & 0.025 & 0.2 & & \\
\hline & & & & & 2.668 & 0.016 \\
\hline \multirow[t]{2}{*}{940.0} & 4088.544 & 4088.544 & 0.008 & 0.0 & & \\
\hline & & & & & 2.677 & 0.016 \\
\hline \multirow[t]{2}{*}{950.0} & 4088.804 & 4088.801 & 0.012 & 0.3 & & \\
\hline & & & & & 2.720 & 0.015 \\
\hline 960.0 & 4089.045 & 4089.046 & 0.004 & -0.2 & & \\
\hline
\end{tabular}


Table 8: C4996 continued

\begin{tabular}{|c|c|c|c|c|c|c|}
\hline $\begin{array}{l}\text { depth } \\
\text { (feet) }\end{array}$ & $\begin{array}{r}\bar{g} \\
(\mathrm{mGals})\end{array}$ & $\begin{array}{r}\hat{g} \\
\text { (mGals) }\end{array}$ & $\begin{array}{r}\sigma_{g} \\
\text { (mGals) }\end{array}$ & Z-score & $\begin{array}{r}\text { density } \\
\left(\mathrm{g} / \mathrm{cm}^{3}\right)\end{array}$ & $\begin{array}{c}\sigma \text { density } \\
\left(\mathrm{g} / \mathrm{cm}^{3}\right)\end{array}$ \\
\hline & & & & & 2.691 & 0.015 \\
\hline \multirow[t]{2}{*}{970.0} & 4089.279 & 4089.298 & 0.015 & -1.3 & & \\
\hline & & & & & 2.421 & 0.014 \\
\hline \multirow[t]{2}{*}{980.0} & 4089.620 & 4089.620 & 0.003 & 0.0 & & \\
\hline & & & & & 2.448 & 0.015 \\
\hline \multirow[t]{2}{*}{990.0} & 4089.927 & 4089.935 & 0.012 & -0.6 & & \\
\hline & & & & & 2.394 & 0.016 \\
\hline \multirow{2}{*}{1000.0} & 4090.271 & 4090.263 & 0.012 & 0.7 & & \\
\hline & & & & & 2.478 & 0.016 \\
\hline \multirow[t]{2}{*}{1010.0} & 4090.581 & 4090.570 & 0.018 & 0.6 & & \\
\hline & & & & & 2.535 & 0.016 \\
\hline \multirow[t]{2}{*}{1020.0} & 4090.867 & 4090.862 & 0.011 & 0.4 & & \\
\hline & & & & & 2.650 & 0.013 \\
\hline \multirow[t]{2}{*}{1030.0} & 4091.126 & 4091.125 & 0.004 & 0.1 & & \\
\hline & & & & & 2.710 & 0.013 \\
\hline \multirow[t]{2}{*}{1040.0} & 4091.372 & 4091.373 & 0.011 & -0.1 & & \\
\hline & & & & & 2.676 & 0.014 \\
\hline \multirow[t]{2}{*}{1050.0} & 4091.627 & 4091.630 & 0.006 & -0.4 & & \\
\hline & & & & & 2.586 & 0.014 \\
\hline \multirow[t]{2}{*}{1060.0} & 4091.887 & 4091.909 & 0.008 & -2.7 & & \\
\hline & & & & & 2.064 & 0.016 \\
\hline \multirow[t]{2}{*}{1070.0} & 4092.324 & 4092.322 & 0.013 & 0.1 & & \\
\hline & & & & & 2.094 & 0.016 \\
\hline \multirow[t]{2}{*}{1080.0} & 4092.728 & 4092.727 & 0.011 & 0.1 & & \\
\hline & & & & & 2.114 & 0.015 \\
\hline \multirow[t]{2}{*}{1090.0} & 4093.126 & 4093.127 & 0.007 & -0.2 & & \\
\hline & & & & & 2.069 & 0.015 \\
\hline \multirow[t]{2}{*}{1100.0} & 4093.537 & 4093.539 & 0.009 & -0.1 & & \\
\hline & & & & & 2.050 & 0.016 \\
\hline \multirow[t]{2}{*}{1110.0} & 4093.957 & 4093.955 & 0.010 & 0.2 & & \\
\hline & & & & & 2.077 & 0.016 \\
\hline \multirow[t]{2}{*}{1120.0} & 4094.359 & 4094.364 & 0.012 & -0.5 & & \\
\hline & & & & & 1.973 & 0.016 \\
\hline \multirow[t]{2}{*}{1130.0} & 4094.795 & 4094.800 & 0.007 & -0.7 & & \\
\hline & & & & & 1.866 & 0.017 \\
\hline \multirow[t]{2}{*}{1140.0} & 4095.273 & 4095.263 & 0.021 & 0.5 & & \\
\hline & & & & & 1.902 & 0.015 \\
\hline \multirow[t]{2}{*}{1150.0} & 4095.773 & 4095.717 & 0.026 & 2.2 & & \\
\hline & & & & & 2.028 & 0.015 \\
\hline
\end{tabular}


Table 8: C4996 continued

\begin{tabular}{|c|c|c|c|c|c|c|}
\hline $\begin{array}{l}\text { depth } \\
\text { (feet) }\end{array}$ & $\begin{array}{r}\bar{g} \\
(\mathrm{mGals})\end{array}$ & $\begin{array}{r}\hat{g} \\
(\mathrm{mGals})\end{array}$ & $\begin{array}{r}\sigma_{g} \\
\text { (mGals) }\end{array}$ & Z-score & $\begin{array}{r}\text { density } \\
\left(\mathrm{g} / \mathrm{cm}^{3}\right)\end{array}$ & $\begin{array}{l}\sigma \text { density } \\
\left(\mathrm{g} / \mathrm{cm}^{3}\right)\end{array}$ \\
\hline \multirow[t]{2}{*}{1160.0} & 4096.220 & 4096.140 & 0.018 & 4.4 & & \\
\hline & & & & & 2.501 & 0.017 \\
\hline \multirow[t]{2}{*}{1170.0} & 4096.474 & 4096.441 & 0.022 & 1.5 & & \\
\hline & & & & & 2.633 & 0.015 \\
\hline \multirow[t]{2}{*}{1180.0} & 4096.718 & 4096.709 & 0.023 & 0.4 & & \\
\hline & & & & & 2.661 & 0.016 \\
\hline \multirow[t]{2}{*}{1190.0} & 4096.977 & 4096.969 & 0.012 & 0.7 & & \\
\hline & & & & & 2.736 & 0.017 \\
\hline \multirow[t]{2}{*}{1200.0} & 4097.211 & 4097.210 & 0.014 & 0.1 & & \\
\hline & & & & & 2.752 & 0.016 \\
\hline \multirow[t]{2}{*}{1210.0} & 4097.451 & 4097.447 & 0.012 & 0.3 & & \\
\hline & & & & & 2.831 & 0.015 \\
\hline \multirow{2}{*}{1220.0} & 4097.664 & 4097.664 & 0.006 & -0.0 & & \\
\hline & & & & & 2.825 & 0.012 \\
\hline \multirow[t]{2}{*}{1230.0} & 4097.883 & 4097.882 & 0.004 & 0.1 & & \\
\hline & & & & & 2.864 & 0.009 \\
\hline \multirow[t]{2}{*}{1240.0} & 4098.091 & 4098.091 & 0.003 & 0.0 & & \\
\hline & & & & & 2.891 & 0.013 \\
\hline \multirow[t]{2}{*}{1250.0} & 4098.292 & 4098.292 & 0.009 & 0.0 & & \\
\hline & & & & & 2.895 & 0.016 \\
\hline \multirow[t]{2}{*}{1260.0} & 4098.491 & 4098.493 & 0.017 & -0.1 & & \\
\hline & & & & & 2.885 & 0.016 \\
\hline \multirow[t]{2}{*}{1270.0} & 4098.695 & 4098.695 & 0.007 & -0.1 & & \\
\hline & & & & & 2.864 & 0.014 \\
\hline \multirow[t]{2}{*}{1280.0} & 4098.904 & 4098.904 & 0.007 & 0.1 & & \\
\hline & & & & & 2.877 & 0.014 \\
\hline \multirow[t]{2}{*}{1290.0} & 4099.108 & 4099.109 & 0.007 & -0.2 & & \\
\hline & & & & & 2.835 & 0.014 \\
\hline \multirow[t]{2}{*}{1300.0} & 4099.324 & 4099.325 & 0.011 & -0.0 & & \\
\hline & & & & & 2.830 & 0.013 \\
\hline \multirow[t]{2}{*}{1310.0} & 4099.541 & 4099.542 & 0.005 & -0.2 & & \\
\hline & & & & & 2.743 & 0.015 \\
\hline \multirow[t]{2}{*}{1320.0} & 4099.757 & 4099.781 & 0.015 & -1.6 & & \\
\hline & & & & & 2.423 & 0.016 \\
\hline \multirow[t]{2}{*}{1330.0} & 4100.109 & 4100.102 & 0.016 & 0.4 & & \\
\hline & & & & & 2.531 & 0.014 \\
\hline \multirow[t]{2}{*}{1340.0} & 4100.397 & 4100.396 & 0.002 & 0.2 & & \\
\hline & & & & & 2.634 & 0.010 \\
\hline 1350.0 & 4100.666 & 4100.663 & 0.008 & 0.3 & & \\
\hline
\end{tabular}


Table 8: C4996 continued

\begin{tabular}{|c|c|c|c|c|c|c|}
\hline $\begin{array}{l}\text { depth } \\
\text { (feet) }\end{array}$ & $\begin{array}{r}\bar{g} \\
\text { (mGals) }\end{array}$ & $\begin{array}{r}\hat{g} \\
\text { (mGals) }\end{array}$ & $\begin{array}{r}\sigma_{g} \\
\text { (mGals) }\end{array}$ & Z-score & $\begin{array}{r}\text { density } \\
\left(\mathrm{g} / \mathrm{cm}^{3}\right)\end{array}$ & $\begin{array}{c}\sigma \text { density } \\
\left(\mathrm{g} / \mathrm{cm}^{3}\right)\end{array}$ \\
\hline & & & & & 2.769 & 0.011 \\
\hline \multirow[t]{2}{*}{1360.0} & 4100.896 & 4100.896 & 0.006 & 0.1 & & \\
\hline & & & & & 2.806 & 0.015 \\
\hline \multirow[t]{2}{*}{1370.0} & 4101.115 & 4101.119 & 0.016 & -0.3 & & \\
\hline & & & & & 2.750 & 0.016 \\
\hline \multirow[t]{2}{*}{1380.0} & 4101.346 & 4101.356 & 0.013 & -0.8 & & \\
\hline & & & & & 2.567 & 0.016 \\
\hline \multirow[t]{2}{*}{1390.0} & 4101.642 & 4101.641 & 0.012 & 0.1 & & \\
\hline & & & & & 2.579 & 0.017 \\
\hline \multirow[t]{2}{*}{1400.0} & 4101.930 & 4101.922 & 0.010 & 0.8 & & \\
\hline & & & & & 2.697 & 0.017 \\
\hline \multirow[t]{2}{*}{1410.0} & 4102.177 & 4102.173 & 0.022 & 0.2 & & \\
\hline & & & & & 2.727 & 0.017 \\
\hline \multirow[t]{2}{*}{1419.9} & 4102.413 & 4102.414 & 0.017 & -0.1 & & \\
\hline & & & & & 2.726 & 0.017 \\
\hline \multirow[t]{2}{*}{1430.0} & 4102.671 & 4102.660 & 0.020 & 0.6 & & \\
\hline & & & & & 2.770 & 0.016 \\
\hline \multirow[t]{2}{*}{1440.0} & 4102.888 & 4102.892 & 0.021 & -0.2 & & \\
\hline & & & & & 2.757 & 0.017 \\
\hline 1450.0 & 4103.095 & 4103.128 & 0.015 & -2.2 & & \\
\hline
\end{tabular}




\section{B.3 C4997}

Table 9: Well C4997 Inversion densities

\begin{tabular}{|c|c|c|c|c|c|c|}
\hline $\begin{array}{l}\text { depth } \\
\text { (feet) }\end{array}$ & $\begin{array}{r}\bar{g} \\
\text { (mGals) }\end{array}$ & $\begin{array}{r}\hat{g} \\
(\mathrm{mGals})\end{array}$ & $\begin{array}{r}\sigma_{g} \\
\text { (mGals) }\end{array}$ & Z-score & $\begin{array}{r}\text { density } \\
\left(\mathrm{g} / \mathrm{cm}^{3}\right)\end{array}$ & $\begin{array}{l}\sigma \sigma \text { density } \\
\left(\mathrm{g} / \mathrm{cm}^{3}\right)\end{array}$ \\
\hline \multirow[t]{2}{*}{0.1} & 4039.495 & 4039.495 & 0.001 & -0.3 & & \\
\hline & & & & & 2.037 & 0.009 \\
\hline \multirow[t]{2}{*}{10.0} & 4039.910 & 4039.911 & 0.007 & -0.2 & & \\
\hline & & & & & 1.979 & 0.010 \\
\hline \multirow{2}{*}{20.0} & 4040.343 & 4040.346 & 0.003 & -0.8 & & \\
\hline & & & & & 1.695 & 0.012 \\
\hline \multirow[t]{2}{*}{30.0} & 4040.880 & 4040.853 & 0.015 & 1.8 & & \\
\hline & & & & & 1.920 & 0.013 \\
\hline \multirow[t]{2}{*}{40.0} & 4041.295 & 4041.303 & 0.005 & -1.6 & & \\
\hline & & & & & 1.574 & 0.013 \\
\hline \multirow[t]{2}{*}{50.0} & 4041.855 & 4041.840 & 0.015 & 1.0 & & \\
\hline & & & & & 1.678 & 0.013 \\
\hline \multirow[t]{2}{*}{60.0} & 4042.361 & 4042.352 & 0.006 & 1.6 & & \\
\hline & & & & & 1.979 & 0.013 \\
\hline \multirow[t]{2}{*}{70.0} & 4042.797 & 4042.786 & 0.017 & 0.7 & & \\
\hline & & & & & 2.053 & 0.012 \\
\hline \multirow[t]{2}{*}{80.0} & 4043.200 & 4043.201 & 0.002 & -0.9 & & \\
\hline & & & & & 1.539 & 0.012 \\
\hline \multirow[t]{2}{*}{90.0} & 4043.747 & 4043.748 & 0.016 & -0.1 & & \\
\hline & & & & & 1.536 & 0.013 \\
\hline \multirow[t]{2}{*}{100.0} & 4044.294 & 4044.296 & 0.006 & -0.4 & & \\
\hline & & & & & 1.470 & 0.011 \\
\hline \multirow[t]{2}{*}{110.0} & 4044.860 & 4044.860 & 0.005 & -0.0 & & \\
\hline & & & & & 1.463 & 0.010 \\
\hline \multirow[t]{2}{*}{120.0} & 4045.433 & 4045.427 & 0.005 & 1.1 & & \\
\hline & & & & & 1.689 & 0.013 \\
\hline \multirow[t]{2}{*}{130.0} & 4045.941 & 4045.935 & 0.010 & 0.5 & & \\
\hline & & & & & 1.786 & 0.014 \\
\hline \multirow[t]{2}{*}{140.0} & 4046.430 & 4046.420 & 0.013 & 0.8 & & \\
\hline & & & & & 1.854 & 0.013 \\
\hline \multirow[t]{2}{*}{150.0} & 4046.838 & 4046.886 & 0.015 & -3.3 & & \\
\hline & & & & & 1.441 & 0.013 \\
\hline \multirow[t]{2}{*}{160.0} & 4047.466 & 4047.458 & 0.005 & 1.7 & & \\
\hline & & & & & 1.825 & 0.011 \\
\hline \multirow[t]{2}{*}{170.0} & 4047.937 & 4047.932 & 0.007 & 0.7 & & \\
\hline & & & & & 2.021 & 0.011 \\
\hline 180.0 & 4048.353 & 4048.356 & 0.005 & -0.5 & & \\
\hline
\end{tabular}


Table 9: C4997 continued

\begin{tabular}{|c|c|c|c|c|c|c|}
\hline $\begin{array}{l}\text { depth } \\
\text { (feet) }\end{array}$ & $\begin{array}{r}\bar{g} \\
\text { (mGals) }\end{array}$ & $\begin{array}{r}\hat{g} \\
\text { (mGals) }\end{array}$ & $\begin{array}{r}\sigma_{g} \\
\text { (mGals) }\end{array}$ & Z-score & $\begin{array}{r}\text { density } \\
\left(\mathrm{g} / \mathrm{cm}^{3}\right)\end{array}$ & $\begin{array}{l}\sigma \text { density } \\
\left(\mathrm{g} / \mathrm{cm}^{3}\right)\end{array}$ \\
\hline & & & & & 1.916 & 0.011 \\
\hline \multirow{2}{*}{190.0} & 4048.808 & 4048.806 & 0.007 & 0.3 & & \\
\hline & & & & & 2.015 & 0.012 \\
\hline \multirow{2}{*}{200.0} & 4049.226 & 4049.231 & 0.007 & -0.7 & & \\
\hline & & & & & 1.893 & 0.012 \\
\hline \multirow[t]{2}{*}{210.0} & 4049.695 & 4049.688 & 0.006 & 1.2 & & \\
\hline & & & & & 2.096 & 0.012 \\
\hline \multirow{2}{*}{220.0} & 4050.091 & 4050.092 & 0.008 & -0.2 & & \\
\hline & & & & & 2.055 & 0.013 \\
\hline \multirow[t]{2}{*}{230.0} & 4050.487 & 4050.507 & 0.015 & -1.3 & & \\
\hline & & & & & 1.869 & 0.012 \\
\hline \multirow{2}{*}{240.0} & 4050.973 & 4050.969 & 0.005 & 0.7 & & \\
\hline & & & & & 2.279 & 0.009 \\
\hline \multirow[t]{2}{*}{250.0} & 4051.321 & 4051.327 & 0.004 & -1.5 & & \\
\hline & & & & & 1.936 & 0.011 \\
\hline \multirow{2}{*}{260.0} & 4051.772 & 4051.772 & 0.007 & 0.0 & & \\
\hline & & & & & 1.941 & 0.012 \\
\hline \multirow[t]{2}{*}{270.0} & 4052.231 & 4052.216 & 0.007 & 2.1 & & \\
\hline & & & & & 2.265 & 0.011 \\
\hline \multirow[t]{2}{*}{280.0} & 4052.577 & 4052.578 & 0.002 & -0.5 & & \\
\hline & & & & & 2.096 & 0.011 \\
\hline \multirow[t]{2}{*}{290.0} & 4052.992 & 4052.983 & 0.009 & 1.0 & & \\
\hline & & & & & 2.282 & 0.013 \\
\hline \multirow[t]{2}{*}{300.0} & 4053.360 & 4053.340 & 0.012 & 1.7 & & \\
\hline & & & & & 2.428 & 0.012 \\
\hline \multirow[t]{2}{*}{310.0} & 4053.657 & 4053.659 & 0.002 & -0.7 & & \\
\hline & & & & & 2.140 & 0.011 \\
\hline \multirow[t]{2}{*}{320.0} & 4054.075 & 4054.053 & 0.010 & 2.1 & & \\
\hline & & & & & 2.472 & 0.011 \\
\hline \multirow[t]{2}{*}{330.0} & 4054.361 & 4054.361 & 0.001 & -0.0 & & \\
\hline & & & & & 2.447 & 0.009 \\
\hline \multirow[t]{2}{*}{340.0} & 4054.675 & 4054.676 & 0.005 & -0.1 & & \\
\hline & & & & & 2.431 & 0.011 \\
\hline \multirow[t]{2}{*}{350.0} & 4054.994 & 4054.995 & 0.007 & -0.1 & & \\
\hline & & & & & 2.395 & 0.013 \\
\hline \multirow[t]{2}{*}{360.0} & 4055.344 & 4055.323 & 0.012 & 1.7 & & \\
\hline & & & & & 2.548 & 0.013 \\
\hline \multirow[t]{2}{*}{370.0} & 4055.608 & 4055.612 & 0.005 & -0.9 & & \\
\hline & & & & & 2.348 & 0.011 \\
\hline
\end{tabular}


Table 9: C4997 continued

\begin{tabular}{|c|c|c|c|c|c|c|}
\hline $\begin{array}{l}\text { depth } \\
\text { (feet) }\end{array}$ & $\begin{array}{r}\bar{g} \\
(\mathrm{mGals})\end{array}$ & $\begin{array}{r}\hat{g} \\
(\mathrm{mGals})\end{array}$ & $\begin{array}{r}\sigma_{g} \\
\text { (mGals) }\end{array}$ & Z-score & $\begin{array}{r}\text { density } \\
\left(\mathrm{g} / \mathrm{cm}^{3}\right)\end{array}$ & $\begin{array}{l}\sigma \text { density } \\
\left(\mathrm{g} / \mathrm{cm}^{3}\right)\end{array}$ \\
\hline \multirow[t]{2}{*}{380.0} & 4055.956 & 4055.952 & 0.007 & 0.6 & & \\
\hline & & & & & 2.523 & 0.012 \\
\hline \multirow[t]{2}{*}{390.0} & 4056.250 & 4056.247 & 0.007 & 0.4 & & \\
\hline & & & & & 2.588 & 0.013 \\
\hline \multirow[t]{2}{*}{400.0} & 4056.533 & 4056.526 & 0.007 & 1.0 & & \\
\hline & & & & & 2.744 & 0.012 \\
\hline \multirow[t]{2}{*}{410.0} & 4056.770 & 4056.765 & 0.006 & 0.8 & & \\
\hline & & & & & 2.879 & 0.011 \\
\hline \multirow[t]{2}{*}{420.0} & 4056.969 & 4056.970 & 0.005 & -0.1 & & \\
\hline & & & & & 2.844 & 0.008 \\
\hline \multirow[t]{2}{*}{430.0} & 4057.183 & 4057.183 & 0.003 & 0.0 & & \\
\hline & & & & & 2.862 & 0.010 \\
\hline \multirow{2}{*}{440.0} & 4057.391 & 4057.392 & 0.005 & -0.1 & & \\
\hline & & & & & 2.850 & 0.011 \\
\hline \multirow[t]{2}{*}{450.0} & 4057.604 & 4057.604 & 0.005 & 0.1 & & \\
\hline & & & & & 2.872 & 0.011 \\
\hline \multirow[t]{2}{*}{460.0} & 4057.811 & 4057.810 & 0.005 & 0.2 & & \\
\hline & & & & & 2.914 & 0.009 \\
\hline \multirow[t]{2}{*}{470.0} & 4058.006 & 4058.005 & 0.003 & 0.1 & & \\
\hline & & & & & 2.949 & 0.008 \\
\hline \multirow[t]{2}{*}{480.0} & 4058.191 & 4058.192 & 0.004 & -0.3 & & \\
\hline & & & & & 2.845 & 0.008 \\
\hline \multirow[t]{2}{*}{490.0} & 4058.400 & 4058.405 & 0.003 & -1.8 & & \\
\hline & & & & & 2.181 & 0.006 \\
\hline \multirow[t]{2}{*}{500.0} & 4058.788 & 4058.788 & 0.001 & -0.4 & & \\
\hline & & & & & 1.853 & 0.007 \\
\hline \multirow[t]{2}{*}{510.0} & 4059.255 & 4059.254 & 0.005 & 0.0 & & \\
\hline & & & & & 1.865 & 0.012 \\
\hline \multirow[t]{2}{*}{520.0} & 4059.723 & 4059.718 & 0.014 & 0.3 & & \\
\hline & & & & & 1.895 & 0.012 \\
\hline \multirow[t]{2}{*}{530.0} & 4060.174 & 4060.174 & 0.003 & 0.1 & & \\
\hline & & & & & 1.940 & 0.010 \\
\hline \multirow[t]{2}{*}{540.0} & 4060.617 & 4060.618 & 0.007 & -0.2 & & \\
\hline & & & & & 1.904 & 0.011 \\
\hline \multirow[t]{2}{*}{550.0} & 4061.073 & 4061.072 & 0.006 & 0.2 & & \\
\hline & & & & & 1.958 & 0.009 \\
\hline \multirow[t]{2}{*}{560.0} & 4061.514 & 4061.512 & 0.003 & 0.6 & & \\
\hline & & & & & 2.207 & 0.010 \\
\hline 570.0 & 4061.894 & 4061.888 & 0.006 & 1.0 & & \\
\hline
\end{tabular}


Table 9: C4997 continued

\begin{tabular}{|c|c|c|c|c|c|c|}
\hline $\begin{array}{l}\text { depth } \\
\text { (feet) }\end{array}$ & $\begin{array}{r}\bar{g} \\
\text { (mGals) }\end{array}$ & $\begin{array}{r}\hat{g} \\
\text { (mGals) }\end{array}$ & $\begin{array}{r}\sigma_{g} \\
\text { (mGals) }\end{array}$ & Z-score & $\begin{array}{r}\text { density } \\
\left(\mathrm{g} / \mathrm{cm}^{3}\right)\end{array}$ & $\begin{array}{l}\sigma \text { density } \\
\left(\mathrm{g} / \mathrm{cm}^{3}\right)\end{array}$ \\
\hline & & & & & 2.387 & 0.013 \\
\hline \multirow[t]{2}{*}{580.0} & 4062.226 & 4062.218 & 0.013 & 0.6 & & \\
\hline & & & & & 2.479 & 0.012 \\
\hline \multirow{2}{*}{590.0} & 4062.529 & 4062.525 & 0.004 & 1.0 & & \\
\hline & & & & & 2.727 & 0.012 \\
\hline \multirow[t]{2}{*}{600.0} & 4062.771 & 4062.769 & 0.007 & 0.4 & & \\
\hline & & & & & 2.786 & 0.013 \\
\hline \multirow{2}{*}{610.0} & 4062.998 & 4062.997 & 0.007 & 0.2 & & \\
\hline & & & & & 2.823 & 0.012 \\
\hline \multirow[t]{2}{*}{620.0} & 4063.217 & 4063.215 & 0.005 & 0.3 & & \\
\hline & & & & & 2.884 & 0.013 \\
\hline \multirow{2}{*}{630.0} & 4063.417 & 4063.419 & 0.011 & -0.2 & & \\
\hline & & & & & 2.848 & 0.013 \\
\hline \multirow[t]{2}{*}{640.0} & 4063.630 & 4063.631 & 0.008 & -0.1 & & \\
\hline & & & & & 2.837 & 0.012 \\
\hline \multirow[t]{2}{*}{650.0} & 4063.847 & 4063.846 & 0.004 & 0.0 & & \\
\hline & & & & & 2.850 & 0.009 \\
\hline \multirow[t]{2}{*}{660.0} & 4064.058 & 4064.058 & 0.003 & 0.0 & & \\
\hline & & & & & 2.858 & 0.011 \\
\hline \multirow[t]{2}{*}{670.0} & 4064.270 & 4064.268 & 0.006 & 0.2 & & \\
\hline & & & & & 2.893 & 0.013 \\
\hline \multirow[t]{2}{*}{680.0} & 4064.467 & 4064.469 & 0.011 & -0.2 & & \\
\hline & & & & & 2.881 & 0.013 \\
\hline \multirow[t]{2}{*}{690.0} & 4064.670 & 4064.673 & 0.010 & -0.3 & & \\
\hline & & & & & 2.839 & 0.012 \\
\hline \multirow{2}{*}{700.0} & 4064.888 & 4064.888 & 0.004 & 0.0 & & \\
\hline & & & & & 2.848 & 0.012 \\
\hline \multirow[t]{2}{*}{710.0} & 4065.102 & 4065.100 & 0.008 & 0.2 & & \\
\hline & & & & & 2.874 & 0.013 \\
\hline \multirow[t]{2}{*}{720.0} & 4065.306 & 4065.306 & 0.007 & -0.0 & & \\
\hline & & & & & 2.872 & 0.013 \\
\hline \multirow[t]{2}{*}{730.0} & 4065.500 & 4065.512 & 0.010 & -1.2 & & \\
\hline & & & & & 2.694 & 0.011 \\
\hline \multirow[t]{2}{*}{740.0} & 4065.763 & 4065.764 & 0.001 & -0.8 & & \\
\hline & & & & & 2.108 & 0.010 \\
\hline \multirow[t]{2}{*}{750.0} & 4066.162 & 4066.165 & 0.006 & -0.6 & & \\
\hline & & & & & 2.011 & 0.012 \\
\hline \multirow[t]{2}{*}{760.0} & 4066.601 & 4066.592 & 0.008 & 1.1 & & \\
\hline & & & & & 2.229 & 0.010 \\
\hline
\end{tabular}


Table 9: C4997 continued

\begin{tabular}{|c|c|c|c|c|c|c|}
\hline $\begin{array}{l}\text { depth } \\
\text { (feet) }\end{array}$ & $\begin{array}{r}\bar{g} \\
\text { (mGals) }\end{array}$ & $\begin{array}{r}\hat{g} \\
\text { (mGals) }\end{array}$ & $\begin{array}{r}\sigma_{g} \\
\text { (mGals) }\end{array}$ & Z-score & $\begin{array}{r}\text { density } \\
\left(\mathrm{g} / \mathrm{cm}^{3}\right)\end{array}$ & $\begin{array}{c}\sigma \text { density } \\
\left(\mathrm{g} / \mathrm{cm}^{3}\right)\end{array}$ \\
\hline \multirow[t]{2}{*}{770.0} & 4066.963 & 4066.962 & 0.002 & 0.6 & & \\
\hline & & & & & 2.655 & 0.007 \\
\hline \multirow[t]{2}{*}{780.0} & 4067.225 & 4067.224 & 0.003 & 0.2 & & \\
\hline & & & & & 2.720 & 0.008 \\
\hline \multirow{2}{*}{790.0} & 4067.469 & 4067.469 & 0.003 & 0.2 & & \\
\hline & & & & & 2.781 & 0.011 \\
\hline \multirow[t]{2}{*}{800.0} & 4067.699 & 4067.699 & 0.009 & 0.0 & & \\
\hline & & & & & 2.792 & 0.013 \\
\hline \multirow[t]{2}{*}{810.0} & 4067.925 & 4067.925 & 0.008 & -0.1 & & \\
\hline & & & & & 2.784 & 0.012 \\
\hline \multirow[t]{2}{*}{820.0} & 4068.153 & 4068.154 & 0.005 & -0.2 & & \\
\hline & & & & & 2.743 & 0.011 \\
\hline \multirow{2}{*}{830.0} & 4068.394 & 4068.393 & 0.005 & 0.2 & & \\
\hline & & & & & 2.784 & 0.012 \\
\hline \multirow[t]{2}{*}{840.0} & 4068.622 & 4068.622 & 0.007 & -0.0 & & \\
\hline & & & & & 2.783 & 0.012 \\
\hline \multirow[t]{2}{*}{850.0} & 4068.834 & 4068.851 & 0.008 & -2.0 & & \\
\hline & & & & & 2.356 & 0.011 \\
\hline \multirow[t]{2}{*}{860.0} & 4069.185 & 4069.189 & 0.003 & -1.0 & & \\
\hline & & & & & 1.994 & 0.011 \\
\hline \multirow[t]{2}{*}{870.0} & 4069.624 & 4069.620 & 0.010 & 0.4 & & \\
\hline & & & & & 2.075 & 0.011 \\
\hline \multirow[t]{2}{*}{880.0} & 4070.029 & 4070.030 & 0.002 & -0.1 & & \\
\hline & & & & & 2.045 & 0.011 \\
\hline \multirow[t]{2}{*}{890.0} & 4070.444 & 4070.448 & 0.010 & -0.4 & & \\
\hline & & & & & 1.978 & 0.011 \\
\hline \multirow[t]{2}{*}{900.0} & 4070.882 & 4070.882 & 0.003 & -0.1 & & \\
\hline & & & & & 1.957 & 0.013 \\
\hline \multirow[t]{2}{*}{910.0} & 4071.308 & 4071.322 & 0.014 & -1.0 & & \\
\hline & & & & & 1.850 & 0.013 \\
\hline \multirow[t]{2}{*}{920.0} & 4071.784 & 4071.790 & 0.012 & -0.5 & & \\
\hline & & & & & 1.810 & 0.013 \\
\hline \multirow[t]{2}{*}{930.0} & 4072.266 & 4072.268 & 0.004 & -0.4 & & \\
\hline & & & & & 1.688 & 0.010 \\
\hline \multirow[t]{2}{*}{940.0} & 4072.780 & 4072.777 & 0.005 & 0.5 & & \\
\hline & & & & & 1.826 & 0.011 \\
\hline \multirow[t]{2}{*}{950.0} & 4073.262 & 4073.250 & 0.006 & 1.9 & & \\
\hline & & & & & 2.311 & 0.010 \\
\hline 960.0 & 4073.603 & 4073.600 & 0.003 & 1.0 & & \\
\hline
\end{tabular}


Table 9: C4997 continued

\begin{tabular}{|c|c|c|c|c|c|c|}
\hline $\begin{array}{l}\text { depth } \\
\text { (feet) }\end{array}$ & $\begin{array}{r}\bar{g} \\
\text { (mGals) }\end{array}$ & $\begin{array}{r}\hat{g} \\
\text { (mGals) }\end{array}$ & $\begin{array}{r}\sigma_{g} \\
\text { (mGals) }\end{array}$ & Z-score & $\begin{array}{r}\text { density } \\
\left(\mathrm{g} / \mathrm{cm}^{3}\right)\end{array}$ & $\begin{array}{l}\sigma \text { density } \\
\left(\mathrm{g} / \mathrm{cm}^{3}\right)\end{array}$ \\
\hline & & & & & 2.631 & 0.007 \\
\hline \multirow[t]{2}{*}{970.0} & 4073.869 & 4073.868 & 0.002 & 0.1 & & \\
\hline & & & & & 2.677 & 0.012 \\
\hline \multirow[t]{2}{*}{980.0} & 4074.125 & 4074.124 & 0.009 & 0.1 & & \\
\hline & & & & & 2.700 & 0.013 \\
\hline \multirow{2}{*}{990.0} & 4074.373 & 4074.374 & 0.011 & -0.1 & & \\
\hline & & & & & 2.684 & 0.014 \\
\hline \multirow[t]{2}{*}{1000.0} & 4074.618 & 4074.629 & 0.007 & -1.6 & & \\
\hline & & & & & 2.507 & 0.014 \\
\hline \multirow[t]{2}{*}{1010.0} & 4074.918 & 4074.928 & 0.010 & -1.1 & & \\
\hline & & & & & 2.366 & 0.013 \\
\hline \multirow[t]{2}{*}{1020.0} & 4075.278 & 4075.264 & 0.015 & 1.0 & & \\
\hline & & & & & 2.439 & 0.013 \\
\hline \multirow[t]{2}{*}{1030.0} & 4075.582 & 4075.581 & 0.003 & 0.5 & & \\
\hline & & & & & 2.612 & 0.007 \\
\hline \multirow[t]{2}{*}{1040.0} & 4075.854 & 4075.854 & 0.003 & 0.2 & & \\
\hline & & & & & 2.695 & 0.013 \\
\hline \multirow[t]{2}{*}{1050.0} & 4076.108 & 4076.105 & 0.010 & 0.3 & & \\
\hline & & & & & 2.723 & 0.012 \\
\hline \multirow[t]{2}{*}{1060.0} & 4076.337 & 4076.349 & 0.026 & -0.5 & & \\
\hline & & & & & 2.695 & 0.012 \\
\hline \multirow[t]{2}{*}{1070.0} & 4076.599 & 4076.601 & 0.007 & -0.2 & & \\
\hline & & & & & 2.669 & 0.013 \\
\hline \multirow[t]{2}{*}{1080.0} & 4076.860 & 4076.859 & 0.006 & 0.3 & & \\
\hline & & & & & 2.708 & 0.014 \\
\hline \multirow[t]{2}{*}{1090.0} & 4077.105 & 4077.107 & 0.012 & -0.2 & & \\
\hline & & & & & 2.694 & 0.014 \\
\hline \multirow[t]{2}{*}{1100.0} & 4077.329 & 4077.359 & 0.016 & -1.9 & & \\
\hline & & & & & 2.540 & 0.014 \\
\hline \multirow[t]{2}{*}{1110.0} & 4077.570 & 4077.650 & 0.016 & -5.1 & & \\
\hline & & & & & 2.205 & 0.014 \\
\hline \multirow[t]{2}{*}{1120.0} & 4078.006 & 4078.027 & 0.019 & -1.1 & & \\
\hline & & & & & 2.128 & 0.012 \\
\hline \multirow[t]{2}{*}{1130.0} & 4078.399 & 4078.423 & 0.031 & -0.8 & & \\
\hline & & & & & 2.084 & 0.013 \\
\hline \multirow[t]{2}{*}{1140.0} & 4078.837 & 4078.831 & 0.012 & 0.5 & & \\
\hline & & & & & 2.130 & 0.015 \\
\hline \multirow[t]{2}{*}{1150.0} & 4079.225 & 4079.227 & 0.014 & -0.1 & & \\
\hline & & & & & 2.117 & 0.012 \\
\hline
\end{tabular}


Table 9: C4997 continued

\begin{tabular}{|c|c|c|c|c|c|c|}
\hline $\begin{array}{l}\text { depth } \\
\text { (feet) }\end{array}$ & $\begin{array}{r}\bar{g} \\
(\mathrm{mGals})\end{array}$ & $\begin{array}{r}\hat{g} \\
(\mathrm{mGals}) \\
\end{array}$ & $\begin{array}{r}\sigma_{g} \\
\text { (mGals) }\end{array}$ & Z-score & $\begin{array}{r}\text { density } \\
\left(\mathrm{g} / \mathrm{cm}^{3}\right)\end{array}$ & $\begin{array}{l}\sigma \text { density } \\
\left(\mathrm{g} / \mathrm{cm}^{3}\right)\end{array}$ \\
\hline \multirow[t]{2}{*}{1160.0} & 4079.612 & 4079.626 & 0.021 & -0.7 & & \\
\hline & & & & & 2.084 & 0.012 \\
\hline \multirow[t]{2}{*}{1170.0} & 4080.032 & 4080.034 & 0.006 & -0.4 & & \\
\hline & & & & & 1.977 & 0.013 \\
\hline \multirow[t]{2}{*}{1180.0} & 4080.462 & 4080.469 & 0.015 & -0.5 & & \\
\hline & & & & & 1.937 & 0.013 \\
\hline \multirow[t]{2}{*}{1190.0} & 4080.913 & 4080.915 & 0.010 & -0.2 & & \\
\hline & & & & & 1.898 & 0.014 \\
\hline \multirow[t]{2}{*}{1200.0} & 4081.402 & 4081.370 & 0.015 & 2.1 & & \\
\hline & & & & & 2.075 & 0.013 \\
\hline \multirow[t]{2}{*}{1210.0} & 4081.833 & 4081.780 & 0.015 & 3.5 & & \\
\hline & & & & & 2.496 & 0.014 \\
\hline \multirow[t]{2}{*}{1220.0} & 4082.090 & 4082.083 & 0.009 & 0.8 & & \\
\hline & & & & & 2.601 & 0.013 \\
\hline \multirow[t]{2}{*}{1230.0} & 4082.362 & 4082.358 & 0.006 & 0.6 & & \\
\hline & & & & & 2.706 & 0.013 \\
\hline \multirow{2}{*}{1240.0} & 4082.611 & 4082.607 & 0.007 & 0.6 & & \\
\hline & & & & & 2.793 & 0.013 \\
\hline \multirow[t]{2}{*}{1250.0} & 4082.836 & 4082.833 & 0.020 & 0.1 & & \\
\hline & & & & & 2.812 & 0.013 \\
\hline \multirow[t]{2}{*}{1260.0} & 4083.055 & 4083.056 & 0.007 & -0.1 & & \\
\hline & & & & & 2.795 & 0.013 \\
\hline \multirow[t]{2}{*}{1270.0} & 4083.282 & 4083.281 & 0.007 & 0.1 & & \\
\hline & & & & & 2.821 & 0.011 \\
\hline \multirow[t]{2}{*}{1280.0} & 4083.501 & 4083.500 & 0.004 & 0.1 & & \\
\hline & & & & & 2.858 & 0.008 \\
\hline \multirow[t]{2}{*}{1290.0} & 4083.710 & 4083.710 & 0.001 & 0.2 & & \\
\hline & & & & & 2.980 & 0.012 \\
\hline \multirow[t]{2}{*}{1300.0} & 4083.885 & 4083.889 & 0.022 & -0.2 & & \\
\hline & & & & & 2.967 & 0.012 \\
\hline \multirow[t]{2}{*}{1310.0} & 4084.068 & 4084.071 & 0.006 & -0.5 & & \\
\hline & & & & & 2.886 & 0.011 \\
\hline \multirow[t]{2}{*}{1320.0} & 4084.274 & 4084.273 & 0.004 & 0.1 & & \\
\hline & & & & & 2.908 & 0.012 \\
\hline \multirow[t]{2}{*}{1330.0} & 4084.466 & 4084.470 & 0.011 & -0.3 & & \\
\hline & & & & & 2.879 & 0.012 \\
\hline \multirow[t]{2}{*}{1340.0} & 4084.676 & 4084.675 & 0.004 & 0.1 & & \\
\hline & & & & & 2.914 & 0.013 \\
\hline 1350.0 & 4084.870 & 4084.870 & 0.008 & -0.1 & & \\
\hline
\end{tabular}


Table 9: C4997 continued

\begin{tabular}{|r|r|r|r|r|r|r|}
\hline $\begin{array}{r}\text { depth } \\
\text { (feet) }\end{array}$ & $\begin{array}{r}\bar{g} \\
(\mathrm{mGals})\end{array}$ & $\begin{array}{r}\hat{g} \\
(\mathrm{mGals})\end{array}$ & $\begin{array}{r}\sigma_{g} \\
(\mathrm{mGals})\end{array}$ & Z-score & $\begin{array}{r}\text { density } \\
\left(\mathrm{g} / \mathrm{cm}^{3}\right)\end{array}$ & $\begin{array}{r}\sigma \text { density } \\
\left(\mathrm{g} / \mathrm{cm}^{3}\right)\end{array}$ \\
\hline \hline & & & & & 2.909 & 0.013 \\
\hline 1360.0 & 4085.052 & 4085.067 & 0.019 & -0.8 & & \\
\hline & & & & & 2.845 & 0.013 \\
\hline 1370.0 & 4085.271 & 4085.280 & 0.005 & -1.8 & & \\
\hline & & & & & 2.491 & 0.012 \\
\hline 1380.0 & 4085.587 & 4085.584 & 0.006 & 0.5 & & \\
\hline & & & & & 2.596 & 0.013 \\
\hline 1390.0 & 4085.868 & 4085.861 & 0.012 & 0.5 & & \\
\hline & & & & & 2.644 & 0.013 \\
\hline 1400.0 & 4086.123 & 4086.126 & 0.004 & -0.6 & & \\
\hline
\end{tabular}




\section{Appendix: C Field operations}

\section{C.1 Equipment}

The equipment used was BHGM Serial Number 14 (\#14) with the Shuttle Sonde, its associated electronics, standard field setup equipment, and spares. The logging was done from a COLOG wireline truck using a 5/16" diameter cable and a GOI, 7 pin, small cablehead with the MGL depth encoder system attached. A natural gamma tool provided information to tie BHGM survey depths to COLOG gamma-gamma density logging runs. PNNL/Energy Solutions provided a mast truck to support the tool and cable in the wells.

\section{C.2 Mobilization}

Testing was performed at the end of October at COLOG in Lakewood, Colorado with winch truck, cable, cablehead and adapter, depth system, and BHGM equipment to make sure any compatibility issues were worked out before the truck and equipment were sent to Hanford. Minor cablehead and depth system problems were encountered and solved.

The freight was sent from MGL, Lafayette, Colorado to PNNL, Hanford, Washington on November 3rd,2006.

The Micro-g Lacoste field personnel were Ryan Albery, Andy Black, and Ethan Mann. Andy and Ethan flew to Portland, Oregon, then drove to Hanford, Washington. Ryan drove from Lafayette to Hanford. The MGL crew arrived in Hanford, Washington on November 10th, 2006. The freight was visually checked and appeared undamaged. The PNNL freight office also checked the equipment with a radiation detector before trucking it out to the W.T.P. site. The gravity meter was run and checked out at the PNNL Guest House that night.

The MGL crew met with Alan Rohay and Tom Brouns early on November 11th before proceeding to the WTP site. After arriving at the site a safety and orientation class was attended by all MGL personnel, followed by a tour of the three well locations on the WTP site. Then the rig-up on the first site, well C4996, started.

\section{C.3 C4996}

A problem with the wireline cablehead occurred (the cable lines shorted out) during truck checkout and was repaired. At 13:00 (all times are local time) the Shuttle tool went into the well. There was a problem with the depth measuring system, but after some checking and rebooting the computer the problem was resolved and the tool was lowered to the bottom of casing (340 feet). Calibrations started at 17:50 and were finished at 22:00. When attempting to move downhole, the depth system failed again. The MGL team spent that night trying to solve the problem. The meter was pulled out of the well on Nov. 13th. The problem appeared to be software related. The manufacturer of depth interface system was contacted but no immediate solution was found. The manufacturer continued to work with MGL personnel on the problem until the problem was solved. On Nov. 14th it was decided to revert to an older version of the software (B5) for the depth system. A Win98 computer was acquired to run the older version of the software, which was not compatible withe newer operating systems. With 
the older software version, the system was working on Nov. 15th, and the crew went back out to the WTP site.

Well C4996 was re-entered for second time at 21:30 Nov. 15th. Calibration checks were run at 340 feet and finished up at 00:30 on Nov. 16th. A natural gamma ray depth tie was run to calibrate the BHGM depth to the COLOG gamma-gamma compensated density log. The tool was then lowered to the bottom station at 1450 feet. Two readings were made, then the Shuttle winch was moved to the bottom station (1458 feet) and a good reading was made. At that time the Shuttle winch failed to move the sensor up to the zero position. The tool was pulled out of the well and it was determined that problem was electronic, not mechanical. The problem may have been caused by the cablehead problem mentioned earlier (shorted cable lines). The MGL team checked out the Shuttle control electronics, and the winch controller was replaced with a spare. At this point, the winch worked on the test bench. It was decided to rig-up and go back into the hole. Further testing of the Shuttle winch downhole near the surface caused the same problem to re-occur. The tool was then pulled out of well and the Shuttle winch controller was checked again on the bench in the office. The same failure mode as seen before occurred again. The Shuttle winch controller was again replaced (with the last spare). Testing managed to re-create the problem on the bench again this time, but further troubleshooting did not identify the cause of the problem.

On Nov. 16th, after talking with the MGL home office, Alan Rohay and Tom Brouns of PNNL were presented with three choices:

1. Send the MGL people and equipment back to Denver for repairs, and return when fixed.

2. Have the crew wait in Hanford for more spare parts and then do further detailed troubleshooting.

3. Continue the survey with non-Shuttle survey techniques. This would entail relying on moving the surface truck winch for depth control. This is not as accurate as the Shuttle depth control, but it should be reasonable considering how shallow these wells are.

The MGL head office in Lafayette did an error analysis and concluded that the error budget can still be under $0.05 \mathrm{~g} / \mathrm{cm}^{3}$, which will meet PNNL's objectives. It was also decided that 10 foot intervals would be better for this survey, as they lessen the effects of depth errors while still meeting the modeling requirements of PNNL.

PNNL gave permission to run the altered survey plan using 10 foot intervals, the surface odometer, and the winch for depth control.

On Nov. 17th, the MGL crew arrived on the WTP site and was rigged up and ready to start downhole at 08:30. Calibrations were performed again at 351 feet. The tool went to bottom of well after checking gamma depth ties at 1108 feet and 1130 feet. The gamma peaks were off 1.7 feet, and a correction was made to the BHGM depth. These are reasonable corrections for a survey of this depth. The gravity survey started at 1450 feet and station readings were made every 10 feet. The procedure used was as follows:

1. move 10 feet on the odometer

2. mark the cable at the odometer with tape 


\section{3. level the gravity sensor}

4. unclamp the sensor

5. take a 4-5 minute reading, recording data at 1 second intervals

6. log the gravity value using a 20 second filter at the 4 minute mark.

The software used to record gravity data was BCON.EXE, ver.1.39.4. The first data file name was C4996B.DAT. Depths were entered by hand from another computer running B5.EXE. All pertinent information was also recorded by hand in a field book as a backup to the computer data. Notes were written in the field book at each station. In well C4996 groups of 5 stations (depths) were recorded, then the meter was lowered at least 50 feet below the starting station, then pulled back up to the first station depth. This group was looped 4 times through, then the meter was moved up to start the next section. On the repeated measurements the offset to the taped mark on the cable was noted for later depth adjustments.

At 1120 feet on Nov. 18th it was discovered that the polymer mud used in this well was glueing itself to the measurement wheels on the depth system, causing depth errors in the 10 foot measurements. The PNNL staff was consulted about the presence of this mud in the other wells to be logged and MGL was assured it would not be present in the other wells. All the previous 10 foot intervals were re-measured by hand using a tape and noted in field book. These depth offsets were used to calculate corrected depths before final processing. From then on all 10 foot depth intervals were measured out with a tape measure and marked on the cable with either tape or paint and the electronic depth system was used only as a check. The winch operator was told to stop when the next 10' mark reached the depth head on the wireline truck. Surface depth errors were usually kept below $1 / 2$ inch using this method. Occasional gamma ray depth ties were performed near log peaks to check the depth measurement errors. Most depth mis-ties noted with this system were under 1 foot and usually only $1 / 2$ foot which is very close to the resolution of the logs used.

A new data file, C4996C.DAT was started at 840 feet on Nov. 20th. The tool was then logged to the surface. Next, it was sent back down to redo some sections to check on repeatability. At 350 feet at 20:00 on Nov. 24th there was a leveling motor (rotate) problem. The tool was pulled out of the well. The motor problem was fixed and the tool went back into well at 22:30 on Nov. 24

Calibrations were done again, and a new data file C4996D.DAT was started. The depth intervals 380-350 feet, 1380-1350 feet, 1330-1300 feet,920-840 feet, and 640-590 feet were repeated. Then the tool was pulled out of the well and the truck rigged down at 12:00 on Nov.26th .

\section{C.4 C4997}

The equipment was then moved to well C4997. At 17:00, Nov. 26th, rig-up started and the tool was at a calibration station near the bottom of the casing (320 feet) at 20:10. After finishing calibrations the tool was lowered in the well. It hung up at 902 feet despite multiple tries. The tool was pulled from the well at 03:45 on Nov. 27th, and a wiper trip was made. 
On Nov. 28th the well was re-entered by the BHGM tool at 13:00. After calibrations it was discovered that the gamma tool was not functioning properly. The tool was pulled out of the well and the gamma tool repaired. At 17:30 the BHGM tool was put back into the well. Calibrations were performed and then a gamma tie was made at 19:30 using a peak at 1114 feet . The first station was recorded at 23:30 at 1400 feet, to data file C4997A.DAT. In this well it was decided to interleave the repeat loops - each loop steps up by one depth interval. Early in the run it was noticed that the long level was more sensitive than normal so the level value was checked at every reading. The logging went well until 19:00 on Dec 2nd . when the long level motor failed at 220 feet. The tool was pulled out and the motor fixed. The tool went back into the well around 23:00.

Calibrations were performed again and also a gamma depth tie. The data was recorded to file C4997B.DAT. The logging went well to the surface. The last station was read at 01:00 on Dec. 4th, at the surface. The equipment was rigged down and moved to well C4993.

It was decided that some routine maintenance should be performed on BHGM \#14 along with replacing the long level sensor before starting the next survey. Replacing the long level sensor would eliminate having to check the long level setting every reading as was done in C4997. Dec 4th was spent doing this and checking out the meter afterwards.

\section{C.5 C4993}

The next day, Dec. 5th, the tool was rigged up and went into the well at 09:10. Calibrations were run near the bottom of the casing and the new Long level appeared to be working perfectly. A gamma ray depth tie was performed using peaks at at 410 feet and 1150 feet. The first reading was made at 1390 foot and recorded to file C4993A.DAT. At reading \#32 a new data file was started (C4993B.DAT) and logging proceeded until 1290 feet at 00:30 on Dec. 6th, when the winch drum brake began slipping. The brake cable was replaced and logging re-started at 04:00. Recordings went well until 880 feet at 11:00 on Dec. 7th when the sensor rotate motor failed. The tool was pulled out of the hole and the rotate motor problem was fixed. It was also noted at this time that there was larger than expected leakage between the cable lines and this was repaired also.

The tool was rigged up again at 14:00 and went back into the well. Calibrations were done again partway down the well. A Gamma tie was performed using a peak at 890 feet, then logging started at 900 feet to data file C4993C.DAT. Readings proceeded well and the surface was reached at 04:10 on Dec. 10th . The tool was lowered back down to check measurements at 70 feet - 40 feet and this data was recorded in data file C4993D.DAT. The tool was pulled out of the well at 06:45 on Dec. 10th, 2006.

\section{C.6 De-mobilization}

Ryan Albery left the WTP site at this time. Later in the morning of Dec. 10th, the downhole equipment was checked for contamination by PNNL staff. After testing, the downhole and support equipment was cleaned up and packed for shipment later by PNNL. Andy Black and Ethan Mann left the WTP site after noon with the BHGM sensor and returned to the guest house, packed up the rest of the equipment, and drove to Portland, Oregon. Airline flights were taken on the morning of Dec. 11th back to Colorado. 
The freight was received back at the MGL offices in Lafayette, Colorado on Dec. 18th, 2006.

\section{Appendix: D Data file formats}

\section{D.1 .dat file format}

The .dat file format is the raw file format written to the hard drive in the field by the BHGM acquisition system during the survey. All time references are in days and fractional days from 12:00:00, May 22, 1960 (the base time used for the tide correction calculations).

The file begins with a header containing relevant well information and borehole meter information

1. the version number of the BCON field program used (version 1.39.4 in the Hanford survey).

2. unused value

3. the date and time of file opening

4. the well name

5. the location of the survey (4 lines)

6. the depth units used: 'f' for feet, 'm' for meters

7. the wellhead latitude (decimal degrees, $+\mathrm{N}$ )

8. the wellhead longitude (decimal degrees, $+\mathrm{E}$ )

9. the survey ground elevation

10. flag for well depth reference: 'GL' is ground level

11. the survey depth reference elevation

12. the MGL meter operators (3 lines)

13. the client representative

14. the logging truck company

15. the logging truck operator

16. blank line

17. the BHGM number

18. the BHGM feedback board number 
19. the electrostatic feedback constants ( 2 lines) (see equation 1)

20. a variable number of time-date-stamped text note lines

For each reading, there is a reading header consisting of:

1. the reading number, relative to the file

2. Station depth in depth units as entered by Micro-g Lacoste crew.

3. two lines of shuttle sonde depths, not used for this survey

4. Counter reading: the gravity meter's screw position which controls the spring tension.

5. KFactor corrected gravity: the gravity value calculated from the counter reading and the calibration table for the meter.

6. Unclamp time: the time when the beam is recognized as free from the clamping mechanism by the computer system.

7. Unclamp date in month/day/year.

8. Long Position: the amount of tilt of the meter element relative to the tool housing in the plane of the meter beam.

9. Long Level Vertical: the bias of the long level at true vertical

10. Long level calibration: coefficient for off-level correction of the long level

11. Cross Position: the amount of tilt of the meter element relative to the tool housing across the plane of the beam.

12. Cross Level Vertical: the bias of the cross level at true vertical

13. Cross level calibration: coefficient for off-level correction of the cross level

14. Rotate Position: the relative angular position of the meter element within the tool housing.

15. Meter temperatures:

Electronics Temperature (Elect): the temperature in degrees celsius measured using a temperature sensor located within the tool housing approximately 1 meter above the gravity meter element

Inside Meter Temp: not used

Bottom Meter Temp: the temperature in degrees celsius measured using a temperature sensor located within the meter box.

16. Beam clamp position: the beam position when clamped 
17. Header information for succeeding lines.

Each succeeding line within a record consists of ten columns of data with the following values:

1. TIME: time of reading

2. TCG: Tide corrected gravity in milliGals relative to the meter's base gravity reading

3. CL: The cross level in arbitrary units which is the readout from the capacitance position level suspended from the bottom of the gravity meter element showing a relative angle to vertical across the plane of the beam.

4. CLError: the cross level off-level correction (not used)

5. LL: The long level in arbitrary units which is the readout from the capacitance position level suspended from the bottom of the gravity meter element showing a relative angle to vertical in the plane of the beam.

6. LLError: the long level off-level correction (not used)

7. EPV: The electrostatic positioning voltage which is the amount of voltage applied to the capacitance position indicator plates to center the beam electrostatically

8. Tide: Tide correction in milliGals calculated using Cartwright and Taylor's 1978 algorithm

9. MTemp: The meter's internal temperature (see above)

10. Beam: the meter beam position (should be in the range 82 to 84 for the electrostatic feedback to function)

At the end of each record the average instantaneous tide corrected gravity (TCG) value is displayed as the Final TCG along with the standard deviation (not used). Notes may be recorded before the next record.

A sample of the .dat file follows. Note that record 1 is not valid: it was recorded to test the system. (note; some long lines have been truncated for display, indicated by ...) 


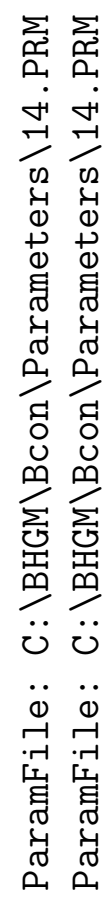

$\stackrel{\sim}{\circ} \stackrel{\sim}{\circ}$

ம்

正

$\ddot{i n}$ is

का

$+$

茎韋

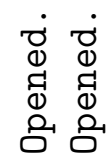

岱

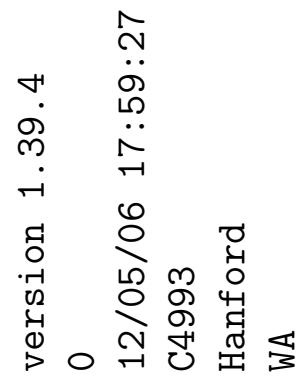

고

$\vec{m} \infty$

ต รั

志

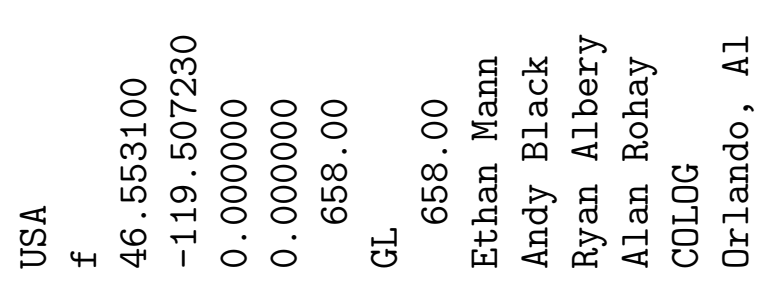

$\circ: \stackrel{\circ}{\circ} \stackrel{1}{\circ}$

ㅇㅇㅇ ஃ

๒

ํํㅇㅇㅇㅇ..

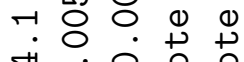

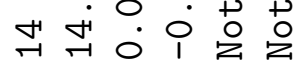




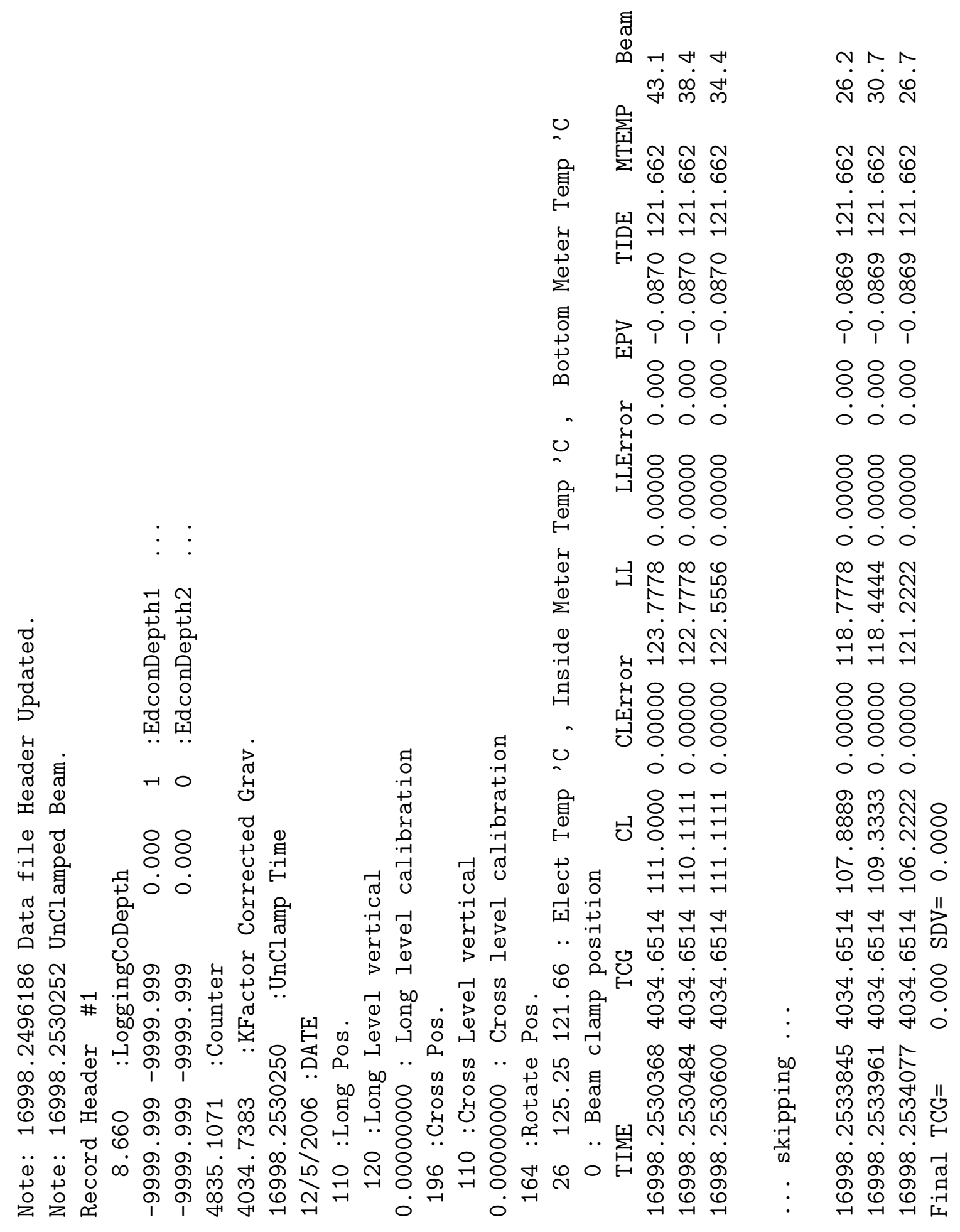




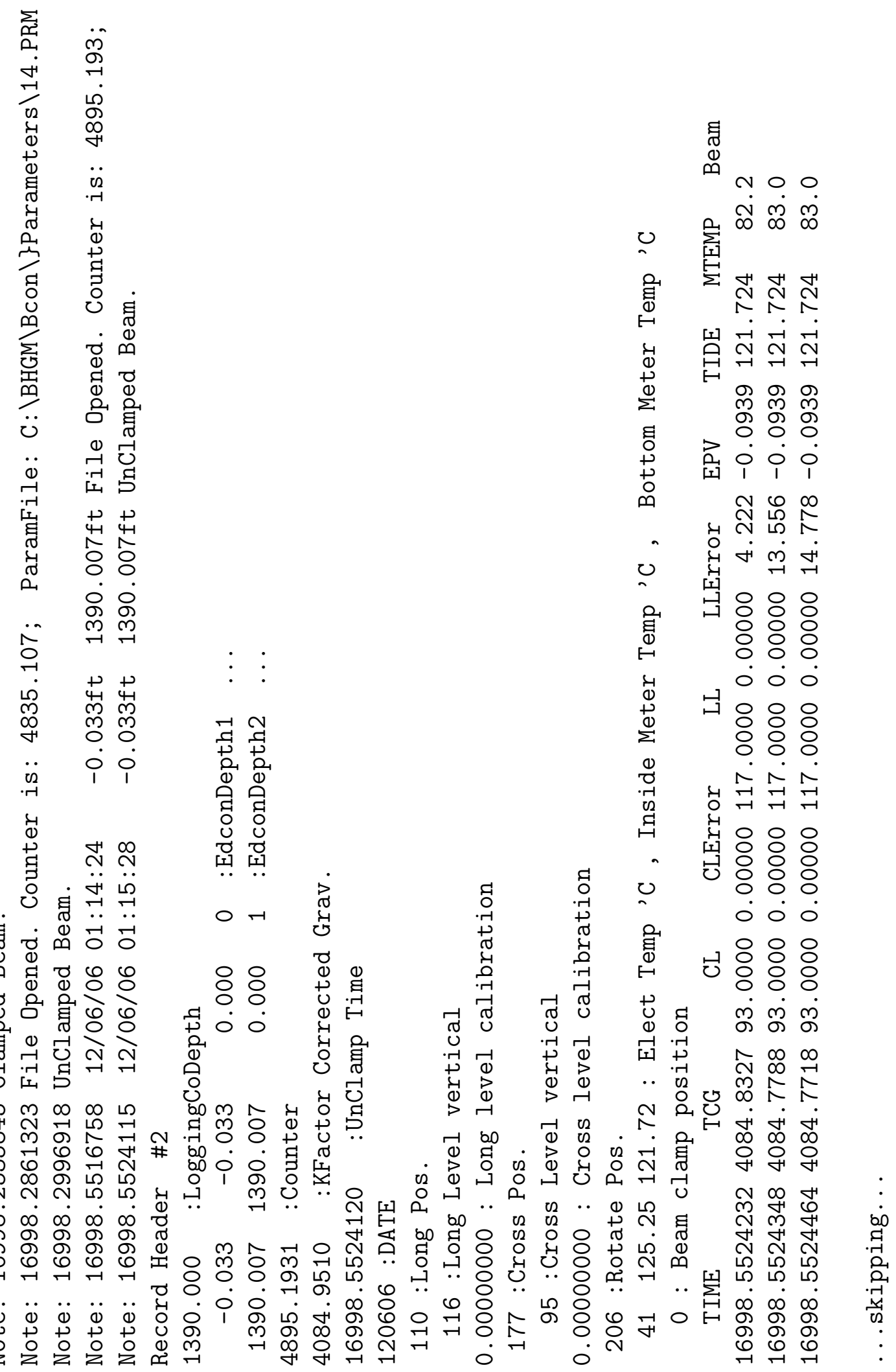




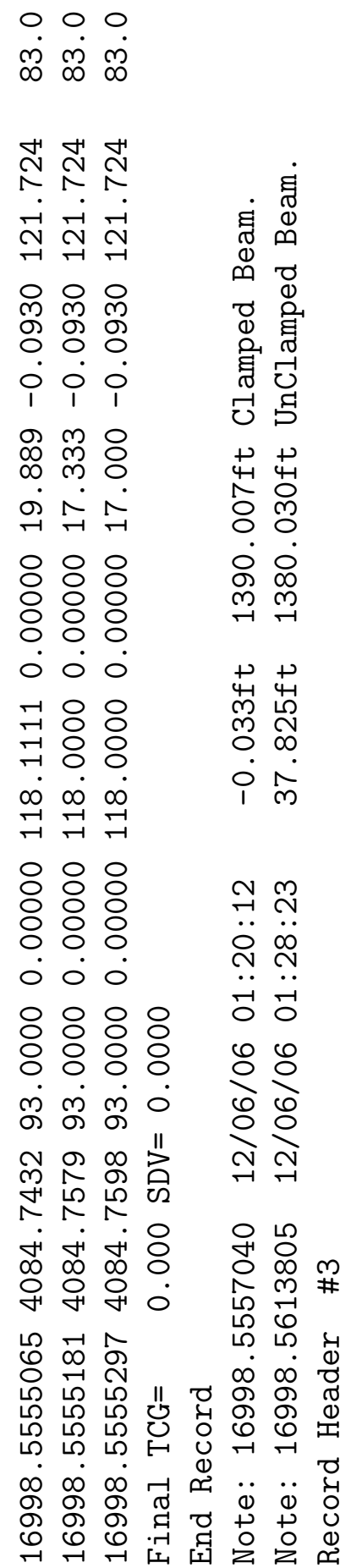




\section{D.2 .rdg file format}

The .dat raw data files are individually run through the data reduction program R8.EXE (see discussion in the Terrain Corrections section). R8.EXE can display the raw data, edit out noise spikes, filter, and pick final gravity value for each individual reading. To minimize drift and hysteresis errors the final gravity value is picked in a window usually centered about 4 minutes after unclamp time. There can be multiple readings in the data file for each reading depth and repeat set; this is usually caused by operator errors or other problems.

This program also outputs a number of files, the most important one is called the "reading" file (*.rdg). This file contains a header (9 lines) with some well and tool information, a two line data header, then data lines with "summaries" of each reading, one line per reading.

The header lines are:

1. the well name

2. unused

3. the wellhead elevation

4. the wellhead latitude (decimal degrees, $+\mathrm{N}$ )

5. the wellhead longitude (decimal degrees, $+\mathrm{E}$ )

6. the depth units used: 'f' for feet, 'm' for meters

7. the electrostatic feedback constants (3 lines) (see equation 1)

8. two lines of column header text

Some reading file numbers are averages from the 1 second data (such as BHGM Temp and Elect temp) and others are just single valued numbers like the Counter or Unclamp time, directly copied from the raw data file. Some of the numbers in the reading file are included for factory diagnostics, such as Rdg Drift, LP,CP, and RP.

1. $\operatorname{Rdg} \#$ : reading number, numbered sequentially from 1 for each data file. (copied)

2. UnClamp Time: time when meter was last unclamped. NOTE: Raw data files may have a one reading delay in this number which was manually corrected in reading files. (copied)

3. Reading Time: center time of the reading data. (averaged)

4. Depth: depth entered manually before each reading. This is the depth used for further processing. (copied)

5. Enc. Offset: Encoder offset, only used with Shuttle surveys. (copied)

6. Gravity Reading: tide corrected gravity reading in mGals. Can be either averaged gravity in a window or hand picked value. (averaged)

7. SDev: standard deviation in mGals of gravity reading. (averaged) 
8. Rdg Drift: drift of the gravity value during the reading (Slope of a least squares fit line through the data) This may be set to zero if the data is hand-picked. (averaged)

9. Drift Corrn: drift Correction. This is a manually or automatically applied drift correction to each reading. (averaged)

10. Elec Temp: electronics temperature as in the .dat file. (averaged)

11. BHGM Temp: BHGM sensor temperature. This should stay constant within $\pm 0.1^{\circ} \mathrm{C}$. The constant can be anywhere from 116 to $131^{\circ} \mathrm{C}$ depending on the "nose" temperature of the meter. (averaged)

12. Counter reading: Counter screw value. This is converted to gravity by the KF table. (copied)

13. EPV: Electrostaic Positioning Voltage (the Feedback voltage in 0-255 A/D units). This is converted to mGals by the A (linear) and $\mathrm{B}$ (squared) calibration terms. (averaged)

14. EDCON Depth1: Depth from one channel of auxillary depth encoder channel. Not used. (copied)

15. Tide: Tide correction, in mGals, from Cartwright and Taylor. This is based on the latitude, longitude and UTC time. (averaged)

16. CL: Cross level value, range $0-255$. This should stay constant to within \pm 3 numbers. The CL value indicates verticality of meter perpendicular to the beam. (averaged)

17. LL: Long level value, range $0-255$. This should stay constant to within \pm 3 numbers. The LL value indicates verticality of meter in the plane of the beam. (averaged)

18. CP: Cross Position. This is a crude measure of mechanical movement in the cross tilt mechanism. (copied)

19. LP: Long Position. This is a crude measure of mechanical movement in the long tilt mechanism. (copied)

20. RP: Rotate Position. This is a crude measure of mechanical rotation in the mechanism that rotates the sensor inside the housing. The rotation aligns the tilt axis of the meter up with the borehole tilt axis. (copied)

21. KFactored Reading: Counter reading converted to gravity (mGals) by the KF table. (copied)

The *.rdg file is used in further processing steps to actually calculate the density and corrections.

A sample of the .rdg file follows. (note; some long lines have been wrapped for display, indicated by ...) 


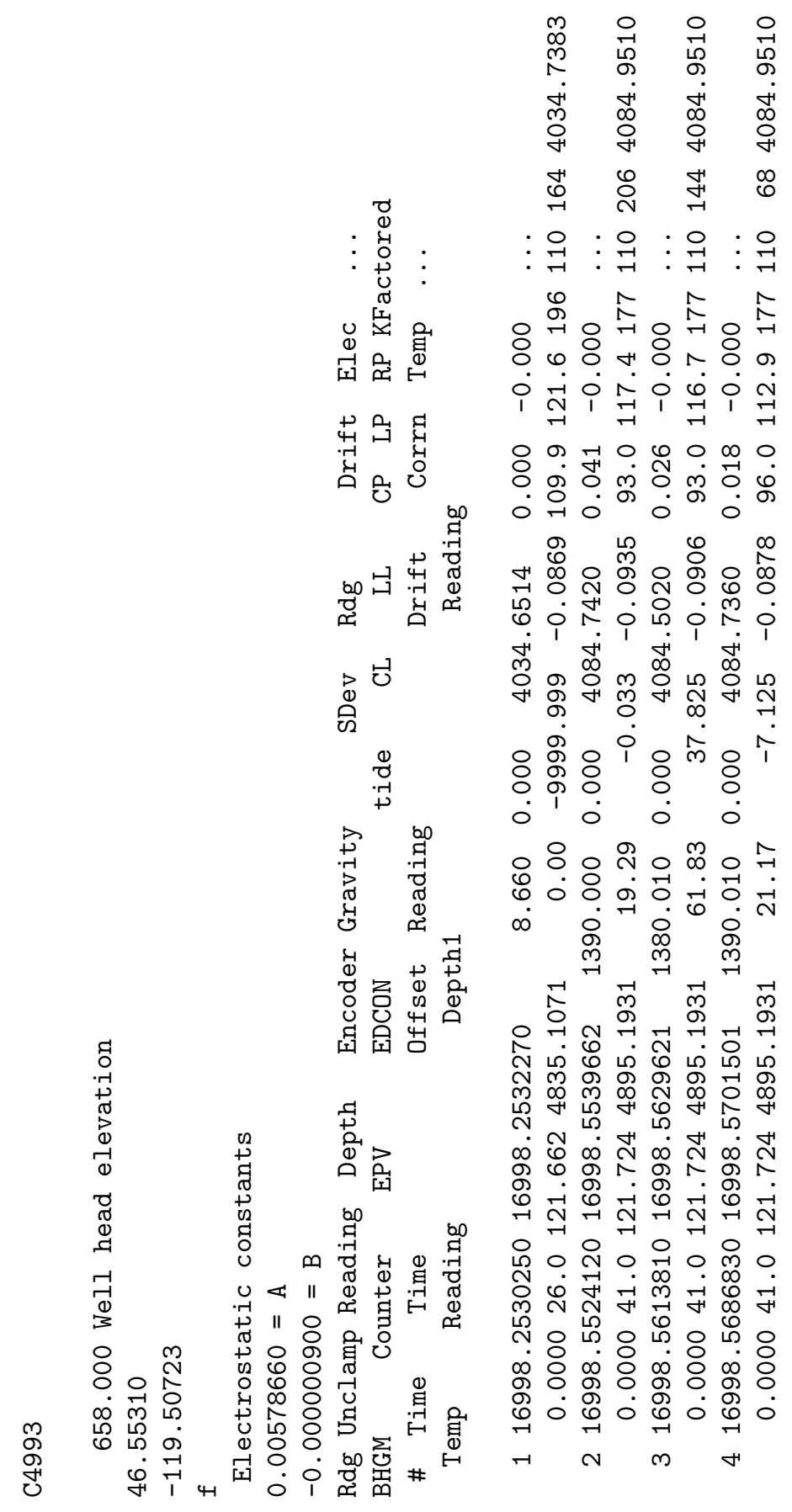




\section{Appendix: E Calibration and test procedures}

Before mobilizing from Lafayette, Colorado to Hanford, Washington, BHGM \#14 went through a standard array of tests and calibrations. The Hanford wells were relatively shallow and cool, which meant that heat testing at high temperatures was not necessary. These wells were vertical wells and not deviated, so sensor tilt would not cause gravity errors. These tests were skipped for this survey. Tests that were performed:

- Physical meter inspection

- Cross and Long level tests

- Clamp/Unclamp tests

- EPV Calibration

- Screw calibration check

The nose test(for meter sensor temperature setting) and magnetization test (for errors due to internal magnetization) were done earlier (8/05). The Pre-Survey Calibration check list is attached.

The in-hole calibrations, done under control of the uphole computer running the BCON program, consisted of:

- Checking Long and Cross level vertical by moving level until gravity is a maximum (verified visually on the uphole computer). Level vertical values in software are reset if necessary.

- Feedback calibration (EPV) - record corrected gravity vs. counter reading for a range of EPV values (30-220). This should be approximately a straight line, except for errors caused by counter non-linearity and drift. This data was analyzed using Microsoft Excel.

- Clamp/Unclamp repeatability. Unclamp meter and record 4 minutes of data, then clamp meter. This is repeated 3-5 times and average gravity values after drift correction are compared using Excel.

These data sets are recorded to data files on the PC and sometimes by hand into the field book. Copies are on the report CD. Long and Cross level settings are changed during surveys as required by testing. No changes were made to the Feedback calibration (EPV) during the survey. There were small errors in each test caused by mechanical counter nonlinearity but overall combination of all tests showed that the calibration used is the correct one. Clamp/Unclamp repeats appeared good (errors under $0.005 \mathrm{mGals}$ after drift correction) during all checks during surveys.

A list of the in-hole calibration files follows: 
C4996-

14L111706.DAT, 14C111706.DAT, 14E111706.DAT, 14A111706.DAT

14L112506.DAT, 14C112506.DAT, 14E112506.DAT, 14A112506.DAT

C4997-

14L112706.DAT, 14C112706.DAT, 14E112706.DAT, 14A112706.DAT

14L112906.DAT, 14C112906.DAT, 14E112906.DAT, 14A112906.DAT

14L120306.DAT, 14C120306.DAT, 14E120306.DAT, 14A120306.DAT

In addition to these files many other level check files were recorded in all three wells at various times whenever the engineer felt it would be prudent. Other calibration file sets were run with no gravity data was recorded afterwards, as when there were tool, truck, or well problems.

Copies of Non-Conformance reports are attached as Appendix $\mathrm{G}$. 


\section{Appendix: F Gravity acquisition procedure}

At each station where a gravity reading was made the meter was first leveled, unclamped, and then the gravity recording was made. The results were written to the computer disc and stored as a raw data file. A copy of the raw data file (XXXXX.DAT) is provided on the CD (back pocket). Several minutes of data are recorded at each station until the operator is satisfied that the meter spring had stabilized and that an accurate reading could be determined.

A combination of the counter screw position and the electrostatic positioning voltage (EPV) or feedback voltage, are used to make a gravity reading. Both the counter screw and the EPV values must be converted using calibration factors to derive a gravity reading.

The calibration table for the counter screw lists counter values, $C(i)$ ], at intervals of 100 numbers and the corresponding gravity values (Table 10 ). To obtain the equivalent gravity value, $G_{C}$, for a counter value $C$, the following formula is used to linearly interpolate over the 100 number counter value range:

$$
G_{c}=G(i)+\frac{(C-C(i))(G(i+1)-G(i))}{100}
$$

where $C(i)$ and $G(i)$ are the equivalent counter and gravity values in 100 counter increments and $C(i)<C<C(i+1)$.

The instantaneous electrostatic positioning voltage or feedback voltage, $V$, is equivalent to a gravitational acceleration, $G_{v}$ given by the formula:

$$
G_{v}=-(A \times V)-\left(B \times V^{2}\right)
$$

where $A$ and $B$ are constants for each meter and feedback board.

In order to filter external accelerations from the readings, the electrostatic positioning voltage is sampled approximately eight times per second over a sample interval of 1 second. Each electrostatic positioning voltage is converted to an equivalent acceleration and added to the gravity value calculated from the counter screw.

The tidal gravity correction value $G_{t}$ is calculated using Cartwright and Taylor's algorithm. The final tide-corrected gravity value $G_{T C}$ is calculated by

$$
G_{T C}=G_{c}+G_{v}+G_{t}
$$

The set of instantaneous gravity values were averaged over the sample interval (1 second) and written to the raw data file along with the information shown in Appendix D.1

Table 10: BHGM \#14 Screw K-factors

\begin{tabular}{|r|r|r|r|}
\hline Counter units & mGals & Counter units & mGals \\
\hline \hline 0 & 0.000 & 4100 & 3420.450 \\
\hline 100 & 83.468 & 4200 & 3503.999 \\
\hline 200 & 166.919 & 4300 & 3587.557 \\
\hline 300 & 250.356 & 4400 & 3671.122 \\
\hline 400 & 333.780 & 4500 & 3754.691 \\
\hline 500 & 417.193 & 4600 & 3838.261 \\
\hline
\end{tabular}


Table 10: BHGM \#14 Screw K-factors continued

\begin{tabular}{|c|c|c|c|}
\hline Counter units & mGals & Counter units & mGals \\
\hline 600 & 500.596 & 4700 & 3921.831 \\
\hline 700 & 583.991 & 4800 & 4005.400 \\
\hline 800 & 667.378 & 4900 & 4088.968 \\
\hline 900 & 750.758 & 5000 & 4172.535 \\
\hline 1000 & 834.133 & 5100 & 4256.101 \\
\hline 1100 & 917.503 & 5200 & 4339.666 \\
\hline 1200 & 1000.871 & 5300 & 4423.230 \\
\hline 1300 & 1084.237 & 5400 & 4506.792 \\
\hline 1400 & 1167.603 & 5500 & 4590.353 \\
\hline 1500 & 1250.966 & 5600 & 4673.912 \\
\hline 1600 & 1334.327 & 5700 & 4757.469 \\
\hline 1700 & 1417.690 & 5800 & 4841.022 \\
\hline 1800 & 1501.059 & 5900 & 4924.570 \\
\hline 1900 & 1584.433 & 6000 & 5008.112 \\
\hline 2000 & 1667.812 & 6100 & 5091.645 \\
\hline 2100 & 1751.196 & 6200 & 5175.169 \\
\hline 2200 & 1834.583 & 6300 & 5258.683 \\
\hline 2300 & 1917.972 & 6400 & 5342.186 \\
\hline 2400 & 2001.362 & 6500 & 5425.678 \\
\hline 2500 & 2084.755 & 6600 & 5509.155 \\
\hline 2600 & 2168.153 & 6700 & 5592.614 \\
\hline 2700 & 2251.560 & 6800 & 5676.053 \\
\hline 2800 & 2334.977 & 6900 & 5759.471 \\
\hline 2900 & 2418.405 & 7000 & 5842.864 \\
\hline 3000 & 2501.845 & 7100 & 5926.229 \\
\hline 3100 & 2585.299 & 7200 & 6009.564 \\
\hline 3200 & 2668.769 & 7300 & 6092.865 \\
\hline 3300 & 2752.257 & 7400 & 6176.132 \\
\hline 3400 & 2835.758 & 7500 & 6259.362 \\
\hline 3500 & 2919.269 & 7600 & 6342.555 \\
\hline 3600 & 3002.787 & 7700 & 6425.711 \\
\hline 3700 & 3086.311 & 7800 & 6508.828 \\
\hline 3800 & 3169.839 & 7900 & 6591.908 \\
\hline 3900 & 3253.371 & 8000 & 6674.948 \\
\hline 4000 & 3336.908 & & \\
\hline
\end{tabular}


Appendix: G Non-Conformance reports (scanned) 
5.1.4. MICROG LACOSTE NON-CONFORMANCE REPORT

Page 1 of 2

Job Number: Kantar Date: $1111 \| 06$

Equipment Category: End Depth Syst Meter \#: 14

Description of Non Conformance: Include Extent of Problem and Impact on other systems or processes.

Depth System wont initialize properly.

Disposition Evaluation: How can this problem be dealt with? Where should this part or process be reassigned? clack hand ware of software

Action to be taken: Investigate 1 Rework Repair Scrap

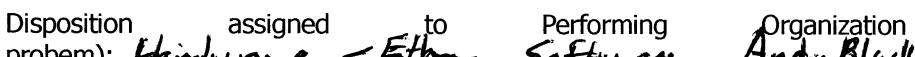

(who

should

fix

Disposition assigned to:

Date for completion:

ASAP

Signature:

Date:

Page 57

158 
MICRO LACOSTE NON-CONFORMANCE REPORT

Page 2 of 2

Investigation Details: Describe the details of the investigation into the source of the problem. Write N/A if an investigation is not applicable.

Gavelwine decked on other geftwane - works!

So problion is Software Re lated with BCON.EXE. vet 1.39 .4

Action to be taken: Investigate more $\quad$ Rework Repair Use-as-is Scrap Signature:

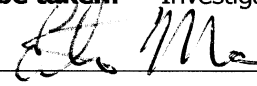
Date:

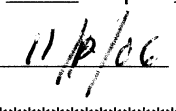

Action Details: Describe the details of how the problem was reworked or repaired and/or why the decision to use-as-is or scrap was chosen.

Supplier of Beard +diver talked to - willinuzitigate.

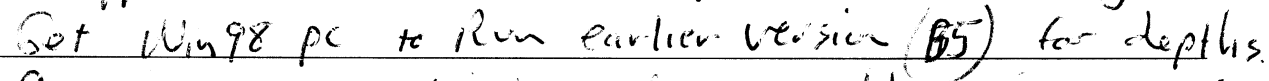

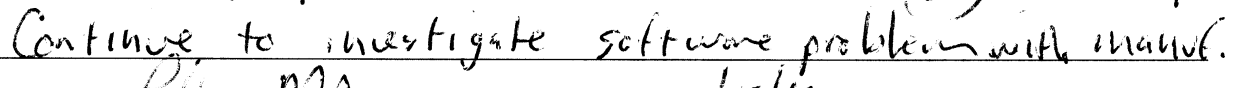
Signature: Date: $1 / 1 / 15 / 6$

VERIFICATION OF ACTION TO NONCONFORMANCE:

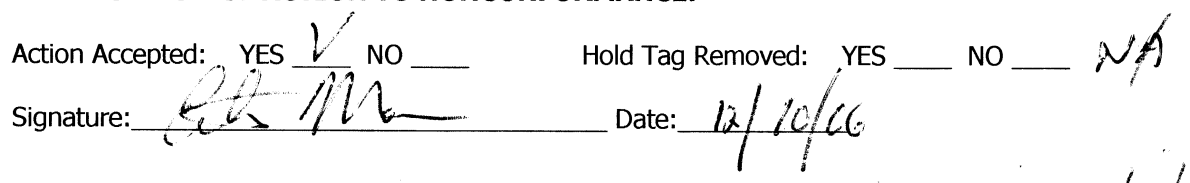

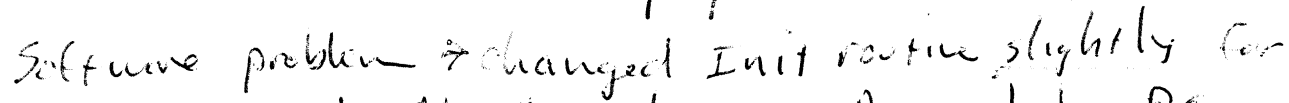
Depth bound. No other changes. Recompiled. Bacon works now.

Page 58

159 


\subsubsection{MICROG LACOSTE NON-CONFORMANCE REPORT}

Page 1 of 2

Job Number: Hau ford $\quad$ Date: $11 / 15 / 06$

Originator: Ethan Mann

Equipment Category: ShirHle Wiuch Meter \#: 14

Description of Non Conformance: Include Extent of Problem and Impact on other systems or processes.

Shuttle winch wen't move up, moves down fin.
multiple spave board failutes $11 / 5,11 / 16$.
Hardwave problem

Disposition Evaluation: How can this problem be dealt with? Where should this part or process be reassigned? Troubleshoot winch contraller boards.

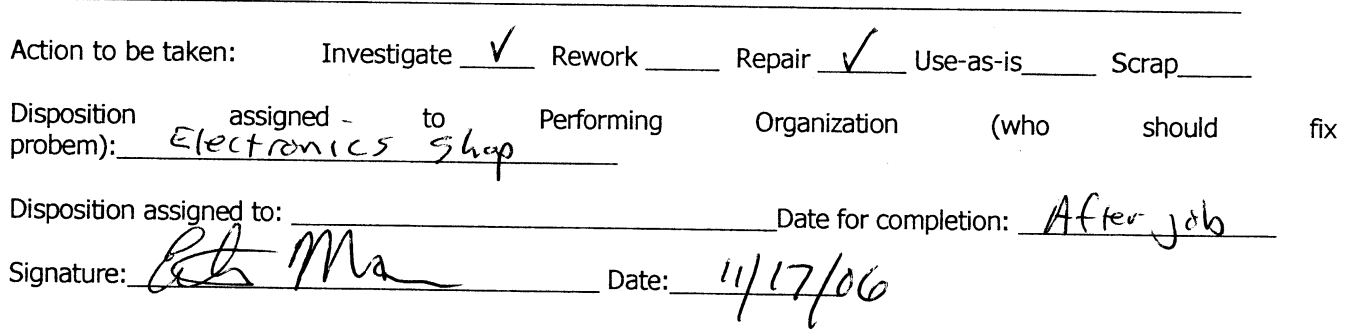


MICROG LACOSTE NON-CONFORMANCE REPORT

Page 2 of 2

Investigation Details: Describe the details of the investigation into the source of the problem. Write N/A if an investigation is not applicable.

Not performed yet. Troubleshort loack at shop.
Now Use tool without shuttle winch.

Action to be taken: Investigate more $\checkmark$ Rework

Signature:

Repair Date: $11 / 17 / 06$

Use-as-is Scrap

Action Details: Describe the details of how the problem was reworked or repaired and/or why the decision to use-as-is or scrap was chosen.

Signature:

Date:

\section{VERIFICATION OF ACTION TO NONCONFORMANCE}

Action Accepted: YES NO

Hold Tag Removed: YES NO

Signature:

Date:

Page 58 


\subsubsection{MICROG LACOSTE NON-CONFORMANCE REPORT}

Page 1 of 2

Job Number: Har fired Date: $2 \mathrm{NOC} / \mathrm{CC}$

Originator: Ethan 1 hanv

Equipment Category: Gamun Toul Meter \#: 14

Description of Non Conformance: Include Extent of Problem and Impact on other systems or processes.

No jawima countrates Gant de ganouna
Upplie.

Disposition Evaluation: How can this problem be dealt with? Where should this part or process be reassigned? Pulledot, Nepain electronics

Action to be taken: Investigate __ Rework __ Repair _

Disposition assigned to Performing Organization (who should fix
probem): Fiela Ciev

Disposition assigned to: Ethin Date for completion: ASAP

Signature: $M$ Date: $11 / 28 / 06$ 
MICROG LACOSTE NON-CONFORMANCE REPORT

Page 2 of 2

Investigation Details: Describe the details of the investigation into the source of the problem. Write N/A if an investigation is not applicable.

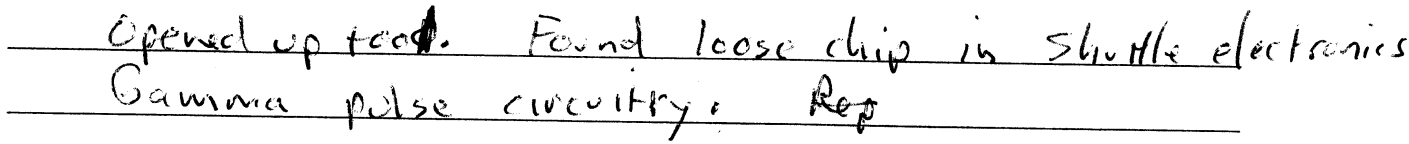

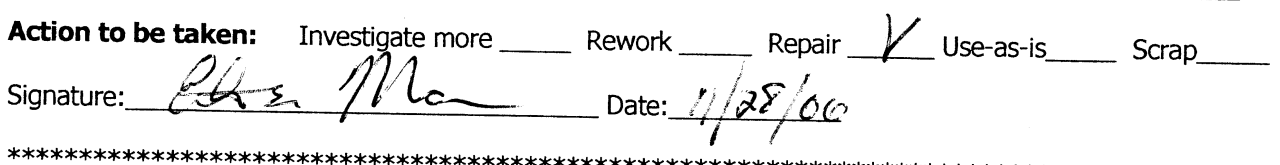

* Action Details: Describe the details of how the problem was reworked or repaired and/or why the decision to
use-as-is or scrap was chosen.

Replaced clip in Gamina circuit o chacked our wirks OK.

Signature: $\mathrm{COS}_{\mathrm{C}} \mathrm{M}$ Date: $11 / 28 / 0 \mathrm{C}$

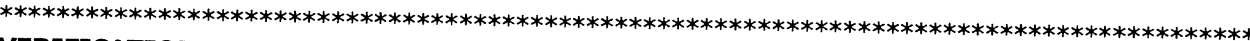

VERIFICATION OF ACTION TO NONCONFORMANCE:

Action Accepted: YES 1 NO

Signature: $\quad 2$

Hold Tag Removed: YES $\checkmark$ NO Date:

Page 58 


\subsubsection{MICROG LACOSTE NON-CONFORMANCE REPORT}

Page 1 of 2

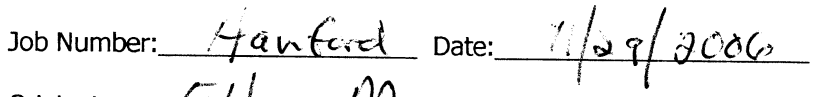

Originator: Ethen Mawn

Equipment Category: Bltom 414 Seviser Meter \#: 14

Description of Non Conformance: Include Extent of Problem and Impact on other systems or processes.

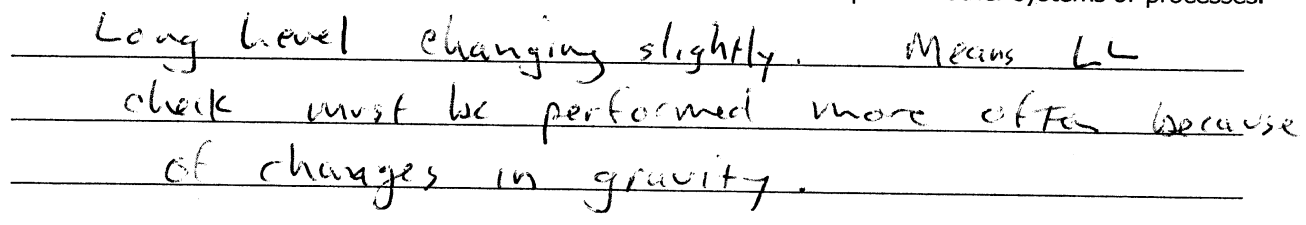

Disposition Evaluation: How can this problem be dealt with? Where should this part or process be reassigned? Do LC checks uore ofteno

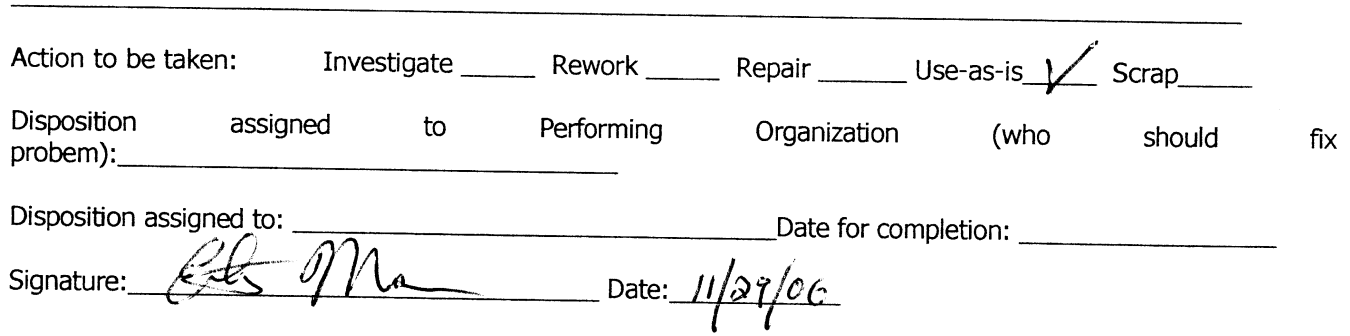


MICROG LACOSTE NON-CONFORMANCE REPORT

Page 2 of 2

Investigation Details: Describe the details of the investigation into the source of the problem. Write N/A if an investigation is not applicable.

$$
N / A \text {. }
$$

Action to be taken: Investigate more Rework Repair Use-as-is Scrap

Signature: Date:

$* * * * * * * * * * * * * * * * * * * * * * * * * * * * * * * * * * * * * * * * * * * * * * * * * * * * * * * * * * * * * * * * * * * * * * * * * * * * * * * * * * * * * * * * * * * * * *$

Action Details: Describe the details of how the problem was reworked or repaired and/or why the decision to use-as-is or scrap was chosen.

iso is is just chack nive ofting Repar is possible bit time consuming and necessitate, pollin olt of lule.

Signature:

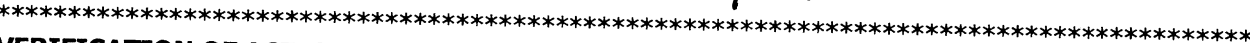
VERIFICATION OF ACTION TO NONCONFORMANCE:

Action Accepted: YES ! NO

Signature:

Hold Tag Removed: YES Date: $11 / 20 \% / 06$ NO<smiles>C1CCC2(CC1)CC2</smiles>

Page 58 


\subsubsection{MICROG LACOSTE NON-CONFORMANCE REPORT}

Page 1 of 2

Job Number: Hanfard Date: $12 / 2 / 2006$

Equipment Category: BHGM Sensor Meter \#: 14

Description of Non Conformance: Include Extent of Problem and Impact on other systems or processes.

Long Level motor faled to move.
caint Level meter.

Disposition Evaluation: How can this problem be dealt with? Where should this part or process be reassigned? Repair motor or find electrical problen and repair.

Action to be taken: Investigate $\quad$ Rework __ Repair $\quad$ Use-as-is__ Scrap_

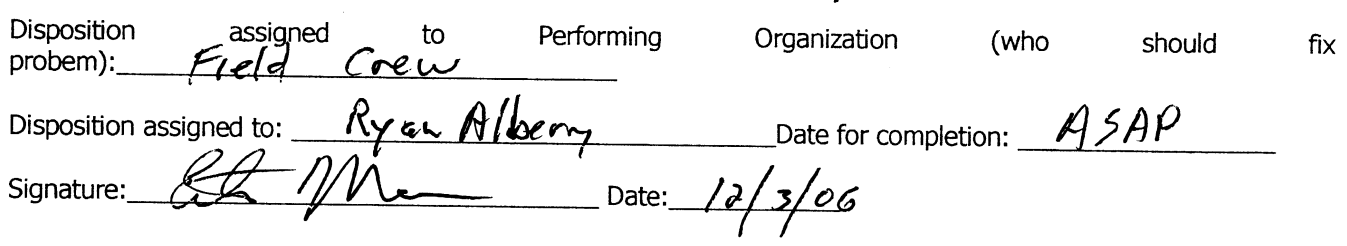


MICRO LACOSTE NON-CONFORMANCE REPORT

Page 2 of 2

Investigation Details: Describe the details of the investigation into the source of the problem. Write N/A if an investigation is not applicable.

Ryan Found bad solder joint on motor wire.

Action to be taken: Investigate more Rework Repair Scrap

Signature: Date:

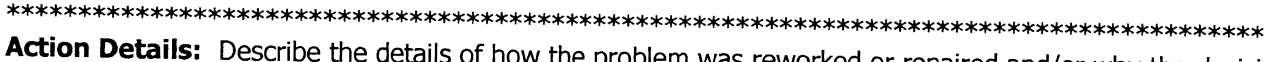

Action Details: Describe the details of how the problem was reworked or repaired and/or why the decision to use-as-is or scrap was chosen.

Clipped wire, resoldered. checked ok.

Re-assembled.

Signature: Date: VERIFICATION OF ACTION TO NONCONFORMANCE:

Action Accepted:

Hold Tag Removed: YES $1 /$ NO

Date: $12 / 3 / 06$

Page 58

167 
Page 1 of 2

Job Number: Hanfird Date: 4 Dee, 2006

originator: Ethan Mann

Equipment Category: BHGM Seusor Meter \#: 14

Description of Non Conformance: Include Extent of Problem and Impact on other systems or processes.

loug hevel. Sensitivity too low. Also center
was moving.

Signature: OL Date: $12 \%$ Da

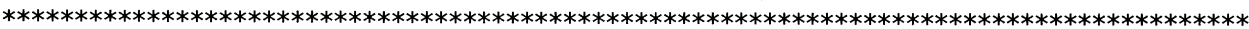
Disposition Evaluation: How can this problem be dealt with? Where should this part or process be reassigned?

Replace level.

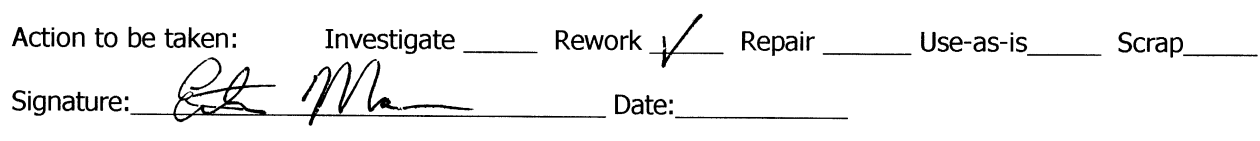

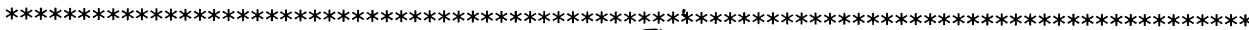
Disposition assigned to Performing Organization:_ Field Crew

Disposition assigned to: Ethan Mann Date for completion: ASAP

Signature: 6 Date: $12 / 4 / 06$ 
Page 2 of 2

MICRO-G LACOSTE NONCONFORMANCE REPORT

\section{Investigation Details:}

Describe the details of the investigation into the source of the problem. Write N/A if an investigation is not applicable.

$$
N / A
$$

Action to be taken: Investigate more

Rework

Repair

Use-as-is

Scrap

Signature:

Date:

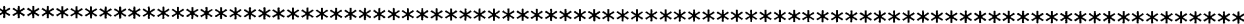

Action Details: Describe the details of how the problem was reworked or repaired and/or why the decision to use-as-is or scrap was chosen.

Level Replaced with spare. Electronics checked.

Long center position (vertical), re-calibrated.

Signature:

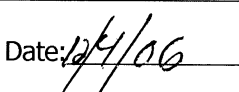

$* * * * * * * * * * * * * * * * * * * * * * * * * * * * * * * * * * * * * * * * * * * * * * * * * * * * * * * * * * * * * * * * * * * * * * * * * * * * * * * * * * * * * * * * * * * * * *$ VERIFICATION OF ACTION TO NONCONFORMANCE:

Action Accepted: YES $\checkmark$ NO

Hold Tag Removed: YES $/$ NO

Signature:

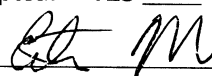
Date: 
Job Number: Hanferd Date: 70 Oe, 2006

Originator: Efhan Nanu

Equipment Category: BHGM sensor

Meter \#: 4

Description of Non Conformance: Include Extent of Problem and Impact on other systems or processes.

Rotate Motor stopped.

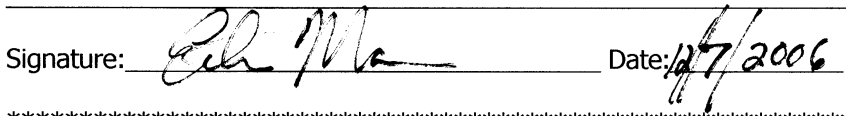

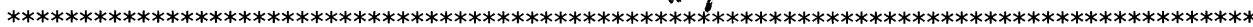
Disposition Evaluation: How can this problem be dealt with? Where should this part or process be reassigned?

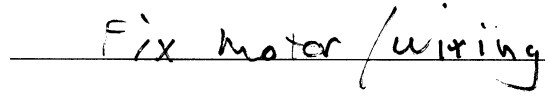

Action to be taken: Investigate $/$ Rework__ Repair Disposition assigned to Performing Organization: Fe'd Crew

Disposition assigned to: Et han Nann Date for completion: A\&AP Signature: 
Page 2 of 2

\section{Investigation Details:}

\section{MICRO-G LACOSTE NONCONFORMANCE REPORT}

Describe the details of the investigation into the source of the problem. Write N/A if an investigation is not applicable.

Wiving ok, chak moter OK. Aecide porblen might be divty moter broshas- cloak up with solvent then lubricate lightly with silicon

Action to be taken: Investigate more Rework - Repair V Use-as-is Scrap

Signature:

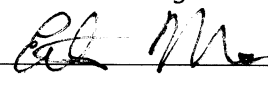
Date: $12 / 7 / 06$

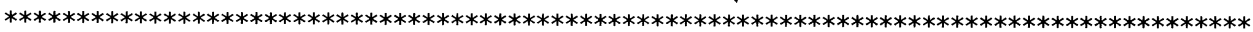
Action Details: Describe the details of how the problem was reworked or repaired and/or why the decision to use-as-is or scrap was chosen.

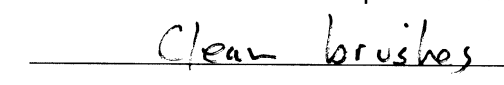

-

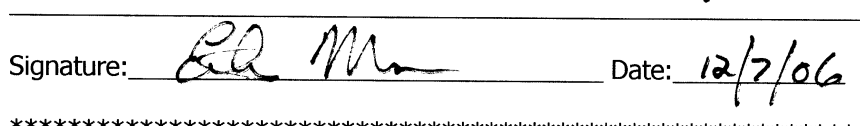

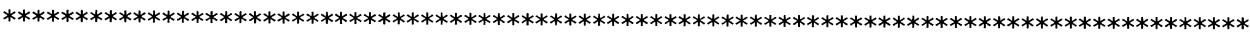
VERIFICATION OF ACTION TO NONCONFORMANCE:

Action Accepted: YES Hold Tag Removed: YES 
